GÖTTINGER SCHRIFTEN ZUR INTERNETFORSCHUNG

Hg.: S. Hagenhoff, D. Hogrefe, E. Mittler, M. Schumann, G. Spindler, V. Wittke

\title{
Rechtliche Rahmenbedingungen von Serviceorientierten Architekturen mit Web Services
}

Judith Nink 

Judith Nink

Rechtliche Rahmenbedingungen von Serviceorientierten Architekturen mit Web Services

This work is licensed under the Creative Commons License 2.0 "by-nd", allowing you to download, distribute and print the document in a few copies for private or educational use, given that the document stays unchanged and the creator is mentioned. You are not allowed to sell copies of the free version.

SORIERIGHISRESERVED 
erschienen als Band 6 in der Reihe „Göttinger Schriften zur Internetforschung“ im Universitätsverlag Göttingen 2010 
Judith Nink

Rechtliche

Rahmenbedingungen von

Serviceorientierten

Architekturen mit

Web Services

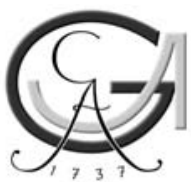

Universitätsverlag Göttingen

2010 


\title{
Bibliographische Information der Deutschen Nationalbibliothek
}

Die Deutsche Nationalbibliothek verzeichnet diese Publikation in der Deutschen Nationalbibliographie; detaillierte bibliographische Daten sind im Internet über $<$ http://dnb.ddb.de $>$ abrufbar.

\section{Reihe}

Band 6 der Reihe „Göttinger Schriften zur Internetforschung“ in der qualitätsgeprüften Sparte des Universitätsverlags Göttingen.

Herausgeber der Reihe: Svenja Hagenhoff, Dieter Hogrefe, Elmar Mittler, Matthias Schumann, Gerald Spindler und Volker Wittke.

\author{
Anschrift des Autors \\ Judith Nink \\ e-mail: judith.nink@web.de
}

Dieses Buch ist auch als freie Onlineversion über die Homepage des Verlags sowie über den OPAC der Niedersächsischen Staats- und Universitätsbibliothek

(http://www.sub.uni-goettingen.de) erreichbar und darf gelesen, heruntergeladen sowie als Privatkopie ausgedruckt werden. Es gelten die Lizenzbestimmungen der

Onlineversion. Es ist nicht gestattet, Kopien oder gedruckte Fassungen der freien

Onlineversion zu veräußern.

Satz und Layout: Judith Nink

(C) 2010 Universitätsverlag Göttingen

http:/ / univerlag.uni-goettingen.de

ISBN: 978-3-941875-29-6

ISSN: 1863-0944 


\section{Vorwort}

Die vorliegende Arbeit ist während meiner Tätigkeit als wissenschaftliche Mitarbeiterin am Lehrstuhl von Prof. Dr. Gerald Spindler entstanden. Sie wurde im Juli 2008 als Dissertationsschrift bei der Juristischen Fakultät der Georg-August-Universität Göttingen eingereicht. Rechtsprechung und Literatur konnten bis Oktober 2009 berücksichtigt werden.

Zum Gelingen dieser Arbeit haben viele Personen beigetragen, die hier leider nicht alle namentlich erwähnt werden können, für deren Unterstützung ich mich aber bedanken möchte. Mein besonderer Dank gilt meinem Doktorvater Prof. Dr. Gerald Spindler, der den ersten gedanklichen Anstoß zur Bearbeitung dieses Themas gab und mich stets hilfreich bei der Erstellung der Arbeit begleitete. Sowohl in menschlicher als auch fachlicher Hinsicht war er ein Doktorvater im besten Sinne.

Ebenso gebührt Herrn Prof. Dr. Andreas Wiebe herzlicher Dank für die Erstellung des Zweitgutachtens in nur einer Woche.

Meinem Bruder Christian, meinem Kollegen Herrn Jörn Heckmann und Frau Stefanie Sieger danke ich für das hilfreiche und konstruktive Korrekturlesen und Frau Ingrid Rosine Floerke für die vielen aufbauenden Gespräche und stetigen Ermunterungen. Besonderer Dank gebührt auch Herrn Jens Nödler für die fachliche Begleitung der Arbeit in technischer Sicht vom ersten Tag an, die jederzeitige Hilfe bei technischen Fragestellungen sowie die Verfeinerung des Themas.

Hervorheben und von ganzem Herzen danken möchte ich weiter meinem Lebensgefährten Herrn Philip Laue, dessen zahlreiche Anmerkungen, Anregungen, Ideen, Aufmunterungen und Unterstützung in erheblicher Weise zum Gelingen dieser Arbeit beigetragen haben.

Schließlich möchte ich mich bei meinem Vater bedanken, der mir immer die notwendigen Freiräume ließ und mich stets in allem unterstützt hat. Ihm widme ich die vorliegende Arbeit.

Köln, im Dezember 2009.

Judith Nink 


\section{Inhaltsüberblick}

$\begin{array}{lll}\text { Vorwort } & \text { V }\end{array}$

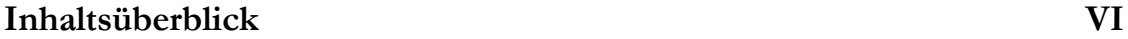

Inhaltsverzeichnis

Abkürzungsverzeichnis $\quad$ XIV

$\begin{array}{ll}\text { Literaturverzeichnis } & \text { XXII }\end{array}$

1. Teil Einleitung und Problemstellung 1

A. EINFÜHRUNG 1

B. GRUNDLAGEN

I. Begrifflichkeiten 5

II. Serviceorientierte Architekturen mit Web Services 10

III. Einsatzbereiche von SOA und Web Services 14

2. Teil Zivilrechtliche Aspekte 17

$\begin{array}{lr}\text { A. Allgemeine Vertragsrechtliche Probleme } & 18\end{array}$

I. Anwendbares Recht im E-Commerce 18

II. Einordnung der Dienste 23

III. Vertragsrechtliche Grundlagen 29

B. Außervertragliche Haftung 121

I. Anwendbares Recht bei außervertraglichen Beziehungen 124

II. Mangel des Web Service 127

III. Missbrauch/Fehlfunktion des UDDI 129

IV. Öffentliche All-Inclusive-Angebote 143

V. Haftung des Web Service-Nutzers 148

VI. Resümee 162

3. Teil Datenschutz bei „Web Service-Portalen“ 164

A. EINFÜHRUNG 164

$\begin{array}{ll}\text { B. RECHTSRAHMEN FÜR DEN DATENSCHUTZ } & 167\end{array}$

I. Internationaler Rahmen 167

II. Recht der Europäischen Union 170 
III. Nationaler Rechtsrahmen 175

IV. Datenschutzrechtliche Anforderungen des TMG und BDSG 205

V. Folgen der Missachtung der Datenschutzvorgaben 260

VI. Rechte der betroffenen Portalnutzer 262

VII. Schutzmöglichkeiten des Betroffenen 270

VIII. Sonstige datenschutzrechtliche Anforderungen 271

C. Resümee: Teil $3 \quad 272$

4. Teil Schlussbetrachtungen 273

$\begin{array}{ll}\text { 5. Teil Anhang } & 277\end{array}$

D. Muster-DAtensChUtZerkLÄrung 277 


\section{Inhaltsverzeichnis}

$\begin{array}{lll}\text { Vorwort } & \text { V }\end{array}$

$\begin{array}{ll}\text { Inhaltsüberblick } & \text { VI }\end{array}$

$\begin{array}{lll}\text { Inhaltsverzeichnis } & \text { VIII }\end{array}$

$\begin{array}{ll}\text { Abkürzungsverzeichnis } & \text { XIV }\end{array}$

$\begin{array}{lll}\text { Literaturverzeichnis } & \text { XXII }\end{array}$

1. Teil Einleitung und Problemstellung $\quad 1$

A. EINFÜHRUNG 1

B. Grundlagen 5

I. Begrifflichkeiten 5

1. Web Services 5

2. Akteure 7

II. Serviceorientierte Arcbitekturen mit Web Services 10

1. Überblick 10

2. Komponenten 11

III. Einsatzbereiche von SOA und Web Services 14

2. Teil Zivilrechtliche Aspekte 17

A. Allgemeine vertragsrechtliche Probleme 18

I. Anwendbares Recht im E-Commerce 18

$\begin{array}{ll}\text { 1. Vereinbarung der Rechtsordnung } & 18\end{array}$

2. Fehlende Rechtswahl 20

3. Zusammenfassender Überblick 23

II. Einordnung der Dienste 23

1. Web Service-Anbieter 27

2. Web Service-Nutzer 28

3. Web Service-Vermittler 29

III. Vertragsrecbtliche Grundlagen 29

1. Vertragstypologische Einordnung 30

a) Web Service-Hersteller - Web Service-Anbieter 30

aa) Kaufvertrag 30

bb) Werkvertrag 32

cc) Miet- und Leasingvertrag 39 
dd) Fazit 41

b) Web Service-Anbieter - Web Service-Vermittler 42

aa) Verhältnis Web Service-Anbieter - Web Service-Vermittler 42

bb) Rolle des Web Service-Vermittlers 43

c) Web Service-Anbieter - Web Service-Nutzer 45

aa) Vergütung der Web Service-Nutzung 46

bb) Unentgeltliche Web Service-Nutzung 55

d) Web Service-Nutzer - Web Service-Vermittler 56

e) Web Service-Nutzer - Dritte 57

2. Zustandekommen des Vertrags 58

a) Vertragsschluss $\quad 59$

aa) Web Service-Anbieter - Web Service-Nutzer 59

bb) Web Service-Nutzer - Dritter 63

cc) Web Service-Anbieter - Dritter 76

b) Sicherheit $\quad 77$

aa) Bedrohungen und Schutzziele $\quad 77$

bb) Mittel zur Gewährung der Schutzziele 79

cc) Resümee 86

3. Vertragliche Pflichten 86

a) Web Service-Anbieter - Web Service-Nutzer 87

b) Web Service-Anbieter - Web Service-Vermittler 88

c) Web Service-Nutzer - Dienstemakler 88

4. Einbeziehung Allgemeiner Geschäftsbedingungen 88

a) Wirksame Einbeziehung von AGB 89

b) AGB des Web Service-Anbieters 90

c) AGB des Web Service-Nutzers 92

5. Irrtumslehre 93

a) Anfechtung 93

aa) Eingabefehler 94

bb) Fehlerhaftes Datenmaterial 98

cc) Übermittlungsfehler und Systemfehler 99

6. Pflichten im E-Commerce 104

a) Verbraucherinformationspflichten 105 
b) Informationspflichten der Telemediendiensteanbieter 106

$\begin{array}{ll}\text { 7. Leistungsstörungen } & 109\end{array}$

a) Mangel des Web Service 110

i) Web Service-Anbieter gegenüber Web Service-Hersteller 113

ii) Web Service-Nutzer gegenüber Web Service-Anbieter 115

b) Fehlerhafte Dienstesuche 116

aa) Web Service-Vermittler - Web Service-Anbieter 116

bb) Web Service-Vermittler - Web Service-Nutzer 119

c) Resümee 120

8. Abrechnungssysteme 121

B. Aubervertragliche Haftung 121

I. Anwendbares Recht bei außervertraglichen Beriebungen 124

1. Deliktsrecht 124

2. Markenrecht 126

3. Wettbewerbsrecht 126

4. Zusammenfassung 127

II. $\quad$ Mangel des Web Service 127

1. Verschuldensabhängige Produzentenhaftung 128

2. Produkthaftung 129

III. Missbrauch/Feblfunktion des UDDI 129

1. Fehlerhafte Dienstesuche 130

2. Unternehmensnamen und fremde Marken 131

a) Marken- und Firmennamensverletzungen 132

b) Unlauterer Wettbewerb 134

aa) Irreführung 135

bb) Anlocken von Interessenten 136

cc) Abfangen von Kunden 137

c) (Störer-)Haftung des Web Service-Vermittlers 138

aa) Verstoß gegen Verkehrspflichten 139

bb) Grundsätze der Störerhaftung 139

cc) Übertragung auf den Web Service-Vermittler 141

d) Verhältnis der Ansprüche 143

IV. Öffentliche All-Inclusive-Angebote 143 
V. Haftung des Web Service-Nutzers 148

1. Eigene oder fremde Informationen 149

a) All-Inclusive-Dienste 150

b) Portale mit Web Services als „Zusatzleistung“ 151

aa) Einordnung im Rahmen der Haftungsprivilegierungen 151

bb) Zwischenergebnis: Haftung bei „Zusatzleistung“ 159

2. Zwischenergebnis 161

VI. Resïmee 162

3. Teil Datenschutz bei „Web Service-Portalen“ 164

A. EINFÜHRUNG 164

B. RECHTSRAHMEN FÜr DEN DATENSCHUTZ 167

I. Internationaler Rabmen 167

1. Europarat 167

2. Vereinte Nationen 168

3. OECD 169

4. Resümee 170

II. Recht der Europäischen Union 170

1. Charta der Grundrechte der Europäischen Union 170

2. Allgemeine Datenschutzrichtlinie 171

3. Datenschutzrichtlinie für elektronische Kommunikation 172

4. Richtlinie über den elektronischen Geschäftsverkehr (E-CommerceRichtlinie) 174

5. Resümee 174

III. Nationaler Rechtsrahmen 175

1. Der Geltungsbereich des deutschen Datenschutzrechts 175

2. Datenübermittlung ins Ausland 177

a) Angemessenes Schutzniveau 178

b) Einwilligung 179

aa) Exkurs: Anforderungen an die Einwilligung 179

i) Zeitpunkt und Dauer $\quad 180$

ii) Form 183

iii) Inhaltliche Anforderungen 188

bb) Empfehlungen zur Ausgestaltung der Einwilligungserklärung 195 
c) Resümee 196

3. Verfassungsrechtlicher Rahmen 197

a) Das Recht auf informationelle Selbstbestimmung 197

b) Eingriff 198

c) Datenschutzgrundsätze 199

aa) Datenvermeidung und Datensparsamkeit 199

bb) Zweckbindung 200

cc) Transparenz 201

dd) Grundsatz der Direkterhebung 202

ee) System- und Selbstdatenschutz 203

ff) Datensicherheit 204

IV. Datenschutzrechtliche Anforderungen des TMG und BDSG 205

1. Personenbezogene Daten 206

a) Definition und Inhalt 206

b) Anonyme und pseudonyme Daten 209

c) Zusammenfassung 210

2. Datenverarbeitungsbefugnisse und Grenzen der Datenverarbeitung 211

a) Web Service-Portale als Telemediendiensteanbieter 211

b) Erlaubnistatbestände 212

aa) Bestandsdaten 212

i) Bestandsdaten bei der Portalnutzung 215

ii) Bestandsdaten bei der Web Service-Nutzung 216

bb) Nutzungs- und Abrechnungsdaten 218

i) Nutzungsdaten 218

ii) Abrechnungsdaten 245

iii) Zusammenfassendes Resümee 251

cc) Inhaltsdaten 252

i) Einordnung 252

ii) Inhaltsdaten und die Web Service-Nutzung 254

c) Exkurs: Die Einwilligung von Minderjährigen 255

aa) Wirksamkeit des Portalnutzungsvertrags 257

bb) Anforderungen 259

V. Folgen der Missacbtung der Datenschutzvorgaben 260 
VI. Rechte der betroffenen Portalnutzer $\quad 262$

1. Unterrichtungspflicht 263

2. Auskunftsrecht 265

$\begin{array}{ll}\text { 3. Datenkorrektur } & 267\end{array}$

4. Zivilrechtliche Ansprüche 269

VII. Schutzmöglichkeiten des Betroffenen $\quad 270$

VIII. Sonstige datenschutzrechtliche Anforderungen $\quad 271$

C. RESÜMEE: TEIL $3 \quad 272$

4. Teil Schlussbetrachtungen $\quad 273$

5. Teil Anhang $\quad 277$

D. Muster-DatenschutZERKLÄRUNG 277 


\section{Abkürzungsverzeichnis}

a.A.

ABl. EG

AcP

A-Drs.

a.F.

AfP

AG

AGB

AktG

Alt.

Anh.

Anm.

AN.ON

Archiv PT

Art.

ASP

AT

AuA

Aufl.

Az.

B2B

BAG

$\mathrm{BB}$

Bd.

BDSG

BGB

BGBl.

BGB-InfoV

BGH

BGHZ andere Ansicht

Amtsblatt der Europäischen Gemeinschaft

Archiv für die civilistische Praxis (Zeitschrift)

Ausschlussdrucksache

alte Fassung

Zeitschrift für Medien- und Kommunikationsrecht

(früher: Archiv für Presserecht)

Amtsgericht

Allgemeine Geschäftsbedingungen

Aktiengesetz

Alternative

Anhang

Anmerkung

Anonymität.Online

Archiv für Post und Telekommunikation (Zeitschrift 1992-1998, Vorgänger der Zeitschrift RTkom, 19992001)

Artikel

Applikation Service Providing (bezieht sich auf die

Bereitstellung von Softwareanwendungen und damit verbundener Dienstleistungen)

Allgemeiner Teil

Arbeit und Arbeitsrecht (Zeitschrift)

Auflage

Aktenzeichen

Business to Business

Bundesarbeitsgericht

Betriebsberater (Zeitschrift)

Band

Bundesdatenschutzgesetz

Bürgerliches Gesetzbuch

Bundesgesetzblatt

Verordnung über Informations- und Nachweispflichten nach bürgerlichem Recht

Bundesgerichtshof

Entscheidungen des Bundesgerichtshofes in Zivilsa- 
chen

Begr.

BRAK-Mitt

Begründung

BR-Drs.

Mitteilungen der Bundesrechtsanwaltskammer

BSI

bspw.

Bundesratsdrucksache

BT-Drs.

Bundesamt für Sicherheit in der Informationstechnik

Btx

beispielsweise

Bundestagsdrucksache

BVerwG

Bildschirmtext

bzgl.

bzw.

Bundesverwaltungsgericht

bezüglich

beziehungsweise

CD

Computer Disk

CR

Computer und Recht (Zeitschrift)

DB

Der Betrieb (Zeitschrift)

DGI

Deutsche Gesellschaft für Informatik

Diss.

Dissertation

DÖV

Die öffentliche Verwaltung (Zeitschrift)

DSB

Datenschutz-Berater (Zeitschrift)

DSRL

Datenschutzrichtlinie

DuD

Datenschutz und Datensicherheit (Zeitschrift)

$\mathrm{E}$

Einleitung

E-Commerce

Electronic Commerce

ECRL

E-Commerce-Richtlinie

EDV

Elektronische Datenverarbeitung

EG

Europäische Gemeinschaft

EG

EGBGB

Vertrag zur Gründung der Europäischen Gemeinschaft

EGG

Einführungsgesetz zum Bürgerlichen Gesetzbuche

E-Government

Elektronischer Geschäftsverkehr-Gesetz

EGV

Electronic Government

Vertrag zur Gründung der Europäischen Gemein-

schaft

Einf.

Einführung

Einl.

Einleitung

EK-DSRL

Datenschutzrichtlinie für elektronische Kommunikation (2002/58/EG)

E-Mail

Electronic Mail 


\section{EMRK}

etc.

EU

$\mathrm{EuGH}$

EuGR

EUV

EuZW

EWG

EWiR

EWR

f.

FAQ

ff.

FIFA

FIT

FS

gem.

GewArch

GG

ggf.

GI

GRUR

GRUR Int.

GRUR-RR

GWB

Habil.

Hdb.

HGB

HK-WettbR
Europäische Konvention zum Schutze der Menschenrechte und Grundfreiheiten

et cetera

Europäische Union

Europäischer Gerichtshof

Europäische Grundrechte und Grundfreiheiten, Lehrbuch, hrsg. v. Dirk Eblers

Vertrag über die Europäische Union

Europäische Zeitschrift für Wirtschaftsrecht

Europäische Wirtschaftsgemeinschaft

Entscheidungen zum Wirtschaftsrecht (Zeitschrift)

Europäischer Wirtschaftsraum

folgende

Frequently Asked Questions

fortfolgende

Fédération Internationale de Football Association (zu deutsch: Internationale Föderation des Verbandsfußballs)

Fraunhofer Institut Angewandte Informationstechnik Festschrift

gemäß

Gewerbearchiv, Zeitschrift

Grundgesetz

gegebenenfalls

Gesellschaft für Informatik e.V.

Gewerblicher Rechtsschutz und Urheberrecht (Zeitschrift)

Gewerblicher Rechtsschutz und Urheberrecht, Internationaler Teil (Zeitschrift)

Gewerblicher Rechtsschutz und Urheberrecht,

Rechtsprechungs-Report (Zeitschrift)

Gesetz gegen Wettbewerbsbeschränkungen

Habilitationsschrift

Handbuch

Handelsgesetzbuch

Heidelberger Kommentar zum Wettbewerbsrecht, 2.

Aufl., 2004, hrsg. von 


$\begin{array}{ll}\text { h.L. } & \text { herrschende Lehre } \\ \text { h.M. } & \text { herrschende Meinung } \\ \text { hrsg. } & \text { herausgegeben } \\ \text { Hrsg. } & \text { Herausgeber } \\ \text { Hs. } & \text { Halbsatz } \\ \text { HTML } & \text { Hypertext Markup Language } \\ \text { HTTP } & \text { Hypertext Transfer Protocol } \\ \text { HTTPS } & \text { Hypertext Transfer Protocol Secure }\end{array}$

i.d.R.

in der Regel

i.d.S.

in diesem Sinne

ing.

Ingenieur

insb.

insbesondere

Int.

Internationales

IP

Internet Protokoll

IPRax

i.S.d.

Praxis des Internationalen Privat- und Verfahrensrechts (Zeitschrift)

iur.

im Sinne des

IT

Iuris (Rechtswissenschaften)

Information Technology (Informationstechnologie)

ITRB IT-Rechtsberater (Zeitschrift)

IUKDG

i.V.m.

Gesetz zur Regelung von Rahmenbedingungen für Informations- und Kommunikationsdienste in Verbindung mit

JGG

Jugendgerichtsgesetz

JMStV

Jugendmedienschutz-Staatsvertrag

JonDonym

Anonymisierungsdienst, der sich aus den Begriffen

John Doe und Anonym zusammensetzt

jurisPK

(s. https://www.jondos.de/de/index)

juris Praxis Kommentar

jurisPR

jurisPR-ITR

juris Praxisreport

juris Praxisreport IT-Recht

JurPC

$\mathrm{JuS}$

JZ

Internet-Zeitschrift für Rechtsinformatik und Infor-

mationsrecht

Juristische Schulung

Juristenzeitung (Zeitschrift)

$\mathrm{K} \& \mathrm{R}$

Kommunikation und Recht (Zeitschrift) 
Kap.

KartellR

$\mathrm{Kfz}$

KG

KWG

LBS

LG

Lit.

LOIS

$\mathrm{m}$.

Marken G

M-Commerce

MDR

MDStV

MMR

MünchKommBGB

MultimediaR

NJW

NJW-RR

Nr.

$\mathrm{NVwZ}$

NVZ

NZA

NZI

OECD

OLG

OSCI

PGP

ProdHaftG

RabelsZ
Kapitel

Kartellrecht

Kraftfahrzeug

Kammergericht

Kreditwesengesetz

Location Based Services

Landgericht

Literatur

Lexical Ontologies for legal Information Sharing

mit

Markengesetz

Mobile Commerce

Monatsschrift für deutsches Recht (Zeitschrift)

Mediendienste-Staatsvertrag

MultiMedia und Recht (Zeitschrift)

Münchner Kommentar zum Bürgerlichen Gesetzbuch

Multimediarecht

Neue Juristische Wochenschrift (Zeitschrift)

Neue Juristische Wochenschrift - Rechtsprechungs-

Report Zivilrecht (Zeitschrift)

Nummer

Neue Zeitschrift für Verwaltungsrecht

Neue Zeitschrift für Verkehrsrecht

Neue Zeitschrift für Arbeitsrecht

Neue Zeitschrift für das Recht der Insolvenz und

Sanierung

Organisation für wirtschaftliche Zusammenarbeit und Entwicklung

Oberstes Landgericht

Online Services Computer Interface

Pretty Good Privacy

Produkthaftungsgesetz

Rabels Zeitschrift für ausländisches und internationa- 


\begin{tabular}{|c|c|}
\hline RDV & Recht der Datenverarbeitung (Zeitschrift) \\
\hline RegE & Regierungsentwurf \\
\hline rer. Nat. & rerum naturalium (Naturwissenschaften) \\
\hline rer. oec. & rerum oeconomicarum (Wirtschaftswissenschaften) \\
\hline RG & Reichsgericht \\
\hline RGZ & $\begin{array}{l}\text { Entscheidungssammlung des Reichsgerichts in Zivil- } \\
\text { sachen }\end{array}$ \\
\hline Rhb. & Rechtshandbuch \\
\hline RL & Richtlinie \\
\hline RMD & $\begin{array}{l}\text { Recht der Multimedia-Dienste, Loseblatt- } \\
\text { Kommentar, hrsg. von Roßnagel, Alexander }\end{array}$ \\
\hline Rn. & Randnummer \\
\hline $\mathrm{RP}$ & Regierungspräsidium \\
\hline $\mathrm{RRa}$ & Reiserecht aktuell (Zeitschrift) \\
\hline Rs. & Rechtssache \\
\hline Rspr. & Rechtsprechung \\
\hline $\mathrm{RStV}$ & $\begin{array}{l}\text { Rundfunkstaatsvertrag (Staatsvertrag für Rundfunk } \\
\text { und Telemedien) }\end{array}$ \\
\hline RTkom & $\begin{array}{l}\text { Zeitschrift für das gesamte Recht der Telekommuni- } \\
\text { kation (Zeitschrift 1999-2001. Nachfolger von Ar- } \\
\text { chiv PT. Vorgänger der Zeitschrift TKMR 2002-- } \\
\text { 2004) }\end{array}$ \\
\hline s. & siehe \\
\hline S. & Satz (bei Paragraphen) \\
\hline S. & Seite \\
\hline SGB V & $\begin{array}{l}\text { Sozialgesetzbuch Fünftes Buch - Gesetzliche Kran- } \\
\text { kenversicherung }\end{array}$ \\
\hline SigG & Signaturgesetz \\
\hline SOA & Serviceorientierte Architektur \\
\hline SOAP & $\begin{array}{l}\text { ursprünglich das Akronym für ,Simple Object Ac- } \\
\text { cess Protocol“", heute wird SOAP als feststehender } \\
\text { Ausdruck für ein bestimmtes Netzwerkprotokoll } \\
\text { verwendet }\end{array}$ \\
\hline StGB & Strafgesetzbuch \\
\hline $\begin{array}{l}\text { STNL } \\
\text { st. Rspr. }\end{array}$ & $\begin{array}{l}\text { Stanford Law Review (Zeitschrift) } \\
\text { ständige Rechtsprechung }\end{array}$ \\
\hline TDDSG & Teledienstedatenschutzgesetz \\
\hline
\end{tabular}

les Privatrecht

Recht der Datenverarbeitung (Zeitschrift)

rerum naturalium (Naturwissenschaften)

rerum oeconomicarum (Wirtschaftswissenschaften)

Reichsgericht

Entscheidungssammlung des Reichsgerichts in Zivil-

$\mathrm{Rhb}$

Rechtshandbuch

Recht der Multimedia-Dienste, Loseblatt-

Randnummer

Regierungspräsidium

Reiserecht aktuell (Zeitschrift)

Rechtssache

Rechtsprechung

Rundfunkstaatsvertrag (Staatsvertrag für Rundfunk und Telemedien)

kation (Zeitschrift 1999-2001. Nachfolger von Ar-

chiv PT. Vorgänger der Zeitschrift TKMR 20022004)

siehe

$$
\text { Satz (bei Paragraphen) }
$$

Seite kenversicherung

Signaturgesetz

Serviceorientierte Architektur

ursprünglich das Akronym für „Simple Object Access Protocol“", heute wird SOAP als feststehender Ausdruck für ein bestimmtes Netzwerkprotokoll verwendet

Strafgesetzbuch

Stanford Law Review (Zeitschrift)

STNL

Teledienstedatenschutzgesetz 
TDG

TK

TK-DSRL

TKG

TKG-E

TKMR

TMG

TU

TV

Tz.

u.

u.a.

Überbl.

UDDI

UKlaG

URI

URL

USA

USB

UWG

v.

Var.

VersR

VertragsR

VG

$\mathrm{VO}$

Vorb.

VPS

VW

VwVfG

W3C
Teledienstegesetz

Telekommunikation

Telekommunikations-Datenschutzrichtlinie

(97/66/EG)

Telekommunikationsgesetz

Entwurf eines Gesetzes zur Änderung telekommunikationsrechtlicher

Vorschriften vom 7.4.2005, BT-Drs. 15/5213

Telekommunikations- und Medienrecht (Zeitschrift

2002-2004. Nachfolger von Archiv PT und RTkom)

Telemediengesetz

Technische Universität

Television

Textziffer

und

und andere

Überblick

Universal Description, Discovery and Integration

Unterlassungsklagegesetz

Uniform Resource Identifier

Uniform Resource Locator

Vereinigte Staaten von Amerika (United States of America)

Universal Serial Bus

Gesetz gegen den unlauteren Wettbewerb

vor

Variante

Versicherungsrecht (Zeitschrift)

Vertragsrecht

Verwaltungsgericht

Verordnung

Vorbemerkung

Virtuelle Poststelle

Volkswagen

Verwaltungsverfahrensgesetz

World Wide Web Consortium 
Web-Dok.

WettbR

WM

WRP

WSDL

WWW

XML

z.B.

ZIP

ZivilR

$\mathrm{ZPO}$

z.T.

ZUM
Web-Dokument

Wettbewerbsrecht

Wertpapier Mitteilungen (Zeitschrift)

Wettbewerb Recht und Praxis (Zeitschrift)

Webservice Description Language

World Wide Web

Extensible Markup Language

zum Beispiel

Zeitschrift für Wirtschaftsrecht und Insolvenzpraxis

Zivilrecht

Zivilprozessordnung

zum Teil

Zeitschrift für Urheber- und Medienrecht 


\section{Literaturverzeichnis}

Aigner, Dietmar/ Hofmann, Dietrich, Fernabsatzrecht im Internet, München 2004.

Ahrens, Hans-Jürgen, Das Herkunftslandprinzip in der E-CommerceRichtlinie, CR 2000, 835-841.

Alexander, Christian, Öffentliche Auftragsvergabe und unlauterer Wettbewerb, WRP 2004, 700-712.

Alonso, Gustavo/ Casati, Fabio/ Kuno, Harumi/ Machiraju, Vijay, Web Services. Concepts, Architectures and Applications, Berlin u.a. 2004.

Apitzsch, Jörg, Mechanismen zur Nachweisbarkeit der Kommunikation bei OSCI Transport, DuD 2007, 744-746.

Arlt, Christian, Datenschutzrechtliche Betrachtung von Onlineangeboten zum Erwerb digitaler Inhalte, MMR 2007, 683-687.

Armgardt, Matthias/ Spalka, Adrian, Der Anscheinsbeweis gemäß \371a Abs. 1 S. 2 ZPO vor dem Hintergrund der bestehenden Sicherheitslücken bei digitalen Signaturen, K\&R 2007, 26-32.

Auer-Reinsdorff, Astrid, Möglichkeiten in Geschäftsbedingungen und individuellen Vereinbarungen, ITRB 2006, 181-186.

Bamberger, Heinz Georg/ Roth, Herbert, Beck`scher Online Kommentar BGB, München Stand 1.9.2009 (zitiert als Bamberger/Roth/Bearbeiter).

Bar, Christian von, Internationales Privatrecht, Zweiter Band, Besonderer Teil, München 1991.

Baumbach, Klaus J./ Merkt, Hanno, Handelsgesetzbuch, 33. Aufl., München 2008.

Bäumler, Helmut, Das TDDSG aus der Sicht eines Datenschutzbeauftragten, DuD 1999, 258-262. 
Bäumler, Helmut (Hrsg.),E-Privacy - Datenschutz im Internet, Braunschweig Wiesbaden 2000 (zitiert als: Verfasser, in: Bäumler).

Bechtold, Stefan, Trusted Computing, CR 2005, 393-404.

Bechtold, Rainer, Kartellgesetz - Gesetz gegen Wettbewerbsbeschränkungen, 4. Aufl., München 2006.

Berger, Arndt/ Janal, Ruth, Suchet und Ihr werdet finden?, CR 2004, 917-925.

Bertsch, Andreas, Digitale Signaturen, Berlin Heidelberg New York 2002.

Bestmann, Sylle, Und wer muss zahlen?, K\&R 2003, 496-502.

Beucher, Klaus/ Leyendecker, Ludwig/ Rosenberg, Oliver von, Mediengesetze, Kommentar zum Rundfunkstaatsvertrag, Mediendienste-Staatsvertrag, Teledienstegesetz und Teledienstedatenschutzgesetz, München 1999.

Bierekoven, Christiane, Der Vertragsschluss via Internet im internationalen Wirtschaftsverkehr, Diss. iur. Universität Köln, Köln u.a. 2001.

Bierekoven, Christiane, Rechtssichere Widerrufsbelehrung im Onlinehandel, ITRB 2007, 73-75.

Bischof, Elke/ Schneider, Jochen, Folgen der Entscheidung des BGH v. 23.3.2005 - III ZR 338/04 für die Vertragsgestaltung..., ITRB 2005, 214-216.

BITKOM, Leitfaden Web Services, Stand Oktober 2005, abrufbar unter: http://www.bitkom.org/de/themen_gremien/36755_34887.aspx $<25.11 .2009>$ (zitiert als: BITKOM, Leitfaden Web Services).

Bitz, Gunter/ Reimer, Helmut, Schöne neue Welt der Services, DuD 2007, 638.

Bizer, Johann, Web Cookies - datenschutzrechtlich, DuD 1998, 277.

Bizer, Johann, Auskunftspflichten über Bestandsdaten, DuD 2002, 429. 
Bizer, Johann, Datenschutz im Telemediengesetz, Stellungnahme zum Entwurf der Bundesregierung Elektronisches-Geschäftsverkehr-Vereinheitlichungsgesetz (ElGVG), BT-Drs. 16/3078, A-Drs. 16(9)530 vom 11.12.2006, (zitiert als: Bizer, A-Drs. 16(9)530).

Bizer, Johann, Sieben Goldene Regeln des Datenschutzes, DuD 2007, 350356.

Booth, David/ Haas, Hugo/ McCabe, Francis/ Newcomer, Eric/ Champion, Michael/ Ferris, Chris/ Orchard, David, Web Service Architecture, W3C Working Group Note 11 February 2004, abrufbar unter:

http://www.w3.org/TR/ws-arch/wsa.pdf <25.11.2009> (zitiert als: W3C Working Group (2004).

Borges, Georg, Verträge im elektronischen Geschäftsverkehr, Habilitationsschrift Universität Köln, München 2003.

Bräutigam, Peter/ Leupold, Andreas (Hrsg.), Online-Handel, München 2003 (zitiert als: Bearbeiter, in: Bräutigam/Leupold).

Bräutigam, Peter/ Rücker, Daniel, Softwareerstellung und \651 BGB - Diskussion ohne Ende oder Ende der Diskussion?, CR 2006, 361-368.

Breinlinger, Astrid, Datenschutzrechtliche Probleme bei Kunden und Verbraucherbefragungen zu Marketingzwecken, RDV 1997, 247-253.

Breyer, Patrick, Neues Internetrecht - Forderungen aus Sicht der Nutzerinnen und Nutzer, A-Drs. 16(9)517 vom 4.12.2006 (zitiert als: Breyer, A-Drs. 16(9)517).

Bruns, Alexander, Informationsansprüche gegen Medien: ein Beitrag zur Verbesserung des Persönlichkeitsschutzes im Medienprivatrecht, Diss. iur. Universität Freiburg, Tübingen 1997.

Brunst, Phillip W., Umsetzungsprobleme der Impressumspflicht bei Webangeboten, MMR 2004, 8-13.

Buchmann, Felix, Widerrufsrecht bei Fernabsatzverträgen: Neues Unheil für gewerbliche ebay Verkäufer, K\&R 2007, 14-20. 
Buchmann, Felix, Die Widerrufsbelehrung im Spannungsfeld zwischen Gesetzgebung und Rechtsprechung, MMR 2007, 347-353.

Büllesbach, Alfred/ Garstka, Hans-Jürgen, Meilensteine auf dem Weg zu einer datenschutzgerechten Gesellschaft, CR 2005, 720-724.

Bundesamt für Sicherheit in der Informationstechnik, Verschlüsselung und Signatur, Kapitel II: Grundlagen, Modul 2 aus: E-Government-Handbuch, 2006 (Stand Kapitel 18.4.2005), abrufbar unter: www.bsi.bund.de $<25.11 .2009>$ (zitiert als: BSI, E-Government-Handbuch, II/2).

Bundesamt für Sicherheit in der Informationstechnik, Rechtliche Rahmenbedingungen für E-Government, Kapitel II: Grundlagen, Modul 3 aus: EGovernment-Handbuch, 2006 (Stand Kapitel 16.3.2005), abrufbar unter: www.bsi.bund.de <25.11.2009> (zitiert als: BSI, E-Government-Handbuch, II/3).

Bundesamt für Sicherheit in der Informationstechnik, VPS-Handbuch für Endanwender, abrufbar unter: www.bsi.bund.de $<25.11 .2009>$ (zitiert als: BSI, VPS-Handbuch Endanwender).

Burghardt, Markus, Web Services, Diss. rer. oec. Universität Göttingen, Wiesbaden 2004.

Burghardt, Markus/ Hagenhoff, Svenja, Web Services - Grundlagen und Kerntechnologien, Arbeitsbericht Nr. 22/2003, Göttingen 2003, abrufbar unter: http://webdoc.sub.gwdg.de/ebook/lm/arbeitsberichte/2003/22.pdf $<25.11 .2009>$ (zitiert als: Burkhardt/Hagenhoff, Arbeitspapier Nr. 22/2003).

Burghardt, Markus/ Hagenhoff, Svenja, Konzeption eines Abrechnungsmodells für Web Services, Arbeitsbericht Nr. 26/2003, Göttingen 2003, abrufbar unter: http://webdoc.sub.gwdg.de/ebook/lm/arbeitsberichte/2003/26.pdf <25.11.2009> (zitiert als: Burkhardt/Hagenhoff, Arbeitspapier Nr. 26/2003).

Buxel, Holger, Die sieben Kernprobleme des Online-Profiling aus Nutzerperspektive, DuD 2001, 579-583.

Calliess, Christian/ Ruffert, Matthias (Hrsg.), EUV/EGV, Kommentar, 3. Aufl., München 2007 (zitiert als: Bearbeiter, in: Callies/Ruffert). 
Cardoso, Jorge, E-Tourism: Creating Dynamic Packages using Semantic Web Processes, abrufbar unter:

http://www.w3.org/2005/04/FSWS/Submissions/16/paper.html $<25.11 .2009>$.

Cichon, Caroline, Internet-Verträge, 2. Aufl., Köln 2005.

Clemens, Rudolf, Die elektronische Willenserklärung - Chancen und Gefahren, NJW 1985, 1998-2005.

Conrad, Yvonne, Kundenkarten und Rabattsysteme, DuD 2006, 405-409.

Däubler, Wolfgang, Die Übermittlung von Arbeitnehmerdaten ins Ausland, CR 1999, 49-57.

Deike, Thies, Open Source Software: IPR-Fragen und Einordnung ins deutsche Rechtssystem, CR 2003, 9-18.

Derleder, Peter/ Thielbar, Carsten, Handys, Klingeltöne und Minderjährigenschutz, NJW 2006, 3233-3239.

Dietrich, Kay, Typisierung von Softwareverträgen nach der Schuldrechtsreform, CR 2002, 473.

Dietrich, Florian/ Hofmann, Ruben, 3... Gerichte, 2... Wochen, 1... Monat?, CR 2007, 318-322.

Dix, Alexander, Vorratsdatenspeicherung von IP-Adressen?, DuD 2003, 234236.

Donaubauer, Andreas Josef, Interoperable Nutzung verteilter Geodatenbanken mittels standardisierter Geo Web Services, Diss. ing. TU München, München 2004.

Döring, Reinhard, Die Haftung für eine Mitwirkung an Wettbewerbsverstößen nach der Entscheidung des BGH "Jugendgefährdende Medien bei eBay", WRP 2007, 1131-1141.

Duhr, Elisabeth/ Naujok, Helga/ Peter, Martina/ Seiffert, Evelyn, Neues Datenschutzrecht für die Wirtschaft, DuD 2002, 5-36. 
Eberle, Carl-Eugen/ Rudolf, Walter/ Wasserburg, Klaus (Hrsg.), Mainzer Rechtshandbuch der Neuen Medien, Heidelberg 2003 (zitiert als: Bearbeiter, in: Eberle/Rudolf/Wasserburg).

Ehlers, Dirk (Hrsg.), Europäische Grundrechte und Grundfreiheiten, 2. Aufl., Berlin 2005 (zitiert als: Bearbeiter, in: Ehlers, EuGR).

Ehmann, Horst, Informationsschutz und Informationsverkehr im Zivilrecht, AcP 188 (1988), 230-380.

Ehret, Susanne, Internet-Auktionshäuser auf dem haftungsrechtlichen Prüfstand, CR 2003, 754-761.

Ekey, Friedrich L./ Klippel, Diethelm/ Kotthoff, Jost/ Meckel, Astrid/ Plaß, Gunda, Heidelberger Kommentar zum Wettbewerbsrecht, 2. Aufl., Heidelberg 2004 (zitiert als: Bearbeiter, in: HK-WettbR).

Elschner, Günter, Rechtsfragen der Internet- und E-Mail-Nutzung am Arbeitsplatz, Lohmar-Köln 2004.

Emmerich, Volker, Unlauterer Wettbewerb, 7. Aufl., München 2004 (zitiert als: Emmerich, Unlauterer Wettbewerb).

Emmerich, Volker, Kartellrecht, 10. Aufl., München 2006 (zitiert als: Emmerich, KartellR).

Engel-Flechsig, Stefan, Teledienstedatenschutz, DuD 1997, 8-16.

Engel-Flechsig, Stefan, Die datenschutzrechtlichen Vorschriften im neuen Informations- und Kommunikationsdienste-Gesetz, RDV 1997, 59-67.

Engels, Stefan/ Jürgens, Uwe/ Fritzsche, Saskia, Die Entwicklung des Telemedienrechts im Jahr 2006, K\&R 2007, 57-68.

Engel-Flechsig, Stefan/ Maennel, Frithjof A./ Tettenborn, Alexander, Das neue Informations- und Kommunikationsdienste-Gesetz, NJW 1997, 29812992.

Enzmann, Matthias/ Roßnagel, Alexander, Realisierter Datenschutz für den Einkauf im Internet, CR 2002, 141-150. 
Erichsen, Hans-Uwe/ Ehlers, Dirk (Hrsg.), Allgemeines Verwaltungsrecht, 13. Aufl., Berlin 2005 (zitiert als Erichsen/Ehlers/Bearbeiter).

Erman, Walter, Bürgerliches Gesetzbuch, Handkommentar, Band I, 12. Aufl., Köln 2008 (zitiert als: Erman/Bearbeiter).

Ernst, Stefan, Beweisprobleme bei E-Mail und anderen OnlineWillenserklärungen, MDR 2003, 1091-1094.

Ernst, Stefan (Hrsg.), Hacker, Cracker \& Computerviren, Köln 2004 (zitiert als: Bearbeiter, in: Ernst).

Ernst, Stefan, Rechtliche Probleme des Suchmaschinen-Marketings, ITRB 2005, 91-93.

Ernst, Stefan, Markenrechtserschöpfung: Verwendung einer fremden Marke als Metatag im HTML-Code oder in "Weiß-auf-Weiß-Schrift" auf Internetseiten ("AIDOL"), jurisPR-ITR 9/2007 Anm. 3.

Ernst, Stefan, Telefonnummer im Internetimpressum, jurisPR-ITR 2/2009 Anm. 2.

Esser, Josef/ Weyers, Hans-Leo, Schuldrecht, Band II, Besonderer Teil, Teilband 1, 8. Aufl., Heidelberg 1998 (zitiert als Esser/Weyers, II/1).

Federrath, Hannes/ Golembiewski, Claudia, Speicherung von Nutzungsdaten durch Anonymisierungsdienste im Internet, DuD 2004, 486-490.

Felke, Klaus/ Jordans, Roman, Der Referentenentwurf für die Umsetzung der Fernabsatzrichtlinie für Finanzdienstleistungen, WM 2004, 166-171.

Ferrari, Franco/ Kieninger, Eva-Maria/ Mankowski, Peter/ Otte, Karsten/ Saenger, Ingo/ Staudinger, Ansgar, Internationales Vertragsrecht, Kommentar, München 2007 (zitiert als Ferrari/Bearbeiter, Int. VertragsR).

Fezer, Karl-Heinz (Hrsg.), Markenrecht, Kommentar, 4. Aufl., München 2009 (zitiert als Fezer, MarkenR). 
Fezer, Karl-Heinz (Hrsg.), Lauterkeitsrecht, Kommentar zum Gesetz gegen den unlauteren Wettbewerb (UWG), Bd. 1, München 2005 (zitiert als: Fezer/Bearbeiter, Bd. 1, UWG).

Fischer-Dieskau, Stefanie, Das elektronisch signierte Dokument als Mittel zur Beweissicherung, Diss. iur. Universität Kassel, Baden-Baden 2006.

Fischer-Dieskau, Stefanie/ Roßnagel, Alexander/ Steidle, Roland, Beweisführung am seidenen Bit-String? - Die Langzeitaufbewahrung elektronischer Signaturen auf dem Prüfstand, MMR 2004, 451-455.

Flume, Werner, Allgemeiner Teil des Bürgerlichen Rechts, Zweiter Band, Das Rechtsgeschäft, 4. Aufl., Berlin u.a. 1992 (zitiert als: Flume, AT 2).

Fragstein, Udo von, Zu den datenschutzrechtlichen Fragen von Kundenbindungsprogrammen, EWiR 2006, 517-518.

Fröhle, Jens, Web Advertising, Nutzerprofile und Teledienstedatenschutz, Diss. iur. Universität Münster, München 2003.

Fröschle, Hans-Peter (Hrsg.), Web-Services, Heidelberg 2003 (zitiert als: Bearbeiter, in: Fröschle).

Führich, Ernst, Dynamic Packaging und virtuelle Reiseveranstalter - Entwicklung und Anwendung des Reisevertragsrechts auf die neue internet-basierte Pauschalreise, RRa 2006, 50-57.

Führich, Ernst, Reiserecht von A-Z, 3. Aufl., München 2006.

Fülbier, Ulrich, Web 2.0 - Haftungsprivilegierungen bei MySpace und YouTube, CR 2007, 515-521.

Gabel, Detlev, Haftung für Hyperlinks im Lichte des neuen UWG, WRP 2005, 1102-1118.

Gaßner, Otto, Rechtsanwendung beim doppeltypischen Vertrag am Beispiel der Werkdienstwohnung, AcP 186 (1986), 325-355. 
Geiger, Andreas, Die Einwilligung in die Verarbeitung von persönlichen Daten als Ausübung des Rechts auf informationelle Selbstbestimmung, NVwZ 1989, 35-38.

Geiger, Gebhard, IT-Sicherheit als integraler Bestandteil des Risikomanagements im Unternehmen, in: Gründer, Torsten/Schrey, Joachim (Hrsg.), Managementhandbuch IT-Sicherheit, Berlin 2007, 27-51.

Geis, Ivo, Die digitale Signatur, NJW 1997, 3000-3004.

Geis, Ivo, Das neue Datenschutzrecht für Teledienste, CR 2002, 667-674.

Geis, Ivo, Der Betroffene als Zahl - Wirtschaftsinteresse contra Betroffenenrechte?, RDV 2007, 1-4.

Geppert, Martin/ Piepenbrock, Hermann-Josef/ Schütz, Raimund/ Schuster, Fabian, Beck`scher TKG-Kommentar, 3. Aufl., München 2006 (zitiert als: BeckTKG-Komm/Bearbeiter).

Gerling, Rainer W., Verschlüsselung, in: Gründer, Torsten/ Schrey, Joachim (Hrsg.), Managementhandbuch IT-Sicherheit, Berlin 2007, 83-103.

Gey, Michael, Zivilrechtliche Haftung von Access-Providern bei Zugangsstörungen, K\&R 2005, 120-126.

Gitter, Rotraud/ Schnabel, Christoph, Die Richtlinie zur Vorratsspeicherung und ihre Umsetzung in das nationale Recht, MMR 2007, 411-416.

Gloy, Wolfgang/ Loschelder, Michael, Handbuch des Wettbewerbsrechts, 3. Aufl., München 2005 (zitiert als Gloy/Loschelder/Bearbeiter, Hdb. WettbewerbsR).

Gola, Peter, Informationelle Selbstbestimmung in Form des Widerspruchsrechts, DuD 2001, 278-281.

Gola, Peter, Datenschutz bei der Kontrolle „mobiler“ Arbeitnehmer - Zulässigkeit und Transparenz, NZA 2007, 1139-1144.

Gola, Peter/ Klug, Christoph,Grundzüge des Datenschutzrechts, München 2003. 
Gola, Peter/ Klug, Christoph, Die Entwicklung des Datenschutzrechts in den Jahren 2006/2007, NJW 2007, 2452-2459.

Gola, Peter/ Müthlein, Thomas, Neuer Tele-Datenschutz - bei fehlender Koordination über das Ziel hinausgeschossen?, RDV 1997, 193-197.

Gola, Peter/ Schomerus, Rudolf, Bundesdatenschutzgesetz, Kommentar, 9. Aufl., München 2007.

Goldmann, Bettina/ Redecke, Rebecca, Gewährleistung bei Softwarelizenzverträgen nach dem Schuldrechtsmodernisierungsgesetz, MMR 2002, 3-8.

Gounalakis, Georgios, Rechtshandbuch Electronic Business, München 2003 (zitiert als: Bearbeiter, in: Gounalakis, Rhb. Electronic Business).

Gounalakis, Georgios/ Mand, Elmar, Die neue EG-Datenschutzrichtlinie Grundlagen einer Umsetzung in nationales Recht (I), GRUR Int. 1997, 431438.

Gounalakis, Georgios/ Mand, Elmar, Die neue EG-Datenschutzrichtlinie Grundlagen einer Umsetzung in nationales Recht (II), CR 1997, 497-504.

Grabitz, Eberhard/ Hilf, Meinhard (Hrsg.), Das Recht der Europäischen Union, Kommentar, Band 1, Loseblatt, München Stand Dezember 2005 (zitiert als Bearbeiter, in: Grabitz/Hilf).

Grunewald, Barbara, Die Anwendbarkeit des AGB-Gesetzes auf Bestimmungen über den Vertragsabschluß, ZIP 1987, 353-357.

Grützmacher, Malte, Gebrauchtsoftware und Übertragbarkeit von Lizenzen, CR 2007, 549-556.

Gundermann, Lukas, E-Commerce trotz oder durch Datenschutz?, K\&R 2000, 225-235.

Günther, Andreas, Produkthaftung für Informationsgüter, Diss. iur. Universität München, Köln 2001.

Hahn, Bernhard, Telekommunikationsdienstleistungs-Recht - Grundfragen, Vertragsrecht und Kundenschutz, Heidelberg 2001. 
Hamel, Miriam, Sicherheitsservices für Service Oriented Architecture (SOA), DuD 2007, 662-665.

Hartl, Robert, Fremde Kennzeichen im Quelltext von Webseiten - Markenund wettbewerbsrechtliche Zulässigkeit, MMR 2007, 12-15.

Härting, Niko, Schnäppchen oder Inhaltsirrtum, ITRB 2004, 61-64.

Härting, Niko, Internetrecht, 2. Aufl., Köln 2005.

Härting, Niko, Schutz von IP-Adressen, ITRB 2009, 35-39.

Härting, Niko/ Schirmbacher, Martin, Internetwerbung und Wettbewerbsrecht, ITRB 2005, 16-18.

Hassemer, Michael, Elektronischer Geschäftsverkehr im Regierungsentwurf zum Schuldrechtsmodernisierungsgesetz, MMR 2001, 635-640.

Heckmann, Dirk (Hrsg.), Juris Praxis Kommentar Internetrecht, Saarbrücken 2007 (zitiert als: jurisPK-Internetrecht/Bearbeiter).

Heermann, Peter W./ Ohly, Ansgar (Hrsg.), Verantwortlichkeit im Netz, Stuttgart u.a. 2003 (zitiert als: Bearbeiter, in: Heermann/Ohly).

Hefermehl, Wolfgang/ Köhler, Helmut/ Bornkamm, Joachim, Wettbewerbsrecht, 27. Aufl., München 2009 (zitiert als: Hefermehl/Köhler/Bornkamm/ Bearbeiter).

Heidemann-Peuser, Heike, Rechtskonforme Gestaltung von Datenschutzklauseln, DuD 2002, 389-394.

Heidrich, Jörg, Die T-Online-Entscheidung des RP Darmstadt und ihre Folgen, DuD 2003, 237-238.

Heim, Andreas, Zur Markenbenutzung durch Meta-Tags, CR 2005, 200-205.

Hellmich, Stefanie, Location Based Services - Datenschutzrechtliche Anforderungen, MMR 2002, 152-158. 
Heun, Sven-Erik (Hrsg.), Handbuch Telekommunikation, 2. Aufl., Köln 2007 (zitiert als: Bearbeiter, in: Heun).

Heussen, Benno, Unvermeidbare Softwarefehler, CR 2004, 1-10.

Heydn, Truiken J./ Schmidl, Michael, Der Handel mit gebrauchter Software und der Erschöpfungsgrundsatz, K\&R 2006, 74-79.

Hillenbrand-Beck, Renate/ Greß, Sebastian, Datengewinnung im Internet, DuD 2001, 389-394.

Hilty, Reto M., Der Softwarevertrag - ein Blick in die Zukunft - Konsequenzen der trägerlosen Nutzung und des patentrechtlichen Schutzes von Software, MMR 2003, 3-15.

Hobert, Guido, Datenschutz und Datensicherheit im Internet: Interdependenz und Korrelation von rechtlichen Grundlagen und technischen Möglichkeiten, Frankfurt am Main 1998.

Hoenike, Mark/ Hülsdunk, Lutz, Die Gestaltung von Fernabsatzangeboten im elektronischen Geschäftsverkehr nach neuem Recht - Gesetzesübergreifende Systematik und rechtliche Vorgaben vor Vertragsschluß, MMR 2002, 415-420.

Hoeren, Thomas, Vorschlag für eine EU-Richtlinie über E-Commerce - Eine erste kritische Analyse, MMR 1999, 192-199.

Hoeren, Thomas, Informationspflichten im Internet - im Lichte des neuen UWG, WM 2004, 2461-2470.

Hoeren, Thomas, Internet- und Kommunikationsrecht, Köln 2008.

Hoeren, Thomas/ Sieber, Ulrich, Handbuch Multimedia-Recht, 21. Ergänzungslieferung Dezember 2008, München 2008 (zitiert als: Bearbeiter, in: Hoeren/Sieber, Hdb. MultimediaR).

Hoffmann, Helmut, Zivilrechtliche Haftung im Internet, MMR 2002, 284-289.

Hoffmann, Helmut, Die Entwicklung des Internet-Rechts bis Mitte 2003, NJW 2003, 2576-2581. 
Hoffmann, Bernd von/ Thorn, Karsten, Internationales Privatrecht, 9. Aufl., München 2007.

Hohmann, Harald, Haftung der Softwarehersteller für das „Jahr 2000“Problem, NJW 1999, 521-526.

Holzer, Johannes, Reaktionsversehen in der Insolvenzordnung?, NZI 1999, 44-47.

Holznagel, Bernd, Recht der IT-Sicherheit, München 2003.

Hornung, Gerrit, Zwei runde Geburtstage: Das Recht auf informationelle Selbstbestimmung und das WWW, MMR 2004, 3-8.

Hornung, Gerrit, Der Personenbezug biometrischer Daten, DuD 2004, 429431.

Hornung, Gerrit, Die digitale Identität, Diss. iur. Universität Kassel, BadenBaden 2005.

Hoß, Dirk, Web-Impressum und Wettbewerbsrecht, CR 2003, 687-691.

Huber, Ulrich, Zur Haftung des Verkäufers bei positiver Vertragsverletzung, AcP 177 (1977), 281-348.

Huppertz, Peter, Handel mit Second Hand-Software, CR 2006, 145-151.

Husch, Gertrud/ Kemmler, Anne/ Ohlenburg, Anna, Die Umsetzung des EU-Rechtsrahmens für elektronische Kommunikation: Ein erster Überblick, MMR 2003, 139-148.

Ihde, Rainer, Cookies - Datenschutz als Rahmenbedingung der Internetökonomie, CR 2000, 413-423.

Imhof, Ralf, One-to-One-Marketing im Internet - Das TDDSG als Marketinghindernis, CR 2000, 110-116.

Immenga, Ulrich/ Mestmäcker, Ernst-Joachim, Wettbewerbsrecht, Band 2 GWB, 4. Aufl., München 2007 (zitiert als: Immenga/Mestmäcker/Bearbeiter, Bd. 2). 
Intveen, Michael, Weitere Einzelheiten zu Haftungsklauseln in Allgemeinen Geschäftsbedingungen im kaufmännischen/unternehmerischen Verkehr, ITRB 2003, 13-15.

Iraschko-Luscher, Stephanie, Einwilligung - ein stumpfes Schwert des Datenschutzes?, DuD 2006, 706-710.

Jacob, Joachim, Stasi-Abhörprotokolle und parlamentarische Untersuchungsausschüsse, DuD 2000, 320-321.

Jacob, Joachim/ Jost, Tanja, Marketingschutz von Kundendaten und Datenschutz - ein Widerspruch?, DuD 2003, 621-624.

Jacobs, Rainer, Markenrechtsverletzungen durch Internetauktionen, in: Ahrens, Hans-Jürgen (Hrsg.), Festschrift für Willi Erdmann, Köln Berlin Bonn München 2002, 327-341 (zitiert als: Jacobs, in: FS Erdmann).

Jandt, Silke, Datenschutz bei Location Based Services - Voraussetzungen und Grenzen der rechtmäßigen Verwendung von Positionsdaten, MMR 2007, 7478.

Jandt, Silke/ Laue, Philip, Voraussetzungen und Grenzen der Profilbildung bei Location Based Services, K\&R 2006, 316-322.

Jarass, Hans D., Das allgemeine Persönlichkeitsrecht im Grundgesetz, NJW 1989, 857-862.

Jarass, Hans D./ Pieroth, Bodo, Grundgesetz, Kommentar, 10. Aufl., München 2009.

Junker, Abbo, Die Entwicklung des Computerrechts in den Jahren 2001/2002, NJW 2003, 2792-2799.

Junker, Abbo, Die Entwicklung des Computerrechts in den Jahren 2003/2004, NJW 2005, 2829-2834.

Junker, Abbo, Die Rom II-Verordnung: Neues Internationales Deliktsrecht auf europäischer Grundlage, NJW 2007, 3675-3682.

Kaller, Paul, Reiserecht, 2. Aufl., München 2005. 
Kaufmann, Noogie C., Metatagging - Markenrecht oder reformiertes UWG?, MMR 2005, 348-352.

Keller, Moritz, Versandhandelskauf und Preisirrtum im Internet, K\&R 2005, 167-174.

Klass, Nadine, Die zivilrechtliche Einwilligung als Instrument zur Disposition über Persönlichkeitsrechte, AfP 2005, 507-518.

Klees, Andreas/ Lange, Timo, Bewerbung, Nutzung und Herstellung von Handyklingeltönen, CR 2005, 684-688.

Klimke, Dominik, Korrekturhilfen beim Online-Vertragsschluss, CR 2005, 582-591.

Koch, Frank A., Internet-Recht, 2. Aufl., München Wien 2005 (zitiert als: Koch, Internet-Recht).

Koch, Frank A., Web Services als neue IT-Vertragsleistung, ITRB 2007, 71-73.

Koch, Frank A., Lizenzrechtliche Grenzen des Handels mit Gebrauchtsoftware, ITRB 2007, 140-144.

Koenig, Christian/ Neumann, Andreas, Telekommunikationsrechtliche Ansprüche auf Leistungen der Fakturierung und des Inkassos für Internet-byCall-Dienstleistungen, K\&R Beilage 3/2004, 1-31.

Köhler, Helmut, Zur Problematik automatisierter Rechtsvorgänge, insbesondere von Willenserklärungen, AcP 182 (1982), 126-171.

Köhler, Helmut, Die wettbewerbsrechtlichen Abwehransprüche (Unterlassung, Beseitigung, Widerruf), NJW 1992, 137-143.

Köhler, Helmut, Die Rechte des Verbrauchers beim Teleshopping (TVShopping, Internet-Shopping), NJW 1998, 185-190.

Köhler, Helmut, Der Rechtsbruchtatbestand im neuen UWG, GRUR 2004, 381-389. 
Köhler, Helmut, „Täter“ und „Störer“ im Wettbewerbs- und Markenrecht Zur BGH-Entscheidung „Jugendgefährdende Medien bei eBay“, GRUR 2008, $1-7$.

Köhntopp, Marit/ Köhntopp, Kristian, Datenspuren im Internet, CR 2000, 248-257.

König, M. Michael, Software (Computerprogramme) als Sache und deren Erwerb als Sachkauf, NJW 1993, 3121-3124.

Kossmann, Donald/ Leymann, Frank, Web Services, Informatik Spektrum 2004, 117- 128.

Kramer, Ernst A., Juristische Methodenlehre, 2. Aufl., München 2005 (zitiert als: Kramer, Methodenlehre).

Kramer, Philipp, Geheime Spione: ist die Verwendung von „Web Bugs“ zulässig?, DSB 11/2001, 8-10.

Kramer, Philipp/ Herrmann, Michael, Datenschutz und E-Commerce, Berlin 2005.

Kremp, Matthias, Wie Unternehmen Sie ausspähen, Spiegel Online 27.6.2007, abrufbar unter: http://www.spiegel.de/netzwelt/web/0,1518,490827,00.html $<25.11 .2009>$.

Krieg, Henning, Unterlassungsanspruch bei Speicherung einer dynamischen IP-Adresse, jurisPR-ITR 14/2007 Anm. 2.

Kröger, Detlef/ Gimmy, Marc A., Handbuch zum Internet-Recht, 2. Aufl., Berlin Heidelberg 2002 (zitiert als: Bearbeiter, in: Kröger/Gimmy, Hdb. InternetR).

Krüger, Wolfgang/ Westermann, Harm Peter, Münchner Kommentar zum Bürgerlichen Gesetzbuch, Band 3, Schuldrecht, Besonderer Teil 1, \$\$ 433-610, 5. Aufl., München 2008 (zitiert als: MünchKommBGB/Bearbeiter, Bd. 3).

Lachmann, Jens-Peter, Ausgewählte Probleme aus dem Recht des Bildschirmtextes, NJW 1984, 405-408. 
Ladeur, Karl-Heinz, Datenverarbeitung und Datenschutz bei neuartigen Programmführern in „virtuellen Videotheken“ - Zur Zulässigkeit der Erstellung von Nutzerprofilen, MMR 2000, 715-721.

Lange, Knut Werner, Neue Marketingstrategien im Internet - ökonomische und rechtliche Analyse, BB 2002, 561-569.

Lange, Knut Werner (Hrsg.), Handbuch zum deutschen und europäischen Kartellrecht, 2. Aufl., Frankfurt am Main 2006 (zitiert als: Lange/Bearbeiter, Hdb. KartellR).

Lange, Raimund, Datentransfer ins Ausland, AuA 2006, 712-714.

Langner, Torsten, Web Services mit Java, München 2005.

Lapp, Thomas, Cookies: Monster oder harmlose Kekse?, ITRB 2001, 113-115.

Larenz, Karl, Lehrbuch des Schuldrechts, Zweiter Band, Besonderer Teil, 1. Halbband, 13. Aufl., München 1986 (zitiert als: Larenz, II/1).

Larenz, Karl, Methodenlehre der Rechtswissenschaft, 6. Aufl., Berlin 1991 (zitiert als: Larenz, Methodenlehre).

Larenz, Karl/ Canaris, Claus-Wilhelm, Lehrbuch des Schuldrechts, Zweiter Band, Besonderer Teil, 2. Halbband, 13. Aufl., München 1994 (zitiert als: Larenz/Canaris, II/2).

Larenz, Karl/ Canaris, Claus-Wilhelm, Methodenlehre der Rechtswissenschaft, 3. Aufl., Berlin u.a. 1995 (zitiert als: Larenz/Canaris, Methodenlehre).

Larenz, Karl/ Wolf, Manfred, Allgemeiner Teil des Bürgerlichen Rechts, 9. Aufl., München 2004 (zitiert als: Larenz/Wolf, AT).

Laue, Philip/ Maidl, Monika/ Peters, Jan, Zweckbindung in ämterübergreifenden Verwaltungsworkflows, DuD 2007, 810-815.

Lehmann, M., Produkt- und Produzentenhaftung für Software, NJW 1992, 1721-1725. 
Lehment, Cornelius, Zur Störerhaftung von Online- Auktionshäusern, WRP 2003, 1058-1065.

Leible, Stefan (Hrsg.), Die Bedeutung des Internationalen Privatrechts im Zeitalter der neuen Medien, Stuttgart u.a. 2003 (zitiert als: Bearbeiter, in: Leible).

Leible, Stefan/ Sosnitza, Olaf (Hrsg.), Versteigerungen im Internet, Heidelberg 2004 (zitiert als: Bearbeiter, in: Leible/Sosnitza).

Leible, Stefan/ Sosnitza, Olaf, „3...2...1...meins!“ und das TDG“, WRP 2004, 592-599.

Leible, Stefan/ Sosnitza, Olaf, Neues zur Störerhaftung von InternetAuktionshäusern, NJW 2004, 3225-3227.

Leible, Stefan/ Sosnitza, Olaf, Haftung von Internetauktionshäusern - reloaded, NJW 2007, 3324-3326.

Leitgeb, Stephan, Virales Marketing - Rechtliches Umfeld für Werbefilme auf Internetportalen wie YouTube, ZUM 2009, 39-49.

Lessig, Lawrence, Code Version 2.0, 2. Aufl., New York 2006.

Lettl, Tobias, Kartellrecht, 2. Aufl., München 2007.

Leverenz, Kent, Auswirkungen des $\ 312 \mathrm{e}$ BGB auf das Versicherungsgeschäft im Internet, VersR 2003, 698-707.

Lewinski, Kai von, Privacy Policies: Unterrichtungen und Einwilligung im Internet, DuD 2002, 395-400.

Litzenburger, Wolfgang, Das Ende des vollständigen Gewährleistungsausschlusses beim Kaufvertrag über gebrauchte Immobilien, NJW 2002, 12441247.

Lonthoff, Jörg/ Ortner, Erich, Klassifikation und Lösungsansätze für Web Services im mobilen Umfeld, in: König-Ries, Brigitta/ Lehner, Franz/ Malaka, Rainer/ Türker, Can, MMS 2007: Mobilität und mobile Informationssysteme, 
Aachen March 6th, 2007, 73-84 (zitiert als: Lonthoff/Ortner, in: KönigRies/Lehner/Malaka/Türker).

Lotz, Volkmar, SOA-Sicherheit für moderne Unternehmen, DuD 2007, 644647.

Lurger, Brigitta/ Vallant, Sonja Maria, Grenzüberschreitender Wettbewerb im Internet, RIW 2002, 188-202.

Lütcke, Jens, Fernabsatzrecht, Kommentar, München 2002.

Mankowski, Peter, Internet und internationales Wettbewerbsrecht, GRUR Int. 1999, 909-921.

Mankowski, Peter, Besondere Formen von Wettbewerbsverstößen im Internet und Internationales Wettbewerbsrecht, GRUR Int. 1999, 995-1003.

Mankowski, Peter, Herkunftslandprinzip und deutsches Umsetzungsgesetz zur e-commerce-Richtlinie, IPRax 2002, 257-266.

Mankowski, Peter, Schuldrechtsreform: Werkvertragsrecht - Die Neuerungen durch $\int 651$ BGB und der Abschied vom Werklieferungsvertrag, MDR 2003, 854-860.

Marly, Jochen, Softwareüberlassungsverträge, 4. Aufl., München 2004.

Martens, Axel, Verteilte Geschäftsprozesse - Modellierung und Verifikation mit Hilfe von Web Services, Diss. rer. nat. Humboldt-Universität Berlin, Stuttgart Berlin 2004.

Martinek, Michael, Moderne Vertragstypen, Band 1: Leasing und Factoring, München 1991 (zitiert als: Martinek, Bd. 1).

Medicus, Dieter, Bürgerliches Recht, 21. Aufl., München 2007.

Meier, Klaus/ Wehlau, Andreas, Die zivilrechtliche Haftung für Datenlöschung, Datenverlust und Datenzerstörung, NJW 1998, 1585-1591.

Meister, Herbert, Datenschutz im Zivilrecht: das Recht am eigenen Datum, 2. Aufl., Bergisch Gladbach 1981. 
Melzer, Ingo/ Werner, Sebastian/ Sauter, Patrick/ Hillinger von Thile, Alexander/ Flehmig, Marcus/ Zengler, Barbara/ Dostal, Wolfgang/ Tröger, Peter/ Stumm, Boris/ Lipp, Matthias/ Jeckle, Mario, Service-orientierte Architekturen mit Web Services, 2. Aufl., München 2007 (zitiert als: Melzer).

Merati-Kashani, Jasmin, Datenschutz im E-Commerce, Diss. iur. Universität Köln, München 2005.

Metzger, Axel, Der neue $\int 651$ BGB - Primat des Kaufrechts oder restriktive Auslegung?, AcP 204 (2004), 231-263.

Meyer, Sebastian, Cookies \& Co. - Datenschutz und Wettbewerbsrecht, WRP 2002, 1028-1035.

Meyer, Uwe, Elektronischer Geschäftsverkehr des Unternehmers mit Verbrauchern und Unternehmern: Anforderungen gemäß \312e BGB und Rechtsfolgen bei Pflichtverstößen, DB 2004, 2739-2742.

Meyerdierks, Per, Sind IP-Adressen personenbezogene Daten?, MMR 2009, 813.

Miedbrodt, Anja/ Mayer, Patrick, E-Commerce - Digitale Signaturen in der Praxis, MDR 2001, 432-436.

Moos, Flemming, Dürfen Access-Provider IP-Nummern speichern?, CR 2003, 385-388.

Moos, Flemming, Unzulässiger Handel mit Persönlichkeitsprofilen? - Erstellung und Vermarktung kommerzieller Datenbanken mit Personenbezug, MMR 2006, 718-723.

Moritz, Hans-Werner/ Dreier, Thomas, Rechts-Handbuch zum E-Commerce, 2. Aufl., Köln 2005 (zitiert als: Bearbeiter, in: Moritz/Dreier).

Möschel, Wernhard, Dogmatische Strukturen des bargeldlosen Zahlungsverkehrs, AcP 186 (1986), 187-236.

Mückl, Stefan, Die Konvergenz der Medien im Lichte des neuen Telemediengesetzes, JZ 2007, 1077-1084. 
Mulch, Joachim, Internet - Konkrete Anforderungen an Informationspflichten der Anbieter, MDR 2007, 309-311.

Müller-Hengstenberg, Claus Dieter, Die Bedeutung des Kaufrechts für Computersoftware, NJW 2000, 3545-3548.

Müller-Hengstenberg, Claus Dieter, Vertragstypologie der Computersoftwareverträge, CR 2004, 161-166.

Müller-Hengstenberg, Claus Dieter/ Kirn, Stefan, Vertragscharakter des Application Service Providing-Vertrags, NJW 2007, 2370-2373.

Müller-Hengstenberg, Claus Dieter/ Krcmar, Helmut, Mitwirkungspflichten des Auftraggebers bei IT-Projekten, CR 2002, 549-557.

Münch, Ingo von/ Kunig, Philip, Grundgesetz-Kommentar, Band 1, Präambel bis Art. 19, 5. Aufl., München 2000 (zitiert als von Münch/Kunig/Bearbeiter).

Musielak, Hans-Joachim, Zivilprozessordnung, Kommentar, 5. Aufl., München 2007 (zitiert als: Musielak/Bearbeiter).

Nordemann, Axel/ Nordemann, Bernd/ Nordemann, Wilhelm, Wettbewerbsrecht Markenrecht, 10. Aufl., Baden-Baden 2004 (zitiert als: Nordemann).

Obergfell, Eva Inés, Das Schutzlandprinzip und „Rom II“, IPRax 2005, 9-13.

Oetker, Hartmut/ Maultzsch, Felix, Vertragliche Schuldverhältnisse, 2. Aufl., Berlin u.a. 2004.

Ohly, Ansgar, Herkunftslandprinzip und Kollisionsrecht, GRUR Int. 2001, 899-908.

Österle, Hubert/ Alt, Rainer/ Heutschi, Roger, WebServices - Hype oder Lösung?, 2003, abrufbar unter: http://de.scientificcommons.org/2558 $<25.11 .2009>$.

Padeck, Ekkehard, Rechtsprobleme bei Schadensfällen in Autowaschanlagen, VersR 1989, 541-556. 
Pahlen-Brandt, Ingrid, Zur Personenbezogenheit von IP-Adressen, K\&R 2008, 288-29.

Palandt, Otto, Bürgerliches Gesetzbuch, Kommentar, 69. Aufl., München 2010 (zitiert als Palandt/Bearbeiter).

Patzak, Andrea, Datenschutzrecht für den E-Commerce, Diss. iur. Universität Wien, Baden-Baden 2006.

Pauli, Dirk, Direktmarketing und die Gewinnung von Kundendaten - Ist die Veranstaltung eines Gewinnspiels ein geeigneter Weg?, WRP 2009, 245-249.

Pawlowski, Hans-Martin, Einführung in die Juristische Methodenlehre, 2. Aufl., Heidelberg 2000.

Petri, B. Thomas, Datenschutzrechtliche Einwilligung im Massengeschäftsverkehr, RDV 2007, 153-158.

Picot, Arnold/ Neuburger, Rahild, Electronic Data Interchange: von EDIFACT zu Webservices, in: Taeger, Jürgen/ Wiebe, Andreas (Hrsg.), Informatik - Wirtschaft - Recht. Regulierung in der Wissensgesellschaft. Festschrift für Wolfgang Kilian zum 65. Geburtstag, Baden-Baden 2004, 513-531 (zitiert als: Picot/Neuburger, in: FS Kilian).

Piper, Henning/ Ohly, Ansgar, Gesetz gegen den unlauteren Wettbewerb, Kommentar, 4. Aufl., München 2006 (zitiert als: Piper/Ohly/Bearbeiter).

Porkert, Kurt, Web-Services - mehr als eine neue Illusion?, Beiträge der Hochschule Pforzheim, Nr. 104, Pforzheim 2002.

Probst, Thomas, Cookies und ihre (unkontrollierten) Alternativen, DSB 6/2007, 11 .

Prokein, Oliver/ Sackmann, Stefan, Der Einsatz von Web Services in deutschen Unternehmen, Ergebnisbroschüre des Projekts EVENT, IIG-Berichte 2/05, abrufbar unter: http://www.telematik.unifreiburg.de/event/pdf/Ergebnisbroschuere_WebServices.pdf <25.11.2009>. 
Püttmann, Friedrich Frank, Rechtliche Probleme der Marktforschung im Internet, K\&R 2000, 492-499.

Raabe, Oliver, Abgrenzungsprobleme bei der rechtlichen Einordnung von Anonymisierungsdiensten im Internet, CR 2003, 268-274.

Raabe, Oliver/ Dinger, Jochen, Telemedienrechtliche Informationspflichten in P2P-Overlay-Netzen und bei Web-Services, CR 2007, 791-797.

Ranke, Johannes S., M-Commerce - Einbeziehung von AGB und Erfüllung von Informationspflichten, MMR 2002, 509-515.

Ranke, Johannes S., M-Commerce und seine rechtsadäquate Gestaltung, Diss. iur. Universität Kassel, Baden-Baden 2004.

Rasmussen, Heike, Datenschutz im Internet, CR 2002, 36-45.

Rasmussen, Heike, Die elektronische Einwilligung im TDDSG, DuD 2002, 406-411.

Räther, Philipp C./ Seitz, Nicolai, Übermittlung personenbezogener Daten in Drittstaaten, MMR 2002, 425-433.

Rauscher, Thomas/Wax, Peter, Wenzel, Joachim, Münchener Kommentar zur

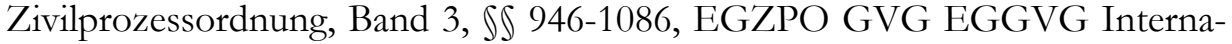
tionales Zivilprozeßrecht, 3. Aufl., München 2008 (zitiert als: MünchKommZPO/Bearbeiter, Bd. 3).

Rebmann, Kurt/ Säcker, Franz Jürgen/ Rixecker, Roland (Hrsg.), Münchner Kommentar zum Bürgerlichen Gesetzbuch, Band 10, Einführungsgesetz zum Bürgerlichen Gesetzbuch (Art. 1-46), Internationales Privatrecht, 4. Aufl., München 2006 (zitiert als: MünchKommBGB/Bearbeiter, Bd. 10).

Redecker, Helmut, Geschäftsabwicklung mit externen Rechnern im Bildschirmtextdienst, NJW 1984, 2390-2394.

Redecker, Helmut, Wer ist Eigentümer von Goethes Werther?, NJW 1992, 1739-1740. 
Redeker, Helmut, Provider-Verträge - ihre Einordnung in die Vertragstypen des BGB, ITRB 2003, 82-86.

Redeker, Helmut, Softwareerstellung und $\ 651$ BGB, CR 2004, 88-91.

Reidenberg, Joel R., Resolving Conflicting International Data Privacy Rules in Cyberspace, Stanford Law Review, Vol. 52, No. 5, Symposium: Cyberspace and Privacy: A New Legal Paradigm? (May, 2000), 1315-1371 (zitiert als: Reidenberg, 52 STNL).

Reithmann, Christoph/ Martiny, Dieter (Hrsg.), Internationales Vertragsrecht, 6. Aufl., Köln 2004 (zitiert als: Bearbeiter, in: Reithmann/Martiny).

Renner, Cornelius, Metatags und Keyword Advertising mit fremden Kennzeichen im Marken- und Wettbewerbsrecht, WRP 2007, 49-54.

Richter, Jan-Peter/ Haller, Harald/ Schrey, Peter, Serviceorientierte Architektur, Informatik Spektrum 2005, 413-416.

Roggenkamp, Jan Dirk, Unanwendbarkeit des Haftungsprivilegs nach TMG auf vorbeugenden Unterlassungsanspruch ("Internet-Versteigerung II"), jurisPR-ITR 11/2007 Anm. 2.

Rössel, Markus, Telemediengesetz, ITRB 2007, 158-162.

Rössel, Markus/ Kruse, Wilhelm, Schadensersatzhaftung bei Verletzung von Filterpflichten, CR 2008, 35-41.

Roßnagel, Alexander, Neues Recht für Multimediadienste - Informations- und Kommunikationsdienste-Gesetz und Mediendienste-Staatsvertrag, NVwZ 1998, 1-8.

Roßnagel, Alexander, Das neue Recht elektronischer Signaturen - Neufassung des Signaturgesetzes und Änderung des BGB und der ZPO, NJW 2001, 18171826.

Roßnagel, Alexander (Hrsg.), Datenschutz beim Online-Einkauf, Braunschweig Wiesbaden 2002 (zitiert als: Bearbeiter, in: Roßnagel, Online-Einkauf). 
Roßnagel, Alexander, Rechtliche Unterschiede von Signaturverfahren, MMR 2002, 215-222.

Roßnagel, Alexander, Die fortgeschrittene elektronische Signatur, MMR 2003, 164-170.

Roßnagel, Alexander (Hrsg.), Handbuch Datenschutzrecht, München 2003 (zitiert als: Roßnagel/Bearbeiter, Hdb. DatenschutzR).

Roßnagel, Alexander (Hrsg.),Technik für Nutzer, Baden-Baden 2004 (zitiert als: Bearbeiter, in: Roßnagel, Technik für Nutzer).

Roßnagel, Alexander (Hrsg.), Recht der Multimedia-Dienste, Kommentar zum IUKDG und zum MDStV, Loseblatt, München Stand April 2005 (zitiert als: Roßnagel/Bearbeiter, RMD).

Roßnagel, Alexander, Datenschutz in der künftigen Verkehrstelematik, NVZ 2006, 281-288.

Roßnagel, Alexander, Das Telemediengesetz, NVwZ 2007, 743-748.

Roßnagel, Alexander/ Banzhaf, Jürgen/ Grimm, Rüdiger, Datenschutz im Electronic Commerce, Heidelberg 2003 (zitiert als: Bearbeiter, in: Roßnagel/ Banzhaf/Grimm).

Roßnagel, Alexander/ Fischer-Dieskau, Stefanie, Elektronische Dokumente als Beweismittel?, NJW 2006, 806-808.

Roßnagel, Alexander/ Laue, Philip, Zweckbindung im Electronic Government, DÖV 2007, 543-549.

Roßnagel, Alexander/ Müller, Jürgen, Ubiquitous Computing - neue Herausforderungen für den Datenschutz, CR 2004, 625-632.

Roßnagel, Alexander/ Pfitzmann, Andreas/ Garstka, Hansjürgen, Modernisierung des Datenschutzrechts, DuD 2001, 253-263.

Roßnagel, Alexander/ Pfitzmann, Andreas/ Garstka, Hansjürgen, Modernisierung des Datenschutzrechts, Gutachten im Auftrag des Bundesministeriums des Inneren, Berlin 2001. 
Roßnagel, Alexander/ Schmücker, Paul (Hrsg.), Beweiskräftige elektronische Archivierung. Bieten elektronische Signaturen Rechtssicherheit?, Heidelberg 2006 (zitiert als: Roßnagel/Schmücker).

Roßnagel, Alexander/ Scholz, Philip, Datenschutz durch Anonymität und Pseudonymität - Rechtsfolgen der Verwendung anonymer und pseudonymer Daten, MMR 2000, 721-731.

Roth-Stielow, Klaus, Grundgesetz und Rechtsanwendung, München 1972.

Rücker, Daniel, Notice and take down-Verfahren für die deutsche Providerhaftung?, CR 2005, 347-355.

Sachs, Michael (Hrsg.), Grundgesetz, Kommentar, 4 Aufl., München 2007 (zitiert als: Sachs/Bearbeiter).

Sack, Rolf, Das internationale Wettbewerbs- und Immaterialgüterrecht nach der EGBGB-Novelle, WRP 2000, 269-289.

Sack, Rolf, Herkunftslandprinzip und internationale elektronische Werbung nach der Novellierung des Teledienstegesetzes (TDG), WRP 2002, 271-283.

Säcker, Franz Jürgen, Berliner Kommentar zum Telekommunikationsgesetz, Frankfurt am Main 2006 (zitiert als: BerlKommTKG/Bearbeiter).

Säcker, Franz Jürgen/ Rixecker, Roland, Münchner Kommentar zum Bürgerlichen Gesetzbuch, Band 1, Allgemeiner Teil, 1. Halbband $\int S$ 1-240, ProstG, 5.Aufl., München 2006 (zitiert als: MünchKommBGB/Bearbeiter, Bd. 1/1).

Säcker, Franz Jürgen/ Rixecker, Roland (Hrsg.), Münchner Kommentar zum Bürgerlichen Gesetzbuch, Band 2, Schuldrecht Allgemeiner Teil, SS 241-432, 5. Aufl., München 2007 (zitiert als: MünchKommBGB/Bearbeiter, Bd. 2).

Säcker, Franz Jürgen/ Rixecker, Roland (Hrsg.), Münchner Kommentar zum Bürgerlichen Gesetzbuch, Band 5, Schuldrecht Besonderer Teil III, SS 705853, Partnerschaftsgesellschaftsgesetz, Produkthaftungsgesetz, 5. Aufl., München 2009 (zitiert als: MünchKommBGB/Bearbeiter, Bd. 5).

Säcker, Franz Jürgen/ Rixecker, Roland (Hrsg.), Münchner Kommentar zum Bürgerlichen Gesetzbuch, Band 4, Schuldrecht Besonderer Teil II, \$S 611-704, 
EFZG, TzBfG, KSchG, 5. Aufl., München 2009 (zitiert als: MünchKommBGB/Bearbeiter, Bd. 4).

Saenger, Ingo, Zivilprozessordnung, Handkommentar, Baden-Baden 2006 (zitiert als: Hk-ZPO/Bearbeiter).

Schaar, Peter, Persönlichkeitsprofile im Internet, DuD 2001, 383-388.

Schaar, Peter, Datenschutzrechtliche Einwilligung im Internet, MMR 2001, 644-648.

Schaar, Peter, Datenschutz im Internet, München 2002.

Schaar, Peter, Datenschutz bei Web-Services, RDV 2003, 59-67.

Schallbruch, Martin, E-Government: Der Staat als Nachfrager und Anbieter, in: Büchner, Wolfgang/ Büllesbach, Alfred (Hrsg.), E-Government, Köln 2003, 1-13.

Schild, Hans-Hermann, Die EG-Datenschutz-Richtlinie, EuZW 1996, 549555.

Schirmbacher, Martin, Metatags und Keyword-Advertising - Zulässigkeit von Metatags und Keywords in der Onlinewerbung, ITRB 2007, 117-118.

Schmidl, Michael, Softwareerstellung und \651 BGB - ein Versöhnungsversuch, MMR 2004, 590-593.

Schmidt, Reimer, Rationalisierung und Privatrecht, AcP 166 (1966), 1-29.

Schmidt-Bleibtreu, Bruno/ Hofmann, Hans/ Hopfauf, Axel, GG - Kommentar zum Grundgesetz, 11. Aufl., Köln München 2008 (zitiert als: SchmidtBleibtreu/Hofmann/Hopfauf/Bearbeiter).

Schmitt, Johannes/ Ackermann, Ralf/ Görtz, Manuel/ Steinmetz, Ralf, VoIPSicherheit - Status quo und neue Aspekte, Darmstadt 2006, abrufbar unter: ftp://ftp.kom.e-technik.tu-darmstadt.de/pub/papers/SAGS06-1-paper.pdf $<25.11 .2009>$. 
Schmitz, Florian/ Laun, Stefan, Haftung kommerzieller Meinungsportale im Internet, MMR 2005, 208-213.

Schmitz, Peter, TDDSG und das Recht auf informationelle Selbstbestimmung, Diss. iur. Universität Mainz, München 2000.

Schmitz, Peter, Datenschutzgerechte Gestaltung von AGB für TelemediaDienste, DuD 2001, 395-399.

Schmitz, Peter/ Eckhardt, Jens, AGB - Einwilligung in Werbung, CR 2006, 533-539.

Schmitz, Peter/ Eckhardt, Jens, Einsatz von RFID nach dem BDSG, CR 2007, 171-177.

Schneider, Annette, Verträge über Internet-Access. Typisierung der Basisverträge mit nicht-kommerziellen Anwendern, München 2001.

Schneider, Jochen, Handbuch des EDV-Rechts, 4. Aufl., München 2009 (zitiert als: Schneider, Hdb. EDV-Recht).

Schneider, Jochen, IT-Vertragsrecht, CR 2005, 695-700.

Schneider, Jochen/ Westphalen, Friedrich Graf von (Hrsg.), SoftwareErstellungsverträge, Köln 2006 (zitiert als: Bearbeiter, in: Schneider/von Westphalen).

Scholz, Philip, Datenschutz beim Internet-Einkauf, Diss. iur. Universität Kassel, Baden-Baden 2003.

Schöttle, Hendrik, Anwaltliche Internet-Rechtsberatung und das Teledienstedatenschutzgesetz, BRAK-Mitt 2004, 253-257.

Schöttler, Ingo, Das neue Telemediengesetz (Teil 2) - Die Informationspflichten der Diensteanbieter, jurisPR-ITR 5/2007 Anm. 6.

Schultze-Melling, Jyn, IT-Sicherheit in der anwaltlichen Beratung, CR 2005, 73-80. 
Schulze, Reiner/ Ebers, Martin, Streitfragen im neuen Schuldrecht, JuS 2004, 462-468.

Schumer, Christian, Design Flow Management mit Web Services in der Mikrotechnik, Diss. rer. nat. Universität Siegen, Siegen 2004, abrufbar unter:

http://www.ub.uni-siegen.de/pub/diss/fb12/2004/schumer/schumer.pdf $<25.11 .2009>$.

Schuppert, Stefan/ Greissinger, Christian, Gebrauchthandel mit Softwarelizenzen, CR 2005, 81-87.

Schuster, Fabian, Der Telekommunikationsvertrag (Festnetz, Internet, Mobilfunk), CR 2006, 444-453.

Schwarz, Mathias/ Peschel-Mehner, Andreas, Recht im Internet, Kommentar, Loseblatt, Augsburg Stand Oktober 2002 (zitiert als: Bearbeiter, in: Schwarz/Peschel-Mehner, Recht im Internet).

Schweinoch, Martin/ Roas, Rudolf, Paradigmenwechsel für Projekte: Vertragstypologie der Neuerstellung von Individualsoftware, CR 2004, 326-331.

Schwenk, Jörg, Sicherheit und Kryptographie im Internet, 2. Aufl., Wiesbaden 2005.

Selk, Robert, Datenschutz und Internet: das TDDSG und dessen Rolle für ein modernes Datenschutzrecht, dargestellt anhand der Technik der Cookies, Diss. iur. Universität Augsburg, Osnabrück 2003.

Senn, Alina/ Schweighofer, Erich/ Liebwald, Doris/ Geist, Anton/ Drachsler, Mathias, LOIS: Erfahrungen und Herausforderungen bei der Weiterentwicklung multilingualer Rechtsontologien, in: Schweighofer, Erich/ Liebwald, Doris/ Drachsler, Mathias/ Geist, Anton (Hrsg.), e-Staat und e-Wirtschaft aus rechtlicher Sicht, Tagungsband des 9. Internationalen Rechtsinformatik Symposions IRIS 2006, Stuttgart u.a. 2006, 393-396 (zitiert als: Senn/Schweighofer/Liebwald/Geist/Drachsler, IRIS 2006).

Simitis, Spiros, Die EU-Datenschutzrichtlinie - Stillstand oder Anreiz?, NJW 1997, 281-288. 
Simitis, Spiros (Hrsg.), Bundesdatenschutzgesetz, 6. Aufl., Baden-Baden 2006 (zitiert als: Simitis/Bearbeiter).

Speck, Andreas, Entwicklungsperspektiven des E-Commerce, in: Hoffmann, Mathis/ Leible, Stefan/ Sosnitza, Olaf, Vertrag und Haftung im E-Commerce, Stuttgart u.a. 2006, 9-27 (zitiert als: Speck, in: Hoffmann/Leible/Sosnitza).

Spiegel, Gerald, Spuren im Netz, DuD 2003, 265-269.

Spindler, Gerald, Haftungsrechtliche Grundprobleme der neuen Medien, NJW 1997, 3193-3199.

Spindler, Gerald, Verschuldensabhängige Produkthaftung im Internet, MMR 1998, 23-30.

Spindler, Gerald, Störerhaftung im Internet, K\&R 1998, 177-183.

Spindler, Gerald, Das Jahr 2000-Problem in der Produkthaftung: Pflichten der Hersteller und der Softwarenutzer, NJW 1999, 3737-3745.

Spindler, Gerald (Hrsg.), Vertragsrecht der Telekommunikationsanbieter, Köln 2000 (zitiert als: Bearbeiter, in: Spindler, Vertragsrecht TK-Anbieter).

Spindler, Gerald, Das Herkunftslandprinzip im neuen Teledienstegesetz, RIW 2002, 183-188.

Spindler, Gerald, Verantwortlichkeit und Haftung für Hyperlinks im neuen Recht, MMR 2002, 495-503.

Spindler, Gerald, Das Gesetz zum elektronischen Geschäftsverkehr - Verantwortlichkeit der Diensteanbieter und Herkunftslandprinzip, NJW 2002, 921927.

Spindler, Gerald, Herkunftslandprinzip und Kollisionsrecht - Binnenmarktintegration ohne Harmonisierung?, RabelsZ 66 (2002), 633-709.

Spindler, Gerald, Neues im Vertragsrecht der Internet-Provider, CR 2004, 203-213. 
Spindler, Gerald, Die Verantwortlichkeit der Provider für „Sich-zu-Eigengemachte“ Inhalte und für beaufsichtigte Nutzer, MMR 2004, 440-444.

Spindler, Gerald, IT-Sicherheit und Produkthaftung - Sicherheitslücken, Pflichten der Hersteller und der Softwarenutzer, NJW 2004, 3145-3150.

Spindler, Gerald (Hrsg.), Vertragsrecht der Internet-Provider, 2. Aufl., Köln 2004 (zitiert als: Bearbeiter, in: Spindler, Vertragsrecht Internet-Provider).

Spindler, Gerald, Verantwortlichkeit eines Plattformbetreibers für fremde Inhalte, JZ 2005, 37-40.

Spindler, Gerald, Haftungs- und vertragsrechtliche Probleme von WebServices, DuD 2005, 139-141.

Spindler, Gerald, Haftung und Verantwortlichkeit im IT-Recht, CR 2005, 741747.

Spindler, Gerald, Das neue Telemediengesetz - Konvergenz in sachten Schritten, CR 2007, 239-245.

Spindler, Gerald, Verantwortlichkeit von IT-Herstellern, Nutzern und Intermediären, Studie im Auftrag des BSI, 2007, abrufbar unter: www.bsi.bund.de $<25.11 .2009>$ (zitiert als: Spindler, Gutachten BSI).

Spindler, Gerald/ Börner, Fritjof (Hrsg.), E-Commerce-Recht in Europa und den USA, Berlin u.a. 2003 (zitiert als: Bearbeiter, in: Spindler/Börner).

Spindler, Gerald/ Klöhn, Lars, Neue Qualifikationsprobleme im E-Commerce, CR 2003, 81-86.

Spindler, Gerald/ Nink, Judith, Verträge via Internetauktionen, DRiZ 2007, 193-197.

Spindler, Gerald/ Schmitz, Peter/ Geis, Ivo, TDG - Teledienstegesetz, Teledienstedatenschutzgesetz, Signaturgesetz, Kommentar, München 2004 (zitiert als: Spindler/Schmitz/Geis/Bearbeiter).

Spindler, Gerald/ Schuster, Fabian, Recht der elektronischen Medien, Kommentar, München 2008 (zitiert als: Spindler/Schuster/Bearbeiter). 
Spindler, Gerald/ Volkmann, Christian, Die Zivilrechtliche Störerhaftung der Internet-Provider, WRP 2003, 1-15.

Spindler, Gerald/ Wiebe, Andreas, Internetauktionen und elektronische Marktplätze, 2. Aufl., Köln 2005 (zitiert als: Bearbeiter, in: Spindler/Wiebe).

Stacke, Rolf B., Der minderjährige Schwarzfahrer: Sind ihm wirklich Tür und Tor geöffnet?, NJW 1991, 875-878.

Stadler, Thomas, Verantwortlichkeit für Hyperlinks nach der Neufassung des TDG, JurPC Web-Dok. 2/2003, Abs. 1-95.

Stadler, Thomas, Haftung für Informationen im Internet, 2. Aufl., Berlin 2005.

Stadler, Tobias, Mobiles Bezahlen, Diss. iur. Universität Kassel, Baden-Baden 2006.

Staudinger, Julius von, Kommentar zum Bürgerlichen Gesetzbuch mit Einführungsgesetz und Nebengesetzen, Buch 1 Allgemeiner Teil, $\int \$$ 90-133; \$\$ 1-54, 63 - Beurkundungsgesetz, Berlin 2004 (zitiert als: Staudinger/Bearbeiter).

Staudinger, Julius von, Kommentar zum Bürgerlichen Gesetzbuch mit Einführungsgesetz und Nebengesetzen, Buch 1 Allgemeiner Teil, Einleitung zu \$S

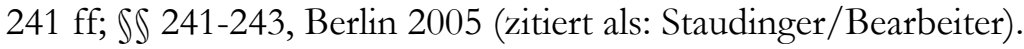

Staudinger, Julius von, Kommentar zum Bürgerlichen Gesetzbuch mit Einführungsgesetz und Nebengesetzen, Buch 2 Recht der Allgemeinen Geschäftsbedingungen, $\iint$ 305-310, Berlin 2006 (zitiert als: Staudinger/Bearbeiter).

Staudinger, Julius von, Kommentar zum Bürgerlichen Gesetzbuch mit Einführungsgesetz und Nebengesetzen, Buch 2 Recht der Schuldverhältnisse, \S 311, 311a, 312, 312a-f, Berlin 2005 (zitiert als: Staudinger/Bearbeiter).

Staudinger, Julius von, Kommentar zum Bürgerlichen Gesetzbuch mit Einführungsgesetz und Nebengesetzen, Buch 2 Recht der Schuldverhältnisse, $\$ \$ 631$ 651, Berlin 2003 (zitiert als: Staudinger/Peters).

Staudinger, Julius von, Kommentar zum Bürgerlichen Gesetzbuch mit Einführungsgesetz und Nebengesetzen, Buch 2 Recht der Schuldverhältnisse, $\int \mathbb{S}$ 651a-651m, Berlin 2003 (zitiert als: Staudinger/Bearbeiter). 
Staudinger, Julius von, Kommentar zum Bürgerlichen Gesetzbuch mit Einführungsgesetz und Nebengesetzen, Buch 2 Recht der Schuldverhältnisse, $\iiint 823$ 825, Berlin 1999 (zitiert als: Staudinger/Bearbeiter).

Staudinger, Julius von, Kommentar zum Bürgerlichen Gesetzbuch mit Einführungsgesetz und Nebengesetzen, Buch 3 Sachenrecht, $\int \mathbb{I}$ 985-1011 - (Eigentum 3), Berlin 2006 (zitiert als: Staudinger/Bearbeiter).

Staudinger, Julius von, Kommentar zum Bürgerlichen Gesetzbuch mit Einführungsgesetz und Nebengesetzen, EGBGB/IPR. Einführungsgesetz zum Bürgerlichen Gesetzbuche/IPR, Art. 27-37 EGBGB; Anhänge - (Internationales Vertragsrecht) -, Berlin 2002 (zitiert als: Staudinger/Bearbeiter).

Steinbeck, Anja, Übertriebenes Anlocken, psychischer Kaufzwang etc. ... gibt es sie noch?, GRUR 2005, 540-547.

Stelkens, Paul/ Bonk, Heinz Joachim/ Sachs, Michael (Hrsg.), Verwaltungsverfahrensgesetz, 7. Aufl., München 2008 (zitiert als: Stelkens/Bonk/Sachs/ Bearbeiter).

Stichtenoth, Jonas, Softwareüberlassungsverträge nach dem Schuldrechtsmodernisierungsgesetz, K\&R 2003, 105-110.

Stögmüller, Thomas, Markenrechtliche Zulässigkeit kontext-sensitiver Werbung im Internet, CR 2007, 446-453.

Stoykova, Daniela, Webanwendungen über XML-Spezifikationen - Verdeutlicht am Beispiel eines holländischen Auktionssystems, Diss. rer. oec. Universität Heidelberg, Heidelberg 2005, abrufbar unter: http://deposit.ddb.de/cgibin $/$ dokserv?idn $=975224247<25.11 .2009>$.

Streinz, Rudolf (Hrsg.), EUV/EGV, Kommentar, München 2003 (zitiert als: Streinz/Bearbeiter).

Stürner, Michael, Access-Provider-Vertrag als Dienstvertrag, jurisPRBGHZivilR 34/2005 Anm. 4.

Stürner, Rolf, Der lediglich rechtliche Vorteil, AcP 173 (1973), 402-449. 
Taylor, Ian J., From P2P to Web Services and Grids. Peers in a Client/Server World, London 2005.

Tettenborn, Alexander, E-Commerce-Richtlinie: Politische Einigung in Brüssel erzielt, K\&R 2000, 59-63.

Thewalt, Stephan, Softwareerstellung als Kaufvertrag mit werkvertraglichem Einschlag, CR 2002, 1-7.

Thorn, Karsten, Internationale Produkthaftung des Zulieferers, IPRax 2001, 561-567.

Tiedtke, Klaus/ Burgmann, Roland,Gewährleistungs- und Haftungsausschluss beim Verkauf gebrauchter Sachen an und zwischen Verbrauchern, NJW 2005, 1153-1157.

Tinnefeld, Marie-Theres, Die Novellierung des BDSG im Zeichen des Gemeinschaftsrechts, NJW 2001, 3078-3083.

Tinnefeld, Marie-Theres/ Ehmann, Eugen/ Gerling, Rainer W., Einführung in das Datenschutzrecht, 4. Aufl., München Wien 2005.

Tosques, Fabio/ Mayr, Philipp, Web Services - Einsatzmöglichkeiten für das Information Retrieval im WWW, Proceedings der 27. Online Tagung der DGI in Frankfurt am Main, abrufbar unter:

http://www.ib.hu-berlin.de/ mayr/arbeiten/tosques_mayr_dgi05.pdf $<25.11 .2009>$.

Ufer, Frederic, Die Haftung der Internet Provider nach dem Telemediengesetz, Hamburg 2007.

Ullmann, Eike (Hrsg.), Juris Praxis Kommentar UWG, Saarbrücken 2006 (zitiert als: jurisPK-UWG/Bearbeiter).

Ullmann, Eike, Wer sucht, der findet - Kennzeichenverletzung im Internet, GRUR 2007, 633-639.

Ulmer, Peter/ Brandner, Hans Erich/ Hensen, Horst-Diether, AGB-Recht, Kommentar zu den $\iint$ 305-310 BGB und zum Unterlassungsklagegesetz, 10. Aufl., Köln 2006 (zitiert als: Ulmer/Brandner/Hensen/Bearbeiter). 
Ulrici, Bernhard, Geschäftsähnliche Handlungen, NJW 2003, 2053-2056.

Volkmann, Christian, Die Unterlassungsvollstreckung gegen Störer aus dem Online-Bereich, CR 2003, 440- 447.

Volkmann, Christian, Haftung des Internet-Auktionsveranstalters für markenverletzende Inhalte Dritter, K\&R 2004, 231-233.

Volkmann, Christian, Der Störer im Internet, Diss. iur. Universität Göttingen, München 2005.

Volkmann, Christian, Aktuelle Entwicklungen in der Providerhaftung im Jahr 2008, K\&R 2009, 361-368.

Volkmann, Christian, Aktuelle Entwicklungen in der Providerhaftung im Jahr 2005, K\&R 2006, 245-252.

Voigt, Paul, Datenschutz bei Google, MMR 2009, 377-382.

Wagner, Rolf, Der Regierungsentwurf eines Gesetzes zum Internationalen Privatrecht für außervertragliche Schuldverhältnisse und für Sachen, IPRax 1998, 429-438.

Wahli, Ueli/ Burroughs, Owen/ Cline, Owen/ Go, Alec/ Tung, Larry, Web Services Handbook for WebSphere Application Server Version 6.1, IBM Redbooks, September 2006, abrufbar unter:

http://www.redbooks.ibm.com/redbooks/pdfs/sg247257.pdf <25.11.2009>.

Wallenberg, Gabriela von, B2B-Onlineshop betriebswirtschaftliche und rechtliche Fragen, MMR 2005, 661-666.

Walpert, Karsten, Analyse von Middleware-Technologien bzgl. Performanz und ihrer Eignung für lose und eng gekoppelte verteilte Anwendungen, Diss. rer. nat. Universität Mainz, Mainz 2005.

Weber, Rolf H./ Willi, Annette, IT-Sicherheit und Recht, Zürich Basel Genf 2006.

Wegmann, Henning, Anforderungen an die Einwilligung in Telefonwerbung nach dem UWG, WRP 2007, 1141-1145. 
Weichert, Thilo, Datenschutzrechtliche Anforderungen an Data-WarehouseAnwendungen bei Finanzdienstleistern, RDV 2003, 113-122.

Wente, Jürgen K., Das Recht der juristischen Recherche: ein Beitrag zum Konflikt zwischen den Medienfreiheiten und der informationellen Selbstbestimmung, Diss. iur. Universität Göttingen, Baden-Baden 1987.

Westphalen, Friedrich Graf von, AGB-Recht im Jahr 2006, NJW 2007, 22282236.

Wichert, Michael, Web-Cookies - Mythos und Wirklichkeit, DuD 1998, 273276.

Wichmann, Thorsten, Web Services für Endkunden: eine realistische Vision?, Spotlight vom 6.3.2002, abrufbar unter:

http://www.berlecon.de/research/spotlights.php?we_objectID $=90$ $<25.11 .2009>$.

Wiebe, Andreas, Die elektronische Willenserklärung, Habilitationsschrift Universität Hannover, Tübingen 2002.

Wiebe, Andreas/ Leupold, Andreas, Recht der elektronischen Datenbanken, Loseblatt, Heidelberg Stand 2003 (zitiert als: Bearbeiter, in: Wiebe/Leupold).

Wiehler, Gerhard, Mobility, Security und Web Services, Erlangen 2004.

Wiesner, Elisabeth, Datenschutzrechtliche Einwilligung zur Werbung: Opt-out ausreichend?, DuD 2007, 604-607.

Woitke, Thomas, Web-Bugs - Nur lästiges Ungeziefer oder datenschutzrechtliche Bedrohung?, MMR 2003, 310-314.

Woitke, Thomas, Das „Wie“ der Anbieterkennzeichnung gemäß \6 TDG, NJW 2003, 871-873.

Worm, Ulrich, Die Verletzung von Urheberrechten und gewerblichen Schutzrechten durch das Setzen von Hyperlinks, Inline-Frames und Meta-Tags, Frankfurt am Main u.a. 2002. 
Wülfing, Thomas/ Dieckert, Ulrich, Praxishandbuch Multimediarecht, Berlin Heidelberg 2002.

Zscherpe, Kerstin, Anforderungen an die datenschutzrechtliche Einwilligung, MMR 2004, 724-727.

Zscherpe, Kerstin, Datenschutz im Internet - Grundsätze und Gestaltungsmöglichkeiten für Datenschutzerklärungen, K\&R 2005, 264-269. 




\section{Teil Einleitung und Problemstellung}

\section{A. Einführung}

Informations- und Kommunikationstechnologien unterliegen dem ständigen Fortschritt der Technik. Das World Wide Web (WWW) bietet zahlreiche Informationsdienste, die sich an den individuellen Bedürfnissen des Nutzers orientieren. Diese Dienste werden zunehmend automatisiert. Gerade im gewerblichen Bereich, dem Ausgangspunkt der Informationsverbreitung, spielt die Möglichkeit der Automatisierung eine große Rolle. Anwendungen zwischen Unternehmern (so genannte Business to Business - B2B - Anwendungen) beruhen indes meist noch auf manuell vereinbarten Schnittstellen und Nachrichtenformaten zwischen den einzelnen Geschäftspartnern, die für jeden Geschäftspartner neu implementiert werden müssen. ${ }^{1}$ Diese Praxis ist nicht nur unflexibel sondern auch zeit- und kostenintensiv. Insbesondere kleinere und mittelständische Unternehmen können sich derartige Anpassungen häufig nicht leisten. Standardisierte Anwendungen sind daher wünschenswert. Eine solche standardisierte Technik zur automatisierten Nutzung von Diensten sind Serviceorientierte Architekturen. Sie stellen allerdings nur ein abstraktes Architekturkonzept dar, das einer konkreten technischen Umsetzung bedarf. Eines der vielversprechendsten Umsetzungskonzepte erfolgt derzeit durch Web Services. ${ }^{2}$ Unter Web Services werden unabhängige, sich selbst

1 Erläuterung von Web Services im Informatiklexikon der GI, abrufbar unter: http://www.giev.de/service/informatiklexikon/informatiklexikon-detailansicht/meldung/95/ <25.11.2009>.

2 Melzer, S. vii. 
beschreibende Anwendungen verstanden, die zum einen innerhalb geschlossener Systeme (zum Beispiel Intranets) und zum anderen in offenen Systemen (dem Internet) publiziert und genutzt werden können. Sie ermöglichen Unternehmen, Medienbrüche vollständig zu vermeiden und eröffnen dem Nutzer die Möglichkeit einer vollautomatisierten Dienstenutzung. Unternehmen können damit verteilte Anwendungen entwickeln und nutzen, ohne sich um Hardware, Systemplattformen, Programmiersprachen und Netz-Typologien der verschiedenen involvierten Bereiche und Geschäftspartner kümmern zu müssen. ${ }^{3}$ Dadurch wird zum einen ein Höchstmaß an Flexibilität gewährleistet und zum anderen Zeit- und Kostenersparnisse erzielt. Web Services ermöglichen damit, vereinfacht ausgedrückt, eine Implementierung verschiedenster Dienste und somit „Interoperabilität für die Massen“"4.

Durch den Einsatz von Serviceorientierten Architekturen wird es Unternehmen möglich, neben der Einbindung ihrer eigenen unternehmensinternen Dienste auch Dienste externer Anbieter in ihre Arbeitsprozesse zu integrieren. Eine typische Landschaft aus Serviceorientierten Architekturen besteht aus vielen kleinen Systemen, die alle einen spezialisierten Dienst (mit Hilfe von Web Services) anbieten, der von ganz verschiedenen Abnehmern genutzt werden kann. ${ }^{5}$ Idealerweise entsteht dadurch ein beinah unerschöpfliches Angebot an Diensten, aus denen man sich dann genau den Dienst aussuchen kann, den man benötigt. ${ }^{6}$ In dieser Idealkonstellation müssen Unternehmen nicht mehr kostenintensive neue Dienste für Prozesse entwickeln und implementieren, sondern können auf bereits vorhandene externe Dienste zugreifen und diese wieder abstoßen, wenn der Bedarf gedeckt ist. Das spart Zeit und senkt vor allem die IT-Kosten des Unternehmens.

Aktuell nutzen viele Unternehmen die Serviceorientierte Architektur noch bevorzugt innerhalb ihrer eigenen „Vertrauenszirkel““. ${ }^{7}$ Dies führt zwar bereits zu einem gewissen $\mathrm{Maß}$ an Kostenersparnissen, eine Idealnutzung der Serviceorientierten Architektur stellt es aber noch nicht dar. Erheblich mehr Flexibilität und Kostenersparnisse wären möglich, wenn Dienste aus der ganzen virtuellen Welt genutzt werden könnten. Das klassische Beispiel für eine sol-

3 Wiebler, S. 13.

4 Küster, in: Fröschle, S. 6.

5 Bitr/Reimer, DuD 2007, 638.

6 Bitz/Reimer, DuD 2007, 638.

7 Bitz/Reimer, DuD 2007, 638. 
che Web Service-Nutzung, das bereits praktisch umgesetzt wird, ${ }^{8}$ sind Reiseportale. Dabei bieten Fluggesellschaften, Hotelanbieter, Reiseversicherungen etc. eigene Web Services an, mit Hilfe derer auf die jeweiligen Dienste (Flüge, Hotelzimmer, Versicherungen) des jeweiligen Unternehmens zugegriffen und eine Buchung vorgenommen werden kann. Reiseportale erbringen diese Leistungen regelmäßig nicht selber. Sie können vielmehr die Web Services der Anbieter durch eine Schnittstelle in das eigene Portal implementieren, so dass Kunden auf dem Portal die Zusammenstellung einer individuellen Reise ermöglicht wird. Idealerweise kann das Reiseportal in einem externen Verzeichnisdienst (UDDI) aus einer Vielzahl derartiger registrierter Dienste wählen und sich so ohne individuelle Vertragsabsprachen die passendsten und gegebenenfalls preisgünstigsten Dienste aussuchen.

Erfolgt der Einsatz Serviceorientierter Architekturen mit Web Services lediglich in Unternehmen oder zwischen bestimmten kooperierenden Unternehmen und damit innerhalb so genannter Vertrauenszirkel, so werden die sich aus der automatisierten Funktionsweise und verteilten Anwendung von Serviceorientierten Architekturen mit Web Services ergebenden Fragen und Problemstellungen meist durch individuelle vertragliche Vereinbarungen der Unternehmen überlagert. Aus diesem Grund wird in der vorliegenden Arbeit der Einsatz Serviceorientierter Architekturen zwischen Unternehmensnetzwerken ausgeklammert. Der Schwerpunkt dieser Untersuchung liegt vielmehr auf dem freien, partnerübergreifenden Einsatz Serviceorientierter Architekturen mit Web Services im World Wide Web.

Die vorliegende Untersuchung befasst sich mit den rechtlichen Rahmenbedingungen der Serviceorientierten Architektur mit Web Services. Dabei wird insbesondere versucht, für diese noch sehr junge Architektur sowie die Web Service-Technik Klarheit und Rechtssicherheit sowohl für die IT-Anwender als auch den Rechtsanwender, der sich mit diesbezüglichen Problemen beschäftigen muss, zu schaffen.

Aus dem partnerübergreifenden verteilten Einsatz Serviceorientierter Architekturen mit Web Services und der Automatisierung der Abläufe resultieren bisher weitestgehend ungeklärte Fragestellungen. ${ }^{9}$ Sowohl für Endkunden als

$8 \quad$ S. nur lastminute.de, abrufbar unter: www.lastminute.de $<25.11 .2009>$.

9 Einen kurzen Einblick hinsichtlich der Probleme beim Vertragsschluss und Haftungsfragen geben Spindler, DuD 2005, 139 ff. u. Koch, ITRB 2007, 71 ff. Einen Einstieg in - allerdings eher sehr allgemeine - datenschutzrechtliche Fragestellungen gibt Schaar, RDV 2003, 59 ff. Aus der Technik resultierende Fragen wirft Speck, in: Hoffmann/Leible/Sosnitza, 9 ff. auf, allerdings ohne Lösungsmöglichkeiten zu bieten. 
auch für Web Service-Nutzer (wie das Reiseportal) stellt sich die Frage, wie sich die Nutzung und Verwendung von Web Services auf Vertragsverhältnisse auswirkt, welche Probleme durch die automatisierten Ablaufe auftreten können und was die Beteiligten tun können, um etwaige Probleme zu vermeiden. Darüber hinaus stellt sich die Frage: welcher der Beteiligten für eventuelle rechtswidrige Handlungen haftet. Dabei spielt insbesondere die Problematik der immer noch aktuellen Diskussion um die Störerhaftung im Internet eine besondere Rolle. ${ }^{10}$ Auch Aspekte zum Umgang mit personenbezogenen Daten, die mit Hilfe der Web Services in großem Umfang automatisiert erhoben und verwendet werden können, spielen eine wichtige Rolle.

Um dem Rechtsanwender den Einstieg in die Materie zu erleichtern und Grundlagen für die folgende rechtliche Untersuchung zu schaffen, werden im 1. Teil die technischen Grundlagen der Serviceorientierten Architektur und Web Services erläutert. Verdeutlicht wird dabei zunächst, was überhaupt unter dem abstrakten Begriff der Serviceorientierten Architektur sowie der Web Service-Technik zu verstehen ist und welche Akteure daran beteiligt sind (B.I.). Zum genaueren Verständnis und vor allem für die rechtliche Qualifikation ist es erforderlich, dafür auch auf die technischen Komponenten und die Funktionsweise von Web Services einzugehen (B.II.). Um Web Services für den Rechtsanwender greifbarer zu machen, wird am Ende des 1. Teils ein Überblick über aktuelle und mögliche Einsatzbereiche von Web Services gegeben (B.III.).

Teil 2 widmet sich den zivilrechtlichen Aspekten der Serviceorientierten Architektur. Dabei werden neben den unmittelbar an der Serviceorientierten Architektur Beteiligten (Web Service-Anbieter, Web Service-Vermittler und Web Service-Nutzer) auch die nicht unmittelbar innerhalb der Serviceorientierten Architektur agierenden, aber doch mit dem Web Service verbundenen oder in Kontakt kommenden, Web Service-Hersteller und Endkunden (Dritter) in die Untersuchung einbezogen. Im Fokus der Untersuchung stehen dabei in Abschnitt A. die Verträge zwischen den Akteuren (III.1. und III.2.) und die daraus resultierenden Rechte (III.5. und III.7.), Pflichten (III.4. und III.6.) sowie die zu beachtenden Sicherheitsaspekte (III. 2.b)) im Umgang mit Web Services; auch hinsichtlich der Erzeugung von Vertrauen in die automatisierte Kommunikation mit Web Services.

10 S. zur Diskussion um die wettbewerbsrechtliche Störerhaftung nur den jüngsten Aufsatz von Köbler, GRUR 2008, 1 ff. 
Abschnitt B. beschäftigt sich demgegenüber mit der außervertraglichen Haftung der an der Serviceorientierten Architektur beteiligten Akteure. Dabei stehen Missbrauchsmöglichkeiten beziehungsweise mögliche Fehlfunktionen des UDDI (III.), als zentrales Medium zur Publikation und Suche von Web Services, und deren rechtliche Behandlung im Vordergrund. Schwerpunkte bilden die kennzeichen- und wettbewerbsrechtliche Beurteilung einer UDDIManipulation durch den Web Service-Anbieter sowie eine etwaige Haftung des Web Service-Vermittlers (III.2.) sowie die Frage der Haftung des Web Service-Nutzers (V.) für rechtswidrige Handlungen durch den Web ServiceAnbieter. Ferner behandelt der Abschnitt die Haftungsmöglichkeiten bei einem, im Falle eines durch einen fehlerhaft agierenden Web Service entstehenden, Schadens und gibt einen Überblick über kartellrechtliche Probleme, die bei der Beteiligung der öffentlichen Hand auftreten können (IV.).

Die datenschutzrechtlichen Rahmenbedingungen bilden den Kern des 3. Teils. Dort ruht der Fokus auf Portalen, die es (externen) Web Service-Anbietern ermöglichen, ihre Dienste mittels Web Services an das Portal zu koppeln und so den Portalnutzern ihre Dienste zu offerieren. Neben Web Servicespezifischen Besonderheiten hinsichtlich des Umgangs mit personenbezogenen Daten, erfolgen im 3. Teil auch allgemeine Hinweise zur datenschutzkonformen Ausgestaltung derartiger Portale.

\section{B. Grundlagen}

I. Begrifflichkeiten

Grundlegend für diese Arbeit sind die Klärung des Begriffs, der Entwicklung und des Umfelds von Web Services, sowie die an der Nutzung und Bereitstellung des Web Service Beteiligten.

\section{Web Services}

Web Services sind Softwarebausteine, über deren offene Kommunikationsschnittstelle Informationen oder Dienste plattform-, programm- und standortunabhängig abgerufen und genutzt werden können. ${ }^{11}$ Der Begriff des Web Service beruht auf der Architektur: Web Services sind im Allgemeinen Dienstleistungen, die mittels einer browserbasierten Software-Anwendung

11 Picot/Neuburger, in: FS Kilian, S. 527; ferner Walpert, S. 221 u. Stoykova, S. 203. 
erbracht werden. ${ }^{12}$ Sie ermöglichen den automatischen Datenaustausch zwischen verteilt verfügbaren Software-Bausteinen, ohne eine direkte Interaktion menschlicher Nutzer vorauszusetzen. Eine einheitliche, konsistente und standardisierte Begriffsdefinition existiert zwar noch nicht, die unterschiedlichen Arbeitsdefinitionen weisen aber gemeinsame Beschreibungselemente auf.

Microsoft zum Beispiel definiert in ihrer Online-Bibliothek Web Services wie folgt:

„Web services are applications that use standard transports, encodings, and protocols to exchange information. With broad support across vendors and businesses, Web services enable computer systems on any platform to communicate over corporate intranets, extranets, and across the Internet with support for end-to-end security, reliable messaging, distributed transactions, and more."13

In einer Kurzdefinition beschreibt IBM Web Services folgendermaßen:

„Web services are self-contained, modular applications that can be described, published, located, and invoked over a network." 14

Das Standardisierungsgremium "World Wide Web Consortium" (W3C) definiert Web Services als:

"[...] software system designed to support interoperable machine-to-machine interaction over a network. It has an interface described in a machine-processable format (specifically WSDL). Other systems interact with the Web service in a manner prescribed by its description using SOAP messages, typically conveyed using HTTP with an XML serialization in conjunction with other Web-related standards."15

Allen Definitionen ist gemein, dass sie Web Services anhand ihrer Architektur umschreiben. Eine grundlegende Eigenschaft ist die Ermöglichung des standardisierten Datenaustauschs zwischen Maschinen innerhalb von geschlosse-

12 Wabli/Burroughs/Cline/Go/Tung, S. 3; i.d.S. auch Langner, S. 72.

13 Microsoft Library, abrufbar unter:

http://msdn.microsoft.com/webservices/default.aspx?pull=/library/en-

us/dnwebsrv/html/wsmsplatform.asp\#wsmsplat_topic2.

14 Wabli/Burroughs/Cline/Go/Tung, S. 7; dem folgend auch Wiebler, S. 99.

15 W3C Working Group (2004), S. 7. 
nen oder offenen Systemen. ${ }^{16}$ Menschen können zwar Initiatoren dieser Kommunikation sein, aber sie nutzen Web Services immer nur mittelbar. ${ }^{17}$ Daraus lässt sich ableiten, dass Web Services als Softwarekomponenten oder unabhängige, modulare, sich selbst beschreibende Dienste aufgefasst werden können, die sich in eine wohldefinierte Funktionalität kapseln und ihre Dienste über standardisierte Schnittstellen anderen Softwarekomponenten oder Anwendungen durch die Veröffentlichung automatisiert zur Verfügung stellen. ${ }^{18}$ Vereinfacht lässt sich ein Web Service auch als Servicezugang beschreiben. ${ }^{19}$

\section{Akteure}

Je nach Art und Weise der Anwendung von Web Services können unterschiedliche Akteure beteiligt sein. Da Web Services im E-Commerce in aller Regel im Rahmen der Serviceorientierten Architektur (SOA) umgesetzt werden, beschränkt sich diese Arbeit auf die Akteure, die im Rahmen der Umsetzung und Nutzung einer Serviceorientierten Architektur beteiligt sind. Relevante Akteure im Rahmen einer Serviceorientierten Architektur können Web Service-Hersteller - denn ohne die entsprechende Software kann die Serviceorientierte Architektur nicht umgesetzt werden, Web Service-Anbieter, Web Service-Vermittler, Web Service-Nutzer und Dritte (Endkunden) sein. Aufgrund zahlreicher unterschiedlicher Begriffsverwendungen ist die Erläuterung der gewählten Bezeichnungen für das Verständnis der Arbeit zwingend erforderlich.

\section{Web Service-Hersteller}

Der Web Service-Hersteller ist der Programmierer oder Produzent des verwendeten Web Service. Bietet er mit dem programmierten Web Service zugleich einen konkreten Dienst an, kann er gleichzeitig auch als Web ServiceAnbieter fungieren. Prominente Hersteller von Web Services sind IBM, Sun Microsystems und Microsoft.

\footnotetext{
16 Tosques/Mayr, S. 3; Melzer, S. 51.

17 Melzer, S. 51.

18 S. Burghardt, S. 12; Walpert, S. 221; i.d.S. auch Wiehler, S. 99; Martens, S. 21; Österle/Alt/Heutschi, S. 1; ferner Küster, in: Fröschle, S. 5.

19 Porkert, S. 5.
} 


\section{Web Service-Anbieter}

Der Web Service-Anbieter, auch Service Provider ${ }^{20}$ oder Web ServiceUnternehmen ${ }^{21}$ genannt, ist derjenige, der einen bestimmten Dienst auf seinem Web Server beziehungsweise seiner Plattform zur Verfügung stellt, ihn beschreibt und publiziert, damit er für Nutzer auffindbar ist. Er übernimmt den Betrieb und die Konzeption des Dienstes und ist für die Wartung dieses sowie der Dienstplattform verantwortlich. ${ }^{22}$

\section{Web Service-Nutzer}

Der Web Service-Nutzer, zum Teil auch Dienstnachfrager, Klient ${ }^{23}$ oder Service Requestor genannt, ${ }^{24}$ ist derjenige, der den Dienst des Web ServiceAnbieters über das UDDI des Web Service-Vermittlers sucht und gebraucht, indem er ihn in seine Geschäftsprozesse und Anwendungen integriert. Web Service-Nutzer sind zum Beispiel Unternehmen, die ein bestimmtes Packet an Diensten für Dritte anbieten, davon aber bestimmte Aufgaben an Web Service-Anbieter auslagern (Outtasking) oder auf die Dienste von externen Web Service-Anbietern zurückgreifen. Dritte können den ausgelagerten Dienst dann über das Portal des Web Service-Nutzers nutzen, erbracht wird der Dienst aber durch den Web Service-Anbieter. Andere Varianten sind Angebote von Web Service-Anbietern, deren sich ein Web Service-Nutzer bedient, um einen Rund-um-Service zu bieten. ${ }^{25}$ Ein solcher praktischer Fall ist die Buchung von Reisen über Webpräsenzen von Reiseportalen:

Die Fluggesellschaften stellen Möglichkeiten zum Nachschlagen beriebungsweise Buchen von Flügen über einen $W$ eb Service bereit. Reiseportale bieten auf ibrer Webpräsenz die Flugmöglichkeiten von verschiedenen Fluggesellschaften (neben Hotel- und Mietwagenbuchungen etc.) an. Welche Unternebmen welche Art von Diensten anbieten, können die Reiseportale über eine Registrierungsdatenbank (dem so genannten UDDI, „Universal Description,

20 Martens, S. 22; Wiebler; S. 101; Stoykova, S. 210; Kossman/Leymann, Informatik Spektrum 2004, 120 .

21 Spindler, DuD 2005, 139.

22 Melzer, S. 14; Burghardt, S. 16.

23 Kossman/Leymann, Informatik Spektrum 2004, 120.

24 Wiebler, S. 101; Burghardt, S. 16; Walpert, S. 231.

25 S. dazu das Anwendungsbeispiel bei Burghardt, S. 14 f. 
Discovery and Integration" 26 erfahren. Der Kunde kann auf der Webpräsenz des Reiseportals nun zentral einen passenden Flug (unter Umständen kombiniert mit Hotel und anderen Leistungen) suchen und buchen. In diesem Fall ist das Reiseportal der Web Service-Nutzer und die Fluggesellschaft Web Service-Anbieter.

\section{Web Service-Vermittler}

Der Web Service-Vermittler, auch Service Broker, ${ }^{27}$ Dienstmakler, Dienstverzeichnis $^{28}$ oder Service Registry ${ }^{29}$ genannt, ermöglicht dem Web ServiceAnbieter die Speicherung seiner zur Nutzung des Web Service erforderlichen Beschreibung in einem zentralen Verzeichnis (dem UDDI). Dieses Verzeichnis lässt sich mit den Gelben Seiten der Telekommunikationsbranche vergleichen, da die primäre Aufgabe des Verzeichnisses das Bereithalten und Finden des Web Service ist. ${ }^{30} \mathrm{Im}$ Gegensatz zu den Gelben Seiten, sind UDDIs nicht zwangsläufig in der Hand eines Web Service-Vermittlers. Es gibt vielmehr zahlreiche UDDIs von Firmen, ${ }^{31}$ in denen eigene oder fremde Dienste angeboten werden. ${ }^{32}$ Durch eine geeignete Katalogisierung erleichtert der Web Service-Vermittler den Kontakt zwischen Web Service-Nutzer und Web Service-Anbieter.

\section{Dritter}

Dritter ist in der Regel der Kunde, der den Web Service über den Web Service-Nutzer (Stichwort Reiseportal) oder den Web Service-Anbieter direkt nutzt, um Informationen zu sammeln, Dienstleistungen zu erhalten oder gar Waren zu kaufen.

\footnotetext{
26 Mehr dazu s. B.II.2 Komponenten.

27 Martens, S. 22; Walpert, S. 231.

28 Melzer, S. 14; Hamel, DuD 2007, 663.

29 Burghardt, S. 16.

30 Melzer, S. 14; Schumer, S. 105; Küster, in: Fröschle, S. 10.

31 S. dazu auch Melzer, S. 15.

32 S. z.B. die UDDIs von www.xmethods.com und http://www.soaprpc.com/ <25.11.2009>.
} 
II. Serviceorientierte Architekturen mit Web Services

\section{1. Überblick}

Das abstrakte Architekturkonzept der Serviceorientierten Architektur zeichnet sich durch leichte Integrierbarkeit aufgrund der vereinheitlichten Schnittstellen aus und ist somit eine der bestgeeigneten Technologien zur Implementierung adaptiver und flexibler Geschäftsprozesse. ${ }^{33}$ Sie ist allerdings lediglich ein Bild der Wirklichkeit, das einer konkreten Umsetzung bedarf und in deren Zentrum das Anbieten, Suchen und Nutzen von Diensten über ein Netzwerk steht. ${ }^{34}$ Web Services realisieren dieses Architekturmodell des Anbietens, Suchens und Nutzens von Diensten am vielversprechendsten. ${ }^{35}$

Web Services verkörpern Dienstleistungen, die auf einer komponentenbasierten verteilten Anwendungsarchitektur mit standardisierten Schnittstellen beruhen. ${ }^{36}$ Der Aufbau eines Web Service lässt sich folgendermaßen darstellen:

1. Die Beschreibung des Dienstes erfolgt über die standardisierte Beschreibungssprache Webservice Description Language (WSDL).

2. Der Dienst wird durch seine Registrierung auf Basis der Beschreibungssprache UDDI (Universal Description, Discovery and Integration) veröffentlicht.

3. Das Auffinden des Dienstes erfolgt über einen standardisierten Mechanismus zur Kompilier- oder zur Laufzeit.

4. Die Nutzung des Dienstes erfolgt über eine wohldefinierte, in XML (Extensible Markup Language) geschriebene Schnittstelle.

5. Jeder Dienst kann grundsätzlich andere Dienste benutzen und lässt sich über den Standard Uniform Resource Identifier (URI) identifizieren. ${ }^{37}$

33 Lotr, DuD 2007, 646.

34 Richter/Haller/Scbrey, Informatik Spektrum 2005, 413; Melzer, S. 7 f.

35 Burghardt, S. 15; ferner Ricbter/Haller/Schrey, Informatik Spektrum 2005, 414; Hamel, DuD 2007, 663.

36 Porkert, S. 4.

37 Walpert, S. 221; Kossman/Leymann, Informatik Spektrum 2004, 120. 
Die zur Benutzung des Web Service notwendigen Informationen sind alle in der öffentlich zugänglichen Schnittstellenbeschreibung enthalten. ${ }^{38}$ Durch diese standardisierten Schnittstellen ist es leicht möglich, den Dienst mit anderen Anwendungen oder Komponenten, unabhängig von den Sprachen, in denen sie geschrieben wurden, lose zu koppeln. ${ }^{39}$ Damit wird ferner die Interoperabilität von Web Services in einer ubiquitären Umgebung ermöglicht. ${ }^{40}$

Die an der Nutzung eines Web Service beteiligten Akteure können ohne weiteres ausgetauscht werden. In ihrer einfachsten Form interagieren zwei Web Services jedoch wie folgt:

1. Ein potenzieller Web Service-Nutzer stellt eine Suchanfrage an einen Verzeichnisdienst.

2. Der Web Service-Vermittler hält in dem Web Service Verzeichnis eine kategorisierte Ansammlung von registrierten, vertrauenswürdigen Web Services.

3. Nachdem der gewünschte Service ausfindig gemacht wurde, können weitere Details über Nachrichtenformate und Protokolle angefragt werden.

4. Basierend auf der Servicebeschreibung, kann eine Protokollanbindung erzeugt werden. Somit können beide Web Services miteinander kommunizieren. ${ }^{41}$

\section{Komponenten}

Grundlegende Komponenten einer Serviceorientierten Architektur mit Web Services sind Kommunikation, Dienstebeschreibung und ein Verzeichnisdienst. ${ }^{42}$ Die wichtigsten, aber nicht zwangsläufig die einzigen, ${ }^{43}$ Spezifikatio-

38 Martens, S. 21; Kossman/Leymann, Informatik Spektrum 2004, 120.

39 Burghardt, S. 18; Alonso/Casati/Kuno/Machiraju, S. 124; Picot/Neuburger, in: FS Kilian, S. 528 f.; Küster, in: Fröschle, S. 6.

40 Porkert, S. 7.

41 Erläuterung von Web Services im Informatiklexikon der GI, abrufbar unter: http://www.giev.de/service/informatiklexikon/informatiklexikon-detailansicht/meldung/95/ <25.11.2009>.

42 Melzer, S. 12. 
nen dieser Komponenten sind bei Web Services SOAP für die Kommunikation, WSDL für die Dienstebeschreibung und das UDDI für den Verzeichnisdienst.

SOAP, früher das Akronym für Simple Object Access Protocol und heute als feststehender Ausdruck verwandt, ${ }^{44}$ ist ein Kommunikationsprotokoll über das Informationen sprach- und plattformunabhängig ausgetauscht werden. ${ }^{45}$ Es beschreibt das XML-basierte Nachrichtenformat der Kommunikation und dessen Einbettung in das Transportprotokoll. ${ }^{46}$ Kommunikationsprotokolle sind exakte Vereinbarungen in Form von Regeln und Formaten, nach denen Daten zwischen Computern, die durch ein Netz miteinander verbunden sind, ausgetauscht werden. ${ }^{47}$

Die Web Service Description Language (WSDL) ist eine XML-basierte Beschreibungssprache für die Schnittstellen von Web Services. ${ }^{48}$ WSDLDokumente sind, vereinfacht ausgedrückt, Dienstbeschreibungen: Web Service-Anbieter beliebiger Art stellen im Internet oder Intranet Web Services zur Verfügung. Damit andere Clients oder Web Service-Nutzer auf der Suche nach einem bestimmten Web Service feststellen können, ob der zur Verfügung gestellte Web Service die vom Client oder Web Service-Nutzer definierten Anforderungen erfüllt, wird das WSDL-Dokument (also die Dienstbeschreibung) analysiert. ${ }^{49}$

Universal Description, Discovery and Integration (UDDI) beschreibt einen Verzeichnisdienst für Web Services, worin allgemeine Anforderungen, Eigenschaften, zum Beispiel die Servicebeschreibung, oder die benötigten Informationen zum Auffinden von Web Services verwaltet werden. ${ }^{50}$ Das UDDI be-

43 Web Services sind nicht zwingend gleichzusetzen mit SOAP, WSDL und UDDI, sie stellen aber Umsetzungen der drei wichtigsten Komponenten der SOA dar, s. Melzer, S. 51 ff., und sollen daher den Schwerpunkt dieser Arbeit bilden.

44 S. http://www.w3.org/TR/2007/REC-soap12-part2-20070427/ <25.11.2009>.

45 Kossman/Leymann, Informatik Spektrum 2004, 120; Walpert, S. 222; Burghardt, S. 50.

46 Melzer, S. $51 \mathrm{ff}$.

47 S. Erläuterung zu Netzwerkprotokoll bei Wikipedia, abrufbar unter http://de.wikipedia.org/wiki/Netzwerkprotokoll.

48 Taylor, S. 53; Küster, in: Fröschle, S, 9; Wiehler, S. 101; Walpert, S. 226; Kossman/Leymann, Informatik Spektrum 2004, 120.

49 Ähnlich auch Schumer, S. 105.

50 Porkert, S. 23; Taylor, S. 53; Melzer, S. 51. 
steht aus einem XML-Schema, das vier Datenstrukturen definiert: den Geschäftszweig, die Service-Kategorie, die Definition bezüglich der Einbindung des Dienstes sowie Programm-Interfaces und operative Interfaces für die genannten Strukturen. ${ }^{51}$ Die Veröffentlichung der Dienste, die öffentlich genutzt werden sollen, im UDDI ist nötig, damit sie überhaupt von Web ServiceNutzern gefunden werden können. ${ }^{52}$ UDDIs sind in so genannte weiße, gelbe und grüne Seiten unterteilt. Weiße Seiten beinhalten die allgemeinen Informationen über den Web Service-Anbieter und stellen damit eine Art Firmenverzeichnis dar. ${ }^{53}$ Die Gelben Seiten ermöglichen, nach Branchen sortiert, eine Suche der im UDDI-Verzeichnis abgelegten Daten und die Grünen Seiten umfassen den technischen Datenbestand, der innerhalb des Verzeichnisses gespeichert wird. ${ }^{54}$ Allerdings muss beachtet werden, dass nicht jeder Web Service in einem UDDI veröffentlicht wird. Das UDDI ist eine für Web Services selbst optionale Komponente, die aber im Rahmen der Abbildung der Serviceorientierten Architektur mit Web Services erforderlich ist.

Alle Komponenten basieren auf XML, welche damit die Kerntechnologie von Web Services darstellt. XML ist ein standardisiertes, kostenloses Datenformat zur Strukturierung und Darstellung von Informationen und zum Austausch zwischen verschiedenen Anwendungen. ${ }^{55} \mathrm{XML}$ ist eine plattform- und betriebssystemunabhängige Metasprache, welche die Definition von neuen Auszeichnungssprachen, wie zum Beispiel WSDL, ermöglicht. ${ }^{56}$

Der Ablauf zwischen Web Service-Komponenten und Akteuren gestaltet sich wie folgt:

Um die Web Service-Nutzung zu ermöglichen, veröffentlicht der Web Service-Anbieter bei dem Web Service-Vermittler im UDDI seinen Web Service, indem er Dienstebeschreibung und Schnittstelle dort einstellt. ${ }^{57}$ Der Web Service-Nutzer kann dann dort nach dem gewünschten Dienst anhand der ver-

\footnotetext{
51 Wiebler, S. 101.

52 Siehe dazu die Beschreibung bei Burghardt, S. 17.

53 Porkert, S. 24; Walpert, S. 232; Martens, S. 25; Burghardt, S. 62.

54 Wiebler, S. 101; Martens, S. 25; Burghardt, S. 62.

55 Burghardt/Hagenhoff, Arbeitspapier Nr. 22/2003, S. 26; Martens, S. 24.

56 Burghardt/Hagenhoff, Arbeitspapier Nr. 22/2003, S. 26.

57 Melzer, S. $14 \mathrm{f}$.
} 
schiedenen Kategorien (weiße, gelbe und grüne Seiten) suchen. ${ }^{58}$ Nach Auffinden kontaktiert er den entsprechenden Web Service-Anbieter mittels SOAP zur Anfrage des Web Service. Nach dieser Anfrage antwortet der Web Service mittels SOAP an den Web Service-Nutzer. ${ }^{59}$ Das alles geschieht - optimaler Weise - voll automatisiert ohne menschliche Zwischenschritte.

III. Einsatzbereiche von SOA und Web Services

Web Services, gegebenenfalls in Serviceorientierten Architekturen, können zum einen innerhalb geschlossener und zum anderen innerhalb offener Systeme eingesetzt werden. Unter geschlossenen Systemen werden hier unter anderem Intranets, hauptsächlich solche von Unternehmen oder sonstigen Betrieben, verstanden. Gängig ist auch die Verwendung zwischen zwei oder mehr Unternehmen (Unternehmensnetzwerke), ${ }^{60}$ die Dritte von der Nutzung ausschließen. Die Registrierung der Dienste erfolgt in diesen Fällen lediglich im unternehmensinternen Intranet oder auf einer Plattform, auf welche nur Mitarbeiter oder sonstige abgrenzbare Personenkreise Zugriff haben. Web Services dienen dort als effizientere Methode der verteilten Verarbeitung. Mögliche Einsatzgebiete im unternehmensinternen Bereich sind die Ermöglichung des Zugriffs auf Terminkalender, Adressbuch und Dokumentenspeicher oder aber das Abrufen bestimmter Daten, Dienste etc. Laut einer Umfrage des vom Bundesministerium für Bildung und Forschung geförderten Projekts „EVENT“, setzen Unternehmen Web Services zur zwischenbetrieblichen Nutzung hauptsächlich in folgenden Bereichen ein: Abwicklung des Zahlungsverkehrs, Unterstützung eines Kunden-/Zuliefererportals, Anbindung an Datenbanken, Unterstützung des unternehmensübergreifenden Reportings, Auslagerung von Teilprozessen (Outtasking), Auslagerung von Geschäftsprozessen (Outsourcing). ${ }^{61}$ Der Einsatz von Serviceorientierten Architekturen mit Web Services oder Web Services allein in geschlossenen Systemen wird im Rahmen dieser Arbeit aus den eingangs genannten Gründen nicht weiter untersucht. ${ }^{62}$

Ein offenes System ist das Internet. Die Dienstebeschreibung wird dabei, im optimalen Fall, in einem öffentlich zugänglichem UDDI abgespeichert und ist

\footnotetext{
58 Burghardt, S. 17 f.

59 Speck, in Hoffmann/Leible/Sosnitza, S. 22 f.; Wiebler, S. 101 f.

60 S. dazu auch Martens, S. 33; Österle/Alt/Heutschi, S. 12 f.

61 Prokein/Sackmann, S. 13.

62 S. A Einführung.
} 
darüber für ,,jedermann“ lokalisierbar. ${ }^{63}$ Nicht jeder Anbieter nutzt aber die Option der öffentlichen UDDI-Registrierung. Auf eine solche öffentliche Registrierung kann in Gänze verzichtet werden oder es erfolgt eine Publikation in privaten UDDIs. Prominente Beispiele für Web Services im Internet sind Amazon Web Services (Ermöglichung der Einbindung von Funktionen und Inhalten von Amazon in Webpräsenzen Dritter, auch Privater), ${ }^{64}$ Yahoo! Search Web Services (Ermöglichung des Zugriffs auf bestimmte YahooDatenbanken mit eigenen Programmen), ${ }^{65}$ Google Web APIs (bei Google AXAJ Search APIs können Verwender die Google Suchfunktion beispielsweise derart in ihre Webpräsenz integrieren, dass User das Internet mittels Google auch über Webpräsenzen Dritter, die Google AXAJ Search APIs integriert haben, durchsuchen können), ${ }^{66}$ AmaTEX (durchsucht Bücher von Amazon über einen Amazon Web Service und extrahiert daraus BIB-Daten für Bibtex/Latex) und Microsofts Virtual Earth (ein virtueller Karten- und Landschaftsdienst). ${ }^{67}$ Nicht ganz so prominente Einsatzbereiche liegen bei Geodiensten, die den verteilten Zugriff auf sowie die Erfassung, Manipulation, Transformation, Analyse und die Präsentation von Geodaten ermöglichen. ${ }^{68}$ Technisch eher einfache Einsatzgebiete sind das Ermöglichen des Abrufens von Börsenkursen, der Temperatur an bestimmten Orten oder das Umrechnen von Währungsbeträgen. ${ }^{69}$ Wenig bekannt, aber dennoch von größerer Relevanz, ist die Verwendung von Web Services zum Beispiel anstelle der Agententechnik beim so genannten „dynamic packaging ${ }^{\text {“70 }}$ durch Reiseportale. ${ }^{71}$

63 Wiebler, S. 99.

64 S. dazu http://www.amazon.com/gp/browse.html?node=3435361 <25.11.2009>; Kossmann/Leymann, Informatik Spektrum 2004, 120.

65 Tosques/Mayr, Proceedings der 27. Online Tagung der DGI, S. 14.

66 S. http://code.google.com/apis/soapsearch/reference.html.

67 S. Heise-Newsmeldung vom 26.5.2007, abrufbar unter: http://www.heise.de/newsticker/result.xhtml?url=/newsticker/meldung/90238\&words=Web $\% 20$ Service\& $\mathrm{T}=$ Web-Service $<25.11 .2009>$.

68 Donaubauer, S. 22.

69 S. bspw. http://www.webservicex.net/WCF/default.aspx <25.11.2009>.

70 S. dazu ausführlich A.III.2.a)bb) Exkurs: Reisevertragsspezifische Besonderheiten.

71 Cardoso, Punkt 3.3, Dynamic Packaging Web Process Generator; vgl. auch die Lösung von GoQuo, abrufbar unter: http://www.goquo.com/online_GDS_Integration.asp <25.11.2009>. 
Die Vorteile von Web Services liegen in der Unabhängigkeit von Plattformen, Programmiersprachen und Protokollen. Es ergeben sich selten Firewallprobleme und die Unternehmen sparen dadurch, dass kostenintensive Implementierungen obsolet sind, zudem Kosten ein. Konkret bedeutet dies, dass zum Beispiel eine in Perl programmierte Software, die auf einem Rechner mit dem Betriebssystem Linux läuft, einen in Java programmierten Web Service, der auf einem Rechner mit dem Betriebssystem Windows zur Verfügung gestellt wird, aufrufen kann. 


\section{Teil Zivilrechtliche Aspekte}

Im Folgenden soll untersucht werden, wie beziehungsweise ob sich das Agieren in einer Serviceorientierten Architektur mit Web Services, die sich durch das Charakteristikum der vollautomatisierten Kommunikation auszeichnet, in das bestehende deutsche Rechtssystem, welchem immer noch die Mensch-zuMensch-Kommunikation als Leitbild zugrunde liegt, eingliedern lässt. Dabei stellt im Abschnitt A. das Vertragsrecht den Schwerpunkt der Untersuchung dar. Insbesondere erfolgt eine Typisierung der verschiedenen Vertragsbeziehungen, wobei außer den klassischen Akteuren, Web Service-Anbieter, Web Service-Vermittler und Web Service-Nutzer, zudem noch der Web ServiceHersteller und der Dritte einbezogen werden, um der Komplexität der verschiedenen Vertragsbeziehungen gerecht zu werden. Darauf aufbauend werden unter anderem die aus den Verträgen resultierenden Rechte und Pflichten der an der Serviceorientierten Architektur Beteiligten behandelt, wobei auch immer Dritter und Web Service-Hersteller im Blick behalten werden. Ferner wird auf Sicherheitsaspekte beim Vertragsschluss eingegangen.

Der Abschnitt B. widmet sich den Fragen der außervertraglichen Haftung, wobei der Missbrauch des UDDI durch den Web Service-Anbieter beziehungsweise Fehlfunktionen des UDDI und daraus resultierende Haftungsmöglichkeiten der Beteiligten der Serviceorientierten Architektur im Vorder- 
grund stehen. Ferner werden Fragen der Produkt- und Produzentenhaftung im Falle eines Mangels beim Web Service sowie kartellrechtliche Fragen bei der Beteiligung der öffentlichen Hand behandelt.

\section{A. Allgemeine vertragsrechtliche Probleme}

\section{Anwendbares Recht im E-Commerce}

Der Einsatz von Web Services ist aufgrund der Ubiquität des Internet unabhängig von Landesgrenzen. Die Akteure einer Serviceorientierten Architektur können von jedem Ort der Welt aus agieren. Inhalte und damit auch die Dienste von Web Service-Anbietern können auf Servern in der ganzen Welt platziert sein und - vorausgesetzt ein Netzzugang ist vorhanden - auch weltweit abgerufen werden. Weist der Einsatz von Web Services in Serviceorientierten Architekturen daher eine Auslandsberührung auf (s. Art. 3 EGBGB), so stellt sich sowohl im vertraglichen Bereich als auch im Rahmen außervertraglicher Beziehungen ${ }^{72}$ immer die Frage nach der anwendbaren Rechtsordnung. Antworten finden sich im so genannten Internationalen Privatrecht, dass im deutschen Recht in Art. 3 ff. EGBGB geregelt ist.

\section{Vereinbarung der Rechtsordnung}

Welches Recht im vertraglichen Bereich anwendbar ist, bestimmt sich maßgeblich nach Art. 27 ff. EGBGB. Eine Möglichkeit, praktisch aus Gründen der Rechtssicherheit wohl auch die empfehlenswerteste, stellt die ausdrückliche oder konkludente Rechtswahl nach Art. 27 Abs. 1 EGBGB als Ausprägung der Privatautonomie dar. Die Möglichkeit der Rechtswahl wird auch im Bereich der Telemediendiensteanbieter nicht durch das Herkunftslandprinzip eingeschränkt, \3 Abs. 2 Nr. 1 TMG. Im Rahmen der Serviceorientierten Architektur, die sich durch Automatismen, regelmäßig ohne menschliches Agieren, auszeichnet, ist die Möglichkeit einer ausdrücklichen Rechtswahlvereinbarung durch Individualvereinbarungen unterzugewichten, da es durch die automatisierten Abläufe regelmäßig vor Vertragsschluss nicht zu individuellen Vereinbarungsmöglichkeiten kommt. Praktisch wesentlich relevanter ist die Rechtswahl durch Allgemeine Geschäftsbedingungen (auch stillschweigend) ${ }^{73}$ oder konkludente Vereinbarungen im Sinne des Art. 27 Abs. 1 S. 2 EGBGB.

72 S. dazu die Erläuterung unter B. Außervertragliche Haftung.

73 BGH, NJW 1990, 242 (244); Bamberger/Roth/Spickhoff; Art. 27 EGBGB Rn. 34. 
Erfolgt die Rechtswahl konkludent, so muss sie sich nach Art. 27 Abs. 1 S. 2, 2. Alt. EGBGB „mit hinreichender Sicherheit aus den Bestimmungen des Vertrags oder aus den Umständen des Falls ergeben“. Indizien für eine stillschweigende Rechtswahl können ausgehend vom Parteiwillen unterschiedlicher Natur sein. Darunter fallen beispielsweise Hinweise auf Rechtsnormen eines bestimmten Staates, ${ }^{74}$ die Vertragssprache, ${ }^{75}$ die vorherige Praxis der Parteien, ${ }^{76}$ Gerichtsstandsvereinbarungen ${ }^{77}$ oder Schiedsgerichtsklauseln ${ }^{78}$. Ob eine Rechtswahl in den jeweiligen Vertragsbeziehungen innerhalb der Serviceorientierten Architektur stillschweigend oder auch durch Allgemeine Geschäftsbedingungen erfolgte, muss immer anhand der Hinweise des Einzelfalls untersucht werden.

Das Zustandekommen sowie die Wirksamkeit der vereinbarten Rechtswahlklausel selbst richten sich nach Art. 27 Abs. 4 in Verbindung mit Art. 31 Abs. 1 EGBGB nach dem Recht, das auf den Vertrag nach der Rechtwahlklausel anwendbar sein soll. ${ }^{79}$ Wird die Vereinbarung durch die Akteure innerhalb von Allgemeinen Geschäftsbedingungen getroffen und ist beispielsweise das deutsche Recht vereinbart, so müssen die Allgemeinen Geschäftsbedingungen wirksam einbezogen werden und die Klausel der Inhaltskontrolle standhalten. 80

Ist das Recht gewählt wurden, so bestimmen sich alle weiteren rechtlichen Fragestellungen nach der gewählten Rechtsordnung. Ausnahmen bestimmen lediglich Art. 7, 11 und 37 Nr. 3 EGBGB. Danach unterliegt beispielsweise die

74 OLG Hamburg, RIW 1986, 462 (463); MünchKommBGB/Martiny, Bd. 10, Art. 27 EGBGB Rn. 57 f.; von Bar, $\$ 4$ Rn. 471; Bamberger/Roth/Spickhoff, Art. 27 EGBGB Rn. 42, dem allerdings die Vertragssprache nicht allein als Indiz genügt.

75 BGH, NJW-RR 1997, 686 (687); i.d.S. auch OLG Düsseldorf, NJW-RR 1991, 55.

76 Bamberger/Roth/Spickhoff, Art. 27 EGBGB Rn. 41; von Bar, \4 Rn. 471.

77 BGH, NJW-RR 2005, 206 (208); NJW 1990, 1420 f.; Bamberger/Roth/Spickhoff, Art. 27 EGBGB Rn. 38; Mankowski, in: Spindler/Wiebe, Kap. 11 Rn. 88; Martiny, in: Reithmann/Martiny, Rn. 86; von Bar, $\int 4$ Rn. 469, der allerdings noch weitere Anhaltspunkte verlangt.

78 BGH， NJW-RR 2005， 206 (208); OLG Hamm, NJW-RR 1993, 1445 (1446); MünchKommBGB/Martiny, Bd. 10, Art. 27 EGBGB Rn. 51.

79 BGH, NJW 1997, 1697 (1698); NJW 1994, 262; NJW 1989, 1431 (1432); Bamberger/Roth/Spickhoff, Art. 27 EGBGB Rn. 30; Ferrari/Ferrari, Int. VertragsR, Art. 27 EGBGB Rn. 6; MünchKommBGB/Martiny, Bd. 10, Art. 27 EGBGB Rn. 100; Härting, Rn. 18.

80 S. ausführlich zu den Anforderungen an eine wirksame Einbeziehung von AGBs A.III.4 Einbeziehung Allgemeiner Geschäftsbedingungen. 
Rechtsfähigkeit und Geschäftsfähigkeit einer Person dem Recht des Staates, dem die Person angehört (Art. 7 Abs. 1 S. 1 EGBGB).

Allerdings kann eine Rechtsordnung nicht unbeschränkt gewählt werden. Denn Schutzvorschriften und Verbraucherschutzvorschriften (Art. 29, 29a EGBGB) des nationalen Rechts sollen nicht umgangen werden. ${ }^{81}$ Dabei ist neben den verbraucherschützenden Vorschriften, die im Rahmen der Serviceorientierten Architektur, in der die Beteiligten häufig als Unternehmer im Sinne des $\int 14$ BGB tätig sind, insbesondere die Vorschrift des Art. 27 Abs. 3 EGBGB von Bedeutung, die besagt, dass wenn „,der sonstige Sachverhalt im Zeitpunkt der Rechtswahl nur mit einem Staat verbunden [ist], [...] die Wahl des Rechts eines anderen Staat[e]s - auch wenn sie durch die Vereinbarung der Zuständigkeit eines Gerichts eines anderen Staat[e]s ergänzt ist - die Bestimmungen nicht berühren [kann], von denen nach dem Recht jenes Staates durch Vertrag nicht abgewichen werden kann". Die freie Rechtswahl wird dadurch bei Binnensachverhalten eingeschränkt, indem zwingende Vorschriften des mit dem Sachverhalt verbundenen Rechts von der Rechtswahl unberührt bleiben. ${ }^{82}$

\section{Feblende Rechtswabl}

Haben die Akteure in der Serviceorientierten Architektur keine Rechtsordnung gewählt, so gilt nach dem Grundsatz des Art. 28 Abs. 1 S. 1 EGBGB, dass der Vertrag dem Recht des Staates unterliegt, mit dem er die engsten Verbindungen aufweist. Das Herkunftslandprinzip aus $₫ 3$ TMG, findet auf das Verbraucherrecht, unabhängig von seiner Qualifikation im Hinblick auf das Kollisionsrecht, ${ }^{83}$ nach $₫ 3$ Abs. 3 Nr. 2 TMG keine Anwendung und muss daher in diesem Bereich auch nicht berücksichtigt werden. ${ }^{84}$

\section{B2C-Verträge}

Fehlt es im Rahmen von Verbraucherverträgen, die bei Umsetzung der Serviceorientierten Architektur nur regelmäßig bei Endkundenverträgen zwischen Web Service-Nutzer und Dritten eine Rolle spielen, an einer Rechtswahl, so

81 S. nur die Vorschriften Art. 27 Abs. 3, 29, 29a EGBGB; von Bar, \4 Rn. 423 ff.; Härting, Rn. 19; von Hoffmann/Thorn, \10 Rn. 28 ff.; Schmidt/Prieß, in: Spindler/Börner, S. 193.

82 MünchKommBGB/Martiny, Bd. 10, Art. 27 EGBGB Rn. 89 ff.; von Hoffmann/Thorn, \10 Rn. 29.

83 S. dazu A.I.2. B2B-Verträge.

84 Härting, Rn. 76. 
kann bei der genaueren Qualifizierung die widerlegbare Regelvermutung des \28 Abs. 2 S. 1 EGBGB helfen. Danach wird vermutet, dass der Vertrag die engsten Verbindungen mit dem Staat aufweist, in dem die Partei, welche die charakteristische Leistung zu erbringen hat, im Zeitpunkt des Vertragsabschlusses ihren gewöhnlichen Aufenthalt oder, wenn es sich um eine Gesellschaft, einen Verein oder eine juristische Person handelt, ihre Hauptverwaltung hat. Als charakteristische Leistung gilt diejenige, welche dem betreffenden Vertragstyp seine Eigenschaft verleiht und seine Unterscheidung von anderen Vertragstypen ermöglicht. ${ }^{85}$ Danach führt dies beim Kaufvertrag zum Landesrecht des Verkäufers, beim Dienstvertrag zum Recht des Dienstleisters und beim Werkvertrag zum Recht des Werkerstellers. ${ }^{86}$ Angeknüpft wird an den Sitz der Vertragspartei, nicht an den Serverstandort oder die Internetadresse. ${ }^{87}$ Bei Verträgen, die in Ausübung einer beruflichen oder gewerblichen Tätigkeit geschlossenen wurden, wie es im Rahmen des Serviceorientierten Architektur regelmäßig der Fall sein wird, kommt zudem Art. 28 Abs. 2 S. 2 EGBGB zum Tragen, wonach bei mehreren Firmenniederlassungen in unterschiedlichen Staaten, auf das Recht des Staates der Hauptniederlassung abgestellt wird. ${ }^{88}$ Ausnahmen können im Sinne des Art. 28 Abs. 5 EGBGB allerdings dann bestehen, wenn ein Anbieter seine Leistung gezielt auf den deutschen Markt ausgerichtet hat und zudem seine Leistungen gezielt in deutscher Sprache bewirbt, dafür müssen sich allerdings Indizien häufen. ${ }^{89}$

Dies gilt aber nur, wenn nicht im Sinne des Art. 29 Abs. 2 in Verbindung mit Art. 29 Abs. 1 Nr. 1 EGBGB dem Vertragsabschluss ein ausdrückliches Angebot oder eine Werbung in dem Staat, in dem der Verbraucher seinen gewöhnlichen Aufenthaltsort hat, vorausgegangen ist und wenn der Verbraucher in diesem Staat die zum Abschluss des Vertrags erforderlichen Rechtshandlungen vorgenommen hat. Denn erfolgte zuvor nur eine dieser Handlungen, so unterliegen Verbraucherverträge nach Art. 29 Abs. 2 EGBGB dem Recht des Staats, in dem der Verbraucher seinen gewöhnlichen Aufenthalt hat. Auf den charakteristischen Vertragsgegenstand kommt es dann nicht an.

85 BGHZ 128, 41 (48); BGH, NJW 1993, 2753 (2754); OLG Düsseldorf, RIW 1997, 780.

86 BGHZ 128, 41 (48); Remien, in: Leible, S. 29; Härting, Rn. 36.

87 Staudinger/Magnus, Art. 28 EGBGB Rn. 78; Remien, in: Leible, S. 29.

88 Staudinger/Magnus, Art. 28 EGBGB Rn. 86; Bamberger/Roth/Spickhoff, Art. 28 EGBGB Rn. 13.

89 S. dazu ausführlich Staudinger/Magnus, Art. 28 EGBGB Rn. 126 ff.; Bamberger/Roth/Spickhoff, Art. 28 EGBGB Rn. 22 ff.; Härting, Rn. 37. 


\section{B2B-Verträge}

Da es sich bei den Beteiligten Akteuren der Serviceorientierten Architektur um Telemediendiensteanbieter handelt, ${ }^{90}$ muss bei Verträgen im Rahmen der Europäischen Union das durch die E-Commerce-Richtlinie (ECRL) ${ }^{91}$ eingeführte und in $\int 3$ TMG verankerte Herkunftslandprinzip berücksichtigt werden. AuBerhalb der Europäischen Union gelten freilich die bereits zu B2C-Verträgen erläuterten Grundsätze. Zwar wird zum Teil davon ausgegangen, dass aufgrund der Ausnahmevorschrift in $\int 3$ Abs. 3 Nr. 1 TMG das Herkunftslandprinzip auf das gesamte Vertragsrecht nicht anwendbar sei, 92 dem kann aber bereits wegen des eindeutigen Wortlauts in $₫ 3$ Abs. 3 Nr. 1 TMG nicht zugestimmt werden. ${ }^{93}$ \ 3 Abs. 3 Nr. 1 TMG spricht ausdrücklich nur von Rechtswahl. Darüber hinaus erwähnt auch $₫ 3$ Abs. 3 Nr. 2 TMG explizit besondere Vertragstatbestände, nämlich in Bezug auf Verbraucherverträge Diese besonderen Erwähnungen wären überflüssig, wenn sich die Ausnahme auf das gesamte Vertragsrecht beziehen sollte. Daher greift das Herkunftslandprinzip im Rahmen von B2B-Verträgen, die innerhalb der Serviceorientierten Architektur unter den Beteiligten die Regel bilden werden.

Allerdings ist das Verhältnis von Herkunftslandprinzip und Kollisionsrecht nicht abschließend geklärt. Während Teile der Literatur die kollisionsrechtliche Qualifikation des Herkunftslandprinzips verneinen und von einer Beeinflussung und Überlagerung des Kollisionsrecht durch das Herkunftslandprinzip ausgehen, ${ }^{94}$ qualifizieren andere das Herkunftslandprinzip kollisionsrechtlich, ${ }^{95}$ so dass allein das Sachrecht des Herkunftsstaates zur Anwendung kommt. Aufgrund des Wortlauts des Art. 3 Abs. 2 ECRL, auf dem $\ 3$ TMG basiert, wird allerdings eine kollisionsrechtliche Einordnung des Herkunfts-

91 Richtlinie 2000/31/EG des Europäischen Parlaments und des Rates v. 8.6.2000 über bestimmte rechtliche Aspekte der Dienste der Informationsgesellschaft, insbesondere des elektronischen Geschäftsverkehrs, im Binnenmarkt („Richtlinie über den elektronischen Geschäftsverkehr"), ABl. EG L178, S. 1-16.

So bspw. Mankowski, IPRax 2002, 264; Borges, 894 ff.; Härting, Rn. 76.

Spindler, RabelsZ 66 (2002), 684; Spindler/Schmitz/Geis/Spindler, \4 TDG Rn. 71; i.d.S. Ahrens, CR 2000, 839; so wohl auch MünchKommBGB/Martiny, Bd. 10, Art. 34 EGBGB Anh. III Rn 42.

Spindler, RabelsZ 66 (2002), 644 ff.; Oby, GRUR Int. 2001, 902; Abrens, CR 2000, 838; Pfeiffer, in: Gounalakis, Rhb. Electronic Business, \12 Rn. 11.

So Mankowski, IPRax 2002, 257; Härting, Rn. 75. 
landprinzips zu verneinen sein, denn dieser spricht nur von einem Einschränkungsverbot. Das bedeutet, dass das Herkunftslandprinzip im Rahmen von B2B-Verträgen ohne Rechtswahlklausel nur als Maßstab für das durch das Internationale Privatrecht bestimmte Sachrecht herangezogen wird. Ist daher das Herkunftslandrecht milder als das Recht des Empfangstaats, muss entweder eine entsprechende Modifikation des Sachrechts des Empfangstaats anhand des Maßstabs des Herkunftslandrechts erfolgen oder das Internationale Privatrecht des Empfangstaats enthält für diesen Fall eine von den allgemeinen Regeln des Internationalen Privatrechts abweichende kollisionsrechtliche Verweisung auf das Recht des Herkunftslands.

\section{Zusammenfassender Überblick.}

Die Beteiligten einer Serviceorientierten Architektur haben umfangreich, sofern einer der Beteiligten aus dem Ausland agiert, und eingeschränkt, wenn es sich um Binnensachverhalte handelt, die Möglichkeit, eine Rechtsordnung nach Art. 27 Abs. 1 EGBGB - auch in Allgemeinen Geschäftsbedingungen $\mathrm{zu}$ vereinbaren. Um Rechtsunsicherheiten gerade bei Auslandsbezügen zu vermeiden, empfiehlt sich eine solche Rechtswahlklausel sogar. Denn fehlt es an einer entsprechenden Rechtswahl, so greift im Rahmen von Verbraucherverträgen und Verträgen außerhalb der Europäischen Union Art. 28 Abs. 1 S. 1 EGBGB, mit unter Umständen nicht erwünschten Folgen. Bei den im Rahmen der Serviceorientierten Architektur häufiger vorkommenden B2BVerträgen muss zudem das Herkunftslandprinzip korrigierend herangezogen werden.

Die vorliegende Untersuchung setzt das Greifen der deutschen Rechtsordnung voraus. Nur sofern kollisionsrechtliche Besonderheiten bestehen, werden diese an den jeweils vertraglich relevanten Stellen erörtert (s. A.III Vertragsrechtliche Grundlagen).

\section{Einordnung der Dienste}

Zunächst einmal stellt sich die Frage, wie die bei der Umsetzung einer Serviceorientierten Architektur mit Web Services Beteiligten überhaupt rechtlich einzuordnen sind. Zu unterscheiden ist grundsätzlich zwischen Telekommunikations-, Telemediendiensten und Rundfunk. Diese Unterscheidung ist insbesondere deshalb relevant, weil eine unterschiedliche rechtliche Einordnung auch unterschiedliche rechtliche Folgen insbesondere im Bereich der Verantwortlichkeit, der Rechte und Pflichten sowie des Datenschutzes mit sich bringt. Telekommunikationsdiensteanbieter werden nach dem Telekommunikationsgesetz (TKG), Telemediendiensteanbieter nach dem Telemediengesetz 
(TMG) und Rundfunk nach dem Staatsvertrag für Rundfunk und Telemedien, kurz Rundfunkstaatsvertrag (RStV), beurteilt. Letzterer stellt eine Vereinbarung der Länder dar, die nach Art. 70 GG die Gesetzgebungskompetenz für das Rundfunkwesen haben. ${ }^{96}$ Der Rundfunkstaatsvertrag erfasst Rundfunk im verfassungsrechtlichen Sinne, der sich zwar nicht abschließend definieren lässt aber nach der Rechtsprechung des Bundesverfassungsgerichts ${ }^{97}$ Rundfunk und massenkommunikative Medien (Telemedien im Sinne des Rundfunkstaatsvertrags) umfasst. ${ }^{98}$

Telekommunikationsdienste sind in $\ 3$ Nr. 24 TKG legaldefiniert als ,,[...] in der Regel gegen Entgelt erbrachte Dienste, die ganz oder überwiegend in der Übertragung von Signalen über Telekommunikationsnetze bestehen, einschließlich Übertragungsdienste in Rundfunknetzen“. Anbieter solcher Dienste (Diensteanbieter) sind nach $\int 3$ Nr. 6 TKG solche, die ganz oder teilweise geschäftsmäßig a) Telekommunikation erbringen oder b) an der Erbringung solcher Dienste mitwirken. Telekommunikation wiederum ist nach der Legaldefinition des $₫ 3 \mathrm{Nr}$. 22 TKG ,[...] der technische Vorgang des Aussendens, Übermittelns und Empfangens von Signalen mittels Telekommunikationsanlagen".

Diensteanbieter im Sinne des Telemediengesetzes sind nach $\int 2$ Nr. 1 TMG alle natürlichen oder juristischen Personen, die eigene oder fremde Telemedien zur Nutzung bereithalten oder den Zugang zur Nutzung vermitteln. Telemedien sind nach $\int 1$ Abs. 1 TMG alle elektronischen Informations- und Kommunikationsdienste, soweit sie nicht Telekommunikationsdienste nach $₫ 3 \mathrm{Nr}$. 24 des Telekommunikationsgesetzes, die ganz in der Übertragung von Signalen über Telekommunikationsnetze bestehen, telekommunikationsgestützte Dienste nach $\int 3$ Nr. 25 des Telekommunikationsgesetzes oder Rundfunk nach $\$ 2$ des Rundfunkstaatsvertrags sind.

Telemedien sind folglich alle übrigen elektronischen Informations- und Kommunikationsdienste (im Sinne einer Residualkategorie), so dass die Abgrenzung zwischen Telekommunikationsdiensten und Telemediendiensten negativ gegenüber den positiv definierten Telekommunikations- und Rund-

96 BVerfGE 12, 205 (228 f.); Schmidt-Bleibtreu/Hofmann/Hopfauf/Sannwald, Art. 70 Rn. 21; Beucher/Leyendecker/v. Rosenberg, \1 TDG 1997 Rn. 2.

97 BVerfGE 74, 297 (350 f.); 83, 238 (302).

98 Begründung zum Neunten Staatsvertrag zur Änderung rundfunkrechtlicher Staatsverträge (Neunter Rundfunkänderungsstaatsvertrag), S. 3, abrufbar unter http://www.lfk.de/gesetzeundrichtlinien/rundfunkstaatsvertrag/main.html <25.11.2009>. 
funkdiensten erfolgen muss. ${ }^{99}$ Bei Telemedien handelt es sich ausweislich der Gesetzesbegründung beispielsweise um Online-Angebote von Waren/Dienstleistungen mit unmittelbarer Bestellmöglichkeit (zum Beispiel Angebot von Verkehrs-, Wetter-, Umwelt- oder Börsendaten, Newsgroups, Chatrooms, elektronische Presse, Fernseh-/Radiotext, Teleshopping), OnlineDienste, die Instrumente zur Datensuche, zum Zugang zu Daten oder zur Datenabfrage bereitstellen (zum Beispiel Internet-Suchmaschinen) sowie die kommerzielle Verbreitung von Informationen über Waren/Dienstleistungsangebote mit elektronischer Post (zum Beispiel WerbeMails). ${ }^{100}$

Entscheidendes Abgrenzungskriterium der Telekommunikation zu Telemedien ist das bei Ersterer überwiegend, das heißt über $50 \%$, die Transportdienstleistung und somit die Übertragung der Signale im Vordergrund steht, währenddessen Telemedien, grob eingeordnet, der Bereitstellung von Informationen, das heißt der inhaltlichen Darstellung, Aufarbeitung oder Verwendung der angebotenen Dienste, dienen. ${ }^{101}$ Dies zeigt aber auch, dass sämtliche Telemedien über Telekommunikation erbracht werden. ${ }^{102} \mathrm{Die}$ Abgrenzung zwischen Telekommunikationsdiensten und Telemedien erfolgt daher richtigerweise nach weit überwiegender Auffassung funktional. Danach stehen die Regelungen des Telekommunikationsgesetzes und des Telemediengesetzes in einem Ergänzungsverhältnis und können funktionsbezogen nebeneinander zur Anwendung kommen. ${ }^{103}$ Die Gegenansicht sieht zum Beispiel den Internetzugang als einheitlichen, lediglich technischen Vorgang an, der als Telekommunikationsdienstleistung lediglich unter das Telekommunikationsgesetz falle. ${ }^{104}$ Gegen diese Auffassung spricht zum einen, dass dem Telemedi-

99 So auch der ausdrückliche Wille des Gesetzgebers: BT-Drs. 16/3078, S. 20; Engels/Jürgens/Fritzsche, K\&R 2007, 58; Spindler, CR 2007, 240; Roßnagel, NVwZ 2007, 744; Rössel, ITRB 2007, 159; Mückl, JZ 2007, 1080.

100 BT-Drs. 16/3078, S. 20.

101 BeckTKG-Komm/Wittern/Schuster, \ 3 Rn. 48; Spindler/Schmitz/Geis/Spindler, \ 2 TDG Rn. 22; BerlKommTKG/Säcker, \3 Rn. 38; Beucher/Leyendecker/v. Rosenberg, \2 TDG Rn. 10; Schols, S. 162; i.d.S. auch BGH, NJW 2002, 361 (362).

102 So auch Spindler/Schmitz/Geis/Spindler, $\$ 2$ TDG Rn. 22.

103 Scholy, S. 163; Spindler/Schmitz/Geis/Spindler, \2 TDG Rn. 22 f.; Beck TKGKomm/Wittern/Schuster, $\ 3$ Rn. 49; BerlKommTKG/Säcker, $\ 3$ Rn. 38; Schmitz, S. 72 ff.; Böhm, in: Gounalakis, Rhb. Electronic Business, $₫ 37$ Rn. 8 f.; so auch Koenig/Neumann, K\&R Beilage 3/2004, 10 u. 24 ff.; BGH, NJW 2002, 361 (362); AG Darmstadt, MMR 2005, 634 (635).

104 Stadler, Rn. 36. 
engesetz dann praktisch kein Anwendungsbereich mehr bliebe ${ }^{105}$ und zum anderen die Gesetzesbegründung zum neuen Telemediengesetz, nach der solche Dienste, die neben der Übertragung von Signalen über Telekommunikationsnetze auch noch inhaltliche Dienstleistungen, wie den Internetzugang oder E-Mail-Dienste, anbieten, zugleich Telekommunikationsdienste sowie Telemedien sein können. ${ }^{106}$ Ebenfalls deutet der Wortlaut des $\$ 1$ Abs. 3 TMG, der davon spricht, dass das Telemediengesetz das Telekommunikationsrecht „unberührt" lasse, auf eine funktionsbezogene Anwendung der beiden Gesetze hin. Das bedeutet, dass auch der Gesetzgeber selbst von einer funktionsbezogenen Abgrenzung ausgeht und insofern den bis dato herrschenden Streit zugunsten der funktionsbezogenen Abgrenzung klargestellt hat.

Rundfunk ist nach $₫ 2$ Abs. $1 \mathrm{RStV}$, ,...] die für die Allgemeinheit bestimmte Veranstaltung und Verbreitung von Darbietungen aller Art in Wort, in Ton und in Bild unter Benutzung elektromagnetischer Schwingungen ohne Verbindungsleitung oder längs oder mittels eines Leiters. Der Begriff schließt Darbietungen ein, die verschlüsselt verbreitet werden oder gegen besonderes Entgelt empfangbar sind.“

In Abgrenzung zum Rundfunkstaatsvertrag orientiert sich das Telemediengesetz an der individuellen Abrufbarkeit der jeweiligen Dienste (zum Beispiel Video-On-Demand), sobald diese nicht mehr vorliegt, wie beispielsweise beim Near-Video-On-Demand, greift der Rundfunkstaatsvertrag. ${ }^{107}$ Die frühere Abgrenzung zwischen Telediensten und Rundfunk nach der redaktionellen Bearbeitung zur Meinungsbildung, ist im Rahmen des Telemediengesetzes gestrichen wurden, allerdings findet sich die Formulierung der journalistischredaktionellen Bearbeitung im Telemedienbereich des Rundfunkstaatsvertrags in den $\iint 54$ Abs. 2, 55 Abs. 2, 56 Abs. 1, 57 wieder. Dabei geht der Gesetzgeber davon aus, dass diese Bearbeitungen als elektronische Presse in Erscheinung treten und es möglich sein soll, dass ,,[...] einseitig und unausgewogen $[\ldots . .]^{“}$ ein Meinungsbeitrag geleistet wird. ${ }^{108}$ Das bedeutet, dass die alte Abgrenzung innerhalb des Rundfunkstaatsvertrags auch noch weiter Geltung hat. 109

\footnotetext{
105 So auch Spindler/Schmitz/Geis/Spindler, \$2 TDG Rn. 22.

106 BT-Drs. 16/3078, S. 20 f.

107 Spindler, CR 2007, 240.

108 Begründung zum Neunten Staatsvertrag zur Änderung rundfunkrechtlicher Staatsverträge (Neunter Rundfunkänderungsstaatsvertrag), S. 16, abrufbar unter http://www.lfk.de/gesetzeundrichtlinien/rundfunkstaatsvertrag/main.html <25.11.2009>.

109 Engels/Jürgens/Fritzsche, K\&R 2007, 57; Spindler, CR 2007, 240 f.; Mückl, JZ 2007, 1081.
} 


\section{Web Service-Anbieter}

Bei dem Web Service-Anbieter muss geschaut werden, welche Leistungen er selbst in Verbindung mit dem Angebot des Web Service erbringt. Bietet er selbst auch die Telekommunikation im Sinne des $₫ 3$ Nr. 22 TKG an, mittels derer der Web Service im Internet angeboten werden kann, so könnte er dafür als Telekommunikationsdienstleister nach $₫ 3 \mathrm{Nr}$. 24 TKG eingeordnet werden, andernfalls ist er selber nur Nutzer der Telekommunikationsdienstleistung im Sinne des $\int 3$ Nr. 14 TKG, ${ }^{110}$ vorausgesetzt es handelt sich um eine natürliche Person.

Darauf wird es aber bei Umsetzung der Serviceorientierten Architektur in aller Regel nicht ankommen, sondern vielmehr auf die Einordnung des Web Service-Angebots im Rahmen der Interaktion selbst. Die Aufgabe des Web Service-Anbieters liegt in der Zurverfügungstellung von Softwarekomponenten, Web Services, und somit in einer Serviceleistung, nicht hingegen im technischen Vorgang des Aussendens, Übermittelns und Empfangens von Signalen mittels Telekommunikationsanlagen im Sinne des $₫ 3$ Nr. 22 TKG. Der Web Service-Anbieter stellt in Form des Web Service einen Dienst zur Suche von Informationen bereit. Zwar erfolgt die Suche dann mittels der Übertragung von Signalen, diese stellt aber nur die Technik, die im Zweifel durch einen Dritten erbracht wird, dar. Im Vordergrund steht jedoch die Suche von Informationen und demnach Inhalten, so dass das Telekommunikationsgesetz für das Angebot des Web Service nicht einschlägig ist. Schon von der Definition her nicht in Betracht kommt die Einordnung als Rundfunkdienst, da die Technik Web Service keine an die Allgemeinheit gerichtete Darbietung in Wort, in Ton und in Bild darstellt und schon gar keine redaktionelle Bearbeitung enthält. Dementsprechend lassen sich Web Services - negativ abgegrenzt - als Telemedien nach $\int 1$ Abs. 1 TMG einordnen. ${ }^{111}$

Telemediendiensteanbieter sind nach $₫ 2$ Abs. 1 TMG alle natürlichen und juristischen Personen, die eigene oder fremde Telemedien zur Nutzung bereithalten oder deren Zugang zur Nutzung vermitteln. Der Web Service-Anbieter stellt das Telemedium Web Service selbst bereit und ermöglicht durch die Publikation der Dienstebeschreibung im UDDI eine Nutzung des Web Service durch den Web Service-Nutzer oder gegebenenfalls einen Dritten. Der Web Service-Anbieter ist daher im Verhältnis zum Web Service-Nutzer und

110 Schaar, RDV 2003, 60.

111 So auch Raabe/Dinger, CR 2007, 796. 
zu Dritten als Telemediendiensteanbieter nach $₫ 2 \mathrm{Nr}$. 1 TMG zu qualifizieren; das Telemediengesetz findet Anwendung.

Im Verhältnis zum Web Service-Vermittler dagegen, ist der Web ServiceAnbieter kein Telemediendiensteanbieter, da der Web Service-Vermittler den Web Service des Web Service-Anbieters nicht im klassischen Sinne selbst nutzt, sondern ihm vielmehr eine Publikationsmöglichkeit für denselben zur Verfügung stellt. Das bedeutet, dass der Web Service-Anbieter gegenüber dem Web Service-Vermittler selbst ein Telemediennutzer sein könnte. Nach $\ 2 \mathrm{Nr}$. 3 TMG ist Nutzer ,jede natürliche oder juristische Person, die Telemedien nutzt, insbesondere um Informationen zu erlangen oder zugänglich zu machen“. Der Web Service-Anbieter gebraucht hier das Telemedium des Web Service-Vermittlers, das UDDI, um seinen Web Service und somit auch Informationen den Web Service-Nutzern oder Dritten zugänglich machen. Gegenüber dem Web Service-Vermittler ist der Web Service-Anbieter folglich lediglich ein Telemediennutzer im Sinne des $₫ 2$ Nr. 3 TMG.

Eine weitergehende Differenzierung muss aber im Rahmen der datenschutzrechtlichen Vorschriften getroffen werden. Nach $₫ 11$ Abs. 1 Nr. 1 TMG finden die Datenschutzvorschriften des Telemediengesetzes bei einer Bereitstellung der Telemedien im Rahmen des Dienst- und Arbeitsverhältnisses zu ausschließlich beruflichen oder dienstlichen Zwecken keine Anwendung. Werden Web Services daher lediglich in geschlossenen Systemen, in Form eines firmeninternen Intranets, angeboten und erfolgt eine Nutzung ausschließlich dienstlich oder beruflich, so finden die Datenschutzvorschriften des Bundesdatenschutzgesetzes (BDSG) Anwendung. ${ }^{112}$ Gleiches gilt für eine rein geschäftliche Kommunikation zwischen zwei Unternehmen, ${ }^{113}$ das heißt werden Web Services lediglich innerhalb von - hier nicht weiter untersuchten - B2B-Intranets angeboten und erfolgt die Nutzung ausschließlich zu geschäftlichen Zwecken, so finden ebenfalls die Vorschriften des Bundesdatenschutzgesetzes und nicht die des Telemediengesetzes für den Datenschutz Anwendung.

\section{Web Service-Nutzer}

Da der Web Service-Nutzer den Web Service in sein eigenes Angebot integriert und damit Dritten die Möglichkeit eröffnet, den Web Service des Web

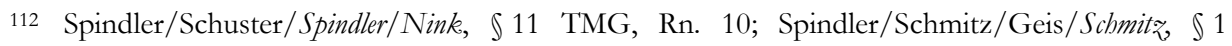
TDDSG Rn. 33.

113 Schaar, RDV 2003, 61. 
Service-Anbieters über den Web Service-Nutzer in Anspruch zu nehmen, hält der Web Service-Nutzer im Sinne des $\ 2$ Nr. 1 TMG ein fremdes Telemedium, den Web Service, zur Nutzung bereit und ist gegenüber Dritten ebenfalls als Telemediendiensteanbieter zu qualifizieren.

Anders verhält sich dies gegenüber dem Web Service-Anbieter, denn der Web Service-Nutzer macht durch die Integration des Web Service auch Informationen, wie die Flugdaten, Dritten zugänglich. Insofern ist der Web ServiceNutzer im Verhältnis zum Web Service-Anbieter ein Telemediennutzer nach \ 2 Nr. 3 TMG.

Gleiches gilt im Verhältnis zum Web Service-Vermittler. Auch hier gebraucht der Web Service-Nutzer ein fremdes Telemedium, das UDDI, um nach Web Services zu suchen und dadurch schließlich fremde Informationen zu erlangen. Daher ist der Web Service-Nutzer auch gegenüber dem Web ServiceVermittler ein Telemediennutzer nach $₫ 2 \mathrm{Nr}$. 3 TMG.

\section{Web Service-Vermittler}

Die Funktion des Web Service-Vermittlers gegenüber Web Service-Anbieter und -Nutzer ergibt sich im Umkehrschluss aus dem bereits Gesagtem: der Web Service-Vermittler stellt sowohl Web Service-Anbieter wie auch -Nutzer das Register zum Publizieren und Suchen von Web Services (das UDDI) bereit und fungiert daher gegenüber beiden als Telemediendiensteanbieter nach $\int 2$ Nr. 1 TMG.

\section{Vertragsrechtliche Grundlagen}

Ein Vertrag wird definiert als die Regelung eines Rechtsverhältnisses, das von zwei oder mehr Parteien einvernehmlich getroffen wird und korrespondierende, auf dieselben Rechtsfolgen gerichtete, Willenserklärungen voraussetzt. ${ }^{114}$ Vom Vertragsbegriff im Sinne der $\$ \int 145$ ff. BGB werden alle Einigungen mit Rechtsbindungswillen im Bereich des Privatrechts erfasst. ${ }^{115}$ Klassischerweise entstehen derartige Einigungen durch persönlichen Kontakt der Parteien, gleich ob unter Anwesenden, schriftlich (unter An- oder Abwesenden) oder fernmündlich. Im Rahmen der Web Service-Nutzung und -Bereitstellung laufen die einzelnen Prozesse unter den Beteiligten aber vollautomatisiert ab. Ein persönlicher Kontakt der Akteure untereinander findet somit regelmäßig nicht

\footnotetext{
114 BGH, NJW 1956, 1313; Bamberger/Roth/Eckert, \145 Rn. 2; Palandt/Heinrichs, Einf. v. \ 145 Rn. 1; Flume, AT 2, §33/2 S. 601 ff.; Oetker/Maultasch, \1 A S. 1 f.

115 Erman/Armbrïster, Vor $\ 145$ Rn. 1, 4.
} 
statt. Jedoch ist mittlerweile allgemein anerkannt, dass derartige Einigungen auch elektronisch oder automatisiert erfolgen können. ${ }^{116}$ Vertragliche Beziehungen zwischen den Akteuren und Beteiligten sind daher denkbar. Fraglich ist jedoch, wie die Beziehungen der Beteiligten vertraglich einzuordnen sind, da daraus unterschiedliche Rechte und Pflichten resultieren.

\section{Vertragstypologische Einordnung}

Die vertraglichen Beziehungen der am Anbieten und Nutzen von Web Services Beteiligten können unterschiedlich ausgestaltet sein. Von Interesse sind die Beziehungen zwischen dem Web Service-Hersteller und dem Web ServiceAnbieter, dem Web Service-Anbieter und dem Web Service-Vermittler, dem Web Service-Anbieter und dem Web Service-Nutzer, dem Web ServiceNutzer und dem Web Service-Vermittler, Web Service-Nutzer und Dritten sowie dem Web Service-Anbieter und Dritten. Unterschieden wird bei den gesetzlichen Vertragstypen nach der Art der geschuldeten Leistung. ${ }^{117}$ Das bedeutet konkret, dass betrachtet werden muss, wie das wirtschaftlichtechnische Leistungsbild in der jeweiligen Beziehung aussieht. ${ }^{118}$

\section{a) Web Service-Hersteller - Web Service-Anbieter}

Zwischen Web Service-Hersteller und Web Service-Anbieter kommen je nach Art der geschuldeten Leistung sowohl Kauf- als auch Werkvertrag in Betracht. Denkbar wären auch Miet- und Leasingvertrag, sofern der Web ServiceHersteller den Web Service lediglich dem Web Service-Anbieter gegen Entgelt zur Nutzung zur Verfügung stellt.

\section{aa) Kaufvertrag}

Der Kaufvertrag nach $\int 433$ BGB ist ein gegenseitig verpflichtendes Schuldverhältnis, durch das der Verkäufer verpflichtet wird, dem Käufer die vereinbarte Sache (oder ein Recht im Sinne des $₫ 453$ BGB) zu verschaffen und der Käufer, dem Verkäufer den vereinbarten Kaufpreis zu zahlen sowie die Sache

\footnotetext{
116 MünchKommBGB/Kramer, Bd. 1/1, Vor $₫ 116$ Rn. 22; Palandt/Heinrichs/Ellenberger, Einf. v. \$116 Rn. 1; Erman/Palm, Vor \116 Rn. 5; Borges, S. 191; Geis, NJW 1997, 3000; dies bereits voraussetzend BGH, NJW 2005, 53 (54); NJW 2005, 976 (977).

117 Larenz, II/1, \38 S. 1; Stichtenoth, K\&R 2003, 109.

118 So auch Koch, ITRB 2007, 73.
} 
abzunehmen. ${ }^{119}$ Sind daher Beschaffung, Abnahme und Kaufpreis des Web Service in standardisierter Form Vertragsgegenstand, könnten die Regeln des Kaufvertrags Anwendung finden. Da es sich bei Web Services aber lediglich um Softwarekomponenten handelt, stellt sich die Frage, ob diese überhaupt Gegenstand eines Kaufvertrages sein können. Dafür müsste Software als Sache zu qualifizieren sein. Sachen im Sinne des Bürgerlichen Gesetzbuchs sind nach $\int 90$ BGB nur körperliche Gegenstände. Teile der Literatur wollen Software, entgegen der hier vertretenen Auffassung, ${ }^{120}$ eine solche Sachqualität abgesprochen. ${ }^{121}$ Im Rahmen des Kaufvertrags ist dieser Streit seit der Schuldrechtsreform von 2002 allerdings nur noch rein akademischer Natur, da Kaufverträge nach $₫ 453$ Abs. 1, 2. Alt. BGB auch über ,sonstige Gegenstände“ abgeschlossen werden können. Unter den Begriff der sonstigen Gegenstände fallen insbesondere auch unkörperliche Objekte, ${ }^{122}$ worunter nach der Gesetzesbegründung auch Software falle, ${ }^{123}$ wie bereits nach der überwiegenden Ansicht bisher die verkörperte Software. ${ }^{124}$ Daher lassen sich die kaufrechtlichen Regeln auf Web Services, gleich ob man sie als Sache oder sonstigen Gegenstand qualifiziert, entweder analog oder direkt anwenden. ${ }^{125}$

Weist ein solcher Vertrag eine Auslandsberührung auf, weil entweder der Hersteller oder der Anbieter ihren Unternehmenssitz im Ausland haben, und erfolgte keine Rechtswahl nach Art. 27 Abs. 1 EGBGB, so muss nach Art. 28 Abs. 1, Abs. 2 EGBGB das Recht des Staats, in dem der Web ServiceHersteller seinen Sitz hat, angewandt werden, da die Übereignung des Web Service die charakteristische Vertragsleistung eines Kaufvertrags darstellt. Bei Kaufverträgen innerhalb der Europäischen Union erfolgt nicht per se eine

119 Palandt/Weidenkaff, Einf. v. \433 Rn. 1; Laren₹ II/1, \39 S. 6; MünchKommBGB/Westermann, Bd. 3, \ 433 Rn. 9.

120 S. ausführlich unter A.III.1.a)bb)Exkurs: Software als Sache?.

121 So Redecker, NJW 1992, 1739 ff.; Dietrich, CR 2002, 473; Hilty, MMR 2003, 3 ff.; MüllerHengstenberg, CR 2004, 164 f.; Bamberger/Roth/Fritzsche, \90 Rn. 26.

122 MünchKommBGB/Westermann, Bd. 3, \453 Rn. 6.

123 BT-Drs. 14/6040, S. 242.

124 Palandt/Weidenkaff, \453 Rn. 8; Bamberger/Roth/Fritzsche, \90 Rn. 27; Diedrich, CR 2002, 473; Müller-Hengstenberg, CR 2004, 164 f.; durch die Betonung, dass eine Verkörperung für die Sacheigenschaft erforderlich ist, ebenfalls davon ausgehend die st. Rspr.: BGH, GRUR 1985, 1055 (1056); NJW 1988, 406 (408); NJW 1990, 320 (321); NJW 1993, 2436 (2437); NJW 2000, 1415 ff.; CR 2007, 75 (76).

125 So auch Junker, NJW 2005, 2831. Zum Streit hinsichtlich der Einordnung von Software als Sache s. ausführlich A.III.1.a)bb)Exkurs: Software als Sache?. 
Modifikation durch das Herkunftslandprinzip. Denn dieses ist nur auf OnlineVerträge, die die Lieferung von unverkörperten Waren zum Gegenstand haben, nicht aber auf die physische Lieferung von verkörperten Waren anwendbar. ${ }^{126}$ Daher ist zu unterscheiden, ob Web Services auf einem Datenträger überlassen werden und physisch versandt beziehungsweise ob sie dem Web Service-Anbieter als Käufer in sonstiger physischer Weise vermittelt werden, oder der Verkauf rein elektronisch erfolgt (Download, via elektronischer Post, etc.). Je nach Transportart des Web Service wird dann das Sachrecht des Empfangsstaats, sofern es milder als das Recht des Herkunftslands ist, durch das Herkunftslandprinzip modifiziert.

\section{bb) Werkvertrag}

Vereinbaren Web Service-Hersteller und Web Service-Anbieter, dass der Web Service individuell für die Bedürfnisse des Web Service-Anbieters hergestellt werden soll, könnte ein Werkvertrag im Sinne des $₫ 631$ BGB vorliegen. Der Werkvertrag ist, wie auch der Kaufvertrag, ein gegenseitiger Vertrag, durch den sich der eine Teil zur Herstellung des Werks und der andere zur Entrichtung der vereinbarten Vergütung verpflichtet. Geschuldet wird ein bestimmter Erfolg, ${ }^{127}$ die Herstellung des Werks. Ein Werk kann sowohl ein körperliches Arbeitsprodukt, wie die Herstellung einer Sache oder ihre Veränderung, als auch ein unkörperliches Arbeitsergebnis, wie Forschungs- und Entwicklungsergebnisse ${ }^{128}$ oder ein Gutachten ${ }^{129}$, sein. ${ }^{130}$ Voraussetzung ist eine schöpferische Leistung des Werkunternehmers. ${ }^{131}$ Eine solche schöpferische Leistung wurde auch bei der individuellen Programmierung von Software anerkannt, 132 so dass unter Umständen auch bei individuell angepassten oder programmierten Web Services die Regeln des Werkvertragsrechts greifen könnten.

126 Spindler/Schmitz/Geis/Spindler, \4 TDG Rn. 10 f.; Spindler/Schuster/Pfeiffer/Weller, \ 3 TMG Rn. 3 f.; Tettenborn, K\&R 2000, 61.

127 BGH, NJW 2002, 3323 (3324); Esser/Weyers, II/1, \31 S. 249; Larenz, II/1, \53 S. 342; Palandt/Sprau, Einf. v. \$ 631 Rn. 1.

128 BGH, NJW 2002, 3323 (3324).

129 BGH, NJW 1995, 392 (393).

130 Palandt/Sprau, Einf. v. \631 Rn. 1; Laren₹, II/1, \53 S. 344.

131 MünchKommBGB/Busche, Bd. 4, \631 Rn. 1; Esser, II/1, \31 S. 250.

132 BGH, NJW 1987, 1259 (1260); NJW 2000, 2812 (2814); NJW 2001, 1718 (1719); davon stillschweigend ausgehend BGH, NJW-RR 2004, 782 ff.; Erman/Schwenker, Vor \$S 631-651 Rn. 22; Palandt/Sprau, Einf. v. \631 Rn. 22; Esser/Weyers \31 S. 251; Redeker, CR 2004, 88. 
Zum Teil wurde jedoch bei der Erstellung von Individualsoftware ein Werklieferungsvertrag nach \$ 651 a.F. BGB angenommen, da Software eine bewegliche Sache im Sinne des $₫ 651$ a.F. BGB sei. ${ }^{133}$ Andere ordneten Software als reinen Werkvertrag nach $\int 631$ BGB ein. ${ }^{134}$ Praktisch wirkte sich die unterschiedliche Einordnung aber kaum aus, da auch bei Werklieferungsverträgen im Wesentlichen Werkvertragsrecht galt. 135 Durch die Schuldrechtsreform, zurückgehend auf die Verbrauchsgüterrichtlinie, ${ }^{136}$ wurde aber die Vorschrift des $₫ 651$ BGB (ehemals Werklieferungsvertrag) vollständig neu gefasst. ${ }^{137}$

\section{Problematik des $\int 651$ S. 1 BGB bei Software}

Nach \651 S. 1 BGB finden bei der Lieferung herzustellender oder zu erzeugender beweglicher Sachen die Vorschriften über den Kauf Anwendung. Konsequenz der Anwendung der kaufrechtlichen Vorschriften für den Vertrag zwischen Web Service-Hersteller und Web Service-Anbieter wäre, dass

- die Sache abgeliefert statt abgenommen wird, was wiederum bedeutet, dass die Gewährleistungsfristen bereits mit der Ablieferung zu laufen beginnen,

- dem Käufer ( $\$ 442$ Abs. 1 Satz 1 BGB) und nicht dem Hersteller ( 640 Abs. 2 BGB) das Wahlrecht bezüglich der Art der Nacherfüllung zusteht,

- der Kaufpreis ab Ablieferung fällig wird,

- Abschlagszahlungen wie in $\int 632$ a BGB nicht existieren

- und die kaufrechtliche Obliegenheit greift. ${ }^{138}$

133 BGH, NJW 1993, 2436 (2437); OLG Köln, CR 2001, 437; Müller-Hengstenberg, NJW 2000, 3545; Marly, Rn. 54.

134 Bartsch, Anm. zu BGH, CR 2000, 424, CR 2000, 427; so auch nach der Schuldrechtsmodernisierung ohne auf die Sachproblematik und \651 BGB einzugehen: OLG Karlsruhe, CR 2003, 95 (96).

135 S. Redecker, CR 2004, 88.

136 Richtlinie 1999/44/EG des Europäischen Parlaments und des Rates v. 25.5.1999 zu bestimmten Aspekten des Verbrauchsgüterkaufs und der Garantien für Verbrauchsgüter, ABl. EG L 171 v. 7.7.1999, S. 12-16.

137 Siehe dazu Erman/Schwenker, $\ 651$ Rn. 1; Bamberger/Roth/Voit, $\ 651$ Rn. 1; Palandt/Sprau, \651 Rn. 1.

138 Dazu ausführlich Schmidl, MMR 2004, 591; Bräutigam/Rücker, CR 2006, 362 ff.; Spindler/Klöhn, CR 2003, 81; Thewalt, CR 2002, 5 f. 
Im Gegensatz zum alten Recht ergeben sich daher durchaus praktisch relevante Konsequenzen, je nachdem, ob $\int 651$ S. 1 BGB auf Software Anwendung findet oder nicht.

Voraussetzung für die Anwendbarkeit des $₫ 651$ S. 1 BGB ist das Vorliegen einer beweglichen Sache. Bewegliche Sachen sind nach $₫ 90$ BGB alle körperlichen Gegenstände, die nicht als Grundstück oder grundstücksgleich zu qualifizieren sind. ${ }^{139}$ Nicht körperliche Gegenstände wie Immaterialgüter, Energien etc. sind dagegen keine Sachen. ${ }^{140}$ Das bedeutet, dass die Frage des Charakters von Software, die im Kaufrecht durch die Einführung des $\int 453$ BGB nur noch eine untergeordnete Rolle spielt, im Werkvertragsrecht entscheidend ist. Während bis zur Schuldrechtsreform, wie bereits erörtert, ${ }^{141}$ weitestgehend Einigkeit bestand, dass zumindest die auf einem Datenträger verkörperte Software Sachqualität hat, entbrannte der Streit um die typologische Einordnung von Software nach der Schuldrechtsreform aufgrund des $₫ 453$ BGB und der Modernisierung des $\int 651$ S. 1 BGB aufs Neue.

Grob lassen sich dabei zwei Auffassungen differenzieren. Ein großer Teil der Literatur bejaht weiterhin die Sachqualität von Software. Diese Auffassung untergliedert sich wiederum in zwei Strömungen: den Teil, der konsequent \651 S. 1 BGB auf individuell hergestellte beziehungsweise angepasste Software anwendet und so zum Kaufrecht gelangt ${ }^{142}$ und den anderen Teil, der \651 S. 1 BGB mit unterschiedlichen Begründungen (zum Beispiel teleologische Reduktion, ${ }^{143}$ keine „sklavische“ Anwendung des Sachbegriffs im Rahmen des $\ 651$ BGB, ${ }^{144}$ Schwerpunkt der Leistung ${ }^{145}$ ) auf Software nicht anwenden will und so zum Werkvertragsrecht gelangt. ${ }^{146}$ Die andere Ansicht dagegen verneint die Sachqualität von Software, so dass $\int 651$ S. 1 BGB nicht greift und Werkvertragsrecht unproblematisch bei individuell hergestellter

139 Palandt/Sprau, \651 Rn. 2; MünchKommBGB/Busche, Bd. 4, \ 651 Rn. 3.

140 MünchKommBGB/Holch, Bd. 1/1, \ 90 Rn.4; Bamberger/Roth/Fritzsche, \ 90 Rn. 18.

141 S. dazu A.III.1.a)aa) Kaufvertrag.

142 Schweinoch/Roas, CR 2004, 330; Mankowski, MDR 2003, 857; Lapp, in: Gounalakis, Rhb. Electronic Business, $\int 43$ Rn. 6.

143 Bräutigam/Rücker, CR 2006, 366 ff.

144 Spindler/Klöhn, CR 2003, 84.

145 Schmidl, MMR 2004, $592 \mathrm{f}$.

146 Marly, Rn. 53 ff. u.119; Bräutigam/Rücker, CR 2006, 366 ff.; Spindler/Klöhn, CR 2003, 84; Schmidl, MMR 2004, 592 f.; Müller-Hengstenberg/Krmar, CR 2002, 549 ff.; MünchKommBGB/Busche, Bd. 4, J 651 Rn. 12. 
Software Anwendung findet. ${ }^{147}$ Daher muss zunächst geklärt werden, ob Software eine Sache ist oder nicht.

\section{Exkurs: Software als Sache?}

Weil Software Gegenstand einer schöpferischen Leistung ist, die man nicht anfassen kann, wird ihr zum Teil die Sachqualität abgesprochen. ${ }^{148}$ Dagegen wird vorgebracht, dass auch ein Buch, dessen Sachqualität nicht angezweifelt werde, das Ergebnis einer schöpferischen Geistestätigkeit sei und ausschließlich wegen seines geistigen Inhalts und nicht wegen seines Informationsträgers, des Papiers, erworben werde. ${ }^{149}$ Das würde bedeuten, dass Software als solche als Sache qualifiziert werden müsste. Gegen die Richtigkeit des Buchbeispiels spricht in letzter Konsequenz aber, dass zwar zweifellos das Buch das Ergebnis einer schöpferischen Geistestätigkeit ist, jedoch wenn Buch und Werk getrennt würden, niemand das lediglich vergeistigte Werk als Sache ansehen würde. ${ }^{150}$ Gleiches gilt für Software - auch hier muss zwischen Programm und Datenträger unterschieden werden. Daher wurde bisher überwiegend die Sachqualität von Standardsoftware zumindest dann bejaht, wenn sie auf einem Datenträger, gleich ob CD, Diskette, USB-Stick, Festplatte oder ähnlichem, verkörpert ist. ${ }^{151}$ Weil der Gesetzgeber in der Gesetzesbegründung bei $\int 453$ BGB Software als sonstigen Gegenstand erwähnte, ${ }^{152}$ will ein Teil der Literatur Software nicht mehr als Sache qualifizieren, sondern generell, unabhängig von der Verkörperung, als sonstigen Gegenstand nach \453 BGB

147 Stichtenoth, K\&R 2003, 106 f.; Junker, NJW 2005, 2831; Heussen, CR 2004, 7; Redeker, CR 2004, 88 f.; Diedrich, CR 2002, 475; vom Ergebnis her auch gegen eine Anwendung des $\int 651$ BGB argumentierend, mit dem Vorschlag doch eher auf das Know-how und damit den Erfolg als auf den Datenträger abzustellen, ohne dabei aber $₫ 453$ BGB als Argument zu erwähnen Thewalt, CR 2002, 7 .

148 So Redecker, NJW 1992, 1739 ff.; Dietrich, CR 2002, 473; Hilty, MMR 2003, 3 ff.; MüllerHengstenberg, CR 2004, 164 f.; Bamberger/Roth/Fritæsche, $₫ 90$ Rn. 26.

149 BGH, CR 2007, 75 (76).

150 So auch Redecker, NJW 1992, 1739 ff.; Bamberger/Roth/Fritzsche, \90 Rn. 26.

151 So die ständige Rspr: BGH, GRUR 1985, 1055 (1056); NJW 1988, 406 (408); NJW 1990, 320 (321); NJW 1993, 2436 (2437); NJW 2000, 1415 ff.; CR 2007, 75 (76); zustimmend in der Literatur: Marly, Rn. 96 ff. insb. Rn. 110; Palandt/Heinrichs/Ellenberger, $\int 90$ Rn. 2; MünchKommBGB/Holch, Bd. 1/1, $\ 90$ Rn. 27; bereits in der Software selbst (auch ohne Verkörperung auf einem Datenträger) eine Sache sehend König, NJW 1993, 3121 ff.; Schneider, Hdb. EDV-Recht, D Rn. 96; offen lassend Erman/Michalski, $\mathbb{S} 90$ Rn. 3.

152 BT-Drs. 14/6040, S. 242. 
einordnen. ${ }^{153}$ Dieser Auffassung ist die systematische Stellung der Erwähnung zu Gute zu halten. Denn warum sollte der Gesetzgeber, der um den bestehenden Streit um die Einordnung von Software und die ergebnisorientierte Lösung über den Umweg der Verkörperung der Rechtsprechung wusste, Software ausgerechnet als sonstigen Gegenstand erwähnen? ${ }^{\text {154 }}$

Bei diesem Gedanken wird aber außer Acht gelassen, dass vor der Schuldrechtsmodernisierung Software nach der überwiegenden Ansicht nur dann als Sache anerkannt wurde, wenn sie in irgendeiner Form verkörpert war, andernfalls wurde Kaufrecht nicht angewandt. Daher erscheint es durchaus denkbar, dass der Gesetzgeber mit der Erwähnung des Softwarebegriffs in der Begründung zu $₫ 453$ BGB lediglich unverkörperte Software meinte, um diese Lücke zu schließen und klarzustellen, dass Kaufrecht einheitlich auf Software, unabhängig von einer etwaigen Verkörperung, angewendet werden kann. ${ }^{155}$ Hinzu kommt, dass eine Klärung derartiger Fragen nicht in den besonderen Teil des Bürgerlichen Gesetzbuchs gehört, sondern dogmatisch eher im allgemeinen Teil des Bürgerlichen Gesetzbuchs hätte gelöst werden müssen. ${ }^{156}$ Dem ließe sich zwar grundsätzlich entgegenhalten, dass der Gesetzgeber dies dann doch auch hätte tun können aber nicht getan hat. Allerdings darf dabei aber nicht übersehen werden, dass es sich lediglich um eine Modernisierung des Schuldrechts und nicht des Bürgerlichen Gesetzbuchs allgemein handelte, so dass der Gesetzgeber diese Regelung im allgemeinen Teil auch nicht bewusst unterlassen hat, weil er Software generell als sonstigen Gegenstand sieht, sondern vielmehr weil eine Neuregelung des allgemeinen Teils gar nicht auf dem Programm stand. Im Übrigen existierte zu diesem Zeitpunkt bereits eine gefestigte Meinung zum Charakter von Software, so dass davon ausgegangen werden muss, dass der Gesetzgeber mit der Erwähnung bei $\int 453$ BGB lediglich die Lücke bei unkörperlicher Software schließen und sie mit der gängigen Praxis der Anwendung des Kaufrechts bei verkörperter Software harmonisieren wollte. Andernfalls wäre es angesichts der herrschenden Auffassung nötig gewesen, ein paar klarstellende Worte zu verlieren, dass durch \453 BGB diese gängige Praxis der Qualifizierung von Software als Sache geändert werden sollte. Derartige Hinweise, die solche Schlüsse zulassen könnten, finden sich aber weder im Gesetzestext noch in der Gesetzesbegründung.

153 Stichtenoth, K\&R 2003, 106 ff.; Junker, NJW 2005, 2832; kritisch zur Einordnung als Sache Müller-Hengstenberg, CR 2004, 164.

154 Ähnlich in der Argumentation Stichtenoth, K\&R 2003, 106 ff. u. Diedrich, CR 2002, 475.

155 So auch Bamberger/Roth/Fritzsche, \90 Rn. 27; Marly, Rn. 117; Schmidl, MMR 2004, 591.

156 Marly, Rn. 117. 
Insofern kann die Verkehrsauffassung beibehalten werden, nach der zwischen Programm und Datenträger differenziert wird. Software ist folglich nach wie vor im Fall einer Verkörperung als Sache im Sinne des $₫ 90$ BGB zu qualifizieren. ${ }^{157}$ Der Unterschied besteht lediglich darin, dass die Vorschriften des Kaufs nun auch auf unverkörperte Software über den Terminus des sonstigen Gegenstands nach \453 BGB angewendet werden können.

\section{Anwendbarkeit des $₫ 651$ S. 1 BGB}

Durch die Bejahung der Sacheigenschaft von Software bleibt nunmehr zu klären, wie mit \651 S. 1 BGB zu verfahren ist. \651 S. 1 BGB kann lediglich in den Fällen außer Acht bleiben, in denen Web Services in keiner Form verkörpert sind, weil sie dem Web Service-Anbieter ausschließlich auf elektronischem Wege übertragen werden. Dann nämlich sind sie keine Sachen und das Werkvertragsrecht findet unproblematisch Anwendung. Rein praktisch betrachtet, wird dies aber aus Sicherheitsgründen so gut wie nie der Fall sein, weil davon ausgegangen werden muss, dass eine Implementierung des individuell angepassten beziehungsweise programmierten Web Service durch den Web Service-Hersteller auf dem System des Web Service-Anbieters erfolgen wird und spätestens dadurch eine Verkörperung erfolgt.

Daher ist es notwendig, die Anwendbarkeit von $₫ 651$ S. 1 BGB genauer zu untersuchen. Der Wortlaut spricht scheinbar eindeutig für die Anwendung der kaufrechtlichen Vorschriften. Hinsichtlich der Ratio des \651 S. 1 BGB erscheint diese Konsequenz allerdings fraglich. Hintergrund des \651 S. 1 BGB ist die Umsetzung der Verbrauchsgüterrichtlinie. ${ }^{158}$ Nach Art. 1 Abs. 4 der Verbrauchsgüterrichtlinie sind auch Verträge über die Lieferung herzustellender und zu erzeugender Verbrauchsgüter Kaufverträge. Aus Gründen der Vereinheitlichung hat der deutsche Gesetzgeber den Anwendungsbereich des \651 BGB auf die Lieferung herzustellender und zu erzeugender Sachen ausgeweitet. ${ }^{159}$ Laut der Gesetzbegründung soll dann aber beispielsweise die Herstellung nicht-körperlicher Werke, wie zum Beispiel die Planung eines Architekten oder die Erstellung von Gutachten, weiter vom Werkvertragsrecht

157 So auch BGH, CR 2007, 75 (76) ohne weiter auf den Streit hinsichtlich $\int 453$ BGB einzugehen.

158 Richtlinie 1999/44/EG des Europäischen Parlaments und des Rates vom 25. Mai 1999 zu bestimmten Aspekten des Verbrauchsgüterkaufs und der Garantien für Verbrauchsgüter („Verbrauchsgüterkauf-Richtlinie"), ABl. EG L 171 v. 7.7.1999, S. 12-16.

159 Gesetzentwurf vom 14.05.2001 zur Modernisierung des Schuldrechts, BT-Drs. 14/6040, S. 268; Metzger, AcP 204 (2004), 251; MünchKommBGB/Busche, Bd. 4, \$ 651 Rn. 2. 
erfasst werden. ${ }^{160}$ Das bedeutet, dass $\int 651$ BGB keine Anwendung auf geistige Werke findet. ${ }^{161}$ Im Fokus des $\ 651$ BGB und der daraus resultierenden Anwendung der kaufrechtlichen Vorschriften steht daher die Übergabe einer beweglichen Sache. Zwar sind auch die nach der Gesetzesbegründung aus \651 BGB herausfallenden Gutachten oder Architektenpläne in aller Regel verkörpert, bei diesen liegt der Schwerpunkt des Vertrags aber nicht auf der Übergabe des verkörperten Gutachtens oder Plans sondern vielmehr in der Herstellung und Anfertigung. ${ }^{162}$ Damit erscheint auch die Entwicklung von Individualsoftware vergleichbar. ${ }^{163}$ Auch hier gibt die Erbringung der geistigen Leistung dem Vertrag das Gepräge und nicht die Herstellung und Lieferung Datenträgers, auf dem die Leistung verkörpert ist. Daraus lässt sich folgern, dass der Gesetzgeber mit dem Anwendungsbereich des $\int 651$ BGB offensichtlich nicht diese Art von Geschäften im Auge hatte, sondern vielmehr das typische auf Warenumsatz gerichtete Rechtsgeschäft, 164 also das Überwiegen des kaufrechtlichen Elements, was auch wiederum der Ratio der Verbrauchsgüterrichtlinie entspricht. 165 Dies alles berücksichtigend, erscheint eine Anwendung des $\int 651$ BGB auf Individualsoftware nicht gewollt und unangemessen.

\section{$\underline{\text { Resümee }}$}

\651 S. 1 BGB findet bei der Herstellung von Individualsoftware und dementsprechend auch der Herstellung von Web Services keine Anwendung, ${ }^{166} \mathrm{da}$ im Vordergrund der vertraglichen Verpflichtung der schöpferische Akt der Entwicklung steht, dieser den Schwerpunkt des Vertrags bildet und es daher

160 BT-Drs. 14/6040, S. 268.

161 So auch Staudinger/Peters, $₫ 651$ Rn. 14; Bamberger/Roth/Voit, $₫ 651$ Rn. 4; Palandt/Sprau, \ 651 Rn. 4; MünchKommBGB/Busche, Bd. 4, \ 651 Rn. 12.

162 S. dazu ausführlich Metəger, AcP 204 (2004), 247 f.; MünchKommBGB/Busche, Bd. 4, \ 651 Rn. 12; Bamberger/Roth/Voit, \651 Rn. 4.

163 So auch Metzger, AcP 204 (2004), 247; Staudinger/Peters, \ 651 Rn. 14; Bamberger/Roth/Voit, \651 Rn. 4; MünchKommBGB/Busche, Bd. 4, \ 651 Rn. 12; Palandt/Sprau, \ 651 Rn. 5; Marly, Rn. 58; Dietrich, CR 2002, 476 f.; Schmidl, MMR 2004, 592; so auch Junker, NJW 2005, 2832, der allerdings bereits Software nicht als Sache ansieht.

164 Marly, Rn. 58.

165 Dazu ausführlich Marly, Rn. 60 f.; Metəgger, AcP 204 (2004), 254 ff.

166 So auch Metzger, AcP 204 (2004), 247; Staudinger/Peters, \ 651 Rn. 14; Bamberger/Roth/Voit, \ 651 Rn. 4; MünchKommBGB/Busche, Bd. 4, \651 Rn. 12; Palandt/Sprau, \651 Rn. 5; Marly, Rn. 58 ff.; Spindler/Klöhn, CR 2003, 84; Bräutigam/Rücker, CR 2006, 368; Dietrich, CR 2002, 476 f.; Schmidl, MMR 2004, 592. 
nicht auf die nebensächliche Verkörperung ankommen kann. Das bedeutet, dass - sofern Web Service-Hersteller und Web Service-Anbieter eine individuelle Herstellung von Web Services vereinbaren - das Werkvertragsrecht nach den $\iint 631 \mathrm{ff}$. BGB einschlägig ist.

Für Verträge mit Auslandsbezug kann auf die Erläuterungen beim Kaufvertrag zurückgegriffen werden. ${ }^{167}$ Fehlt es an einer Rechtswahlklausel, so greift Art. 28 Abs. 1, 2 EGBGB. Charakteristische Leistung des Werkvertrags ist die Erstellung des Web Service, so dass regelmäßig das Recht des Staats in dem der Web Service-Hersteller seinen Sitz hat Anwendung findet; im Europäischen Raum gegebenenfalls modifiziert durch das Herkunftslandprinzip; je nachdem ob der Web Service physisch in verkörperter Form zum Web Service-Anbieter transferiert wird oder unverkörpert in elektronischer Form.

\section{cc) Miet- und Leasingvertrag}

Fraglich erscheint, ob die Miete beziehungsweise das Leasing eines Web Service möglich ist. Zunächst einmal wird es praktisch relativ selten vorkommen, dass Web Service-Anbieter und Web Service-Hersteller überhaupt Interesse an einer Überlassung auf Zeit haben. Web Services werden zwar in der Regel mit Hilfe von Standardkomponenten entwickelt, jedoch erfolgt die Programmierung des eigentlichen Web Service meist individuell, da diese anhand der individuellen Funktionalität des Web Service, also des Charakters der zu erbringenden Dienstleistung, definiert werden muss. ${ }^{168}$ Erfolgt regelmäßig eine individuelle Programmierung, so erscheint die Überlassung eines Web Service auf Zeit mit der darauf folgenden Rückgabe zumindest für den Hersteller wenig lukrativ, da er den konkreten Web Service nach Ablauf des Zeitvertrags dann kaum anderweitig ohne größere Veränderungen oder Anpassungen erneut vermieten oder verkaufen könnte. Sinnvoll erscheint eine Miete oder das Leasing von Web Services daher lediglich bei simplen standardisiert programmierten Diensten, ${ }^{169}$ wie Wetterabrufdiensten oder Terminkalenderfunktionen. In diesen Fällen erscheint es denkbar, dass standardisierte Web Services auch bei mehreren Web Service-Anbietern ohne Veränderung der Programmstruktur Verwendung finden können.

Gegenstand eines Mietvertrags sind nach $\int 535$ BGB Mietsachen. Darunter versteht man Wohnräume, Geschäftsräume etc. aber auch sonstige bewegliche

\footnotetext{
167 S. A.III.1.a)aa) Kaufvertrag.

168 Melzer, S. 63 f.

169 S. nur Melzer, S. 63 f.
} 
Sachen; entscheidend ist lediglich, dass es sich um eine Sache nach $₫ 90$ BGB handelt. ${ }^{170} \mathrm{Da}$ Web Services, wenn sie verkörpert sind, Sachen im Sinne des $\int 90$ BGB darstellen, ${ }^{171}$ könnten sie grundsätzlich auch Gegenstand eines Mietvertrags sein. ${ }^{172}$ Problematisch an einer solchen Qualifikation ist indes, dass - wie schon bei der Parallelproblematik hinsichtlich der Qualifikation des Application Service Providing-Vertrags als Mietvertrag ${ }^{173}$ kritisiert wird -174 der Web Service lediglich den Zugriff auf Ressourcen ermöglicht. Verglichen mit einem Produkthersteller von Sachgütern, könnte man daran denken, einen Vergleich zu einer Speditionsbeauftragung für den Transport der Sachgegenstände vom Hersteller an den Händler zu ziehen, bei dem die Speditionsfirma eine Dienstleistung erbringt. ${ }^{175}$ Das würde übertragen auf Serviceorientierte Architekturen mit Web Services bedeuten, dass der Web Service des Herstellers für den Web Service-Anbieter jeweils den Zugriff auf dessen Ressourcen ermöglicht, ohne das dieser eine Kontrolle über das Zugriffsmedium hat. Damit einher geht aber auch die permanente Verantwortung des Herstellers, dass die Zugriffe auch erfolgreich verlaufen, keine Datenverluste entstehen etc. Ordnet man dagegen den Web Service als gemietetes Transportmittel durch den Web Service-Anbieter ein, so muss der Hersteller nach $\int 535$ Abs. 1 S. 2 BGB den Web Service dem Web Service-Anbieter in einem zum vertragsgemäßen Gebrauch geeigneten Zustand überlassen und sie während der Mietzeit in diesem Zustand zu erhalten. Dabei kann dem Web Service-Anbieter die Erhaltungspflicht des Web Service übertragen werden. ${ }^{176}$ Betrachtet man diese beiden Möglichkeiten, so wird es der Ratio einer Web Service-Überlassung durch den Web Service-Hersteller grundsätzlich eher nicht entsprechen, eine

170 BGH, NJW-RR 1989, 589; Palandt/Weidenkaff, Einf. v. \535 Rn. 78; Erman/Jendrek, \535 Rn. 25; MünchKommBGB/Häublein, Bd. 3, \535 Rn. 62.

171 S. A.III.1.a)bb)Exkurs: Software als Sache?.

172 So auch für ASP- Verträge BGH, CR 2007, 75 (76) m. Anm. Lejeune und für Software im Allgemeinen LG Köln, CR 1996, 154; Palandt/Weidenkaff, Einf. v. \535 Rn. 103; Marly, Rn. 201. Anders ist dies natürlich zu beurteilen, wenn man Software nicht als Sache ansieht. Mit dieser Ansicht zieht z.B. Stichtenoth, K\&R 2003, 108 die mietrechtlichen Vorschriften allenfalls analog in Betracht; Müller-Hengstenberg/Kirn, NJW 2007, 2370 ff.

173 So der BGH, CR 2007, 75 (76) m. Anm. Lejeune; Junker, NJW 2003, 2797; Poble/Schmeding, Anm. zu BGH, CR 2007, 75 ff., K\&R 2007, 385 (386).

174 Müller-Hengstenberg/Kirn, NJW 2007, 2370 f.

175 So Müller-Hengstenberg/Kirn, NJW 2007, 2371.

176 Bamberger/Roth/Ehlert, \535 Rn. 187; Palandt/Weidenkaff, \535 Rn. 32; BGH, NJW 2004, 2961 (2962); so auch BGH, NJW 2002, 2383 f. insbesondere, wenn dies im Mietzins berücksichtigt wird. 
Dienstleistung mit allen Pflichten anzubieten, sondern vielmehr einen Gebrauchsgegenstand zu überlassen und dem Web Service-Anbieter die entsprechenden Instandhaltungspflichten aufzubürden. Freilich muss hier immer eine Auslegung des konkreten Vertrags unter Berücksichtigung aller eingeräumten Rechte und Pflichten erfolgen, in der Regel wird aber bei der Überlassung von Web Services durch den Web Service-Hersteller an den Web Service-Anbieter ein Mietvertrag anzunehmen sein.

Auch beim Leasingvertrag ist anerkannt, dass Software, und somit auch Web Services, leasingfähig sind. ${ }^{177}$ Vereinbaren Web Service-Hersteller und Web Service-Anbieter einen solchen Miet- oder Leasingvertrag muss aber beachtet werden, dass in diesem Falle die klassische Rückgabe nach $\ 546$ Abs. 1 BGB bei Web Services, und Software generell, nicht den gleichen Effekt hat, wie eine Rückgabe einer Wohnung. Im Falle der Wohnungsrückgabe erfolgt durch die körperliche Rückgabe ein vollständiger Ausschluss von der Nutzung. Web Services als Softwarekomponenten sind aber kopierbar, so dass eine Rückgabe der Web Services auf ihrem ursprünglichen Datenträger keine Gewähr für einen vollständigen Nutzungsausschluss liefert. Daher muss die Rückgabepflicht des $\int 546$ BGB sachgemäß in eine Verpflichtung zur vollständigen Löschung der Web Services sowie etwaiger Sicherheitskopien umgedeutet werden oder durch eine vorher eingebaute Programmsperre erfolgen. ${ }^{178}$

Sowohl für Miet- als auch Leasingvertrag ist die charakteristische Leistung des Vertrags die Überlassung des Web Service. Fehlt es daher an einer Rechtswahlklausel bei Verträgen mit Auslandsberührung, so findet in der Regel das Recht des Staats in dem der Web Service-Hersteller seinen Unternehmenssitz hat Anwendung. Im Europäischen Raum wird das Recht gegebenenfalls durch das Herkunftslandprinzip modifiziert, wenn der Überlassungsakt des Web Service in unverkörperter elektronischer Form erfolgt.

dd) Fazit

Je nachdem wie die Vereinbarungen zwischen Web Service-Hersteller und dem Web Service-Anbieter konkret aussehen, kommen die Typen des Kauf-, Werk-, Miet- und Leasingvertrags in Betracht. Um im jeweiligen konkreten Vertragsverhältnis eine genaue Einordnung vornehmen zu können, müssen letztlich die jeweiligen Parteivereinbarungen genau untersucht und ausgelegt

177 BGH, NJW 1993, 461 (462); NJW 1984, 2938; OLG Celle, NJW-RR 1993, 432 (433 f.); Schneider, Hdb. EDV-Recht, F Rn. 303 ff.; Marly, Rn. 201.

178 So auch Marly, Rn. 202; ferner BGH, NJW 1987, 2004 (2006 f.). 
werden. Der konkrete Vertragstyp ist dann entscheidend für weitere Rechte und Pflichten der Beteiligten. ${ }^{179}$

\section{b) Web Service-Anbieter - Web Service-Vermittler}

Im Verhältnis Web Service-Anbieter und Web Service-Vermittler steht der Eintrag der Dienstebeschreibung in den verschiedenen Kategorien (weiße, gelbe und grüne Seiten) ${ }^{180}$ im Mittelpunkt der vertraglichen Beziehungen. Die konkrete vertragliche Einordnung hängt davon ab, ob zwischen Web ServiceVermittler und Web Service-Anbieter ein Entgelt für den Eintrag vereinbart wurde. Darüber hinaus stellt sich die Frage, wie der Web Service-Vermittler auf das Vertragsverhältnis zwischen Web Service-Anbieter und Web ServiceNutzer einwirkt. Ist die Suche des Web Service-Nutzers erfolgreich verlaufen, so bezieht er die Dienstebeschreibung, die alle notwendigen technischen Informationen zur Dienstenutzung oder einen Verweis auf diese beinhaltet, um den Dienst in seine eigene IT-Landschaft einzubinden oder zu nutzen. ${ }^{181}$ Insofern kommt dem Web Service-Vermittler, wie bereits die Begrifflichkeit nahe legt, eine Art Vermittleraufgabe zu, was wiederum zeigt, dass der Web Service-Vermittler jedenfalls nicht selbst Vertragspartner im Rahmen des Web Service-Nutzer- und Web Service-Anbietervertrags wird. Denkbar wären eine so genannte Nachweismaklerschaft oder eine Stellvertretung durch den Web Service-Vermittler.

aa) Verhältnis Web Service-Anbieter - Web Service-Vermittler

Sofern es sich um einen externen Web Service-Vermittler handelt, muss aus Gründen der Ökonomie davon ausgegangen werden, dass regelmäßig Entgelte vereinbart werden. In diesen Fällen lässt sich der Eintrag mit einem so genannten Anzeigenvertrag vergleichen. Anzeigenverträge werden in der Regel dem Typus des Werkvertrags zugeordnet. ${ }^{182}$ Geschuldet wird die fehlerfreie Veröffentlichung der vom Besteller nach Form und Inhalt festgelegten Anzeige. ${ }^{183}$ Eine Abnahme des Werks ist zwar nach der Beschaffenheit des Werks

Dazu mehr unter A.III.3 Vertragliche Pflichten.

S. B.II.2 Komponenten.

Burghardt, S. 18; ferner Melzer, S. 53.

Erman/Schwenker, Vor \$S 631-651 Rn. 24; MünchKommBGB/Busche, Bd. 4, \ 631 Rn. 236; Bamberger/Roth/Voit, \ 631 Rn. 11; Palandt/Sprau, Einf. v. \631 Rn. 18; OLG Düsseldorf, MDR 1972, 688; LG Lübeck, NJW-RR 1999, 1655; LG Köln, NJW-RR 1999, 563; LG Mainz, NJW-RR 1998, 631; LG Hannover, NJW-RR 1989, 1525; AG Pinneberg, NJW-RR 1999, 1574. 
ausgeschlossen, nach $\ 646$ BGB tritt aber an ihre Stelle die Vollendung des Werks. ${ }^{184}$ Ähnlich verhält es sich auch bei dem Eintrag der Dienstebeschreibung des Web Service in dem Verzeichnis des Web ServiceVermittlers. Auch hier ist nach dem objektiven Empfängerhorizont eine fehlerfreie Veröffentlichung der Dienstebeschreibung Vertragsgegenstand, so dass in Anlehnung an den Anzeigenvertrag im Verhältnis Web ServiceAnbieter - Web Service-Vermittler bei Vereinbarung eines Entgelts ein Werkvertrag nach $\int 631 \mathrm{BGB}$ vorliegt. Wie auch beim Anzeigenvertrag ist eine Abnahme des Eintrags aus der Beschaffenheit der Eintragung im Sinne des $\int 646$ BGB nicht möglich, so dass an diese Stelle die Vollendung des Eintrags tritt. Mit Vollendung des Eintrags wird dann gemäß SS 641 Abs. 1, 646 BGB auch die Vergütung gegenüber dem Web Service-Vermittler fällig.

Vereinbaren Web Service-Anbieter und Web Service-Vermittler für den Eintrag in das Verzeichnis kein Entgelt, so handelt es sich, sofern bei den Parteien ein Rechtsbindungswille vorliegt, ${ }^{185}$ um einen Auftrag nach $\int 662$ BGB, der sich vom Werkvertrag durch die Unentgeltlichkeit unterscheidet. ${ }^{186}$

In beiden Fällen ist der Eintrag im UDDI charakteristische Vertragsleistung, so dass im Falle einer fehlenden Rechtswahlklausel bei Verträgen mit Auslandsbezug regelmäßig das Recht des Staats, in dem der Web ServiceVermittler seinen Unternehmenssitz hat, Anwendung findet. Im Europäischen Raum wird dieses Sachrecht, sofern es milder als das Herkunftslandrecht ist, durch das Herkunftslandprinzip modifiziert, da der Eintrag aus der Natur des UDDI heraus auf elektronischem Wege erfolgt.

bb) Rolle des Web Service-Vermittlers

Dadurch, dass der Web Service-Vermittler dem Web Service-Nutzer das Auffinden des Dienstes ermöglicht und ihm bei einer erfolgreichen Suche die Dienstebeschreibung zukommen lässt, drängt sich die Überlegung auf, dass der Web Service-Vermittler durch diese Leistungen für den Web ServiceAnbieter tätig wird und die bereits angesprochene Nachweismaklerschaft in

184 MünchKommBGB/Busche, Bd. 4, \631 Rn. 236; LG Hannover, NJW-RR 1989, 1525 f.; AG Königstein, NJW-RR 1999, 1355 (1356); a.A. AG Dresden, NJW-RR 1999, 562 (nicht rechtskräftig), das die Fälligkeit des Lohns erst in der Abnahme sieht, da alle körperlichen Gegenstände abnahmefähig seien. Dabei übersieht das Gericht aber, dass nach Druck des Anzeigenblattes (Zeitung etc.) eine Kontrolle mit darauf folgender Abnahme durch den Besteller vor der Verbreitung an Dritte praktisch nicht mehr möglich ist.

185 Esser/Weyers, II/1, \35 I/3 S. 312.

186 Palandt/Sprau, Einf. v. \ 631 Rn. 12; Larenz, II/1, \56 I S. 409. 
Betracht kommen könnte. Dabei wird der so genannte Nachweismakler derart tätig, dass er seinen Auftraggeber, hier der Web Service-Anbieter, durch das Nennen von Interessenten über die Gelegenheit eines Vertragsschlusses informiert und der Vertragspartner dadurch in die Lage versetzt wird, in konkrete Verhandlungen über den von ihm angestrebten Hauptvertrag einzutreten, ohne das bereits alle Einzelheiten des Vertrags festgelegt sein müssen. ${ }^{187} \mathrm{Da}$ rüber hinaus muss der geführte Nachweis dem Maklerauftrag entsprechen und die nachgewiesene Gelegenheit muss mit der tatsächlich Wahrgenommenen identisch sein. ${ }^{188} \mathrm{Da}$ der Web Service-Vermittler, ähnlich wie die Betreiber von Branchenverzeichnissen im Sinne der Gelben Seiten, lediglich dem Web Service-Nutzer die Suche nach geeigneten Diensten ermöglicht und diesem im Anschluss die Dienstebeschreibung zukommen lässt (ähnlich wie die Anzeige der Adresse und Telefonnummer des gewünschten Unternehmens), fehlt es in diesem Fall an der Nennung des potentiellen Vertragspartners gegenüber dem Web Service-Anbieter. Vielmehr wird der Web Service-Nutzer von sich aus, aufgrund der erhaltenen Dienstebeschreibung, automatisiert mit dem Web Service-Anbieter in Vertragsbeziehung treten. Es mangelt daher an der notwendigen Erbringung des Nachweises gegenüber dem Auftraggeber. Insofern scheidet eine Nachweismaklerschaft aus.

Auch eine Stellvertretung durch den Web Service-Vermittler bei Abschluss des Web Service-Nutzungsvertrags kommt nicht in Betracht. Eine solche würde nach $\int 164$ Abs. 1 BGB die Abgabe einer fremden Willenserklärung in fremden Namen mit Vertretungsmacht erfordern. Da das Ermöglichen des Suchens des Dienstes sowie das Übermitteln der Dienstebeschreibung aber, wenn überhaupt, Vorbereitungshandlungen darstellen und schon keine Willenserklärung des Web Service-Vermittlers bezüglich des Abschlusses eines Web Service-Nutzungsvertrags ersichtlich ist, geschweige denn eine Vertretungsvollmacht, liegt keine Stellvertretung durch den Web Service-Vermittler vor. Das Vertragsverhältnis zwischen Web Service-Anbieter und Web ServiceVermittler beschränkt sich auf den bereits ${ }^{189}$ erläuterten Eintrag im UDDI.

\footnotetext{
187 St. Rspr. BGH, NJW-RR 1988, 1397 (1398); NJW-RR 1990, 1008; NJW-RR 1991, 950; NJWRR 1996, 113 (114); Oetker/Maultzsch, \10 B I S. 570; Erman/Werner, \652 Rn. 12.

188 BGH, NJW-RR 1990, 1008.

189 S. A.III.1.b)aa) Verhältnis Web Service-Anbieter - Web Service-Vermittler.
} 


\section{c) Web Service-Anbieter - Web Service-Nutzer}

Die vertraglichen Verhältnisse zwischen Web Service-Anbieter und Web Service-Nutzer hängen zum einen entscheidend von der Art der Beziehung ab, je nachdem ob es sich um einen öffentlich nutzbaren Web Service innerhalb eines offenen Systems handelt, wo direkte Absprachen nicht zwangsläufig existieren, oder ob es sich um die Web Service-Nutzung innerhalb eines geschlossenen Systems handelt, bei dem direkte Absprachen naturgemäß notwendig sind, und zum anderen wie sich das Leistungsbild gestaltet. Ausschlaggebend für die vertragliche Typisierung ist, dass der Web Service technisch auf dem System des Web Service-Anbieters verbleibt und eine Übertragung an den Web Service-Nutzer nicht erfolgt. ${ }^{190}$ Denn die Nutzung des Web Service erfolgt lediglich über die öffentlich (offenes System) beziehungsweise zumindest dem Web Service-Nutzer (geschlossenes System) zugängliche Schnittstelle, wobei allerdings der Web Service in die laufende Anwendung des Web Service-Nutzers - trotz des physischen Verbleibens auf dem Server des Web Service-Anbieters - eingebunden wird. Indes erfolgt die dauerhafte Speicherung der bearbeiteten oder zu bearbeitenden Daten allein auf dem System des Web Service-Nutzers, der Web Service-Anbieter bleibt während des ganzen Nutzungsprozesses inaktiv. ${ }^{191}$

Zieht man das eingangs erläuterte Reisebuchungsportalbeispiel als klassisches Beispiel für die Nutzung von Web Services innerhalb offener Systeme heran, so stellt sich zunächst die Frage, ob die Fluggesellschaft - als Web ServiceAnbieter - von dem Reiseveranstalter - als Web Service-Nutzer - für die Nutzung des Web Service zum Suchen und Finden von Flügen eine Vergütung verlangt. In diesem Beispiel ist nicht grundsätzlich von einer Entgeltpflicht für die bloße Nutzung auszugehen, da die Fluggesellschaften in der Regel daran interessiert sein werden, mit den Reiseportalen zu kooperieren, um sich Wettbewerbsvorteile zu verschaffen und dadurch ihre Flüge vermehrt zu besetzen. Gleiches gilt auch für die Amazon Web Services: dort zahlt Amazon teilweise sogar Provisionen, wenn ein Kaufvertrag durch die Vermittlung eines Web Service-Anbieters erfolgte. ${ }^{192}$ Dennoch sind aber auch Fälle denkbar, insbesondere in Bereichen, in denen es auf die bloße Information als solche an-

190 Koch, ITRB 2007, 71.

191 Koch, ITRB 2007, 72.

192 S. http://developer.amazonwebservices.com/connect/entry.jspa?externalID=687 u. http://www.joomlaos.de/option,com_remository/Itemid,41/func,fileinfo/id,3838.html $<25.11 .2009>$. 
kommt, ${ }^{193}$ bei denen der Web Service-Anbieter bereits für die bloße Nutzung des Web Service ein Entgelt verlangt. Insofern muss hier zwischen entgeltlichen und unentgeltlichen Web Services, mit der Option der Vermittlungsgebühr, unterschieden werden.

\section{aa) Vergütung der Web Service-Nutzung}

Verlangt der Web Service-Anbieter vom Web Service-Nutzer ein Nutzungsentgelt, so muss genau differenziert werden, wodurch das Leistungsbild des Web Service-Anbieters geprägt wird, vereinfacht ausgedrückt, was er dem Web Service-Nutzer als Gegenleistung für dessen Entgelt schuldet. Der geschuldete Gegenstand bestimmt sich nach den Willen der Parteien ${ }^{194}$ und ist nach den $\$ \int 133,157$ BGB auszulegen. Grundsätzlich gestaltet sich das Leistungsbild wie folgt: der Web Service-Anbieter stellt mittels des Web Service eine Dienstleistung über eine standardisierte Schnittstelle zur Verfügung. 195 Genauer gesagt, bietet er den Zugriff auf einen Dienst mittels Web Service. Weiter ist der Web Service-Anbieter in aller Regel für die Verfügbarkeit des Dienstes verantwortlich, ${ }^{196}$ da die Erreichbarkeit des Dienstes über die Schnittstelle eine zentrale Anforderung darstellt. ${ }^{197}$ Das Aufrechterhalten des von ihm angebotenen Service stellt eine ihm obliegende Pflicht dar. ${ }^{198}$ Für den Web Service-Nutzer ist es notwendig zu wissen, wie er den Dienst des Web Service-Anbieters abrufen kann, so dass eine exakte und korrekte Dienstebeschreibung für ihn zur erfolgreichen Web Service-Nutzung erforderlich ist. ${ }^{199}$ Konkret bedeutet dies, dass man grundsätzlich zwischen dem Funktionieren des Web Service, worunter natürlich auch das Bereithalten der Schnittstelle fällt, und der Verfügbarkeit sowie dem Funktionieren des Diens-

193 Denkbar sind hier Wetterdienste, Suchdienste, etc. die sich bspw. nicht mittels Werbung finanzieren.

194 Larenz, Methodenlehre, S. 298 ff.; s. auch Schneider, S. 132.

195 Burghardt/Hagenhoff, Arbeitspapier Nr. 26/2003, S. 3; Speck, in: Hoffmann/Leible/Sosnitza, S. 22 f.; Melzer, S. 15 f.

196 Melzer, S. 14; Burghardt, S. 16.

197 Burghardt, S. 18.

198 Melzer, S. 14.

199 Melzer, S. 15 f.; ferner Burghardt, S. 18. 
tes unterscheiden muss. Denkbar sind daher Werk-, Dienst-, 200 aber auch Mietverträge.

\section{Festnetzuertrag}

Betrachtet man das aufgezeigte Leistungsbild, so könnte man den vertraglichen Schwerpunkt im Zugänglichmachen von Funktionalitäten sehen. ${ }^{201} \mathrm{Da}$ bei könnte man daran denken, zwischen zwei unterschiedlichen Leistungen, dem Zurverfügungstellen der öffentlichen Schnittstelle und der Ermöglichung der Nutzung des Web Service, um die gewünschte Information zu erhalten, zu differenzieren, ähnlich wie beim Festnetzvertrag. ${ }^{202}$ Eine derartige Differenzierung geht indes fehl, da im Unterschied zum Festnetzvertrag, bei dem die Anschlusskomponente als solche bereits eine für den Anschlussinhaber brauchbare Komponente darstellt - denn durch den vorhandenen Anschluss kann der Anschlussinhaber zumindest angerufen werden, so dass zumindest die einseitige Erreichbarkeit ermöglicht wird -, die bloße Bereithaltung der öffentlichen Schnittstelle für den Web Service-Nutzer indes keinerlei Wert hat. Insofern kann keine vertragliche Differenzierung zwischen dem Bereithalten der Schnittstelle und der Ermöglichung der Dienstenutzung erfolgen, diese müssen vielmehr im Zusammenhang betrachtet werden.

\section{Access-Provider-Vertrag}

Fraglich ist, wie man dieses Bündel aus Zugriff auf den Dienst durch den Web Service und Nutzung des Dienstes selber vertraglich einordnen kann. Dabei stellt sich die Frage, was genau der geschuldete Gegenstand bei diesem Bündel ist. Im Falle der Leistungen durch Access-Provider, die sowohl den Internetanschluss als auch die Internetverbindung vertraglich anbieten - insofern ebenfalls ein Bündel an Leistungen, bei dem der Anschluss allein aber ebenfalls, wie auch bei dem Bereithalten der Schnittstelle bei den Web Services, für den Nutzer keinerlei Wert hat -, sieht der Bundesgerichtshof neben der Bereithaltung des Anschlusses lediglich das sachgerechte Bemühen um die Herstellung der Verbindung in das Internet als geschuldet an und geht insofern

200 Spindler, DuD 2005, 140.

201 So auch Koch, ITRB 2007, 73.

202 Beim Festnetzvertrag wird zwischen einer Anschluss- (Bereitstellung des Anschlusses) und einer Verbindungskomponente (geschuldet wird der Transport der Daten zum Zweck der Entgegennahme und des Herstellens von Verbindungen) differenziert, s. Schuster, CR 2006, 446; i.d.S. auch Imping, in: Spindler, Vertragsrecht TK-Anbieter, Teil II Rn. 3 u. 7 sowie Teil VI Rn. 1. 
von einem Dienstvertrag aus, ${ }^{203}$ bei dem die reine Tätigkeit als solche geschuldet wird und ein sich Bemühen genügt. 204 Dabei muss aber beachtet werden, dass im Falle der Access-Provider ein geschuldeter Erfolg, der dann zum Werkvertragsrecht führen würde, mit dem Argument der Netzüberlastung abgelehnt wird und der Kunde daher eine jederzeitig gleich bleibende Verfügbarkeit nicht erwarten könne. ${ }^{205} \mathrm{Ob}$ dieses Argument indes auch bei der Bereitstellung von Web Services verfängt, erscheint fraglich. Eine Überlastung des Web Service durch extensive Nutzung zahlreicher Web Service-Nutzer erscheint aufgrund der Vielzahl unterschiedlicher Web Services aktuell noch eher fern liegend. Im Übrigen stellt die Zugangsgewährung durch das Bereitstellen von Einwahlknoten, um die es in dem vom Bundesgerichtshof entschiedenen Fall ging, bei Access-Providern die typische Vertragsleistung dar. ${ }^{206}$ Alle übrigen Leistungen wie Navigationshilfen, Mailbox, OnlineDienste etc. sind optional und richten sich nach der Art des Vertragsbündels. 207 Bei Web Services lässt sich zwar das Bereithalten der öffentlichen Schnittstelle mit dem Bereitstellen der Einwahlknoten bei Access-ProviderVerträgen vergleichen, darüber hinaus ist für den Web Service-Nutzer aber auch immer das Zurverfügungstellen der Dienstenutzung entscheidend, da die Schnittstelle allein, wie bereits erörtert, für ihn wertlos ist. Das bedeutet, dass sich ein konkreter Vertragsschwerpunkt im Falle der Web Services nicht herauskristallisieren lässt. Daher kann die Einordnung der Web Service-Nutzung nicht ausschließlich anhand der Rechtsprechung zum Access-Provider-Vertrag erfolgen.

203 BGH, NJW 2005, 2076; ähnlich Spindler, in: Spindler, Vertragsrecht Internet-Provider, Teil IV Rn. 93 u. Spindler, CR 2004, 207 f., der den Access-Provider-Vertrag zwar eher dem Dienstvertrag zuordnet, jedoch eigentlich einen Vertragstypus eigener Art für sachgerechter hält; für einen Vertrag sui generis Schuster, CR 2006, 450 ff.

Esser/Weyers, II/1, \27 II/3 S. 232; Erman/Edenfeld, \$ 611 Rn. 1; Bamberger/Roth/Fuchs, \ 611 Rn. 11. BGH, NJW 2005, 2076; Spindler, CR 2004, 207; Stürner, jurisPR-BGHZivilR 34/2005 Anm. 4. Bernütr/Weinreich, in: Gounalakis, Rhb. Electronic Business, \33 Rn. 192; Schneider, S. 147; i.d.S. auch Schuster, CR 2006, 447.

207 Bernütr/Weinreich, in: Gounalakis, Rhb. Electronic Business, \33 Rn. 192; Bischof/Schneider, ITRB 2005, 214; i.d.S. auch Schneider, S. 157. 


\section{Differenzierung nach Art und Weise der Vergütung?}

Denkbar wäre aber die Art und Weise der Vergütung in die Vertragstypisierung einfließen zu lassen. Bei der Nutzung von Web Services im Rahmen von offenen Netzen sind sowohl leistungsbezogene, jede Nutzung wird einzeln vergütet, als auch leistungsunabhängige Vergütungssysteme - Dauernutzungsvertrag zum Festpreis - vorstellbar. Ist eine leistungsbezogene Vergütung vorgesehen, erscheint ein Vergleich mit dem Call-by-Call Internetzugang nahe liegend. Bei Call-by-Call-Verträgen zahlt der Nutzer nur die tatsächlich zustande gekommene Verbindung, es erfolgt daher eine leistungsbezogene Vergütung. Aufgrund dieses leistungsabhängigen Vergütungssystems wird vertreten, dass bei Call-by-Call-Verträgen der Erfolg im Vordergrund stehe und deshalb Werkvertragsrecht anzuwenden sei.208 An dieser Ansicht wird aber kritisiert, dass die Frage des Vertragstyps sich nicht nach der Form der Vergütung richte sondern immer nach dem Leistungsinhalt und dieser unterscheide sich nicht von anderen (leistungsunabhängig vergüteten) Access-ProviderVerträgen, da bei allen der Datentransport Leistungsinhalt sei. ${ }^{209}$ Die Vergütungsform könne aber in der Auslegung des Parteiwillens, der die Typisierung prägt, berücksichtigt werden. ${ }^{210}$

Dieses Problem ist mit der Typisierung eines Web Service-Vertrags allerdings nicht in Gänze vergleichbar. Denn wie bereits festgestellt ist nicht allein die Bereitstellung der öffentlichen Schnittstelle für die Typisierung ausschlaggebend, was der Zugangsgewährung bei Call-by-Call-Verträgen entspräche, sondern vielmehr das Zusammenspiel des Bereithaltens und der Ermöglichung der Dienstenutzung. Insofern umfasst auch die Vergütung, gleich ob einmalig von Nutzung zu Nutzung oder in Form eines monatlichen Festbetrags, eine Kombination von Leistungen. Daher kann die Vergütung allenfalls im Rahmen des Parteiwillens berücksichtigt werden, ${ }^{211}$ nicht aber allein über den Vertragstyp entscheiden.

208 Spindler, in: Spindler, Vertragsrecht Internet-Provider, Teil IV Rn. 87; Spindler, CR 2004, 208; i.d.S. auch Gey, K\&R 2005, 123; ähnlich Schneider, CR 2005, 700, der auch auf die leistungsabhängige Vergütung bei der Einordnung des Vertragstyps abstellt.

209 Schuster, CR 2006, 448.

210 Schuster, CR 2006, 449.

211 Oetker/Maultzsch, \ 7 S. 397; i.d.S. auch Bamberger/Roth/Voit, \631 Rn. 6, der die erfolgsabhängige Vergütung lediglich als ein Indiz zur Bestimmung des Vertragstyps sieht. 


\section{Gemischttypischer Vertrag?}

Da der Web Service-Nutzungsvertrag offensichtlich aus mehreren untrennbar zusammenhängenden Vertragsteilen besteht, soll untersucht werden, ob der Nutzungsvertrag als gemischttypischer Vertrag qualifiziert werden kann. Bei gemischttypischen Verträgen sind verschiedene Vertragstypen derart miteinander verbunden, dass sie nur in ihrer Gesamtheit ein sinnvolles Ganzes ergeben. ${ }^{212}$ Das bedeutet zunächst einmal, dass der Web ServiceNutzungsvertrag aus verschiedenen Vertragstypen bestehen muss, wofür die Vertragselemente des Web Service-Nutzungsvertrags untersucht werden müssen.

Einordnung der verschiedenen Vertragselemente

\section{Zugriff auf den Dienst mittels des Web Service}

Wie bereits erörtert, ${ }^{213}$ könnte das Bereitstellen der öffentlichen Schnittstelle und der Zugriff auf den Dienst über den Web Service mit dem AccessProvider-Vertrag verglichen werden. Daher könnte dieser Bestandteil entweder als Dienst- oder als Werkvertrag eingeordnet werden, je nachdem ob man das Bemühen der Zugriffsermöglichung als ausreichend ansieht oder der Zugriff als spezifischer Erfolg Gegenstand des Vertrags ist. Geschuldet ist beim Dienstvertrag die ordnungsgemäße Erbringung der Dienstleistung, ${ }^{214}$ wobei Gegenstand des Dienstvertrags nach $\ 611$ Abs. 2 BGB explizit Dienste jeder Art sein können. Dagegen schuldet beim Werkvertrag der Werkunternehmer einen Erfolg. ${ }^{215}$ Ein stichhaltigeres und greifbareres Kriterium zur Abgrenzung ist aber die Risikoverteilung: Während der Werkunternehmer bis zur Abnahme oder Vollendung des Werks auf eigene Gefahr arbeitet und insofern solange Herstellungsversuche oder Nachbesserungen vornehmen muss, bis der Erfolg eintritt, hat der Dienstleister bereits nach erbrachter Leistung, unabhängig davon ob das sinngebende Ziel erreicht wurde oder nicht, einen

212 Erman/Kindl, Vor \311 Rn. 15; MünchKommBGB/Emmerich, Bd. 2, \311 Rn. 45; Palandt/Grüneberg, Überbl. v. \311 Rn. 19; Bamberger/Roth/Gehrlein/Sutschet, \311 Rn. 20; Schneider, S. 136; Gaßner, AcP 186 (1986), 333.

213 A.III.1.c) Access-Provider-Vertrag.

214 BGH, NJW 1970, 1596 (1597); NJW 1984, 2406 (2407); NJW 2002, 3323 (3324); Bamberger/Roth/Fuchs, \$ 611 Rn. 11; Oetker/Maultzsch, \ 7 S. 396.

215 BGH, NJW 1960, 431; OLG Düsseldorf, NJW-RR 1998, 345 (346); MünchKommBGB/Busche, Bd. 4, \ 631 Rn. 14. 
Vergütungsanspruch. ${ }^{216}$ Dem steht auch nicht die Art der Vergütung, die beim Dienstvertrag zwar typischerweise zeitbezogen bemessen wird, aber auch zeitunabhängig beziehungsweise sogar, obwohl ein solcher nicht geschuldet ist, erfolgsbezogen erfolgen kann, ${ }^{217}$ entgegen, denn wie bereits festgestellt, hilft die Art der Vergütung bei der Typisierung der Web Service-Nutzung lediglich bei der Auslegung des Parteiwillens. ${ }^{218}$

Da das Argument der Netzüberlastung beim Zugriff auf Web Services wohl eher nicht greifen wird, spricht einiges dafür, dass die Vertragsparteien den Zugriff auf den Dienst mittels des Web Service als Erfolg qualifizieren wollen und demnach ein bloßes sich Bemühen um das Erbringen des Web Service nicht genügt. Problematisch ist jedoch, worin das Werk, das für die Qualifikation als Werkvertrag erforderlich ist, zu sehen ist. Ein Werk liegt nach $\ 631$ Abs. 2, 1. Alt. BGB regelmäßig in der Herstellung oder Veränderung einer Sache. Eine solche ist die Zugriffsermöglichung mittels Web Service aber eindeutig nicht. $\ 631$ Abs. 2, 2. Alt. BGB lässt darüber hinaus auch den durch Arbeit oder Dienstleistung herbeigeführten Erfolg als Werk zu. Dennoch wird im parallelen Bereich der Telekommunikationsdienstleistungen kritisiert, dass durch die Telekommunikationsverbindung, übertragen auf Web Services durch den Zugriff auf den Dienst, kein individuelles Werk hergestellt werde, das zwingend Gegenstand des Werkvertrags sei. ${ }^{219}$ Diese Argumentation ist indes aufgrund des $₫ 631$ Abs. 2, 2. Alt. BGB fraglich, denn können auch Dienstleistungen dem $\ 631$ BGB entsprechende Werke sein, so leuchtet nicht ein, warum bei der Telekommunikationsverbindung beziehungsweise hier der Zugriffsermöglichung durch das Anbieten eines Web Service das individuelle Werk fehlen soll. Vielmehr verfängt aber ein weiterer Kritikpunkt aus dem Bereich der Access-Provider: zwar erscheint die Überlastung eines Web Service unwahrscheinlicher als die eines Zugangsproviders, dennoch handelt es sich um eine Software, bestehend aus verschiedenen Komponenten, so dass immer ein gewisses Ausfall-Restrisiko bezüglich der Verfügbarkeit bleibt. Dieses lässt sich zwar minimieren, insbesondere durch höchst sorgfältige Wartung, aber technisch wohl kaum vollständig beseitigen. Qualifiziert man nun

216 BGH, NJW 2002, 3323 (3324); MünchKommBGB/Busche, Bd. 4, \ 631 Rn. 17; Esser/Weyers, II/1, \27 II/3 S. 233; Oetker/Maultzsch, \ 7 S. 397 f.

217 Als Beispiel für die erfolgsbezogene Vergütung trotz des Schuldens der bloßen Arbeitskraft, wird der Akkordlohn genannt: so Larenz, II/1, \52 I/1 S. 310; Esser/Weyers, II/1, \27 II/3 S. 233; Oetker/Maultæssch, \ 7 B S. 397; MünchKommBGB/Müller-Glöge, Bd. 4, \611 Rn. 26.

218 S. A.III.1.c) Differenzierung nach Art und Weise der Vergütung?.

219 So Habn, Rn. 225; dieses Argument als nachvollziehbar ansehend Schuster, CR 2006, 449. 
den Zugriff auf den Dienst mittels des Web Service als Werkvertrag, würde ein technischer Ausfall die teilweise Nichterfüllung der Leistungspflicht bedeuten, da der Zugriff stets geschuldet und nicht nach $\int 634$ Nr. 1 BGB nachholbar ist. ${ }^{220}$ Dies würde zum einen der gewollten Risikoverteilung zwischen Web Service-Anbieter und Web Service-Nutzer widersprechen und zum anderen an die Grenzen des Werkvertragsrechts stoßen. Insofern kann das System des Werkvertrags zumindest nicht in der im Gesetz vorgegebenen Konstellation auf derartige Systeme angewandt werden. ${ }^{221}$ Daher muss der Zugriff auf den Dienst mittels eines Web Service vielmehr in Anlehnung an die AccessProvider-Rechtsprechung 222 dem Dienstvertragsrecht zugeordnet werden, so dass das sachgerechte Bemühen um den Zugriff auf den Dienst, unabhängig von der Art der Vergütung, ausreichend ist.

\section{Zurverfügungstellung des Dienstes}

Die andere Komponente im Rahmen der Web Service-Nutzung durch den Web Service-Nutzer ist die Notwendigkeit der Zurverfügungstellung des Dienstes, auf den der Web Service-Nutzer mittels des Web Service zugreifen kann. Dieser Dienst ist trotz der technischen Trennung vom Web Service selbst, notwendiger Bestandteil der vertraglichen Nutzung. Denn der Web Service selbst ist für den Web Service-Nutzer ohne den dazugehörigen Dienst nutzlos, da so keinerlei Daten vorhanden wären, die der Web Service-Nutzer mittels des Web Service abrufen könnte. Daher muss auch die Zurverfügungstellung des Dienstes bei der Typisierung berücksichtigt werden. Die reine Zurverfügungstellung des Dienstes muss, wie auch schon die Zugriffsermöglichung, dem Dienstvertragsrecht zugeordnet werden, denn auch hier ist der geschuldete Gegenstand nicht die erfolgreiche Nutzung sondern das bloße Anbieten des Dienstes. Das bedeutet, dass die bloße Tätigkeit, die Zurverfügungstellung des Dienstes, geschuldet ist.

\section{Dienstenutzung}

Kernelement der Vertragsbeziehungen zwischen Web Service-Anbieter und Web Service-Nutzer ist die eigentliche Nutzung des Dienstes. Darunter fällt das Abspeichern und Bereithalten von Informationen (zum Beispiel die Temperatur) zum Abruf. Diese Informationen können dann mittels Web Service

\footnotetext{
220 Spindler, CR 2004, 207; Schuster, CR 2006, 449; BGH, CR 2005, 816 m. Anm. Schuppert.

221 Spindler, CR 2004, 207; dem zustimmend Schuster, CR 2006, 449.

222 BGH, CR 2005, 816 m. Anm. Schuppert.
} 
vom Web Service-Nutzer abgerufen werden. Insofern erfolgt ein Datentransfer. Sowohl das Abspeichern und Bereithalten von Informationen als auch der Datentransfer liegen im Macht- und somit auch im Risikobereich des Web Service-Anbieters, da der Dienst ein von ihm unterhaltenes System darstellt. ${ }^{223}$ Insofern ist es hier systemgerecht, dass der Web Service-Anbieter einen Erfolg schuldet 224 und somit bezüglich der Dienstenutzung Werkvertragsrecht greift. $^{225}$

\section{Gesamtheit als sinnvolles Ganzes}

Die unterschiedlichen genannten Vertragsbestandteile - die Ermöglichung des Zugriffs auf den Dienst mittels Web Service, die Bereithaltung des Dienstes sowie die Dienstenutzung - sind auch logisch miteinander untrennbar verknüpft. Denn mit der Bereithaltung der öffentlichen Schnittstelle allein kann der Web Service-Nutzer in praxi nichts anfangen.226 Bildlich dargestellt, lässt sich das alleinige Bereithalten der öffentlichen Schnittstelle mit dem bloßen Bereithalten eines Tisches in einem (nicht Selbstbedienungs-) Restaurant vergleichen: weist der Kellner dem hungrigen und durstigen Besucher lediglich einen Tisch zu, bedient aber nicht und verwehrt dem Besucher dadurch Speis und Trank, so ist der Tisch allein für ihn regelmäßig nutzlos. Das Zurverfügungstellen eines Tisches (mietvertragliche Elemente) ${ }^{227}$, der Service (Dienstvertrag) 228 und die Speisen oder Getränke (Kauf- beziehungsweise Werkvertrag) ${ }^{229}$ gehören daher untrennbar zusammen und ergeben nur in

223 Schuppert, in: Spindler, Vertragsrecht Internet-Provider, Teil II Rn. 20; Redecker, ITRB 2003, 83; den Datentransfer durch Web Services ohne Begründung als Werkvertrag einordnend Spindler, $\operatorname{DuD} 2005,140$.

224 So zum Datentransfer allgemein Schuppert, in: Spindler, Vertragsrecht Internet-Provider, Teil II Rn. 20; Redecker, ITRB 2003, 83.

225 Spindler, DuD 2005, 140.

226 S. dazu bereits A.III.1.c)Festnetzvertrag.

227 Laren $2 /$ Canaris, II/2, \63 II/1 S. 46.

228 RGZ 65, 11 (13 f.); 85, 185 (187 f.); AG Garmisch-Partenkirchen, NJW 1969, 608 (609); LG Karlsruhe, NJW 1994, 947; Erman/Kindl, Vor \311 Rn. 17.

229 AG Burgwedel, NJW 1986, 2647; Erman/Kindl, Vor \311 Rn. 17. 
ihrer Gesamtheit ein sinnvolles Ganzes. Der Speisenrestaurantvertrag ${ }^{230}$ wird dementsprechend als gemischttypischer Vertrag eingeordnet. ${ }^{231}$

Gleiches muss für den Vertrag über die Nutzung von Web Services gelten. Denn ohne die Bereithaltung eines funktionierenden Dienstes und die schlussendlich erfolgende Datenübertragung, bleibt die zugängliche öffentliche Schnittstelle, die den Zugriff auf den Dienst ermöglicht, für den Web ServiceNutzer uninteressant. Die Bereithaltung der Schnittstelle (Dienstvertrag) ergibt nur in Kombination mit der Zurverfügungstellung eines funktionierenden Dienstes (Dienstvertrag) und der Datenübertragung (Werkvertrag) ein für den Web Service-Nutzer sinnvolles Ganzes. Der Vertrag über die Nutzung von Web Services ist folglich als gemischttypischer Vertrag mit dienst- und werkvertraglichen Elementen einzuordnen.

Gemischttypische Verträge werden in Typenkombinationen und Typenverschmelzungen untergliedert. ${ }^{232}$ Typenkombinationen wiederum lassen sich unterteilen in solche, bei denen für mindestens eine Seite Leistungen vereinbart werden, die zu verschiedenen gesetzlichen Vertragstypen gehören und solche mit atypischer Gegenleistung. ${ }^{233}$ Typenverschmelzungen dagegen zeichnen sich dadurch aus, dass eine einzige Leistung gleichzeitig die Merkmale verschiedener Vertragstypen erfüllt. ${ }^{234}$ Da der Web Service-Nutzungsvertrag verschiedene Leistungen - Bereithalten der öffentlichen Schnittstelle, Zurverfügungstellen eines funktionierenden Dienstes und die Ermöglichung der Dienstenutzung (Datentransfer) - umfasst, die unterschiedlichen Vertragstypen zuzuordnen sind - Dienst- und Werkvertrag -, handelt es sich beim Web Service-Nutzungsvertrag um eine Typenkombination mit unterschiedlichen gesetzlichen Vertragstypen.

230 Auch Bewirtungsvertrag genannt.

231 RGZ 65, 11 (13 f.); 85, 185 (187 f.); AG Garmisch-Partenkirchen, NJW 1969, 608; LG Karlsruhe, NJW 1994, 947; Larenz/Canaris, \ 63 II/1 S. 46; a.A. AG Hamburg, NJW 1973, 2253, das den Speisenrestaurantvertrag als atypischen Vertrag qualifiziert.

232 Martinek, Bd. 1, \2 S. 20; Bamberger/Roth/Gebrlein/Sutschet, 』 311 Rn. 22 ff.; Staudinger/Löwisch (2005), \311 Rn. 37 ff.; Palandt/Grüneberg, Überbl. v. \311 Rn. 21 ff.; Larenz/Canaris, II/2, S 63 I/1 S. 42, der auch die Typenneuschaffung als Untertypus des gemischten Vertrages sieht. Da diese Kategorie im Rahmen der Web Service-Nutzung aber keinerlei Rolle spielt, soll hier nicht weiter darauf eingegangen werden, ob die Typenneuschaffung eine Unterkategorie des gemischten Vertrages darstellt.

233 Staudinger/Löwisch (2005), \ 311 Rn. 37; Larenz/Canaris, II/2, \63 I/1 S. 42; Martinek, Bd. 1, S 2 S. 20.


renz/Canaris, II/2, S 63 I/1 S. 42; Staudinger/Löwisch (2005), J 311 Rn. 44. 


\section{Resümee}

Wird für die Web Service-Nutzung innerhalb offener Systeme ein Entgelt verlangt, so handelt es sich um einen gemischt-typischen Vertrag in Form einer Typenkombination mit dienst- und werkvertraglichen Elementen. Nach der heute in der Literatur vorherrschenden Theorie der interessengerechten Rechtsanwendung werden solche Verträge immer flexibel anhand des Sinn und Zwecks des Einzelfalls und der jeweiligen Interessenlage beurteilt. ${ }^{235}$ Führt diese Variante zu keiner sachgerechten Lösung, so ist hilfsweise das Recht desjenigen Vertragstyps heranzuziehen, das den rechtlichen oder wirtschaftlichen Schwerpunkt bildet. ${ }^{236}$

Charakteristische Vertragsleistung ist sowohl die Ermöglichung des Zugriffs auf den Dienst mittels Web Service, die Bereithaltung des Dienstes sowie die Ermöglichung der Dienstenutzung. Alle Vertragsleistung fallen in die Sphäre des Web Service-Anbieters, daher ist bei Verträgen, bei denen mindestens ein Beteiligter seinen Unternehmenssitz im Ausland hat, im Fall des Fehlens einer Rechtswahlklausel, regelmäßig das Sachrecht des Web Service-Anbieters anzuwenden. Alle Vertragsleistungen erfolgen elektronisch, deshalb wird im Europäischen Raum das nach Art. 28 Abs. 1, 2 EGBGB anzuwendende Sachrecht zudem gegebenenfalls durch das Herkunftslandprinzip modifiziert.

\section{bb) Unentgeltliche Web Service-Nutzung}

Anders fällt die Betrachtung aus, wenn der Web Service-Anbieter auf ein Entgelt verzichtet. Dies ergibt sich schon aus dem Wortlaut der $\int \$ 611$ Abs. 1 und 631 Abs. 1 BGB, die als Gegenleistung von der vereinbarten Vergütung sprechen und somit unzweifelhaft eine entgeltliche Gegenleistung voraussetzen. Unentgeltliche Web Service-Nutzungsmöglichkeiten sind denkbar, wenn sich der Web Service-Anbieter anderweitig finanziert, zum Beispiel durch Schaltung von Werbung. Gerade aufgrund des technischen und wirtschaftlichen

235 Staudinger/Löwisch, \311 Rn. 36; MünchKommBGB/Emmerich, Bd. 2, 『311 Rn. 46; Erman/Kindl, Vor $₫ 311$ Rn. 16; Martinek, Bd. 1, \2 S. 25; Schneider, S. 139; OLG Köln, NJW 1980, 1395 (1396). Nach diesem Prinzip werden wahlweise das früher vertretene Absorptionsprinzip (der Vertragstyp der Hauptleistung bestimmt die Natur des Vertrags) oder das Kombinationsprinzip (jede Leistung wird nach den für sie geltenden Vertragstyp beurteilt) angewandt. 
Aufwands kann dabei aber nicht von einem bloßen Gefälligkeitsverhältnis, bei dem ein Rechtsbindungswille fehlt, ${ }^{237}$ ausgegangen werden.

Ein unentgeltlicher Vertrag, der Rechte und Pflichten beinhaltet, ${ }^{238}$ ist der Auftrag ( $\$ 662$ BGB). Dabei verpflichtet sich der Beauftragte, ein ihm von dem Auftraggeber übertragenes Geschäft für diesen unentgeltlich zu besorgen. Dabei ist eine Geschäftsbesorgung jedes Tätigwerden im Interesse eines anderen, sei es wirtschaftlicher, ideeller, rechtsgeschäftlicher oder tatsächlicher Natur. ${ }^{239}$ Ermöglicht der Web Service-Anbieter dem Web Service-Nutzer die Nutzung eines funktionierenden Dienstes durch das Bereitstellen einer öffentlichen Schnittstelle, so stellt dies zweifellos eine Tätigkeit im Interesse des Web Service-Nutzers dar, sofern es sich um eine unentgeltliche Geschäftsbesorgung handelt. Bei dem Vertrag hinsichtlich der unentgeltlichen Dienstenutzung zwischen Web Service-Anbieter und Web Service-Nutzer handelt es sich daher um einen Auftrag im Sinne des $₫ 662$ BGB.

\section{d) Web Service-Nutzer - Web Service-Vermittler}

Technisch betrachtet, bietet der Web Service-Vermittler dem Web ServiceNutzer die Möglichkeit, die vom Web Service-Anbieter im UDDI hinterlegten Dienstebeschreibungen zu durchsuchen, was durch die Kategorisierung der Beschreibungen erleichtert wird. ${ }^{240}$ Im Mittelpunkt dieses Verhältnisses stehen das Suchen und Finden der Dienste.

$\mathrm{Da}$ es darauf ankommt, dass der Web Service-Nutzer zum einen erfährt, ob der gewünschte Dienst registriert ist oder nicht, und es zum anderen im Falle einer erfolgreichen Suche auf den tatsächlichen Erhalt der Dienstebeschreibung ankommt, also eine korrekte Auskunft geschuldet wird ein bloßes sich Bemühen ausreichen zu lassen, würde Sinn und Zweck eines solchen Dienstes konterkarieren -, schuldet der Web Service-Vermittler dem Web Service-Nutzer einen Erfolg, so dass im Falle einer entgeltlichen Nut-

237 Flume, AT 2, $\int 7 / 5$ S. 87; MünchKommBGB/Kramer, Bd. 2, Einl. Rn. 31; Bamberger/Roth/Eckert, \$145 Rn. 6.

238 S. Palandt/Sprau, Einf. v. \662 Rn. 1 ff.; Larenz, II/2, \56 I/1 S. 411 ff.; Esser/Weyers, II/2, \35/I S. 308 f.; Flume, AT 2, \7/5 S. 87.

239 St. Rspr. BGH, NJW 1963, 390 (391); NJW 1963, 1825 (1826); NJW 1965, 1271 (1272); NJW 1971, 1404; Larenz, II/1, \56 I/1 S. 410; kritisch zur Weite dieser Definition MünchKommBGB/Seiler, Bd. 4, \662 Rn. 13; Oetker/Maultzssch, \11 B II 1 S. 593 f.

240 S. bereits B.II.2 Komponenten. 
zung ein Werkvertrag ${ }^{241}$ vorliegt und im Falle der unentgeltlichen Nutzung ein Auftrag.

In beiden Fällen ist die Ermöglichung des Suchens und Findens von Web Services charakteristische Vertragsleistung, so dass im Falle einer fehlenden Rechtswahlklausel bei Verträgen mit Auslandsbezug regelmäßig das Recht des Staats, in dem der Web Service-Vermittler seinen Unternehmenssitz hat, Anwendung findet. Im Europäischen Raum wird dieses Sachrecht, sofern es milder als das Herkunftslandrecht ist, durch das Herkunftslandprinzip modifiziert, da das Suchen und Finden von Web Services elektronisch erfolgt.

\section{e) Web Service-Nutzer - Dritte}

Unmittelbare Beziehungen zu dem Dritten, der letztlich, wie im Beispiel der Reisebuchung, ${ }^{242}$ nur die Service-Komponenten des Web Service-Nutzers nutzt, bestehen lediglich bei dem Web Service-Nutzer, der dem Dritten seine Plattform und den dort integrierten Web Service zur Verfügung stellt. Dabei muss zwischen der Grundleistung, zum Beispiel dem Reisevertrag, auf der einen, und der Nutzung des integrierten Web Service als Hilfsmittel zur Verwirklichung des Grundvertrags auf der anderen Seite, unterschieden werden. Der Grundvertrag richtet sich immer nach der jeweils gewünschten Leistung, im Falle der Reisebuchung über ein Reiseportal kommt ein Reisevertrag im Sinne des $\int \$ 651a ff. BGB zustande, 243 bei der Wetterauskunft ein Werkvertrag und so weiter. Der Web Service, der dabei in die Prozesse des Web Service-Nutzers integriert ist, dient aus Sicht des Dritten lediglich als Mittel zum Zweck des Abschlusses des Grundvertrags. In den meisten Fällen wird der Dritte gar nicht wissen, welche technischen Hilfsmittel verwandt werden, so dass es für ihn gleichgültig ist, ob ein Web Service verwendet wird oder nicht. Auch findet keinerlei Übertragung des Web Service an den Dritten statt. Dieser bleibt vielmehr auf dem System des Web Service-Anbieters und wird lediglich über die öffentliche Schnittstelle in die Plattform des Web ServiceNutzers integriert, so dass der Dritte lediglich (unbewusster) Nutzer des Web Service ist. Auch muss der Dritte regelmäßig keine direkte Vergütung für die Web Service-Nutzung bezahlen. Diese erfolgt lediglich, wenn überhaupt, im Verhältnis Web Service-Anbieter - Web Service-Nutzer. Zwar ist denkbar,

241 Die Situation ist insofern vergleichbar mit dem Auskunftsvertrag, der ebenfalls als Werkvertrag eingeordnet wird, s. MünchKommBGB/Busche, Bd. 4, \$ 631 Rn. 246.

242 S. B.I.2 Web Service-Nutzer.

243 S. dazu detailliert A.III.1.a)bb) Exkurs: Reisevertragsspezifische Besonderheiten. 
dass der Web Service-Nutzer die mit der Web Service-Nutzung verbundenen Kosten im Rahmen des Grundvertrags auf den Dritten umlegt, das ist aber für die Einordnung des Vertrags zwischen Web Service-Nutzer und Web ServiceAnbieter irrelevant. Der Web Service spielt daher im Rahmen der Beziehungen zwischen Web Service-Nutzer und Dritten keinerlei Rolle und ändert auch nichts an der Einordnung des Grundvertrags. ${ }^{244}$ Der Web Service ist lediglich das Mittel zum Zweck und nicht Hauptgegenstand des Vertrags. Wie der Service erbracht wird, kann und wird dem Dritten in der Regel gleichgültig sein.

\section{Zustandekommen des Vertrags}

$\mathrm{Da}$ Verträge für mindestens einen der Beteiligten regelmäßig eine Verpflichtung begründen und im Falle von Leistungsstörungen die Geltendmachung von Sekundärrechten ermöglichen, ${ }^{245}$ ist es notwendig zu untersuchen, wie und wann die oben genannten Verträge zustande kommen. Weil Verträge nach $\int 145$ BGB auf Angebot und Annahme beruhen und grundsätzlich bereits das Angebot nach $\int 145$ BGB Bindungswirkung hat, muss in einem ersten Schritt untersucht werden, in welchen Handlungen Angebot und Annahme bei der Nutzung von Web Services gesehen werden können. In einem weiteren Schritt werden Anforderungen an eine sichere Übermittlung von rechtsverbindlichen Erklärungen mittels Web Services aufgestellt.

Dabei sollen lediglich die Beziehungen zwischen Web Service-Anbieter und Web Service-Nutzer, Web Service-Nutzer und Dritten sowie ferner zwischen Web Service-Anbieter und Dritten beleuchtet werden, da nur bei diesen der Web Service eine unmittelbare Rolle spielt. Die vertraglichen Beziehungen mit dem Web Service-Vermittler stellen lediglich Vorbereitungshandlungen zur Bereitstellung und Nutzung des Web Service dar; das der Web Service Gegenstand des Vertrags ist, ändert nichts an Zeitpunkt und Art der Abgabe von Angebot und Annahme. Spielt dagegen der Web Service bei Vertragsschluss eine entscheidende Rolle, so stellt sich die Frage, worin die das Angebot und die Annahme begründenden Willenserklärungen zu sehen sind.

\footnotetext{
244 So auch Koch, ITRB 2007, 72.

245 Staudinger/Löwisch, \311 Rn. 7; Erman/Armbrüster, Vor \145 Rn. 2.
} 


\section{a) Vertragsschluss}

\section{aa) Web Service-Anbieter - Web Service-Nutzer}

Da Web Service-Anbieter und Web Service-Nutzer vor der konkreten Nutzung des Web Service in der Regel keinerlei persönlichen Kontakt haben werden, muss genauer untersucht werden, in welchen „Erklärungen“ Angebot und Annahme zu sehen sind und ob überhaupt Willenserklärungen abgegeben werden. Grundsätzlich ist es erforderlich, dass sowohl Angebot als auch Annahme einen übereinstimmenden Rechtsbindungswillen enthalten, der notwendige Voraussetzung für das Zustandekommen eines Vertrags ist. ${ }^{246}$

\section{Elektronische Willenserklärungen}

Sowohl die Registrierung des Web Service als auch dessen Nutzung erfolgen elektronisch. Eine persönliche Kommunikation der Beteiligten erfolgt nicht, insofern können die für Angebot und Annahme erforderlichen Willenserklärungen lediglich in elektronischer Form erfolgen. Während früher strittig war, ob eine elektronische Erklärung den Erfordernissen einer rechtsgeschäftlichen Willenserklärung gerecht wird, ${ }^{247}$ ist heute einhellig anerkannt, dass elektronische Erklärungen durchaus Willenserklärungen sein können. ${ }^{248}$ Dabei wird nach Mebrings ${ }^{249}$ sinnvollerweise zwischen der elektronisch übermittelten Willenserklärung, der automatisierten Willenserklärung und der Computererklärung unterschieden, wobei die Grenzen zwischen der automatisierten Willenserklärung und der Computererklärung allerdings fließend sind. 250

Elektronisch übermittelte Willenserklärungen sind „normale“ Willenserklärungen, die lediglich auf elektronischem Wege, durch ein Medium, übermittelt

246 BGH, NJW 1956, 1313; NJW 1971, 1404 (1405); MünchKommBGB/Kramer, Bd. 1/1, Vorb. \ 145 Rn. 26; Medicus, Rn. 31.

247 Kritisch zur Einordnung als Willenserklärung bspw. Clemens, NJW 1985, 2001 ff.; für den Bereich der „vollautomatisierten Willenserklärungen“ beim bargeldlosen Zahlungsverkehr Möschel, AcP 186 (1986), 195.

248 BGH, NJW 2002, 363 (364 f.); OLG Hamm, NJW 2001, 1142; Wiebe, in: Gounalakis, Rhb. Electronic Business, \15 Rn. 2; Borges, S. 191; Koch, Internet-Recht, \3 S. 99; Mebrings, in: Hoeren/Sieber, Hdb. MultimediaR, Teil 13.1 Rn. 107; Palandt/Heinrichs/Ellenberger, Einf. v. \116 Rn. 1; Spindler/Schuster/Spindler/Weber, Vor \116 BGB Rn. 1 ff.; Spindler, DuD 2005, 139.

249 Mehrings, in: Hoeren/Sieber, Hdb. MultimediaR, Teil 13.1 Rn. 89 ff. Dem folgend Bierekoven, S. 22; Spindler/Schuster/Spindler/Weber, Vor $\$ 116$ BGB Rn. 1.

250 Spindler/Schuster/Spindler/Weber, Vor $\$ 116$ BGB Rn. 1. 
werden. Sie sind im Kern menschliche Willenserklärungen, bei deren Transport ein Medium als Mittel zum Zweck genutzt wird, und müssen folglich als solche behandelt werden. ${ }^{251}$ Bei der automatisierten Willenserklärung wird eine Erklärung aufgrund vorheriger manueller Dateneingabe vom Computerprogramm automatisch erzeugt. Da der Mensch hier als Initiator der Willenserklärung fungiert und er der geistige Schöpfer der Erklärung ist, wird die automatische Erklärung ebenfalls als Willenserklärung anerkannt. ${ }^{252}$ Die Computererklärung zeichnet sich dadurch aus, dass sie zum einen automatisiert erzeugt und zum anderen elektronisch übermittelt wird; ein bekanntes Beispiel ist die beim Internetversandhandel automatisch erzeugte und übermittelte Bestellbestätigungs-E-Mail. Obwohl weder im Zeitpunkt der Erzeugung noch der Übermittlung eine menschliche Beteiligung erfolgt, ist heutzutage unstrittig, dass auch Computererklärungen echte Willenserklärungen sein können, da die eingesetzten Programme letztlich immer auf den Willen des Betreibers zurückgehen und dieser den allgemeinen Willen hat, sich die damit erzeugten Erklärungen zurechnen zu lassen. ${ }^{253}$

\section{Angebot}

Das Angebot könnte entweder in dem Einstellen der Dienstebeschreibung im UDDI durch den Web Service-Anbieter gesehen werden oder aber konkludent in der Nutzung durch den Web Service-Nutzer. ${ }^{254}$ Möglicherweise ist das Einstellen der Dienstebeschreibung im UDDI indes eine bloße ,invitatio ad offerendum", bei der der notwendige Rechtsbindungswille fehlt, ${ }^{255}$ so dass ein

251 Mebrings, in: Hoeren/Sieber, Hdb. MultimediaR, Teil 13.1 Rn. 98; Bierekoven, S. 23; Koch, Internet-Recht, $₫ 3$ S. 99.

252 BGH, NJW 2005, 53 (54); NJW 2005, 976 (977); Spindler/Schuster/Spindler/Weber, Vor $\ 116$ BGB Rn. 5; Wiebe, in: Gounalakis, Rhb. Electronic Business, \15 Rn. 16 ff; dem i.E. zustimmend Borges, S. 195.

253 BGH, NJW 2002, 363 (364); davon ausgehend BGH, NJW 2005, 976 ff. = JZ 2005, 791 ff. m. Anm. Spindler; OLG Hamm, NJW 2004, 2601; OLG München, MMR 2003, 274 (275) m. Anm. Hoffmann; OLG Frankfurt, MMR 2003, 405; AG Westerburg, MMR 2003, 609; Mebrings, in: Hoeren/Sieber, Hdb. MultimediaR, Teil 13.1 Rn. 100 ff.; Koch, Internet-Recht, \ 3 S. 101; Borges, S. 195; Spindler/Schuster/Spindler/Weber, Vor $\$ 145$ BGB Rn. 6; Wiebe, S. 214 ff.; Palandt/Heinrichs/Ellenberger, Einf. v. \$116 Rn. 1.

254 Diese Möglichkeiten aufwerfend, mit dem Hinweis, dass dies anhand des Einzelfalls geprüft werden müsste, Koch, ITRB 2007, 72.

255 BGH, NJW 2005, 976 = JZ 2005, 791 m. Anm. Spindler, Palandt/Heinrichs, \145 Rn. 2; Mehrings, in: Hoeren/Sieber, Hdb. MultimediaR, Teil 13.1 Rn. 137 ff.; Flume, AT 2, \35/1 S. 636; Spindler/Schuster/Spindler/Weber, Vor \145 BGB Rn. 4; Koch, Internet-Recht, \3 S. 103; Wiebe, S. 450 . 
Angebot erst durch den Web Service-Anbieter erfolgen müsste, was wiederum frühestens konkludent durch die Nutzung des Web Service möglich wäre. Problematisch an dieser Konstellation ist allerdings, dass dadurch Angebot und Annahme in einer Handlung lägen, der Nutzung des Web Service, so dass die Annahme in der „Duldung“ der Nutzung gesehen werden müsste. Die Nutzung und Duldung der Nutzung lassen sich im Rahmen der Web ServiceNutzung aber zeitlich faktisch nicht trennen, höchstens durch eine juristische Sekunde. Das würde für den Web Service-Anbieter bedeuten, dass er de facto gar keine Möglichkeit mehr hat, dass Angebot abzulehnen und dem Web Service-Nutzer keine Widerrufsmöglichkeit seines Angebots im Sinne des $\ 130$ Abs. 1 S. 2 BGB bleibt. Zwar ist der Widerruf nach $\$ 130$ Abs. 1 S. 2 BGB im E-Commerce aufgrund der Schnelligkeit der Übertragung oft tatsächlich ausgeschlossen, ${ }^{256}$ die Annahme bleibt aber auch im E-Commerce grundsätzlich in der Sphäre des Betroffenen, so dass dem Angebotsempfänger regelmäßig die Option bleibt, das Angebot abzulehnen. Dies wäre in der genannten Konstruktion aber gerade nicht der Fall, was der Ratio eines freien Willensentschlusses bei Abgabe einer Willenserklärung widersprechen würde. Daher kann das Angebot nicht in der Nutzung des Web Service durch den Web Service-Nutzer gesehen werden. Das bedeutet aber auch, dass dem Web ServiceNutzer keine anderweitige Möglichkeit zur Abgabe eines Angebots bliebe, so dass bereits die Annahme, dass Einstellen des Web Service in das UDDI sei eine bloße invitatio ad offerendum, mangels praktischer Handhabbarkeit abgelehnt werden muss.

Vielmehr kommt daher, sofern es sich nicht um eine private Nutzungsvereinbarung handelt, nur das Einstellen der Dienstebeschreibung als Angebot durch den Web Service-Nutzer in Frage. Denn realiter ist aus Sicht des Web Service-Anbieters das Einstellen der Dienstebeschreibung zugleich das Bereitstellen des Dienstes für die Öffentlichkeit, da es von dessen Seite keines weiteren Zwischenschritts mehr bedarf. Ein solches Bereitstellen einer Leistung ist grundsätzlich eine offerta ad incertas personas, wobei die Annahme durch die Inanspruchnahme der Leistung erfolgt. ${ }^{257}$ Eine solche Abgabe eines Angebotes zugunsten eines noch unbestimmten Vertragspartners wird zum Beispiel auch durch das Aufstellen eines Automaten ${ }^{258}$ oder das Einstellen eines Arti-

256 Mebrings, in: Hoeren/Sieber, Hdb. MultimediaR, Teil 13.1 Rn. 135.

257 Flume, AT 2, \35/1 S. 636; Spindler/Schuster/Spindler/Weber, Vor $₫ 145$ BGB Rn. 5; Mehrings, in: Hoeren/Sieber, Hdb. MultimediaR, Teil 13.1 Rn. 135; Koch, Internet-Recht, $\ 3$ S. 104; Erman/Armbrïster, $\int 145$ Rn. 8.

258 Padeck, VersR 1989, 542; Palandt/Heinrichs, \145 Rn. 7; Koch, Internet-Recht, \3 S. 104; Flume, AT 2, \35/1 S. 636. 
kels bei Internetauktionen angenommen.259 $\mathrm{Ob}$ das Einstellen der Dienstebeschreibung und die Registrierung im UDDI mit diesen Konstellationen verglichen werden können, muss anhand von Kriterien, wie zum Beispiel der Bestimmtheit des Vertragsvorschlags, dem Interesse des Anbieters, eine Haftung aufgrund einer seine Leistungsfähigkeit übersteigenden vertraglichen Verpflichtung zu vermeiden, und dem Interesse an einer Bonitätsprüfung des Vertragspartners, ausgelegt werden. ${ }^{260}$ Zwar spricht abseits der praktischen Handhabbarkeit auch der Vergleich mit den Gelben Seiten für eine invitatio ad offerendum. So kommt dort durch das Einstellen der Adresse eines Handwerkers in die Gelben Seiten auch kein Vertrag durch einen telefonischen Anruf eines potentiellen Kunden zustande. Vielmehr ist die Registrierung in den Gelben Seiten eine bloße invitatio ad offerendum. Der Unterschied zu der Registrierung in den Gelben Seiten besteht allerdings darin, dass kein persönliches, telefonisches oder anderweitiges Gespräch über den Gegenstand des Vertrags mehr stattfindet. Durch die Dienstebeschreibung erhält der Web Service-Nutzer bereits alle notwendigen Informationen zur Nutzung des Dienstes. Der Web Service-Anbieter trägt dem Web Service-Nutzer, den er noch nicht personalisieren kann, ein derart konkretes Angebot zur Dienstenutzung an, dass der Web Service-Nutzer nur noch akzeptieren muss. Dem steht auch keinerlei Kapazitätsbeschränkung entgegen, da es sich um eine weitgehend unbegrenzte Dienstleistung handelt. Durch das Einstellen der Dienstebeschreibung und der dadurch erfolgenden Ermöglichung der direkten Dienstenutzung zeigt der Web Service-Anbieter auch seine Bereitschaft, den aus dem Nutzungsvertrag resultierenden Pflichten nachzukommen.

Insofern lässt sich die Situation mit derjenigen des Aufstellens von Warenautomaten oder dem Einstellen von Artikeln auf Internetauktionsplattformen vergleichen. Die Registrierung und das Einstellen der Dienstebeschreibung im UDDI stellen ein verbindliches Angebot des Web Service-Anbieters ad incertas personas dar. Auch an der notwendigen Willenserklärung bestehen keine Zweifel, da der Web Service-Anbieter den Registrierungsvorgang selber vornimmt und lediglich die Übermittlung auf elektronischem Wege erfolgt. ${ }^{261}$

259 BGH, MMR 2005, 37 (38) m. Anm. Spindler, Spindler/Nink, DRiZ 2007, 193 f.; Koch, InternetRecht, \3 S. 104; Wiebe, in: Spindler/Wiebe, Kap. 4 Rn. 128.

260 Borges, S. 206; ferner Wiebe, S. 450.

261 Dies ändert nichts am Vorliegen einer echten Willenserklärung, s. A.III.2.a)aa) Elektronische Willenserklärungen. 


\section{Annabme}

Als Annahme dieser Vertragsofferte kommt rein praktisch, außerhalb individueller Verhandlungen, lediglich die Nutzung des Web Service, als konkludente Erklärung in Betracht. Die Annahme einer Vertragsofferte kann grundsätzlich formlos, also auch durch schlüssiges Verhalten, erfolgen. ${ }^{262}$ Entscheidend für die Einordnung als konkludente Willenserklärung ist, dass der Vertragsgegner nach der Verkehrssitte und den Gesamtumständen dem Verhalten des Handelnden eine Willenserklärung entnehmen konnte. ${ }^{263}$ Zunächst einmal kann die Nutzung des Web Service unproblematisch eine Willenserklärung darstellen, denn die Nutzung wird vom Web Service-Nutzer initiiert und lediglich elektronisch übermittelt. Da der Web Service-Anbieter durch seine Registrierung (unter Umständen auch durch das Hinterlegen seiner Allgemeinen Geschäftsbedingungen, Preise etc.) und das Einstellen seiner Dienstebeschreibung alle zum Vertragsschluss notwendigen Informationen und Vorbereitungen getroffen hat, kann er eine darauf folgende Nutzung des Web Service objektiv lediglich als Einverständnis zum Abschluss eines Vertrags zu den von ihm genannten Konditionen deuten. Insofern muss die Nutzung des Web Service als konkludente Annahme der Vertragsofferte des Web Service-Anbieters durch den Web Service-Nutzer ausgelegt werden.

\section{bb) Web Service-Nutzer - Dritter}

Wie bereits erläutert,264 dient der Web Service im Rahmen der Vertragsbeziehungen zwischen Web Service-Nutzer und Dritten als Mittel zum Abschluss des Grundvertrags (zum Beispiel des Reise- beziehungsweise Werkvertrags). Hier muss danach differenziert werden, wie die Leistungen des Web ServiceNutzers ausgestaltet sind. Eine genaue Bestimmung des Vertragsschlusses kann daher immer nur anhand des Einzelfalls erfolgen. Betrachtet werden sollen hier beispielhaft so genannte „All-Inclusive-Dienste“, wie die praktisch relevante Ermöglichung der Reisebuchungen über die Plattform eines Reiseveranstalters sowie einzelne Dienste wie Wetter- oder Verkehrsnachrichtendienste.

262 Palandt/Heinrichs/Ellenberger, Einf. v. $\$ 116$ Rn. 6; Erman/Palm, Vor $\$ 116$ Rn. 7; Flume, AT 2, $\int 5 / 3$ S. 69 f.

263 BGH, NJW 1963, 1248; MünchKommBGB/Kramer, Bd. 1/1, Vorb. \116 Rn. 22; Erman/Palm, Vor $\$ 116$ Rn. 7.

264 S. A.III.1.e) Web Service-Nutzer - Dritte. 
„All-Inclusive-Dienste“

Betrachtet man das eingangs ${ }^{265}$ dargestellte Beispiel der Reisebuchung, so erfolgt die Nutzung des Web Service lediglich für Teile der Komplettreise, wie die Buchung von Flügen, unter Umständen Hotelzimmern oder auch Transportdienstleistungen. Der Dritte nutzt die Web Services nur mittelbar durch die Eingabe seiner Reisepräferenzen. Der „Kontakt“ besteht lediglich zwischen Reiseportal und Dritten, nicht zwischen Einzelanbieter und Dritten. Praktisch funktionieren Reiseportale regelmäßig derart, dass der Dritte auf der Plattform seine Präferenzen (Reisezeitraum, Reiseziel, Komfort- und Leistungsumfang etc.) eingibt und daraufhin das System via Web Services eine automatische Abfrage der Kooperationspartner bezüglich der Verfügbarkeit der gewünschten Leistungen startet. Die gewünschten Informationen werden in Form einer SOAP-Nachricht an den Web Service-Nutzer zurückgesandt, der dann dem Dritten die verschiedenen Einzelinformationen als Gesamtpaket, welches der Dritte entweder akzeptieren oder ablehnen kann, präsentiert. Insofern stellt das daraus resultierende Angebot eine Kombination aus individuellen Wünschen, aber auch fremdbestimmten Komponenten dar, so dass sich das konkrete Angebot als eine Art „individuelle Pauschalreise“ zusammenfassen lässt. In der Literatur wird für diese Art der Reisebuchung, anlehnend an die Bezeichnung in der Praxis, ${ }^{266}$ zum Teil auch der Begriff des „dynamic packaging" verwendet. ${ }^{267}$

\section{Exkurs: Reisevertragsspezifische Besonderheiten}

Dennoch ist fraglich, ob das Reiseportal überhaupt Vertragspartner des Dritten wird oder ob es nicht lediglich eine Vermittlerrolle zwischen den verschiedenen Web Service-Anbietern und dem Dritten einnimmt. Das Reisevertragsrecht ist in den $\iint 651 \mathrm{a}$ ff. BGB geregelt. Allerdings ist zu beachten, dass die \S 651a ff. BGB nur Pauschalreisen regeln und insofern wohl die Bezeichnung Reiseveranstaltervertrag passender wäre. ${ }^{268}$ Innerhalb des Reisevertragsrechts wird durch $\int 651 \mathrm{a}$ Abs. 2 BGB klar bestimmt, dass der Reiseveranstalter grundsätzlich nicht nur eine Vermittlerposition einnimmt, sondern selbst Vertragspartner des Reisenden wird.

\footnotetext{
265 S. B.I.2 Web Service-Nutzer.

266 S. nur bei lastminute.com, abrufbar unter: http://www.de.lastminute.com/de <25.11.2009>.

267 So Bamberger/Roth/Gleib, \651a Rn. 19; Fübrich, RRa 2006, 50 ff.

268 S. dazu Bamberger/Roth/Gleib, \ 651a Rn. 1; Kaller, Rn. 8 ff.
} 
Um feststellen zu können, ob das Reisevertragsrecht auch auf die vorliegende Konstellation anwendbar ist, müssen zunächst einige Begrifflichkeiten geklärt werden. Reiseveranstalter sind solche Personen, die in eigener Verantwortung eine Gesamtheit an Reiseleistungen als eigene anbieten, wobei sie sich dabei eines Dritten als Leistungsträger bedienen können. ${ }^{269}$ Eine Gesamtheit an Leistungen, eine so genannte Pauschalreise, wiederum ist eine im Voraus festgelegte Verbindung von mindestens zwei gleichartigen Dienstleistungen, die zu einem Gesamtpreis verkauft oder zum Verkauf angeboten werden. ${ }^{270}$ Leistungsträger werden in \651a Abs. 2 BGB legaldefiniert.

Reiseveranstalter können unabhängig von ihrer Bezeichnung und ihrer grundsätzlichen bloßen Vermittlertätigkeit auch Reisebüros sein, wenn sie die Leistungen in eigener Verantwortung erbringen, wofür immer die Erklärungen und das Verhalten des Reiseportals ausgelegt werden müssen. ${ }^{271}$ Allerdings ist die bloße Zusammenstellung von Reiseleistungen nach Wunsch des Reisenden noch nicht zwangsläufig eine Reise im Sinne des $\ 651$ a. ${ }^{272}$ Im Falle der Reiseportale liegt die Crux darin, dass sie zum einen keine fertigen Reisepakete bereithalten, sondern diese individuell durch den Web Service erst nach der Anfrage des Kunden zusammenstellen, so dass die Gewährleistung der Voraussetzung „im Voraus festgelegte Verbindung“ unklar ist, und sie zum anderen de facto die Reise nach den Wünschen des Kunden zusammenstellen. Während der Kunde einer klassischen Katalogbestellung daher maximal Einfluss auf die Zimmerart, die Verpflegung und sonstige Zusatzleistungen ausüben kann, ist es dem Kunden des Reiseportals selbst möglich, das Reisemittel, den Anbieter der Unterkunft etc. zusammenzustellen.

Der Europäische Gerichtshof hat im Rahmen einer Vorabentscheidungsfrage zur Auslegung von bestimmten Aspekten der Pauschalreiserichtlinie ${ }^{273}$, nach der die $\iint 651 \mathrm{a}$ ff. BGB ausgerichtet sind, entschieden, dass der Begriff der „im Voraus festgelegten Verbindung“ dahingehend auszulegen ist, dass er

269 BGH, NJW 1974, 37 (38); MünchKommBGB/Tonner, Bd. 4, Vorb. \S 651a ff. Rn. 10; Kaller, Rn. 16.

270 OLG Frankfurt, NJW-RR 1988, 1328 (1329); Kaller, Rn. 9.

271 BGH, NJW 1974, 37 (38); OLG Düsseldorf, NJW-RR 1998, 50; OLG Frankfurt, NJW-RR 1988, 1328 (1329); MünchKommBGB/Tonner, Bd. 4, Vorb. JS 651a ff. Rn. 10; Bamberger/Roth/Geib, \651a Rn. 19.

272 Bamberger/Roth/Gleib, \651a Rn. 19; a.A. MünchKommBGB/Tonner, Bd. 4, \651a Rn. 22, der die im Reisebüro individuell zusammengestellte Reise regelmäßig als Reise im Sinne der $\iint 651 \mathrm{a}$ ff. qualifiziert.

273 Richtlinie 90/314/EWG des Rates v. 13.6.1990 über Pauschalreisen, ABl. EG L 158, S. 59-64. 
auch ,[...] Verbindungen von touristischen Dienstleistungen einschließt, die in dem Zeitpunkt vorgenommen werden, zu dem der Vertrag zwischen dem Reisebüro und dem Verbraucher geschlossen wird.“274 Gleiches muss daher auch für Reiseportale gelten, da die Bezeichnung der Unternehmensart für die Auslegung der Voraussetzungen für die Annahme einer Pauschalreise irrelevant ist. Insofern stellt zum einen die über das Reiseportal ,individuell“ zusammengestellte Reise eine Pauschalreise im Sinne der $\iint$ 651a ff. BGB dar, zum anderen wird das Reiseportal durch die Zusammenstellung hier auch zum Reiseveranstalter nach $\int 651$ a BGB. ${ }^{275}$ Etwas anderes ergibt sich lediglich dann, wenn die Reise nicht im Rahmen des so genannten dynamic packaging gebucht, sondern in separaten Schritten durchgeführt und nicht zu einem Gesamtpreis gebündelt wird (so genanntes dynamic bundling). ${ }^{276} \mathrm{Da}$ dies im Falle der Reiseportalbuchung via Web Services aber nicht dem angebotenen Leitbild entspricht, ist hier vom dynamic packaging ausgehend das Reisevertragsrecht anwendbar, so dass das Reiseportal (Web Service-Nutzer) direkter Vertragspartner des Dritten (Kunde) wird. Die Leistungsträger und somit die Web Service-Anbieter (Hotels, Fluggesellschaften etc.) sind indes nur die Erfüllungsgehilfen des Reiseportals. ${ }^{277}$ Einen Vertrag haben die Leistungsträger selber lediglich mit dem Reiseportal. ${ }^{278}$ Zum Schutz des Reisenden wird aber von einem Vertrag zugunsten Dritter ausgegangen, bei dem ein Einwendungsdurchgriff Leistungsträger - Reisender als stillschweigend abbedungen gilt. ${ }^{279}$

\section{Angebot und Annahme}

Zu welchem Zeitpunkt der Vertrag zwischen Reiseportal und Drittem zustande kommt, das heißt in welchen Erklärungen beziehungsweise Handlungen Angebot und Annahme zu sehen sind, ist aufgrund der Vielzahl an Handlun-

274 EuGH, EuZW 2002, 402 (403) - Club-Tour, Viagens e Tourismo SA.

275 Fübrich, S. 78; Bamberger/Roth/Gleib, \651a Rn. 19; wohl dafür plädierend MünchKommBGB/Tonner, Bd. 4, \$651a Rn. 23a. Dies durch die eigenen AGBs in Abs. 2 der Präambel anerkennend bspw. lastminute.de, abrufbar unter: http://www.lastminute.de $<25.11 .2009>$.

276 Bamberger/Roth/Gleib, \651a Rn. 19.

277 Kaller, Rn. 27.

278 BGH, NJW 2006, 3268 (3269); NJW 1988, 1380 (1381). Die Vertragsart richtet sich dabei wiederum nach dem jeweiligen Vertragsgegenstand.

279 BGH, NJW 1985, 1457 f.; MünchKommBGB/Tonner, Bd. 4, 』 651a Rn. 40; Kaller, Rn. 28; Erman/Seiler, \651a Rn. 11, der im Einzelfall aber auch einen Vertrag mit Schutzwirkung zugunsten Dritter annimmt; Staudinger/Eckert, \651a Rn. 56 f. 
gen der Beteiligten nicht eindeutig und bedarf daher der genaueren Untersuchung. Dabei werden zunächst die verschiedenen Erklärungen der Beteiligten betrachtet und sodann die Allgemeinen Geschäftsbedingungen des Reiseportals untersucht.

Die Web Präsenz des Web Service-Nutzers (Reiseportal) stellt lediglich eine invitatio ad offerendum dar. Ein verbindliches Angebot ist nicht gewollt, da die Verfügbarkeit der Einzelleistungen zum einen limitiert ist und zum anderen auch gar nicht in seinem Machtbereich liegt. Das Vertragsangebot könnte daher in der Anfrage des Dritten durch Eingabe seiner Präferenzen und der daraufhin folgenden Suche gesehen werden. Dies erscheint aber realitätsfern, da der Dritte zu diesem Zeitpunkt noch gar nicht weiß, welche Modalitäten verfügbar sind, das heißt, wann und wo der in Frage kommende Flug startet, welches Hotel verfügbar ist etc. Das würde bedeuten, dass er dem Web Service-Nutzer ein Angebot über eine zu erbringende Leistung macht, die er nicht genauer kennt. Ein Angebot ist grundsätzlich nach $\int 145$ BGB bindend, so dass der Dritte an jegliche Zusammenstellung durch den Web ServiceNutzer mit allen Rechten und Pflichten gebunden wäre. Da dies aber objektiv betrachtet so von beiden Seiten nicht gewollt sein kann, kann die Eingabe der Präferenzen des Dritten lediglich als unverbindliche Anfrage beziehungsweise eigene Einladung des Dritten an den Web Service-Nutzer, diesem eine Offerte zu unterbreiten, gewertet werden.

Vielmehr kommt die Zusammenstellung des Reiseportals aufgrund der Anfrage des Dritten als Angebot in Frage. Dadurch, dass der Web Service-Nutzer via Web Services die Verfügbarkeit der Teilleistungen geprüft und diese zu einem Gesamtpaket zusammengestellt hat, könnte man annehmen, dass der Web Service-Nutzer dem Dritten eine konkrete verbindliche Offerte machen möchte. Allerdings stellt sich die Frage, ob das vollautomatisierte Anbieten des Gesamtpakets eine rechtsverbindliche Willenserklärung des Web ServiceNutzers darstellen kann. Die Parameter für die Eckdaten der Reise bestimmt der Dritte und auf der Basis dieser Parameter sucht der Web Service bei den Vertragspartnern automatisch nach den passenden Teilleistungen, die dann wiederum durch das System des Web Service-Nutzers automatisiert zusammengestellt werden. Der Web Service-Nutzer ist daher nicht direkt in die Zusammenstellung und Präsentation des „Angebots“ eingebunden. Das bedeutet, dass die Erklärung automatisch generiert und sodann elektronisch an den 
Dritten übermittelt wird. Die Situation entspricht in ihren Grundzügen denen der Computererklärung, die allgemein als Willenserklärung anerkannt wird. ${ }^{280}$

Der Unterschied zur „klassischen“ Computererklärung ist allerdings, dass im Falle des Web Service-Nutzers lediglich der Web Service des Web ServiceAnbieters genutzt wird, es sich also nicht um eigene, durch den Web ServiceNutzer selbst programmierte oder zumindest zurechenbar programmierte Software handelt, sondern um fremde Software, dessen Funktionalitäten er nicht beeinflussen kann. Die Computererklärung wird allerdings gerade deswegen als eigene Erklärung des Softwareverwenders eingeordnet, weil die Funktionsweise und die durch die Software generierte Erklärung letztlich auf dem Willen des Softwareverwenders beruhen, da dieser die Leitlinien der Programmierung festlegt. Dennoch muss auch im Falle der bloßen Web ServiceVerwendung das gleiche gelten. Zwar hat der Web Service-Nutzer keinen Einfluss auf die Programmierung des Web Service, er weiß aber aufgrund der Dienstebeschreibung welche Art von Leistungen der Web Service umfasst und bindet ihn dann bewusst in sein Portal ein. Integriert er somit beispielsweise den Web Service einer Fluggesellschaft, so entscheidet er sich bewusst dafür, dass er einen Web Service einbindet, der Flugverbindungen sucht, findet und diese bei entsprechendem Anstoß auch bucht. Die Situation lässt sich insofern mit der Angestellten im Reisebüro vergleichen, die händisch die Flugdaten ermittelt, sie dem Kunden anbietet und gegebenenfalls bucht. Diese Arbeit übernimmt im vorliegenden Fall der Web Service, der wie auch die Angestellte, für das Reiseportal eingesetzt und diesem zugerechnet wird. Daher beruht die Zusammenstellung durch den oder die eingebundenen Web Services letztlich doch auf dem Willen des Web Service-Nutzers, der dem Dritten diese gebündelten Reisezusammenstellungen anbieten will, 281 und stellt insofern eine Willenserklärung des Web Service-Nutzers dar.

Ob diese Willenserklärung aber bereits als Angebot durch den Web ServiceNutzer gewertet werden kann, ist eine davon unabhängige Frage. Die Situation ist zwar eindeutig eine andere als bei der bloßen Präsentation der Website, dennoch könnte auch hier vom Web Service-Nutzer unter Umständen eine zusätzliche Überprüfung der Verfügbarkeit sowie eine Bonitätsprüfung erwünscht sein, so dass dieser noch kein rechtsverbindliches Angebot durch die Zusammenstellung der Reisekomponenten machen möchte. Die geheimen

280 S. A.III.2.a)aa) Elektronische Willenserklärungen.

281 Dieses Problem im Falle eines Übermittlungsirrtums anreißend und dabei offensichtlich ebenfalls eine Willenserklärung des Web Service-Nutzers annehmend Spindler, Anm. zu BGH, JZ 2005, 791 ff., JZ 2005, 793 (795). 
Wünsche des Web Service-Nutzers spielen aber bei der Auslegung von Willenserklärungen keine Rolle. Entscheidend ist, wie der konkrete beziehungsweise nachrangig der objektive Empfänger die Erklärung des Erklärenden verstehen durfte. ${ }^{282} \mathrm{Da}$ der Web Service-Nutzer durch die Suche und Buchung beim Web Service-Anbieter aber gerade eine Verfügbarkeitsprüfung vornimmt ${ }^{283}$ und dementsprechend objektiv betrachtet eine weitere Verfügbarkeitsprüfung aufgrund des besonderen Systems nicht mehr erforderlich ist, stellt sich die Zusammenstellung durch den Web Service-Nutzer für den objektiven Empfängerhorizont nicht mehr als invitatio ad offerendum sondern vielmehr als rechtsverbindliches Angebot bezogen auf das Argument der Verfügbarkeit dar.

Anders verhält es sich bei der fehlenden Möglichkeit der Überprüfung der Zahlungsfähigkeit des Dritten. Eine solche Überprüfung kann der Web Service-Nutzer erst durch die Eingabe der entsprechenden Daten des Dritten vornehmen. Diese Datenpreisgabe erfolgt aber erst nach Zusammenstellung der Reisemodalitäten. Läge in der Zusammenstellung der Reisemodalitäten daher bereits ein Angebot durch den Web Service-Nutzer, so würde diesem die Möglichkeit der Überprüfung der Zahlungsfähigkeit des Dritten de facto beschnitten, da sie für ihn nach Abgabe eines rechtsverbindlichen Angebots kein wirksames Mittel mehr darstellt. Die Bonitätsprüfung ist kein neues oder unbekanntes Mittel, sie wurde und wird vor allem in zahlreichen Verkaufsbereichen genutzt, in denen das Vertrauen durch persönlichen Kontakt und/oder langjährige Geschäftsbeziehungen fehlt, wie zum Beispiel beim Btx. ${ }^{284}$ Eine solche Zahlungsüberprüfung wäre dem Kunden - objektiv betrachtet - demnach auch nicht völlig fremd. Daher könnte man dahin tendieren, dass die Zusammenstellung trotz der Prüfung der Verfügbarkeit der Reisemodalitäten doch nur eine invitatio ad offerendum darstellt. Bei einer solchen Auslegung bliebe aber außer Acht, dass der Web Service-Nutzer gerade durch die Überprüfung der Verfügbarkeit ein völlig neues Konzept - jenseits der bloßen Warenpräsentation auf einer Website - und dem Dritten dadurch eine gewisse „Buchungssicherheit“ suggeriert, insofern also sowohl

282 Flume, AT 2, \16/3 S. 312 f.; Laren₹/Wolf; AT, \28 Rn. 19; Medicus, Rn. 45; Palandt/Heinrichs, \145 Rn. 2; Härting, Rn. 170; Mehrings, in: Hoeren/Sieber, Hdb. MultimediaR, Teil 13.1 Rn. 146; ferner Larenz, Methodenlehre, S. 299.

283 S. dazu auch das ähnliche Modell der „Seminarbuchung“ bei Burghardt, S. 14.

284 S. zur Überprüfung der Zahlungsfähigkeit des Kunden bereits Redecker, NJW 1984, 2391; Palandt/Heinrichs, \145 Rn. 2. 
starke Leistungsfähigkeit als auch -bereitschaft vermittelt. ${ }^{285}$ Will der Web Service-Nutzer einen solchen Eindruck vermeiden, so könnte er diesen - objektiv stark vermittelten - Eindruck dadurch unterbinden, dass er deutlich darauf hinweist, dass er ein Angebot noch von weiteren Prüfungen, wie der Zahlungsfähigkeit, abhängig machen will. ${ }^{286}$ Daher verfängt das Argument der Bonitätsprüfung zugunsten der Annahme einer invitatio ad offerendum nicht.

Insofern stellt die Zusammenstellung der Reisemodalitäten nach Auslegung der Erklärungen für den objektiven Empfänger ein auf einer Willenserklärung des Web Service-Nutzers beruhendes Angebot dar, welches der Dritte durch das Anklicken eines entsprechenden Buttons annehmen kann.

Gegen diese Zuordnung von Angebot und Annahme sprechen wiederum oft die Allgemeinen Geschäftsbedingungen (AGBs) ${ }^{287}$ der Reiseportale. In deren Klauseln wird häufig darauf hingewiesen, dass der Dritte selbst, durch die Eingabe seiner Daten, ein wirksames Angebot abgebe und dieses erst durch eine später erfolgende Buchungsbestätigung via E-Mail durch das Reiseportal angenommen werde. 288

\section{Informationspflichten im Fernabsatz}

Eine solche Regelung innerhalb der Allgemeinen Geschäftsbedingungen ist zwar grundsätzlich, insbesondere vor dem Hintergrund von begrenzten Vorräten etc., möglich, jedoch ergeben sich für den Verwender darüber hinaus bei Fernabsatzverträgen gegenüber Verbrauchern, was die Dritten regelmäßig sein werden, besondere Pflichten. Nach $\iint 312 \mathrm{c}$ Abs. 1 S. 1, 312e Abs. 1 Nr. 2, Abs. 3 S. 1 BGB in Verbindung mit $\ 3$ Nr. 1 BGB-InfoV, der im Wesentlichen Art. 10 der E-Commerce-Richtlinie ${ }^{289}$ umsetzt, $^{290}$ muss der Fernabsatz-

Lachmann, NJW 1984, 408; MünchKommBGB/Kramer, Bd. 1/1, Vorb. \145 Rn. 31 u. \145 Rn. 10; Mehrings, in: Hoeren/Sieber, Hdb. MultimediaR, Teil 13.1 Rn. 147; Holžbach/Süßenberger, in: Moritz/Dreier, C Rn. 205; Borges, S. 206.

So auch Lachmann, NJW 1984, 408; so auch für Verbraucher Mehrings, in: Hoeren/Sieber, Hdb. MultimediaR, Teil 13.1 Rn. 150, 153.

Zur wirksamen Einbeziehung in Verträge allgemein s. A.III.4.a) Wirksame Einbeziehung von Allgemeinen Geschäftsbedingungen.

288 S. nur Nr. 1 der AGBs von lastminute.de, abrufbar unter: http://www.lastminute.de/ $<25.11 .2009>$ und Nr. 1 der AGBs von Andano, abrufbar unter: http://www.andano.de/agb.htm $<25.11 .2009>$.

289 Richtlinie 2000/31/EG des Europäischen Parlaments und des Rates v. 8.6.2000 über bestimmte rechtliche Aspekte der Dienste der Informationsgesellschaft, insbesondere des elektronischen Geschäftsverkehrs, im Binnenmarkt, ABl. EG L 178 v. 17.7.2000 S. 1-16. 
unternehmer im elektronischen Geschäftsverkehr rechtzeitig vor Abgabe der Bestellung des Verbrauchers, diesem Informationen über die einzelnen technischen Schritte, die zu einem Vertragsschluss führen, zur Verfügung stellen.

Damit gemeint sind keineswegs die Informationen über die einzelnen Stadien der Datenverarbeitung, sondern vielmehr welche Handlungen und Erklärungen welche rechtlichen Wirkungen haben, insbesondere durch welche Handlungen/Erklärungen Angebot und Annahme erfolgen. ${ }^{291}$ Vor diesem Hintergrund stellt sich die Frage, wann die Information zum einen klar und verständlich und zum anderen rechtzeitig erfolgt. Vor dem Hintergrund der gängigen Praxis der Reiseportale, diese Informationen in Allgemeine Geschäftsbedingungen einzubinden, stellt sich daher die konkrete Frage, ob die Einbindung in Allgemeine Geschäftsbedingungen überhaupt diesen Anforderungen genügt.

So könnte man argumentieren, dass gerade die Betonung der Klarheit und Verständlichkeit der Information eher gegen eine ausreichende Information durch Einbindung in Allgemeinen Geschäftsbedingungen spräche, da die Informationen dort unterzugehen drohen beziehungsweise die Informationspflicht sogar bewusst durch überlange Bedingungen umgangen werden könnte. ${ }^{292}$ Indes muss sich diese Argumentation aber entgegenhalten lassen, dass gerade bei Allgemeinen Geschäftsbedingungen das Transparenzgebot (Klarheit und Verständlichkeit) zwingend verlangt wird. Stützt man sich immer auf das Argument, dass Allgemeine Geschäftsbedingungen bewusst missbraucht oder durch die Länge generell ungeeignet sind, so könnten sie nie das erforderliche Transparenzgebot erfüllen und wären immer unwirksam. Insofern kann auch im Rahmen der vorvertraglichen Informationspflichten eine Einbindung dieser Informationen in Allgemeinen Geschäftsbedingungen nicht schaden. ${ }^{293}$ Darüber hinaus sollten die Informationen über das Zustandekommen des Vertragsschlusses aber aus Gründen des Verbraucherschutzes tatsächlich nicht in überlangen Allgemeinen Geschäftsbedingungen versteckt

290 Felke/Jordans, WM 2004, 168 Fn. 25; Palandt/Grüneberg, $\$ 3$ BGB-InfoV Rn. 1; Bamberger/Roth/Masuch, § 3 BGB-InfoV Rn. 2; Härting, Rn. 515.

291 MünchKommBGB/Wendehorst, Bd. 2, \$312e Rn. 78; Palandt/Grüneberg, \ 3 BGB-InfoV Rn. 2; Bamberger/Roth/Masuch, \ 3 BGB-InfoV Rn. 4; Aigner/Hofmann, Rn. 345; Meyer, DB 2004, 2741; a.A. Leveren₹, VersR 2003, 701, der es als ausreichend ansieht, wenn der Kunde über den Ablauf der Antragsaufnahme aufgeklärt wird.

292 So z.B. Lütcke, $₫ 312$ e Rn. 45.

293 Aigner/Hofmann, Rn. 345; MünchKommBGB/Wendehorst, Bd. 2, 』312e Rn. 74; Leßmann/Leßmann, in: Gounalakis, Rhb. Electronic Business, \ 11 Rn. 54. 
werden, sondern vielmehr an prominenter Stelle im ersten Abschnitt der Bedingungen platziert werden.

Rechtzeitig erfolgt die Information, wenn sie dem Verbraucher auf Seiten, die er zwingend vor dem Erscheinen eines Buchungsbuttons aufrufen muss, bereitgestellt wird, ${ }^{294}$ wobei eine tatsächliche Kenntnisnahme nicht erforderlich ist.

\section{Regelung des Vertragsschlusses durch Allgemeine Geschäftsbedingungen}

Jenseits der Problematik der zulässigen Information in Allgemeinen Geschäftsbedingungen ist aber fraglich, ob Allgemeine Geschäftsbedingungen überhaupt wirksam den Vertragsabschluss, präziser die Frage wie der Vertrag zustande kommt, regeln können. Denn eine Geltung der Allgemeinen Geschäftsbedingungen setzt nach $₫ 305$ Abs. 2 BGB einen Vertragsschluss voraus. Legt man dies zugrunde, so können Klauseln, die erst nach dem Zustandekommen des Vertrags wirksam einbezogen werden, grundsätzlich nicht regeln durch welche Handlungen und Erklärungen der Vertrag zustande kommt. ${ }^{295}$

Zwar kann die Geltung der Allgemeinen Geschäftsbedingungen für alle künftigen Verträge über so genannte Rahmenvereinbarungen im Sinne des $\ 305$ Abs. 3 BGB vereinbart werden. Zum einen ist aber Voraussetzung für die Wirksamkeit einer solchen Rahmenvereinbarung, dass der Verwender den Kunden unmissverständlich darauf hingewiesen hat, dass seine Allgemeinen Geschäftsbedingungen allen künftig abzuschließenden Verträgen zugrunde liegen sollen. ${ }^{296}$ Nicht ausreichend ist daher der bloße Hinweis auf die Allgemeinen Geschäftsbedingungen generell. Ein entsprechender Hinweis auf eine Vertragsabschlussklausel lässt sich in der Praxis allerdings eher nicht bei Reiseportalen finden. ${ }^{297}$ Zum anderen dienen solche Rahmenvereinbarungen eher der Erleichterung eines steten Geschäftskontakts, um nicht bei jedem Vertrag erneut die Einbeziehungsvoraussetzungen des $₫ 305$ Abs. 2 BGB erfüllen zu

294

MünchKommBGB/Wendehorst, Bd. 2, \312e Rn. 70.

KG, NJW 1981, 2822; Palandt/Heinrichs, \305 Rn. 5; Bamberger/Roth/Becker, \305 Rn. 68; diese Überlegung gleichfalls in ihre Argumentation einbeziehend jedoch am Ende zu einem Ergebnis kommend: OLG Düsseldorf, NJW 2005, 1515.

BGH, NJW-RR 1987, 112; MünchKommBGB/Basedow, Bd. 2, \305 Rn. 87, 96; Ulmer/Brandner/Hensen/Ulmer, \305 Rn. 206.

S. dazu bspw. das Buchungsverfahren von www.lastminute.de $<25.11 .2009>$. 
müssen, nicht aber der vorvertraglichen Einbeziehung von Allgemeinen Geschäftsbedingungen.

Dennoch wird zum Teil ohne Begründung davon ausgegangen, dass innerhalb von Allgemeinen Geschäftsbedingungen auch im vorvertraglichen Verhältnis Bedingungen, sei es für das spätere Vertragsverhältnis oder den Vertragsschluss als solchen, ohne Rahmenvereinbarungen aufgestellt werden können. ${ }^{298}$ Danach wäre die gängige Praxis, in den Allgemeinen Geschäftsbedingungen zu bestimmen, dass der Reisevertrag erst durch die Buchungsbestätigung des Reiseportals zustande kommt und der Kunde selbst durch das Klicken des Buchungsbuttons das Angebot abgibt, zulässig. Der Zeitpunkt des Vertragsschlusses könnte demnach bei entsprechend klarer, verständlicher und rechtzeitiger Information wirksam geregelt werden.

Dass eine derartige Bedingung in Allgemeinen Geschäftsbedingungen den Vertragsschluss aber keineswegs wirksam regeln kann, zeigt wiederum die Tatsache, dass Allgemeine Geschäftsbedingungen erst nach Vertragsschluss zur Wirksamkeit gelangen. ${ }^{299}$ Lediglich bestimmte Modalitäten im Rahmen des Gesetzesspielraums, wie der Ausschluss der Bindungswirkung an ein Angebot oder der Verzicht auf den Zugang der Annahmeerklärung, lassen sich durch Allgemeine Geschäftsbedingungen regeln, ${ }^{300}$ nicht aber die Festlegung durch welche Handlungen Angebot und Annahme zustande kommen. ${ }^{301}$

\section{Zusammenfassung}

Die gängige Praxis, das Zustandekommen des Vertrags zugunsten des Reiseportals soweit wie möglich nach hinten zu verlagern, zugleich aber den Nutzer an sein Angebot zu binden, ist durch die Regelung in Allgemeinen Geschäftsbedingungen nicht möglich. Gleiches gilt freilich auch für Anbieter in anderen Branchen. Derartige Klauseln erlangen erst nach Vertragsschluss Geltung und können nicht mehr rückwirkend in den objektiv ermittelten Zeitpunkt der Abgabe von Angebot und Annahme hinein wirken. Entgegen den Allgemeinen Geschäftsbedingungen erfolgt das Angebot daher durch die Zusammenstellung des Reiseportals, da dieses, anders als auf normalen Verkaufsplattfor-

\footnotetext{
298 BGH, NJW 1996, 2574 (2575); OLG Düsseldorf, NJW 2005, 1515 f.

299 Ulmer/Brandner/Hensen/Ulmer, \305 BGB Rn. 13 u. 163; Staudinger/Schlosser, \305 Rn. 163; Grunewald, ZIP 1987, 356; AG Freudenstadt, NJW-RR 1994, 239 (239).

300 Staudinger/Scblosser, \305 Rn. 165; Ulmer/Brandner/Hensen/Ulmer, \305 BGB Rn. 163.

301 So ausdrücklich das AG Freudenstadt, NJW-RR 1994, 239 (239).
} 
men, eine Verfügbarkeitsprüfung via Web Services vornimmt und insofern das Argument der Vorratsschuld nicht mehr verfängt. Durch das Klicken des „Buchungs-Buttons“ erfolgt dann die Annahme durch den Nutzer.

Diese Qualifizierung kann im Falle der Nichterfüllung für das Reiseportal bedeutende Konsequenzen, wie Schadensersatzansprüche des Kunden, nach sich ziehen. Dennoch ist dieses Ergebnis durchaus sachgerecht: gerade im Lastminute-Buchungsbereich ist der Reisende auf eine gewisse Sicherheit bezüglich des wirksamen Vertragsschlusses angewiesen, sind derartige Reisen doch auch schon am gleichen beziehungsweise nächsten Tag möglich, so dass der Nutzer unter Umständen bereits Aufwendungen in Anbetracht der bevorstehende Reise tätigt, die er bei einer nachträglichen Feststellung der mangelnden Verfügbarkeit der Reise durch das Reiseportal umsonst getätigt hätte.

Will das Reiseportal dennoch Angebot und Annahme nach hinten verlagern, so muss sie den Kunden darauf ausdrücklich und klar verständlich - außerhalb der Allgemeinen Geschäftsbedingungen - vor dem Ingangsetzen des Zusammenstellungsprozesses darauf hinweisen. In diesem Falle ist der Rechtssicherheit genüge getan und der Hinweis stellt einen Teil der Willenserklärung des Reiseportals dar.

Auch im Rahmen von Verbraucherverträgen ist grundsätzlich eine Rechtswahl nach Art. 27 Abs. 1 EGBGB möglich, wenn der Web Service-Nutzer seinen Unternehmenssitz im Ausland hat. Allerdings wird diese Möglichkeit im Rahmen von Verbraucherverträgen durch Art. 29 Abs. 1 EGBGB eingeschränkt, der besagt, dass dem Verbraucher nicht der Schutz durch zwingende Vorschriften seines Aufenthaltslandes entzogen werden dürfen, wenn nach Nr. 1 dem Vertragsabschluss ein ausdrückliches Angebot oder eine Werbung in diesem Staat vorausgegangen ist und wenn der Verbraucher in diesem Staat die zum Abschluss des Vertrags erforderlichen Rechtshandlungen vorgenommen hat. Darüber hinaus enthält auch Art. 29a Abs. 1 EGBGB eine starke Einschränkung der Rechtswahlfreiheit für das EU-Ausland. Danach sind trotz gegenläufiger Rechtswahl dennoch die deutschen Verbraucherschutzvorschriften anzuwenden, wenn der Vertrag einen engen Zusammenhang mit Deutschland aufweist. Ein solcher enger Zusammenhang ist nach Art. 29a Abs. 2 EGBGB insbesondere anzunehmen, wenn der Vertrag auf Grund eines öffentlichen Angebots, einer öffentlichen Werbung oder einer ähnlichen geschäftlichen Tätigkeit zustande kommt, die in einem Mitgliedstaat der Europäischen Union oder einem anderen Vertragsstaat des Abkommens über den Europäischen Wirtschaftsraum entfaltet wird, und der andere Teil bei Abgabe seiner auf den Vertragsschluss gerichteten Erklärung seinen gewöhnlichen Aufenthalt in einem Mitgliedstaat der Europäischen Union oder einem ande- 
ren Vertragsstaat des Abkommens über den Europäischen Wirtschaftsraum hat. Unter den Begriff der öffentlichen Werbung fällt auch Werbung des Web Service-Nutzers im Internet, die in Deutschland abrufbar ist. ${ }^{302}$ Erfolgt daher beispielsweise Werbung für individuelle Reisen durch den Web Service-Nutzer im Internet und hat dieser seinen gewöhnlichen Aufenthaltsort in einem Mitgliedsstaat der Europäischen Union, so sind die deutschen Verbraucherschutzvorschriften anwendbar, gleich welches Sachrecht vereinbart wurde.

Fehlt es an einer Rechtswahl und liegen die Voraussetzungen des Art. 29 Abs. 1 Nr. 1 EGBGB vor, so unterliegen Verbraucherverträge nach Art. 29 Abs. 2 EGBGB dem Recht des Staats, in dem der Verbraucher seinen gewöhnlichen Aufenthalt hat. Wirbt der Web Service-Nutzer daher für seine Leistungen im Internet und ist diese Werbung auch in Deutschland abrufbar, so liegen die Voraussetzungen des Art. 29 Abs. 1 Nr. 1 EGBGB regelmäßig vor und es muss deutsches Recht angewandt werden. 303

\section{Einzelne Dienste}

Anders als beim Vertragsschluss bei All-Inclusive-Angeboten verhält es sich bei typischen Einzeldiensten, wie Wetter- oder Verkehrsnachrichtendiensten. Hier bietet der Web Service-Nutzer dem Dritten auf seiner Webpräsenz einen Dienst zur Abfrage des Wetters, der Verkehrsnachrichten etc. an, dessen Abfrage auf Web Services basiert. Diese Arten von Services sind, abgesehen von Überlastungen bei extremen Zugriffen, weitgehend unbegrenzt nutzbar. Eine „Verfügbarkeitslimitierung“" gibt es insofern nicht, daher kann bereits das Anbieten des Service auf der Webpräsenz als verbindliches Angebot durch den Web Service-Nutzer gewertet werden, das der Dritte dann konkludent durch die Nutzung annimmt. Hinsichtlich des Kollisionsrechts gilt das bereits zu AllInclusive-Diensten Gesagte. 304

Etwas anderes kann allerdings dann gelten, wenn die Dienste begrenzte Ressourcen, wie den Verkauf bestimmter Waren, zum Gegenstand haben. Hier könnte man an den klassischen E-Commerce der elektronischen Marktplätze denken, wo die Website selbst regelmäßig nur eine invitatio ad offerendum

\footnotetext{
302 MünchKommBGB/Martiny, Bd. 10, Art. 29a EGBGB Rn. 59; Schmidt/Prieß, in: Spindler/Börner, S. 193; Bamberger/Roth/Spickhoff, Art. 29 EGBGB Rn. 12.

303 So grds. für Werbung im Internet Schmidt/Prieß, in: Spindler/Börner, S. 193; Bamberger/Roth/Spickhoff, Art. 29 EGBGB Rn. 12.

304 A.III.2.a)bb) Zusammenfassung.
} 
darstellt. ${ }^{305} \mathrm{Da}$ es dem automatisierten Ablauf durch den Web Service allerdings immanent ist, dass er gerade eine Verfügbarkeitsprüfung in Echtzeit vornehmen kann, 306 ist wie auch schon bei der Zusammenstellung der Reisemodalitäten durch das Reiseportal, sofern sich dies für den Dritten nach dessen Verständnishorizont auch derart darstellt, ${ }^{307}$ regelmäßig keine invitatio ad offerendum anzunehmen. Ist eine Ware daher nach Abgleich durch den Web Service noch verfügbar, muss dies als Angebot durch den Web Service-Nutzer gewertet werden, das der Dritte annehmen kann.

\section{Geschlossene Systeme}

Im Rahmen von B2B-Lösungen dagegen wird die konkrete Nutzung des Web Service in der Regel für den Vertragsschluss irrelevant sein. Gerade im Bereich der geschlossenen Systeme müssen vor Nutzung und Implementierung des Web Service zwangsläufig individuelle Verhandlungen über die Art und Weise des Web Service-Einsatzes, den Nutzerkreis etc. geführt werden. Daher muss der Vertragsschluss bereits viel früher erfolgen, nämlich aufgrund einer individuellen Vereinbarung der Parteien, in der Regel wohl Unternehmen, unter Umständen sogar durch persönliche Kommunikation, unabhängig von den Besonderheiten des elektronischen Geschäftsverkehrs. Daher bedarf der Vertragsschluss beim Einsatz von Web Services innerhalb geschlossener Systeme keiner weiteren Betrachtung.

\section{cc) Web Service-Anbieter - Dritter}

Im Rahmen der Serviceorientierten Architektur kommt ein Vertragsschluss zwischen Web Service-Anbieter und Dritten aufgrund der verteilten Architektur lediglich dann in Betracht, wenn durch den Web Service-Nutzer deutlich gemacht wird, dass er dem Dienst des Web Service-Anbieters nur eine Plattform bietet, er selber indes bei der Web Service-Nutzung kein Vertragspartner werden möchte. Technisch betrachtet, erreichen die Informationen des Web Service immer zuerst die Plattform des Web Service-Nutzers, der dann diese Informationen dem Dritten automatisiert auf seiner Plattform bereitstellt. Da der Web Service-Nutzer durch den Vorgang keine eigene Willenserklärung

\footnotetext{
305 OLG Frankfurt, MMR 2003, 405 (406); AG Butzbach, NJW-RR 2003, 54 f.; AG HamburgBarmbek, NJW-RR 2004, 1284 f.; Spindler/Schuster/Spindler/Weber, Vorb. \$S 145 ff. BGB Rn. 4; Härting, Rn. 171; Thot/Gimmy, in: Kröger/Gimmy, Hdb. InternetR, S. 6.

306 S. nur das Beispiel der „Seminarbuchung“ bei Burghardt, S. 14.

307 S. A.III.2.a)bb) Web Service-Nutzer - Dritter.
} 
abgibt, sondern lediglich die Willenserklärung des Web Service-Anbieters übermittelt, handelt es sich um eine Erklärungsbotenschaft durch den Web Service-Nutzer für den Web Service-Anbieter. ${ }^{308}$ Das Angebot zum Vertragsschluss zwischen Web Service-Anbieter und Dritten erfolgt, je nach Ausgestaltung des Dienstes, entweder durch das Bereitstellen des Web Service und die Annahme entsprechend durch die Web Service-Nutzung oder umgekehrt. Handelt es sich um unbegrenzt verfügbare Informationen, so kann das Angebot gegebenenfalls bereits in der Bereitstellung des Web Service gesehen werden. Handelt es sich indes um Vorratsschulden und ist eine vorherige Bonitätsabfrage erforderlich, so kann das Angebot regelmäßig erst in der Web Service-Nutzung beziehungsweise einer späteren Zustimmung zu einem Suchergebnis durch den Dritten liegen.

b) Sicherheit

Elektronische Kommunikationsnetze zeichnen sich dadurch aus, dass unterschiedliche Akteure handeln. Da diese aber nicht immer kooperieren, sondern durchaus auch entgegengesetzte Interessen verfolgen, kann es dazu kommen, dass manche Akteure die Kommunikation behindern oder blockieren, Daten verändern oder fremde Identitäten zur Täuschung im Rechtsverkehr nutzen oder aber die Kommunikation abhören. ${ }^{309}$ Diese Fremdeinwirkungen können auch bei der Kommunikation via Web Services auftreten.

aa) Bedrohungen und Schutzziele

Gerade bei der Übertragung von Willenserklärungen durch Web Services in Form von SOAP-Nachrichten ist die Datensicherheit nicht ausreichend gewährleistet, vielmehr bestehen gewisse Schwachstellen. So ist es potenziellen Angreifern grundsätzlich möglich, sich in den Kommunikationsfluss zwischen Web Service-Anbieter und Web Service-Nutzer einzuklinken und dadurch die in der SOAP-Nachricht verankerten Erklärungen auszulesen, zu verändern und diese verfälschten Erklärungen an den Empfänger weiterzuleiten. ${ }^{310}$

308 S. zur Abgrenzung zwischen Stellvertretung und Botenschaft Laren₹/Wolf, AT, \46 Rn. 71 ff.; Staudinger/Schilken, Vorb. \$S 164 ff. Rn. 73 ff.; MünchKommBGB/Schramm, Bd. 1/1, Vorb. \\ 164 ff. Rn. 42.

309 Federrath/Pfitzmann, in: Moritz/Dreier, F Rn. 1.

310 Burghardt, S. 77 f.; ausführlich zu denkbaren Angriffsszenarien Taylor, S. 133 ff.; ferner Melzer, S. 188 f. Diese mangelnde Sicherheit resultiert daher, dass SOAP auf HTTP beruht und SOAP daher auch wie HTTP keine Sicherungsfunktionen gegen das unberechtigte Lesen der SOAP- 
Solche Missbräuche, Verfälschungen und Verluste können das Vertrauen in die Kommunikation via Web Services zerstören. ${ }^{311}$ Um den Beteiligten ein vertrauenswürdiges IT-System zu bieten, sollten gewisse Schutzziele erfüllt werden. Solche mit den Bedrohungen der Sicherheit korrespondierenden Schutzziele sind: Vertraulichkeit, Integrität, Authentizität. ${ }^{312}$ Die Gewährleistung, dass eine Erklärung tatsächlich vom angegeben Aussteller stammt und infolgedessen der Schutz vor gefälschten Identitäten, wird als Authentizität bezeichnet. ${ }^{313}$ Diese ist ein notwendiges Kriterium für den elektronischen Rechts- und Geschäftsverkehr, da man Verträge grundsätzlich nur schließen kann, wenn man weiß mit wem. ${ }^{314}$ Dagegen wird die Möglichkeit, Änderungen des Inhalts einer Erklärung erkennen zu können als Integrität bezeichnet. ${ }^{315}$ Wie auch das Wissen um den Absender einer Willenserklärung, ist auch der Inhalt einer Willenserklärung entscheidend für den Vertragsschluss, 316 so dass auch die Integrität ein wichtiges Kriterium für den elektronischen Vertragsschluss darstellt. Bei der „ungeschützten“ Kommunikation via Web Services sind die übermittelten Daten öffentlich zugänglich und können somit von Unbefugten gelesen und verändert werden, so dass man nie wirklich sicher sein kann, von wem die Inhalte tatsächlich kommen. ${ }^{317}$ Aus diesen Gründen werden weder Authentizität noch Integrität gewährleistet. Sind die übermittelten Erklärungen nicht vor unbefugter Kenntnisnahme geschützt - wie bei der Kommunikation mittels der SOAP-basierten Web Services -, 318 mangelt es darüber hinaus an der Vertraulichkeit. ${ }^{319}$ Auch dies ist ein entscheidendes Kri-

Nachrichten enthält. Daher wird zur Verwendung von HTTPS geraten, die zumindest gewisse Angriffe unterbinden können, s. dazu bspw. Melz̨er, S. 215 f.

311 S. dazu allgemein Lapp, in: Gounalakis, Rhb. Electronic Business, $\ 18$ Rn. 1 f.

312 Lapp, in: Gounalakis, Rhb. Electronic Business, $\ 18$ Rn. 3; Weber/Willi, S. 12 ff.; Geiger, in: Gründer/Schrey, S. 32 f.

313 Weber/Willi, S. 13 f.; Bertsch, S. 3; Geiger, in: Gründer/Schrey, S. 33; BSI, VPS-Handbuch Endanwender, S. 4; BSI, E-Government-Handbuch, II/2, S. 12.

314 Lapp, in: Gounalakis, Rhb. Electronic Business, \18 Rn. 4.

315 S. Roßnagel/Roßnagel, RMD, Teil 5, Einl. SigG Rn. 15; Weber/Willi, S. 13; BSI, E-GovernmentHandbuch, II/2, S. 11.

316 Lapp, in: Gounalakis, Rhb. Electronic Business, \18 Rn. 5.

317 S. allgemein zur ungeschützten Datenübermittlung im Internet: Schwenk, S. 6.

318 Weber/Willi, S. 13; Geiger, in: Gründer/Schrey, S. 33; Bertsch, S. 3.

319 Roßnagel/Roßnagel, RMD, Teil 5, Einl. SigG Rn. 13; zum Trusted Computing allgemein Bechtold, CR 2005, 393 ff.; ferner BSI, VPS-Handbuch Endanwender, S. 4. 
terium für den elektronischen Vertragsschluss, da nicht jede Erklärung für Jedermann zugänglich kundgetan werden soll. ${ }^{320}$ Hinzu kommen ferner Fragen der Beweisbarkeit der Authentizität unter den Akteuren und der Integrität des Nachrichteninhalts sowie die Erfüllung der gesetzlich vorgeschriebenen Form, deren Ermöglichung (Beweisbarkeit) und Einhaltung (Form) über das Vertrauen hinaus zusätzlich Anreize zum Einsatz von Web Services schaffen können.

bb) Mittel zur Gewährung der Schutzziele

Eines der wirksamsten Mittel zur Sicherung des Aspekts der Vertraulichkeit ist die Verwendung von kryptographischen Verfahren zur Verschlüsselung von Daten. ${ }^{321}$ Zum Nachweis von Integrität, Authentizität, Beweisbarkeit sowie der Formerfüllung eignen sich elektronische Signaturen. 322

\section{Kryptographische Verfahren}

Verschlüsselungsverfahren zur Lösung der genannten Sicherheitsprobleme lassen sich auf verschiedenen Ebenen in der Kommunikation via Web Services implementieren. Eine Umsetzung kann sowohl auf der Transportebene (Punkt-zu-Punkt-Sicherheit) als auch in der Anwendungsschicht (Ende-zuEnde-Sicherheit) erfolgen. ${ }^{323}$ Sicherheit auf der Transportebene zeichnet sich dadurch aus, dass Nachrichten, Willenserklärungen etc. nicht abhör- und veränderbar an den Empfänger, vergleichbar mit der Durchleitung durch ein Rohr, übermittelt werden. ${ }^{324}$ Es wird folglich ein sicherer Kanal zwischen zwei Parteien, beziehungsweise zwei Punkten, ermöglicht, so dass eine so genannte Punkt-zu-Punkt-Sicherheit gewährleistet wird. Problematisch ist die Punkt-zuPunkt-Sicherheit aber im Rahmen von verteilten Anwendungen, die über das Zwei-Parteien-Verhältnis hinausgehen, wie der Serviceorientierten Architektur. Innerhalb dieser Kommunikation wird die Übermittlung in der Regel von

\footnotetext{
320 S. Lapp, in: Gounalakis, Rhb. Electronic Business, $\int 18$ Rn. 9.

321 Holz̨nagel, \4 Rn. 5 f.; Weber/Willi, S. 32; Gerling, in: Gründer/Schrey, S. 89; Bertsch, S. 3; Lapp, in: Gounalakis, Rhb. Electronic Business, \18 Rn. 20.

322 Lapp, in: Gounalakis, Rhb. Electronic Business, \18 Rn. 10; Bižer, in: Kröger/Gimmy, Hdb. InternetR, S. $41 \mathrm{ff}$.

323 Burghardt, S. 88 f.

324 Melzer, S. 194.
} 
Intermediären unterbrochen. ${ }^{325}$ Diese können dann die Informationen einsehen, auslesen, in die Rolle eines Angreifers schlüpfen etc., so dass Integrität und Vertraulichkeit nicht mehr gewährleistet sind. 326

Das Ende-zu-Ende-Sicherheitskonzept dagegen schützt den kompletten Pfad zwischen den kommunizierenden Endsystemen. ${ }^{327}$ Intermediäre erhalten dabei lediglich Zugriff auf die für sie notwendigen Daten im Header der Nachricht, ${ }^{328}$ hingegen bleiben bezüglich des SOAP-Bodies ${ }^{329}$ Integrität und Vertraulichkeit gewahrt - jede Veränderung an dem Inhalt wird durch eine Signatur dokumentiert. 330

Aufgrund dieser erhöhten Sicherheit empfiehlt es sich, die Verschlüsselung bei der Kommunikation via Web Services auf Anwendungsebene im Rahmen eines Ende-zu-Ende-Sicherheitskonzepts zu implementieren.

Bei den kryptographischen Verfahren wird unterschieden zwischen symmetrischen und asymmetrischen Verschlüsselungsverfahren. Diese beiden Verfahren unterscheiden sich dadurch, dass bei symmetrischen Verschlüsselungsverfahren sowohl für die Ver- als auch die Entschlüsselung derselbe Schlüssel verwandt wird, währenddessen bei asymmetrischen Verschlüsselungsverfahren für Verschlüsselung (öffentlicher Schlüssel) und Entschlüsselung (geheimer Schlüssel) unterschiedliche Schlüssel verwendet werden. ${ }^{331}$ Bei dem symmetrischen Verfahren zur Entschlüsselung ist es notwendig, den geheimen gemeinsamen Schlüssel auszutauschen. Dies geschieht in der Regel über das offene Internet. Daher ist es irrelevant wie sicher der Schlüssel letztlich ist, da potenzielle Angreifer den Schlüssel beim Austausch über das Internet abfangen

325 Buchbolz, in: Fröschle, S. 71 f. Intermediäre sind Vermittlungsstellen hinsichtlich der Daten, so dass die Daten immer eine Zwischenstation durchlaufen, s. Apitzsch, DuD 2007, 744.

Burghardt, S. 88; Melzer, S. 194 f.

Schmitt/Ackermann/Görtr/Steinmetr, S. 6.

Buchboly, in: Fröschle, S. 72. Im Header befinden sich die sogenannten Nutzungsdaten. Das sind Daten die zur Abwicklung der Nachricht notwendig sind, s. dazu im Bereich der Telemedien, Spindler/Schuster/Spindler/Nink, J 15 TMG Rn. 2.

Im SOAP-Body befinden sich Inhaltsdaten. Unter Inhaltsdaten versteht man die Informationen, die mittels der SOAP-Nachricht transportiert werden.

Burghardt, S. 89.

Schwenk, S. 13 f.; Gerling, in: Gründer/Schrey, S. 89 ff.; Lapp, in: Gounalakis, Rhb. Electronic Business, \ 18 Rn. 17 ff.; Bertsch, S. 8 ff. 
können. ${ }^{332}$ Insofern sind symmetrische Verschlüsselungsverfahren zur Gewährleistung der Vertraulichkeit lediglich bedingt geeignet.

\section{Die elektronische Signatur}

Asymmetrische Verschlüsselungsverfahren werden in der Regel durch die elektronische Signatur umgesetzt. Dabei kann durch die Verwendung des geheimen Schlüssels, der sich nicht aus dem öffentlich bekannt gegebenen Schlüssel errechnen lässt, und die gegenseitige Abhängigkeit der Schlüssel voneinander bezüglich der Lesbarkeit des Inhalts, eine vertraulichere Kommunikation als bei symmetrischen Verfahren ermöglicht werden. ${ }^{333}$ Wie auch beim symmetrischen wird bei dem asymmetrischen Verschlüsselungsverfahren der Signaturschlüssel mit dem zu signierenden Inhalt mathematisch verknüpft. ${ }^{334}$ Dabei wird mittels eines Hashalgorithmus ein so genannter HashWert (ein Stellvertreter fester Länge) aus den zu signierenden Daten berechnet, der das Dokument dann als eine Art Fingerabdruck eindeutig kennzeichnet. ${ }^{335}$ Wird die Datei in irgendeiner Form verändert, so fällt dies auf, sobald der Empfänger den Hash überprüft. Das bedeutet, dass der jeweilige HashWert immer nur für genau eine Datei steht und Veränderungen an der Datei erkannt werden können, indem bei der Prüfung durch den Empfänger das Dokument erneut gehasht wird und zugleich der erstmalige Hash-Wert entschlüsselt und dann mit dem späteren Hash-Wert verglichen wird. ${ }^{336}$ Stimmen beide Hash-Werte überein, wurde der Inhalt des Dokuments nicht verändert. Dieser Abgleich dient der Sicherung der Integrität. Nach der Verschlüsselung der Daten wird der Hash-Wert mittels des geheimen Schlüssels signiert. ${ }^{337}$ Diese Signatur kann dann vom Empfänger mit Hilfe des öffentlichen Schlüssels überprüft werden, ${ }^{338}$ wodurch die Authentizität gesichert wird. Elektroni-

332 Weber/Willi, S. 86; Lapp, in: Gounalakis, Rhb. Electronic Business, \ 18 Rn. 19; Bertsch, S. 9.

333 Engel-Flechsig, in: Moritz/Dreier, F Rn. 84 f.; Lapp, in: Gounalakis, Rhb. Electronic Business, \18 Rn. 19 f.; Brisch/Brisch, in: Hoeren/Sieber, Hdb. MultimediaR, Teil 13.3 Rn. 4 ff.; Holznagel, Rn. 8 ff.; Melzer, S. 190 f.

334 Weber/Willi, S. $96 \mathrm{f}$.

335 Roßnagel/Schmücker, S. 13; Brisch/Brisch, in: Hoeren/Sieber, Hdb. MultimediaR, Teil 13.3 Rn. 1; Fischer-Dieskau, S. 61; Engel-Flechsig, in: Moritz/Dreier, F Rn. 87.

336 Melzer, S. 192; Fischer-Dieskau, S. 61; Lapp, in: Gounalakis, Rhb. Electronic Business, \ 18 Rn. 26; Bertsch, S. 9 f.

337 Schwenk, S. 14; Roßnagel/Schmücker, S. 13.

338 Burghardt, S. 84; Bertsch, S. 9; Schwenk, S. 14. 
sche Signaturen lassen sich auch in offenen Architekturen wie Serviceorientierten Architekturen verwenden. ${ }^{339}$

Das Signaturgesetz (SigG) enthält vier Sicherheitsstufen von elektronischen Signaturen: die einfache ( $\$ 2 \mathrm{Nr} .1 \mathrm{SigG})$, die fortgeschrittene ( $\$ 2 \mathrm{Nr} .2 \mathrm{SigG})$, die qualifizierte $(\$ 2 \mathrm{Nr} .3 \mathrm{SigG})$ und die qualifiziert elektronische Signatur mit Anbieterakkreditierung - kurz akkreditierte Signatur - (\$\$ 2 Nr. 15, 15 SigG). An die einfache Signatur werden bei besonderen keine besonderen Sicherheitsanforderungen (sogar die eingescannte, kopierbare Unterschrift wird davon erfasst) gestellt, ${ }^{340}$ insbesondere muss sie nicht dem asymmetrischen Verschlüsselungsverfahren entsprechen. Insofern hat sie keinerlei nennenswerten Sicherheitswert. Die einfache Signatur wird daher zwar im Signaturgesetz erwähnt und definiert, jedoch werden an sie keine Rechtsfolgen geknüpft, vielmehr unterfallen einfache Signaturen den folgenlosen sonstigen Verfahren nach $\int 1$ Abs. 2 SigG, ${ }^{341}$ bei denen weder eine Kontrolle stattfindet noch gesetzliche Anforderungen gestellt werden. ${ }^{342}$

\section{Die fortgeschrittene elektronische Signatur}

Gleiches gilt für die fortgeschrittene Signatur. Nach der Gesetzesbegründung entspricht zum Beispiel die Softwarelösung „Pretty Good Privacy (PGP)“ der fortgeschrittenen elektronischen Signatur. ${ }^{343}$ Bei Pretty Good Privacy erfolgt die Veröffentlichung des öffentlichen Schlüssels privat ohne staatliche Kontrolle. Dies birgt die Gefahr der so genannten „Man-in-the-Middle-Attack“, bei der ein Dritter seinen eigenen öffentlichen Schlüssel als den eines anderen ausgibt und so die an den anderen gerichtete Kommunikation entschlüsseln kann. ${ }^{344}$ Die fortgeschrittene Signatur hat demnach zwar ein deutlich höheres Sicherheitsniveau als die einfache Signatur, allerdings verbleiben vielerlei Manipulationsmöglichkeiten, ${ }^{345}$ so dass die korrekte Zuordnung zum Erzeuger

Buchboly, in: Fröschle, S. 74.

S. Roßnagel, MMR 2003, 164; Fischer-Dieskau, S. 72; Koch, Internet-Recht, 』 4 S. 134.

Roßnagel, NJW 2001, 1819; Spindler/Schmitz/Geis/Geis, \1 SigG Rn. 2; Begr. RegE zum SigG 2001 v. 16.11.2000, BT-Drs. 14/4662, S. 18.

Roßnagel/Roßnagel, RMD, Teil 5, Einl. SigG Rn. 184.

Begr. RegE BT-Drs. 14/4662, S. 18; Härting, Rn. 192.

Weber/Willi, S. 87; Holznagel, S 6 Rn. 10.

Neben der Man-in-the-Middle-Attack sind vor allem Manipulationen bei der Beantragung des Zertifikats oder bei der Übergabe der Signaturerstellungseinheit nicht auszuschließen, durch welche die Signatur einem Falschen zugeordnet wird. Durch eine mangelhafte Identifizierung 
der Signatur nicht gewährleistet werden und eine Manipulation bei der Signaturbeantragung bezüglich des scheinbaren Antragstellers nicht ausgeschlossen werden kann, ebenso wenig wie die Ausspähung und Verwendung des privaten Schlüssels. ${ }^{346}$ Insofern können Integrität und Authentizität nicht ausreichend gewährleistet werden. ${ }^{347}$ Diese technischen Unsicherheiten führen dazu, dass eine mit einer fortgeschrittenen Signatur signierte Willenserklärung nicht die Schriftform im Sinne des $\int \mathbb{S} 126$ Abs. 3, 126a BGB ersetzen kann, da sie lediglich als Objekt des Augenscheins nach $₫ 371$ ZPO als Beweismittel im Prozess einführbar ist. ${ }^{348}$ Insofern sind die Beteiligten auf die Unwägbarkeiten der freien richterlichen Beweiswürdigung angewiesen. Gerade für den Signaturempfänger stellt die fortgeschrittene elektronische Signatur demnach kein sehr verlässliches Beweismittel im Prozess dar, um etwaige Haftungen zu verhindern, die der Nutzer des Web Service beispielsweise aufgrund manipulierter Informationen erlitten hat. 349

\section{Die qualifizierte und akkreditierte elektronische Signatur}

Die qualifizierte elektronische Signatur muss nach $₫ 2 \mathrm{Nr}$. 3 SigG die Voraussetzungen der fortgeschrittenen elektronischen Signatur nach $₫ 2$ Nr. 2 a-d SigG erfüllen, mit der Auflage, dass lediglich von einem Zertifizierungsdiensteanbieter erstellte Schlüsselpaare verwendet werden dürfen, wobei sich der private Schlüssel in der Regel auf einer Chipkarte befindet. ${ }^{350}$ Technisch wird die Sicherheit dadurch gewährleistet, dass die Komponenten nach $\int 17$ Abs. 1 SigG so zu gestalten sind, dass sich Fälschungen der Signatur und der signierten Daten zuverlässig erkennen lassen. Darüber hinaus müssen sie so gestaltet werden, dass sie vor einer unberechtigten Nutzung des Signaturschlüssels schützen und nach $₫ 17$ Abs. 3 Nr. 1 SigG müssen die Zertifizierungsdiensteanbieter sicherstellen, dass die Einmaligkeit sowie die Geheimhaltung der Schlüssel gewährleistet ist. ${ }^{351}$ Die Schlüsselpaare der ak-

des Antragstellers könnte der Falsche im Zertifikat genannt worden und es ist auch nicht die Ausspähung und Verwendung des privaten Schlüssels auszuschließen, s. dazu Roßnagel, MMR 2003, 166.

$346 \mathrm{Zu}$ den Schwächen der fortgeschritten elektronischen Signatur ausführlich Roßnagel, MMR 2003, 164 ff.; ferner allgemein die Sicherheitsproblematik ansprechend Härting, Rn. 193.

347 Härting, Rn. 194.

348 Weiterführend Roßnagel, MMR 2003, 168 ff.

349 Roßnagel, MMR 2003, 168 ff.

350 S. Koch, Internet-Recht, $\int 4$ S. 135.

351 Ausführlich zu den technischen Sicherheitserfordernissen Fischer-Dieskau, S. 76. 
kreditierten elektronischen Signatur unterscheiden sich dadurch von der qualifizierten elektronischen Signatur, dass sie von einem Zertifizierungsdiensteanbieter erstellt worden sind, der von einer zuständigen

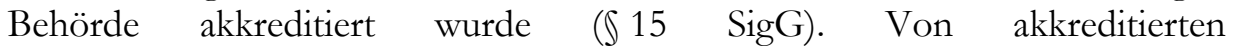
Zertifizierungsdiensteanbietern ausgestellte Zertifikate zeichnen sich durch eine langfristige Prüfbarkeit von mindestens 30 Jahren aus, ${ }^{352} \int 4$ Abs. $2 \mathrm{SigV}$. Darüber hinaus werden akkreditierte Zertifizierungsdiensteanbieter bei einer Insolvenz nach $\int 14 \mathrm{Abs}$. $2 \mathrm{SigG}$, sofern keine Übernahme von einem anderen Zertifizierungsdiensteanbieter erfolgt, im Gegensatz zu normalen Zertifizierungsdiensteanbietern von der zuständigen Behörde übernommen, 353 solche Zertifikate sind daher auch konkursresistent. 354

Durch diese technischen Sicherheitsanforderungen bei qualifizierter und akkreditierter Signatur können sowohl Integrität als auch Authentizität gewährleistet werden. ${ }^{355}$ Allerdings ist die Verwendung von qualifizierten oder akkreditierten elektronischen Signaturen - trotz der hohen Sicherheitsstandards keineswegs verbindlich. Vielmehr ist auch die Anwendung anderer Verfahren nach $\int 1$ Abs. 2 SigG freigestellt. ${ }^{356}$ Einen konkreten Anreiz zur Verwendung der qualifizierten oder akkreditierten elektronischen Signatur hat der Gesetzgeber aber durch die $\$ ₫ 126$ Abs. 3, 126a BGB, $\$ 371$ a Abs. 1 S. 2 ZPO und $\int 3 \mathrm{a} \mathrm{VwVfG} 357$ geschaffen.

\section{Gesetzliche Anreize}

\126 Abs. 3 BGB stellt klar, dass eine dem \126a BGB entsprechende elektronische Signatur die Schriftform ersetzen kann. Solche dem \126a BGB

Roßnagel/Roßnagel, RMD, Teil 5, Einl. SigG Rn. 198; BT-Drs. 14/4662, S. 28.

Spindler/Schmitz/Geis/Geis, $\$ 13$ SigG Rn. 3.

Roßnagel/Roßnagel, RMD, Teil 5, Einl. SigG Rn. 204.

Brisch/Brisch, in: Hoeren/Sieber, Hdb. MultimediaR, Teil 13.3 Rn. 8 ff.; Fischer-Dieskau, S. 61 f.; Roßnagel/Schmücker, S. 13; Lapp, in: Gounalakis, Rhb. Electronic Business, $\$ 18$ Rn. 28; Spindler/Schuster/Spindler/Weber, \$126a Rn. 1; Fischer-Dieskau/Roßnagel/Steidle, MMR 2004, 451 f.; Roßnagel, NJW 2001, 1817; Miedbrodt/Mayer, MDR 2001, 432; so auch Begr. RegE BT-Drs. 14/4662, S. 14.

356 Die Anwendung bestimmter technischer Verfahren wurde bewusst nicht vorgeschrieben, um die Prüfung verschiedener technischer Verfahren im Wettbewerb zu ermöglichen: Begr. RegE BR-Drs. 966/96, S. 19; s. dazu auch Roßnagel/Roßnagel, RMD, Teil 5, Einl. SigG Rn. 3; Spindler/Schmitz/Geis/Geis, \1 SigG Rn. 2.

$357 \int 3 \mathrm{a}$ VwVfG spielt im privaten Bereich, der hier behandelt wird, aber keine Rolle, so dass darauf nicht weiter eingegangen wird. 
entsprechende Signaturen sind lediglich die qualifizierte und akkreditierte elektronische Signatur im Sinne des Signaturgesetzes, ${ }^{358}$ da nur diese derart hohe technische und organisatorische Sicherheitsanforderungen erfüllen, das ein Substitut der Schriftform möglich erscheint. ${ }^{359}$ Das hieße für den Vertragsabschluss mittels Web Services, dass - bei Verwendung dieser Signaturformen - auch solche Verträge abgeschlossen werden könnten, die der Schriftform bedürfen. Dies wird in der Regel jedoch gar nicht erforderlich sein. Die meisten Verträge können formlos geschlossen werden. Bei vielen Vertragstypen, die die Schriftform vorsehen, wie Verbraucherdarlehensverträ-

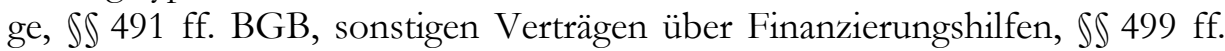
BGB, oder Bürgschaften ( $\iint 765$ ff. BGB), ist die elektronische Form von Gesetzes wegen ausgeschlossen. ${ }^{360}$ Ein praktischer Fall der Anwendbarkeit der elektronischen Signatur wäre der Wohnraummietvertrag. ${ }^{361}$ Dennoch ist der Bereich in dem die elektronische Form die Schriftform ersetzen kann denkbar gering. Allerdings kann sie in den Fällen, in denen keine besondere Form vorgeschrieben ist, durch ihre Verwendung ein gewisses Vertrauen schaffen, insbesondere bei Verträgen die - obwohl nicht von Gesetzes wegen erforderlich - häufig schriftlich abgeschlossen werden, wie zum Beispiel bei Reiseverträgen ( $(551 \mathrm{a}$ BGB).

\371a Abs. 1 S. 2 ZPO enthält bei Verwendung qualifizierter oder akkreditierter elektronischer Signaturen eine gesetzliche Beweiserleichterung in Form eines Anscheinsbeweises zugunsten der Echtheit des Dokuments und der Zuordnung zum Signaturschlüsselinhaber. Geschützt wird dadurch der regelmäßig beweispflichtige Erklärungsempfänger vor unbegründeten Einwänden des Signaturverwenders, das übermittelte Dokument stamme nicht von ihm, es sei manipuliert worden oder ähnlichem. ${ }^{362}$ Diesen prima-facie-Beweis kann der Signaturverwender nur dadurch erschüttern, dass er Tatsachen vorträgt, die ernstliche Zweifel an der Abgabe der Erklärung durch den Signaturschlüs-

358 Roßnagel, MMR 2002, 217; Engel-Flechsig, in: Moritz/Dreier, F Rn. 153; Spindler/Schuster/Spindler/Weber, \126a BGB Rn. 4 f.; ferner Koch, Internet-Recht, \3 S. 124.

359 S. Engel-Flecbsig, in: Moritz/Dreier, F Rn. 153.

360 S. \492 Abs. 1 S. 2 BGB (Verbraucherdarlehensvertrag), \500 BGB (Finanzierungsleasing), \501 BGB (Teilzahlungsgeschäft) und \766 S. 2 BGB (Bürgschaft).

361 \550 BGB, s. auch Palandt/Weidenkaff; \550 Rn. 8; BGH, NJW 2004, 2963 (2963).

362 Musielak/Huber, \371a ZPO Rn. 6; Hk-ZPO/Eichele, \371a Rn. 4; Roßnagel/Fischer-Dieskau, NJW 2006, 807. 
sel-Inhaber begründen. ${ }^{363}$ Der Empfänger der mittels Web Service übermittelten Willenserklärung hat dadurch eine gewisse Sicherheit, dass er sich auf den Erklärungsinhalt verlassen kann und später auch nicht in Beweisnöte gerät. Dies kann das Vertrauen beider Parteien in die Kommunikation via Web Services erheblich stärken und stellt insofern einen starken Anreiz dar, das durch den Gesetzgeber als weitgehend sicher eingestufte Sicherungsmittel der qualifizierten oder akkreditierten elektronischen Signatur zu verwenden. ${ }^{364}$

cc) Resümee

Sollen beim Vertragsschluss Authentizität und Integrität gewährleistet und dadurch das Vertrauen in die Web Service-Nutzung gestärkt werden, ist zu raten, qualifizierte, im Sinne des $₫ 2 \mathrm{Nr} .3 \mathrm{SigG}$, oder akkreditierte, nach $\rrbracket \S 2$ Nr. 15, 15 SigG, elektronische Signaturen bei der Übermittlung zu verwenden. ${ }^{365}$ Dies würde die Missbrauchsgefahr stark eindämmen und darüber hinaus dem Empfänger ein im Prozess hilfreiches Beweismittel an die Hand geben.

Durch die Spezifikation XML Signature ${ }^{366}$ lassen sich solche elektronischen Signaturen auch innerhalb von den bei der Web Service-Kommunikation verwendeten XML-Dokumenten implementieren und ermöglichen so die Realisierung des eingangs favorisierten Ende-zu-Ende-Sicherheitskonzepts auf Anwendungsebene. ${ }^{367}$

\section{Vertragliche Pflicten}

Aus den unterschiedlichen Vertragsverhältnissen im Rahmen der Serviceorientierten Architektur ergeben sich in den jeweiligen Vertragsbeziehungen regelmäßig unterschiedliche Leistungspflichten. Derartige Leistungspflichten lassen sich in Haupt- und Nebenleistungspflichten untergliedern. Unter Hauptleis-

363 Roßnagel/Fischer-Dieskau, NJW 2006, 807; Fischer-Dieskau/Roßnagel/Steidle, MMR 2004, 452; Ernst, MDR 2003, 1092; Musielak/Huber, \371a ZPO Rn. 10; Spindler/Schmitz/Geis/Geis, Einf. SigG Rn. 37; Spindler/Schuster/Gramlich, \1 SigG Rn. 17; BGH, NJW 1991, 230 (231); NJW 2004, 3623 (3624).

364 Kritisch Armgardt/Spalka, K\&R 2007, 27 ff., die die qualifizierte elektronische Signatur als wesentlich unsicherer sehen, als vom Gesetzgeber angenommen und daher eine Berücksichtigung dieser Unsicherheit in der Auslegung des $\ 371$ a Abs. 1 S. 2 ZPO fordern.

365 So auch Porkert, S. 14.

366 S. http://www.w3.org/Signature/ <25.11.2009>.

367 Ausführlich zur Technik von XML Signature Burghardt, S. 94 ff. u. Melzer, S. 200 ff. 
tungspflichten versteht man diejenigen Pflichten, mit denen ein Schuldverhältnis als solches zur Entstehung gelangt und durch die es in seiner besonderen Eigenart gekennzeichnet wird. ${ }^{368}$ Dagegen dienen Nebenleistungspflichten der Vorbereitung, Durchführung und Sicherung der Hauptleistung. ${ }^{369}$

\section{a) Web Service-Anbieter - Web Service-Nutzer}

Haben Web Service-Anbieter und Web Service-Nutzer einen Vertrag über die Nutzung des Web Service geschlossen, so sind die Hauptleistungspflichten des Web Service-Anbieters entsprechend der unterschiedlichen vertraglichen Elemente $^{370}$ in dem Bereithalten der öffentlichen Schnittstelle, dem Zurverfügungstellen eines funktionierenden Dienstes sowie der Ermöglichung der Dienstenutzung zu sehen. ${ }^{371}$ Darüber hinaus obliegen ihm auch die Sicherstellung der weiteren Verfügbarkeit sowie die Verwaltung des Web Service. ${ }^{372}$ Unter die Verwaltung des Web Service fällt neben der Fehlerprüfung auch eine mögliche Weiterentwicklung. ${ }^{373}$ Gegebenenfalls sollte der Web Service-Anbieter den Web Service-Nutzer auch über eine eventuelle Rücknahme des Web Service informieren.

Das Bereithalten der öffentlichen Schnittstelle, das Zurverfügungstellen eines funktionierenden Dienstes, die Ermöglichung der Dienstenutzung sowie naturgemäß die Sicherstellung der weiteren Verfügbarkeit sind den gemischttypischen Vertrag der Web Service-Nutzung kennzeichnende, essentielle Elemente ${ }^{374}$ und daher als Hauptleistungspflichten zu qualifizieren, was wiederum bedeutet, dass der Web Service-Nutzer diesbezüglich einen Erfüllungsanspruch hat. Die Verwaltung der Web Services dagegen dient der Förderung der Nutzung der Web Services und stellt insofern eine Nebenleistungspflicht dar. Ein Erfüllungsanspruch auf Verwaltung der Web Services besteht nicht,

368 Staudinger/Olžen, \241 Rn. 147; Palandt/Heinrichs, \241 Rn. 5. Zum Spezialfall der kaufrechtlichen Hauptleistungspflichten Huber, AcP 177 (1977), 296; ferner Medicus, Rn. 206.

369 Palandt/Heinrichs, \241 Rn. 5; Staudinger/Olzen, \241 Rn. 152; Erman/Westermann, Einl. \ 241 Rn. 7.

370 S. dazu A.III.1.c) Gemischttypischer Vertrag?.

371 I.d.S., wenn auch nicht derart differenziert: Melzer, S. 14; Burghart, S. 19.

372 Melzer, S. 14; Burghart, S. 19.

373 Melzer, S. 14.

374 S. dazu A.III.1.c) Gemischttypischer Vertrag?. 
indes kommen bei deren Verletzung in der Regel Schadensersatzansprüche in Betracht. 375

Den Web Service-Anbieter dagegen treffen jenseits der üblichen Pflichten, wie die Entrichtung des Entgelts, sofern es sich um einen entgeltlichen Vertrag handelt, sowie den sonstigen Schutzpflichten, keine besonderen Pflichten.

b) Web Service-Anbieter - Web Service-Vermittler

Im Verhältnis zum Dienstemakler liegt die Hauptleistungspflicht, sofern Gegenstand des Vertrags, in der Entrichtung des Werklohns für die Eintragung im UDDI. Darüber hinaus trifft ihn als Nebenleistung die Pflicht, dem Web Service-Vermittler umgehend mitzuteilen, wenn er seinen Dienst zurückgezogen hat, so dass dieser den Eintrag im UDDI umgehend entfernen kann.

Der Web Service-Vermittler indes muss neben seiner Hauptleistungspflicht, der Veröffentlichung der Dienstebeschreibung, lediglich die üblichen Schutzpflichten im Sinne des \241 Abs. 2 BGB erfüllen.

\section{c) Web Service-Nutzer - Dienstemakler}

Auch dem Web Service-Nutzer obliegt, je nach Vertragstypus, zuvörderst die Entrichtung des Entgelts für die Suchmöglichkeit. Der Web ServiceVermittler ist neben der Ermöglichung der Suche nach entsprechenden Diensten zudem gehalten, dem Web Service-Nutzer die entsprechende Dienstebeschreibung des Web Service-Anbieters zukommen zu lassen. Darüber hinaus ist er verpflichtet das UDDI aktuell zu halten.

\section{Einberiebung Allgemeiner Geschäftsbedingungen}

Die Nutzung von Web Services wird im Rahmen der Allgemeinen Geschäftsbedingungen regelmäßig an bestimmte Auflagen des Web Service-Anbieters, wie möglichen Haftungsbeschränkungen, geknüpft sein. Daher stellt sich die Frage zu welchem Zeitpunkt Web Service-Anbieter und gegebenenfalls Web Service-Nutzer ihre Allgemeinen Geschäftsbedingungen wirksam in den Vertrag einbeziehen können. Diese Frage ist ein klassisches Problem der Umsetzung der Serviceorientierten Architektur, die den vollautomatisierten Vertragsschluss mit sich bringt. Außerhalb der Serviceorientierten Architektur können die Allgemeinen Geschäftsbedingungen regelmäßig im Rahmen der persönli-

375 MünchKommBGB/Kramer, Bd. 2, \241 Rn. 18; Bamberger/Roth/Grüneberg/Sutschet, \241 Rn. 43. Mehr zu den Ansprüchen bei Leistungsstörungen unter A.III.7.a)ii) Web Service-Nutzer gegenüber Web Service-Anbieter. 
chen vorvertraglichen Gespräche ausgetauscht oder gegebenenfalls sogar ausgehandelt werden, so dass in diesen Fällen die Wirksamkeit der Einbeziehung keine neuen Fragen aufwirft.

Die Besonderheit bei der Serviceorientierten Architektur liegt darin, dass Web Service-Anbieter und -nutzer vor Vertragsschluss keinerlei Kontakt miteinander haben. Der Web Service-Anbieter registriert den Dienst im UDDI und stellt dort seine Dienstebeschreibung bereit, anhand dieser implementiert der Web Service-Nutzer den Web Service in seine Plattform und nutzt ihn, erst dadurch kommt der Vertrag zustande. ${ }^{376}$ Für die wirksame Einbeziehung von Allgemeinen Geschäftsbedingungen gelten im Rahmen des $₫ 305$ Abs. 2 BGB allerdings besondere Voraussetzungen, die der Verwender erfüllen muss.

\section{a) Wirksame Einbeziehung von AGB}

Erforderlich ist zum einen, dass der Verwender bei Vertragsabschluss ausdrücklich auf die Allgemeinen Geschäftsbedingungen hinweist, \ 305 Abs. 2 Nr. 1 BGB, und der anderen Partei eine zumutbare Möglichkeit der Kenntnisnahme verschafft, $\int 305$ Abs. 2 Nr. 2 BGB. Das bedeutet nach der mittlerweile herrschenden Meinung für Vertragsschlüsse im Internet, dass ein deutlich sichtbarer Hyperlink auf die Allgemeinen Geschäftsbedingungen bei Vertragsschluss erfolgt, wobei es genügt, dass die Allgemeinen Geschäftsbedingungen am Bildschirm dargestellt werden und die Möglichkeit der wiedergabefähigen Speicherbarkeit eingeräumt wird, siehe $\int 312$ e Abs. 1 S. 1 Nr. 4 BGB. ${ }^{377}$ Mit dem Kriterium „bei Vertragsschluss“ ist der unmittelbare zeitliche Zusammenhang von Angebot und Annahme gemeint, ${ }^{378}$ das heißt der Einbeziehungswille muss spätestens im Augenblick des Vertragsschlusses zum Ausdruck gebracht werden. ${ }^{379}$

Die Pflichten nach $₫ 305$ Abs. 2 BGB gelten allerdings nur gegenüber Verbrauchern, nicht aber wenn die Allgemeinen Geschäftsbedingungen gegenüber Unternehmern gestellt werden, siehe $₫ 310$ Abs. 1 S. 1 BGB. Von dieser „Pri-

376 S. A.III.2.a)aa) Web Service-Anbieter - Web Service-Nutzer.

377 BGH, NJW 2006, 2976 (2977); OLG Hamburg, MMR 2002, 677 (678); von Westphalen, NJW 2007, 2229; Wiebe, in: Spindler/Wiebe, Kap. 4 Rn. 120; MünchKommBGB/Basedow, Bd. 2, \305 Rn. 65; Ulmer/Brandner/Hensen/Ulmer, \305 Rn. 135b; Spindler, in: Spindler, Vertragsrecht Internet-Provider, Teil IV Rn. 34 ff.; Spindler/Schuster/Spindler/Weber, \312e BGB Rn. 11, 16; Borges, S. 275 f.

378 Borges, S. 277.

379 Staudinger/Schlosser, \305 Rn. 114. 
vilegierung“ bei der Stellung von Allgemeinen Geschäftsbedingungen gegenüber Unternehmern unberührt bleibt aber $\ 305$ Abs. 1 BGB, der unabhängig von den jeweiligen persönlichen Eigenschaften von Verwender und Empfänger gilt. ${ }^{380}$ Die Einbeziehung von Allgemeinen Geschäftsbedingungen gegenüber Unternehmern erfolgt derart, dass der Verwender in seinem Vertragsangebot auf die Allgemeinen Geschäftsbedingungen hinweist und der andere Teil dieses Angebot annimmt, ohne der Einbeziehung zu widersprechen. 381 Bei diesem Hinweis genügt jede stillschweigend erklärte Willensübereinstimmung, die Voraussetzungen der $\$ ₫ 305$ Abs. 2 und 3 BGB brauchen nicht erfüllt zu werden. ${ }^{382}$ Dies zeigt, dass auch bei der Verwendung von Allgemeinen Geschäftsbedingungen gegenüber Verbrauchern die Stellung der Allgemeinen Geschäftsbedingungen de facto, wenn auch abgemildert in Form eines Hinweises auf diese, ganz im Sinne des Wortlauts von $\ 305$ Abs. 1 S. 1 BGB bei Vertragsschluss erfolgen muss. Erleichtert wird daher nicht die zeitliche Komponente, das „Wann“ der Stellung, sondern lediglich das „Wie“.

\section{b) AGB des Web Service-Anbieters}

Der erste Kontakt des Web Service-Anbieters zum Web Service-Nutzer ist die Übermittlung der gewünschten Informationen mittels des Web Service. Zu diesem Zeitpunkt ist indes bereits ein wirksamer Vertrag zwischen den beiden zustande gekommen. Stellt der Web Service-Anbieter die Allgemeinen Geschäftsbedingungen, insbesondere wenn es sich um einen Verbraucher als Web Service-Nutzer handelt, erst zu diesem Zeitpunkt, so liegt keine wirksame Einbeziehung mehr vor. Die Stellung muss daher früher erfolgen. Sinnvoll erscheint dabei nur eine Stellung im UDDI zusammen mit der Dienstebeschreibung, denn das ist die einzige Möglichkeit, dem Web ServiceNutzer die Allgemeinen Geschäftsbedingungen noch vor Vertragsschluss zu stellen, um die wirksame Einbeziehung zu ermöglichen. ${ }^{383}$ Dabei genügt auch ein Hyperlink auf die Allgemeinen Geschäftsbedingungen, ${ }^{384}$ die dann auf dem Server des Web Service-Anbieters platziert sein sollten, da der Web Service-Vermittler wohl kaum gesonderten Speicherplatz zum Ablegen der ver-

380 Ulmer/Brandner/Hensen/Ulmer, \305 Rn. 83.

381 BGH, NJW 1992, 1232; Ulmer/Brandner/Hensen/Ulmer, $\int 305$ Rn. 170; BGH, BB 1976, 1289; WM 1977, 1353 (1354).

382 BGH, NJW 1985, 1838 (1839); NJW 1992, 1232; Palandt/Grüneberg, §310 Rn. 4; Ulmer/Brandner/Hensen/Ulmer, $\int 310$ BGB Rn. 29.

383 So Koch, ITRB 2007, 72.

384 Spindler, in: Spindler, Vertragsrecht Internet-Provider, Teil IV Rn. 36. 
schiedenen Allgemeinen Geschäftsbedingungen des Anbieters gewähren wird, sofern dem Web Service-Nutzer ermöglicht wird, diese in wiedergabefähiger Form zu speichern. Der Hyperlink sollte, um dem Erfordernis der deutlichen Wahrnehmbarkeit zu genügen, in der Dienstebeschreibung platziert werden, denn die Informationen innerhalb der Dienstebeschreibung muss der Web Service-Nutzer zwangsläufig wahrnehmen, um den Web Service nutzen zu können. Ist es dem Web Service-Anbieter nicht möglich seine Allgemeinen Geschäftsbedingungen über einen Hyperlink in wiedergabefähiger und speicherbarer Form zu hinterlegen, so ist ihm $\mathrm{zu}$ raten, diese an die Dienstebeschreibung unübersehbar gekoppelt direkt im UDDI bereit zu halten. Diese Variante könnte unter Umständen ebenfalls an dem vorgesehenen Speicherplatz des Web Service-Vermittlers scheitern: da sich das UDDI als eine Art „Gelbe Seiten“, dementsprechend als ein Branchenverzeichnis, versteht, wird es nicht im Sinne des Web Service-Vermittlers sein, für Informationen, die für sein Vertragsverhältnis mit dem Web Service-Anbieter und somit die Publikation des Dienstes überflüssig sind, größere Speicherkapazitäten zur Verfügung zu stellen, die ihm nur zusätzliche Kosten bescheren. Insofern wird es darauf hinauslaufen, dass der Web Service-Anbieter auf die Variante des Hyperlinks zurückgreifen muss.

Das gerade Gesagte gilt in dieser strikten Form indes nur, wenn der AGBEmpfänger, der Web Service-Nutzer, ein Verbraucher ist. Im Rahmen der Serviceorientierten Architektur besteht aber die Besonderheit, dass der Web Service-Nutzer regelmäßig, wie das Reiseportalbeispiel zeigt, ebenfalls als Unternehmer den Web Service in sein Angebot integriert. Insofern muss nicht notwendig bereits ein Hyperlink auf wiedergabe- und speicherfähige Allgemeine Geschäftsbedingungen in der Dienstebeschreibung im UDDI erfolgen, ausreichend ist bereits, dass innerhalb der Dienstebeschreibung, als Vertragsangebot, ein Hinweis auf die Allgemeinen Geschäftsbedingungen erfolgt. Deswegen ist auch im Falle der Stellung gegenüber einem Unternehmer das UDDI der Dreh- und Angelpunkt, wenn auch auf den Hyperlink verzichtet werden kann. Da es sich aber bei dem Einstellen der Dienstebeschreibung im UDDI um ein Angebot ad incertas personas handelt und aufgrund der automatisierten Abläufe keinerlei Rückmeldung an den Web Service-Anbieter darüber erfolgt, ${ }^{385}$ mit wem dieser es künftig zu tun haben wird, kann nicht ausgeschlossen werden, dass nicht doch ausnahmsweise ein Verbraucher sein Angebot annimmt. Daher ist zu empfehlen, dass der Web Service-Anbieter seine Allgemeinen Geschäftsbedingungen grundsätzlich bereits im UDDI 
durch einen deutlichen Hyperlink, der auf die Allgemeinen Geschäftsbedingungen in wiedergabe- und speicherfähiger Form verweist, in der Dienstebeschreibung stellt. 386 Wird der Web Service nicht im Rahmen einer Serviceorientierten Architektur angeboten und fehlt es somit an der Zwischenstation des Web Service-Vermittlers, so bereitet die Stellung der Allgemeinen Geschäftsbedingungen keine weiteren Probleme, da der Web ServiceAnbieter einfach einen deutlichen Link auf seiner Webpräsenz, direkt an der Stelle, wo die Nutzung des Web Service erfolgen kann, auf seine Allgemeinen Geschäftsbedingungen legen kann, wie auch bei herkömmlichen elektronischen Marktplätzen.

\section{c) $\mathrm{AGB}$ des Web Service-Nutzers}

Der Web Service-Nutzer nimmt erst durch die Web Service-Nutzung Kontakt mit dem Web Service-Anbieter auf. Darin liegt zugleich die Annahme des Angebots und somit der Vertragsschluss. Will der Web Service-Nutzer daher selber auch Allgemeine Geschäftsbedingungen verwenden, so müsste er bei der erstmaligen Web Service-Nutzung, sprich bei Vertragsschluss, deutlich auf diese hinweisen, um die zeitliche Komponente der Einbeziehungsvoraussetzung zu erfüllen. Problematisch ist daran aber, dass Web Services nach einem vollautomatisierten Art Frage-Antwort-Mechanismus funktionieren und agieren. Sofern es dem Web Service-Nutzer also überhaupt technisch möglich wäre, den Hinweis auf seine Allgemeinen Geschäftsbedingungen in die erstmalige Nutzung zu integrieren, so wird dies den gesetzlichen Erfordernissen an einen deutlichen Hinweis kaum genügen. Die Anfrage des Web ServiceNutzers wird nämlich nur automatisiert erfüllt, indem durch den Web Service die gewünschten Informationen übermittelt werden. Insofern wird schon die Integration des Hinweises in die erstmalige Dienstenutzung in der Regel für den Web Service-Nutzer keine wirksame Einbeziehung der Allgemeinen Geschäftsbedingungen darstellen. ${ }^{387}$

Darüber hinaus ist aber auch das Kriterium „bei Vertragsschluss“ nicht erfüllt. Der räumliche und zeitliche Zusammenhang muss so verstanden werden, dass dem Empfänger noch die Möglichkeit bleibt, den Vertrag zu den Bedingungen des Verwenders abzulehnen. Andernfalls bleibt eine derartige Regelung zweck-

386 So auch Koch, ITRB 2007, 72, allerdings ohne Begründung und nur vom Stellen im UDDI sprechend.

387 Ausnahmen wären freilich denkbar, wenn der Diensteanbieter dem Web Service-Nutzer eine Einbeziehung seiner AGB auf diese Weise gestatten würde, wobei dann auch wieder fraglich ist, wann diese Gestattung stattfinden soll. 
los. Insofern genügen erst nach oder mit Vertragsschluss zugesandte (Briefpost) oder zugefaxte Allgemeine Geschäftsbedingungen nicht den Anforderungen an eine wirksame Einbeziehung. ${ }^{388}$ Nichts anderes kann im Bereich der Kommunikation via Web Services gelten: integriert der Web ServiceNutzer seine Allgemeinen Geschäftsbedingungen innerhalb der erstmaligen Web Service-Nutzung, so ist dies nichts anderes als die Zusendung mittels Post oder Fax zeitgleich mit dem Vertragsschluss, so das von einer Einbeziehung keine Rede sein kann. Eine derartige Einbindung würde sich allenfalls als Angebot auf Änderung des Vertrags verstehen lassen. ${ }^{389}$ Daneben bleibt dem Web Service-Nutzer lediglich eine Einbeziehung durch persönlichen Kontakt mit dem Web Service-Anbieter vor Vertragsschluss.

\section{Irrtumslebre}

Werden im Rahmen von elektronischen Vertragsschlüssen Web Services zur Übertragung von Willenserklärungen eingesetzt, so können zahlreiche Fehler auftreten. Solche Fehler lassen sich unterteilen in Fehler, die auf die Technik Web Service zurückzuführen sind und solche, die auf menschlichen Versagen beruhen. Fehler die auf der fehlerhaften Funktionsweise von Web Services basieren, lassen sich wiederum untergliedern in Übermittlungs- und Systemfehler. Auf menschlichem „Versagen“ beruhende Fehler basieren regelmäßig auf Tippfehlern. Diese Fehler wirken sich auf die Willenserklärungen der Beteiligten der Serviceorientierten Architektur aus. Für alle Beteiligten ist es daher wichtig, zu erfahren, wie und ob sie sich von den für sie meist ungünstigen Erklärungen lösen können. Besonders relevant wird die rechtliche Beurteilung solcher Fehler, bei denen aufgrund des Fehlers ein Vertrag mit einem Endkunden (Dritter) über eine Sache geschlossen wurde, die so nicht mehr oder grundsätzlich nicht verfügbar ist und damit für Web Service-Anbieter und Web Service-Nutzer nicht beschaffbar ist.

\section{a) Anfechtung}

Wird durch den Web Service eine fehlerhafte Erklärung abgegeben, der ein Irrtum im Sinne der $\iint 119$ ff. BGB zugrunde liegt, kann der Web ServiceNutzer in einer Serviceorientierten Architektur grundsätzlich nach $\int 142$ BGB anfechten und dadurch die Willenserklärung ex tunc vernichten. Für eine wirksame Anfechtung des Vertrags ist aber auch das tatsächliche Vorliegen

388 Spindler, in: Spindler, Vertragsrecht Internet-Provider, Teil IV Rn. 38.

389 Spindler, in: Spindler, Vertragsrecht Internet-Provider, Teil IV Rn. 38; Ulmer/Brandner/Hensen/Ulmer, \305 BGB Rn. 157; i.d.S. auch BGH, NJW 1983, 816 (817). 
eines anfechtbaren Irrtums, der auf einer fehlerhaften Willenserklärung beruht, Voraussetzung. Aufgrund der vollautomatisierten Funktionsweise von Web Services, erfolgt der Fehler allerdings nicht dadurch, dass dem Web ServiceNutzer selbst ein Fehler im Angebot unterläuft. Der Fehler beruht vielmehr auf einem technischen Fehler. Allerdings haben bereits die vorangegangenen Untersuchungen gezeigt, dass die Zusammenstellung durch den Web Service, obschon es im Zeitpunkt der Erzeugung selbst an einer menschlichen Handlung mangelt, eine Willenserklärung des Web Service-Nutzers darstellt - denn sie ist letztlich auf dessen Willen zurückzuführen. ${ }^{390}$ Die Zusammenstellung durch den Web Service ist folglich grundsätzlich anfechtungsfähig. ${ }^{391}$

Ein Irrtum liegt im Falle der $\$ ₫ 119,120$ BGB vor, wenn Wille und Erklärung unbewusst auseinander fallen. ${ }^{392}$ Dabei ist zwischen verschiedenen Irrtumsarten zu differenzieren, nach Irrtümern in der Willensbildung und solchen in der Willensumsetzung. Während Fehler in der Willensumsetzung grundsätzlich anfechtbar sind, sind es Fehler in der Willensbildung (als Motivirrtümer), mit Ausnahme des $\int 119$ Abs. 2 BGB, grundsätzlich nicht. ${ }^{393}$ Liegen Willensmängel durch den Einsatz von Web Services vor, so muss man, wie üblicherweise bei allen elektronisch abgeschlossenen Verträgen, zwischen Eingabefehlern, fehlerhaftem Datenmaterial, Übermittlungsfehlern und Systemfehlern als möglichen Irrtumsvarianten unterscheiden. ${ }^{394}$

\section{aa) Eingabefehler}

Weicht der äußere Erklärungstatbestand vom inneren Willen des Erklärenden ab, wie bei Eingabefehlern durch Vertippen oder Verklicken, so handelt es

390 S. bereits A.III.2.a)bb) Angebot und Annahme.

391 S. BGH, NJW 2005, 976 (977) = JZ 2005, 791 m. Anm. Spindler, OLG Hamm, NJW 2004, 2601; OLG Frankfurt, MMR 2003, 405 (406 f.); Hoffmann, NJW 2003, 2577.

392 Staudinger/Singer, $\int 119$ Rn. 6; Spindler/Schuster/Spindler/Weber, $\int \mathbb{S} 119,120$ BGB Rn. 1; MünchKommBGB/Kramer, Bd. 1/1, Vorb. \$S 116 ff. Rn. 7 ff.; Flume, AT 2, \$21/1 S. 415 f.

393 Zur Unbeachtlichkeit des Motivirrtums (Fehler in der Willensbildung) ausführlich Flume, AT 2, \ 25 S. 491 ff.; Erman/Palm, Vor \$116 Rn. 22 ff.

394 S. Mehrings, in: Hoeren/Sieber, Hdb. MultimediaR, Teil 13.1 Rn. 226; Wiebe, in: Spindler/Wiebe, Kap. 4 Rn. 70 ff.; so auch schon Köbler, AcP 182 (1982), 134, allerdings noch mit der Ansicht „Maschinenfehler“ (Systemfehler) seien nach dem Stand der Technik so gut wie ausgeschlossen. 
sich regelmäßig um einen anfechtbaren Erklärungsirrtum nach $\int 119$ Abs. 1, 2. Alt. BGB. 395

\section{Irrtum des Web Service-Anbieters}

Beruht der Irrtum auf einer fehlerhaften Eingabe von Daten, etwa von Preisen, in die Datenbank, erfolgt der Irrtum nicht bei Abgabe der Willenserklärung, wie $\int 119$ Abs. 1 BGB fordert, sondern bereits im Vorfeld. Fehler in der Willensbildung berechtigen aber grundsätzlich nicht zur Anfechtung. Zum Teil wird zwar angenommen, dass auch Übertragungsfehler bei der manuellen Dateneingabe zum Beispiel. in Datenbanken anfechtbare Irrtümer sein können, wenn sich der Fehler unmittelbar auf den Inhalt der Erklärung auswirkt. ${ }^{396}$ Die Grenzen, wann ein solcher Fehler sich aber tatsächlich unmittelbar auf den Inhalt der Erklärung auswirkt, erscheinen äußerst schwammig. So ist unklar, ob es dafür genügt, wenn das Datenbankmaterial lediglich einen Rechnungsposten bildet oder ob das Material ohne weitere Rechnungen unverändert in die Willenserklärung einfließen muss.

Daher erscheint die Anwendung dieser Ansicht auf Willensmängel durch die Verwendung von Web Services im Rahmen einer Serviceorientierten Architektur praktisch mangels Klarheit nicht brauchbar. Denn bei Zugrundelegung dieser Ansicht wäre eine Abgrenzung zum unbeachtlichen Motivirrtum kaum mehr möglich. Letztlich stellt das Vertrauen des Web Service-Nutzers in die Richtigkeit des Datenbankmaterials lediglich eine Motivation dar, auf der basierend der Web Service-Nutzer den Web Service eine Willenserklärung abgeben lässt. ${ }^{397}$ Im Übrigen stößt eine Erweiterung des anfechtbaren Irrtums nach $\int 119$ Abs. 1 BGB an die Grenzen des Wortlauts, der eindeutig einen Fehler „bei Abgabe der Willenserklärung“" verlangt. Die Einspeisung der für die Abgabe der Willenserklärung zugrunde gelegten Daten, auf welche der Web Service zugreift, gehört aber lediglich zur Willensbildung. Die so gespeicherten Daten stellen den Willen des Web Service-Nutzers dar, der durch die Übertragung mittels Web Service gegenüber dem Dritten geäußert wird. Zwar können dadurch zwischen Willen und Willensäußerung gewisse Zeiträume entstehen. Nicht erfolgt damit aber ein dem Irrtum immanentes unbewusstes Auseinan-

395 OLG Nürnberg, MMR 2003, 183 (184); OLG Hamm, NJW 1993, 2321; AG Lahr, NJW 2005, 991 (992); AG Fürth, Urt. v. 11.08.2009 - 360 C 2779/08, Tz. 25; Flume, AT 2, \23/2 S. 450 f.; Erman/Palm, \199 Rn. 33; MünchKommBGB/Kramer, Bd. 1/1, \119 Rn. 48.

396 OLG Hamm, NJW 1993, 2321; Palandt/Heinrichs/Ellenberger, \$119 Rn. 10; Mebrings, in: Hoeren/Sieber, Hdb. MultimediaR, Kap. 13.1 Rn. 229; Staudinger/Singer, \119 Rn. 36.

397 S. auch Köbler, AcP 182 (1982), 136. 
derfallen von Wille und Erklärung. ${ }^{398}$ Denn Wille und Erklärung stimmen überein, lediglich in der Willensbildung, der Dateneinspeisung unterläuft dem Web Service-Nutzer ein - rechtlich unbeachtlicher - Irrtum. ${ }^{399}$ Bei der Verwendung von Web Services zur Abgabe von Willenserklärungen wird daher keine Anfechtung wegen Eingabefehlern in Betracht kommen, da diese immer im Vorfeld erfolgen. 400

Tritt der Web Service-Nutzer als eine Art „All-Inclusive-Dienst“ auf, zum Beispiel als Reiseportal, so können durch das Anbieten von kombinierten Leistungen, die zwar vom Web Service-Anbieter erbracht werden, aber Vertragspartner des Dritten der Web Service-Nutzer ist, ${ }^{401}$ weitere Probleme entstehen. Denn anfechtbare Rechtsgeschäfte sind nach \143 Abs. 1, 2 BGB gegenüber dem Vertragspartner zu erklären. Zwar wird zwischen dem Web Service-Nutzer und dem eigentlichen Leistungserbringer, dem Web ServiceAnbieter, beispielsweise im Rahmen von Reiseverträgen von einem Vertrag zugunsten des Dritten ausgegangen, allerdings ist dabei ein Einwendungsdurchgriff regelmäßig stillschweigend abbedungen, 402 so dass es dem Web Service-Anbieter auch nicht möglich wäre, die Anfechtung gegenüber dem Dritten zu erklären. Würde man entgegen der hier vertretenen Auffassung einen anfechtbaren Irrtum aufgrund eines Eingabefehlers annehmen, wäre der Web Service-Anbieter lediglich zur Anfechtung gegenüber dem Web ServiceNutzer als seinem Vertragspartner berechtigt. Allerdings müsste er dann sofern vertragliche Vereinbarungen diesbezüglich fehlen - dem Web ServiceNutzer die Schadensdifferenz, die diesem durch die zu niedrige Preisangabe entstanden ist, gegebenenfalls neben etwaigen Ansprüchen wegen Vertragsverletzung nach $₫ 122$ Abs. 1 BGB ersetzen.

LG Frankfurt, NJW-RR 1997, 1273.

LG Frankfurt, NJW-RR 1997, 1273.

So auch LG Frankfurt, NJW-RR 1997, 1273; LG Köln, MMR 2003, 481 (482); AG Frankfurt, NJW-RR 1990, 116 (117); Spindler, Anm. zu BGH, JZ 2005, 791 ff., JZ 2005, 793 (794); Köbler, AcP 182 (1982), 136; Bamberger/Roth/Wendtland, \ 119 Rn. 29; a.A. Keller, K\&R 2005, 171 f., der zwar richtig darauf abstellt, dass die Software selbst keinen Willen hat, aber verkennt, dass nach dem Wortlaut des $\$ 119$ Abs. 1 BGB der Irrtum bei Abgabe der Willenserklärung erfolgen muss und diese Willensentäußerung durch Übermittlung kann eine Software sehr wohl vornehmen.

S. A.III.2.a)bb) Exkurs: Reisevertragsspezifische Besonderheiten.

S. A.III.2.a)bb) Exkurs: Reisevertragsspezifische Besonderheiten. 


\section{Irrtum des Dritten (Kunde)}

Unterläuft dem Kunden ein Irrtum, etwa ein Tippfehler bei der Suche nach Angeboten mittels Web Service, gibt er zumindest im Rahmen von komplexeren All-Inclusive-Diensten zunächst nur eine unverbindliche invitatio ad offerendum ab. Je nachdem wie der konkrete Service ausgestaltet ist, gibt der Web Service-Nutzer mittels des Web Service, der auf Grundlage der (fehlerhaften) Suchdaten des Kunden eine Zusammenstellung vornimmt, entweder ein Angebot ab, was der Kunde nur noch annehmen oder ablehnen kann, oder er fordert den Kunden auf Grundlage der Zusammenstellung auf, ein Angebot abzugeben, indem dieser einen „Buchen-“, „Kaufen-“ Button oder ähnliches drückt. ${ }^{403}$ Das Angebot wird dann wiederum nach bestimmten Überprüfungen, wie beispielsweise der Bonität des Kunden, durch den Web ServiceNutzer angenommen. Aber gleich durch welche Handlung Angebot und Annahme erfolgen, stellt jedenfalls die Eingabe der Suchdaten noch keine rechtsverbindliche Willenserklärung des Kunden dar, sondern vielmehr einen grundsätzlich unbeachtlichen Fehler im Vorfeld seiner Willenserklärung.

Allerdings fallen auch bei der Annahme des Angebots beziehungsweise der Abgabe des Angebots - und somit der Abgabe seiner verbindlichen Willenserklärung - Gewolltes und Erklärtes auseinander. Denn der Kunde will das Angebot $\mathrm{X}$ annehmen beziehungsweise abgeben, tatsächlich nimmt er aber das Angebot $\mathrm{Y}$ an beziehungsweise gibt es ab. Auf den Eingabefehler, der lediglich einen unbeachtlichen Irrtum in der Willensbildung darstellt, kommt es insofern gar nicht mehr an. Vielmehr handelt es sich hier um einen klassischen Inhaltsirrtum, der ihn zur Anfechtung gegenüber dem Web ServiceNutzer nach $\ 119$ Abs. 1, 1. Alt. BGB berechtigt.

Innerhalb anderer Geschäftsmodelle, bei denen bereits das Anbieten der Web Service-Nutzung ein Angebot des Web Service-Nutzers, weil der Dienst nicht an einen begrenzten Warenbestand geknüpft ist, und die Nutzung des Web Service eine Annahme durch den Kunden darstellt (oder auch umgekehrt), können freilich auch durch Tippfehler des Kunden bei Abgabe seines Angebots oder seiner Annahme anfechtbare Erklärungsirrtümer nach $\ 119$ Abs. 1 2. Alt BGB vorliegen.

Nach $\int 312$ e Abs. 1 S. 1 Nr. 1 BGB wird der Web Service-Nutzer aber auch verpflichtet, dem Kunden ein Mittel zur Verfügung zu stellen, womit dieser Eingabefehler vor Abgabe seiner verbindlichen Willenserklärung erkennen

403 S. dazu A.III.2.a)bb) Web Service-Nutzer - Dritter. 
und berichtigen kann, indem diese Korrekturmöglichkeit in den Bestell/Buchungsvorgang integriert wird. ${ }^{404}$ Stellt der Web Service-Nutzer dem Kunden keine entsprechende Korrekturmöglichkeit zur Verfügung, so ist der Vertrag zwar nicht unwirksam, jedoch erhält der Web Service-Nutzer im Falle einer Anfechtung durch den Kunden keinen Vertrauensschaden nach $\ 122$ Abs. 1 BGB. ${ }^{405}$ Darüber hinaus beginnt auch die Widerrufsfrist nach dem sowohl im B2B- als auch B2C-Bereich geltenden 406 \$312e Abs. 3 S. 2 BGB abweichend von $₫ 355$ Abs. 2 S. 1 BGB nicht vor Erfüllung der in $₫ 312 \mathrm{e}$ Abs. 1 S. 1 geregelten Pflichten, wodurch dem Kunden bei fehlender Korrekturmöglichkeit auch eine längere Widerrufsmöglichkeit gewährt wird.

Hat der Web Service-Nutzer indes eine entsprechende Korrekturmöglichkeit zur Verfügung gestellt, so lassen sich Eingabefehler dennoch nicht immer vermeiden. ${ }^{407}$ Die Anfechtung, bei deren Ausübung der Kunde dem Web Service-Nutzer den Vertrauensschaden nach $\int 122$ Abs. 1 BGB ersetzen muss, stellt aber gegenüber dem im elektronischen Geschäftsverkehr nach den \\355, 312d, 312e Abs. 3 S. 2 BGB bestehenden Widerrufsrecht die für den Kunden deutlich ungünstigere Alternative dar. Bemerkt der Kunde seinen Irrtum daher innerhalb der Widerrufsfrist, ${ }^{408}$ so ist ihm zu raten, den Widerruf anstelle der Anfechtung auszuüben.

bb) Fehlerhaftes Datenmaterial

Bei einer fehlerhaften Willenserklärung, die auf Grundlage von fehlerhaftem Datenmaterial zustande kommt, liegt die Problematik darin, dass der Fehler im Datenmaterial, der letztlich zu der fehlerhaften Willenserklärung führte, der Willenserklärung vorgelagert ist. ${ }^{409}$ Es handelt sich dabei lediglich um einen Fehler in der Willensbildung, der - wie auch der Eingabefehler - nicht zur

404 S. dazu ausführlich Klimke, CR 2005, 583; MünchKommBGB/Wendehorst, Bd. 2, \312e Rn. 63 ff.; Bamberger/Roth/Masuch, \$312e Rn. 21.

405 Begr. RegE BT-Drs. 14/6040, S. 173; Klimke, CR 2005, 590; Bamberger/Roth/Masuch, \312e Rn. 31.

406 MünchKommBGB/Wendehorst, Bd. 2, \312e Rn. 13; Bamberger/Roth/Masuch, \312e Rn. 3; Hassemer, MMR 2001, 635; Leverenz, VersR 2003, 699.

407 So auch Härting, Rn. 212.

408 Zur Länge der Widerrufsfrist ausführlich Dietrich/Hofmann, CR 2007, 318 ff.; Bucbmann, K\&R 2007, 14 ff.; speziell bei Internetauktionen: Spindler/Nink, DRiZ 2007, 197.

409 LG Köln, MMR 2003, 481 (482); Köbler, AcP 182 (1982), 135; Mehrings, in: Hoeren/Sieber, Hdb. MultimediaR, Teil 13.1 Rn. 240; Wiebe, in: Spindler/Wiebe, Kap. 4 Rn. 70; Spindler, Anm. zu BGH, JZ 2005, 791 ff., JZ 2005, 793 (794). 
Anfechtung berechtigt, denn letztlich beruht der Fehler im Datenmaterial nur auf einem Eingabefehler.

cc) Übermittlungsfehler und Systemfehler

Oftmals wird zwischen Fehlern in Hard- und Software, so genannten Systemfehlern, und Übermittlungsfehlern unterschieden. Während Erstere lediglich als unbeachtliche Motivirrtümer eingeordnet werden, ${ }^{410}$ gelten Letztere als beachtliche Übermittlungsirrtümer nach $\ 120$, 2. Alt. BGB. ${ }^{411}$ Bei genauerer Betrachtung der beiden Fehlerkategorien ergeben sich jedoch zahlreiche Fragen und Überschneidungen.

Nach $\int 120$ BGB liegt ein Übermittlungsfehler nur dann vor, wenn sich der Erklärende für die Übermittlung seiner Willenserklärung einer Person oder Einrichtung bedient. Dieser Fehler wird dem Erklärungsirrtum nach \119 Abs. 1, 2. Alt. BGB gleichgestellt und das Übermittlungsrisiko dem Erklärenden aufgebürdet. ${ }^{412}$ Unter den Begriff der Einrichtung in $\$ 120,2$. Alt. BGB fällt auch die Einschaltung verschiedener Dienste, wie Netzbetreiber und Internetserviceprovider. ${ }^{413}$ Entscheidend ist daher, dass sich der Erklärende eines Dritten bedient - sei es Mensch oder Maschine - auf den er keinen Einfluss hat. Der Web Service-Anbieter mit Hilfe dessen Web Service Informationen übermittelt werden, kann grundsätzlich ein solcher Dritter sein, dessen sich der Web Service-Nutzer zum Erhalt von Informationen bedient. Wird indes mit Hilfe

410 BGH, NJW 1998, 3192 (3193); Wiebe, in: Spindler/Wiebe, Kap. 4 Rn. 70; Wiebe, in: Gounalakis, Rhb. Electronic Business, $\ 15$ Rn. 62; Mehrings, in: Hoeren/Sieber, Hdb. MultimediaR, Teil 13.1 Rn. 244; Härting, Rn. 219; Larenz/Wolf, AT, \ 36 Rn. 19; Köbler, AcP 182 (1982), 135; z.T. a.A. Schmidt, AcP 166 (1966), 21.

411 So die ganz h.M.: OLG Hamm, NJW 2004, 2601 - karstadt.de; OLG Frankfurt, MMR 2003, 405 (406 f.); MünchKommBGB/Kramer, Bd. 1/1, \$120 Rn. 3; Spindler/Schuster/Spindler/Weber, IS 119, 120 Rn. 7; Bamberger/Roth/Wendtland, \120 Rn. 2; Mehrings, in: Hoeren/Sieber, Hdb. MultimediaR, Teil 13.1 Rn. 245; Wiebe, in: Spindler/Wiebe, Kap. 4 Rn. 72; Wiebe, S. 376 f.; Härting, Rn. 221.

412 Palandt/Heinrichs/Ellenberger, $\mathbb{\int 1 2 0}$ Rn. $\quad 1 ; \quad$ Staudinger/Singer, $\$ 120 \quad$ Rn. 1; MünchKommBGB/Kramer, Bd. 1/1, \120 Rn. 1; Bamberger/Roth/Wendtland, \120 Rn. 1; Flume, AT 2, \23/3, S. 456; Larenף/Wolf, AT, \36 Rn. 14 ff.

413 BGH, JZ 2005, 791 (792) m. Anm. Spindler; OLG Frankfurt, MMR 2003, 405 (406); zustimmend Dümig, Kurzkommentar zu OLG Frankfurt/M., MMR 2003, 405 ff., EWiR 2003, 953 (954); Wiebe, in: Gounalakis, Rhb. Electronic Business, \15 Rn. 73; Mehrings, in: Hoeren/Sieber, Hdb. MultimediaR, Teil 13.1 Rn. 245; Palandt/Heinricbs/Ellenberger, $\int 120$ Rn. 2; Bamberger/Roth/Wendtland, $\int 120$ Rn. 2; MünchKommBGB/Kramer, Bd. 1/1, \120 Rn. 3; Staudinger/Singer, $\int 120$ Rn. 5; Larenz/Wolf, AT, $\ 36$ Rn. 14; Keller, K\&R 2005, 172; für Web Services auch Spindler, Anm. zu BGH, JZ 2005, 791 ff., JZ 2005, 793 (795). 
eigener technischer Einrichtungen des Erklärenden fehlerhaft übermittelt, so kann $\ 120$, 2. Alt. BGB schon vom Wortlaut keine Anwendung mehr finden, ${ }^{414}$ so dass der Web Service-Anbieter sich gegenüber dem Web ServiceNutzer nicht auf einen Übermittlungsfehler berufen kann. Übertragen auf die Serviceorientierte Architektur mit Web Services bedeutet dies, dass wenn der Web Service-Nutzer entgegen der grundsätzlichen Konstellation der Serviceorientierten Architektur Informationen mit eigenen, ihm gehörenden Web Services übermittelt, $\int 120$, 2. Alt. BGB keine Anwendung findet.

Werden tatsächlich fremde technische Einrichtungen verwendet, die eine fehlerhafte Übermittlung verursachen, so ist $₫ 120$ BGB für den Bereich des Verlassens der Erklärung des Absenders bis zum Erreichen des Empfängers anzuwenden. ${ }^{415}$ Erfolgt der Irrtum aber bereits vor der Übermittlung, so kann kein Übermittlungsirrtum mehr vorliegen, denn die Übermittlung selbst erfolgt einwandfrei, es wird lediglich ein vorher erfolgter Fehler falsch weiter übermittelt. 416

Wendet man diese Grundsätze auf Web Services im Rahmen der Serviceorientierten Architektur an, ergibt sich folgendes Bild: im Rahmen der Serviceorientierten Architektur bedient sich der Nutzer des Web Service des Anbieters und somit einer fremden Einrichtung zur Abgabe seiner Erklärung (beispielsweise ein Angebot über eine individualisierte Pauschalreise). Der Web ServiceNutzer integriert den Web Service dabei regelmäßig derart für seine Zwecke, dass der Vertragsschluss allein zwischen ihm und dem Kunden, nicht aber zwischen Web Service-Anbieter und Kunden zustande kommt. Daher käme ein anfechtbarer Übermittlungsirrtum bei entsprechenden Fehlern in Betracht. ${ }^{417}$ Aufgrund der verteilten Struktur der Nutzung von Web Services im Rahmen der Serviceorientierten Architektur wirft dieses Ergebnis aber Zweifel auf. Denn die Erklärung wird nicht vom Web Service des Anbieters direkt an den Dritten (beziehungsweise Kunden) abgegeben, sondern technisch vielmehr zunächst an den Web Service-Nutzer übermittelt, der den Web Service

414 So für den gegenteiligen Fall auch OLG Frankfurt, MMR 2003, 405 (406); Keller, K\&R 2005, 172, 174; Laren₹/Wolf, AT, $\$ 36$ Rn. 14 f.

415 Wiebe, in: Gounalakis, Rhb. Electronic Business, \15 Rn. 74; Wiebe, S. 403; Bamberger/Roth/Wendtland, \$120 Rn. 3; Larenz/Wolf, AT, \36 Rn. 14 f.

416 Mebrings, in: Hoeren/Sieber, Hdb. MultimediaR, Teil 13.1 Rn. 246; LG Köln, MMR 2003, 481 (482); ferner Härting, ITRB 2004, 63; a.A. OLG Frankfurt, MMR 2003, 405 (406), das den durch eine Software erfolgten Übertragungsfehler in ein Angebot bei der späteren automatischen Vertragsannahme fortwirken lassen möchte und einen Übermittlungsirrtum annimmt.

417 So auch Spindler, Anm. zu BGH, JZ 2005, 791 ff., JZ 2005, 793 (795). 
in seine Plattform integriert hat. Erst dieser gibt dann die fehlerhaft an ihn übermittelte Willenserklärung durch die Bündelung als Gesamtpaket an den Dritten (Kunden) weiter. Der Übermittlungsirrtum findet indes seine Grenzen beim Empfänger der Willenserklärung und Empfänger ist der Dienstenutzer, nicht der Dritte. Eine Anfechtung wegen eines Übermittlungsirrtums gegenüber dem Dritten (Kunden) bleibt dem Nutzer verwehrt. Dem Nutzer bleibt gegenüber dem Kunden daher nur die Berufung auf Unmöglichkeit der Beschaffung der gewünschten Leistung, wenn diese nicht verfügbar sein sollte.

Anders gestaltet sich die Situation, wenn der Web Service-Nutzer keinen eigenen Vertrag mit dem Kunden schließen möchte, sondern dem Web ServiceAnbieter lediglich seine Plattform zur Verfügung stellt und dies Dritten gegenüber deutlich macht. Die Erklärung des Web Service erreicht dann zwar wieder zunächst technisch den Web Service-Nutzer als Plattformbetreiber, jedoch agiert er dabei lediglich als Bote, ${ }^{418}$ der die vom Web Service-Anbieter technisch entgegengenommene Erklärung dem Kunden sichtbar macht und dadurch übermittelt. Ist die Erklärung nun fehlerhaft durch den Web Service übermittelt worden, so kann dennoch kein Übermittlungsirrtum angenommen werden, weil der Fehler bei der Verwendung der eigenen Technik des Web Service-Anbieters unterlaufen ist und nicht durch die Übermittlung des Web Service-Nutzers. Anstelle des Übermittlungsirrtums kommt hier aber ein Erklärungsirrtum in Betracht. ${ }^{419}$

Unter Systemfehlern werden dagegen Fehler verstanden, die in der Programmierung der Software liegen und die zur Abgabe beziehungsweise zur Erzeugung fehlerhafter Willenserklärungen führen. ${ }^{420}$ Der Unterschied zum Übermittlungsirrtum liegt darin, dass bei der Übermittlung die Programmierung des Diensteanbieters korrekt verlaufen ist, dagegen die Übermittlung durch externe Einflüsse oder die Einschaltung eines Dritten gestört wird und deshalb fehlerhaft verläuft. Während also beim Übermittlungsfehler der Wille korrekt gebildet und lediglich falsch abgegeben wurde und somit ein klassischer Erklärungsirrtum in Form eines Übermittlungsirrtums vorliegt, wird bei dem Systemfehler bereits der Wille falsch gebildet. Dies erklärt auch die Einordnung des Systemfehlers als unbeachtlichen Motivirrtum. Für die Web ServiceNutzung im Rahmen der Serviceorientierten Architektur bedeutet dies, dass

418 S. A.III.2.a)cc) Web Service-Anbieter - Dritter.

419 S. A.III.5.a)cc) Übermittlungsfehler und Systemfehler.

420 Köbler, AcP 182 (1982), 134; Mehrings, in: Hoeren/Sieber, Hdb. MultimediaR, Teil 13.1 Rn. 244; Wiebe, in: Gounalakis, Rhb. Electronic Business, \15 Rn. 62; BGH, NJW 1998, 3192 (3193). 
der Web Service-Anbieter auch nicht gegenüber dem Web Service-Nutzer im Falle der fehlerhaften Übermittlung durch seinen Web Service anfechten könnte, denn der Fehler liegt in der Software des Anbieters, so dass ein Übermittlungsirrtum nicht in Betracht kommt.

Allerdings ordnet die Rechtsprechung die Übertragung mittels Software als bloße Willensentäußerung ein, da der Wille bereits vorher durch die Eingabe in ein Datenbanksystem korrekt gebildet wurde. ${ }^{421}$ Der Bundesgerichtshof grenzt dabei den Übertragungsfehler vom verdeckten (unbeachtlichen) Kalkulationsirrtum ab, qualifiziert ihn aber letztlich als Erklärungsirrtum nach $\ 119$ Abs. 1, 2. Alt. BGB, ${ }^{422}$ wobei er zwischen fehlerhaft übertragender Software und solcher die fehlerhaft „berechnet“ unterscheidet. ${ }^{423}$ Während die fehlerhaft übertragende Software Parallelen zum Übermittlungsirrtum aufweisen soll, wird die fehlerhaft berechnende Software dem unbeachtlichen Kalkulationsirrtum gleichgestellt. Allerdings beruht der klassische Übermittlungsfehler gerade auf unbeherrschbaren Fremdeinflüssen und nicht auf internen Fehlern, wie bei dem Softwarefehler.

Dennoch erscheint diese Gleichstellung im Ergebnis, wenn auch nicht in der Begründung, sachgerecht. Wie der Bundesgerichtshof selbst darlegt, ${ }^{424}$ erfolgt durch die nur übermittelnde Software gerade keine Willensbildung mehr, sondern lediglich eine Übertragung des bereits gebildeten Willens. Der Erklärende entäußert dabei seinen bereits (korrekt) gebildeten Willen, der in der Datenbank „ruht", durch das „Sprachrohr" der Software, bei der dann ein Fehler unterläuft, so dass scheinbar tatsächlich Parallelen zum Übermittlungsirrtum vorliegen. Es handelt sich allerdings, wie auch der Bundesgerichtshof annimmt, nicht um den Irrtum eines Übermittlers (Dritten) sondern den „Irrtum“ der eigenen Software und somit keinen Übermittlungsirrtum. ${ }^{425}$ Verläuft die Willensbildung demnach beim Web Service-Anbieter korrekt und unterläuft der Fehler in der Datenübertragung und demnach in der Willensentäußerung, so liegt kein unbeachtlicher Motivirrtum aber auch kein Übermittlungs-

421 So der BGH, JZ 2005, 791 (792 f.) m. Anm. Spindler. Im entschiedenen Fall ging es um einen Übertragungsfehler aus einer Datenbank, so dass auf der Homepage - die unumstritten eine invitatio ad offerendum darstellte - ein zu niedriger Preis angegeben wurde, zu dem ein Käufer bestellte. Der BGH sah in der Übertragung durch die Software einen Erklärungsirrtum, der in der Vertragsannahme fortwirke.

BGH, JZ 2005, 791 (792 f.) m. Anm. Spindler.

Spindler, Anm. zu BGH, JZ 2005, 791 ff., JZ 2005, 793 (794).

424

BGH, JZ 2005, 791 (792) m. Anm. Spindler.

425

S. bereits A.III.5.a)cc) Übermittlungsfehler und Systemfehler. 
irrtum vor, es handelt sich vielmehr um einen klassischen Fall des Erklärungsirrtums bei der Abgabe der Willenserklärung. ${ }^{426}$

Damit ist indes nicht die Frage beantwortet, warum nicht auch die fehlerhaft berechnende Software als beachtlicher Erklärungsirrtum eingeordnet werden kann. ${ }^{427}$ Die fehlende Anfechtungsmöglichkeit von Irrtümern in der Willensbildung beruht auf dem Gebot der Verkehrssicherheit, ${ }^{428}$ insbesondere auch darauf, dass Fehler in der Willensbildung im Gegensatz zu solchen in der Willensäußerung noch korrigierbar und insofern deutlich beherrschbarer sind, so dass damit ein höheres $\mathrm{Maß}$ an Verantwortung einhergeht. ${ }^{429}$ Im Falle der fehlerhaft berechnenden Software kann aber gerade keine Korrektur mehr stattfinden, ${ }^{430}$ so dass auch die tatsächliche Beherrschbarkeit der Fehler fraglich ist. Freilich ist es dem Erklärenden zumutbar, die Software, deren er sich für seine Erklärungen bedient, regelmäßig auf mögliche Fehler zu überprüfen. Bei technischen Produkten sind derartige Fehleranalysen aber immer auf den Stand der Technik beschränkt. Hat der Erklärende das seinerseits Erforderliche getan, kann die fehlende Korrekturmöglichkeit nicht zwangsläufig zu seinen Lasten gehen. Denn derartige Fehler sind für ihn nicht mehr beherrschbar, ebenso wenig wie die fehlerhafte übertragende Software. Damit sind sowohl die fehlerhaft übertragende als auch die fehlerhaft berechnende Software als beachtliche Irrtümer einzuordnen, wobei der Berechnungsirrtum dem Erklärungsirrtum gleichzustellen ist.

Dies bedeutet für die Web Service-Nutzung im Rahmen der Serviceorientierten Architektur, dass der Web Service-Anbieter bei der fehlerhaften Übertragung durch seinen Web Service - als fehlerhaft übertragende oder unter Umständen sogar fehlerhaft berechnende Software - gegenüber dem Nutzer aufgrund eines Erklärungsirrtums zur Anfechtung berechtigt ist. Außerhalb der Serviceorientierten Architektur sind Web Service-Anbieter und Web Service-Nutzer regelmäßig ein und dieselbe Person, daher erfolgt die fehlerhafte Erklärung durch den Web Service des Anbieters direkt gegenüber dem Dritten (Kunden), so dass innerhalb einer solchen Struktur ein anfechtbarer Erklä-

\footnotetext{
426 So auch MünchKommBGB/Kramer, Bd. 1/1, \119 Rn. 89.

427 Dies bereits kritisch hinterfragend Spindler, Anm. zu BGH, JZ 2005, 791 ff., JZ 2005, 793 (794 f.).

428 Staudinger/Knothe, Vorb. SS 116-144 Rn. 32; Bamberger/Roth/Wendtland, \$119 Rn. 37.

429 MünchKommBGB/Kramer, Bd. 1/1, \119 Rn. 10; Staudinger/Singer, \119 Rn. 5; Larenף/Wolf, AT, $\$ 36$ Rn. 2.

430 Anders als beim Eingabefehler in die Datenbank durch einen Mitarbeiter.
} 
rungsirrtum des Anbieters/Nutzers (als identische Person) gegenüber dem Dritten (Kunden) im Sinne des $₫ 119$ Abs. 1, 2. Alt. BGB vorläge.

Wie gezeigt, sind die Grenzen zwischen den Anfechtungsmöglichkeiten innerhalb der Serviceorientierten Architektur mit Web Services fließend. Festzuhalten bleibt aber, dass Fehler, die bei der Erklärungsübermittlung durch den Web Service auftreten, lediglich im Verhältnis zwischen Web Service-Anbieter und Web Service-Nutzer beachtliche Irrtümer in der Willensäußerung darstellen, die nach $\int 119$ Abs. 1, 2. Alt. BGB anfechtbar sind. Gegenüber dem Dritten (Kunden) spielen diese Fehler keine Rolle. Außerhalb der Serviceorientierten Architektur sind derartige Fehler auch gegenüber Dritten (Kunden) anfechtbare Erklärungsirrtümer.

\section{Pflichten im E-Commerce}

Der elektronische Geschäftsverkehr bringt dem Verbraucher sowohl Vor- als auch Nachteile. Während sich die Überwindung von räumlichen Distanzen, eine große Angebotsvielfalt einhergehend mit leichteren Zugangsmöglichkeiten, schnellere Transaktionen, jederzeitige Aktualisierbarkeit der Angebote sowie Kostenersparnisse als Vorteile nennen lassen, so stehen diesen Vorteilen Informationsdefizite bezüglich der Identität des Verkäufers und der Qualität der Ware (keine Augenscheinnahme möglich), unüberlegte Bestellungen, Risiken der Abwicklung und Rückabwicklung des Vertrags, aggressive oder irreführende Verkaufsmethoden, Schutzrechtsgefälle durch grenzüberschreitende Transaktionen, Rechtsdurchsetzungsprobleme sowie Datenmissbrauchsmöglichkeiten als Nachteile gegenüber. ${ }^{431}$ Insofern ist der Verbraucher dem Unternehmer technisch und strukturell unterlegen. Dem Ausgleich dieses Defizits dienen die $\int \mathbb{S} 312 \mathrm{~b}-312 \mathrm{~d}$ BGB sowie $\int 312 \mathrm{e}$ BGB ${ }^{432}$, der technische Mindeststandards für eine faire Vertragsanbahnung und einen fairen Vertragsschluss sicherstellen soll. ${ }^{433}$ Nach diesen Vorschriften werden dem Unternehmer be-

431 BGH, NJW-RR 2005, 1058 (1059); Köbler, NJW 1998, 185; Leßmann/Leßmann, in: Gounalakis, Rhb. Electronic Business, $\ 11$ Rn. 6; MünchKommBGB/Wendehorst, Bd. 2, Vorb. $\int \S 312 \mathrm{~b} f f$. Rn. 4; Begr. RegE BT-Drs. 14/2658, S. 15; ferner Ultsch, in: Schwarz/Peschel-Mehner, Recht im Internet, Kap. 3-G Rn. 4; zu den Vorteilen Waldenberger, in: Hoeren/Sieber, Hdb. MultimediaR, Teil 13.4 Rn. 1.

$432 \rrbracket 312 \mathrm{e}$ BGB hat gegenüber $₫ 312 \mathrm{~b}$ BGB sogar einen weiteren Adressatenkreis, da er nicht explizit von Verbrauchern, sondern lediglich von Kunden - worunter daher auch Unternehmer fallen können - spricht.

433 MünchKommBGB/Wendehorst, Bd. 2, Vorb. $\iint 312 \mathrm{~b}$ ff. Rn. 5; Leßmann/Leßmann, in: Gounalakis, Rhb. Electronic Business, \11 Rn. 36. 
sondere Informationspflichten auferlegt und dem Verbraucher bestimmte Rechte, wie das Widerrufsrecht, gewährt. Daneben hat der Gesetzgeber insbesondere Informationspflichten in den $\iint 5,6$ TMG (sowie dem bereits erwähnten $\ 312 \mathrm{c}$ Abs. 1 S. 1 BGB) für die Telemediendiensteanbieter eingeführt, die einer umfassenden Aufklärung des Verbrauchers dienen sollen. Die Erfüllung dieser Pflichten soll die Identifizierung und die umgehende Kontaktaufnahme ermöglichen, um dem Verbraucher die Überprüfung des Telemediendiensteanbieters vor Abschluss eines Geschäfts zu ermöglichen und ihn nicht völlig rechtlos zu stellen. ${ }^{434}$

\section{a) Verbraucherinformationspflichten}

Im Rahmen der Serviceorientierten Architektur sind regelmäßig alle Beteiligten Unternehmer, so dass eine Untersuchung spezieller verbraucherschützender Vorschriften außen vor bleiben kann. Der Verbraucherschutz spielt allerdings im Verhältnis zwischen Web Service-Nutzer und Dritten eine Rolle, da der Web Service-Nutzer als Unternehmer (zum Beispiel Reiseportal) den Dienst sowohl Unternehmern als auch Verbrauchern zur Verfügung stellt.

Da der Web Service in diesem Verhältnis aber lediglich ein Mittel zum Zweck der Vertragserfüllung beziehungsweise des Vertragsabschlusses darstellt, ergeben sich hier keine Besonderheiten im Vergleich zu anderen Verträgen im elektronischen Geschäftsverkehr. Dazu gehören insbesondere die Informationspflichten nach $\int 312 \mathrm{c}$ BGB in Verbindung mit der Verordnung über Informations- und Nachweispflichten nach bürgerlichem Recht (BGB-InfoV). Dabei muss der Web Service Nutzer insbesondere für den Dritten erkennbar seinen Geschäftszweck und seine Identität darlegen, darüber hinaus ist ihm auch die Angabe einer ladungsfähigen Anschrift zumutbar, $₫ 1$ Abs. 1 Nr. 2 BGB-InfoV. Handelt es sich bei dem Web Service Nutzer, wie im Beispiel der Reisebuchung, um ein Reiseportal, das als Reiseveranstalter zu qualifizieren ist, ${ }^{435}$ so muss dieser zusätzlich die Informations- und Nachweispflichten des Abschnitts 3 der BGB-InfoV (\$S 4-11) beachten und erfüllen. Ferner sind die Belehrung über das Widerrufsrecht des Verbrauchers ( $\$ 355$ Abs. 2 S. 1 BGB in Verbindung mit $\int \mathbb{S} 312 \mathrm{c}, \int 312 \mathrm{e}$ Abs. $\left.3 \mathrm{BGB}\right)^{436}$ sowie die Beachtung der

434 Spindler/Schmitz/Geis/Spindler, \6 TDG Rn. 1; Mulch, MDR 2007, 309; Schöttler, jurisPR-ITR 5/2007 Anm. 6.

435 S. dazu die Ausführungen unter A.III.2.a)bb) Exkurs: Reisevertragsspezifische Besonderheiten.

436 Zur Form der Widerrufsbelehrung allgemein Bierekoven, ITRB 2007, 73 ff.; Buchmann, MMR 2007, 347 ff. Zur Länge der Widerrufsfrist ausführlich Dietrich/Hofmann, CR 2007, 318 ff.; Buchmann, K\&R 2007, 14 ff.; Spindler/Nink, DRiZ 2007, 197. 
Einbeziehungsvoraussetzungen von Allgemeinen Geschäftsbedingungen) \305 Abs. 2 BGB) zu nennen.

b) Informationspflichten der Telemediendiensteanbieter

Die Pflichten der $\iint 5,6$ TMG dienen nicht allein dem Verbraucherschutz, denn auch geschäftlich tätige Nutzer haben ein vitales Interesse daran, zu erfahren, mit wem sie in Kontakt treten und gegebenenfalls Geschäftsbeziehungen aufbauen. ${ }^{437}$ Diesem Interesse entspricht das in Art. 5 Abs. 1 ECRL geschaffene und in $\ 5$ Abs. 1 TMG umgesetzte Transparenzgebot, wonach Telemediendiensteanbieter die in $₫ 5$ Abs. 1 Nr. 1-7 TMG genannten Informationen leicht erkennbar, unmittelbar erreichbar und ständig verfügbar zu halten haben. Mindestmaß der Angaben sind E-Mail-Adresse sowie die Zurverfügungstellung eines weiteren schnellen, unmittelbaren und effizienten Kommunikationswegs, ${ }^{438}$ der im Einzelfall nicht notwendigerweise auch die Angabe einer Telefonnummer umfasst. ${ }^{439}$

Im Rahmen der Serviceorientierten Architektur sind alle drei Beteiligten Telemediendiensteanbieter - der Web Service-Anbieter gegenüber dem Web Service-Nutzer, der Web Service-Vermittler gegenüber Web Service- Anbieter sowie -Nutzer und der Web Service-Nutzer gegenüber den Dritten - und müssen daher die Informationspflichten des $\int 5$ TMG umsetzen. Leicht erkennbar sind Informationen, wenn sie einfach und effektiv, ohne langes Suchen, ${ }^{440}$ optisch wahrnehmbar sind. ${ }^{441}$ Angaben in Allgemeinen Geschäftsbedingungen genügen dem Erfordernis daher nicht. ${ }^{442}$ Pflichtangaben sind unmittelbar erreichbar, wenn der Zugriff ohne wesentliche Zwischenschritte erfolgen kann, wobei das Anbieten auf einer gesonderten Seite, die der Nutzer ler/Schmitz/Geis/Spindler, \& 6 TDG Rn. 2; Spindler/Schuster/Micklits, \& 5 TMG Rn. 2 f.; von Wallenberg, MMR 2005, 662 f.; Woitke, NJW 2003, 871; so wohl auch Ranke, MMR 2002, 512, der lediglich von Nutzer spricht; insofern nicht ganz eindeutig Schöttler, jurisPR-ITR 5/2007 Anm. 6, der sowohl von „Verbraucher“ als auch der „Allgemeinheit“ spricht.

438 Darunter kann auch, je nach den Umständen des Einzelfalls, eine elektronische Anfragemaske fallen, s. auch Ernst, jurisPR-ITR 2/2009 Anm. 2.

439 EuGH, MMR 2009, 25 (26) m. Anm. Ott.

440 Begr. RegE BT-Drs. 14/6098, S. 21.

441 BGH, NJW 2006, 3633 (3634 f.); OLG Hamburg, MMR 2003, 105; Spindler/Schuster/Micklitr, § 5 TMG Rn. 16; Hoenike/Hülsdunk, MMR 2002, $416 \mathrm{f.}$

442 
über einen, gegebenenfalls sogar zwei, ${ }^{443}$ Links von der Website aufrufen kann, genügt. ${ }^{444}$ Ständig verfügbar sind die Informationen, wenn sie jederzeit abgerufen werden können, was beispielsweise dann erfüllt ist, wenn sie dauerhaft über einen funktionstüchtigen Link abrufbar sind. ${ }^{445}$ Dabei genügt aber ein mit dem Begriff „Impressum“ gekennzeichneter Link, über den die Anbieterangaben aufgerufen werden können, der nur in sehr kleiner Schrift und drucktechnisch nicht hervorgehoben am rechten unteren Ende der Homepage platziert ist, den Anforderungen des $\ 5$ TMG aufgrund der Ratio des $\ 5$ TMG, zu wissen mit wem man in (geschäftlichen) Kontakt tritt, nicht. ${ }^{446}$

Umstritten bei grenzüberschreitenden Sachverhalten, wie es im Rahmen der Serviceorientierten Architektur häufig der Fall sein kann, ist die Sprache in der die Informationen nach $\int 5$ Abs. 1 TMG gehalten werden müssen. Während ein Teil der Literatur auf das Herkunftslandprinzip verweist, wonach die Sprache anhand des Staats erfolgen muss, in dem der Telemedienanbieter seine Niederlassung hat, ${ }^{447}$ wollen andere Teile des Schrifttums die Information in der Sprache halten, in der die Website gefasst ist. ${ }^{448}$ Verlangt man, dass die Information in der gleichen Sprache gehalten werden muss, wie die Website selbst, so stellt man zumindest sicher, dass der Nutzer die Sprache auch versteht, da er sich ja offensichtlich auch auf der Website selbst zurecht findet. Andererseits überlässt man so dem Anbieter der Website das Bestimmungsrecht über die Sprache, was auch keine interessengerechte Lösung darstellt. ${ }^{449}$ Letztlich wäre es wünschenswert, wenn der Europäische Gesetzgeber sich um eine Lösung bemüht. Bis dahin erscheint es sinnvoll auf das Herkunftslandprinzip abzustellen, da dieses der E-Commerce-Richtlinie zu Grunde liegt. Im B2B-Bereich, wie zwischen Web Service-Anbieter und -Nutzer sowie zwi-

\footnotetext{
443 So ausdrücklich der BGH, NJW 2006, 3633 (3635).

444 BGH, NJW 2006, 3633 (3635); Hoenike/Hülsdunk, MMR 2002, 417; Woitke, NJW 2003, 873; von Wallenberg, MMR 2005, 663.

445 Spindler/Schuster/Micklitz, \5 TMG Rn. 29; Hoß, CR 2003, 689.

446 So zu Recht OLG Frankfurt, K\&R 2009, 197 (199). Das Revisionsverfahren ist aktuell beim BGH anhängig (Az: I ZR 11/09).

447 Spindler/Schmitz/Geis/Spindler, \6 TDG Rn. 6.

448 Hoeren, MMR 1999, 197; ders., WM 2004, 2463; Brunst, MMR 2004, 12; von Wallenstein, MMR 2005, 663 .

449 Spindler/Schuster/Micklitz, \$5 TMG Rn. 23.
} 
schen Web Service-Vermittler und -Anbieter beziehungsweise -Nutzer könnte allerdings auch Englisch als lingua franca hinnehmbar sein. ${ }^{450}$

Im Rahmen von kommerzieller Kommunikation müssen darüber hinaus die Informationspflichten des $\ 6$ Abs. 1 TMG erfüllt werden, damit insbesondere Werbung bzw. der Werbecharakter des Angebots klar erkennbar wird. ${ }^{451}$ Der Begriff der kommerziellen Kommunikation richtet sich nach $₫ 2$ S. 1 Nr. 5 TMG. Kommerzielle Kommunikation wird danach sehr weit gefasst, der Begriff umfasst ,jede Form der Kommunikation, die der unmittelbaren oder mittelbaren Förderung des Absatzes von Waren, Dienstleistungen oder des Erscheinungsbilds eines Unternehmens, einer sonstigen Organisation oder einer natürlichen Person dient, die eine Tätigkeit im Handel, Gewerbe oder Handwerk oder einen freien Beruf ausübt". Insofern wird fast jede Art der Werbung und Selbstdarstellung erfasst, die in irgendeiner Weise mit einer wirtschaftlichen Tätigkeit verbunden ist. ${ }^{452} \mathrm{Da}$ die Beteiligten der Serviceorientierten Architektur regelmäßig wirtschaftlich tätig sind, indem sie Dienste anbieten, wie die UDDI-Nutzung oder die Web Service-Nutzung, müssen Web Service-Anbieter, -Vermittler und -Nutzer auch die Pflichten des $\ 6$ Abs. 1 TMG erfüllen.

Bei Nichteinhaltung dieser Pflichten setzt sich der Telemediendiensteanbieter einer Unterlassungsklage nach $₫ 2 \mathrm{UKlaG}$ oder nach $₫ 4 \mathrm{Nr}$. $11 \mathrm{UWG}$ durch die klagebefugten Verbände aus. ${ }^{453}$ Darüber hinaus stellt ein Verstoß gegen $\ 5$ Abs. 1 TMG eine Ordnungswidrigkeit dar, die nach $\$ 16$ Abs. 2 Nr. 1, Abs. 3 mit einem Bußgeld bis zu 25.000 geahndet werden kann. Ein Verstoß gegen \6 Abs. 1 TMG hingegen ist nicht bußgeldbewährt. Keine Auswirkungen hat der Pflichtenverstoß auf die Wirksamkeit des Vertragsschlusses. ${ }^{454}$ \& 5 Abs. 1 TMG stellt jedoch ein Schutzgesetz im Sinne des $\int 823$ Abs. 2 BGB dar, so

450 Spindler/Schuster/Micklitz, \5 TMG Rn. 24.

451 Spindler/Schmitz/Geis/Spindler, \ 7 TDG Rn. 1; Leitgeb, ZUM 2009, 43.

452 Spindler/Schuster/Schmitr, $\ 2$ TMG Rn. 11; Spindler/Schmitz/Geis/Spindler, 33 TDG Rn. 21.

453 OLG Hamm, MMR 2009, 552 f.; OLG Düsseldorf, MMR 2009, 266; OLG Frankfurt, MMR 2009, 194; OLG Düsseldorf, MMR 2008, 682 f.; OLG München, MMR 2004, 36 (38); LG Frankfurt, Urt. v. 13.05.2009 - 2-06 O 61/09; LG Essen, MMR 2008, 196; Ranke, MMR 2002, 512; Spindler/Schuster/Micklits \5 TMG Rn. 69; Spindler/Schuster/Micklits \6 TMG Rn. 121. 
dass der Betroffene im Falle eines Schadens, Schadensersatz geltend machen kann. ${ }^{455}$

Für den Web Service-Nutzer, der diese Pflichten gegenüber den Dritten erfüllen muss, stellt die Erfüllung der Pflichten kein besonderes Problem - wie auch bei klassischen Website-Betreibern - dar, weil er die Informationen entsprechend in seine Website integrieren kann. Ähnlich verhält es sich mit dem Web Service-Vermittler, auch dieser wird eine Möglichkeit finden, die Informationen bei der UDDI-Oberfläche entsprechend anzubringen. Problematisch indes ist die Pflichtenerfüllung für den Web Service-Anbieter, der sie gegenüber dem Web Service-Nutzer erfüllen muss. Denn der Web ServiceNutzer erhält alle relevanten Informationen zur Nutzung des Web Service lediglich über den Web Service-Vermittler aus dem UDDI. Um eine entsprechende Information vor Vertragsschluss und somit vor der Web ServiceNutzung ermöglichen zu können, wird der Web Service-Anbieter die nach $\ 5$ Abs. 1 Nr. 1-7 TMG erforderlichen Informationen bereits im UDDI platzieren müssen. Dies könnte entweder direkt in der Unternehmensbeschreibung erfolgen oder durch einen optisch hervorgehoben, gut erkennbaren Link in der Unternehmensbeschreibung, bei dem explizit darauf hingewiesen wird, dass der Web Service-Nutzer durch Anklicken des Links die entsprechenden Informationen erhält. Das entspricht auch dem Kriterium der ständigen Verfügbarkeit. Zwar wird der Web Service-Nutzer immer eine erneute UDDISuche starten müssen, um die Informationen oder zumindest den Link abrufen zu können, aber diesem Prozedere steht der Telos der Regelung nicht entgegen. Vor Vertragsschluss wird eine leichte Erkennbarkeit und unmittelbare Erreichbarkeit garantiert. Denn der Zeitpunkt der Betrachtung der Dienstebeschreibung ist im Rahmen der Serviceorientierten Architektur der erste Berührungspunkt mit dem Web Service-Anbieter. Und durch eine dauerhafte Anzeige im UDDI wird auch die ständige Verfügbarkeit garantiert.

\section{Leistungsstörungen}

Kommen Softwarekomponenten, wie Web Services, im Rahmen von Vertragsbeziehungen zum Einsatz, so stellt sich im Falle des Auftretens von Mängeln bei der Software die Frage, wer wofür haftet und wie sich diese Mängel bewältigen lassen. Konkret ergeben sich dabei folgende Fragestellungen: wer ist verantwortlich wenn ein Dienst unvollständig456 oder fehlerhaft erbracht

\footnotetext{
455 AG Mönchengladbach, MMR 2003, 606 (608); Spindler/Schmitz/Geis/Spindler, \6 TDG Rn. 43; Spindler/Schuster/Micklitz, \5 TMG Rn. 73.

456 Speck, in: Hoffmann/Leible/Sosnitza, S. 23.
} 
wird und bestehen in diesem Fall noch Entgeltansprüche gegenüber dem Nutzer? Wer trägt die Verantwortung, wenn die Information vollständig und korrekt vom Web Service-Anbieter abgesandt, aber lediglich unvollständig oder fehlerhaft bei dem Web Service-Nutzer ankommt? Derartige Fehler können entweder auf technischen Übertragungsfehlern oder auf Softwaremängeln beruhen. Übertragungsfehler fallen in die Risikosphäre des Access-Providers. Dieser wirkt aber lediglich durch die Übermittlung auf die Ausführung des Web Service ein; es ergeben sich daher im Bereich der Übertragung keine Web Service-spezifischen Rechte bei Störungen und daraus resultierenden Mängeln. ${ }^{457}$ Daher ist für diese Arbeit lediglich die Konstellation von Interesse, dass die genannten Mängel auf einen Fehler des Web Service, sprich einem Softwaremangel, beruhen.

Neben Mängeln in der Web Service-Architektur spielen Fehler bei der Dienstesuche eine relevante Rolle. Dabei sind diverse Konstellationen denkbar. So könnte der Dienstemakler dem Web Service-Nutzer, bei dessen Suche anhand bestimmter Kriterien, Dienste anzeigen, die den Kriterien des Web Service-Nutzers nicht entsprechen aber aufgrund der Anzeige als Suchergebnis dann durch den Web Service-Nutzer irrtümlich genutzt werden. ${ }^{458}$ Weiter wäre denkbar, dass der Dienstemakler trotz korrekter Registrierung des Web Service durch den Web Service-Anbieter X im UDDI bei der Suche durch einen Web Service-Nutzer anhand der entsprechenden Kriterien den konkreten Dienst des X dem Web Service-Nutzer nicht anzeigt, währenddessen seine Konkurrenten Y und $\mathrm{Z}$ angezeigt und vom Web Service-Nutzer daraufhin auch genutzt werden. Ein weiterer Fall wäre die Konstellation, dass der Web Service-Anbieter seinen Dienst korrekt im UDDI registriert, der Web ServiceNutzer diesen aber fehlerhaft veröffentlicht, indem er ihn in eine falsche Branche oder Dienstekategorie einordnet oder aber ihm die Dienstebeschreibung eines anderen Dienstes zuordnet, so dass der Web Service-Nutzer einen für ihn ungeeigneten, unerwünschten Dienst integriert und schlimmstenfalls sogar zur Nutzung für Dritte freigibt.

\section{a) Mangel des Web Service}

Ist die Web Service-Architektur mangelhaft, so können sich zahlreiche praktische Probleme ergeben, die hier keinesfalls abschließend sondern vielmehr beispielhaft erwähnt sein sollen. So erscheint es möglich, dass der Web Service

\footnotetext{
457 S. zu den Störungsfällen im Rahmen der Telekommunikation Sörup, in: Heun, K Rn. 34 ff.

458 S. Speck, in: Hoffmann/Leible/Sosnitza, S. 23.
} 
„übersieht", dass eine bestimmte Ware vorübergehend oder dauerhaft nicht verfügbar ist. Darüber hinaus können aber auch falsche Waren bestellt werden, indem der Web Service gegenüber dem Kunden zwar die richtige Ware anbietet aber dennoch eine andere bestellt. ${ }^{459}$ Ferner können sich Mängel auch derart auswirken, dass der Web Service einen Dienst nicht vollständig erbringt, indem er eine Buchung nur zum Teil abschließt. 460

Agiert der Web Service fehlerhaft, so kann der Mangel zum einen auf einen Fehler durch den Web Service Hersteller beruhen, zum anderen aber auch ein nachträglich - zum Beispiel durch unzureichende Wartung des Web ServiceAnbieters oder auch durch unsachgemäße Implementierung des Web ServiceNutzers - im Gebrauchszeitraum auftreten. Da bei Leistungsstörungen immer nur der Vertragspartner in Verantwortung genommen werden kann und dieser nicht zwangsläufig mit dem tatsächlich für den Fehler Verantwortlichen identisch ist, muss sich der durch den Mangel Betroffene zumindest bei der Geltendmachung von vertraglichen Ansprüchen an diesen halten. ${ }^{461}$ Das bedeutet, dass sich der Dritte (der den beim Web Service-Nutzer implementierten Web Service in Anspruch nimmt) als Endanwender im Falle von Mängeln in der Web Service Architektur auf vertraglicher Ebene nicht an den in der Web Service-Wertschöpfungskette am Anfang stehenden Web Service Hersteller halten kann. Solche Ansprüche kommen lediglich im Rahmen der außervertraglichen Haftung im Bereich der Produkt- und Produzentenhaftung in Betracht. ${ }^{462}$ Welche Ansprüche der Betroffene im Falle von Leistungsstörungen konkret geltend machen kann, ergibt sich aus dem jeweiligen Vertragsverhältnis.

Im Rahmen der Serviceorientierten Architektur bestehen unmittelbare Vertragsbeziehungen bezüglich der dauerhaften Überlassung der Web Services als Hauptvertragsgegenstand lediglich zwischen Web Service-Hersteller und Web

459 Auch hier sind zahlreiche Konstellationen denkbar: der Kunde will ein Auto reservieren, der Web Service reserviert aber ein Motorrad und bestätigt dem Kunden die Reservierung eines Autos. Derartige Fehler können noch gravierender ausfallen, wenn ein Diensteanbieter nicht nur eine Warenkategorie, wie motorisierte Fortbewegungsmittel, anbietet sondern diverse, die völlig unterschiedliche Waren beinhalten und der Web Service aus einer anderen Warenkategorie als der gewünschten bestellt.

460 Wie im Beispiel der Reisebuchung, wenn z.B. Hotel und Mietwagen gebucht wurden, die Buchung des Fluges aber trotz Anforderung durch den Kunden nicht erfolgte.

461 Das ergibt sich zum Beispiel aus $₫ 280$ Abs. 1 BGB, der ausdrücklich ein „Schuldverhältnis“ voraussetzt, oder $\$ 323$ Abs. 1 BGB, der von ,gegenseitigem Vertrag“ spricht.

462 S. dazu B.II Mangel des Web Service. 
Service-Anbieter. Die Beziehungen zwischen Web Service-Anbieter, selbst wenn dieser zugleich Web Service-Hersteller sein sollte, und Web ServiceNutzer dagegen sind auf das Funktionieren und die Verfügbarkeit des Web Service sowie das Funktionieren des Dienstes gerichtet; 463 Vertragsgegenstand bei der Beziehung Web Service-Nutzer - Dritter ist indessen die Erfüllung des Grundvertrags. ${ }^{464}$ Ansprüche wegen Mängeln in der Web Service-Architektur können daher grundsätzlich nur vom Web Service-Anbieter gegenüber dem Web Service-Hersteller geltend gemacht werden.

Wann ein Mangel vorliegt, bestimmt sich nach den gesetzlichen Negativdefinitionen der $\iint 434,633$ BGB, wonach zusammengefasst dann ein Mangel vorliegt, wenn der Web Service negativ von den für ihn geltenden Vorgaben abweicht, das heißt, die Ist-Beschaffenheit negativ von der Soll-Beschaffenheit des Web Service abweicht. 465 Wie bei Softwarekomponenten die SollBeschaffenheit auszusehen hat, kann nicht pauschal gesagt werden. Liegt ein Pflichtenheft zur Vertragsabwicklung vor, so richtet sich die SollBeschaffenheit nach diesem, ${ }^{466}$ fehlt es allerdings an einem solchen, so muss die Soll-Beschaffenheit individuell anhand der greifbaren Faktoren beurteilt werden. Zu den Bestimmungsfaktoren zählt im Falle des Kaufs von StandardWeb Services nach $\int 434$ Abs. 1 S. 3 BGB auch eine etwaige Leistungsbeschreibung des Verkäufers. Weitere Faktoren bei fehlenden Vereinbarungen über die Beschaffenheit sind die gewöhnliche Verwendung von Web Services sowie eventuelle gesetzliche Vorgaben. ${ }^{467} \mathrm{Da}$ Web Services aber äußerst vielfältig eingesetzt werden, ist die gewöhnliche Verwendung zumindest bei standardisierten Web Services auf das ordnungsgemäße Suchen und Finden von Informationen sowie den Transport dieser Informationen zu beschränken. Dieser gesetzlichen Mangelbestimmung wird allerdings häufig in Bezug auf Software entgegengehalten, dass Software nie mangelfrei sei und die Definition des Mangels daher für Software so nicht generell gelten dürfe. ${ }^{468} \mathrm{Da}$ dies aber auch für andere komplexe Entwicklungen aus Wissenschaft und Technik

\footnotetext{
463 S. dazu A.III.1.c)aa) Vergütung der Web Service-Nutzung.

464 Wie z.B. die Erfüllung eines Reisevertrags, s. A.III.1.e) Web Service-Nutzer - Dritte.

465 Redecker, in: Schneider/von Westphalen, D Rn. 280.

466 Goldmann/Redecke, MMR 2002, 3; Redecker, in: Schneider/von Westphalen, D Rn. 281.

467 Redecker, in: Schneider/von Westphalen, D Rn. 285 f.

468 Ablehnend zu dieser Argumentation: Marly, Rn. 839; Redecker, in: Schneider/von Westphalen, D Rn. 288; Spindler, CR 2005, 741.
} 
gilt ${ }^{469}$ und für einen Schadensersatzanspruch, als härteste Folge, zusätzlich ein Verschulden erforderlich ist, was nicht vorliegen dürfte, wenn die Web Services nach dem Stand der Technik entwickelt wurden, kann dieses Argument nicht greifen. Auch für Web Services, wie auch Software generell, muss daher der allgemeine Mangelbegriff der $\$ \int 434,633$ BGB gelten. Erfolgt demnach eine Suche mittels Web Services fehlerhaft oder gar nicht, so weicht die IstBeschaffenheit von der Soll-Beschaffenheit ab und es liegt ein Mangel im Sinne der $\iint 434,633 \mathrm{BGB}$ vor.

\section{i) Web Service-Anbieter gegenüber Web Service-Hersteller}

Bestehen Mängel in der Architektur des Web Service, so kann lediglich der Web Service-Anbieter gegenüber dem Web Service-Hersteller vertragliche Mängelansprüche geltend machen. Hier werden neben der Nacherfüllung bezüglich der Mängelbeseitigung in erster Linie Ansprüche auf Ersatz des Mangelschadens insbesondere aber auch die Schadensersatzansprüche bei mangelbezogenen Folgeschäden, eine Rolle spielen. Gerade aber die Haftung für derlei Schäden wird in aller Regel vom Hersteller entweder im Rahmen von Individualvereinbarungen oder - im elektronischen Geschäftsverkehr wohl häufiger - in Allgemeinen Geschäftsbedingungen abbedungen. Während sich Individualvereinbarungen lediglich an den Grenzen der Sittenwidrigkeit $(\mathbb{\$} 138$ BGB) und des gesetzlichen Verbots ( $\$ 134$ BGB) messen lassen müssen, ${ }^{470}$ bildet für Allgemeine Geschäftsbedingungen die Inhaltskontrolle (\$S 307-309 BGB), die Grenze der Privatautonomie. ${ }^{471} \mathrm{Da}$ es sich bei dem Vertragsverhältnis zwischen Web Service-Hersteller und Web Service-Anbieter allerdings in aller Regel um ein B2B-Verhältnis handelt, unterliegen die Allgemeinen Geschäftsbedingungen des Unternehmers lediglich dem allgemeinen Kontrollmaßstab des $₫ 307$ Abs. 2 Nr. 2 BGB; die $\int \mathbb{S} 308-309$ BGB finden dann nach $\int 310$ Abs. 1 BG B keine Anwendung.

Es ist höchst umstritten, ob die Freizeichnung von mangelbezogenen Folgeschäden nach dem Maßstab des $₫ 307$ Abs. 2 Nr. 2 BGB zulässig ist. Während

469 OLG Düsseldorf, CR 1995, 269; Redecker, in: Schneider/von Westphalen, D Rn. 288; Marly, Rn. 840. Das OLG Köln, NJW 1996, 1683 betont sogar, dass selbst dann, wenn die geschuldete Eigenschaft technisch überhaupt nicht erreichbar ist, ein Mangel vorliegt.

470 Von Westphalen, in: Schneider/von Westphalen, H Rn. 6; Ulmer/Brandner/Hensen/Fuchs, Vorb. v. \ 307 BGB Rn. 3; BVerfG, NJW 1990, 1469 (1470).

471 Staudinger/Schlosser, Vorb. zu \$S 307-309 Rn. 13; Spindler, Gutachten BSI, Rn. 99; von Westphalen, in: Schneider/von Westphalen, H Rn. 6; Palandt/Grüneberg, Vorb. v. \307 Rn. 1; Goldmann/Redecke, MMR 2002, 7. 
ein Teil der Lehre einen solchen Ausschluss nach $\iint 310$ Abs. 1 S. 2, 307 Abs. 2 Nr. 2 BGB für unzulässig erachtet, da diese eine Kardinalpflicht darstelle, 472 bejahen andere eine solche Freizeichnung für leichte Fahrlässigkeit. ${ }^{473}$ Wiederum andere wollen den Haftungsausschluss zumindest bei gebrauchten Sachen außerhalb des Verbrauchsgüterkaufs zulassen, da der Verkäufer in diesem Fall auch das Rücktrittsrecht nach $\$ 309 \mathrm{Nr} .8 \mathrm{~b}$ bb) BGB ausschließen könne. ${ }^{474}$ $\mathrm{Da}$ die Softwarekomponenten der Web Services erst in Kombination mit einem Dienst, der wiederum vom Web Service-Anbieter und nicht vom Web Service-Hersteller mit dem Web Service verbunden wird, sinnvoll nutzbar sind, werden diese Komponenten in der Regel, im Gegensatz zu herkömmlicher Standardsoftware, wo Nutzungslizenzen als so genannte „Second-HandLizenzen“" weiterveräußert werden können, ${ }^{475}$ eher nicht vom Erstkäufer an Dritte weiterveräußert. Daher kann die Ansicht, ein Haftungsausschluss sei zumindest bei gebrauchten Waren möglich, für die Freizeichnung bei Web Services außen vor bleiben.

Würde man mit der ersten Ansicht ein absolutes Freizeichnungsverbot für mangelbezogene Folgeschäden annehmen, so würde dies den Hersteller von Web Services unangemessen benachteiligen, denn er weiß zum einen nicht, wie der Web Service-Anbieter den Web Service einsetzen will und demnach ist es für ihn auch unabsehbar, welcher Art mögliche Folgeschäden und wie weitreichend sie sein könnten. Zum anderen zeichnen sich Web Services in Umsetzung der Serviceorientierten Architektur gerade durch eine verteilte Anwendung aus. Wer den Web Service nutzt und welche Folgeschäden daraus resultieren können, ist schon für den Web Service-Anbieter nicht ersichtlich, erst recht aber nicht für den Web Service-Hersteller. Im Übrigen greift auch das Argument der Kardinalpflicht nicht, da bei einer solchen an die obliegende Pflicht nicht indes die Art des Schadens angeknüpft werden muss. ${ }^{476}$ Darüber hinaus hat der Gesetzgeber im Falle des Verbrauchsgüterkaufs, der den Käu-

472 Bamberger/Roth/Becker, $₫ 309$ Rn. 30, 35; i.d.S. auch MünchKommBGB/Kieninger, Bd. 2, \307 Rn. 67; von Westphalen, in: Schneider/von Westphalen, I Rn. 45; Auer-Reinsdorff, ITRB 2006, 185; Schulze-Ebers, JuS 2004, 466; Intveen, ITRB 2003, 13 f.

473 BGH, NJW 2005, 422 (424); Staudinger/Coester, $\int 307 \quad$ Rn. 450; Ulmer/Brandner/Hensen/Fuchs, \307 Rn. 290; Tiedtke/Burgmann, NJW 2005, 1155 f.

474 Litżenburger, NJW 2002, 1245; Palandt/Grüneberg, \ 307 Rn. 34a.

475 S. zum Verkauf von gebrauchten Softwarelizenzen Grützmacher, CR 2007, 549 ff.; Koch, ITRB 2007, 140 ff.; Huppertz, CR 2006, 145 ff.; Heydn/Schmidl, K\&R 2006, 74 ff.; Schuppert/Greissinger, CR 2005, $81 \mathrm{ff}$.

476 Tiedtke/Burgmann, NJW 2005, 1155. 
fer schützen soll, in $\ 475$ Abs. 3 BGB gerade den Ausschluss der Schadensersatzhaftung mit Ausnahme von $\iint 308,309$ BGB zugelassen. Dies zeigt, dass ein solcher Haftungsausschluss dann doch erst recht auch allgemein (freilich besonders im B2B-Bereich, wo bereits die $\$ \int 308,309$ BGB nicht greifen) möglich sein muss. Insofern kann diese Ansicht sachgerechterweise nicht angewendet werden. Daher muss dem Web Service-Hersteller zumindest der Ausschluss der Haftung für mangelbezogene Folgeschäden bei leichter Fahrlässigkeit möglich sein.

Derartige Einschränkungen verlieren aber dann ihre Geltung, wenn sich Web Service-Hersteller und Web Service-Anbieter, wie im B2B-Bereich nach der Rechtswahlklausel des Art. 27 EGBGB zulässig, auf eine fremde Rechtsordnung einigen. Eine solche Vereinbarung ist sogar im Rahmen von Allgemeinen Geschäftsbedingungen möglich. ${ }^{477}$ Ist die Rechtswahl nach dem jeweiligen gewählten Recht wirksam (Art. 31 Abs. 1 in Verbindung mit 29 Abs. 4 EGBGB), so wird sowohl die Inhaltskontrolle als auch die daraus resultierende Wirksamkeit oder Unwirksamkeit dem deutschen Recht entzogen. ${ }^{478}$

\section{ii) Web Service-Nutzer gegenüber Web Service-Anbieter}

Eine ganz andere Problematik ist das Geltendmachen von Mangelfolgeschäden - aufgrund eines Fehlers in der Web Service-Architektur - des Web Service-Nutzers gegenüber dem Web Service-Anbieter, der nicht zugleich Hersteller der Web Services ist. Auch hier wird der Web Service-Anbieter mangels Wissen um seinen Vertragspartner regelmäßig einen Haftungsausschluss für leicht fahrlässige Schäden und mangelbezogene Folgeschäden in seine Allgemeinen Geschäftsbedingungen aufnehmen. Der übrige Haftungsrahmen beschränkt sich dann lediglich auf Schäden und mangelbezogene Folgeschäden, die der Web Service-Anbieter grob fahrlässig oder vorsätzlich zu vertreten hat. Wann das bei fremder Software, die für die eigenen Belange genutzt wird, allerdings der Fall ist, erscheint fraglich. Zwar kann der Web Service-Anbieter die übliche Pflege der Web Services durchführen beziehungsweise durchführen lassen. Jedoch wird er sich bei Unkenntnis des genauen Programmierungscode lediglich auf übliche Kontrollen beschränken können und man wird ihm

477 Palandt/Heldrich, Art. 27 EGBGB Rn. 6; Spindler, Gutachten BSI, Rn. 101; Schmidt/Prieß, in: Spindler/Börner, S. 175.

478 BGHZ 123, 380 (383); MünchKommBGB/Spellenberg, Bd. 10, Art. 31 EGBGB Rn. 21; Spindler, Gutachten BSI, Rn. 101; Palandt/Heldrich, Art. 31 EGBGB Rn. 3; Staudinger/Hausmann, Art. 31 EGBGB Rn. 72. 
demnach im Falle eines Fehlers kaum mehr als leichte Fahrlässigkeit vorwerfen können.

\section{b) Fehlerhafte Dienstesuche}

Ist die Dienstesuche fehlerhaft, kann dies unterschiedliche Gründe und Auswirkungen haben. Hintergrund für die fehlerhafte Dienstesuche wird, neben technischen Defiziten, die fehlerhafte Veröffentlichung des Web Service durch den Web Service-Anbieter sein. Wird ein Dienst fehlerhaft gesucht, so hat dies keinerlei unmittelbare Auswirkungen für den Web Service-Nutzer, sofern er eine Fehlermeldung erhält. Anders verhält es sich jedoch, wenn die Dienstesuche derart fehlerhaft verläuft, dass eine völlig andere Diensteart als die gewünschte als Ergebnis geliefert wird und der Web Service-Nutzer diesen Fehler nicht bemerkt/nicht bemerken kann, er ihn daraufhin in seine eigene Webpräsenz integriert und zur Nutzung zur Verfügung stellt. Für den Web Service-Anbieter wirkt sich eine derartige fehlerhafte Dienstesuche dann negativ aus, wenn sein eigener Web Service bei der Suche nicht angezeigt wird, wohl aber diejenigen von Konkurrenten.

Im Rahmen der fehlerhaften Dienstesuche muss zwischen den jeweiligen Akteuren, Web Service-Vermittler im Verhältnis zum Web Service-Anbieter und Web Service-Vermittler im Verhältnis zum Web Service-Nutzer, unterschieden werden. Da dem Verhältnis Web Service-Vermittler - Web ServiceAnbieter ein Anzeigenvertrag beziehungsweise bei unentgeltlicher Veröffentlichung des Web Service ein Auftrag zugrunde liegt, ${ }^{479}$ richten sich die vertraglichen Ansprüche nach dem Werkvertrags- beziehungsweise Auftragsrecht. Dies gilt auch für das Verhältnis Web Service-Vermittler - Web ServiceNutzer, da auch dieser Vertragsbeziehung ein Werkvertrag beziehungsweise bei Unentgeltlichkeit ein Auftrag zugrunde liegt. ${ }^{480}$

aa) Web Service-Vermittler - Web Service-Anbieter

Wird der Web Service im Rahmen eines entgeltlichen Werkvertrags fehlerhaft veröffentlicht, so ist der Web Service-Vermittler zur Nacherfüllung durch eine berichtigte Veröffentlichung des Web Service im UDDI oder einen fehlerfreien Wiederholung der Veröffentlichung verpflichtet, sofern der Veröffentli-

\footnotetext{
479 S. A.III.1.b)aa) Verhältnis Web Service-Anbieter - Web Service-Vermittler.

480 S. A.III.1.d) Web Service-Nutzer - Web Service-Vermittler.
} 
chungszweck noch erreichbar ist. ${ }^{481} \mathrm{Da}$ im UDDI veröffentlichte Web Services meist auf einen dauerhaften Dienst gerichtet sind, lässt sich der Zweck der Veröffentlichung, das Finden dieses speziellen Dienstes durch Dritte, zumindest noch für künftige Web Service-Nutzer erreichen. Insofern stellt die Nacherfüllung ein durchaus wichtiges Recht des Web Service-Anbieters im Rahmen der fehlerhaften Veröffentlichung des Web Service dar. Ist der Zweck nicht mehr erreichbar, so kann der Web Service-Anbieter den Vertrag auch kündigen, mit dem Nachteil nach $\$ 649$ BGB die volle Vergütung entrichten zu müssen. ${ }^{482}$

Interessanter für den Web Service-Anbieter ist indes die Geltendmachung von Schadensersatz für mangelbezogene Folgeschäden wegen entgangenen Gewinns, wenn sein Web Service aufgrund einer fehlerhaften Veröffentlichung oder eines technischen Defizits auf Seiten des Web Service-Vermittlers nicht gefunden wird. Zum einen wird der Web Service-Vermittler aber, wie auch der Web Service-Hersteller gegenüber dem Web Service-Anbieter, eine Haftung für Schäden und mangelbezogene Folgeschäden in seinen Geschäftsbedingungen ausschließen, was nach der hier vertretenen Auffassung zumindest für leichte Fahrlässigkeit möglich ist. ${ }^{483}$ Zum anderen ist es - jenseits eines etwaigen Haftungsausschlusses durch den Web Service-Vermittler - aber fraglich, ob dem Web Service-Anbieter diesbezüglich überhaupt ein Schadensersatzanspruch zusteht. Verdienstausfälle fallen unter den Anwendungsbereich des \252 BGB und können danach als entgangener Gewinn geltend gemacht werden. \252 S. 2 BGB enthält eine Beweiserleichterung ${ }^{484}$ bezüglich des Vorliegens von entgangenen Gewinn, wenn ein solcher nach dem gewöhnlichen Lauf der Dinge oder nach den besonderen Umständen, insbesondere

481 S. zum Anzeigenvertrag: MünchKommBGB/Busche, Bd. 4, \631 Rn. 237; Staudinger/Peters, \631 Rn. 26; Palandt/Sprau, Einf. v. \631 Rn 18. Zur Bannerwerbung im Internet: Cichon, \5 Rn. 577.

482 MünchKommBGB/Busche, Bd. 4, \631 Rn. 237; Staudinger/Peters, \ 631 Rn. 26.

483 S. zur Zulässigkeit der Freizeichnung von mangelbezogenen Folgeschäden in AGBs sowie möglichen Rechtswahlklauseln: A.III.7.a)i) Web Service-Anbieter gegenüber Web ServiceHersteller.

484 \252 S. 2 BGB begrenzt nicht den Umfang des entgangenen Gewinns, sondern schafft vielmehr eine Beweiserleichterung: wenn ein solcher Gewinn dem gewöhnlichen Lauf der Dinge entsprechen würde, dann wird der Gewinn vermutet, gleichgültig ob er zum entsprechenden Zeitpunkt tatsächlich erwartbar war oder nicht. Ist das nicht der Fall, so können dennoch potenzielle Posten ersetzt werden, wenn der Geschädigte dafür den vollen Beweis erbringt, s. BGH, NJW 1959, 1079; NJW 1979, 1403 (1404); NJW 1983, 758; NJW-RR 1996, 1077 (1078 f.); NJW 2005, 3348; Staudinger/Schiemann, \252 Rn. 18; MünchKommBGB/Oetker, Bd. 2, \252 Rn. 4 ff. u. 31; Erman/Kuckuk, \252 Rn. 10. 
nach den getroffenen Anstalten und Vorkehrungen, mit Wahrscheinlichkeit erwartet werden konnte. Vor dem Hintergrund, dass die Web ServiceNutzung im Rahmen einer Serviceorientierten Architektur bislang noch kaum praktische Anwendungsfälle aufweist, erscheint es schwierig von einem gewöhnlichen Lauf der Dinge zu sprechen. Da aber das UDDI Ähnlichkeiten mit Branchenbüchern wie den Gelben Seiten aufweist und hinzu kommt, dass das UDDI im Rahmen der Serviceorientierten Architektur das einzige Mittel zum Auffinden eines Web Service und demnach auch zum späteren Vertragsschluss zwischen Web Service-Anbieter und Web Service-Nutzer darstellt, 485 ist durchaus anzunehmen, dass eine korrekte Eintragung im UDDI zu Vertragsschlüssen zwischen Web Service-Anbieter und Web Service-Nutzer und somit auch zu gewissen Einkünften beim Web Service-Anbieter führt. Insofern wird wohl die Beweiserleichterung des $\ 252$ S. 2 BGB zugunsten der Annahme eines entgangenen Gewinns als Schaden greifen. Wie hoch dieser Schaden letztlich zu beziffern wäre, hängt jeweils von den Umständen des Einzelfalls und insbesondere des sich herauskristallisierenden Marktpreises für die Nutzung des jeweiligen Dienstes ab. Abseits des Vorliegens eines Schadens, stellt sich jedoch das Problem des Zurechnungszusammenhangs zwischen fehlerhafter beziehungsweise Nichteintragung im UDDI oder auch bloBen Nichtanzeigens des Web Service-Anbieters nach einer entsprechenden Suche des Web Service-Nutzers und dem entgangenen Gewinn. Hier könnte man die Rechtsprechung zum Unterlassen von Einträgen im Telefonbuch oder in den Gelben Seiten heranziehen. Danach muss der Geschädigte die Kausalität dezidiert nachweisen, denn der fehlende Eintrag allein soll eher nicht zu Umsatzeinbußen führen. ${ }^{486}$ Ähnlich derartigen Verzeichnisdiensten ist auch bei einer erfolgreichen Suche im UDDI noch nicht gewährleistet, dass der Web Service-Nutzer auch gerade diesen Web Service wählt. Eine solche Wahl wird sich eher nach den sonstigen Angeboten anderer vergleichbarer Web Service-Anbieter richten. Etwas anderes gilt freilich, wenn der Web Service-Anbieter der einzige Anbieter ist, der einen bestimmten Service anbietet.

485 Damit soll indes nicht impliziert werden, dass der Diensteanbieter seinen Web Service auch nicht in anderen UDDIs veröffentlichen kann, gemeint ist lediglich, dass ein Auffinden lediglich über Dienstverzeichnisse möglich ist, nicht hingegen durch Anzeigen in Zeitungen, Schaufensterbummel oder ähnlichem. Auch eine Mund-zu-Mund-Propaganda wird aufgrund der Anonymität des Internet eher nicht die Regel sein, so dass der Diensteanbieter im Rahmen der SOA als Verbreitungsmedium auf das UDDI angewiesen ist.

486 AG Köln, Archiv PT 1996, 345; LG Bonn, Archiv PT 1996, 343 (344); dem zustimmend allerdings wohl mit a.A. bei Telefonbüchern Statz, Anm. zu OLG Düsseldorf, Archiv PT 1996, 342 f.; LG Bonn, Archiv PT 1996, 343 f. und AG Köln, Archiv PT 1996, 345, Archiv PT 1996, 344 (345). 
Der Unterschied zu herkömmlichen Branchenverzeichnissen besteht aber darin, dass Web Services im Rahmen der Serviceorientierten Architektur lediglich UDDIs als Möglichkeit haben, auf sich aufmerksam zu machen und die Kausalität sich bei fehlenden Einnahmen daher dann in den meisten Fällen zwangsläufig ergibt. Insofern wäre ein Schadensersatzanspruch des Web Service-Anbieters gegen den Web Service-Vermittler wegen entgangenen Gewinns möglich, wenn dieser nicht die Haftung für mangelbezogene Folgeschäden ausgeschlossen hat oder der Web Service-Vermittler die fehlerhafte Dienstesuche, den fehlerhaften oder nicht erfolgten Eintrag grob fahrlässig beziehungsweise gar vorsätzlich verschuldet hat. Dies gilt grundsätzlich auch wenn der Web Service-Vermittler unentgeltlich für den Web Service-Anbieter tätig wird, da im Rahmen von unentgeltlichen Aufträgen weitestgehend die gleichen Haftungsmaßstäbe wie bei entgeltlichen Verträgen gelten. 487

Ein Anspruch aus $\int 823$ Abs. 1 BGB wegen eines Eingriffs in das Recht am eingerichteten und ausgeübten Gewerbebetrieb kommt dagegen mangels Betriebsbezogenheit des Eingriffs nicht in Betracht. ${ }^{488}$ Ein $\int 45 \mathrm{~m}$ Abs. 1 TKG ${ }^{489}$ vergleichbares Schutzgesetz zur Eintragung in ein UDDI existiert nicht, so dass auch ein Schadensersatzanspruch wegen der Verletzung eines Schutzgesetzes nach $\int 823$ Abs. 2 BGB nicht in Betracht kommt.

\section{bb) Web Service-Vermittler - Web Service-Nutzer}

Verläuft die Dienstesuche des Web Service-Nutzers beim Web ServiceVermittler fehlerhaft, so kann der Web Service-Nutzer im Falle eines entgeltlichen Werkvertrags zunächst eine fehlerfreie erneute Suche verlangen, bei weiteren erfolgslosen Suchen mindern (sofern sinnvoll) oder Schadensersatz verlangen, wobei auch hier wieder Haftungsausschlüsse im Rahmen der Allgemeinen Geschäftsbedingungen vereinbart sein werden. Ein Rücktritt wird dagegen bei einer abrufbasierten Abrechnung aus der Natur der Sache nicht in Frage kommen.

487 S. dazu ausführlich MünchKommBGB/Seiler, Bd. 4, \662 Rn. 53 ff.; Medicus, Rn. 369; BGH, NJW 1959, 1221 (1223).

488 OLG Celle, Urt. v. 07.09.2006 - 8 U 99/06, Juris-Dokument, Rn. 23 ff.; LG Kleve, Archiv PT 1996, 256 (258) m. Anm. Staťr, zu diesem Punkt bestätigt durch OLG Düsseldorf, Archiv PT 1996, 342 (343).

$489 \int 45 \mathrm{~m}$ Abs. 1 S. 1 u. 2 TKG gewähren dem Teilnehmer einen Eintragungs- und Berichtigungsanspruch bzgl. der Aufnahme in öffentliche Telefonteilnehmerverzeichnisse, s. dazu BeckTKGKomm/Dablke, \45m TKG-E 2005 Rn. 11. 
Handelt es sich um einen unentgeltlichen Auftrag, so hat der Web ServiceNutzer lediglich einen Anspruch auf Schadensersatz, sofern dieser nicht in den Allgemeinen Geschäftsbedingungen ausgeschlossen ist.

c) Resümee

Ansprüche der Akteure im Rahmen der Serviceorientierten Architektur rund um fehlerhaft agierende Web Services und eine fehlerhafte Dienstesuche sind, wie die vorangegangenen Untersuchungen zeigen, durchaus vorhanden. Allerdings werden sie in den für die Beteiligten finanziell interessantesten Bereichen, der Schadensersatzhaftung, in der Praxis entweder in Individualvereinbarungen 490 oder häufiger in Allgemeinen Geschäftsbedingungen regelmäßig ausgeschlossen. Ein solcher Ausschluss hält der Inhaltskontrolle stand, sofern er sich lediglich auf leicht fahrlässig bezogene Schäden bezieht. Schadensersatz können die Beteiligten in den Fällen der wirksamen Freizeichnung daher nur bei grober Fahrlässigkeit oder gar Vorsatz geltend machen, was allerdings nicht sehr häufig vorkommen wird, so dass die Schadensersatzhaftung bei Leistungsstörungen im Rahmen der Web Service-Nutzung eine eher untergeordnete Rolle spielen wird.

Daher wird sich der Großteil der Mängelansprüche bei entgeltlichen Verträgen auf Nacherfüllung (sofern noch möglich) oder eine Minderung (sofern diese sinnvoll ist) beschränken. Im Verhältnis Web Service-Anbieter - Web ServiceVermittler ist zudem eine Kündigung möglich, wenn eine Nachholung nicht mehr sinnvoll ist. Dabei trägt der Web Service-Anbieter aber das volle Vergütungsrisiko. Bei unentgeltlichen Verträgen kommen grundsätzlich nur Schadensersatzansprüche in Frage. Sind solche in den Allgemeinen Geschäftsbedingungen ausgeschlossen, so stehen dem Geschädigten keine anderen Rechtsbehelfe zur Verfügung.

Noch beschränkter können die Ansprüche der Beteiligten ausfallen, wenn die Beteiligten, auch in Allgemeinen Geschäftsbedingungen, die Geltung einer fremden Rechtsordnung vereinbart haben. In derartigen Fällen sind die Klauseln des Verwenders der Inhaltskontrolle entzogen, so dass die Freizeichnung bei Leistungsstörungen je nach Rechtsordnung durchaus breiter ausfallen kann und somit die Ansprüche des Geschädigten sehr begrenzt sind.

490 Solche werden nur im Verhältnis Web Service-Anbieter - Web Service-Hersteller geschlossen, da in den anderen Beziehungen in der Regel kein persönlicher Kontakt erfolgt. 


\section{Abrechnungssysteme}

Werden Web Services entgeltlich angeboten, so stellt sich die Problematik eines sinnvollen Abrechnungssystems. Abrechnungssysteme für die Web Service-Nutzung sind in ganz unterschiedlicher Art und Weise umsetzbar. Welches System letztlich genutzt wird, liegt an den Präferenzen und im Ermessen des Web Service-Anbieters. Eine genaue Darstellung derartiger Systeme kann im Rahmen dieser Arbeit nicht geleistet werden, zumal sie überwiegend von technischem nicht aber rechtlichem Interesse sind. Daher soll hier nur ein kurzer Überblick gewährt werden.

Der Sinn und Zweck der Serviceorientierten Architektur ist der hohe Automatisierungsgrad hinsichtlich der Implementierung und Nutzung des Web Service, daher ist eine Interaktion der Akteure bei der Abrechnung nicht erwünscht. ${ }^{491}$ Praktisch sinnvoll ist deshalb nur die Verwendung von automatisierten Abrechnungssystemen. Dabei kommen sowohl eigene Abrechungssysteme des Web Service-Anbieters als auch zentrale Abrechnungssysteme in Frage, wobei unterschiedliche Abrechnungsmodelle, wie eine monatliche Grundgebühr, verbrauchsabhängige Systeme oder Volumenabrechnungen (so genannte „pay as you use“-Systeme) ${ }^{492}$ und ähnliches möglich sind. Zentrale Abrechungssysteme setzen eine Registrierung sowohl auf Web ServiceAnbieter- als auch auf Web Service-Nutzerseite voraus, damit die Abrechnung dann tatsächlich vollautomatisiert ablaufen kann. ${ }^{493}$ Zentrale Abrechnungssysteme haben den Vorteil, dass durch ihren Einsatz Transaktionskosten gesenkt werden und das Abrechnungssystem standardisiert für alle Web ServiceAnbieter und -Nutzer verwendet werden kann. ${ }^{494}$

\section{B. Außervertragliche Haftung}

Treten Störungen im Rahmen der Serviceorientierten Architektur durch rechtswidrige oder fehlerhafte Handlungen der Beteiligten oder technische Fehler auf, so können auch außerhalb der vertraglichen Beziehungen Ansprüche der Beteiligten aber auch Dritter bestehen. Berühren rechtswidrige Handlungen der Beteiligten Konkurrenten, so erlangen insbesondere - aber nicht

\footnotetext{
491 Burkhardt/Hagenhoff, Arbeitspapier Nr. 26/2003, S. 4; dies voraussetzend Melzer, S. 299.

492 S. BITKOM, Leitfaden Web Services, S. 25.

493 Burkhardt/Hagenhoff, Arbeitspapier Nr. 26/2003, S. 8 ff.

494 Burkhardt/Hagenhoff, Arbeitspapier Nr. 26/2003, S. 6.
} 
allein - wettbewerbsrechtliche Ansprüche an Bedeutung. Dabei können die Betroffenen - sofern nicht wettbewerbsrechtliche Ansprüche Gegenstand sind - sowohl gegen den Verletzer als auch, je nach Sachverhalt, nach den Grundsätzen der so genannten Störerhaftung gegen den die Rechtsverletzung fördernden Betreiber des UDDI (Web Service-Vermittler), des Portals (Web Service-Nutzer) etc. vorgehen. Denn, wie der Bundesgerichtshof 495 erst jüngst klargestellt hat, besteht keine formelle Subsidiarität des mittelbaren Störers gegenüber dem unmittelbaren Täter. Im Rahmen wettbewerbsrechtlicher Verstöße hat der BGH in einer neueren Entscheidung von seiner bisherigen Rechtsprechung, in der er stets die Grundsätze der Störerhaftung anwandte, 496 offensichtlich aufgegeben und prüft dort, ob eine Verletzung gegen allgemeine Verkehrspflichten vorliegt. ${ }^{497}$ Zwar fehlt es aktuell noch an einer weiteren Entscheidung des BGH, die die Aufgabe der Störerhaftung im Wettbewerbsrecht bestätigt, jedoch spricht aufgrund der plötzlichen Abkehr ohne weitere Begründung durch den BGH einiges dafür, dass im Bereich des Wettbewerbsrechts das Institut der Störerhaftung aufgegeben wurde. ${ }^{498}$ Für den Bereich des Urheberrechts und des gewerblichen Rechtsschutzes hält der BGH aber offensichtlich weiterhin an der verschuldensunabhängigen Störerhaftung fest. 499

Ziel der verschuldensunabhängigen Störerhaftung ist entweder die dauerhafte Sperrung oder aber die Entfernung beziehungsweise Löschung des Inhalts. 500 Für die vorliegende Arbeit relevante zivilrechtliche Anspruchsgrundlagen, die Beseitigung und Unterlassung gegenüber dem Störer gewähren, sind die $\int \mathbb{S} 1004,12$ BGB, $\ 37$ Abs. 2 HGB sowie die $\int \mathbb{S} 14,15$ MarkenG. Diese Anspruchsgrundlagen mit unterschiedlicher Schutzrichtung, ähneln sich in ihrer Grundstruktur. 501 Zugunsten des Web Service-Vermittlers als

BGH, MMR 2007, 518 (519).

Statt vieler z.B. noch BGH, GRUR 2002, 618 (619) - Meißner Dekor.

BGH, MMR 2007, 634 (635 ff.), der in diesem Fall die Grundlage nur allgemeinen Rechtsinstitut der deliktischen Verkehrspflichten sucht, ohne aber explizit die wettbewerbsrechtliche Störerhaftung aufzugeben. So auch Hefermehl/Köhler/Bornkamm/Köbler, \8 UWG Rn. 2.14a; Köbler, GRUR 2008, 1 ff.; Döring, WRP 2007, 1137 ff.

98 S. Volkmann, K\&R 2009, 362.

So jedenfalls jüngst BGH, CR 2009, 450 (451) Tz. 16 - Halzband und BGH, GRUR 2009, 841 (842 f.) - Cybersky.

Volkmann, S. 53.

Volkmann, S. 53. 
Telemediendiensteanbieter nach $₫ 2 \mathrm{Nr} .1 \mathrm{TMG}^{502}$ scheint allerdings die Haftungsprivilegierung des $\int 10$ TMG zu greifen, nach welcher der Web ServiceVermittler nicht haften müsste. Beseitigungs- und Unterlassungsansprüche werden im Telemediengesetz aber nicht positiv geregelt. ${ }^{503}$ \ 7 Abs. 2 S. 2 TMG hält lediglich fest, dass „Verpflichtungen zur Entfernung oder Sperrung der Nutzung von Informationen nach den allgemeinen Gesetzen [...] auch im Falle der Nichtverantwortlichkeit des Diensteanbieters nach den $\iint 8$ bis 10 [TMG] unberührt" bleiben. Daher entbrannte in Literatur und Rechtsprechung ein Streit um die Anwendbarkeit der Haftungsprivilegierungen auf die Störerhaftung. ${ }^{504}$ Durch die stetige Rechtsprechung ${ }^{505}$ nach dem Grundsatzurteil „Internetversteigerung“ des Bundesgerichtshofs ${ }^{506}$ dürfte nun aber weitestgehend unbestritten sein, dass die Haftungsprivilegierung gerade keine Anwendung auf die Störerhaftung findet. Dies zeigt sowohl $\ 7$ Abs. 2 S. 2 TMG als auch die Entstehungsgeschichte des Telemediengesetzes. ${ }^{507}$ Insofern können Web Service-Anbieter - je nach Rechtsverletzung - nach den jeweils einschlägigen allgemeinen Normen in Anspruch genommen werden. Da die

502 S. A.II.3 Web Service-Vermittler.

503 S. zu diesem Dilemma ausführlich Volkmann, S. 100; Spindler/Volkmann, WRP 2003, 3; Spindler, in: Spindler/Wiebe, Kap. 6 Rn. 35; Leible/Sosnitza; WRP 2004, 598; ferner Hoffmann, MMR 2002, 286.

504 S. zum Streit samt der Argumentationen ausführlich: Spindler, in: Spindler/Wiebe, Kap. 6 Rn. 36. Für eine Anwendbarkeit der Haftungsprivilegierung auf die Störerhaftung noch OLG Düsseldorf, MMR 2004, 315 (316); OLG Brandenburg, MMR 2004, 330 (332); LG Düsseldorf, MMR 2003, 120 (123); LG Berlin, MMR 2004, 195 (197); Hoeren, Anm. zu BGH, MMR 2004, 668 ff., MMR 2004, 672 f.; Ehret, CR 2003, 759 f.; v. Samson-Himmelstjerna/Rücker, in: Bräutigam/Leupold, B V Rn. 129 ff.

505 BGH, MMR 2007, 634 (665) - Jugendgefährdende Medien bei eBay; MMR 2007, 507 (508) Internetversteigerung II m. Anm. Spindler, MMR 2007, 518; OLG Köln, MMR 2007, 786 (787); OLG Hamburg, MMR 2006, 744 (745) u. MMR 2006, 238 (239); OLG München, MMR 2006, 739 (741); OLG Düsseldorf, MMR 2006, 618 (619) u. MMR 2006, 553 (555); OLG Brandenburg, MMR 2006, 107.

506 BGH, NJW 2004, 3102 (3103 ff.) - Internetversteigerung.

507 So auch Spindler, in: Spindler/Wiebe, Kap. 6 Rn. 36; Hoeren, Internet- und KommunikationsR, Rn. 711; Ufer, S. 140 ff.; Härting, Rn. 1061; Neubauer, in: Moritz/Dreier, D Rn. 55 ff.; Fülbier, CR 2007, 518 f.; Staudinger, in: Leible/Sosnitza, Teil 3, F Rn. 498 ff.; Rössel, Anm. zu BGH, CR 2007, 523 ff., CR 2007, 527 (528); s. auch die Rspr.-Übersicht von Volkmann, K\&R 2006, 245 ff. So bereits vor der Grundsatzentscheidung des BGH: Spindler/Volkmann, WRP 2003, 3; Spindler/Schmitz/Geis/Spindler, $\$ 8$ TDG Rn. 18 ff.; Freytag, in: Hermann/Ohly, S. 152; Volkmann, K\&R 2004, 231; Wülfing/Dieckert, S. 90; Jacobs, in: FS Erdmann, S. 340; Lehment, WRP 2003, 1062. Kritisch Rücker, CR 2005, 347 ff.; a.A. Gercke, Anm. zu AG Potsdam, CR 2005, 232, CR $2005,233 \mathrm{f}$. 
Störerhaftung aber nicht über Gebühr auf Dritte erstreckt werden soll, unterliegt sie gewissen Einschränkungen. Zum einen muss der Störer analog $\mathbb{\$} 10$ Nr. 1 TMG Kenntnis (Kennenmüssen genügt) von der Rechtsverletzung erlangt haben ${ }^{508}$ und zum anderen muss er zumutbare Prüfungs- und Kontrollpflichten verletzt haben. 509 Wann Prüfungs- und Kontrollpflichten zumutbar sind, ist immer anhand des Einzelfalls zu bestimmen. Betrachtet man allerdings die Rechtsprechung der vergangenen Jahre, so geht die Tendenz hin zu keinen allzu strengen Anforderungen an die Zumutbarkeit. ${ }^{510}$

\section{Anwendbares Recht bei außervertraglichen Beziehungen}

\section{Deliktsrecht}

Auf Sachverhalte mit Auslandsberührung im Rahmen von außervertraglichen Schuldverhältnissen finden die Vorschriften der Rom II-Verordnung511 Anwendung. Die Rom II-Verordnung, die für schadensbegründende Ereignisse seit dem 11.1.2009 unmittelbar gilt, hat das allgemeine Kollisionsrecht um die für den vorliegenden Untersuchungsgegenstand relevanten Spezialregeln für das Internationale Privatrecht der Produkthaftung (Art. 5 Rom II-VO), des unlauteren Wettbewerbs und der Wettbewerbsbeschränkungen (Art. 6 Rom II-VO) sowie des Immaterialgüterschutzes (Art. 8 Rom II-VO) ergänzt.

Nach Art. 4 Abs. 1 Rom II-VO gilt der Grundsatz des Tatorts. Danach ist grundsätzlich das Recht des Staats anzuwenden, in dem der Schaden eintritt, unabhängig davon, in welchem Staat das schadensbegründende Ereignis oder indirekte Schadensfolgen eingetreten ist. Ausnahmen bestehen nach Art. 4

508 Spindler, in: Spindler/Wiebe, Kap. 6 Rn. 39; s. auch die Gesetzesbegründung des $\ 11$ TDG zum Schadensersatz: BT-Drs. 14/6098, S. 22; a.A. Standinger, in: Leible/Sosnitza, Teil 3, F Rn. 507.

509 BGH, GRUR 1997, 313 (315) - Architektenwettbewerb; GRUR 1999, 418 (420) - Möbelklassiker; NJW 2001, 3265 (3266) - ambiente.de; NJW 2004, 3102 (3105 f.) - Internetversteigerung. Die Voraussetzungen dogmatisch ausführlich aufzeigend OLG München, MMR 2006, 739 (740 f.).

510 S. nur BGH, MMR 2007, 518 ff. zur Haftung eines Verlags für Meinungsforen; OLG Hamburg, MMR 2006, 398 ff. - Cybersky, zur Haftung eines Softwareherstellers dessen Programm die Umgehung des Pay-TV ermöglicht; LG Köln, ZUM 2007, 568 ff. - Rapidshare, zur Haftung eines Web-Hosters, der den Upload von Musikstücken ohne weiteren Verzeichnisdienst ermöglicht; LG Hamburg, MMR 2007, 333 ff. - Usenet m. Anm. Hoeren, zur Haftung des Zugangsvermittlers zu einem Netzwerk auf dem rechtswidriges Material veröffentlicht wurde.

511 Verordnung (EG) Nr. 864/2007 des Europäischen Parlaments und des Rates v. 11.7.2007 über das auf außervertragliche Schuldverhältnisse anzuwendende Recht („Rom II“), ABl. EG L $199 / 40$ v. 31.7.2007, S. 40-49. 
Abs. 2 Rom II-VO nur im Falle eines gemeinsamen gewöhnlichen Aufenthaltsorts des Schädigers und des Geschädigten oder nach Abs. 3 aufgrund einer engeren Verbindung zu einem anderen Staat.

Für die Produkthaftung gelten nach aktueller Rechtslage zwar die allgemeinen Anknüpfungsregeln, allerdings bestehen Besonderheiten hinsichtlich der Bestimmung des Handlungs- und Erfolgsorts. ${ }^{512}$ So ist der Handlungsort der Sitz des Herstellers. ${ }^{513}$ Hinsichtlich des Erfolgsorts wird auf den Geschädigten abgestellt: ist der Erwerber, hier der Web Service-Anbieter, Geschädigter, so wird allein auf den Erwerbsort abgestellt, ist der Geschädigte ein unbeteiligter Dritter, Web Service-Nutzer, Web Service-Vermittler (eher selten) oder Dritter, so wird an den Ort der Rechtsverletzung angeknüpft. ${ }^{514}$ Das Herkunftslandprinzip des $₫ 3$ TMG ist für den Untersuchungsgegenstand nur insoweit relevant, als es um produkthaftungsrelevante Pflichten geht, die online erbracht werden, was lediglich dann der Fall der ist, wenn die Web Services vom Hersteller elektronisch geliefert werden. ${ }^{515}$

Die Tatortregelung wird im Bereich der Produkthaftung allerdings durch die drei Stufenregelung in Art. 5 Abs. 1 Rom II-VO modifiziert. ${ }^{516}$ Grundsätzlich ist nach Art. 5 Abs. 1 S. 1 lit. a Rom II-VO das Recht des Staats anzuwenden, in dem der Geschädigte zum Zeitpunkt des Schadenseintritts seinen gewöhnlichen Aufenthalt hatte, sofern das Produkt auch in diesem Staat in Verkehr gebracht wurde. Wenn es allerdings an der letzteren Voraussetzung fehlt, kommt nach Art. 5 Abs. 1 S. 1 lit. b Rom II-VO das Recht des Staats zur Anwendung, in dem das Produkt erworben wurde, falls das Produkt in diesem Staat in Verkehr gebracht wurde. Ist auch das nicht der Fall, so ist nach Art. 5 Abs. 1 S. 1 lit c Rom II-VO das Recht des Staats anzuwenden, in dem der Schaden eingetreten ist, sofern das Produkt auch in diesem Staat in Verkehr gebracht wurde. Eine Ausnahme greift nach Art. 5 Abs. 1 S. 2 Rom II-VO nur dann, wenn die geschädigte Person das Inverkehrbringen des Produkts oder eines gleichartigen Produkts in dem Staat, dessen Recht nach den Buchstaben

512 Thorn, IPRax 2001, 562 f.; von Hoffmann/Thorn, \ 11 Rn. 49.

513 BGH, NJW 1981, 1606 f.; Thorn, IPRax 2001, 684; MünchKommBGB/Junker, Bd. 10, Art. 40 EGBGB Rn. 155; für verschiedene Handlungsorte Bamberger/Roth/Spickhoff, Art. 40 EGBGB Rn. 46.

514 Staudinger/von Hoffmann, Art. 40 EGBGB Rn. 95 f.; MünchKommBGB/Junker, Bd. 10, Art. 40 EGBGB Rn. 156; von Hoffmann/Thorn, \11 Rn. 49.

515 Spindler, RabelsZ 66 (2002), 392; Lurger/Vallant, RIW 2002, 190; Bamberger/Roth/Spickhoff, Art. 40 EGBGB Rn. 45.

516 S. Junker, NJW 2007, 3679. 
$\mathrm{a}, \mathrm{b}$ oder $\mathrm{c}$ anzuwenden ist, vernünftigerweise nicht voraussehen konnte. In diesem Fall ist das Recht des Staats anzuwenden, in dem die Person, deren Haftung geltend gemacht wird, ihren gewöhnlichen Aufenthalt hat.

\section{Markenrecht}

Im gesamten Bereich des internationalen Immaterialgüterrechts wird das Schutzlandprinzip, das nunmehr auch Art. 8 Abs. 1 Rom II-VO verwirklicht wird, angewandt, wonach das Recht des Staats angewendet werden soll, für dessen Territorium Schutz vor Verletzungshandlungen oder Verletzungserfolgen begehrt wird.517 Die Möglichkeit einer entgegenlaufenden Rechtsordnungsvereinbarung wird durch Art. 8 Abs. 3 Rom II-VO ausgeschlossen. Das Herkunftslandprinzip findet nach $₫ 3$ Abs. 4 Nr. 6 TMG keine Anwendung auf das Markenrecht und muss daher nicht modifizierend berücksichtigt werden.

\section{Wettbewerbsrecht}

Im Bereich des Wettbewerbsrechts gilt, wie bisher, auch nach Inkrafttreten der Rom II-VO das Marktortprinzip. Nach dem Marktortprinzip, das in Art. 6 Abs. 1 Rom II-VO verankert ist, wird an den Ort der wettbewerbsrechtlichen Interessenkollision angeknüpft. ${ }^{518}$ Art. 6 Abs. 4 Rom II-VO schließt die Möglichkeit einer Rechtswahl durch die Parteien aus. Bei europäischen Sachverhalten wird das Marktortprinzip im Bereich der Telemediendienste durch das Herkunftslandprinzip korrigiert. ${ }^{519}$

Da das Marktortprinzip aber dazu führt, dass eine Interessenkollision überall dort stattfindet, wo der Web Service angeboten wird, was gegebenenfalls aufgrund der Globalität des Internet weltweit sein kann, und Wettbewerber ihre

517 BGH, NJW 1998, 1395 (1396); NJW 1994, 2888 (2889); NJW 1992, 2824; Pfeiffer, in: Gounalakis, Rhb. Electronic Business, \12 Rn. 182; Spindler/Schuster/Pfeiffer/Weller, Art. 40 EGBGB Rn. 21; Mankowski, GRUR Int. 1999, 996 f.; Sack, WRP 2000, 279; Obergfell, IPRax 2005, 11.

518 BGH, NJW 1962, 37 (38) - Kindersaugflaschen; GRUR 1971, 153 (154) - Tampax; NJW 1991, 1054 - Kaffeefahrt ins Ausland; NJW 1998, 1227 (1228); GRUR 2004, 1035 (1036); WRP 2006, 736 (738) - Arzneimittelwerbung im Internet; GRUR 2007, 245 - Schulden Hulp; Hefermehl/Köhler/Bornkamm/Köbler, Einl. UWG Rn. 5.5; jurisPK-UWG/Ullmann, Einleitung Rn. 71; Spindler/Schuster/Pfeiffer/Weller, Art. 40 EGBGB Rn. 17; Härting, Rn. 71; Mankowski, GRUR 1999, 909; Sack, WRP 2000, 272; Spindler, RabelsZ 66 (2002), 693.

519 S. dazu ausführlich Spindler, RabelsZ 66 (2002), 694 f.; ders., RIW 2002, 185 f.; Sack, WRP 2002, 273 ff.; Spindler/Schuster/Pfeiffer/Weller, Art. 40 EGBGB Rn. 18. 
Ansprüche dann ebenfalls nach allen Rechtsordnungen geltend machen können, in denen die Handlung eine Rechtsverletzung darstellt, muss, um einer Uferlosigkeit des Internationalen Wettbewerbsrechts entgegen zu wirken, das Marktortprinzip im Bereich des Internet zurückhaltend angewendet werden. ${ }^{520}$ Daher ist bei Wettbewerbsverletzungen im Internet der Erfolgsort im Inland belegen, wenn sich der Internet-Auftritt bestimmungsgemäß dort auswirken soll oder ein nicht unwesentlicher Teil der Bevölkerung gezielt als mögliche Kunden angesprochen werden sollen. ${ }^{521}$ Wann und ob dies der Fall ist, wird anhand der Umstände des Einzelfalls ermittelt, wobei Sprache, Ausgestaltung der Homepage, Produkt- und Angebotsbesonderheiten, Disclaimer und ähnliches Anhaltspunkte bieten können. ${ }^{522}$

\section{Zusammenfassung}

Je nach Rechtscharakter der jeweiligen Beziehungen, sind bei Sachverhalten mit Auslandberührung die jeweiligen Besonderheiten des Internationalen Vertragsrechts zu beachten und gegebenenfalls ist ein fremdes Sachrecht anzuwenden. Im Rahmen der vorliegenden Untersuchung wird davon ausgegangen, dass deutsches Sachrecht Anwendung findet. Lediglich zu beachtende Besonderheiten des Internationalen Privatrechts werden an den entsprechenden Stellen erörtert.

\section{Mangel des Web Service}

Werden Softwarekomponenten wie Web Services im Rahmen der Serviceorientierten Architektur, aber auch außerhalb, eingesetzt, so stellt sich im Falle des Auftretens von Mängeln an der Web Service-Architektur neben den vertraglichen Ansprüchen 523 die Frage, welche außervertraglichen Ansprüche gegenüber wem bestehen. Dabei kann hinsichtlich der grundsätzlichen Problematik von Mängeln bei Web Services auf die Ausführungen zum Vertrags-

520 BGH, WRP 2006, 736 (738) - Arzneimittelwerbung im Internet; Hefermehl/Köhler/Bornkamm/Köbler, Einl. UWG Rn. 5.8; Freitag, in: Kröger/Gimmy, Hdb. InternetR, S. 453; Härting, Rn. 72 f.

521 BGH, WRP 2006, 736 (738) - Arzneimittelwerbung im Internet; GRUR 2005, 431 (432) HOTEL MARITIME; Hefermehl/Köhler/Bornkamm/Köbler, Einl. UWG Rn. 5.8; jurisPKUWG/Ullmann, Einleitung Rn. 75.

522 Hefermehl/Köhler/Bornkamm/Köbler, Einl. UWG Rn. 5.8; jurisPK-UWG/Ullmann, Einleitung Rn. 76; Freitag, in: Kröger/Gimmy, Hdb. InternetR, S. 454.

523 S. A.III.7 Leistungsstörungen. 
recht verwiesen werden. ${ }^{524}$ Für die vorliegende Arbeit geht es konkret um die Situation, dass die Mängel auf einen Fehler des Web Service und somit einem Softwaremangel beruhen. Es werden daher mögliche Ansprüche aus der verschuldensabhängigen Produzentenhaftung sowie der verschuldensunabhängigen Produkthaftung untersucht.

\section{Verschuldensabhängige Produzentenhaftung}

Die deliktischen Ansprüche nach den Grundsätzen der Produzentenhaftung können neben den vertraglichen Ansprüchen geltend gemacht werden. ${ }^{525}$ Die Produzentenhaftung hat gegenüber den vertraglichen Ansprüchen den Vorteil, dass auch Dritte außerhalb des Vertragsverhältnisses Schadensersatz geltend machen können. Im Rahmen der Serviceorientierten Architektur können auch Web Service-Nutzer und sogar der Dritte mit Hilfe der Produzentenhaftung Ansprüche gegen den Web Service-Hersteller geltend machen. Erforderlich für die Anwendung der Produzentenhaftung ist, neben der Eröffnung einer Gefahrenquelle durch die Inverkehrgabe eines Produkts, ${ }^{526}$ die vom Hersteller zu verantwortende Verletzung eines in $\ 823$ Abs. 1 BGB geschützten Rechtguts.

Software, und somit auch Web Services als Softwarekomponenten, lässt sich nach nunmehr herrschender Auffassung unter den Produktbegriff fassen.527 Werden jedoch Informationen durch Web Services nicht oder fehlerhaft übermittelt, so wird dies in der Regel nicht zu vorhandenen Datenverlusten, Schäden an der Hardware oder gar Personenschäden, als weitgehend anerkannte Rechtsgutverletzungen durch Software, ${ }^{528}$ sondern vielmehr zu Einkommenseinbußen und ähnlichem bei den Verwendern führen. Das bei Einkommenseinbußen geschädigte Vermögen gehört aber gerade nicht zu den von $\int 823$ Abs. 1 BGB geschützten Rechtsgütern. ${ }^{529}$ Insofern wird die Pro-

S. A.III.7 Leistungsstörungen.

BGH, NJW 1977, 377 (380); NJW 1976, 1505 (1506); MünchKommBGB/Wagner, Bd. 5, 』 823 Rn. 594; Palandt/Sprau, \823 Rn. 167/168.

Bamberger/Roth/Spindler, \823 Rn. 478; BGH, NJW 1988, 2611.

Spindler, Gutachten BSI, Rn. 104; ders., NJW 2004, 3145; ders., NJW 1999, 3737; ders., MMR 1998, 24; Deike, CR 2003, 15; Hohmann, NJW 1999, 524; Schneider, Hdb. EDV-Recht, J Rn. 294 ff.; Marly, Rn. 1303, 1309 ff.; Mankowski, in: Ernst, Kap. 3 Rn. 439.

S. dazu Spindler, Gutachten BSI, Rn. 107 ff.

Ganz h.M.: RGZ 51, 92 (93); 52, 365 (366); 58, 24 (28); 62, 315 (317); 95, 173 (174); 102, 223 (225); BGH, NJW 1958, 1041 (1042); NJW 1964, 720 (722); NJW 1983, 2313 f.; MünchKommBGB/Wagner, Bd. 5, \ 823 Rn. 184. 
duzentenhaftung bei Fehlern, die durch die Verwendung von Web Services auftreten, keine große Rolle spielen.

\section{Produkthaftung}

Im Gegensatz zur Produzentenhaftung ist die Haftung nach dem Produkthaftungsgesetz (ProdHaftG) verschuldensunabhängig. Web Services als Softwarekomponenten fallen zwar unter den Anwendungsbereich des Produkthaftungsgesetzes, 530 jedoch ist der Kreis der geschützten Rechtsgüter noch enger als der des $₫ 823$ Abs.1 BGB. Nach $\ 1$ ProdHaftG muss der Hersteller dem Geschädigten den Schaden ersetzen, wenn durch den Fehler des Produkts jemand getötet, sein Körper oder seine Gesundheit verletzt oder eine Sache beschädigt wird, wobei es sich dabei nach $₫ 1$ Abs. 1 S. 2 ProdHaftG um eine andere Sache als den Web Service selbst handeln muss. Auch hier gilt folglich, dass, wenn durch das fehlerhafte Suchen und Finden des Web Service keine andere Sache beschädigt wird - was eher selten vorkommt -, die Produkthaftung keinen besonderen Anwendungsbereich bei Web Services hat. Insofern kann auch eine Haftung nach dem Produkthaftungsgesetz untergewichtet werden. 531

\section{Missbrauch/Fehlfunktion des UDDI}

Die Konstellation der Serviceorientierten Architektur rund um das UDDI und den Web Service-Vermittler ermöglicht einen Wettbewerb der Web ServiceAnbieter. Aufgrund der Struktur des UDDI, das sich in weiße, gelbe und grüne Seiten unterteilt, worin allgemeine Informationen über den Web ServiceAnbieter sortiert nach Branchen und der technische Datenbestand des Service

530 Marly, Rn. 1303; Escher-Weingart, in: Gounalakis, Rhb. Electronic Business, $\ 38$ Rn. 45; Günther, S. 193 ff.; Spindler, NJW 1999, 3742; Lehmann, NJW 1992, 1724; s. auch BGH, NJW 2009, 2952 ff. zu einer fehlerhaften Steuerungsgerätesoftware für Airbags.

531 Sollte der Fall eintreten, dass eine entsprechende Rechtsverletzung auftritt, und weist der Sachverhalt Auslandsbezüge auf, so ist nach aktueller Rechtslage grds. das Recht des Staats, in dem der Web Service-Hersteller seinen Unternehmenssitz hat, anzuwenden, auf Verlangen aber auch das Sachrecht des Schädigungsorts, was bei dem Web Service-Anbieter der Erwerbsort und bei Web Service-Nutzer, Web Service-Vermittler oder Dritten der Ort der Rechtsverletzung ist. Eine Modifikation des anzuwenden Rechts durch das Herkunftslandprinzip erfolgt lediglich bei Rechtsordnungen von Mitgliedsstaaten der Europäischen Union und auch nur dann, wenn die Web Services elektronisch geliefert wurden. Nach Inkrafttreten der drei Stufenregelung in Art. 5 Abs. 1 Rom II-VO ist indes grundsätzlich das Recht des Staats anzuwenden, in dem der Geschädigte zum Zeitpunkt des Schadenseintritts seinen gewöhnlichen Aufenthalt hatte, sofern der Web Service-Hersteller den Web Service auch in diesem Staat in Verkehr gebracht hat, wobei hinsichtlich des Orts der Inverkehrgabe ggf. Folgeprobleme auftreten können. 
gespeichert und abgerufen werden können, ${ }^{532}$ wird eine Dienste- und somit auch eine für die Web Service-Nutzer günstige Marktvielfalt ermöglicht, die bei gesteigerter Nutzung durch Web Service-Anbieter und -nutzer einen echten Preis- und Servicewettbewerb zulässt. Der dadurch zwischen den Web Service-Anbietern entstehende Konkurrenzkampf um Preise und Qualität der Dienste, birgt aber zum einen die Gefahr der Nutzung unlauterer Praktiken, um sich gegen die Konkurrenz im Markt durchzusetzen beziehungsweise einen Vorteil zu verschaffen. Zum anderen können sich Fehler des Web Service-Vermittlers oder der technischen Suche im UDDI im Rahmen dieses Konkurrenzkampfs negativ für den betroffenen Web Service-Anbieter auswirken, der durch eine mögliche Nichtanzeige bei der UDDI-Suche Umsatzeinbußen erleidet, weil dem Web Service-Nutzer praktisch nur seine Konkurrenten zur Auswahl stehen. Wie die Betroffenen sich bei solchen Fehlern und gegen derartige Manipulationen rund um das UDDI wehren können, soll im folgenden Abschnitt untersucht werden.

\section{Feblerbafte Dienstesuche}

Verläuft die Dienstesuche durch den Web Service-Nutzer fehlerhaft, indem ein bestimmter Web Service-Anbieter, der Dienstleistungen mit seinem Web Service anbietet, bei den Ergebnissen der UDDI-Suche nicht angezeigt wird, so stellt sich für den Web Service-Anbieter neben der Geltendmachung von vertraglichen Ansprüchen die Frage, ob er noch andere Möglichkeiten hat, gegen den Web Service-Vermittler vorzugehen. Hintergrund einer solchen fehlerhaften Suche ist die fehlerhafte oder nicht erfolgte Veröffentlichung des Web Service durch den Web Service-Vermittler - trotz korrekter Dateneingabe des Web Service-Anbieters - oder ein technischer Fehler in der Web Service-Architektur, der zu fehlerhaften Suchergebnissen führt. ${ }^{533}$ Verläuft die Suche indes nicht derart, dass der suchende Web Service-Nutzer gar keine Ergebnisse erhält und so merken könnte, ${ }^{534}$ dass ein Fehler vorliegt, sondern dass ihm vielmehr andere den Suchkriterien entsprechende Web ServiceAnbieter angezeigt werden, so könnten wettbewerbsrechtliche Ansprüche, wie Unterlassung, Beseitigung vor allem aber Schadensersatz, wegen Übervorteilung der Konkurrenz und eigener Benachteiligung gegen den Web ServiceVermittler in Frage kommen.

532 S. B.II.2 Komponenten.

533 S. dazu bereits A.III.7 Leistungsstörungen u. A.III.7.b) Fehlerhafte Dienstesuche.

534 Freilich nicht zwangsläufig, da es auch möglich ist, dass ein solcher Dienst tatsächlich nicht existiert oder zumindest nicht im genutzten UDDI. 
Die für die nach den $\iint 8 \mathrm{ff}$. UWG erforderliche geschäftliche Handlung im Sinne des $₫ 2$ Nr. 1 UWG zur Geltendmachung etwaiger Ansprüche, liegt durch die Schaltung der Einträge im UDDI zugunsten der Web ServiceAnbieter, als andere Unternehmen, durch den Web Service-Vermittler vor. Der Web Service-Vermittler kommt auch als Anspruchsgegner solcher wettbewerbsrechtlicher Ansprüche in Betracht, da Verletzer jeder sein kann, der als Täter einer Zuwiderhandlung gegen die $\iint 3-7$ UWG selbst oder in mittelbarer Täterschaft die wettbewerblichen Interessen eines anderen verletzt oder zu verletzen droht. 535 Darüber hinaus muss die Wettbewerbshandlung aber auch unlauter sein. Unlauter sind im Allgemeinen alle Handlungen, die den anständigen Gepflogenheiten im Handel, Gewerbe, Handwerk oder selbstständiger beruflicher Tätigkeit zuwiderlaufen.536 Darunter fällt auch die Marktbehinderung in jeglicher Form. ${ }^{537}$ Die nicht erfolgte Anzeige des speziellen Web Service-Anbieters bei der Suche im UDDI beruht aber in der Regel auf einem technischen oder gegebenenfalls menschlichen Fehler. Gegen die anständigen Gepflogenheiten im Handelsverkehr verstößt dieser Fehler keineswegs. Daher kann sich der Web Service-Anbieter nicht auf wettbewerbsrechtliche Ansprüche gegen den Web Service-Vermittler stützen.

\section{Unternebmensnamen und fremde Marken}

Eine andere mögliche Situation entsteht, wenn ein Web Service-Anbieter im UDDI falsche Angaben macht, indem er bei seiner eigenen Beschreibung den Namen eines fremden, beispielsweise in diesem Bereich sehr bekannten Unternehmens angibt oder auch in der Dienstebeschreibung fremde Kennzeichen verwendet, 538 die einen großen Marktzulauf haben. Dabei wird das fremde Renommee genutzt, um selber häufiger von Web Service-Nutzern gefunden zu werden oder die Chancen zu erhöhen, dass der eigene Dienst in mehr Plattformen von Web Service-Nutzern eingebunden wird, der Web Service-Anbieter de facto also das UDDI missbraucht, damit sein Dienst häufiger genutzt wird, als die Dienste der Konkurrenz.

535 Piper/Ohly/Piper, \8 UWG Rn. 145; Hefermehl/Köhler/Bornkamm/Köbler, \8 UWG Rn. 2.5.

536 Piper/Ohly/Piper, $\$ 3$ UWG Rn. 6.

537 \4 Nr. 10 UWG nennt spezielle Marktbehinderungsfälle als Beispiel für unlautere Wettbewerbshandlungen. Die allgemeine Marktbehinderung wird von der Generalklausel des $₫ 3$ UWG erfasst, s. Begr. RegE zu \4 Nr. 10, BT-Drs. 15/1487, S. 19 - Entwurf eines Gesetzes gegen den unlauteren Wettbewerb (UWG) v. 22.8.2003.

538 S. zu den ungenügenden Verifikationsmechanismen in den vorhandenen UDDIs auch Küster, in: Fröschle, S. 10. 
Da die Kategorien des UDDI (Branche, Art der Leistung etc.) zunächst einmal die Grundlage der Suche des Web Service-Nutzers durch den Web ServiceVermittler bilden, lässt sich zur Untersuchung die Problematik der Metatags oder der „Weiß-auf-Weiß-Schrift“"539 in Verbindung mit Suchmaschinen heranziehen. Metatags sind Bestandteile der Seitenbeschreibung im HTML-Code einer Website, die einen Teil der Suchkriterien, an denen sich Suchmaschinen bei der Indizierung von Internetseiten im Falle einer Suchanfrage orientieren, darstellen - Metatags sind für den normalen Internetnutzer nicht ohne Weiteres sichtbar. ${ }^{540}$ Aus diesem Grunde nehmen Unternehmen gerne Namen und Marken von Konkurrenten in ihre Metatags auf, um auch bei Suchanfragen speziell zu diesen Wettbewerbern gelistet zu werden. Diese Praktik verstößt allerdings nach der überwiegenden Auffassung regelmäßig gegen markenrechtliche Vorschriften ${ }^{541}$ - sofern es sich nicht bloß um beschreibende Angaben handelt ${ }^{542}$ und auch tatsächlich eine Verwechslungsgefahr besteht ${ }^{543}$-sowie das Namens- und Firmenbezeichnungsrecht. Darüber hinaus ist eine derartige Verwendung fremder Kennzeichen in aller Regel auch nach den $\iint$ 3, 4 Nr. 10 UWG unlauter. ${ }^{544}$

\section{a) Marken- und Firmennamensverletzungen}

Durch ein Metatagging mit unzulässigen Kennzeichen werden in der Regel, sofern in den Metatags eine eingetragene Marke verwendet wurde, die Rechte

539 Die Weiß-auf-Weiß-Schrift auf HMTL-Seiten ist für die Suchergebnisse von noch größerer Bedeutung als Metatags, da sie von Suchmaschinen viel häufiger als Suchkriterium herangezogen werden, s. dazu Ernst, jurisPR-ITR 9/2007 Anm. 3; Ott, Anm. zu LG Braunschweig, MMR 2007, 121 f., MMR 2007, 123.

540 Hartl, MMR 2007, 12; Schirmbacher, ITRB 2007, 117; Renner, WRP 2007, 49; Stögmüller, CR 2007, 446 f.; Heim, CR 2005, 201; Fezer/Mankowski, Bd. 1, UWG, 『 4-S12 Rn. 76; Gloy/Loschelder/Schulte-Beckhausen, Hdb. WettbewerbsR, \ 29 Rn. 57.

541 S. die Rspr.: BGH, MMR 2007, 648 f.; NJW 2007, 153 ff. - Impuls; OLG Celle, MMR 2006, 817 f.; OLG Hamburg, MMR 2005, 186 ff.; zum Aspekt der Störerhaftung LG Köln, MMR 2006, 115 f.; a.A. OLG Düsseldorf, MMR 2006, 396 ff., die eine solche Verwendung als zulässig ansehen; OLG Frankfurt, GRUR-RR 2008, 292 f. H.L.: Nordemann, Rn. 1431; Gloy/Loschelder/Schulte-Beckhausen, Hdb. WettbewerbsR, I 29 Rn. 58 f.; Heim, CR 2005, 203; Hartl, MMR 2007, 14; Ullmann, GRUR 2007, 636.

So jüngst der BGH, MMR 2009, 331 (332 f.) - pcb zu AdWords. BGH, MMR 2009, 329 (330) - Beta Layout m. Anm. Hoeren zu Adwords.

OLG Hamm, GRUR-RR 2009, 186 (187); Gloy/Loschelder/Schulte-Beckhausen, Hdb. WettbewerbsR, \29 Rn. 60; Heim, CR 2005, 201; Hartl, MMR 2007, 14 f.; Renner, WRP 2007, 51. 
aus $\iint 14$ Abs. 1, 2, 15 Abs. 1, 2 MarkenG verletzt. Bei der Verwendung fremder Unternehmensbezeichnungen wird zugleich auch das Namensrecht des $\S 12$ BGB und bei Vorliegen eines Handelsgewerbes $₫ 37$ Abs. 2 HGB verletzt. ${ }^{545}$ Selbige Rechte kommen auch bei dem Missbrauch des UDDI in Betracht. Eine Verletzung dieser Rechte zieht Beseitigungs-, Unterlassungs- und Schadensersatzansprüche ${ }^{546}$ des Verletzten nach sich (s. $\iint 14$ Abs. 5, 6, 15 Abs. 1, 2, 4 und 5 MarkenG sowie $\int S$ 1004, 823 BGB). Im Gegensatz zu Metatags sind aber die Angaben im UDDI für den Suchenden auch ohne Weiteres sichtbar. Insofern stellt sich, entgegen der bei Metatags aufgekommenen Diskussion, nicht die Frage, ob überhaupt eine Nutzung im geschäftlichen Verkehr vorliegt. ${ }^{547}$ Freilich greifen die markenrechtlichen Ansprüche nur, wenn keine Schranken zugunsten des Verwenders vorliegen. Eine solche Schranke wäre $\ 23$ Nr. 2 und 3 MarkenG. $\int 23$ MarkenG schützt allerdings lediglich deskriptive Angaben über Merkmale und Eigenschaften von Waren und Dienstleistungen. ${ }^{548}$ Sollten solche deskriptiven Angaben im UDDI nicht notwendig sein - wobei regelmäßig keine Notwendigkeit vorliegen wird - ist die Verwendung fremder Kennzeichen oder Firmenbezeichnungen nicht von \23 MarkenG gedeckt und dem Kennzeicheninhaber stehen Unterlassungsund gegebenenfalls sogar Schadensersatzansprüche zu.

Agiert (mindestens) einer der beteiligten Akteure aus dem Ausland, so muss das Recht des Staats angewendet werden, in dem der Markeninhaber seine Marke angemeldet hat, das heißt im Falle einer deutschen Marke deutsches Recht und bei einer Gemeinschaftsmarke die Gemeinschaftsmarkenverordnung. Im Übrigen gilt der Schutz nur für den Staat in dem die Marke auch angemeldet wurde; 549 die Gemeinschaftsmarke genießt Schutz in ganz Europa, die deutsche Marke indes nur in der Bundesrepublik Deutschland.

545 OLG Celle, MMR 2006, 817 m. differenzierender Anm. Kazemi; Schirmbacher, ITRB 2007, 118; $\mathrm{zu}$ den Anforderungen des Namensschutzes durch $\ 12$ BGB bei Firmen Palandt/Heinrichs/Ellenberger, \12 Rn. 9.

546 Schadensersatzansprüche greifen allerdings nur im Falle eines Verschuldens des Schädigers.

547 So das OLG Düsseldorf, MMR 2006, 396 (397); MMR 2004, 319 (320 f.); Fezer, MarkenR, G Rn. 84. S. dazu allerdings die ablehnende Auffassung der Lit.: Renner, WRP 2007, 50 f.; Hartl, MMR 2007, 13; Heim, CR 2005, 201.

548 S. statt aller Fezer, MarkenR, \23 Rn. 1.

549 Nordemann, Rn. 2111; EuGH, GRUR 1994, 286 (288) - Quattro/Quadra. 


\section{b) Unlauterer Wettbewerb}

Als unlautere Wettbewerbshandlungen bei einem solchen Verhalten des Web Service-Anbieters kommen irreführende Werbung ( $\$ 5 \mathrm{UWG})$, Anlockung von Interessenten $(\mathbb{} 4$ Abs. $1 \mathrm{UWG})$ sowie ferner das Abfangen von Kunden $(\mathbb{\$} 4$ Abs. 10 UWG) in Betracht. Stellt die Nutzung fremder Begriffe, wie Firmenbezeichnungen oder sonstiger Kennzeichen, durch den Web Service-Anbieter eine unlautere Wettbewerbshandlung nach den $\$ \int 3$ ff. UWG dar, so können Unterlassungs- und Beseitigungsansprüche nach $\int 8$ UWG sowie Schadensersatzansprüche ( 9 UWG) geltend gemacht werden. Handelt der Web ServiceAnbieter hinsichtlich der unlauteren Wettbewerbshandlung vorsätzlich und erzielt hierdurch zu Lasten einer Vielzahl von Abnehmern einen Gewinn, so kann nach $\ 10$ Abs. 1 UWG darüber hinaus auch der damit erzielte Gewinn heraus verlangt werden.

Unterlassungs- und Beseitigungsansprüche können neben der Geltendmachung durch den Mitbewerber nur kollektiv durch die Verbände geltend gemacht werden, denen nach $\int 8$ Abs. 3 Nr. 2, Nr. 3 und Nr. 4 UWG eigene Unterlassungs- und Beseitigungsansprüche zustehen. Individuelle Unterlassungs- oder Beseitigungsansprüche eines Verbrauchers oder eines Nutzers, wie dem Web Service-Nutzer, können daher nicht geltend gemacht werden. Die Ansprüche sind insofern abschließend. ${ }^{550}$ Gleiches gilt für eine etwaige Gewinnabschöpfung, da hier nach $\ 10$ Abs. 1 UWG ausdrücklich auf die Anspruchsberechtigten des $\int 8$ UWG verwiesen wird. Schadensersatzansprüche nach $₫ 9$ UWG stehen sogar nur dem Mitbewerber, und demnach anderen Web Service-Anbietern, zu. Wird der Web Service-Nutzer daher von falschen Angaben des Web Service-Anbieters im Rahmen der Trefferliste berührt und entstehen ihm dadurch Schäden, so kann er sich nicht auf Ansprüche nach dem UWG berufen. Diese stehen nur Mitbewerbern und zum Teil entsprechenden Verbänden zur Verfügung.

Da das UDDI regelmäßig weltweit abgerufen werden kann, muss im Fall des unlauteren Wettbewerbs im Ausland nach dem modifizierten Marktortprinzip anhand des konkreten UDDI-Eintrags untersucht werden, ob sich der UDDIEintrag bestimmungsgemäß in diesem Staat auswirken sollte oder ein nicht unwesentlicher Teil der Bevölkerung gezielt als mögliche Kunden angesprochen werden sollte. Trifft eine dieser Möglichkeiten zu, so muss das Recht dieses Staats angewandt werden.

550 S. Begr. RegE zu \8, BT-Drs. 15/1487, S. 22; Hefermehl/Köhler/Bornkamm/Bornkamm, \5 UWG Rn. 1.11; jurisPK-UWG/Ernst, $\$ 8$ Rn. 90. 


\section{aa) Irreführung}

Mit Marken- und Firmennamenverletzungen können auch Irreführungen im Sinne der $\int \S 3,5$ UWG einhergehen. ${ }^{551} \mathrm{Ob}$ eine solche Irreführung im Falle des UDDI-Eintrags mit Verwendung fremder Kennzeichen oder Firmennamen vorliegt, muss anhand eines verständigen, durchschnittlich informierten Nutzers beurteilt werden. ${ }^{552}$ Während man bei einem Suchmaschinennutzer noch davon ausgehen kann, dass dieser durchaus auch mit irrelevanten Treffern rechnet, so dass ein bloßes Erscheinen in der Trefferliste noch nicht zwangsläufig für eine Irreführung ausreicht, ${ }^{553}$ erscheint eine derartige Annahme bei dem Nutzer der UDDI-Suchfunktion sehr fraglich. UDDIs haben im Gegensatz zu Suchmaschinen einen viel kleineren Suchbereich. Dieser beschränkt sich, statt auf das World Wide Web, lediglich auf die eingetragenen Inhalte des UDDI. Der Eintrag im UDDI dient zweifellos der Förderung der Nutzung seiner Dienste, ${ }^{554}$ so dass es sich um Werbung im Sinne des $₫ 5$ UWG handelt. Für eine Einstufung als irreführende Werbung kann jede täuschende Handlung oder zur Täuschung geeignete Handlung, die den Werbeadressaten in seiner wirtschaftlichen Entscheidung beeinflusst beziehungsweise beeinflussen kann oder die Mitbewerber schädigt beziehungsweise schädigen kann, genügen, so dass es für eine Irreführung bereits ausreicht, wenn sich der Verkehr aufgrund dieses Verhaltens überhaupt erst mit der Werbung befasst. ${ }^{555}$ Trägt der Web Service-Anbieter im UDDI eine fremde Firma oder (bekannte) fremde Kennzeichen unbefugt ein, um seine eigene Trefferquote bei der Suche zu erhöhen, so kann dieses Verhalten sehr wohl dazu geeignet sein, den Web Service-Nutzer in seiner Entscheidung um die Wahl eines Web Service-Anbieters - insbesondere wenn sich die benutzten Kennzeichen und Firmen im Rahmen der gleichen Dienstekategorie befinden - zu beeinflussen und dadurch die benutzte Firma mitsamt ihrer Kennzeichen zu schädigen. Zumindest aber wird sich der Web Service-Nutzer, der den Web

551 S. z.B. das Urteil des LG Mannheim, MMR 1998, 217 (218); i.d.S. auch LG Hamburg, MMR 2000, 46 (47); Hartl, MMR 2007, 14 f. der das Vorliegen einer Irreführung bei Metatags in der Regel aber ablehnt; grds. a.A. OLG Düsseldorf, MMR 2004, 319 (321).

552 BGH, NJW-RR 2000, 1490 (1492); NJW 2001, 3262 (3263); Begr. RegE zu \4 Nr. 10, BT-Drs. 15/1487, S. 19.

553 So Hartl, MMR 2007, 14 f.; Kaufmann, MMR 2005, 351; Härting/Schirmbacher, ITRB 2005, 17.

554 S. zum Begriff der Werbung Gloy/Loschelder/Gloy, Hdb. WettbewerbsR, \15 Rn. 1 ff.; jurisPK-UWG/Link, \5 Rn. 95; Piper/Ohly/Piper, \5 UWG Rn. 77.

555 BGH, NJW 1995, 3124 (3125); NJW 1991， 3030; NJW-RR 1989, 1382 (1383); Piper/Ohly/Piper, \5 UWG Rn. 77; Ernst, ITRB 2005, 91. 
Service-Anbieter durch dessen „Eintragungsoptimierung“ in der Trefferliste vorfindet, mit diesem befassen, indem er sein Service- sowie gegebenenfalls das Preis-/Leistungsangebot sondiert. Aufgrund der begrenzten Vielfalt derartiger Serviceleistungen durch Web Services wird eine solche Beschäftigung des Web Service-Nutzers, im Gegensatz zu Suchmaschinentreffern, zwangsläufig erfolgen. Insofern liegt durch die Verwendung fremder Firmenbezeichnungen oder Kennzeichen eine irreführende Werbung im Sinne des $\int 5$ UWG vor. Dies ergibt sich zudem vor dem Hintergrund, dass, wenn die Verwendung fremder geschützter Begriffe immer zulässig wäre, dann jeder Web ServiceAnbieter seine Trefferquote durch entsprechende Begriffe optimieren könnte und eine Suche im UDDI für den Web Service-Nutzer kaum noch effektiv und verwertbar wäre. 556

bb) Anlocken von Interessenten

\ 4 Nr. 1 UWG schützt lediglich Verbraucher und sonstige Marktteilnehmer, nicht aber die Mitbewerber, denn Gegenstand des Schutzes aus $₫ 4$ Nr. 1 UWG ist die Entscheidungsfreiheit der den Wettbewerbern auf der Marktgegenseite gegenüberstehenden Marktteilnehmer. ${ }^{557} \mathrm{Ob}$ daher das Verwenden fremder Begriffe durch den Web Service-Anbieter ein unlauteres Anlocken nach $\int 4$ Nr. 1 UWG durch den Web Service-Anbieter darstellt, ist höchst fraglich. ${ }^{558}$ Eine mögliche Variante des $₫ 4$ Nr. 1 UWG wäre eine Beeinträchtigung durch sonstigen unangemessenen unsachlichen Einfluss $(\mathbb{} 4$ Nr. $1,3$. Var. UWG). Der Unterfall der irreführenden Werbung als unangemessener unsachlicher Einfluss ist aber bereits Regelungsgegenstand von $\int 5$ UWG und fällt somit nicht unter den Anwendungsbereich des $\int 4$ Nr. 1 UWG. ${ }^{559}$ Als weitere Fallgruppen gelten beispielsweise psychischer Zwang, übertriebenes Anlocken, ${ }^{560}$ unzureichende Information, Verleitung zum Vertrags- und

S. dazu schon bei Metatags: Ernst, ITRB 2005, $91 \mathrm{f}$.

Begr. RegE zu \4 Nr. 1, BT-Drs. 15/1487, S. 17; Piper/Ohly/Piper, \ 4 UWG Rn. 1/1 u. 1/4.

So aber grds. Hartl, MMR 2007, 15, der allerdings anmerkt, dass die Schwelle von einer der Werbung immanenten Beeinflussung zur unangemessenen, unsachlichen Beeinflussung regelmäßig nicht erreicht werden dürfte, zumal für die relevante rechtsgeschäftliche Entscheidungsfreiheit noch weitere Handlungen auf den jeweiligen Webseiten erforderlich wären. Grds. ebenfalls ein übertriebenes Anlocken verneinend jurisPK-UWG/Link, $₫ 4$ Nr. 1 Rn. 126, allerdings darauf hinweisend, dass im Extremfall eine andere Beurteilung gerechtfertigt sein kann.

59 S. dazu Fezer/Steinbeck, \4-1 Rn. 4; Hasselblatt, in: Gloy/Loschelder, Hdb. WettbewerbsR, \ 63 Rn. 14.

Anders für Extremfälle jurisPK-UWG/Link, \4 Nr. 1 Rn. 126. 
Rechtsbruch sowie die Überrumpelung. 561 Die bloße Eingabe fremder Begriffe im UDDI tangiert aber regelmäßig keine dieser Fallgruppen. Eine Verletzung des $\int 4$ Nr. 1 UWG kommt daher nicht in Betracht.

\section{cc) Abfangen von Kunden}

Der Tatbestand der gezielten Behinderung von Mitbewerbern ( $\$ 4$ Abs. 10 UWG) umfasst auch das unlautere Abfangen von Kunden. ${ }^{562}$ Das Schmücken des UDDI-Eintrags durch den Web Service-Anbieter mit dem Namen oder den geschützten Dienstleistungsbezeichnungen seiner Konkurrenten, könnte eine Zuwendung der Kunden zum besagten Web Service-Anbieter zur Folge haben. Der Tatbestand des unlauteren Abfangens von Kunden nach $\int 4$ Abs. 10 UWG könnte dementsprechend durch das Verhalten des Web ServiceAnbieters tangiert sein. Grundsätzlich ist der Konkurrenzkampf um Kunden aber Bestandteil und sogar Wesensmerkmal des Wettbewerbs und daher nicht per se unlauter. ${ }^{563}$ Damit man von einem unlauteren Abfangen sprechen kann, müssen zusätzliche Momente hinzutreten. Verhalten, das dem Anlocken von Kunden dient, ist nämlich nur dann als unlauter anzusehen, wenn gezielt der Zweck verfolgt wird, den Mitbewerber an seiner Entfaltung zu hindern und ihn dadurch zu verdrängen. ${ }^{564}$ Das zielgerichtete Abfangen, mit der Absicht die Kunden anstelle des beabsichtigten Kaufs zum Geschäftsabschluss in eigenen Räumlichkeiten zu verleiten, wird davon erfasst. ${ }^{565}$ Es genügt bereits ein Abfangen potenzieller Kunden des Mitbewerbers in unmittelbarer Nähe zu dessen Ladengeschäft. ${ }^{566}$ Unlauter ist ein solches Abfangen aber erst dann, wenn dem Kunden die Möglichkeit genommen wird, die Angebote der Kon-

561 Zu den Fallgruppen: Steinbeck, GRUR 2005, 540 ff.; Hefermehl/Köhler/Bornkamm/Köbler, $\ 4$ UWG Rn. 1.35 ff.; z.T. mit anderen Fallgruppen Plaß, in: HK-WettbR, $₫ 4$ Rn. 56 ff.

562 Plaß, in: HK-WettbR, \4 Rn. 425; Hefermehl/Köhler/Bornkamm/Köbler, \4 UWG Rn. 10.25 ff.; jurisPK-UWG/Seichter, \4 Nr. 10 Rn. 36 ff.; Piper/Ohly/Obly, \4 UWG Rn. 10/44 ff.

563 BGH, GRUR 1986, 547 (548) - Handzettelwerbung; Emmerich, Unlauterer Wettbewerb, \& 6 S. 116; Nordemann, Rn. 1427; Piper/Ohly/Obly, \ 4 UWG Rn. 10/44; Plaß, in: HK-WettbR, § 4 Rn. 425; jurisPK-UWG/Seichter, \4 Nr. 10 Rn. 36.

564 BGH, GRUR 2001, 1061 (1062) - mitwohnzentrale.de; Hefermehl/Köhler/Bornkamm/Köbler, \4 UWG Rn. 10.25; Hasselblatt, in: Gloy/Loschelder, \45 Rn. 13.

565 BGH, GRUR 1986, 547 (548) - Handzettelwerbung; GRUR 1963, 197 (201) - Zahnprothese Pflegemittel.

566 BGH, GRUR 1987, 532 (533) - Zollabfertigung; GRUR 1960, 431 (433) - KfzNummernschilder; Hasselblatt, in: Gloy/Loschelder, \45 Rn. 14. 
kurrenten zu prüfen. ${ }^{567}$ Unzulässig ist auch das Inserat im Branchenbuch unter dem Anfangsbuchstaben des Konkurrenten, insbesondere wenn es drucktechnisch hervorgehoben wird, obwohl die eigene Firma mit einem anderen Buchstaben beginnt. 568 Jede Handlung muss allerdings immer anhand der Umstände des Einzelfalls bewertet werden. Die Beispiele zeigen jedoch, dass ein tatsächliches Abfangen ermöglicht werden muss. Durch die Verwendung fremder Kennzeichen oder Firmennamen im UDDI erfolgt ein solches Abfangen dagegen noch nicht. Vielmehr räumt sich der Web Service-Anbieter „nur“ einen Platz in einer Liste von Diensten ein, die er nicht anbietet, oder er taucht neben einer fremden Firma auf. Dem Kunden bleibt aber sehr wohl der Vergleich mit den anderen Listenergebnissen und somit auch ein Vergleich der Angebote. ${ }^{569}$ Eine drucktechnische Hervorhebung in der Listung, wie im Fall des Branchenbuchs, ${ }^{570}$ ist bei der UDDI-Suche nicht ersichtlich. Insofern werden derartige Handlungen in der Regel nicht unter den Anwendungsbereich des $₫ 4 \mathrm{Nr}$. 10 UWG fallen, sondern sind vielmehr nach dem Markenoder Namensrecht zu beurteilen. ${ }^{571}$

\section{c) (Störer-)Haftung des Web Service-Vermittlers}

Liegt eine unlautere oder sonst rechtswidrige Handlung des Web ServiceAnbieters durch eine fremde Firmennamen- oder Kennzeicheneingabe im UDDI vor, so sind zwar die direkten Ansprüche gegen selbigen durchaus wichtig, da es sich ja um den unmittelbaren Verletzer handelt, schneller und zur Beseitigung der Störung des Markts effektiver wird aber häufig das Vorgehen gegen den Web Service-Vermittler sein. Als „Herr über das UDDI“ hat regelmäßig nur dieser die Möglichkeit der Löschung oder Änderung von UDDI-Einträgen. Daher ist es für konkurrierende Web Service-Anbieter ebenso bedeutsam, direkte Ansprüche gegen den rechtsverletzenden Web Service-Anbieter wie auch Ansprüche gegen den Web Service-Vermittler geltend machen zu können.

567 Piper/Ohly/Obly, \4 UWG Rn. 10/47; Hasselblatt, in: Gloy/Loschelder, $₫ 45$ Rn. 14; BGH, GRUR 1986, 547 (548) - Handzettelwerbung.

568 OLG Bamberg, NJW-RR 1993, 50; OLG Düsseldorf, NJW 1956, 64.

569 So auch für Metatags OLG Düsseldorf, GRUR-RR 2004, $353 \mathrm{f}$.

570 OLG Bamberg, NJW-RR 1993, 50.

571 Emmerich, Unlauterer Wettbewerb, \6 S. 122 f. Zu Metatags: Piper/Ohly/Obhy, \4 UWG Rn. 10/53; Plaß, in: HK-WettbR, \ 4 UWG Rn. 426; OLG Düsseldorf, GRUR-RR 2003, 48. 
aa) Verstoß gegen Verkehrspflichten

Im Falle eines Wettbewerbsverstoßes durch den Web Service-Anbieter haftet der Web Service-Vermittler nach der neueren BGH-Rechtsprechung als Täter gemäß $\ 8,3$ UWG auf Unterlassen, wenn er gegen die Generalklausel des $\ 3$ UWG verstoßen hat. ${ }^{572}$ Danach kann derjenige, der durch sein Handeln im geschäftlichen Verkehr in einer ihm zurechenbaren Weise die Gefahr eröffnet, dass Dritte Interessen von Marktteilnehmern verletzen, die durch das Wettbewerbsrecht geschützt sind, eine unlautere geschäftliche Handlung begehen, wenn er diese Gefahr nicht im Rahmen des Möglichen und Zumutbaren begrenzt. ${ }^{573}$ Dies ist immer nur dann der Fall, wenn der Web Service-Vermittler geschützte Interessen von schutzbedürftigen Marktteilnehmern verletzt, dies für ihn erkennbar war und ihm die Gefahrenabwehr möglich und zumutbar ist. ${ }^{574}$ Diese im Wettbewerbsrecht deutlich höheren Anforderungen an die Haftung gegenüber der Störerhaftung dürften durch den Web ServiceVermittler regelmäßig nicht erfüllt sein.

\section{bb) Grundsätze der Störerhaftung}

Für Verstöße gegen fremde Firmennamen- oder Kennzeichen kommen die Grundsätze der mittelbaren Störerhaftung zum Tragen. ${ }^{575}$ Danach können die von der rechtswidrigen Handlung Betroffenen vom Web Service-Vermittler Löschung des verwendeten Firmennamen, der Marke etc. sowie - bei Vorliegen von zumutbaren Prüfungs- und Kontrollpflichten -576 auch Unterlassung verlangen. Bisher wurde bezüglich des Unterlassungsanspruchs davon ausgegangen, dass ein solcher nur dann greift, wenn bereits zuvor eine kerngleiche $^{577}$ Rechtsverletzung stattgefunden hat. Nach jüngster Rechtsprechung des Bundesgerichtshofs ${ }^{578}$ ist in besonderen Fällen aber auch ein vorbeugender Unterlassungsanspruch denkbar. Der Bundesgerichtshof hat einen solchen

572 BGH, MMR 2007, 634 (635) - Jugendgefährdende Medien bei eBay.

573 BGH, MMR 2007, 634 (635) - Jugendgefährdende Medien bei eBay.

574 Köhler, GRUR 2008, 4.

575 S. dazu bereits den Überblick unter B. Außervertragliche Haftung.

576 S. B Außervertragliche Haftung.

577 S. zur Modifizierung der Prüfungs- und Kontrollpflichten durch die Kerntheorie Spindler, JZ $2005,39 \mathrm{f}$.

578 BGH, MMR 2007, 507 (509 ff.) - Internetversteigerung II m. Anm. Spindler. In diese Richtung tendierend, wobei allerdings noch die ausstehende Begründung abzuwarten bleibt, BGH, Urt. v. 30.4.2008 - I ZR 73/0- Internet-Versteigerung III. 
vorbeugenden Unterlassungsanspruch bejaht, nachdem das Unternehmen, welches bereits gegen Verletzungen seiner deutschen Marke auf einer Internetauktionsplattform vorgegangen ist, zwischenzeitlich die Gemeinschaftsmarke anmeldete und trotz fehlender Verletzung dieser auch für die Gemeinschaftsmarke vorbeugend Unterlassung verlangte. Das Gericht begründete das Vorliegen der für den Unterlassungsanspruch erforderlichen Erstbegehungsgefahr mit der Verletzung der identischen nationalen Marken. ${ }^{579}$ Da es sich hier um einen sehr speziellen Fall der Anwendung der vorbeugenden Unterlassungsklage handelt, ${ }^{580}$ bleibt abzuwarten, ob die Rechtsprechung zu einer weiteren Ausdehnung des Unterlassungsanspruch führen wird oder das Urteil, wie zu hoffen bleibt, 581 einen Spezialfall bezüglich des vorbeugenden Unterlassungsanspruchs darstellt. Eine grundsätzliche Ausdehnung der Haftung auf den vorbeugenden Unterlassungsanspruch erscheint $\mathrm{zu}$ weitgehend. Es ist bereits höchst fraglich, ob eine derartig weitgehende Haftung des Web Service-Anbieters noch mit den Vorgaben der E-Commerce-Richtlinie ${ }^{582}$ vereinbar ist. ${ }^{583}$ Denn während die Prüfpflichten des Störers beim allgemeinen Unterlassungsanspruch auf einer anderen Ebene angesiedelt sind als das Verbot allgemeiner Überwachungspflichten nach Art. 15 Abs. 1 ECRL, dessen deutsche Umsetzung in $\int 7$ Abs. 2 S. 1 TMG erfolgte, so dass es zu keinem Widerspruch kommt, kann dies mit der Annahme eines vorbeugenden Unterlassungsanspruchs nur schwerlich in Einklang gebracht werden. Insbesondere wird dadurch ein Wertungswiderspruch zum Schadensersatzanspruch, der erst nach Kenntnisnahme möglich ist, ${ }^{584}$ hervorgerufen. ${ }^{585}$ Eine ganz andere The-

BGH, MMR 2007, 507 (510) - Internetversteigerung II m. Anm. Spindler

So auch Jürgens, Anm. zu BGH, K\&R 2007, 387 ff., K\&R 2007, 392 (393) u. Roggenkamp, jurisPR-ITR 11/2007 Anm. 2, die die Voraussetzungen für die Bejahung einer Erstbegehungsgefahr daher als denkbar eng ansehen.

581 Andernfalls wären für Plattformbetreiber die Risiken kaum noch überschaubar und die Rentabilität einer solchen Plattform müsste in Frage gestellt werden. Anders aber scheinbar BGH, Urt. v. 30.4.2008 - I ZR 73/05 - Internet-Versteigerung III, wobei hier allerdings noch die Entscheidungsbegründung aussteht.

Richtlinie 2000/31/EG des Europäischen Parlaments und des Rates v. 8.6.2000 über bestimmte rechtliche Aspekte der Dienste der Informationsgesellschaft, insbesondere des elektronischen Geschäftsverkehrs, im Binnenmarkt („Richtlinie über den elektronischen Geschäftsverkehr - ECommerce-Richtlinie“) ABl. EG L 178 v. 17.7.2000, S. 1-16.

583 Strikt gegen eine solche Ausdehnung Leible/Sosnitza, NJW 2007, 3324; ebenfalls kritisch Spindler, MMR 2007, 512; Leible/Sosnitra, NJW 2004, 3226 in Besprechung des Urteils Internetversteigerung I; Rücker, CR 2005, 355; Rachlock, Anm. zu LG Hamburg, MMR 2005, 326 ff., MMR 2005, $328(330)$. 
matik, die hier aber nicht weiter behandelt werden kann, ist die Frage, ob der Bundesgerichtshof die Problematik über die Vereinbarkeit eines vorbeugenden Unterlassungsanspruchs mit der E-Commerce-Richtlinie dem Europäischen Gerichtshof (EuGH) hätte vorlegen müssen. ${ }^{586}$

\section{cc) Übertragung auf den Web Service-Vermittler}

Unabhängig von diesen Unklarheiten kann der Web Service-Vermittler bei Rechtsverletzungen des Web Service-Anbieters im UDDI aber zumindest auf Beseitigung und bei Vorliegen von zumutbaren Prüf- und Kontrollpflichten auch auf Unterlassung in Anspruch genommen werden. Der Beseitigungsanspruch richtet sich dabei auf Löschung der rechtswidrigen Inhalte, das bedeutet konkret verwendete fremde Firmenbezeichnungen, Kennzeichen oder ähnliches. Dagegen ist die Löschung des gesamten UDDI-Eintrags des Web Service-Anbieters nicht erforderlich, um den rechtswidrigen Zustand zu beseitigen, und daher auch nicht Gegenstand des Beseitigungsanspruchs. ${ }^{587}$

Ob dem Web Service-Vermittler die für den Unterlassungsanspruch erforderlichen Prüfpflichten obliegen, hängt auch davon ab, ob er das UDDI zumindest auf einer Seite (gegenüber dem Web Service-Anbieter und/oder dem Web Service-Nutzer) entgeltlich beziehungsweise in sonstiger Weise kommerziell betreibt oder nicht. Bei gemeinnützigen Organisationen wie der DENIC hat der Bundesgerichtshof Prüfpflichten in der Phase der Erstregistrierung für unzumutbar gehalten und in der zweiten Phase nach Kenntnis Prüfpflichten dahingehend eingeschränkt, dass eine Registrierung nur dann gelöscht werden muss, wenn die Verletzung des Rechts offenkundig ist. ${ }^{588}$ Kommerziellen Betreibern, die unter Umständen sogar an der Rechtsverletzung verdienen,

585 Spindler, MMR 2007, 512; Leible/Sosnitza, NJW 2007, 3324 f.; kritisch auch Roggenkamp, jurisPRITR 11/2007 Anm. 2.

586 So bspw. Leible/Sosnitza, NJW 2007, 3324, da nach Art. 234 Abs. 3 EG letztinstanzliche Gerichte, wenn sie Zweifel an der Auslegung von Gemeinschaftsrecht haben und eine Entscheidung darüber zum Erlass des Urteils für erforderlich halten, eine Vorabentscheidung des Europäischen Gerichtshofs einholen müssen. A.A. Rössel, Anm. zu BGH, CR 2007, 523 ff., CR 2007, 527 (528), der meint, dass es sich dabei lediglich um eine politische Entscheidung handele.

587 BGH, NJW 1990, 1986 (1988) - Emil Nolde; GRUR 1966, 272 (274) - Arztschreiber; Staudinger/Gursky, \1004 Rn. 144. Für wettbewerbsrechtliche Beseitigungsansprüche BGH, GRUR 1981, 60 (64) - Sitex; Köbler, NJW 1992, 139; Kotthoff/Gabel, in: HK-WettbR, \ 8 Rn. 71.

588 BGH, NJW 2001, 3265 (3266 ff.) - ambiente.de; s. dazu die Erläuterungen von Staudinger, in: Leible/Sosnitza, Teil 3 F Rn. 505 ff. 
dagegen wird in der Rechtsprechung deutlich mehr zugemutet. ${ }^{589} \mathrm{Da}$ es sich bei Web Service-Vermittlern aber in aller Regel um private Unternehmen handeln wird, die durch das Betreiben des UDDI Geld verdienen möchten und regelmäßig nicht gemeinnützig handeln, wird ein kommerzielles Betreiben des UDDI grundsätzlich zu bejahen sein. Daher ist dem Web Service-Vermittler grundsätzlich bereits ein gewisses Maß an Prüfungs- und Kontrollpflichten zumutbar. Ein weiteres Kriterium ist die technische Möglichkeit der UDDIKontrolle durch den Web Service-Vermittler. Kriterien für die technische Zumutbarkeit sind von den Umständen des Einzelfalls abhängig, wie beispielsweise der Anzahl der Mitarbeiter, dem technischen Fortschritt sowie dem finanziell zumutbaren Aufwand. ${ }^{590} \mathrm{Da}$ UDDIs lediglich die Einträge von Web Service-Anbietern enthalten, erscheint es durchaus personell wie auch finanziell zumutbar, dass UDDI bei Kenntnis eines rechtswidrigen Inhalts auf künftige kerngleiche Rechtsverletzungen hin manuell oder auch automatisiert durch Filtersoftware hin zu durchsuchen.

Probleme bereitet auf den ersten Blick der Umfang der Prüfungspflichten, denn UDDIs sind derart ausgestaltet, dass die Registrierung durch den Web Service-Anbieter selbst erfolgt. Nachdem dieser alle erforderlichen Informationen angegeben hat, speichert er seinen Eintrag, dieser wird dann sogleich für Web Service-Nutzer publiziert und kann von Dritten gesucht werden. ${ }^{591}$ Das bedeutet, dass eine vorherige Kontrolle des Eintrags durch den Web ServiceVermittler, ebenso wie bei der DENIC und dem Internetauktionshaus eBay etc., nicht erfolgt. Eine solche ist aber, wie auch das Verbot allgemeiner Überwachungspflichten nach $\ 7$ Abs. 2 S. 2 TMG zeigt, gar nicht erforderlich und vom Gesetzgeber auch nicht gewollt. Gerade an diesem Punkt, wo noch jegliche konkrete Kenntnis bezüglich etwaiger Rechtsverletzungen fehlt, soll der Telemediendiensteanbieter privilegiert werden. Insofern erstreckt sich eine Prüfpflicht lediglich auf bekannte Rechtsverletzungen, die dem Web Service-

589 S. nur BGH, MMR 2007, 634 ff. - Jugendgefährdende Medien bei eBay; MMR 2007, 507 ff. Internetversteigerung II m. Anm. Spindler, MMR 2007, 518; NJW 2004, 3102 ff. - Internetversteigerung; OLG Köln, MMR 2007, 786 ff. z.T. kommerzieller Dienst; OLG Hamburg, MMR 2006, 398 ff. - Cybersky u. MMR 2006, 744 ff. u. MMR 2006, 238 ff.; OLG München, MMR 2006, 739 ff.; OLG Brandenburg, MMR 2006, 107.

590 S. zu diesem Aspekt OLG Brandenburg, MMR 2004, 330 (332); ferner BGH, NJW 2004, 3102 (3105) - Internetversteigerung; jurisPK-Internetrecht/Heckmann, Vorb. Kap. 1.7 Rn. 73 ff.

591 S. eine ausführliche Beschreibung zum Eintrag im UDDI unter: http://www.virtualuniversity.ch/software/soap/7.html $\quad<25.11 .2009>$ u. http://www.microsoft.com/germany/msdn/library/xmlwebservices/WebdienstbeschreibungU ndErmittlungMitHilfeVonUDDITeil1.mspx?mfr=true <25.11.2009>. 
Vermittler durch Hinweise, zum Beispiel in Form einer Abmahnung, vermittelt wurden.

Je nach Art der Rechtsverletzung des Web Service-Anbieters kann der Web Service-Vermittler nach $\ 1004$ BGB in Verbindung mit $\ 12$ BGB, $\int 37$ Abs. 2 HGB beziehungsweise in Verbindung mit $\iint 14$ Abs. 5, 6, 15 Abs. 1, 2, 4 und 5 MarkenG oder, sofern man eine Störerhaftung auch bei der Verletzung wettbewerbsrechtlicher Pflichten annimmt, auch nach $\int 8$ UWG in Verbindung mit $\iint 3,5 \mathrm{UWG}$ von den Betroffenen in Anspruch genommen werden.

\section{d) Verhältnis der Ansprüche}

Das Kennzeichenrecht ist ein vorrangiger sondergesetzlicher Schutz. Verwendet der Web Service-Anbieter daher fremde Kennzeichen im UDDI, so greifen die markenrechtlichen Ansprüche vor denen des Bürgerlichen Gesetzbuchs (Verletzung des Namensrechts, $\iint 1004,823$ Abs. 1 BGB in Verbindung mit $\int 12$ BGB), des $₫ 37$ Abs. 2 HGB ${ }^{592}$ und des Lauterkeitsrechts. ${ }^{593}$ Liegt eine Verletzung des Namensrechts vor, so werden die Ansprüche aus $\iint 1004,823$ Abs. 1 BGB in Verbindung mit $\int 12$ BGB sowie die des \37 Abs. 2 HGB durch die $\iint 8$ ff. UWG verdrängt. ${ }^{594}$ Eine eigene Bedeutung erlangen die $\iint 1004,823$ Abs. 1 BGB bei Verletzung des Namensrechts lediglich dann, wenn keine Wettbewerbshandlung vorliegt,595 was aber im Rahmen der Angabe fremder Namen im UDDI regelmäßig nicht der Fall sein wird. Zu beachten gilt, dass der mittelbare Störer gegenüber dem unmittelbaren Störer nicht subsidiär haftet, ${ }^{596}$ so dass es dem Betroffenen freigestellt ist, ob er zunächst den Web Service-Anbieter oder lieber den Web ServiceVermittler in Anspruch nimmt.

IV. Öffentliche All-Inclusive-Angebote

Unabhängig von der Web Service-Anbieter - UDDI-Problematik, ergeben sich Fragen der lauterkeitsrechtlichen Zulässigkeit im Rahmen von öffentlichen „All-Round-Services“. Die automatisierten Abläufe und die flexible In-

592 Baumbach/Hopt/Hopt, \37 Rn. 10.

593 BGH, GRUR 1999, 161 (162) - MacDog; Piper/Ohly/Piper, Vorb. \4 UWG Rn. 8; Fezer/Götting, \4-10 Rn. 92

594 Hefermehl/Köhler/Bornkamm/Köbler, Einl. UWG Rn. 7.27; Klippel, in: HK-WettbR, E2 Rn. 8; Baumbach/Hopt/Hopt, \ 37 Rn. 10.

595 Piper/Ohly/Piper, Vorb. \4 UWG Rn. 8.

596 BGH, MMR 2007, 518 (519). 
tegration von Web Services bieten sich für eine Nutzung im Bereich des so genannten Electronic Government (E-Government) an, da sie Abläufe beschleunigen und Kosten senken können. ${ }^{597}$ Nutzt eine Gemeinde/Stadt oder auch das Land nun Web Services, um ihr Angebot zu verbessern und zu verschnellern sowie gegebenenfalls Kosten einzusparen, so könnten dabei auch eigene Angebote, wie zum Beispiel die Anmeldung eines Kraftfahrzeugs, die Baugenehmigung etc., mit externen, sich ergänzenden Diensten, wie dem Abschluss einer Kraftfahrzeugversicherung, dem Angebot eines Architekten, Bauunternehmers etc., gekoppelt werden. Ermöglicht eine Gemeinde nun beispielsweise die elektronische Anmeldung eines Kraftfahrzeugs und bietet auf der eigenen Web-Präsenz zugleich den Abschluss einer Kfz-Versicherung durch das Versicherungsunternehmen V an, indem ein Web Service des Versicherungsunternehmens in die Web-Präsenz der Verwaltung eingebunden wird, so drängt sich die Frage der Zulässigkeit einer solchen Kooperation auf. Davon abgesehen werden konkurrierende Versicherungsunternehmen, die nicht integriert wurden, gegen eine solche Kooperation vorgehen wollen, da die Kooperation das Versicherungsunternehmen V sicher in vielen Fällen übervorteilen wird. Kunden sparen sich den weiteren Schritt einer Versicherungsunternehmens-Suche und können bequem ihr Auto in einem Schritt anmelden. Wenn das Versicherungsunternehmen V auch akzeptable Preise anbietet, wird man davon ausgehen können, dass es einen großen Zulauf in der entsprechenden Gemeinde erhalten wird.

Hat die Gemeinde nur einen oder eine geringe Zahl an Versicherungsunternehmen die Integration ihres Web Service angeboten, so wird dieses Verhalten in aller Regel gegen die wettbewerbsrechtlichen Vorschriften des Gesetzes gegen Wettbewerbsbeschränkungen (GWB) verstoßen. Dieses erfordert zwar, dass die Gemeinde als Unternehmen handelt, allerdings wird auch das Handeln eines Hoheitsträgers als Unternehmen gewertet und ist am Gesetz gegen Wettbewerbsbeschränkungen zu messen, wenn es - auch unter Beachtung gemeinschaftsrechtlicher Maßstäbe - den ihm durch das öffentliche Recht zugewiesenen Aufgabenbereich verlässt und der Sache nach eine in den Wettbewerb eingreifende Maßnahme trifft. ${ }^{598}$ Dass mit einer Tätigkeit auch öffent-

597 S. zur Optimierung von Verwaltungsabläufen ausführlich Laue/Maidl/Peters, DuD 2007, 810 ff.; Schallbruch, in: Büchner/Büllesbach, S. 3.

598 Lange/Lange, Hdb. KartellR, \1 Rn. 657; Bechtold/Becbtold, \1 Rn. 10; BGH, WRP 1987, 310 (311) - Guten Tag-Apotheke II; WRP 1991, 393 (396 f.); GRUR 1993, 407 (408) - Selbstzahler. S. ferner auch die Formulierung des $₫ 130$ Abs. 1 S. 1 GWB, wonach das Gesetz auch ,auf Unternehmen, die ganz oder teilweise im Eigentum der öffentlichen Hand stehen oder die von ihr verwaltet oder betrieben werden" Anwendung findet. 
liche Aufgaben erfüllt werden, hindert nicht per se die Bindung an das Kartellrecht. ${ }^{599}$ Die elektronische Zulassung von Kraftfahrzeugen stellt zwar eine hoheitliche Maßnahme dar, ${ }^{600}$ jedoch geht es im Falle des KfzZulassungsportals nicht primär um die Kfz-Zulassung sondern vielmehr um die Einbindung externer Unternehmen auf dem Portal. Geht man davon aus, dass eine solche Einbindung gegen Entgelt erfolgt, kann es sich um einen Mietvertrag für die Einbindung des Web Service des Versicherungsunternehmens auf der Plattform (Miete der Einbindung) oder auch einen Kaufvertrag (Kauf des Speichers) handeln. Unabhängig vom Vertragstypus ist aber entscheidend, dass hier die Entscheidung der Gemeinde, wessen Web Service im Kfz-Zulassungsportal eingebunden wird, Gegenstand des Konkurrentenausschlusses ist. Die Gemeinde als „Herrin der Vergabe der Einbindung externer Dienste" ${ }^{601}$ handelt diesbezüglich auch als Unternehmen im Sinne des Gesetzes gegen Wettbewerbsbeschränkungen.

Wie das Verhalten der Gemeinde wettbewerbsrechtlich exakt zu beurteilen ist, hängt zunächst davon ab, ob die Gemeinde die Einbindung des Web Service im Wege einer Ausschreibung nach transparenten Kriterien im Sinne des $\int 97$ GWB vergeben hat ${ }^{602}$ oder auf eine solche verzichtet hat und die Einbindung des Web Service in für Konkurrenten undurchsichtiger Weise vergeben hat. $\mathrm{Da}$ die Unternehmen gemäß $\ 97$ Abs. 7 GWB einen Anspruch auf Einhaltung der Vorschrift des $₫ 97$ Abs. 1-6 GWB haben, können sie im Falle der Nichtbeachtung durch die Gemeinde im Wege des Nachprüfungsverfahrens ( $\iint 102$ ff. GWB) dagegen vorgehen. Darüber hinaus können Verstöße gegen das Vergaberecht auch über $\$ 3$ in Verbindung mit $₫ 4 \mathrm{Nr}$. 11 UWG erfasst werden, sofern es sich bei der verletzten Vorschrift nicht nur um eine bloße Ordnungsvorschrift handelt. ${ }^{603} \mathrm{Im}$ Falle der Verletzung des $₫ 3$ in Verbindung mit $\ 4$ Nr. 11 UWG können Unterlassungs- und Beseitigungsansprüche nach \8 UWG sowie Schadensersatzansprüche ( $\$ 9$ UWG) geltend gemacht werden.

599 Bechtold/Bechtold, \1 Rn. 10; Immenga/Mestmäcker/Zimmer, Bd. 2, \1 Rn. 30.

600 S. zum Begriff der hoheitlichen Maßnahme Stelkens/Bonk/Sachs/Stelkens/Stelkens, $₫ 35$ Rn. 68 ff.; Erichsen/Ehlers/Ruffert, \20 Rn. 17.

601 S. zu der Vermietung von Gewerbeflächen für Schilderpräger BGH, GRUR 2003, 167 (168); GRUR 1999, 278 (280) - Schildpräger im Landratsamt.

602 BGH, GRUR 2006, 608 (609) - Hinweis auf konkurrierende Schilderpräger; GRUR 1999, 278 (280) - Schildpräger im Landratsamt.

603 Nordemann, Rn. 1745; Alexander, WRP 2004, 706 ff.; Köbler, GRUR 2004, 387. 
Vor diesem Hintergrund ist der Gemeinde, sofern sie externen Diensten auf ihrem Portal die Möglichkeit zur Implementierung ihrer Web Service geben, dringend zu raten, dass sie vorab, um dem Transparenzgebot gerecht zu werden, immer eine Ausschreibung vornehmen. Erfolgt eine Ausschreibung, so muss die Vergabe den Grundsätzen des $\int 97$ GWB entsprechen. ${ }^{604}$

Dennoch kommen auch im Falle einer transparenten, nicht zu beanstandenden Vergabe - je nachdem ob die Gemeinde eine marktbeherrschende Stellung einnimmt oder nicht $-{ }^{605}$ Verletzungen der $\$ \$ 19,20$ GWB in Betracht, wenn die Gemeinde nur einen oder eine begrenzte Zahl an Versicherungsanbietern zulässt. Die Verbotsnorm des $₫ 1$ GWB findet hier keine Anwendung, da diese eine Vereinbarung zwischen Unternehmen erfordert, um übergeordnete gemeinsame Interessen wahrzunehmen. ${ }^{606}$ Ein solches übergeordnetes gemeinsames Interesse existiert aber nicht. Zwar geht es letztlich sowohl dem Versicherungsunternehmen als auch der Gemeinde um Autos, die Tätigkeit erfolgt aber unabhängig voneinander auf unterschiedlichen Ebenen. Das Interesse der Gemeinde beschränkt sich auf die Zulassung von Kraftfahrzeugen, während sich das Interesse des Versicherungsunternehmens auf die Versicherung von Kraftfahrzeugen bezieht. Durch die Einbindung des Web Service des Versicherungsunternehmens wird lediglich das Interesse einer zusätzlichen Einnahmequelle für die Einbindung verfolgt, dagegen bei dem Versicherungsunternehmen das Interesse, mehr Versicherungsabschlüsse zu erzielen. Daher beschränkt sich die Untersuchung auf die $\iint 19,20$ GWB.

Bei der Beurteilung, ob eine für die Anwendbarkeit der $\$ \int 19,20$ GWB erforderliche marktbeherrschende Stellung der Gemeinde vorliegt oder nicht, ist allein an den relevanten Markt der Zulassung der Einbindung von Web Services von Versicherungsunternehmen auf dem Kfz-Zulassungsportal zu knüpfen. ${ }^{607}$ Zwar können Versicherungsunternehmen sowohl im Internet als auch analog quasi uneingeschränkt angesiedelt sein und werben. Im aufgezeigten Fall ist aber zusätzlich der Standortfaktor, das heißt der räumlich relevante

604 Entscheidend ist, ob die Auswahl unter angemessenen und fairen Bedingungen erfolgte, s. BGH, GRUR 1999, 278 (281) - Schilderpräger im Landratsamt.

605 Die $\iint 19-20$ GWB knüpfen an eine absolute oder relative Marktstellung des Unternehmens an und verbieten damit in Zusammenhang stehende bestimmte Verhaltensweisen, s. Bechtold/Bechtold, Vorb. SS 19 ff. Rn. 1.

606 Lange/Lange, Hdb. KartellR, \1 Rn. 659.

607 So auch bei der Anmietung von Gewerbeflächen bei Schilderprägern: BGH, GRUR 2003, 167 (168); GRUR 1999, 278 (280) - Schilderpräger im Landratsamt. 
Markt, ${ }^{608}$ entscheidend, da dieser gerade eine neue Möglichkeit der Werbung für Versicherungsunternehmen eröffnet. Da das Internet keine Grenzen kennt, ist auch nur auf den Markt der Kfz-Zulassungsportale, die eine Implementierung von Web Services anbieten, relevant. Bezüglich ihres eigenen kommunalen Kfz-Zulassungsportals hat die Gemeinde zweifellos ein Monopol und daher auch nach $\ 19$ Abs. 2 S. 1 Nr. 1 GWB eine marktbeherrschende Stellung.

Darüber hinaus muss die Gemeinde diese marktbeherrschende Stellung aber auch durch die Zulassung des Versicherungsunternehmens $\mathrm{V}$ missbraucht haben. Ein solcher Missbrauch könnte in der Beeinträchtigung anderer Versicherungsunternehmen ohne sachlich gerechtfertigten Grund ( $\mathbb{1} 19$ Abs. 4 Nr. 1 GWB oder auch $₫ 20$ Abs. 1 GWB), der sich weitgehend mit dem Behinderungsverbot des $₫ 19$ Abs. 4 Nr. 1 GWB deckt, ${ }^{609}$ liegen. Eine Behinderung im Sinne des $₫ 20$ Abs. 1, 1. Alt. GWB erfasst jedes Marktverhalten, das objektiv nachteilige Auswirkungen auf die wettbewerbliche Entfaltungsfreiheit des davon Betroffenen hat. ${ }^{610}$ Lässt die Gemeinde nur die Einbindung des Web Service eines bestimmten Versicherungsunternehmens zu - auch die Auswahl aufgrund einer Ausschreibung erfolgte -, so behindert dies zweifellos andere Versicherungsunternehmen. Die zudem erforderliche Unbilligkeit einer Behinderung wird allgemein anhand einer „Abwägung der beiderseitigen Interessen unter Berücksichtigung der auf die Freiheit des Wettbewerbs gerichteten Zielsetzung"“611 des Gesetzes gegen Wettbewerbsbeschränkungen konkretisiert. Dabei ist auch die Marktmacht des handelnden Unternehmens zu berücksichtigen. Zwar stellt beispielsweise die Vermietung von Gewerbefläche an Schilderpräger in unmittelbarer räumlicher Nähe zur Kfz-Zulassungsstelle durch die Gemeinde keine unbillige Behinderung anderer Schilderpräger dar, ${ }^{612}$ jedoch ist die Gemeinde gehalten, anderen Schilderprägern die Gelegenheit zu geben, an geeigneter Stelle auf ihr Angebot hinzuweisen. ${ }^{613} \mathrm{Die}$ Vermietung von Gewerbefläche an Schilderpräger unterscheidet sich aber

608 S. zu den verschiedenen Marktabgrenzungsformen Immenga/Mestmäcker/Möschel, $\ 19$ Rn. 23 ff.; Emmerich, KartellR, \ 27 Rn. 11 ff.; Lange/Hübschle, Hdb. KartellR, \2 Rn. 849 ff.

609 Emmerich, KartellR, \27 Rn. 4; Lange/Hübscble, Hdb. KartellR, \3 Rn. 935.

610 BGH, GRUR 1982, 60 (61) - Original-VW-Ersatzteile II; OLG Düsseldorf, GRUR 2002, 831 f.; Lettl, \$ 9 Rn. 109.

611 BGHZ 38, 90 (102) - Treuhandbüro.

612 BGH, GRUR 1999, 278 (281) - Schilderpräger im Landratsamt.

613 BGH, GRUR 2006, 608 (609) - Hinweis auf konkurrierende Schilderpräger. 
insofern vom vorliegenden Fall, als dass es im Fall der Schilderpräger um begrenzte Kapazitäten geht (Gewerbefläche), die vermietet werden sollen. Bei der Einbindung von Web Services im Portal der Kfz-Zulassungsstelle handelt es sich indes nur um Speicherplatz, der bezüglich der Implementierung des Web Service sehr gering sein wird. Insofern ist es der Gemeinde durchaus möglich, allen entsprechend ihren Festlegungen zahlenden Versicherungsunternehmen die Möglichkeit der Implementierung ihres Web Service zu offerieren. Eine solche Marktöffnung würde die Gemeinde nicht belasten, ein eventueller personeller Aufwand bezüglich der Implementierung kann durch ein entsprechend hohes Entgelt für die Einbindung ausgeglichen werden. Die Gemeinde trifft eine entsprechende Öffnung für den Markt der KfzVersicherungen nicht hart, wohl aber die Versicherungsunternehmen, wenn ihnen im Gegensatz zu einem Konkurrenten die Möglichkeit der Implementierung ihrer Web Services verwehrt würde. Insofern würde eine Beschränkung der Einbindung lediglich eines Web Service eines Versicherungsunternehmens unter Abwägung der beiderseitigen Interessen eine unbillige Behinderung der konkurrierenden Versicherungsunternehmen gemäß \ 20 Abs. 1, 1. Alt. GWB darstellen, gegen welche sich die konkurrierenden Versicherungsunternehmen nach $\int 33$ GWB mit Unterlassung wehren oder gegebenenfalls bei Vorsatz oder Fahrlässigkeit sogar Schadensersatz geltend machen können.

Eröffnet die öffentliche Hand im Rahmen der Erfüllung ihrer öffentlichen Aufgaben daher Portale und lässt dabei auch die Implementierung von Web Services externer Unternehmen zu, so sollte sie zur Vermeidung von VerstöBen gegen das Gesetz gegen den unlauteren Wettbewerb entweder die Implementierung der Web Services jeweils für den gesamten entsprechenden Markt zulassen und nicht bloß auf ein spezielles Unternehmen beschränken oder aber zumindest eine Ausschreibung zur Vergabe der Implementierung vornehmen.

\section{Haftung des Web Service-Nutzers}

Auch der Web Service-Nutzer als Portalbetreiber kann für eigene und fremde Rechtsverletzungen, die in Form von Inhalten durch den Web Service des Web Service-Anbieters übermittelt werden, ${ }^{614}$ in Anspruch genommen wer-

614 Solche können je nach Art des Dienstes ganz unterschiedlicher Natur sein: übermittelt der Web Service bspw. Bilder, Software oder sonstige Werke so kommen Urheberrechtsverletzungen bei fehlenden Lizenzen ins Spiel, bei der Übermittlung von Texten, können diese das Allgemeine Persönlichkeitsrecht tangieren, gleiches kann u.U. auch bei Bildern berührt sein etc. 
den. Je nachdem wie man die Integration des Web Service einordnet, genauer gesagt, ob es sich um eigene oder fremde Inhalte des Web Service-Nutzer handelt, so ist der Web Service-Nutzer entweder im Sinne des $₫ 7$ Abs. 1 TMG nach den allgemeinen Gesetzen verantwortlich und haftet somit uneingeschränkt oder er haftet lediglich als Störer auf Unterlassung und Beseitigung. Bei der Geltendmachung darüber hinausgehender Ansprüche wird der Web Service-Nutzer nach den $\int S$ 8-10 TMG privilegiert.

Der Web Service-Nutzer, der lediglich den Web Service als Telemedium zur Nutzung für Dritte bereithält, ist nach $₫ 2$ Nr. 1 TMG gegenüber Dritten dennoch ein Telemediendiensteanbieter. ${ }^{615}$ Nichts desto Trotz ist der Web Service-Nutzer nur eine Art Zugangsvermittler zum Telemedium Web Service. Ein solcher wird nach den $\int \S 7$ ff. TMG hinsichtlich der Verantwortlichkeit privilegiert. ${ }^{616} \mathrm{Um}$ die Haftungsprivilegierung jedoch anwenden zu können, ist es erforderlich, dass die mittels des Web Service übermittelten Informationen fremde Informationen im Sinne des $\int 7$ Abs. 2 TMG sind. Handelt es sich indes um eigene Informationen, worunter auch zu Eigen gemachte Informationen fallen, ${ }^{617}$ so ist der Web Service-Nutzer nach $\ 7$ Abs. 1 TMG nach den allgemeinen Gesetzen verantwortlich, die Haftungsprivilegierung greift nicht.

\section{Eigene oder fremde Informationen}

$\mathrm{Ob}$ es sich noch um fremde oder bereits zu Eigen gemachte Informationen handelt, muss aufgrund der Besonderheit der verteilten Anwendung und der Funktionsweise von Web Services genauer untersucht werden. Eine Abgrenzung muss immer anhand des Einzelfalls erfolgen, dabei kann jedoch auf folgende Kriterien zurückgegriffen werden: die Art der Datenübernahme (will er sich die Inhalte zurechnen lassen oder nicht), ${ }^{618} \mathrm{ihr}$ Zweck sowie die konkrete Präsentation der fremden Daten durch den Übernehmenden. ${ }^{619}$ Insbesondere muss der Anbieter darlegen und beweisen, dass er keinen Einfluss auf den

615 S. A.II.2 Web Service-Nutzer.

616 Spindler/Schmitz/Geis/Spindler, \2 TDG Rn. 25; Spindler/Schuster/Schmitz, \1 TMG Rn. 25; LG Frankfurt, MMR 2008, 121 (122 f.) - You Porn; so konkludent durch die Anwendung des \8 TMG LG Köln, ZUM 2007, 873 (875).

617 BT-Drs. 14/6098, S. 23; Lempold/Rücker, in: Wiebe/Leupold, Teil IV Rn. 120; Spindler, in: Spindler/Wiebe, Kap. 6 Rn. 11; Spindler/Schuster/Hoffmann, \ 7 TMG Rn. 15; OLG Köln, MMR 2002, 548 - Steffi Graf m. Anm. Spindler.

618 Schmitr/Laun, MMR 2005, 210; Spindler/Schuster/Hoffmann, \ 7 TMG Rn. 17.

619 OLG Köln, MMR 2002, 548 - Steffi Graf m. Anm. Spindler. 
betreffenden Inhalt hatte. ${ }^{620}$ Zwar hat der Web Service-Nutzer auf die Informationen, die durch den Web Service übermittelt werden keinerlei Einfluss, indes hat er im Rahmen eines Vertragsverhältnisses mit dem Web ServiceAnbieter den Web Service selbst in sein Portal integriert. Telos der Implementierung ist je nach Portalzweck entweder das Angebot eines Leistungspakets aus einer Hand oder das Anbieten von Zusatzdiensten, -leistungen als eine Art „Gimmick“ zu den sonstigen Angeboten des Portals. In die Art der Präsentation der Daten muss auch der Umstand berücksichtigt werden, inwiefern es dem Nutzer des Portals erkennbar ist, welche Inhalte vom Portalbetreiber und welche von externen Dienstleistern (Web Service-Anbietern) stammen. ${ }^{621} \mathrm{Ob}$ dies für den Nutzer erkennbar ist, hängt davon ab wie das Portal ausgestaltet ist.

\section{a) All-Inclusive-Dienste}

Bei Portalen, die verschiedene Dienste als Gesamtleistung anbieten, wie zum Beispiel Reiseportalen oder ähnlichem, ist es dem Dritten, dem Nutzer des Portals, kaum möglich zu erkennen, welche Dienstleistungen von welchem Anbieter stammen. Die späteren Pakete, die aus einem Sammelsurium an Dienstleistungen bestehen, werden Dritten als eine Gesamtleistung verkauft. Insofern spielt es für den Dritten auch gar keine unmittelbare Rolle, von wem die einzelnen Dienstleistungen tatsächlich stammen. Vertragspartner und damit derjenige an den sich der Dritte bei Problemen wendet, ist in erster Linie der Web Service-Nutzer. Vor diesem Hintergrund und der Tatsache, dass die von verschiedenen Web Service-Anbietern stammenden Dienstleistungen zuletzt als Gesamtpaket durch den Web Service-Nutzer an den Dritten veräuBert werden, muss davon ausgegangen werden, dass der Web Service-Nutzer sich die übermittelten Inhalte zurechnen lassen möchte, so dass ein zu Eigenmachen der Inhalte bejaht werden kann. Daher haftet der Web Service-Nutzer hinsichtlich der mittels der Web Services übermittelten Informationen im Sinne des $₫ 7$ Abs. 1 TMG nach den allgemeinen Gesetzen. Das bedeutet, dass er neben der Haftung auf Beseitigung und Unterlassung auch kenntnisunabhängig auf Schadensersatz haftet und unter Umständen sogar strafrechtlich für die mittels der Web Services übermittelten Inhalte zur Verantwortung gezogen werden kann. Auf eine Haftungsprivilegierung nach den $\$ \int 8$ 8-10 TMG kann er sich nicht berufen.

620 Spindler, MMR 2004, 442.

621 So bereits zu $\int 5$ TDG 1997: Spindler, NJW 1997, 3196; Wülfing/Dieckert, S. 80; LG Potsdam, MMR 2002, 829 (830). 
b) Portale mit Web Services als „Zusatzleistung“

Ist der Web Service dagegen deutlich als externer Dienst sichtbar in das Portal integriert, wird die Erkennbarkeit der Fremdheit von Informationen in der Regel gegeben sein. Das ist beispielsweise bei Integration der Google AXAJ Search $A P I s^{622}$, die innerhalb einer Website die Suche im gesamten Internet ermöglichen, ohne das die Google-Homepage besucht werden muss, der Fall, da hier das Google-Logo in der Suchfunktion deutlich integriert ist. Dem objektiven Betrachter ist dadurch deutlich erkennbar, dass es sich nicht um einen Dienst des Website-Betreibers sondern vielmehr einen solchen von Google handelt. Sind die Web Services derart deutlich als externe Dienste integriert, ist es für den objektiven Nutzer offensichtlich, dass der Web Service-Nutzer sich die über den Web Service erhaltenen Informationen nicht zurechnen lassen möchte. Distanziert sich der Web Service-Nutzer darüber hinaus noch von den Inhalten, die der Web Service übermittelt, so kann nicht von einem zu Eigenmachen der Inhalte gesprochen werden. Dies gilt umso mehr, da der Web Service-Nutzer tatsächlich auch keine Einwirkungsmöglichkeit auf die Inhalte, die mittels des Web Service übermittelt werden, hat. Es liegt sogar in der Hand des Web Service-Anbieters, den Diensteinhalt ohne Wissen des Web Service-Nutzers zu ändern, indem er den Web Service auf einen anderen Informationspool zugreifen lässt. In derartigen Fällen handelt es sich daher um fremde Informationen im Sinne des $₫ 7$ Abs. 2 TMG und der Web ServiceNutzer könnte hinsichtlich des Web Service den Haftungsprivilegierungen der

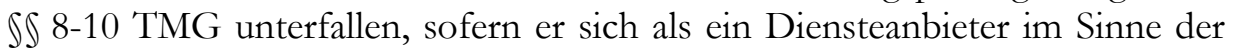

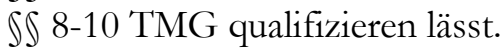

aa) Einordnung im Rahmen der Haftungsprivilegierungen

Zugangsprovider fallen nach herrschender Meinung unter die Haftungsprivilegierung des $₫ 8$ TMG. ${ }^{623} \mathrm{Ob}$ die Situation des Web Service-Nutzers hinsichtlich des Web Service allerdings tatsächlich mit der eines in $\int 8$ TMG geregelten Access Providers vergleichbar ist, erscheint fraglich. Denn klassischerweise zeichnet sich ein Access Provider dadurch aus, dass er geringere Möglichkeiten zur Abstellung einer Störung hat als beispielsweise ein Hostprovider. ${ }^{624}$ Indes besteht eine vertragliche Beziehung zwischen dem Web Service-Anbieter, von

622 Mehr Informationen unter: http://code.google.com/apis/ajaxsearch/ <25.11.2009>.

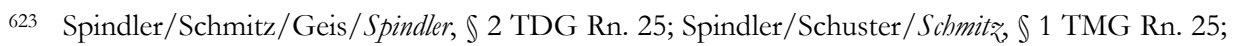
LG Frankfurt, MMR 2008, 121 (122) - You Porn; so konkludent durch die Anwendung des $\ 8$ TMG LG Köln, ZUM 2007, 873 (875).

624 Spindler/Schmitz/Geis/Spindler, § 9 TDG Rn. 32. 
dem der rechtswidrige Inhalt stammt, und dem Web Service-Nutzer, was hingegen bei Access Providern gegenüber den rechtsverletzenden Angeboten im Internet in aller Regel nicht der Fall ist. Hier handelt es sich daher um einen anderen Sachverhalt, der zwar auf den ersten Blick der Situation eines klassischen Zugangsproviders ähnelt aber bei näherer Betrachtung diverse Unterschiede aufweist. Indes kommt hinzu, dass durch den Web Service-Nutzer keinerlei Einfluss auf die mittels des Web Service übermittelten Inhalte besteht, die Inhalte fallen ausschließlich in die Sphäre des Web ServiceAnbieters. Vereinfacht bedeutet dies, dass realiter der Web Service samt dem dahinter stehenden Dienst zwar vom Web Service-Nutzer selbst in dessen Portal integriert wird, was eher auf eine Einordnung als Host Provider hindeuten würde, dieser jedoch keinerlei Einfluss auf die übermittelten Inhalte hat, was wiederum für eine Qualifikation als Access Provider spricht. Hinzu kommt die Besonderheit, dass der Web Service über die Schnittstelle dauerhaft mit dem Portal verbunden ist, was der Konstellation des $₫ 10$ TMG ähnelt, dagegen die übermittelten Inhalte technisch nicht mehr als einer zeitlich begrenzten Zwischenspeicherung im Sinne des $\$ 9$ TMG (Caching) bedürfen. Insofern handelt es sich um eine besondere Konstellation der Serviceorientierten Architektur, die in dieser Form noch nicht rechtlich eingeordnet wurde und scheinbar zunächst mit allen Providerformen etwas gemein beziehungsweise nicht gemein hat.

Die vorliegende Problematik weist zum Teil Gemeinsamkeiten mit der Problematik des so genannten Framing auf. Dabei werden fremde Webseiten derart innerhalb der eigenen Webseite übernommen, dass zwar keine physikalische Kopie der Dateien auf dem eigenen Server erfolgt, aber diese dergestalt eingebunden werden, dass beim Aufruf der Seite durch einen Internetnutzer dessen Browser veranlasst wird, den fremden Inhalt direkt von einem externen Server auf einen zugewiesenen Unterabschnitt auf dem Bildschirm zu laden. ${ }^{625} \mathrm{Da}$ Frames letztlich technisch jedoch weitgehend Inline-Links gleichen und die \\ 7 ff. TMG auf solche nicht anwendbar sind, ${ }^{626}$ kann die Problematik nicht vollständig auf Web Services in Serviceorientierten Architekturen übertragen

625 LG München, MMR 260 (261); Gabel, WRP 2005, 1104; Spindler/Schuster/Müller, \14 MarkenG Rn. 50; Gounalakis/Rhode, in: Gounalakis, Rhb. E-Business, \22 Rn. 59; Worm, S. 184.

626 Art. 21 Abs. 2 der Richtlinie 2000/31/EG ABl. EG L 178 v. 17.7.2000, S. 1-16 sparte Hyperlinks von der Haftungsprivilegierung aus, dies wurde vom deutschen Gesetzgeber übernommen. Bestätigt durch BGH, Urt. v. 18.10.2007 - I ZR 102/05, Rn. 20 - ueber18.de; NJW 2004, 2158 (2159) - Schöner Wetten. S. auch Koch, Internet-Recht, \13 S. 688; Härting, Rn. 1092; Spindler, MMR 2004, 440; ders., NJW 2002, 924; Leupold/Rücker, in: Wiebe/Leupold, Teil IV Rn. 216f.; Gersdorf, in: Eberle/Rudolf/Wasserburg, Rn. 258. 
werden. Jedoch wurden noch zu $\int 5$ TDG 1997, der die Anwendbarkeit der Haftungsprivilegierungen auf Links nicht ausschloss, bereits Überlegungen angestellt, inwiefern sich Frames in das System der Privilegierungen eingliedern lassen. Diese Überlegungen können aufgrund der gewissen Ähnlichkeit der Struktur herangezogen werden.

\section{Anwendbarkeit der $\iiint$, 9 TMG?}

So wird argumentiert, dass das Setzen von Links und somit erst Recht von Frames über die einfache Access-Leistung des reinen Zugangverschaffens beziehungsweise Durchleitens und der daraus resultierenden automatischen Zwischenspeicherung hinausgeht. Denn durch das bewusste und gewollte Setzen des Links/Frames bestehe eine willensgetragene Einbeziehung des fremden Inhalts in das eigene Angebot. ${ }^{627}$ Dies entspricht auch der Ratio der Haftungsprivilegierung, die gerade darauf beruht, dass die ,[...]Tätigkeit des Diensteanbieters beim bloßen Durchleiten auf den technischen Vorgang beschränkt ist, ein Kommunikationsnetz zu betreiben und den Zugang zu diesem zu vermitteln, über das von Dritten zur Verfügung gestellte Informationen übermittelt oder zum alleinigen Zweck der Übermittlung kurzzeitig zwischengespeichert werden.“ “628 Der Gesetzgeber führt dazu weiter aus, dass diese Tätigkeit [...] automatischer Art [ist], bei welcher der Diensteanbieter in der Regel keine Kenntnis über die weitergeleitete oder kurzzeitig zwischengespeicherte Information hat und diese auch nicht kontrolliert. Bei diesem automatisiert ablaufenden Prozess trifft der Diensteanbieter im Hinblick auf die Informationen keine eigene Entscheidung." "629 Diese Ausführungen decken sich mit den Erwägungen des Richtliniengebers: „Diese Tätigkeit ist rein technischer, automatischer und passiver Art, was bedeutet, dass der Anbieter eines Dienstes der Informationsgesellschaft weder Kenntnis noch Kontrolle über die weitergeleitete oder gespeicherte Information besitzt.“630

Überträgt man die Ausführungen auf den Web Service-Nutzer in der Serviceorientierten Architektur, so scheint es zwar zu passen, dass auch der Web Ser-

627 Worm, S. 188.

628 BT-Drs. 14/6098, S. 24.

629 BT-Drs. 14/6098, S. 24.

630 Erwägungsgrund 42 der Richtlinie 2000/31/EG des Europäischen Parlaments und des Rates v. 8.6.2000 über bestimmte rechtliche Aspekte der Dienste der Informationsgesellschaft, insbesondere des elektronischen Geschäftsverkehrs, im Binnenmarkt („Richtlinie über den elektronischen Geschäftsverkehr - E-Commerce-Richtlinie“) ABl. EG L 178 v. 17.7.2000, S. 1-16. 
vice-Nutzer bei dem vollautomatisiert ablaufenden Web Service keine Entscheidung bezüglich der übermittelten Informationen trifft, denn diese stammen allein aus der Sphäre des Web Service-Anbieters, und realiter auch keine konkrete Kenntnis von diesen Informationen hat. Jedoch tritt, ebenso wie bei dem Setzen von Links/Frames, die bewusste und gewollte Einbindung des Web Service hinzu. Eine solche willensgetragene Einbindung hatte der Gesetzgeber bei der Schaffung des Privilegierungstatbestands aber nicht vor Augen. Dies zeigen auch die folgenden Klarstellungen des Richtliniengebers: „Ein Diensteanbieter kann die Ausnahmeregelungen für ,reine Durchleitung““ und das „Caching“ in Anspruch nehmen, wenn er in keiner Weise mit der übermittelten Information in Verbindung steht. Dies bedeutet unter anderem, dass er die von ihm übermittelte Information nicht verändert. Unter diese Anforderung fallen nicht Eingriffe technischer Art im Verlauf der Übermittlung, da sie die Integrität der übermittelten Informationen nicht verändern[.]“631 sowie des Gesetzgebers: „Ein Diensteanbieter, der absichtlich mit einem der Nutzer seines Dienstes zusammenarbeitet, um rechtswidrige Handlungen zu begehen, leistet hingegen mehr als „reine Durchleitung“ und kann daher den hierfür festgelegten Haftungsausschluss nicht in Anspruch nehmen."632 Zwar wirkt der Web Service-Nutzer weder technisch auf die Übermittlung durch den Web Service ein noch wirkt er (normalerweise) absichtlich mit dem Web Service-Anbieter zusammen, um rechtswidrige Handlungen zu begehen. Jedoch zeigt der Einstieg der Ausführungen, dass der Provider mit den Informationen, die er übermittelt beziehungsweise zu denen er Zugang verschafft, nicht in Verbindung stehen darf. Eine solche Verbindung besteht aber gerade in Form des Vertragsverhältnisses zwischen Web Service-Nutzer und Web Service-Anbieter, ${ }^{633}$ sei es auch in Form eines unentgeltlichen Auftrags. ${ }^{634}$ Denn dieses hat gerade die Web Service-Nutzung zum Vertragsgegenstand. Darüber hinaus wählt der Web Service-Nutzer durch das Einbinden des Web Service den nach der Dienstebeschreibung zugrunde liegenden Dienst mit samt seinen Inhalten aus. Insofern hat der Web Service-Nutzer grundsätzlich Kenntnis, zumindest abstrakte, bezüglich der übermittelten Inhalte. Dass der Web Service-Anbieter technisch und tatsächlich die Möglichkeit hat, diesen Inhalte-Pool durch andere, rechtswidrige Inhalte auszutau-

631 Erwägungsgrund 43 der Richtlinie 2000/31/EG ABl. EG L 178 v. 17.7.2000, S. 1-16.

632 Erwägungsgrund 44 der Richtlinie 2000/31/EG ABl. EG L 178 v. 17.7.2000, S. 1-16 sowie BTDrs. 14/6098, S. 24.

633 S. zum entgeltlichen Vertrag bereits A.III.1.c)aa) Vergütung der Web Service-Nutzung.

634 S. A.III.1.c)bb) Unentgeltliche Web Service-Nutzung. 
schen, kann nicht dazu führen, eine grundsätzliche Kenntnis bezüglich der Inhalte, auf die der Web Service zugreift, zu verneinen. Unter Zugrundelegung dieser Überlegungen und dem Sinn und Zweck der Privilegierungen der $\iint 8$, 9 TMG kann weder $\int 8$ noch $\int 9$ TMG auf die Web Service-Nutzung durch den Web Service-Nutzer, der den Web Service als „fremde“ Zusatzleistung auf seiner Website anbietet, Anwendung finden.

\section{Anwendbarkeit des $\int 10 T M G$ ?}

Demnach bleibt nur noch ein mögliches Greifen des $₫ 10$ TMG zu Gunsten des Web Service-Nutzers. Problematisch dabei ist aber zum einen, dass technisch keine längerfristige Speicherung der mittels des Web Service übermittelten Inhalte durch den Web Service-Nutzer erforderlich ist und in aller Regel wohl auch nicht erfolgen wird. ${ }^{635}$ Gerade aber eine Speicherung, die über eine bloße Zwischenspeicherung hinausgeht, ist Voraussetzung der Anwendbarkeit von $₫ 10$ TMG. ${ }^{636}$ Zum anderen setzt $\ 10$ S. 1 TMG voraus, dass die Speicherung für einen Nutzer erfolgt. Mit Nutzer ist allerdings derjenige gemeint, der die Informationen zugänglich macht und zwischen diesem sowie dem Hostprovider muss ein irgendwie geartetes Rechtsverhältnis bestehen. ${ }^{637}$ Darüber hinaus muss die Speicherung vom Nutzer veranlasst worden sein. ${ }^{638}$ Derjenige, der die Informationen zugänglich macht, ist in der vorliegenden Konstellation der Web Service-Anbieter. Zwischen diesem und dem Web ServiceNutzer besteht ein Vertrag und dementsprechend das erforderliche Rechtsverhältnis. Allerdings erfolgt die kurzzeitige Speicherung praktisch nicht für den Web Service-Anbieter, sondern für Dritte, die Besucher des Portals, die die Informationen abrufen. ${ }^{639}$ Mit dieser Argumentation wird zumindest eine Anwendung des $\ 10$ TMG auf Frames und Hyperlinks, wenn man vertreten würde, dass die $\iint 7 \mathrm{ff}$. TMG diesbezüglich analogiefähig sind, abgelehnt. ${ }^{640}$ Darüber hinaus erscheint auch die Voraussetzung der Veranlassung der Spei-

635 Eine längerfristige, über die bloße Zwischenspeicherung hinausgehende, Speicherung bspw. der Suchergebnisse der Google Web APIs könnte nach einer gewissen Anzahl von Suchanfragen, den Speicherplatz des Web Service-Nutzers unnötig auslasten.

636 Spindler/Schuster/Hoffmann, $\mathbb{1} 10$ TMG Rn. 13; Spindler/Schmitz/Geis/Spindler, $\mathbb{1} 11$ TDG Rn. 5; Leupold/Rücker, in: Wiebe/Leupold, Teil IV Rn. 139.

637 Spindler/Schuster/Hoffmann, \10 TMG Rn. 15; i.d.S. auch Koch, Internet-Recht, \13 S. 688.

638 Spindler/Schmitz/Geis/Spindler, \11 TDG Rn. 7; Spindler, NJW 2002, 923.

639 So auch für Frames Koch, Internet-Recht, \13 S. 688.

640 S. Spindler, MMR 2002, 497; Koch, Internet-Recht, \13 S. 666, 688. 
cherung durch den Web Service-Anbieter fraglich. Entscheidender Unterschied zu der Setzung von Frames oder Hyperlinks ist aber, dass die Einbindung des Web Service einvernehmlich erfolgt. Durch die Publikation der Dienstebeschreibung im UDDI oder auf der eigenen Homepage ${ }^{641}$ macht der Web Service-Anbieter deutlich, dass er eine Einbindung seines Web Service wünscht und er bei erfolgreicher Einbindung mittels des Web Service die Nutzung der angegebenen Dienste ermöglicht. Aufgrund dieses Wissens integriert der Web Service-Nutzer den Web Service in seine Plattform. Wird der Web Service dann durch einen Dritten angestoßen, so speichert - wenn auch nur temporär - der Web Service-Nutzer die übermittelten Inhalte natürlich in erster Linie für den Dritten. Aufgrund der vertraglichen Beziehung zwischen Web Service-Anbieter und Web Service-Nutzer erfolgt die temporäre Zwischenspeicherung aber zumindest auch für den Web Service-Anbieter. Dies lässt sich damit erklären, dass die Dienstenutzung Gegenstand des Vertrags zwischen Web Service-Anbieter und Web Service-Nutzer ist und es sowohl im Sinne des Web Service-Anbieters als auch Web Service-Nutzers ist, alles zur Erfüllung dieses Vertrags Erforderliche zu tun. Und darunter fällt auch die Zwischenspeicherung für den Web Service-Anbieter, da diese zur Erfüllung des Vertrags technisch notwendig ist. Insofern lässt sich dem Telos des Vertragsverhältnisses entnehmen, dass die Speicherung zumindest auch auf Veranlassung des Web Service-Anbieters als Nutzer geschieht. Damit besteht technisch wie auch aus der Besonderheit der verteilten Architektur heraus ein kleiner aber nicht zu vernachlässigender Unterschied zu der Konstellation rund um Frames und Hyperlinks. Daher erscheint eine Anwendung des $\ 10$ TMG auf den Web Service-Nutzer durchaus möglich. Da es aber an einer von \10 TMG vorausgesetzten längerfristigen Speicherung der Inhalte mangelt, kann lediglich eine analoge Anwendung in Betracht kommen.

\section{Analoge Anwendung des $\$ 10 \mathrm{TMG}$}

Voraussetzung für die analoge Anwendung von Vorschriften ist eine planwidrige Regelungslücke. ${ }^{642}$ Eine solche wurde bei der analogen Anwendbarkeit der Haftungsprivilegierungen auf Hyperlinks stets damit abgelehnt, dass dem Gesetzgeber die Problematik der Hyperlinks zum einen durch die bewusste Auslassung in der E-Commerce-Richtlinie und die Bitte des Bundesrats in der

641 So liefert bspw. Google die Dienstebeschreibung ihrer Web APIs auf der eigenen Homepage, abrufbar unter: http://code.google.com/.

642 Laren₹, Methodenlehre, S. 381. 
Gesetzesbegründung ${ }^{643}$ - der durch die Bundesregierung nicht entsprochen wurde ${ }^{644}$ - und zum anderen durch die vorherige hitzige Diskussion rund um die Hyperlinkproblematik durchaus bewusst war; der Gesetzgeber daher keineswegs planwidrig sondern vielmehr bewusst nicht reguliert hat. ${ }^{645}$

Ganz anders ist die Situation aber hinsichtlich der Web Service-Nutzung im Rahmen der Serviceorientierten Architektur. Die Haftungsvorschriften stammen aus dem Jahr 2001. Zu diesem Zeitpunkt hatte weder der Richtliniengeber noch der Gesetzgeber vor Augen, dass eine solche Problematik aufkommen könnte. Der Boom hinsichtlich Web Services setzte erst ein Jahr später $e^{\text {ein }}{ }^{646}$ und selbst zu diesem Zeitpunkt wurden derartige Einsatzmöglichkeiten überwiegend in der Theorie der Semantik-Web-Bücher aufgeworfen. ${ }^{647}$ Ein breiterer vor allem praktischer Einsatz von Web Services erfolgte erst nach und nach, keinesfalls war eine solche Entwicklung aber bereits 2001 absehbar. Zwar hat der Gesetzgeber das Telemediengesetz $2006^{648}$ erneut novelliert, dabei wurden die Vorschriften über die Verantwortlichkeit aber ohne Änderungen übernommen. ${ }^{649}$ Der Gesetzgeber konzentrierte sich im Rahmen der Novellierung aufgrund der zahlreichen Unstimmigkeiten hauptsächlich auf die Zusammenführung des Mediendienstestaatsvertrags mit dem Teledienstesowie dem Teledienstedatenschutzgesetz. Insofern kann man hier durchaus von einer planwidrigen Regelungslücke sprechen.

Die weitere Voraussetzung für eine Analogie ist das Vorliegen eines vergleichbaren Sachverhalts. ${ }^{650}$ Dafür muss der Telos der Haftungsprivilegierungen genauer betrachtet werden. Ziel der Haftungsprivilegierung ist es, rein technische Kommunikationsvorgänge aus der Verantwortlichkeit herauszuneh-

643 Stellungnahme des BR zum RegE BT-Drs. 14/6098, S. 34.

644 Gegenäußerung der Bundesregierung, BT-Drs. 14/6098, S. 37.

645 BGH, NJW 2004, 2158 (2159) - Schöner Wetten; OLG Stuttgart, MMR 2006, 387 (388) m. Anm. Liesching; Spindler, MMR 2002, 498; Stadler, JurPC Web-Dok. 2/2003, Abs. 10 ff.; Spindler/Schmitz/Geis/Spindler, Vor $\int 8$ TDG Rn. 33; Koch, Internet-Recht, \13 S. 666 f.; Neubauer, in: Moritz/Dreier, D Rn. 70 f.; Gersdorf, in: Eberle/Rudolf/Wasserburg, Rn. 258.

646 S. zum einsetzenden Hype Wichmann, v. 6.3.2002, abrufbar unter: http://www.berlecon.de/research/spotlights.php?we_objectID=90<25.11.2009>.

647 S. z.B. Jablonski/Meiler/Petrov, in Fröschle, S. 78 ff.

648 Der Gesetzesentwurf trat am 1.3.2007 in Kraft.

649 S. BT-Drs. 16/3078, S. 15.

650 Laren₹, Methodenlehre, S. 381; Pawlowski, Rn. 165. 
men. ${ }^{651}$ Damit soll insbesondere ein für den Provider nicht leistbarer Kontrollaufwand vermieden und Rechtssicherheit geschaffen werden. ${ }^{652}$ Dies zugrunde gelegt, muss es sich auch bei der kurzzeitigen Speicherung der Informationen, die von dem Web Service übertragen wurden, um einen rein technischen Vorgang handeln, was aber zweifellos bejaht werden kann, da die kurzzeitige Speicherung zwangsläufig im Cache erfolgt. Darüber hinaus müssen die Kontrollmöglichkeiten der gespeicherten Inhalte für den Web ServiceNutzer ebenso schwer leistbar sein, wie bei einem klassischen Hoster. Bevor der Web Service durch den Dritten in Gang gesetzt wird, weiß der Web Service-Nutzer lediglich abstrakt um die Inhalte, die übermittelt werden können. $\mathrm{Ob}$ diese Inhalte aber auch die in der Dienstebeschreibung angepriesenen sind, kann der Web Service-Nutzer in diesem Stadium nicht kontrollieren. Sobald die Inhalte dann vom Web Service des Web Service-Anbieters übermittelt wurden, erfolgt automatisch die Zwischenspeicherung beim Web Service-Nutzer, da die Inhalte sonst gar nicht für den Dritten sichtbar angezeigt werden könnten. Das heißt, aufgrund der Technik besteht auch zu diesem Zeitpunkt keine Möglichkeit der Kontrolle der übermittelten Inhalte. Daher ist die Kontrollmöglichkeit des Web Service-Nutzers hinsichtlich der Zwischenspeicherung mit den Kontrollmöglichkeiten des klassischen Hosters vergleichbar. Darüber hinaus leistet der Web Service-Nutzer sogar weniger als ein klassischer Hoster, da er eben gerade nicht längerfristig speichert sondern nur kurzzeitig zwischenspeichert. Daher muss er erst Recht in den Genuss der Haftungsprivilegierung des \10 TMG kommen. \10 TMG kann daher analog zugunsten des Web Service-Nutzers Anwendung finden.

\section{Sonderproblem der Suchmaschinen-Web Services}

Eine analoge Anwendung des $\int 10$ TMG kann allerdings nicht pauschal angenommen werden, denn wie Hyperlinks werden auch Suchmaschinen nicht von den Haftungsprivilegierungen erfasst. ${ }^{653}$ Handelt es sich daher um einen Web Service für die Einbindung von Suchmaschinen, wie beispielsweise dem Google AXAJ Search API, so erscheint es fraglich, ob die Haftungsprivilegierung dennoch (analog) Anwendung finden kann.

651 Erwägungsgrund 42 der E-Commerce-Richtlinie, 2001/31/EG v. 8.6.2000, ABl. EG L 178 v. 17.7.2000, S. 1-16; BT-Drs. 14/6098, S. 22 f.

652 Spindler, MMR 2002, 497.

653 S. Art. 21 ECRL, 2001/31/EG v. 8.6.2000, ABl. EG L 178 v. 17.7.2000, S. 1-16; Gegenäußerung der Bundesregierung, BT-Drs. 14/6098, S. 37; so auch KG, MMR 2006, 393 (394) m. Anm. Spieker. 
Im vorliegenden Fall soll indes nicht der Suchmaschinenbetreiber privilegiert werden, sondern derjenige, der den Suchdienst in seiner Plattform einbindet und die Suchergebnisse kurzzeitig zwischenspeichert. Da der Web ServiceNutzer nicht selbst Betreiber des Suchdienstes ist, sondern lediglich den Zugang und die Plattform dafür schafft, ist die Situation eine völlig andere und es kann bei der Beurteilung, ob die Haftungsprivilegierung greift oder nicht, lediglich auf die Tätigkeit des Web Service-Nutzers ankommen. Daher spielt es für eine Anwendung der Haftungsprivilegierung keine Rolle, welche Art von Dienst der Web Service-Nutzer in seine Website einbindet. \10 TMG kann daher unabhängig von der Art des eingebundenen Dienstes analog Anwendung finden.

bb) Zwischenergebnis: Haftung bei „Zusatzleistung“

Da zugunsten des Web Service-Nutzers $\int 10$ TMG analog greift, haftet er als Störer theoretisch auf Beseitigung und im Falle des Vorliegens zumutbarer Prüfungs- und Kontrollpflichten auch auf Unterlassung hinsichtlich der Übermittlung kerngleicher rechtswidriger Inhalte durch die Web Services. In den Fällen der rechtswidrigen Inhalteübermittlung durch einen Web Service wird der Beseitigungsanspruch hinsichtlich der Inhalte praktisch allerdings kaum eine Rolle spielen, da der Web Service von Dritten selbst in Gang gesetzt wird und diesem die entsprechenden Inhalte automatisiert übermittelt werden. Zwar erhält der Dritte die Inhalte innerhalb des Portals des Web Service-Nutzers, dieser unterzieht die übermittelten Inhalte aber keiner Prüfung, bevor sie der Dritte einsehen kann. Daher kann der Dritte diese abspeichern, bevor der Web Service-Nutzer Kenntnis von der Rechtswidrigkeit der Inhalte erlangt. Hat er dann durch Abmahnung oder ähnliches Kenntnis erlangt, ist es für eine Beseitigung zu spät, da er nur schwerlich alle Kopien bei Dritten löschen kann. Auf seinem eigenen Portal kann er lediglich die im Zwischenspeicher hinterbliebenen Kopien löschen, sofern eine solche Kopie überhaupt noch im Cache vorhanden sein sollte, denn eine längerfristige Speicherung erfolgt nicht, die Inhalte werden immer neu durch Auslösung eines Dritten vom Web Service des Web Service-Anbieters übermittelt.

Insofern bleibt den Betroffenen praktisch lediglich ein in die Zukunft gerichteter Unterlassungsanspruch bezogen auf die Möglichkeit des Abrufs des rechtswidrigen Inhalts durch den Web Service des Web Service-Anbieters. Eine solche Unterlassung wird aber aus den bereits genannten Gründen ebenfalls technisch nicht möglich sein, weil eine Kontrolle der Übermittlung nicht möglich ist und dann zwangsläufig eine neue Rechtsverletzung vor einer möglichen Kontrolle durch den Web Service-Nutzer auftritt. Darüber hinaus kann er die de facto erneut erfolgte Rechtsverletzung erneut aus dem eigenen Cache 
beseitigen und damit zumindest das weitere Andauern der Rechtsverletzung vermeiden. Indes kann er rechtsverletzende Inhalte, die gegebenenfalls bei Dritten gespeichert wurden, mangels Zugriffsmöglichkeit nicht beseitigen. Daher würde der Web Service-Nutzer jedes Mal aufs Neue gegen den Unterlassungstitel verstoßen, ${ }^{654}$ so dass ihm nach $\int 890$ ZPO Ordnungsgeld oder sogar -haft drohen. ${ }^{655}$ De facto müsste er also alle vom Web Service übermittelten Inhalte zunächst manuell betrachten und kontrollieren bevor er sie zur Ansicht an den Dritten freigibt, um den Zwangsvollstreckungsmaßnahmen zu entgehen, ${ }^{656}$ denn eine rein technische Bewertung, ob ein Inhalt rechtswidrig ist oder nicht, ist nicht möglich. ${ }^{657}$ Dies widerspricht allerdings zum einem dem Sinn und Zweck der Technik der Web Services, die gerade von Automatismen und vereinfachten Nutzungsmöglichkeit lebt, und würde zum anderen das Verbot allgemeiner Überwachungspflichten in \ 7 Abs. 2 S. 1 TMG konterkarieren. ${ }^{658}$ Die einzige Möglichkeit, weitere Rechtsverletzungen und somit auch einen Verstoß gegen den Unterlassungstitel zu verhindern wäre es, den Web Service des Web Service-Anbieters aus dem Portal zu entfernen. Ob eine solche Maßnahme allerdings im Rahmen des Unterlassungstitels erwartet werden kann und demnach vom Unterlassungstitel erfasst ist beziehungsweise in den Tenor mit aufgenommen werden kann, ist eine Frage der Zumutbarkeit im Rahmen des materiellen Anspruchs. ${ }^{659} \mathrm{Da}$ es sich allerdings lediglich um

654 \890 ZPO erfordert einen schuldhaften Verstoß, da dieser aber i.S.v. Vorsatz und Fahrlässigkeit zu verstehen ist, wird ein Verschulden in aller Regel gegeben sein, s. Musielak/Lackmann, \890 Rn. 5; MünchKommZPO/Schilken, Bd. 3, \890 Rn. 10; Hk-ZPO/Pukall, \890 Rn. 7; BVerfG, NJW 1991, 3139; BGH, NJW 1994, 45 (46).

655 Denn die Unterlassungsvollstreckung wird auch dann betrieben, wenn sie nur durch eine bestimmte Maßnahme des Schuldners erfüllt werden kann, s. MünchKommZPO/Schilken, Bd. 3, \890 Rn. 3.

656 Spindler, K\&R 1998, 181; Pichler, Anm. zu OLG München, MMR 1998, 539, MMR 1998, 540 (542); Volkmann, CR 2003, 442.

657 Berger/Janal, CR 2004, 923. Zwar wäre eine solche Bewertung mittels Ontologien denkbar, diese haben sich in der Rechtswissenschaft zu Recht allerdings bisher nicht durchsetzen können, da viele juristische Begrifflichkeiten einer Abwägung bedürfen, die technisch nicht umsetzbar ist. S. dazu auch die Berichte des LOIS-Projekts, wobei festgestellt wurde, dass Ontologien für eine juristische Anwendung oft die Tiefe und Exaktheit fehlt und die juristische Begriffsstruktur daher nicht vollständig abgebildet werden konnte, Senn/Schweighofer/Liebwald/Geist/Drachsler, IRIS 2006, S. 292.

Volkmann, CR 2003, 442; Spindler, K\&R 1998, 181.

659 Eine Verschiebung der Zumutbarkeitsprüfung in das Vollstreckungsverfahren wird nur ausnahmsweise gestattet, wenn ,weder die Art der zukünftigen Angebote noch die in der Zukunft bestehenden technischen Möglichkeiten, klare Verdachtsfälle herauszufiltern, abzusehen sind“, s. BGH, MMR 2007, 507 (511) - Internetversteigerung II; Rössel/Kruse, CR 2008, 40. 
die Entfernung eines speziellen Web Service eines bestimmten Web ServiceAnbieters handelt, dürfte der Zumutbarkeit nichts im Wege stehen, zumal zu befürchten ist, dass der Web Service-Anbieter seinen Web Service erneut zum Übermitteln rechtswidriger Inhalte missbrauchen wird. Die Entfernung des Web Service ist daher vergleichbar mit der Sperrung von Nutzern von Internetauktionshäusern, die Waren mit rechtswidrigen Hintergrund zum Verkauf anbieten. Auch dort muss mit Wiederholungen gerechnet werden, deshalb genügt es in der Regel nicht, zur Vermeidung des Verstoßes gegen den Unterlassungstitel lediglich das konkrete Angebot zu sperren, es muss vielmehr die Ursache beseitigt werden. ${ }^{660} \mathrm{Um}$ einen Verstoß gegen den Unterlassungstitel zu vermeiden, sollte der Web Service-Nutzer daher den Web Service des Web Service-Anbieters aus seiner Web-Präsenz entfernen.

\section{Zwischenergebnis}

Verbreitet der Web Service-Anbieter mittels seines Web Service rechtswidrige Inhalte und ist dieser Web Service innerhalb der Web-Präsenz des Web Service-Nutzers integriert, so dass Dritte diese rechtswidrigen Inhalte abrufen können, so hängt die Haftung davon ab, auf welche Art und Weise der Web Service in die Web-Präsenz des Web Service-Nutzers integriert ist. Handelt es sich um ein Portal, dass mit Hilfe verschiedener Web Services ein Paket an Leistungen anbietet, ohne das sofort und eindeutig für Dritte erkennbar ist, von wem die jeweilige Leistung tatsächlich stammt, da der Vertrag lediglich mit dem Portalbetreiber (Web Service-Nutzer) geschlossen wird, so macht sich der Web Service-Nutzer die mittels der Web Services übermittelten Informationen zu Eigen. Daher haftet er als Content-Provider im Sinne des $\int 7$ Abs. 1 TMG ohne Privilegierungen voll für die übermittelten Inhalte. Wird der Web Service dagegen lediglich als Zusatzleistung für Dritte erkennbar als fremder Dienst in die Web-Präsenz des Web Service-Nutzers integriert, so handelt es sich bei den durch den Web Service übermittelten Inhalten um fremde Inhalte. Zugunsten des Web Service-Nutzers, gleichgültig welcher Art der implementierte Web Service ist, greift dann \10 TMG analog, so dass er lediglich als Störer haftet; darüber hinausgehend ist er privilegiert. Praktisch wird allerdings aufgrund der Besonderheit der Web Service-Nutzung lediglich eine Haftung auf Unterlassung in Frage kommen, da eine Beseitigung in aller Regel - abgesehen von der Löschung des Cache - nicht möglich sein wird.

660 Wobei das Beispiel der Internetauktionsplattform insofern nicht vollständig übereinstimmt, als das derartige Nutzer, mangels geeigneter Identifizierungssysteme bei der Anmeldung, sich dann einfach wieder mit einem neuen Profil anmelden. Dieser Aspekt kann aber hier untergewichtet werden, da es darauf für die Zumutbarkeit nicht ankommt. 
Um im Rahmen der Unterlassung wiederholte Verstöße gegen den Titel zu vermeiden, ist eine Unterlassung derart auszugestalten, dass der Web ServiceNutzer den Web Service des besagten Web Service-Anbieters aus seiner WebPräsenz entfernt und es auch weiterhin unterlässt, ihn erneut einzubinden.

\section{Resümee}

Die vorangegangenen Ausführungen zeigen, dass das Wettbewerbsrecht rund um die Web Service-Nutzung eine nicht zu vernachlässigende Bedeutung hinsichtlich der Haftung hat. Während öffentlich-rechtliche Portale, die externe Dienste in ihr Portal integrieren, in der Praxis in den nächsten Jahren, bis zur vollständigen Akzeptanz und vor allem Verbreitung von Diensten, die mittels Web Service genutzt werden können, wohl eher die Minderheit der praxisrelevanten Fälle sein und somit auch aus wettbewerbsrechtlicher Perspektive eher eine untergeordnete Rolle spielen werden, ${ }^{661}$ könnte die Problematik rund um die unlautere Nutzung des UDDI durch irreführende Werbung nach $\ 3$ UWG in Verbindung mit $\ 5$ UWG eine durchaus große Rolle spielen. Mangels Subsidiaritätsverhältnis hinsichtlich des unmittelbaren und mittelbaren Störers, werden bei Wettbewerbsverletzungen durch den Web ServiceAnbieter - neben den unmittelbaren Ansprüchen aus den $\iint 9$ ff. UWG gegen diesen - insbesondere auch Ansprüche gegen den Web Service-Vermittler nach den Grundsätzen der Störerhaftung von Relevanz sein.

Aufgrund der Masse an Störerhaftungsfällen im Endkundenbereich in den letzten Jahren wird den Großteil der praktischen Fälle aber vermutlich die Haftung des Web Service-Nutzers für rechtswidrige Inhalte, die der Web Service-Anbieter mit Hilfe des Web Service übermittelt, einnehmen, sofern sich ein solcher Markt für Web Service-Anbieter als lukrativ erweisen sollte. Der Web Service-Anbieter hat, aufgrund der Funktionsweise der Web ServiceNutzung im Rahmen der Serviceorientierten Architektur, eine durchaus verantwortungsvolle Position inne, da er den alleinigen Zugriff hinsichtlich der Informationen, auf die der Web Service zugreift, hat und somit auch über Rechtmäßigkeit beziehungsweise Rechtswidrigkeit der übermittelten Inhalte entscheidet. Um diese Machtposition - auch hinsichtlich der Haftung des Web Service-Vermittlers für wettbewerbswidrige Registrierungen des Web ServiceAnbieters - zumindest zu minimieren und „schwarzen Schafen“ entgegenzuwirken, würde es sich empfehlen, die Web Service-Anbieter einer gewissen Kontrolle zu unterwerfen. Eine solche lässt sich in ganz unterschiedlicher

661 Eröffnet die öffentliche Hand aber einen solchen Dienst, so ist sie gehalten, die Grundsätze des Vergaberechts und des GWB zu beachten, s. B.IV Öffentliche All-Inclusive-Angebote. 
Weise, zum Beispiel durch die Vergabe von Zertifikaten durch eine unabhängige Stelle, Nutzerbewertungen, ein Post-Ident-Verfahren bei der Registrierung im UDDI (auch freiwillig, so dass der Web Service-Nutzer selbst entscheiden kann, ob er das Risiko, an ein schwarzes Schaf zu geraten, auf sich nehmen möchte oder er lieber einen geprüften Web Service-Anbieter wählt) oder ähnliches, schaffen. Eine genauere Erörterung derartiger Systeme kann im Rahmen dieser Arbeit nicht erfolgen. 


\section{Teil Datenschutz bei „Web Service-Portalen“}

\section{A. Einführung}

Unabhängig von den Besonderheiten des Einsatzes von Web Services in Umsetzung einer Serviceorientierten Architektur, können Web Services auch zur Übermittlung von Daten und Informationen von externen Diensten zu Portalen eingesetzt werden. Praktisch könnte ein solches Anwendungsfeld derart aussehen, dass ein Portal zunächst einmal eine Anwendungsoberfläche bietet, die von einem geschlossenen oder offenen Personenkreis (im Folgenden Portalnutzer) genutzt werden kann. Auf dem Portal können sich eigene Inhalte des Portalbetreibers befinden oder aber der Portalbetreiber stellt lediglich eine Plattform mit Rahmenbedingungen zur Verfügung und überlässt die inhaltliche Ausgestaltung externen Anbietern von Diensten, die mittels Web Services lose an die Plattform gekoppelt werden. Diese externen Dienste unterschiedlicher Anbieter (im Folgenden Web Service-Anbieter) können, müssen aber nicht, einen groben Sachzusammenhang aufweisen. Sie bieten ihre Leistungen mit Hilfe von Web Services auf dem Portal auf eigene Rechnung an. Dabei sind sie lediglich durch eine Schnittstelle lose an das Portal gekoppelt, das eigentliche Agieren erfolgt von eigenen Servern der Web Service-Anbieter. Der Web Service stellt insofern die technische Verbindung zwischen dem Portal und dem davon unabhängigen Dienst her. Der Portalbenutzer kann 
durch diese Koppelung den Dienst des Web Service-Anbieters direkt vom Portal aus nutzen, ohne den Web Service-Anbieter gesondert kontaktieren zu müssen.

Zieht man das eingangs erläuterte Beispiel des Reiseportals heran, ${ }^{662}$ so ändert sich der Sachverhalt derart, dass nicht das Reiseportal ein Gesamtpaket an Leistungen (Flug, Hotel, Mietwagen etc.) verkauft, sondern die Anbieter der Fluggesellschaften, der Hotels und Mietwagenunternehmer das Reiseportal lediglich als Oberfläche für eigene Verkaufstätigkeiten benutzen, um eigene Verträge mit den Portalnutzern über das Portal abzuschließen. ${ }^{663}$ Ob für die Portalnutzer erkennbar ist, dass es sich um externe Dienste handelt, die nicht vom Portalbetreiber angeboten werden, hängt vom Geschäftsmodell des Web Service-Portals ab. Portalnutzer können direkt nach der gewünschten Dienstleistung, Information oder Ware von dem Portal aus suchen. Die Lieferung erfolgt bei Suchanfragen, die Daten als Gegenstand haben, dann direkt mit Hilfe des Web Service auf dem Portal und im Falle von gegenständlichen Waren auf dem postalischen Wege. Der Vertrag wird mit dem jeweiligen Web Service-Anbieter geschlossen, wobei das Anbieten des Web Service das Angebot und die Nutzung des Web Service die Annahme darstellen, soweit unbeschränkt verfügbare Informationen Vertragsgegenstand sind. ${ }^{664}$ Handelt es sich um Waren, die nur beschränkt vorrätig sind, so stellt regelmäßig die Nutzung das Angebot und die Lieferungsbestätigung die Annahme dar. ${ }^{665} \mathrm{Um}$ die Abwicklung kostengünstiger und schneller zu gestalten, könnten die Portale derart gestaltet sein, dass sie zunächst eine Registrierung des Nutzers erfordern und sodann die Web Service-Anbieter auf die beim Portalbetreiber hinterlegten Registrierungsdaten, beispielsweise zum Zwecke der Abrechnung, mittels Web Service zugreifen können.

Je nach Ausgestaltung derartiger Portale können diese einen sehr großen und vielseitigen Pool an Daten der Portalnutzer bieten. Bei den an der Portalgestaltung Beteiligten (Web Service-Anbieter aber auch Portalbetreiber) wird aus

662 S. B.I.2 Web Service-Nutzer.

663 Derartige Portale können in den unterschiedlichsten Bereichen entstehen, s. dazu Bodendorf/Schobert, in: Fröschle, S. 44 ff., der ein Kfz-Versicherungsportal (Versicherungsgesellschaft, Routenplaner, Mietwagenvermietung, Wetterdienst etc.) nennt. Auch Portale für geschlossene Nutzerkreise, wie bestimmte Berufs- oder Interessengruppen, sind denkbar. Die Möglichkeiten sind dabei unbegrenzt und durch die Möglichkeit der losen Koppelung von Web Services leicht und kostengünstig umsetzbar.

664 S. dazu bereits die Erläuterungen bei A.III.2.a)bb) Einzelne Dienste.

665 S. dazu A.III.2.a)bb) „All-Inclusive-Dienste“. 
kommerziellen Gesichtspunkten naturgemäß häufig auch der Wunsch bestehen, diese zahlreichen Daten, insbesondere in Verbindung mit dem Nutzungsverhalten innerhalb des Portals, zu verwerten, indem Interessen, Präferenzen, Kaufkraft, Kaufgewohnheiten und Preisniveau der Einkäufe genau ausgewertet und analysiert werden. Dies kann verschiedene Hintergründe haben, zum Beispiel das Interesse den Markt zu analysieren, aber auch die Kunden genau kennen zu lernen und ihnen individualisierte Werbung zu übermitteln, indem beispielsweise das Portalprofil auf den Kunden und seine Präferenzen abgestimmt oder gar die gesammelten Daten an Externe verkauft werden. Solche Datensammlungen können auf unterschiedliche Art und Weise und mit Hilfe verschiedener Techniken angelegt werden, wie zum Beispiel mit Cookies, Web-Bugs oder durch die Speicherung von Log-Files. Der Portalnutzer hat dabei realiter keine Kontrolle über den Umgang mit seinen Daten. Häufig wird er nicht einmal bemerken, durch welche Erfassungsmaßnahmen wann und in welchem Umfang Daten über ihn gesammelt und darüber hinaus gegebenenfalls an einen nicht in Gänze überschaubaren Kreis von Web Service-Anbietern übermittelt werden. Gerade der Einsatz von Web Services und die daraus resultierende Möglichkeit, Daten vollautomatisiert zu sammeln, bergen zusätzliche Gefahren der Unüberschaubarkeit. Daher drängen sich Fragen der Zulässigkeit derartiger Verwertungshandlungen bezüglich der Daten von Portalnutzern auf. Der Hauptuntersuchungsgegenstand dieses Teils wird daher die Zulässigkeit des Umgangs mit den Daten durch den Portalbetreiber und vor allem die Web Service-Anbieter darstellen. Dabei wird insbesondere untersucht, welche Handlungen konkret zulässig sind und wo der Datenschutz Grenzen aufzeigt.

Die vorliegende Untersuchung über den Umgang mit personenbezogenen Daten durch die an der Web Service-Portal-Nutzung beteiligten Akteure und die Rechte der Betroffenen in Kapitel B.IV beschränkt sich auf die Analyse des deutschen Hoheitsbereichs, der umfangreiche und konkrete Regelungen für den Umgang mit personenbezogenen Daten durch die an der Web ServicePortal-Nutzung beteiligten Akteure im deutschen Datenschutzrecht bietet. Da das Internet eine grenzüberschreitende und somit internationale Materie ist, kann das deutsche Datenschutzrecht jedoch nicht völlig losgelöst von internationalen und vor allem nicht von den europäischen Rechtsentwicklungen betrachtet werden. Daher wird im Folgenden, zum besseren Verständnis des vorliegenden Untersuchungsgegenstands hinsichtlich der einschlägigen nationalen Regelungen, ein Überblick über den internationalen und europäischen Regelungsrahmen gegeben. 


\section{B. Rechtsrahmen für den Datenschutz}

I. Internationaler Rahmen

Aufgrund der Internationalität des Internet wird und wurde stets nach Koordinierung auf internationaler Ebene verlangt. ${ }^{666} \mathrm{Im}$ Bereich des Datenschutzes wurde diese Kodifizierung und Harmonisierung bereits vorangetrieben, ${ }^{667}$ wenn auch noch nicht in befriedigender Weise, wie die unterschiedlichen Schutzniveaus und Regelungskonzepte letztlich zeigen. ${ }^{668}$

\section{Europarat}

Für eine nachhaltige Beeinflussung der Entwicklung des internationalen Datenschutzes sorgte bis zur Einführung der Europäischen Datenschutzrichtlinie 95/47/EG in erster Linie der Europarat. ${ }^{669}$

Erste Ansätze zum Schutz der Privatsphäre lieferte die Europäische Konvention zum Schutz der Menschenrechte und Grundfreiheiten (EMRK) vom 4.11.1950, die als völkerrechtlicher Vertrag in Deutschland den Rang eines Bundesgesetzes genießt, ${ }^{670}$ in Art. 8 Abs. 1, wonach jede Person ein Recht auf Achtung ihres Privat- und Familienlebens, ihrer Wohnung und ihrer Korrespondenz hat, worunter auch Aspekte des Rechts auf informationelle Selbstbestimmung fallen. ${ }^{671}$ Ein ausdrückliches Recht auf Datenschutz existierte indes nicht. ${ }^{672}$ Diese Lücke wurde durch das Übereinkommen zum Schutz des Men-

666 S. nur Reidenberg, 52 STNL, 1362 ff., der bereits Strategien zur Koordination und Kooperation vorgeschlagen hat; zu den diesbezüglichen Bemühungen der EU-Kommission und der Art. 29Datenschutzgruppe, die sich hinsichtlich einer Vereinfachung der Datenübermittlung an Stellen in so genannten Drittländern bemühen Gola/Klug, NJW 2007, 2458; Positionen des Düsseldorfer Kreises zum internationalen Datenverkehr, v. 28.3.2007, abrufbar unter: https://www.ldi.nrw.de/mainmenu_Service/submenu_Entschliessungsarchiv/Inhalt/Beschlue sse_Duesseldorfer_Kreis/Inhalt/2007/20070419_Internationaler_Datenverkehr/Positionspapi er.pdf <25.11.2009>; eine Selbstregulierung mittels „Code“ vorschlagend Lessig, Code 2.0, S. 83 ff.

667 Patzak, S. 208.

668 S. dazu ausführlich Reidenberg, 52 STNLR , 1336 ff.; Schaar, Rn. 73 ff.

669 Simitis/Simitis, Einleitung Rn. 151; Tinnefeld/Ehmann/Gerling, S. 100; Patzak, S. 212; Schaar, Rn. 83.

670 BVerfGE 74, 358 (370); 82, 106 (119 f.); BVerfG, NJW 2004, 3407 (3408); Eblers, in: ders., EuGR, $₫ 2$ Rn. 6.

671 Patzak, S. 216; Scholz, S. 113 f.

672 Tinnefeld/Ehmann/Gerling, S. 103; Scholz, S. 113. 
schen bei der automatischen Verarbeitung personenbezogener Daten vom 28.1.1981673, das völkerrechtliche Bindungswirkung hat, geschlossen. ${ }^{674}$ Das Übereinkommen ergänzt damit die Europäische Konvention zum Schutz der Menschenrechte und Grundfreiheiten im Hinblick auf den Datenschutz. ${ }^{675}$ Darin werden Datenschutzgrundsätze aufgestellt (Art. 5), die Behandlung von sensiblen Daten geregelt (Art. 6), dem Betroffenen Rechte auf Auskunft, Berichtigung und Löschung an die Hand gegeben (Art 8) sowie die Möglichkeit, innerstaatliche Sanktionen festzulegen (Art.10), eröffnet. Darüber hinaus werden Regelungen zum grenzüberschreitenden Datenverkehr getroffen. Das Übereinkommen wurde 2001 durch ein Zusatzprotokoll ergänzt, das festlegt, dass jede Vertragspartei Kontrollstellen gewährleisten muss sowie Regelungen zum grenzüberschreitenden Verkehr mit Nicht-Vertragsparteien trifft. ${ }^{676}$

Ergänzend zu diesem Übereinkommen beschloss der Europarat zusätzlich Empfehlungen, wobei für den vorliegenden Untersuchungsgegenstand insbesondere die Empfehlung zum Schutz personenbezogener Daten im Internet vom 23.2.1999 relevant ist. ${ }^{677}$ Diese hat zwar keine unmittelbar bindende Wirkung, aber durch die Konkretisierung unbestimmter Rechtsbegriffe und Generalklauseln genießt sie zumindest indirekte Bindungswirkung. ${ }^{678}$ Darin werden vor allem frühzeitige und umfassende Aufklärung durch die Provider über die mit ihren Diensten verbundenen Risiken verlangt und ein besserer Selbstschutz angeregt.

\section{Vereinte Nationen}

Sich bereits seit 1968 mit den Konsequenzen automatisierter Datenverarbeitung, aus Furcht diese könnte eine Gefährdung für die Menschenrechte darstellen, befassend, ${ }^{679}$ beschloss die Generalversammlung der Vereinten Natio-

673 Inkraft getreten am 1.10.1985.

674 Scholz, S. 114; Patzak, S. 224 f.; Schaar, Rn. 84 f.; ausführlich zum Übereinkommen Simitis/Simitis, Einleitung Rn. 151 ff.

675 Patzak, S. 224.

676

Das

Zusatzprotokoll

ist

abrufbar

unter:

http://www.conventions.coe.int/Treaty/ger/Treaties/Html/181.htm <25.11.2009>.

677 Empfehlung Nr. R (99) 5, des Ministerkommitees an die Mitgliedsstaaten über den Schutz des Privatlebens im Internet, abrufbar unter: http://www.unipotsdam.de/u/ls_klein/egmr/minkom/ch/rec1999-5.pdf. Abdruck der Leitlinien in Schaar, Rn. $88 \mathrm{f}$.

Scholy, S. 115.

Simitis/Simitis, Einleitung Rn. 192; Patzak, S. 209; Schaar, Rn. 79. 
nen am 15.12.1990 Richtlinien über personenbezogene Daten in automatisierten Dateien mit Empfehlungscharakter. ${ }^{680} \mathrm{Da}$ es sich aber nur um Empfehlungen handelt, sind diese nicht bindend. ${ }^{681}$ Nach den Empfehlungen sollen Mitgliedsstaaten - aber auch internationale staatliche Organisationen - in ihren nationalen Rechtsvorschriften besondere Vorgaben berücksichtigen (zum Beispiel Richtigkeit der Daten, Möglichkeit der Einsichtnahme durch den Betroffenen, Rechtmäßigkeit und die Beachtung von Treu und Glauben etc.). Zum Zwecke der humanitären Hilfe oder dem Schutz der Menschenrechte und der Grundfreiheiten können sie aber davon abweichen, siehe Punkt 6. Bemerkenswert ist die erstmalige Forderung in Punkt 8 nach einer kompetenten und unabhängigen Kontrollinstanz. Aufgrund der Ungenauigkeit dieser Richtlinien und fehlenden Präzisierung auf die Online-Welt sind sie allerdings ungeeignet als Grundlage für eine bereichsspezifische Regelung; eine Lösung der datenschutzrechtlichen Probleme im Internet wäre damit nicht möglich. 682

\section{3. $O E C D$}

Neben dem Europarat hat auch die Organisation für wirtschaftliche Zusammenarbeit und Entwicklung (OECD) einen wichtigen Beitrag zur Datenschutzdiskussion geleistet, indem der Rat am 23.9.1980 die Richtlinien für den Schutz der Privatsphäre und den grenzüberschreitenden Datenverkehr personenbezogener Daten verabschiedete. ${ }^{683}$ Diese völkerrechtlich nicht bindenden Richtlinien ${ }^{64}$ enthalten materielle und verfahrensrechtliche Regelungen für den öffentlichen und privaten Sektor, wobei der Grundsatz der Transparenz einen besonderen Stellenwert genießt. ${ }^{685}$ Zugrunde liegende Idee dieser Empfehlungen war das Bemühen, durch Harmonisierung der Datenschutzbestimmungen, dem Entstehen von Handelshemmnissen durch unterschiedliche Anforderungen nationaler Datenschutzgesetze vorzubeugen. ${ }^{686}$

\footnotetext{
680 Guidelines Concerning Computerized Personal Data Files, v. 14.12.1990, abrufbar unter: http:/ $/$ www.legislationline.org/legislation.php?tid $=219 \& l i d=6157<25.11 .2009>$.

681 Tinnefeld/Ehmann/Gerling, S. 99; Roßnagel/Burkert, Hdb. DatenschutzR, Kap. 2.3 Rn. 40.

682 So auch Patrak, S. 209; Scholv, S. 118.

683 Abrufbar unter: http://www.oecd.org/dataoecd/16/7/15589558.pdf <25.11.2009>; ausführlich zur Bedeutung dieser Empfehlungen Roßnagel/Burkert, Hdb. DatenschutzR, Kap. 2.3 Rn. $30 \mathrm{ff}$.

684 Tinnefeld/Ehmann/Gerling, S. 98.

685 Scholv, S. 116; Schaar, Rn. 81.

686 Simitis/Simitis, Einleitung Rn. 184; Schaar, Rn. 81; Patzak, S. 210.
} 


\section{Resümee}

Auf internationaler Ebene mangelt es an einem allgemeinen rechtsverbindlichen Regelungswerk zum Datenschutz. Zwar gibt es, wie die vorangegangenen Ausführungen zeigen, gewisse Regulierungsansätze, denen fehlt es aber zum einen an Präzession und zum anderen meist an Rechtsverbindlichkeit. Wichtigster Anknüpfungspunkt ist das Recht auf Privatsphäre in Art. 8 EMRK. Ein weltweit rechtsverbindliches Regelungswerk zum Datenschutz im elektronischen Geschäftsverkehr ist daher noch in weiter Ferne. Ein solches wäre aber gerade für den grenzüberschreitenden elektronischen Datenverkehr, den das Internet einhergehend mit zahlreichen diesbezüglichen Problemen mit sich bringt, von immenser Bedeutung. ${ }^{687}$

\section{Recht der Europäischen Union}

\section{Charta der Grundrechte der Europäischen Union}

Trotz Fehlens eines entsprechenden gemeinsamen Grundrechtekatalogs, wurde vom Europäischen Gerichtshof stets unter Hinweis auf Art. 8 EMRK das Recht auf Privatleben als ein von der Gemeinschaftsordnung geschütztes Grundrecht eingeordnet. ${ }^{688}$ Schließlich wurde am 7.12.2000 auf dem Gipfel der Europäischen Union in Nizza die Charta der Grundrechte verkündet, ${ }^{689}$ die im Vertrag von Lissabon, ${ }^{690}$ der am 1.12.2009 in Kraft getreten ist, in Art. 6 Abs. 1 als mit den Verträgen gleichrangiges Primärrecht anerkannt werden. Mit Art. 8 der Charta wurde ein Grundrecht auf Schutz personenbezogener Daten geschaffen. Danach hat jede Person das Recht auf Schutz der sie betreffenden personenbezogenen Daten. Diese Daten dürfen nur nach Treu und Glauben für festgelegte Zwecke und mit Einwilligung der betroffenen Person oder auf einer sonstigen gesetzlich geregelten, legitimen Grundlage verarbeitet werden. Dies wird untermauert durch einen Auskunfts- und Berichtigungsanspruch. Die Einhaltung der Vorgaben überwacht eine unabhän-

$687 \mathrm{Zu}$ der aktuellen Handhabung des grenzüberschreitenden Datenverkehrs s. B.III.2 Datenübermittlung ins Ausland. EuGH, NJW 1992, 1553 (1554); NJW 1994, 3005 (3006).

689 Der Vertrag von Nizza wurde am 26.2.2001 ratifiziert und ist am 1.2.2003 in Kraft getreten, abgedruckt in: ABl. EG C 80 v. 10.3.2001.

690 Der Vertrag wurde am 13.12.2007 von den Staats- und Regierungschef ratifiziert, die Ratifizierung durch die Bundesrepublik Deutschland steht noch aus, abgedruckt in: ABl. EG C 306/10 v. 7.12.2007. 
gige Stelle. Seit dem 1.12.2009 gibt es nunmehr endlich ein eigenes Datenschutzgrundrecht der Europäischen Union.

\section{Allgemeine Datenschutzrichtlinie}

Von entscheidender Prägung für das deutsche Datenschutzrecht, war und ist die Richtlinie zum Schutz natürlicher Personen bei der Verarbeitung personenbezogener Daten und zum freien Datenverkehr (Datenschutzrichtlinie) ${ }^{691}$, die am 23.5.2001, deutlich nach Ablauf der Umsetzungsfrist am 24.10.1998, im Bundesdatenschutzgesetz und später auch im Teledienstedatenschutzgesetz sowie dem Mediendienstestaatsvertrags (heute Telemediengesetz) umgesetzt wurde. Die Richtlinie, die zur Gewährleistung eines hohen Datenschutzniveaus verpflichtet ${ }^{692}$ hat so erstmals durch die Festlegung der Rechtsangleichung in den Gemeinschaftsstaaten ein einheitliches Schutzniveau in der Europäischen Union geschaffen. ${ }^{693}$ Dadurch wurde und wird der grenzüberschreitende innereuropäische Datenverkehr deutlich erleichtert. ${ }^{694}$

In Erwägungsgrund 10 der Datenschutzrichtlinie wird festgehalten, dass die Mitgliedsstaaten nicht unter dem in der Richtlinie vorgegebenen Mindestschutzniveau bleiben dürfen. Dies zeigt aber auch, dass die Mitgliedsstaaten sehr wohl über die dort festgelegten Maßnahmen hinausgehen dürfen. ${ }^{695}$ Kern des Schutzniveaus bilden die in den Art. 5-9 der Richtlinie festgelegten Grundsätze der Zweckbindung, der Datenqualität und -verhältnismäßigkeit, der Transparenz sowie der Sicherheit. Darüber hinaus kommt den Art. 10-17 der Richtlinie, die die Rechte der Betroffenen, die Beschränkung der Datenübermittlung in Drittländer sowie einen besonderen Schutz für sensible Daten regeln, besondere Bedeutung zu. Der Absicherung der Harmonisierung dient die durch Art. 29 der Richtlinie vorgesehene Datenschutzgruppe, auch Art. 29-Gruppe genannt, die Stellungnahmen und Empfehlungen zu allen Fragen abgeben und der Kommission übermitteln kann, die eine personenbezogene Datenverarbeitung in der Gemeinschaft betreffen (Art. 30 Abs. 1).

691 Richtlinie 95/46/EG des Europäischen Parlaments und des Rates v. 24.10.1995, ABl. EG L 281 v. 23.11.1995, S. 31-50.

692 Erwägungsgrund 10 der Richtlinie 95/46/EG; Simitis, NJW 1997, 283; Roßnagel/Brühann, Hdb. DatenschutzR, Kap. 2.4 Rn. 15; Schaar, Rn. 97.

693 Erwägungsgrund 5 und 8 der Richtlinie 95/46/EG

694 S. nur Erwägungsgrund 6 der Richtlinie 95/46/EG.

695 Schaar, Rn. 104; Scholz, S. 120; i.d.S. auch Simitis, NJW 1997, 282. 
Aufgrund der umfassenden Umsetzung der Datenschutzrichtlinie in das nationale Datenschutzrecht, kann hier auf eine weitergehende Darstellung und Diskussion der Richtlinieninhalte verzichtet werden. ${ }^{696}$ Bedeutung hat die Datenschutzrichtlinie daher für die folgende Untersuchung hauptsächlich für die Interpretation nationaler Vorschriften. Dies entspricht dem im Art. 10 EG verankerten Prinzip des „effet utile“, wonach nationale Rechtsvorschriften eine gemeinschaftskonforme Auslegung erfordern und eine solche Auslegung nur unter Heranziehung der einschlägigen Richtlinien möglich ist. ${ }^{697}$

\section{Datenschutzrichtlinie für elektronische Kommunikation}

Als bereichspezifische Ausprägung ${ }^{698}$ der Datenschutzrichtlinie folgte 1997 auf die rasanten Entwicklungen im Bereich der Telekommunikation die Richtlinie über die Verarbeitung personenbezogener Daten und den Schutz der Privatsphäre im Bereich der Telekommunikation (TelekommunikationsDatenschutzrichtlinie) ${ }^{699}$.

Um dem gesamten Spektrum der elektronischen Kommunikationsdienste auch über Telefondienste hinaus zu entsprechen, wurde die Telekommunikations-Datenschutzrichtlinie überarbeitet und am 30.5.2002 als Richtlinie über die Verarbeitung personenbezogener Daten und den Schutz der Privatsphäre in der elektronischen Kommunikation (Datenschutzrichtlinie für elektronische Kommunikation ${ }^{700}$ neu erlassen. Durch die Neufassung fällt auch die $\mathrm{Zu}-$ gangsverschaffung zum Internet unter den Geltungsbereich.

Die Richtlinie bezieht zwar sich vor allem auf Telekommunikationsdienstleistungen, aber in zwei Vorschriften auch auf den hier relevanten Telemediendatenschutz. ${ }^{701}$ So erlaubt Art. 5 Abs. 3 die Speicherung von Informationen, die im Endgerät des Nutzers gespeichert sind, sofern eine ausreichende Information des Nutzers erfolgt und diesem ein Widerspruchsrecht eingeräumt wird.

696 S. dazu weiterführend Schild, EuZW 1996, 549; Simitis, NJW 1997, 281; Gounalakis/Mand, GRUR Int. 1997, 431; dies., CR 1997, 431; Jacob, DuD 2000, 5.

697 Kabl, in: Calliess/Ruffert, Art. 10 EGV Rn. 58; Streinz/Streinz, Art. 10 EGV Rn. 35; von Bogdandy, in: Grabitz/Hilf, Art. 10 EGV Rn. 55; Helfrich, in: Hoeren/Sieber, Hdb. MultimediaR, Teil 16.1 Rn. 97; Scholz, S. 123; Tinnefeld, NJW 2001, 3080.

698 S. Art. 1 Abs. 2 S. 1 TK-DSRL.

699 Richtlinie 97/66/EG des Europäischen Parlaments und des Rates vom 15.12.1997, ABl. EG L 24 v. 30.1.1998, S. 1-8.

700 Richtlinie 2002/58/EG v. 12.7.2002, ABl. EG L 201, v. 31.7.2002, S. 37-47.

701 Patzak, S. 248; dies voraussetzend Husch/Kemmler/Oblenburg, MMR 2003, 147. 
Dabei hatte der Richtliniengeber insbesondere Identifizierungsmittel wie WebBugs und Cookies vor Augen. ${ }^{702} \mathrm{Im}$ deutschen Recht findet sich in $\int 13$ Abs. 1 S. 2 TMG eine Regelung für Cookies, da diese sich jedoch nur auf automatisierte Verfahren bezieht und diesbezüglich keine ausdrückliche Widerspruchsmöglichkeit vorgesehen ist, muss die Vorschrift entsprechend den Richtlinienvorgaben erweitert werden. ${ }^{703}$

Darüber hinaus bezieht sich auch Art. 13 Abs. 1, der besagt, dass die Verwendung von automatischen Anrufsystemen ohne menschlichen Eingriff (automatische Anrufmaschinen), Faxgeräten oder elektronischer Post für die Zwecke der Direktwerbung nur bei vorheriger Einwilligung der Teilnehmer gestattet werden darf, auf Telemediendiensteanbieter. Art. 13 Abs. 1 trifft demnach eine Opt-In-Regelung für die Zusendung von Nachrichten zu Zwecken der Direktwerbung. Zu Werbezwecken gilt indes nach Art. 13 Abs. 2 das OptOut-Prinzip. Diese Regelung wurde im deutschen Recht im Gesetz gegen den unlauteren Wettbewerb umgesetzt.

Jüngst erfolgte eine weitere Änderung der Datenschutzrichtlinie für elektronische Kommunikation durch die Richtlinie über die Vorratsspeicherung von Daten, die bei der Bereitstellung öffentlich zugänglicher elektronischer Kommunikationsdienste oder öffentlicher Kommunikationsnetze erzeugt oder verarbeitet werden (Vorratsdatenspeicherungs-Richtlinie). ${ }^{704}$ Danach müssen bei allen Telekommunikationsdiensten umfassende Informationen zum $\mathrm{Ob}$ und Wie der elektronischen Kommunikationsverbindungen, bei der Nutzung mobiler Endgeräte insbesondere zum jeweiligen Aufenthaltsort des Nutzers dauerhaft vorgehalten werden. ${ }^{705}$ Der deutsche Gesetzgeber hat sich in der Umsetzung aber auf das Telekommunikationsgesetz und die Strafprozessordnung beschränkt. ${ }^{706}$ Eine Änderung des Telemedienrechts soll nicht erfolgen, dementsprechend ist eine Erlaubnis zur Vorratsdatenspeicherung von Nutzer-

\footnotetext{
702 S. nur Erwägungsgründe 24 und 25 der EK-DSRL; Husch/Kemmler/Oblenburg, MMR 2003, 147; Patzak, S. 250.

703 Husch/Kemmler/Oblenburg, MMR 2003, 147; Patzak, S. 250.

704 Richtlinie 2006/24/EG des Europäischen Parlaments und des Rates v. 15.3.2006, ABl. EG L 105/54 v. 13.4.2006, S. 54-63.

705 S. dazu ausführlich Gitter/Schnabel, MMR 2007, 411.

706 S. nur den Gesetzentwurf BT-Drs. 16/5846 v. 27.6.2007.
} 
daten nicht vorgesehen. ${ }^{707}$ Eine weitere Untersuchung kann daher für die vorliegende Arbeit unterbleiben.

\section{Richtlinie über den elektronischen Geschäftsverkehr (E-Commerce-Ricbtlinie)}

Die am 8.6.2000 erlassene Richtlinie über bestimmte rechtliche Aspekte der Dienste der Informationsgesellschaft, insbesondere des elektronischen Geschäftsverkehrs, im Binnenmarkt (Richtlinie über den elektronischen Geschäftsverkehr - E-Commerce-Richtlinie) ${ }^{708}$ bezieht sich auf Dienste der Informationsgesellschaft. Darunter wird jede in der Regel gegen Entgelt elektronisch im Fernabsatz und auf individuellen Abruf eines Empfängers erbrachte Dienstleistung verstanden, ${ }^{709}$ so dass die E-Commerce-Richtlinie grundsätzlich Bedeutung für den Untersuchungsgegenstand der vorliegenden Arbeit hat.

Wie Art. 1 Abs. 5 lit. b der E-Commerce-Richtlinie festlegt, finden die Datenschutzrichtlinie sowie die Telekommunikations-Datenschutzrichtlinie uneingeschränkt Anwendung auf die Dienste der Informationsgesellschaft. Einen darüber hinausgehenden Regelungsbedarf hat der Richtliniengeber allerdings verneint und hervorgehoben, dass die Grundsätze des Schutzes personenbezogener Daten auch bei der Umsetzung und Anwendung der E-CommerceRichtlinie zu beachten sind. ${ }^{710}$ Insofern ergeben sich durch die E-CommerceRichtlinie keine besonderen Anforderungen für den Datenschutz.

\section{Resïmee}

Wenn auch die datenschutzrechtliche Regulierung im Bereich der Europäischen Union deutlich weiter fortgeschritten ist als im internationalen Bereich, so fehlt es dennoch an einer gemeinschaftsrechtlichen (datenschutzrechtlichen) Ordnung für den Bereich der so genannten Dienste der Informationsge-

707 S. dazu http://www.daten-speicherung.de/index.php/telemediengesetz-tritt-in-kraftvorratsspeicherung-von-nutzerdaten-bleibt-unzulaessig/ <25.11.2009>; i.d.S. auch BT-Drs. 16/5846, S. 26, 72.

708 Richtlinie 2000/31/EG des Europäischen Parlaments und des Rates v. 8.6.2000, ABl. EG L $178 / 1$ v. 17.7.2000, S. 1-16.

709 Diese Definition ergibt sich aus Art. 1 Nr. 2 der Richtlinie 98/34/EG über Informationsverfahren auf dem Gebiet der Normen und technischen Vorschriften und der Vorschriften für die Dienste der Informationsgesellschaft v. 22.6.1998, ABl. EG L 204 v. 21.7.1998, S. 37-48, geändert durch 98/48/EG v. 20.7.1998, ABl. EG L 217 v. 5.8.1998, S. 18-26, der über Art. 2 lit. a der ECRL Geltung beansprucht.

710 So ausdrücklich Erwägungsgrund 14 der ECRL; Schols, S. 128. 
sellschaft. Das vorhandene Regelungswerk bezieht sich entweder nicht spezifisch auf Online-Dienste (Datenschutzrichtlinie) oder weitgehend auf Telekommunikationsdienstleistungen (Datenschutzrichtlinie für elektronische Kommunikation). Die Richtlinie, die sich explizit auch an die hier betrachteten Telemediendienste wendet (E-Commerce-Richtlinie), hält die Aufstellung spezieller Datenschutzregelungen für unnötig. Telemediendienste werden daher lediglich von den allgemeinen Datenschutzquellen erfasst. ${ }^{711}$

III. Nationaler Rechtsrahmen

\section{Der Geltungsbereich des deutschen Datenschut_rechts}

Da bei Web Service-Portalen sowohl Portalbetreiber als auch Web ServiceAnbieter ihren Sitz außerhalb von der Bundesrepublik Deutschland haben und von dort Deutsche Bürger aufgrund der fehlenden Grenzen des Internet ansprechen und erreichen können, ist die erste Hürde bei der Bestimmung der datenschutzrechtlichen Rechte und Pflichten der beteiligten Akteure, die Lokalisierung der Datenverarbeitung und die daraus resultierende Frage der Anwendbarkeit des deutschen Datenschutzrechts.

Eine Klarstellung enthält $\int 1$ Abs. 5 BDSG, ${ }^{712}$ der Art. 4 der Datenschutzrichtlinie Rechnung trägt. Danach findet das deutsche Datenschutzrecht keine Anwendung, sofern eine in einem anderen Mitgliedstaat der Europäischen Union oder in einem anderen Vertragsstaat des Abkommens über den Europäischen Wirtschaftsraum (EWR) belegene verantwortliche Stelle personenbezogene Daten im Inland erhebt, verarbeitet oder nutzt, es sei denn, dies erfolgt durch eine Niederlassung im Inland. ${ }^{713}$ Das bedeutet, dass die Datenschutzrichtlinie und darauf aufbauend das Bundesdatenschutzgesetz nicht auf das Territorialprinzip sondern auf das Sitzprinzip (dem Sitz der datenverarbeitenden Stelle) abstellen. ${ }^{714}$ Die Ausnahme des $\int 1$ Abs. 5 S. 1 BDSG („,es sei denn") lässt aber das Bundesdatenschutzgesetz dann Anwendung finden,

711 Patzak, S. 256.

$712 \int 1$ Abs. 5 BDSG findet über $\int 12$ Abs. 4 TMG auch bei Telemedien Anwendung.

713 Konkret bedeutet dies, dass neben den Staaten der EU noch Island, Liechtenstein und Norwegen darunter fallen, wodurch die Verpflichtung besteht, die Richtlinie umzusetzen, s. dazu Simitis/Dammann, \$1 Rn. 211.

714 Dubr/Naujok/Peter/Seiffert, DuD 2002, 6; Gola/Schomerus, \1 Rn. 27; Moos, in: Kröger/Gimmy, Hdb. InternetR, S. 503. 
wenn die Datenerhebung durch eine Niederlassung 715 im Inland erfolgt, daher kann man exakter auch vom Niederlassungsprinzip oder abgeschwächten Sitzprinzip sprechen. ${ }^{716}$

Da aber alle Mitgliedsstaaten dem Schutzniveau der Datenschutzrichtlinie entsprechen müssen, um ein angemessenes und einheitliches Schutzniveau zu gewährleisten, wird der Datenaustausch innerhalb der Gemeinschaft durch die europarechtlichen Richtlinien erleichtert. Die datenschutzrechtlichen Konsequenzen werden in allen Mitgliedsstaaten ähnlicher Natur sein, daher erfolgt die weitere Darstellung lediglich anhand des deutschen Rechts, eine Abgrenzung ist nur zum EU/EWR-Ausland erforderlich. ${ }^{717}$

Für Drittstaaten, dazu gehören alle Staaten, die nicht Mitglied der Europäischen Union oder des Europäischen Wirtschaftsraums sind, kommt es nach $\$ 1$ Abs. 5 S. 2 BDSG auf den Ort der Datenverarbeitung an, es gilt das Territorialprinzip. ${ }^{718}$ Konkret bedeutet dies, dass wenn die Datenverarbeitung in Deutschland erfolgt, obwohl sich der Sitz des Unternehmens beispielsweise in Indien befindet, deutsches Datenschutzrecht Anwendung findet.

Online-Angebote können unabhängig vom Ort der Einspeisung weltweit abgerufen werden, eine Datenverarbeitung der verantwortlichen Akteure der Web Service-Portale im Ausland ist demnach durchaus wahrscheinlich. An das deutsche Datenschutzrecht sind die beteiligten Akteure nur dann gebunden, wenn sie ihre Niederlassung in der Bundesrepublik Deutschland haben oder im Falle von Drittstaaten wenn sie die Datenverarbeitung in der Bundesrepublik Deutschland vornehmen. Allerdings findet das deutsche Datenschutzrecht nach $\int 1$ Abs. 5 S. 4 BDSG auf Drittstaaten auch dann keine Anwendung, wenn Datenträger nur zum Zweck des Transits durch das Inland eingesetzt werden; dies ist zum Beispiel beim Inlandsrouting ohne Sender oder Empfänger der Datenpakete mit Sitz im Inland der Fall. ${ }^{719}$ Nach dem Schutzzweck der Norm muss das deutsche Datenschutzrecht aber auch dann Anwendung fin-

715 Hinsichtlich des Niederlassungsbegriffs wird auf die Definition des $\int 42$ Abs. 2 GewO verwiesen, so BT-Drs. 14/4329, S. 31, wonach eine solche dann vorliegt, wenn der Gewerbetreibende im Geltungsbereich dieses Gesetzes einen zum dauernden Gebrauch eingerichteten, ständig oder in regelmäßiger Wiederkehr von ihm benutzten Raum für den Betrieb seines Gewerbes besitzt.

716 So bspw. Simitis/Dammann, \1 Rn. 199.

717 S. auch B.III.2 Datenübermittlung ins Ausland.

718 Püttmann, K\&R 2000, 494; Moos, in: Kröger/Gimmy, Hdb. InternetR, S. 504.

719 Moos, in: Kröger/Gimmy, Hdb. InternetR, S. 505. 
den, wenn sich das Angebot erkennbar an deutsche Nutzer richtet und dabei personenbezogene Daten erhoben werden, die durch ein von dem Anbieter gesteuertes automatisiertes Verfahren auf dem Rechner des Nutzers an den Anbieter übermittelt werden - das heißt, das System des Betroffenen für fremde Zwecke funktionalisiert wird, was auch durch Cookies erfolgen kann. ${ }^{720}$

Das deutsche Datenschutzrecht enthält umfangreiche und verbindliche Regelungen, deren Geltung nicht vertraglich ausschließbar ist, dementsprechend lässt Art. 34 EGBGB eine Rechtswahlklausel nach Art. 27 EGBGB, mit der die Anwendbarkeit des deutschen Datenschutzrechts außer Kraft gesetzt würde, nicht zu. ${ }^{721}$

\section{Datenübermittlung ins Ausland}

Erfolgen Datenübermittlungen über die Grenzen der Bundesrepublik Deutschland hinaus, weil beispielsweise ein im Web Service-Portal integrierter Web Service-Anbieter seinen Sitz außerhalb von Deutschland hat und auch die Datenverarbeitung dort erfolgt oder die Daten aus anderen Gründen (zum Beispiel Kostenersparnisse) im Ausland verarbeitet werden (Outsourcing von Daten), so muss untersucht werden, welche rechtlichen Voraussetzungen ein Unternehmen erfüllen muss, damit eine solche Übermittlung zulässig ist. Dabei unterscheidet das Bundesdatenschutzgesetz zwischen Übermittlungen innerhalb der EU/EWR ( $\ 4$ b Abs. 1 BDSG) und solchen in Drittstaaten, $\ 4 \mathrm{~b}$ Abs. 2, 2. Var. BDSG.

Der Datentransfer in EU- und EWR-Staaten ist aufgrund der Harmonisierung des Datenschutzrechts unproblematisch zu handhaben: es sind die gleichen Regeln zu beachten wie bei Übermittlungen an inländische Stellen. ${ }^{722}$ Daher bedarf es keiner gesonderten Ausführungen, wenn ein oder mehrere Beteiligte im EU-/EWR-Raum ansässig sind und Datenübermittlungen dorthin erfolgen.

Indes hat bei Drittstaaten die Datenübermittlung nach $₫ 4$ b Abs. 2 S. 2 BDSG zu unterbleiben, wenn in diesen Ländern kein angemessenes Schutzniveau gewährleistet wird. Wird ein solches gewährleistet, so gelten nach $\ 4 \mathrm{~b}$ Abs. 2

\footnotetext{
720 Simitis/Dammann, S 1 Rn. 226 f.; Duhr/Naujok/Peter/Seiffert, DuD 2002, 7.

721 Moos, in: Kröger/Gimmy, Hdb. InternetR, S. 505; Püttmann, K\&R 2000, 494; Däubler, CR 1999, 51.

722 Simitis/Simitis, \ 4b Rn. 26 f.; Gola/Schomerus, \ 4b Rn. 2 ff.
} 
S. 1 BDSG, die gleichen Regeln, wie bei Datenübermittlungen in EU/EWRStaaten. Die Zulässigkeit der Datenübermittlung in Drittstaaten steht und fällt somit grundsätzlich mit einem angemessenen Datenschutzniveau ${ }^{723}$ Ausnahmen sind möglich im Falle des Greifens der Ausnahmetatbestände des $\ 4 \mathrm{c}$ BDSG, wozu insbesondere die Einwilligung des Betroffenen oder die Abgabe von Datenschutzgarantien gehören.

\section{a) Angemessenes Schutzniveau}

Auf Grundlage des Art. 25 der Datenschutzrichtlinie entscheidet die Kommission, ob ein angemessenes Schutzniveau vorliegt. Kriterien für die Bestimmung der Angemessenheit werden in $\int 4 \mathrm{~b}$ Abs. 3 BDSG genannt. Bis heute hat die Kommission festgestellt, dass ein angemessener Schutz für personenbezogene Daten bisher lediglich in der Schweiz, Kanada, Argentinien, Guernsey und der Insel Man, bei Anwendung der vom Handelsministerium der Vereinigten Staaten vorgelegten Grundsätze des ,sicheren Hafens“ (Safe HarborPrinciples) sowie bei der Übermittlung von Fluggastdatensätzen an die Zollund Grenzschutzbehörde der Vereinigten Staaten, besteht. ${ }^{724}$ In diesen Ländern kann eine Datenübermittlung ohne gesonderte Prüfung der Angemessenheit des Datenschutzniveaus bei der verarbeitenden Stelle erfolgen. ${ }^{725}$ In allen anderen Drittländern, insbesondere auch den Vereinigten Staaten (USA), denen es an einem einheitlichen Datenschutzrecht vollständig mangelt, muss im Rahmen des jeweiligen Einzelfalls gesondert anhand der Kriterien des $\int 4 \mathrm{~b}$ Abs. 3 BDSG überprüft werden, ob ein angemessenes Schutzniveau bei der verarbeitenden Stelle vorliegt. ${ }^{726} \mathrm{Da}$ ein Datenübermittlungsverbot als mögliche Konsequenz den Datenhandel zwischen der Europäischen Union und den USA erheblich erschweren würde, haben das amerikanische Handelsministerium und die Kommission der Europäischen Union als Ergebnis eines Dialogs die so genannten Safe Harbor-Principles vom 27.7.2000 hervorgebracht.727 Nach diesen Grundsätzen verpflichten sich amerikanische Unternehmen

723 Lange, AuA 2006, 712; Niedermeier, in: Gounalakis, Rhb. Electronic Business, \19 Rn. 80; Gola/Schomerus, \4b Rn. 7 ff.

724 S. zum aktuellen Stand http://ec.europa.eu/justice_home/fsj/privacy/thridcountries/index_de.htm.

725 Gola/Schomerus, \4 $4 \mathrm{~b}$ Rn. 14.

726 Simitis/Simitis, \4b Rn. 46 ff.; Gola/Schomerus, \4b Rn. 10 ff.; Lange, AuA 2006, 712; speziell zu den USA Räther/Seitz, MMR 2002, 427.

727 S. Räther/Seitz, MMR 2002, 427; Simitis/Simitis, \4b Rn. 70 ff. 
selbst, bestimmte europäische Datenschutzanforderungen einzuhalten, ${ }^{728}$ indem sie sich auf freiwilliger Basis auf einer durch das amerikanische Handelsministerium geschaffenen öffentlichen Liste eintragen. Damit gehen die eingetragenen Unternehmen ${ }^{729}$ bindende rechtliche Verpflichtungen ein, deren Einhaltung das US-Handelsministerium überwacht. Bei Zuwiderhandeln drohen rechtliche Konsequenzen. Die Kommission hat ein angemessenes Schutzniveau anerkannt, wenn die Übermittlung von Daten an amerikanische Unternehmen unter Zugrundelegung der Safe Harbor-Principles erfolgt. ${ }^{730}$

\section{b) Einwilligung}

Fehlt es sowohl an einem angemessenen Schutzniveau als auch dem Beitritt zu den Safe Harbor-Principles, bleibt nur noch ein Rückgriff auf die Ausnahmetatbestände des $\int 4 \mathrm{c}$ BDSG. Eine in der Praxis relevante Ausnahme ist die Einholung einer Einwilligung in die Datenübermittlung durch den Betroffenen $(\llbracket 4 c$ Abs. 1 Nr. 1 BDSG). Die sonstigen Ausnahmetatbestände des $\ 4 \mathrm{c}$ BDSG decken eher Teilaspekte, die meist im Zusammenhang mit der Vertragsbegründung stehen, ab. Insbesondere aber die Datenübermittlung für Werbezwecke, die für die Akteure der Web Service-Portale regelmäßig am interessantesten sein dürfte, wird davon nicht abgedeckt.

Die Einwilligung setzt die Einhaltung der Voraussetzungen des $₫ 4$ a BDSG und dabei insbesondere voraus, dass der Betroffene in Kenntnis der Sachlage eingewilligt hat und demnach sowohl Kenntnis der Sachlage hat als auch über den Gegenstand der Einwilligung informiert ist. ${ }^{731}$

aa) Exkurs: Anforderungen an die Einwilligung

Aufgrund der technischen Ausgestaltung von Web Services, die systemunabhängig, kostengünstig und ohne komplizierte Anpassungen lose an Portale gekoppelt werden können, spricht vieles dafür, dass auch Web ServiceAnbieter aus Drittstaaten ihre Dienste an Web Service-Portale, die sich (zu-

728 S. zum Inhalt Räther/Seitz, MMR 2002, 428; Simitis/Simitis, \4b Rn. 71 ff.

729 Eine Liste der eingetragenen Unternehmen kann unter http://web.ita.doc.gov/safeharbor/shlist.nsf/webPages/safe+harbor+list eingesehen werden.

730 2000/520/EG v. 26.7.2000, Entscheidung der Kommission vom 26.7.2000 gem. der Richtlinie 95/46/EG des Europäischen Parlaments und des Rates über die Angemessenheit des von den Grundsätzen des „,sicheren Hafens“ und der diesbezüglichen „Häufig gestellten Fragen“ (FAQ) gewährleisteten Schutzes, vorgelegt vom Handelsministerium der USA, ABl. EG L 215/7 v. 25.8.2000, S. 7-47.

731 Däubler, CR 1999, 51 f.; Räther/Seitz, MMR 2002, 431; Simitis/Simitis, \4c Rn. 8 ff. 
mindest auch) an deutsche Nutzer richten, koppeln. Die Frage der Zulässigkeit der Datenübermittlung an Web Service-Anbieter in Drittstaaten ohne angemessenes Schutzniveau, wird daher bei der Ausgestaltung solcher Portale eine bedeutende Rolle spielen. Da die Einwilligung bei einer Datenübermittlung an Web Service-Anbieter in solchen Staaten dementsprechend eine große Relevanz hat, werden an dieser Stelle die Voraussetzungen, an die eine wirksame Einwilligung geknüpft wird, genauer betrachtet.

\section{i) Zeitpunkt und Dauer}

Die Einwilligung des deutschen Datenschutzrechts ist an die Terminologie des Bürgerlichen Gesetzbuchs entlehnt und stellt demnach eine antezipierte Erlaubnis dar, ${ }^{732}$ die der Datenverwendung vorangehen muss ( $(183 \mathrm{BGB})$. Eine nachträgliche Genehmigung ( $\$ 184$ BGB) der Datenverwendung, die nach der Systematik des Bürgerlichen Gesetzbuchs gerade keine Einwilligung ( $\mathbb{1} 183$ BGB) ist, oder eine zu einem beliebigen Zeitpunkt erteilbare Zustimmung genügen den Anforderungen an die Einwilligung nicht. ${ }^{733}$ Andernfalls könnte die Einwilligung nie eine freie Entscheidung des Betroffenen ermöglichen, denn den Vorgang der Datenverarbeitung selbst kann der Betroffene nicht mehr rückgängig machen. Erfolgt zuerst die Datenübermittlung - gleiches gilt freilich für eine etwaige Datenverarbeitung - und wird erst anschließend nach der Zustimmung des Portalnutzers gefragt, so genügt dies nicht den Anforderungen an eine wirksame Einwilligung.

Im Rahmen der Portalnutzung sollte die Einwilligung daher derart ausgestaltet sein, dass die Zustimmung bestmöglich noch vor Absenden der Anmeldedaten des Portalnutzers erfolgt. Denn vor dem Absenden der Daten liegt technisch noch keine Datenbeschaffung und dementsprechend auch keine Datenerhebung oder Datenübermittlung im Sinne der Legaldefinition des $₫ 3$ Abs. 3 und Abs. 4 BDSG vor. ${ }^{734}$ Sie kann daher auch nach der Dateneingabe am Ende eines Online-Formulars erfolgen, dabei muss nur gewährleistet werden, dass sie tatsächlich vor dem Absenden der Daten, und damit dem Datenverarbeitungsvorgang, abgegeben wird. Ist eine Registrierung für die Portalnutzung nicht erforderlich, so müssen Portalbetreiber und Web Service-Anbieter den-

732 Gola/Schomerus, \4a Rn. 2; Roßnagel/Holznagel/Sonntag, Hdb. DatenschutzR, Kap. 4.8 Rn. 19; Spindler/Schuster/Spindler/Nink, I 4a BDSG Rn. 1; Moos, in: Kröger/Gimmy, S. 519; Zscherpe, MMR 2004, 724.

733 Simitis/Simitis, J 4a Rn. 27; Scholy, S. 282; Ranke, S. 107; Merati-Kashani, S. 110.

734 Spindler/Schuster/Spindler/Nink, \ 4a BDSG Rn. 1. 
noch - trotz mangelnder Möglichkeit, die Einwilligung in den Registrierungsprozess zu integrieren - den Grundsatz einhalten, dass die Einwilligung vor der Datenverarbeitung - worunter auch die Datenübermittlung fällt - eingeholt wird.

Zeitlich gilt die Einwilligung grundsätzlich für die Dauer der konkreten Datenverarbeitung, wenn der Betroffene sie nicht zuvor widerrufen hat. Im Rahmen der Dauer der konkreten Datenverarbeitung kommt der Grundsatz der Zweckbindung zum Tragen, \4a Abs. 1 S. 2 BDSG. Die Daten müssen immer dann gelöscht werden, wenn der vorher festgelegte Zweck erreicht wurde, dies ergibt sich aus $\ 20$ Abs. 2 Nr. 1 und $\ 35$ Abs. 2 Nr. 1 BDSG. ${ }^{735}$

Eine weitere zeitliche Begrenzung bildet die Möglichkeit des Betroffenen, seine zunächst erteilte Einwilligung zu widerrufen. Zwar enthalten weder das Bundesdatenschutzgesetz noch das Telemediengesetz eine explizite Regelung hinsichtlich eines Widerrufsrechts bezüglich der Einwilligung, ein solches steht dem Betroffenen jedoch bereits als Betätigung des Rechts auf informationelle Selbstbestimmung, das die verfassungsrechtliche Grundlage des deutschen Datenschutzrechts bildet, ${ }^{736}$ zur Verfügung. ${ }^{737}$ Darüber hinaus setzt das Telemediengesetz in $\$ 13$ Abs. 2 Nr. 4 eine solche Möglichkeit für eine elektronische Einwilligung voraus. Der Widerruf stellt daher eine Korrekturmöglichkeit des Betroffenen dar. Übt der Betroffene sein Widerrufsrecht aus, so führt dies nicht zur rückwirkenden Rechtswidrigkeit der erfolgten Speicherung, ${ }^{738}$ der Widerruf kann nur in die Zukunft (ex nunc) gerichtet sein. ${ }^{739}$ Durch die Widerrufsausübung verliert die verarbeitende Stelle die Berechtigung, die Daten für ihre Zwecke zu nutzen, daher müssen sie gelöscht werden (\$\$ 20 Abs. 2 Nr. 1, 35 Abs. 2 Nr. 1 BSDG) Erfolgt eine Übermittlung an Dritte, so sind diese über den Widerruf zu informieren. ${ }^{740}$ Der Widerruf kann indes nicht

735 Simitis/Simitis, \4a BDSG Rn. 29.

736 B.III.3.a) Das Recht auf informationelle Selbstbestimmung.

737 Roßnagel/Holznagel/Sonntag, Hdb. DatenschutzR, Kap. 4.8 Rn. 64; Simitis/Simitis, $\$ 4a BDSG Rn. 94.

738 OLG Düsseldorf, ZIP 1985, 1319 (1320).

739 Simitis/Simitis, \4a BDSG Rn. 101; Gola/Schomerus, \4a BDSG Rn. 18; Gola, DuD 2001, 279; Schaar, MMR 2001, 647; Iraschko-Luscher, DuD 2006, 709. Dies zeigt auch die Formulierung in \13 Abs. 2 Nr. 4 TMG, wonach der Diensteanbieter für eine elektronische Einwilligung sicherzustellen hat, dass der Nutzer die Einwilligung jederzeit mit Wirkung für die Zukunft widerrufen kann.

740 Simitis/Simitis, \4a BDSG Rn. 103; Roßnagel/Holznagel/Sonntag, Hdb. DatenschutzR, Kap. 4.8 Rn. 67; Ranke, S. 108. 
unbeschränkt ausgeübt werden, die Grenzen von Treu und Glauben müssen beachtet werden. ${ }^{741}$ So fehlt es schon an einem Eingriff in das Recht auf informationelle Selbstbestimmung, wenn die Daten anonymisiert wurden, in diesem Fall ist daher ein Widerruf ausgeschlossen. ${ }^{742}$

Je nach Ausgestaltung der Web Service-Portale können für den Betroffenen Unklarheiten hinsichtlich des richtigen Widerrufsempfängers aufkommen. Dabei ist fraglich, ob es genügt, den Widerruf gegenüber dem Portalbetreiber zu erklären, der die Einwilligung für die Datenverarbeitung und demnach auch für die Datenübermittlung für sich selbst sowie die im Portal integrierten Web Service-Anbieter vom Betroffenen eingeholt hat, oder ob er verpflichtet ist, den Widerruf jeweils einmal gegenüber dem Portalbetreiber sowie den Web Service-Anbietern zu erklären.

Wurde die Einwilligung tatsächlich nur gegenüber dem Portalbetreiber abgegeben, so ist es aus Gründen der Rechtssicherheit und, um durch den Widerruf nicht das Recht auf informationelle Selbstbestimmung in sein Gegenteil zu kehren, lediglich erforderlich, den Widerruf gegenüber dem Portalbetreiber zu erklären. Da dieser bereits der direkte Empfänger der Einwilligung war, genügt es auch, wenn er der alleinige direkte Empfänger des Widerrufs ist. Während es für den Betroffenen schwer absehbar ist, wer tatsächlich seine personenbezogenen Daten verarbeitet hat und wie er den jeweiligen Web ServiceAnbieter am Besten kontaktiert, ist es für den Portalbetreiber ein Leichtes, die Web Service-Anbieter als Vertragspartner, genau wie bei der vorherigen Einwilligung, auch über den Widerruf zu informieren. Die Interessenlage ist insofern keine andere, als wenn er Dritten die personenbezogenen Daten des Betroffenen übermittelt hätte. In diesem Fall müsste er die Dritten ebenfalls über den Widerruf informieren. Das Risiko der erfolgreichen Widerrufsübermittlung obliegt daher dem Portalbetreiber. Dies entspricht auch der Wertung des $\ 13$ Abs. 2 Nr. 4 TMG, der fordert, dass der Web Service-Anbieter für eine elektronische Einwilligung sicherzustellen hat, dass der Nutzer die Einwilligung jederzeit mit Wirkung für die Zukunft widerrufen kann. Dahinter verbirgt sich nichts anderes, als das derjenige, der den ,gefährlicheren“"Weg der elektronischen Einwilligung eröffnet hat, auch für eine einfache (,,jederzeit") Widerrufsmöglichkeit sorgen muss. Diese Wertung würde

741 Gola/Schomerus, \4a BDSG Rn. 18; Roßnagel/Holznagel/Sonntag, Hdb. DatenschutzR, Kap. 4.8 Rn. 66; i.d.S. auch Iraschko-Luscher, DuD 2006, 709.

742 Roßnagel/Scholr, MMR 2000, 723; Roßnagel/Holznagel/Sonntag, Hdb. DatenschutzR, Kap. 4.8 Rn. 66. 
konterkariert, wenn der Betroffene selbst die Web Service-Anbieter kontaktieren müsste.

Die Wertung des $₫ 13$ Abs. 2 Nr. 4 TMG ist auch für die Fälle heranzuziehen, in denen die Web Service-Anbieter die Einwilligung vor der Datenübermittlung oder Web Service-Nutzung selber eingeholt haben, zum Beispiel mit Hilfe des Web Service. Auch diese müssen dem Betroffenen einen einfachen und schnellen Widerruf ermöglichen. Wie sie dies technisch bewerkstelligen, liegt allein in ihrer Sphäre.

\section{ii) Form}

\section{Schriftform}

Nach \4a Abs. 1 S. 3 BDSG hat die Einwilligung grundsätzlich schriftlich zu erfolgen. Dieses Erfordernis soll zum einen den Betroffenen vor Übereilung schützen und zum anderen im Streitfall, der verantwortlichen Stelle den Beweis über den Umfang ihrer Legitimation zu erleichtern. ${ }^{743}$ Die Schriftform richtet sich dabei nach $\int 126$ BGB. Nach dessen Absatz 3 ist, um den modernen Anforderungen des elektronischen Geschäftsverkehrs gerecht zu werden, insbesondere wenn Ländergrenzen überschritten werden und die tatsächliche Abgabe der Einwilligung durch die Unwägbarkeiten des postalischen Weges in die Länge gezogen wird, auch die elektronische Form unter den strengen Voraussetzungen des $\ 126 \mathrm{a}$ BGB möglich. $\ 126 \mathrm{a}$ in Verbindung mit $\ 126$ Abs. 3 BGB ermöglicht die Ersetzung der Schriftform durch eine qualifiziert elektronische Signatur. Da sich diese aber noch nicht flächendeckend, gerade im Bereich der Verbraucher, durchgesetzt hat, gelten für das Telemedien- $(₫ 13$ Abs. 2 TMG) und das Telekommunikationsgesetz (\$ 94 TKG) vereinfachte Formerfordernisse, $\ 4$ a Abs. 1 S. 3 BDSG (,soweit nicht wegen besonderer Umstände eine andere Form angemessen ist"), nach denen eine elektronische Einwilligung unter bestimmten Voraussetzungen zulässig ist. ${ }^{744}$ Insbesondere durch die Herabsenkung der Anforderungen im Teledienstedatenschutzgesetz von 2001 sollte die elektronische Einwilligung für die breite Anwendung attraktiver gestaltet werden. ${ }^{745}$

743 Roßnagel/Holznagel/Sonntag, Hdb. DatenschutzR, Kap. 4.8 Rn. 28; Ranke, S. 108; Conrad, DuD 2006, 407; Heidemann-Peuser, DuD 2002, 393.

744 BT-Drs. 14/6098, S. 28; Scholz, S. 283; Ranke, S. 108; jurisPK-Internetrecht/Heckmann, Kap. 1.13 Rn. 24.

745 BT-Drs. 14/6098, S. 28. 


\section{Elektronische Form}

Als Telemediendiensteanbieter ${ }^{746}$ können Portalbetreiber und Web ServiceAnbieter die vereinfachte Form der elektronischen Einwilligung des $₫ 13$ Abs. 2 TMG verwenden. Daher spielt das Erfordernis der Schriftform in der vorliegenden Arbeit keine weitere Rolle. Da die elektronische Form aufgrund der fehlenden Verkörperung (klassische Schriftform) und dem Fehlen von biometrischen Kennzeichen gewissen Risiken ausgesetzt ist, wird die Wirksamkeit an besondere Voraussetzungen gekoppelt. ${ }^{77}$ \& 13 Abs. 2 TMG knüpft die Wirksamkeit der elektronischen Einwilligung an vier Voraussetzungen. Der Diensteanbieter muss sicherstellen, dass der Nutzer seine Einwilligung bewusst und eindeutig erteilt hat (Nr. 1), die Einwilligung protokolliert wird (Nr. 2), der Nutzer den Inhalt der Einwilligung jederzeit abrufen kann (Nr. 3) und der Nutzer die Einwilligung jederzeit mit Wirkung für die Zukunft widerrufen kann (Nr. 4). 748 Darüber hinaus muss der Diensteanbieter nach $₫ 13$ Abs. 3 S. 1 TMG den Nutzer vor Erklärung der Einwilligung auf dessen Widerrufsrecht hinweisen. Dieser Hinweis muss nach $₫ 13$ Abs. 3 S. 2 in Verbindung mit $\ 13$ Abs. 1 S. 3 TMG für den Nutzer jederzeit abrufbar sein.

Die in Nr. 1 geforderte eindeutige und bewusste Erklärungshandlung soll einer übereilten Entscheidung vorbeugen. ${ }^{749}$ Die Schutzbedürftigkeit resultiert aus den technikspezifischen Gefahren, konkret der Anwendung eines flüchtigen Mediums (Bildschirm) und des Handelns durch einfachen Knopfdruck oder Mausklick, das nicht zwischen wichtigen und unwichtigen Handlungen unterscheidet. ${ }^{750}$ Kurz gesagt, soll ein zufälliges und unbeabsichtigtes Auslösen einer elektronischen Einwilligung verhindert werden.

Die Formulierung „bewusst und eindeutig“ zeigt, dass die allgemeinen Anforderungen an eine rechtsgeschäftliche Erklärung aufgegriffen werden sollen. ${ }^{751}$ Entlehnt an diesen Anforderungen wird subjektiv vorausgesetzt, dass die

S. dazu B.IV.2.a) Web Service-Portale als Telemediendiensteanbieter

Engel-Flechsig, DuD 1997, 13; ders., RDV 1997, 64; Engel-Flechsig/Maennel/Tettenborn, NJW 1997, 2986; Fröble, S. 236.

Die Parallelregelung des $\int 94$ TKG hat einen identischen Wortlaut.

BT-Drs. 13/7385, S. 23; Roßnagel/Bizer, RMD, Teil 3, \ 4 TDDSG Rn. 22; Scholz, S. 284; Rasmussen, DuD 2002, 408.

BT-Drs. 13/7385, S. 23 u. BT-Drs. 14/6098, S. 28, wo auf den Schutzzweck der Vorgängernorm verwiesen wird.

LG Potsdam, DuD 2005, 302 (304); Schmitz, S. 99; Spindler/Schmitz/Geis/Schmitz, \ 4 TDDSG Rn. 15; Roßnagel/Bizer, RMD, Teil 3, \ 4 TDDSG Rn. 242; Scholz, S. 284. 
elektronische Einwilligung mit Handlungswillen und Erklärungsbewusstsein erfolgt sowie eines auf die Erlaubnis der Verarbeitung der eigenen Daten in einem bestimmten Verwendungszusammenhang gerichteten Geschäftswillens bedarf. ${ }^{752}$ Erforderlich aber auch ausreichend ist dafür, dass sich der jeweilige Nutzer bewusst ist, überhaupt eine rechtsverbindliche Einwilligung hinsichtlich der Verarbeitung seiner personenbezogenen Daten abzugeben sowie das Wissen, welche genauen Daten zu welchem Zweck verarbeitet werden sollen. ${ }^{753} \mathrm{Nach}$ der Gesetzesbegründung genügt für eine solche Autorisierung beispielsweise bereits eine bestätigende Wiederholung des Übermittlungsbefehls bei gleichzeitiger zumindest auszugsweiser Darstellung der Einwilligungserklärung auf dem Bildschirm. ${ }^{754}$ Danach soll ausreichend sein, wenn der Nutzer zunächst ein Kontrollkästchen mit dem Text „Ich willige in die Verarbeitung und Nutzung meiner personenbezogenen Daten gemäß der vorstehenden Datenschutzerklärung ein" und anschließend ein Schaltfeld mit dem Text „Ich akzeptiere und willige ein“ aktivieren muss. 755 In der Gesetzesbegründung zu der gleichlautenden Vorschrift des \94 TKG wird allerdings vorgeschlagen, das Erfordernis zum Beispiel dadurch umzusetzen, „dass eine entsprechende Eingabemaske vorgegeben und die Einwilligung vom Teilnehmer mit einer qualifizierten elektronischen Signatur nach dem Signaturgesetz vorgesehen wird." 756 Zwar bleibt es dem Telemediendiensteanbieter grundsätzlich selbst überlassen, wie er diese Anforderung technisch umsetzt, ${ }^{757}$ dieses Beispiel deutet aber darauf hin, dass die elektronische Einwilligung an erhöhte Voraussetzungen zu knüpfen ist. ${ }^{758}$ Indes kann der Maßstab aber auch

752 LG Potsdam, DuD 2005, 302 (304); Scholz, S. 284; Ranke, S. 109; Roßnagel/Bizer, RMD, Teil 3 , \ 4 TDDSG Rn. 242; Spindler/Schmitz/Geis/Schmitz, \4 TDDSG Rn. 15; jurisPKInternetrecht/Heckmann, Kap. 1.13 Rn. 25.

753 OLG Brandenburg, MMR 2006, 405 (406) m. Anm. Breyer, LG Potsdam, DuD 2005, 302 (304); Spindler/Schmitz/Geis/Schmitz \4 TDDSG Rn. 17; jurisPK-Internetrecht/Heckmann, Kap. 1.13 Rn. 26 f.; Geis, CR 2002, 672.

754 BT-Drs. 13/7385, S. 23; dies aufgreifend OLG Brandenburg, MMR 2006, 405 (406) m. Anm. Breyer, LG Potsdam, DuD 2005, 302 (304); kritisch zum Erfordernis der doppelten Bestätigung, da im alten $₫ 4$ Abs. 2 TDDSG nur der Singular ,eine bewusste $[\ldots]$ “ verwendet wurde Schmity/Eckhardt, CR 2006, 533 f. Dieses Argument hat sich aber mit $₫ 13$ Abs. 2 Nr. 1 TMG erledigt, der nur verlangt, dass der Betroffene die Einwilligung „bewusst und eindeutig erteilt hat".

755 Diese Vorgehensweise wurde vom OLG Brandenburg, MMR 2006, 405 (406) m. Anm. Breyer als ausreichend erklärt.

756 BT-Drs. 15/2316, S. 88.

757 S. die Formulierung „kann z.B.“ BT-Drs. 15/2316, S. 88.

758 So auch Wegmann, WRP 2007, 1143 f. 
nicht die qualifiziert elektronische Signatur sein, da andernfalls $₫ 13$ Abs. 2 TMG, wie ursprünglich intendiert, keine Erleichterung mehr gegenüber $\int 4 \mathrm{a}$ BDSG, der bereits die elektronische Form im Sinne des $₫ 126$ a BGB in Verbindung mit $₫ 126$ Abs. 3 BGB zulässt, darstellen würde. Insofern sollte man sich weiterhin an den bereits zu $₫ 4$ Abs. 2 TDDSG a.F. entwickelten Grundsätzen orientieren.

Die in Nr. 2 verlangte Protokollierung der Einwilligung dient zum einen der Transparenz und zum anderen der Nachweisbarkeit, der vom Nutzer erlaubten Datenverarbeitung, denn dadurch kann der Nutzer jederzeit nachprüfen, wann er wem und in welchem Umfang eine Einwilligung in die Datenverarbeitung erteilt hat. ${ }^{759}$ Darüber hinaus dient sie dem Telemediendiensteanbieter als Nachweis zur Legitimation der Datenverarbeitung. ${ }^{760} \mathrm{Da}$ die Protokollierung selbst ein personenbezogenes Datum darstellt, darf es nur zweckgebunden verwendet werden, nämlich zur Kontrolle der vom Nutzer legitimierten Datenverarbeitung. ${ }^{761}$

Mit der Protokollierungspflicht einher geht die Pflicht in Nr. 3 es dem Nutzer zu ermöglichen, den Inhalt der Einwilligung jederzeit abrufen zu können. Die Anforderung ,,jederzeit“ ist dabei wörtlich zu verstehen. Dem Nutzer muss es demnach möglich sein, den Inhalt seiner Einwilligung 24 Stunden am Tag, solange das Vertragsverhältnis weiter besteht, abrufen zu können. ${ }^{762}$ Dieser „Service“ stellt eine Gewährleistung des Transparenzgebots ${ }^{763}$ dar und gewährt dem Nutzer einen zusätzlichen Schutz. ${ }^{764}$

Schließlich ist der Telemediendiensteanbieter nach Nr. 4 gehalten, dem Nutzer den jederzeitigen Widerruf seiner Einwilligung zu ermöglichen. Auch dabei hat der Telemediendiensteanbieter technisch freie Hand. Auf die Möglichkeit zum Widerruf muss der Telemediendiensteanbieter nach $₫ 13$ Abs. 3 S. 1 TMG vor der Abgabe der Einwilligung hinweisen und der Inhalt dieser Unterrichtung muss für den Nutzer nach $₫ 13$ Abs. 3 S. 2 TMG jederzeit abrufbar sein. Der

759 Scholz, S. 285; Roßnagel/Holznagel/Sonntag, Hdb. DatenschutzR, Kap. 4.8 Rn. 94; Rasmussen, DuD 2002, 409; i.d.S. auch Geis, CR 2002, 672.

760 Roßnagel/Holznagel/Sonntag, Hdb. DatenschutzR, Kap. 4.8 Rn. 94; Geis, CR 2002, 672.

761 Scholz, S. 285.

762 Rasmussen, DuD 2002, 409; Schaar, MMR 2001, 646; Spindler/Schmitz/Geis/Schmitz, \ 4 TDDSG Rn. 22; Ranke, S. 109. Ausführlich zu den Möglichkeiten den Zugriff nur auf die Person zu beschränken, die die Einwilligung auch abgegeben hat Scholz, S. 285 f.

763 S. zum Transparenzgrundsatz B.III.3.c)cc) Transparenz.

764 Roßnagel/Holžnagel/Sonntag, Hdb. DatenschutzR, Kap. 4.8 Rn. 95. 
Widerruf selber kann formlos erklärt werden und ist unabhängig von der Form der Einwilligung. ${ }^{765}$

Missachten Portalbetreiber und Web Service-Anbieter die durch $₫ 13$ Abs. 2 Nr. 1-4 TMG auferlegten Pflichten, hat dies zur Konsequenz, dass die Einwilligung unwirksam und die darauf basierende Datenverarbeitung unzulässig ist. ${ }^{766}$

\section{Formularmäßige Einwilligung}

$\mathrm{Da}$ es den organisatorischen Aufwand deutlich verringert, wenn die Einwilligung zusammen mit anderen Erklärungen abgegeben wird, stellt dies eine sehr beliebte Variante der Einholung der Einwilligung dar. Im Rahmen der Web Service-Portale würde es sich, im Falle einer Registrierungspflicht, anbieten, die Einwilligung für die Datenverarbeitung in den Registrierungsvorgang zu integrieren. Eine solche Vorgehensweise hat den Vorteil, dass bereits keine Daten in unzulässiger Art und Weise erhoben werden, sondern bereits im Voraus der zulässige Rahmen abgesteckt wird. Gegen eine solche Kombination ist grundsätzlich nichts einzuwenden, allerdings muss dafür gesorgt werden, dass für den Betroffenen erkennbar ist, dass es sich um eine Einwilligungserklärung handelt. Diese muss also optisch hervorgehoben und als OptOut-Variante ausgestaltet sein. ${ }^{767}$ Die gängige Methode, Datenschutzerklärungen in einem unüberschaubaren Paket zusammen mit Allgemeinen Geschäftsbedingungen zu verstecken, lässt den Betroffenen in aller Regel nicht erkennen, dass es sich um eine Einwilligungserklärung handelt. Der formularmäßigen Verortung der Einwilligung in Allgemeinen Geschäftsbedingungen steht das Datenschutzrecht grundsätzlich zwar nicht entgegen, ${ }^{768}$ wobei sich der Inhalt freilich an den $\$ \int 307 \mathrm{ff}$. BGB messen lassen muss und die Klauseln nach $\ 305$ BGB wirksam einbezogen werden müssen. ${ }^{769}$ Jedoch bestimmt $\int 4 \mathrm{a}$ Abs. 1 S. 4 BDSG das die Einwilligung in ihrem äußeren Erscheinungsbild hervorzuheben ist, wenn sie zusammen mit anderen Erklärungen abgegeben werden soll. Dieser Gedanke beansprucht auch für die elektro-

765 Spindler/Schuster/Spindler/Nink, \ 13 TMG Rn. 7; Schaar, MMR 2001, 647.

766 Roßnagel/Holznagel/Sonntag, Hdb. DatenschutzR, Kap. 4.8 Rn 87.

767 BGH, MMR 2008, 731 (733) m. Anm. Grapetin; a.A. noch OLG München, MMR 2007, 47 (48); Pauli, WRP 2009, 246.

768 BGH, NJW 2677 (2678); Ranke, S. 109; Schmitz, DuD 2001, 397.

769 BGH, NJW 2677 (2678); OLG Brandenburg, MMR 2006, 405 f. m. Anm. Breyer, LG Potsdam, DuD 2005, 302 ff.; Wegmann, WRP 2007, 1145; Schmitz, DuD 2001, 397. 
nische Einwilligung im Bereich der Telemedien über $₫ 12$ Abs. 4 TMG Geltung. ${ }^{770}$ Die Einwilligung muss dementsprechend deutlich visuell hervorgehoben werden und darf nicht in klein gedruckten Klauseln verschwinden; ${ }^{771}$ das entspricht auch dem Transparenzgebot des $\int 307$ Abs. 1 S. 2 BGB.

\section{iii) Inhaltliche Anforderungen}

Im Hinblick auf das Recht auf informationelle Selbstbestimmung ${ }^{772}$ stellt das Datenschutzrecht nicht nur die bereits aufgezeigten formalen Anforderungen an eine wirksame Einwilligung, sondern darüber hinaus auch inhaltliche Anforderungen. Konkret bedeutet dies, dass die Portalbetreiber und/oder Web Service-Anbieter die Einwilligung hinreichend bestimmt ausgestalten müssen und die Einholung frei von Zwang erfolgen muss. Weiter darf die Erbringung eines Diensteangebots nicht an die Bereitschaft zur Erteilung einer Einwilligung durch den Nutzer in die Datenverarbeitung für andere Zwecke gekoppelt werden.

\section{Bestimmtheit}

Das Erfordernis der hinreichenden Bestimmtheit der Einwilligung ist logische Konsequenz des Ausflusses der Einwilligung aus dem Recht auf informationelle Selbstbestimmung. Denn nur durch eine Zweckbestimmung ist es dem Betroffenen möglich, die Datenverarbeitung einzuschränken. Dementsprechend genügen weder pauschal gehaltene Erklärungen noch Blankoeinwilligungen dem Erfordernis der Bestimmtheit. ${ }^{773}$ Im Rahmen der Einwilligung durch den Portalbetreiber oder die Web Service-Anbieter muss folglich konkretisiert werden, wie die Daten genutzt und welche Techniken (Cookies, Web-Bugs etc.) eingesetzt werden, an welche Web Service-Anbieter Daten weiter übermittelt werden sollen sowie zu welchem Zeitpunkt und an welchem Ort die Datenverarbeitung erfolgt. ${ }^{.74}$ Schreibt der Portalbetreiber in

770 Scholi, S. 293; Ranke, S. 110.

771 LG München, DuD 2006, 309 (310); AG Elmshorn, MMR 2005, 870 (871 f.); Simitis/Simitis, \4a Rn. 40 f.; Roßnagel/Holznagel/Sonntag, Hdb. DatenschutzR, Kap. 4.8 Rn. 41; Spindler/Schuster/Spindler/Nink, \4a BDSG Rn. 8; Scholv, S. 293; Ranke, S. 109 f.; Wegmann, WRP 2007, 1145 .

772 S. dazu B.III.3.a) Das Recht auf informationelle Selbstbestimmung.

773 BGHZ 95, 362 (367 f.); OLG Schleswig, MMR 1998, 41 (44); Petri, RDV 2007, 156 f.; Heidemann-Peuser, DuD 2002, 393; Ranke, S. 110; Scholz, S. 295.

774 S. Ranke, S. 110. 
seine Datenschutzerklärung, dass die erhobenen Daten an „vertrauenswürdige Web Service-Anbieter" übermittelt werden, genügt dies nicht dem Erfordernis der Bestimmtheit. ${ }^{775}$ Ein Grenzfall dürfte die Formulierung darstellen, dass die Daten an „externe Web Service-Anbieter, außerhalb des Bereichs der Europäischen Union, deren Dienste im Portal implementiert sind“, übermittelt werden beziehungsweise diese Web Service-Anbieter auf die Daten zugreifen. Je nach Portalgröße und Anzahl der implementierten Web Services kann es sich um eine überschaubare Anzahl an Web Service-Anbietern handeln, die der Betroffene schnell überblicken kann, oder aber eine sehr große Zahl, die für den Betroffenen nicht in Gänze überschaubar ist. Auch können die Portale derart ausgestaltet sein, dass Web Service-Anbieter schnell wechseln können, ohne dass der Betroffene davon etwas mitbekommt. Insgesamt erscheint eine solche Formulierung daher nicht genau genug. Von einer Konkretisierung der Web Service-Anbieter kann durch diese Formulierung jedenfalls nicht gesprochen werden, dafür wäre es vielmehr erforderlich, gleich wie groß das Portal tatsächlich ist, die jeweiligen Web Service-Anbieter, an welche Daten übermittelt werden sollen, exakt zu bezeichnen, indem zumindest der Firmenname, der Sitz des Unternehmens und sonstige erforderliche Identifizierungsmerkmale mitgeteilt werden. ${ }^{776}$ Da nur durch eine exakte Bezeichnung eine Konkretisierung erfolgt, kann auch nur dann das Kriterium der Bestimmtheit erfüllt sein. Darüber hinaus sollte auch der jeweilige Charakter des Dienstes beschrieben werden, damit der Betroffene tatsächlich ermessen kann, wer seine Daten zu welchem Zweck erhält.

\section{Freinilligkeit}

Im ersten Satz der Einwilligungsvorschrift in $\int 4 a$ Abs. 1 S. 1 BDSG heißt es, dass die Einwilligung nur wirksam ist, wenn sie auf der freien Entscheidung des Betroffenen beruht. Die Freiwilligkeit der Einwilligung stellt aufgrund der systematischen Stellung im Bundesdatenschutzgesetz daher einen der wichtigsten zu beachtenden Grundsätze bei der Einholung einer Einwilligung dar. Das Erfordernis der Freiwilligkeit ist ein Ausfluss des informationellen Selbstbestimmungsrechts ${ }^{777}$ und entspricht darüber hinaus den Vorgaben der Daten-

\footnotetext{
775 So Ranke, S. 110.

776 Ähnlich Weichert, RDV 2003, 121, der hohe Anforderung an die „Beschreibungspräzession“ fordert.

777 Iraschko-Luscher, DuD 2006, 707 f.; Geiger, NVwZ 1989, 37; Jarass, NJW 1989, 860; Scholz, S. 299.
} 
schutzrichtlinie. ${ }^{778}$ Nach Art. 2 lit. h DSRL muss die Einwilligung ,ohne Zwang" erfolgen. Wird eine Einwilligung unter Zwang abgegeben, so ist sie unwirksam sowie die darauf begründete Datenverarbeitung und demnach auch die Datenübermittlung in Drittstaaten unzulässig. 779

Probleme bereitet die Eingrenzung des Grundsatzes der Freiwilligkeit, da die Zustimmung in die Datenverarbeitung zumeist auch mit dem Bedürfnis oder Wunsch des Betroffenen einhergeht, das Portal und die angebotenen Dienste nutzen zu dürfen. ${ }^{780}$ Dieser Problematik wird allerdings bereits durch das in \28 Abs. 3 b) BDSG verankerte Koppelungsverbot, das nunmehr einheitlich für alle datenschutzrechtlichen Vorschriften gilt, ${ }^{781}$ abgeholfen. ${ }^{782}$ Danach darf der Diensteanbieter die Bereitstellung von Telemedien nicht von der Einwilligung des Nutzers in eine Verwendung seiner Daten für andere Zwecke abhängig machen, wenn dem Nutzer ein anderer Zugang zu diesen Telemedien nicht oder in nicht zumutbarer Weise möglich ist. ${ }^{783}$ Praktisch wird die Freiwilligkeit daher häufig an den Grenzen des Koppelungsverbots zu messen sein.

Kriterien für die Freiwilligkeit sind der freie Wille des Betroffenen ohne Zwang, das bedeutet ohne physischen und ohne, für den Online-Bereich relevanter, psychischen Zwang, hinsichtlich des $\mathrm{Ob}$ der Datenübermittlung (und dementsprechend auch der Datenübermittlung) sowie das Wissen um den Umfang der Datenübermittlung, da andernfalls eine freie Entscheidung nicht möglich ist. ${ }^{784}$ Freilich ist dafür auch eine umfassende Information über den Zweck der Übermittlung personenbezogener Daten erforderlich. 785 Darüber

778 Richtlinie 95/46/EG des Europäischen Parlaments und des Rates v. 24.10.1995 zum Schutz natürlicher Personen bei der Verarbeitung personenbezogener Daten und zum freien Datenverkehr, ABl. EG L 281 v. 23.11.1995, S. 31-50.

Simitis/Simitis, \4a Rn. 64; Schaar, MMR 2001, 644; Scholv, S. 300.

So lässt sich bspw. das Internetauktionshaus eBay von jedem Benutzer bei der Anmeldung eine Einwilligung in die Übermittlung sämtlicher persönlicher Daten in den USA geben, s. http://pages.ebay.de/help/policies/privacy-policy.html.

BT-Drs. 16/12011, S. 43.

Schol, S. 303; Schaar, MMR 2001, 644. Ein solches aus diesem Grunde für das BDSG fordernd Irascko-Luscher, DuD 2006, 708; i.d.S auch Engel-Flechsig, DuD 1997, 13; Simitis/Simitis, \ 4a Rn. 63.

S. ausführlich zum KoppelungsgebotB.III.2.b)aa) Koppelungsverbot.

Roßnagel/Holzzagel/Sonntag, Hdb. DatenschutzR, Kap. 4.8 Rn. 54; Petri, RDV 2007, 156; Schaar, MMR 2001, 644 f.; Ranke, S. 110.

Schaar, MMR 2001, 644; Weichert, RDV 2003, 120; Petri, RDV 2007, 156. 
hinaus resultiert daraus auch die Erforderlichkeit, die Datenübermittlung verweigern zu können. ${ }^{786}$ Denn die Verweigerung stellt eine Ausübung des informationellen Selbstbestimmungsrechts dar, indem der Betroffene frei darüber entscheidet, wer Zugang zu seinen Daten erhalten soll und wer nicht. ${ }^{787}$ Regelmäßig fehlt es an der Freiwilligkeit, wenn dem Betroffenen keine andere Wahl bleibt, als in die Datenverarbeitung einzuwilligen. ${ }^{788}$ Daher darf auch nicht der Eindruck vermittelt werden, dass dem Betroffenen im Falle einer Verweigerung der Einwilligung grundlegende vertragliche Leistungen vorenthalten würden, ${ }^{789}$ gewisse Einschränkungen bei Verweigerung der Datenverarbeitung (Datenübermittlung) können indes zulässig sein.

\section{Koppelungsverbot}

Das in $\ 28$ Abs. 3 b) BDSG verankerte, das Freiwilligkeitserfordernis konkretisierende, Koppelungsverbot soll den Tausch von personenbezogenen Daten gegen Dienstleistungen verhindern. ${ }^{790}$ Konkret zielt das Koppelungsverbot darauf ab, dass die Bereitschaft des Nutzers nicht daran geknüpft werden soll, einer Zweckänderung seiner Daten zustimmen zu müssen („, für andere Zwecke). Mit Verwendung der Daten zu anderen Zwecken ist hauptsächlich die Verwendung zu Zwecken der Werbung, Marktforschung und Übermittlung an Dritte gemeint. ${ }^{791}$ Durch dieses Verbot sollen insbesondere die Schwächen der elektronischen Einwilligung abgemildert werden. ${ }^{792}$ So soll beispielsweise eine Einwilligung dann nicht mehr freiwillig sein und gegen das Koppelungsverbot verstoßen, wenn das Ausfüllen eines Fragebogens mit der gleichzeitigen Teilnahme an einem Gewinnspiel und der damit verbundenen Aussicht auf Geldgewinne verbunden worden ist. ${ }^{793}$ Nicht erforderlich für das Greifen des

\footnotetext{
786 Roßnagel/Holznagel/Sonntag, Hdb. DatenschutzR, Kap. 4.8 Rn. 54; Roßnagel/Bizer, RMD, Teil 3, § 3 TDDSG Rn. 184; Irascko-Luscher, DuD 2006, 708; dies voraussetzend Weichert, RDV 2003, 120.

787 Irascko-Luscher, DuD 2006, 708.

788 Roßnagel/Holznagel/Sonntag, Hdb. DatenschutzR, Kap. 4.8 Rn. 54; LG Stuttgart, DuD 1999, 294 (295).

789 Weichert, RDV 2003, 120; Roßnagel/Holznagel/Sonntag, Hdb. DatenschutzR, Kap. 4.8 Rn. 63.

790 Scholy, S. 304; Roßnagel/Bizer, RMD, Teil 3, § 3 TDDSG Rn. 183 ff.

791 Roßnagel/Bizer, Teil 3, RMD, \3 TDDSG Rn. 189; Spindler/Schuster/Spindler/Nink, \12 TMG Rn. 8; Elschner, S. 252; Schaar, Rn. 593.

792 Roßnagel/Holznagel/Sonntag, Hdb. DatenschutzR, Kap. 4.8 Rn. 71.

793 LG Stuttgart, DuD 1999, 294 (295).
} 
Koppelungsverbots ist, dass zwischen dem gewünschten Telemediendienst und dem primären Datenerhebungszweck ein Zusammenhang besteht, die Daten können auch aus der Nutzung eines anderen Telemediendienstes stammen, das heißt unterschiedliche Diensteangebote, die über ein Webportal erbracht und miteinander verknüpft werden können, werden hiervon erfasst. ${ }^{794}$ Allerdings greift das Koppelungsverbot dann nicht, wenn eine Speicherung der Daten für bestimmte Zwecke erforderlich ist. ${ }^{795}$

Fraglich erscheint, wann kein anderer (zumutbarer) Zugang zu Telemediendiensten im Sinne des $\ 28$ Abs. 3 b), 2. Hs. BDSG existiert. Aus dem Gesetzeswortlaut der Vorgängervorschrift in $\ 12$ Abs. 3 TMG, der von ,diesen Telemedien“ sprach, wurde zum Teil geschlossen, dass ein anderer zumutbarer Zugang nur dann vorliegt, wenn dem Nutzer zumindest eine andere $\mathrm{Zu}$ gangsmöglichkeit hinsichtlich der von demselben Diensteanbieter angebotenen Telemedien besteht. ${ }^{796}$ Gegen eine solche Auslegung spricht aber, dass dann bereits der Anwendungsbereich der Einschränkung ausgeschlossen wäre. ${ }^{797}$ Denn müsste der einzelne Anbieter immer andere Zugangsmöglichkeiten anbieten, so wäre das Verbot in den Fällen, in denen der Anbieter bereits andere Zugangsmöglichkeiten anbietet, sinnlos. ${ }^{798}$ Darüber hinaus wären im Fall einer derartigen Auslegung die Regelungsgehalte der beiden Halbsätze des \28 Abs. 3 b) BDSG deckungsgleich: erlaubt der Diensteanbieter den Zugang zu seinen Telemedien auch ohne Einwilligung, macht er bereits die Erbringung von Telemedien nicht von der Einwilligung des Nutzers abhängig, so dass $\ 28$ Abs. 3 b), 1. Hs. BDSG keine Anwendung findet. ${ }^{799}$

Daher muss die Möglichkeit des Zugangs nach der jeweiligen Marktsituation beurteilt werden. Fehlt es demnach an einer Monopolstellung, weil dem Nutzer mindestens ein entsprechend gleichwertiger Dienst zur Verfügung steht, existiert eine andere Zugangsmöglichkeit im Sinne des $\ 28$ Abs. 3 b), 2. Hs.

794 Spindler/Schuster/Spindler/Nink, \12 TMG Rn. 8; Roßnagel/Bizer, RMD, Teil 3, \3 TDDSG Rn. 199.

795 So sah das LG Frankfurt, MMR 2006, 769 (770) die Speicherung der Personalausweisnummer bei der FIFA Weltmeisterschaft 2006 zur Erfüllung der vertragsgemäßen gefahrlosen Teilnahme als erforderlich an.

796 Roßnagel/Bizer, RMD, Teil 3, \$ 3 TDDSG Rn. 204.

797 Scholv, S. 304 f.; Schmitr, S. 110.

798 Schmitz, S. 110; Scholz, S. 304 f.; Zscherpe, MMR 2004, 727.

799 OLG Brandenburg, MMR 2006, 405 (407); dem zustimmend Spindler/Schuster/Spindler/Nink, \12 TMG Rn. 9. 
BDSG und das Koppelungsverbot greift nicht. ${ }^{800}$ Die grammatikalische Auslegung der Vorschrift würde zwar eher dafür sprechen, dass sie sich lediglich an der Zugangsmöglichkeit des einzelnen Anbieters orientiert. Indes sprechen die Ratio des Paragraphen und der historische Wille des Gesetzgebers, ${ }^{801}$ der die Einschränkung erst später, nur zur Präzisierung des Koppelungsgebots und zur Verhinderung des Ausnutzens einer marktbeherrschenden Stellung beziehungsweise einer Monopolstellung, ${ }^{802}$ eingefügt hat, für die hier vertretene Auffassung.

Unklar ist allerdings, wie absolut die marktbeherrschende oder die Monopolstellung einzuordnen sind. Nach Auffassung des OLG Brandenburg genügt für den Ausschluss des Koppelungsverbots, dass andere Diensteanbieter ${ }^{803}$ vergleichbare Leistungen anbieten und lässt dabei sogar $73 \%$ der Marktanteile nicht als Monopolstellung genügen. ${ }^{804}$ In diesem konkreten Fall verbleiben 27 $\%$ Marktanteile, die sich auf andere Diensteanbieter derselben Branche verteilen. Faktisch betrachtet, verbleibt dem Nutzer tatsächlich die Möglichkeit andere Diensteanbieter zu nutzen. Eine solche Einordnung lässt sich aber nicht anhand von starren Grenzen, die sich in Prozentsätzen ausdrücken, festmachen. Hat im Falle der Web Service-Portale beispielsweise ein Portalbetreiber einen Marktanteil von $49 \%$, so bleiben den Nutzern zwar tatsächlich noch 51 $\%$ Marktanteile verteilt auf andere Portalbetreiber, bei denen sie sich anmelden können. Orientiert an einer starren Grenze, dabei das Urteil des OLG Brandenburg heranziehend, würde dies bedeuten, eine marktbeherrschende Stellung verneinen zu müssen und beim Abhängigmachen der Portalnutzung von der Einwilligung in eine zweckfremde Datenverarbeitung das Greifen des Koppelungsverbots ebenfalls mangels Ausnutzen einer marktbeherrschenden Stellung verneinen zu müssen. Dabei bliebe aber unberücksichtigt, wie die Portale tatsächlich ausgestaltet sind. So kann es sein, dass nur das Web Service-Portal mit dem Marktanteil von $49 \%$ bestimmte Web Service-Anbieter implementiert hat, die exklusive Dienste anbieten, währenddessen die übrigen Web Service-Portale lediglich Such-Web Services und Wetterdienste oder ähn-

800 OLG Brandenburg, MMR 2006, 405 (407); Spindler/Schmitz/Geis/Schmitz \ 3 TDDSG Rn. 36 ff.; Spindler/Schuster/Spindler/Nink, $\$ 12$ TMG Rn. 9; Schaar, Rn. 594; Elschner, S. 252; Fröble, S. 239; Scholv, S. 305; Schmitz, S. 109; Zscherpe, MMR 2004, 727.

801 BT-Drs. 13/7385, S. 6.

802 Spindler/Schmitz/Geis/Schmitz, \ 3 TDDSG Rn. 37.

803 Im Fall des OLG Brandenburg handelte es sich um ein Internetauktionshaus, konkret das Auktionshaus eBay.

804 OLG Brandenburg, MMR 2006, 405 (407). 
liches anbieten. Da es dann an vergleichbaren Leistungen fehlt, dürfte in diesem Fall ein Monopol vorliegen, so dass das Abhängigmachen der Portalnutzung von der Einwilligung in die Datenverarbeitung gegen das Koppelungsverbot des $₫ 28$ Abs. 3 b) BDSG verstößt.

Dieses Beispiel zeigt, dass gerade im Fall der Web Service-Portale starre Grenzen hinsichtlich einer marktbeherrschenden Stellung ungeeignet sind, um das Greifen oder Nichtgreifen des Koppelungsverbots zu beurteilen. Vielmehr muss immer die konkrete Marktsituation anhand des speziellen Einzelfalls untersucht werden, ${ }^{805}$ wobei auch die Art der implementierten Web ServiceAnbieter - und nicht allein die grobe Angebotsgattung „Web Service-Portale“ - eine wichtige, unter Umständen sogar entscheidende, Rolle spielen müssen. Auch eine Monopolstellung, sofern es sich nicht um ein absolutes Monopol handelt, darf lediglich ein Indiz für das Greifen des Koppelungsverbots sein; letztlich muss aber immer der konkrete Einzelfall betrachtet werden.

Greift das Koppelungsverbot, weil im konkreten Einzelfall die Ausnutzung einer Monopolstellung erfolgt, muss der Telemediendiensteanbieter den $\mathrm{Zu}-$ gang sowohl mit als auch ohne Einwilligung in andere Verwendungszwecke anbieten. ${ }^{806}$

\section{Sensible Daten}

\3 Abs. 9 BDSG nennt in einem Katalog besondere Daten, bei denen im Bundesdatenschutzgesetz eine erhöhte Schutzbedürftigkeit des Betroffenen angenommen wird, da dort die diskriminierende Verwendung besonders nahe liegt. 807 Dabei handelt es sich im Sprachgebrauch um „sensible“ oder „sensitive" Daten. Besondere Arten personenbezogener Daten sind danach Angaben über die rassische und ethnische Herkunft, politische Meinungen, religiöse oder philosophische Überzeugungen, Gewerkschaftszugehörigkeit, Gesundheit oder Sexualleben. $\int 3$ Abs. 9 BDSG setzt Art. 8 Abs. 1 der Datenschutzrichtlinie ${ }^{808} \mathrm{um}$, danach sollen diese Daten grundsätzlich nicht verarbeitet werden dürfen. Ausnahmen dieses Grundsatzes enthält Art. 8 Abs. 2 lit. a DSRL, wenn die betroffene Person ausdrücklich in die Verarbeitung der ge-

805 So auch Spindler/Schuster/Spindler/Nink, \12 TMG Rn. 9; Schaar, MMR 2001, 648; Rasmussen, DuD 2002, 410.

806 Schmit\%, in: Hoeren/Sieber, Hdb. MultimediaR, Teil 16.4 Rn. 81; Spindler/Schuster/Spindler/Nink, $\ 12$ TMG Rn. 10.

807 Roßnagel/Holznagel/Sonntag, Kap. 4.8 Rn. 56.

808 95/46/EG, ABl. EG L 281 v. 23.11.1995, S. 31-50. 
nannten Daten eingewilligt hat. Dem entspricht $\int 4 a$ Abs. 3 BDSG, der bestimmt, dass soweit besondere Arten personenbezogener Daten ( $\mathbb{3}$ Abs. 9 BDSG) erhoben, verarbeitet oder genutzt werden, sich die Einwilligung darüber hinaus ausdrücklich auf diese Daten beziehen muss. Wollen Portalbetreiber und Web Service-Anbieter daher diese sensiblen Daten erheben und verarbeiten, müssen sie zum einen in ihrer Datenschutzerklärung gesondert darauf hinweisen und auch die Einwilligungserklärung entsprechend formulieren, damit das ausdrückliche Beziehen auf diese Daten auch gewährleistet wird. ${ }^{809}$

bb) Empfehlungen zur Ausgestaltung der Einwilligungserklärung

Wollen Portalbetreiber und Web Service-Anbieter die Einwilligung innerhalb ihrer Allgemeinen Geschäftsbedingungen platzieren, so muss die Einwilligung darstellungstechnisch hervorgehoben werden. ${ }^{810}$ Nur so kann der Pflicht des \13 Abs. 2 Nr. 1 TMG, das es sich um eine bewusste und eindeutige Erklärung handeln muss, entsprochen werden. Neben den allgemeinen Anforderungen an die wirksame Einbeziehung von Allgemeinen Geschäftsbedingungen muss weiter die besondere Hinweispflicht bezüglich der Einwilligungserklärung erfüllt werden. Will man allerdings eventuelle Probleme mit der Transparenz vermeiden, so empfiehlt es sich, die Einwilligungserklärung in einem von den Allgemeinen Geschäftsbedingungen getrennten Formular zu platzieren. ${ }^{811}$ Gerade bei umfangreichen Einwilligungserklärungen, die sich sowohl auf die Verarbeitungsbefugnisse des Portalbetreibers als auch die der Web Service-Anbieter beziehen, empfiehlt sich eine derartige Trennungslösung aus Gründen der Übersichtlichkeit und um PflichtenverstöBe zu vermeiden. Eine solche Lösung könnte beispielsweise derart ausgestaltet sein, dass über oder unter der Zustimmung zu den Allgemeinen Geschäftsbedingungen im Laufe des Registrierungsprozesses die Zustimmung hinsichtlich der Datenschutzbestimmungen, die durch einen direkten Link abgerufen werden können, erfolgt. Fehlt es am Registrierungserfordernis oder wollen die Web Service-Anbieter die Einwilligungserklärung getrennt vom Portalbetreiber einholen, so könnte die Einwilligungserklärung zum Beispiel in einem

809 Der restriktivere Umgang mit den sensiblen Daten muss sich unmittelbar auf den Einwilligungstext auswirken, Simitis/Simitis, \4a Rn. 86; Roßnagel/Holznagel/Sonntag, Kap. 4.8 Rn. 56.

810 LG Berlin, VuR 2002, 413 (417); AG Elmshorn, MMR 2005, 870 (871); Spindler/Schmitz/Geis/Schmitz \4 TDDSG Rn. 17; Ranke, S. 117; von Lewinski, DuD 2002, 397 f.; Heidemann-Peuser, DuD 2002, 393.

811 So auch Simitis/Simitis, \ 4a Rn. 41; Scholv, S. 295; i.d.S. auch OLG Celle, NJW 1980, 347 (348). 
Pop-Up-Fenster platziert werden, ${ }^{812}$ sofern sichergestellt wird, dass eine aktive Zustimmung Voraussetzung für die weitere Nutzung darstellt. Andernfalls wäre durch die Möglichkeit der Pop-Up-Unterdrückung in den Browsereinstellungen eine Einwilligung nicht immer gewährleistet.

Hinsichtlich des „Wie“ der Ausgestaltung, stellt die so genannte Opt-OutKlausel in der Praxis eine beliebte Methode dar, ${ }^{813}$ bei welcher der Betroffene die Datenverarbeitung beispielsweise durch Auskreuzen ausschließen kann. Allerdings wird die Opt-Out-Variante häufig nicht als ausreichend angesehen und stattdessen eine Opt-In-Klausel gefordert, ${ }^{814}$ bei der die Einwilligung durch aktive Zustimmung zum Beispiel in Form des Ankreuzens erfolgt.

Für die Opt-Out-Variante lässt sich freilich anbringen, dass dabei auf einen verständigen, sorgsamen Leser abzustellen ist, der keine Erklärung ungelesen akzeptiert, ${ }^{815}$ so dass eine Opt-Out-Klausel eigentlich ausreichend sein dürfte. Jedoch ist ein solcher Leser in der Praxis wohl eher die Minderheit. Gerade wenn die Erklärung in Allgemeinen Geschäftsbedingungen integriert ist, wird ein sorgsames Lesen und Auskreuzen wohl eher selten erfolgen. Zieht man weiter die Pflicht des $\int 13$ Abs. 2 Nr. 1 TMG heran, wonach der Diensteanbieter sicherstellen muss, dass der Nutzer seine Einwilligung bewusst und eindeutig erteilt hat, wird deutlich, dass dieser Pflicht - realistisch betrachtet - nur dann nachgekommen wird, wenn der Nutzer gezwungen ist, sich zu der Verarbeitung seiner Daten aktiv zu äußern. Ein aktives und bewusstes Äußern erfolgt in aller Regel aber nicht durch Opt-Out-Klauseln. Daher ist es erforderlich, die Einwilligung, insbesondere zur Erfüllung der Pflicht aus $\int 13$ Abs. 2 Nr. 1 TMG, als Opt-In-Klausel auszugestalten. ${ }^{816}$

\section{c) Resümee}

Sollen personenbezogene Daten in Nicht-EU/EWR-Staaten oder Staaten ohne angemessenes Schutzniveau übermittelt werden, so können Daten an

812 S. zum Pop-Up-Fenster ausführlich Banæ̧haf, in: Roßnagel/Banzhaf/Grimm, Teil C S. 263 f.

813 Für das Ausreichen einer Opt-Out-Klausel OLG München, MMR 2007, 47 (49).

814 So LG München, DuD 2006, 309 (311); Schaar, Rn. 584 f.; von Fragstein, EWiR 2006, 518; in Richtung Opt-In-Klausel tendierend Wiesner, DuD 2007, 606 f.; i.d.S. auch OLG Brandenburg, MMR 2006, 405 (406), das von „bestätigende[r] Wiederholung des Übermittlungsbefehls“ spricht, und das OLG Bremen, DuD 2002, 433 (434), die in AGBs ausdrückliche Erklärungen fordern.

815 So die Argumentation des OLG München, MMR 2007, 47 (49).

816 A.A. BGH, MMR 2008, 731 (733) m. Anm. Grapetin, der eine Opt-Out-Klausel genügen lässt. 
amerikanische Unternehmen, die sich den Safe Harbor-Principles unterworfen haben, ohne weitere Anforderungen entsprechend dem Rahmen der Datenschutzgesetze übermittelt werden. Bei den übrigen Staaten ist regelmäßig, zumindest für eine umfassende Datenübermittlung, lediglich die Einholung einer Einwilligung in die Datenübermittlung durch den Betroffenen notwendig. Sollen daher die auf einem Web Service-Portal erhobenen Daten zum Zwecke einer kostengünstigeren Datenverarbeitung beispielsweise nach Indien transferiert werden, oder ist ein integrierter Web Service-Anbieter in China ansässig, so müssen die Akteure vom Betroffenen eine Einwilligung in die Datenübermittlung, die den oben genannten Anforderungen entsprechen muss, einholen. Handeln die Akteure den Vorgaben zur Datenübermittlung zuwider, so drohen ihnen nach $\int 43$ Abs. 2 Nr. 3, Abs. 3 BDSG Bußgelder in Höhe bis zu 250.000 Euro oder, sofern die Übermittlung gegen Entgelt erfolgt, gegebenenfalls sogar eine strafrechtliche Verfolgung nach \44 Abs. 1, 1. Alt. BDSG, die mit Freiheitsentzug bis zu zwei Jahren bestraft wird.

\section{Verfassungsrechtlicher Rabmen}

\section{a) Das Recht auf informationelle Selbstbestimmung}

Verfassungsrechtliche Grundlage des deutschen Datenschutzrechts ist das Grundrecht auf informationelle Selbstbestimmung. Das Bundesverfassungsgericht erkannte im Volkszählungsurteil817 1983 den Verfassungsrang des Datenschutzes eindeutig an, indem es aus Art. 2 Abs. 1 in Verbindung mit Art. 1 Abs. 1 GG das Recht auf informationelle Selbstbestimmung ableitete. ${ }^{818} \mathrm{Da}$ von umfasst wird das Recht grundsätzlich selbst über die Preisgabe und Verwendung seiner persönlichen Daten zu bestimmen und zu wissen „,wer, was, wann und bei welcher Gelegenheit über ihn weiß““.819 Im Zeitalter der Informationsgesellschaft, wo personenbezogene Daten immer wichtiger und wertvoller werden, insbesondere weil jede Bewegung in der Online-Welt, anders als in der analogen Welt, Datenspuren hinterlässt, ${ }^{820}$ und ihre Erhebung, Ver-

\footnotetext{
817 BVerfGE 65, 1 - Volkszählung.

818 BVerfGE 65, 1 (42 f.) - Volkszählung. Zuvor wurde bereits im Mikrozensus-Beschluss von 1969 anerkannt, dass „,wenn der Staat das Recht für sich in Anspruch nehmen könnte, den Menschen zwangsweise in seiner ganzen Persönlichkeit zu registrieren und zu katalogisieren, sei es auch in der Anonymität einer statistischen Erhebung, und ihn damit wie eine Sache zu behandeln, die einer Bestandsaufnahme in jeder Beziehung zugänglich ist“", die Menschenwürde verletzt ist, s. BVerfGE 27, 1 (6).

819 BVerfGE 65, 1 (43) - Volkszählung; BVerfG, MMR 2006, 217 (221).

820 Roßnagel, in: Roßnagel/Banzhaf/Grimm, Teil B S. 119.
} 
arbeitung und Nutzung in allen gesellschaftlichen Handlungsfeldern von enormer Bedeutung ist, ${ }^{821}$ wird das Recht auf informationelle Selbstbestimmung, dessen Schutz die Datenschutzgesetze dienen, 822 immer wichtiger.

Gerade auf elektronischen Portalen, wie Web Service-Portalen, hinterlässt der Nutzer mit jeder Bewegung Datenspuren, durch Klicks, Betrachtungen von Angeboten und Dienstleistungen, ganz zu schweigen von der Nutzung etwaiger Dienste; diese Datenspuren können und werden regelmäßig festgehalten und ausgewertet. ${ }^{823}$ Durch die Auswertung dieser Datenmengen können zahlreiche Auskünfte über den Betroffenen gewonnen werden. Dies birgt das Risiko, dass der Betroffene in bestimmten Bereichen nicht mehr in eine Rolle schlüpfen kann, weil der Kommunikationspartner bereits so viel über ihn weiß, dass ein Verstellen oder sich auf eine bestimmte Art und Weise Verhalten nicht mehr möglich ist. Darüber hinaus wird der Betroffene regelmäßig noch nicht einmal wissen, dass sein Kommunikationspartner bereits dieses Wissen über ihn hat, da er keine wirkliche Kontrolle über den Umgang mit seinen Daten hat. ${ }^{824}$ Dem Betroffenen wird dadurch die Freiheit genommen, sich in unterschiedlichen gesellschaftlichen Kreisen auch jeweils unterschiedlich geben zu können. Dem muss der Datenschutz entgegenwirken.

\section{b) Eingriff}

Jeder Umgang mit personenbezogenen Daten, ${ }^{825}$ der die Selbstbestimmung der betroffenen Person nicht beachtet, stellt einen Eingriff in das Recht auf informationelle Selbstbestimmung dar. ${ }^{826}$ Werden daher personenbezogene Daten der Nutzer gegen deren Willen beziehungsweise ohne deren Zustimmung durch den Portalbetreiber und die Web Service-Anbieter erhoben, so stellt dies einen Eingriff in das Recht auf informationelle Selbstbestimmung

821 Roßnagel/Roßnagel, Hdb. DatenschutzR, Einleitung Rn. 2; Schmitz, in: Spindler/Wiebe, Kap. 13 Rn. 2; s. zu den zahlreichen Verwertungs- und Nutzungsmöglichkeiten der Daten Hornung, MMR 2004, 5.

822 Spindler/Schuster/Spindler/Nink, \11 TMG Rn. 2; Schmitr, S. 4; ders., in: Spindler/Wiebe, Kap. 13 Rn. 1.

823 S. zu den Sammlungs- und Auswertungsmöglichkeiten im elektronischen Geschäftsverkehr ausführlich Grimm, in: Roßnagel/Banzhaf/Grimm, Teil A, S. 44 ff. u. 55 ff.; Wiese, in: Bäumler, S. $9 \mathrm{ff}$.

824 Roßnagel, in: Roßnagel/Banzhaf/Grimm, Teil B S. 120.

$825 \mathrm{Zu}$ personenbezogenen Daten s. B.IV.1 Personenbezogene Daten.

826 Roßnagel/Pfitzmann/Garstka, S. 70 f. 
dar. Das Grundrecht wird aber nicht unbeschränkt gewährleistet, sondern kann nach Art. 2 Abs. 1 GG gesetzlich eingeschränkt werden. ${ }^{827}$ Solche Einschränkungen, die den Eingriff rechtfertigen, finden sich beispielsweise in den Erlaubnistatbeständen des Bundesdatenschutzgesetzes sowie des Telemediengesetzes. Darüber hinaus kann ein Eingriff auch durch eine wirksame Einwilligung des Betroffenen gedeckt sein. Der Umgang mit personenbezogenen Daten darf daher nur aufgrund eines Gesetzes oder mit der Einwilligung des Betroffenen erfolgen.

\section{c) Datenschutzgrundsätze}

Soweit personenbezogene Daten ${ }^{828}$ vorliegen, müssen beim Umgang mit diesen bestimmte datenschutzrechtliche Grundsätze beachtet werden. Untergliedern lassen sich die für den Untersuchungsgegenstand relevanten datenschutzrechtlichen Schutzkonzepte in: Datenvermeidung und Datensparsamkeit, Zweckbindung, Transparenz, Direkterhebung der Daten, System- und Selbstdatenschutz sowie Datensicherheit. Die allgemeinen Datenschutzgrundsätze wurden zum Teil in verschiedenen einfachgesetzlichen Datenschutzregelungen konkretisiert.

aa) Datenvermeidung und Datensparsamkeit

Die Grundsätze der Datenvermeidung und Datensparsamkeit werden in $\ 3 \mathrm{a}$ BDSG legaldefiniert. Danach haben sich Gestaltung und Auswahl von Datenverarbeitungssystemen an dem Ziel auszurichten, keine oder so wenig personenbezogene Daten wie möglich zu erheben oder zu verwenden. Dabei wird das Prinzip der Datenvermeidung und Datensparsamkeit durch \3a S. 2 BDSG konkretisiert, wonach die Daten des Betroffenen nach (technischer) Möglichkeit in anonymisierter oder pseudonymisierter Form erhoben oder verarbeitet werden sollen. ${ }^{829}$ Dies entspricht der Festlegung des Bundesverfassungsgerichts im Volkszählungsurteil, dass ,sich alle Stellen, die zur Erfüllung ihrer Aufgaben personenbezogene Daten sammeln, auf das zum Erreichen des angegebenen Ziels erforderliche Minimum beschränken müssen." "830 \3a

827 BVerfGE 65, 1 (43 f.) - Volkszählung; Schaar, Rn. 120; Simitis/Simitis, Einleitung Rn. 33; Hornung, S. 152.

$828 \mathrm{Zu}$ personenbezogenen Daten s. B.IV.1 Personenbezogene Daten.

829 Gola/Schomerus, \3a Rn. 8; Simitis/Bizer, \3a BDSG Rn. 1 u. 68 ff.; Roßnagel/Müller, CR 2004, 631.

830 BVerfGE 65, 1 (46). 
BDSG präzisiert den datenschutzrechtlichen Erforderlichkeitsgrundsatz in Form eines technischen Gestaltungsrechts. ${ }^{831}$ Nach diesem Grundsatz darf ein Umgang mit personenbezogenen Daten nur im Rahmen des erforderlichen Maßes stattfinden, ${ }^{832}$ kurz: überflüssige personenbezogene Daten dürfen nicht erhoben, verwendet oder genutzt werden. ${ }^{833}$ Dieser Grundsatz wird durch \3a BDSG zur Gestaltung und Auswahl von Datenverarbeitungssystemen eingesetzt, was bedeutet, dass der Grundsatz der Erforderlichkeit bereits im Rahmen der Technikgestaltung Berücksichtigung finden muss. ${ }^{834}$ Der Gesetzgeber nimmt dadurch bereits im Vorfeld der Technikentwicklung Einfluss auf ihre Ausgestaltung und fördert so die Integration datenschutzrechtlicher Anforderungen in technische Gestaltungsprozesse. ${ }^{835}$ Eingang hat dieser Grundsatz auch in $\int 13$ Abs. 6 TMG gefunden, wo bestimmt ist, dass der Web Service-Anbieter die Nutzung von Telemedien und ihre Bezahlung nach technischer und zumutbarer Möglichkeit anonym oder unter Pseudonym zu ermöglichen hat. Für Web Service-Portale bedeutet dies, dass die Nutzung der Plattform zumindest unter Pseudonym ermöglicht werden sollte, da dies technisch grundsätzlich keine Schwierigkeiten bereiten dürfte.

\section{bb) Zweckbindung}

Je größer die Datensammlungen sind, desto mehr Verknüpfungs- und Auswertungsmöglichkeiten bieten sich für das sammelnde Unternehmen sowie für andere Unternehmen, die diese Sammlungen als Datenquelle nutzen können. ${ }^{836} \mathrm{Um}$ die Zusammenführung von Daten durch datenerhebende Behörden oder Unternehmen zu vermeiden, gilt der strenge Grundsatz der Zweckbindung, ${ }^{837}$ der im Rahmen jeder Datenverarbeitung beachtet werden muss. Nach dem Grundsatz der Zweckbindung dürfen Daten nur zu dem Zweck verarbeitet und genutzt werden, für den sie erhoben oder gespeichert worden

831 Simitis/Bizer, \3a BDSG Rn. 2; Begr. zum Entwurf eines Gesetzes zur Änderung des Bundesdatenschutzgesetzes und anderer Gesetze v. 13.10.2000, BT-Drs. 14/4329, S. 33; ferner Roßnagel/Müller, CR 2004, 631.

S. Simitis/Bizer, \3a Rn. 2; Schaar, Rn. 129 f.; Scholz, S. 374.

833 Bizer, DuD 2007, 353.

834 Schaar, Rn. 888; ferner Bizer, DuD 2007, 353.

835 Scholv, S. 376.

836 Roßnagel/von Zęschwitz, Hdb. DatenschutzR, Kap. 3.1 Rn. 2.

837 Roßnagel/von Zeæschwitz, Hdb. DatenschutzR, Kap. 3.1 Rn. 1; Spindler/Schmitz/Geis/Schmitz, \5 TDDSG Rn. 7; Scholv, S. 236; jurisPK-Internetrecht/Heckmann, Kap. 1.14 Rn. 16; Roßnagel, NVwZ 1998, 4; ferner Engel-Flechsig/Maennel/Tettenborn, NJW 1997, 2986. 
sind. ${ }^{838}$ Daraus folgt, dass zum einen der Zweck bereits zu Beginn der Datenerhebung oder -verarbeitung festgelegt sein muss und zum anderen die Daten dann nur zu diesem Zweck verarbeitet und genutzt werden dürfen. ${ }^{839}$ Eine nachträgliche Zweckänderung ist lediglich in den Fällen des Greifens eines entsprechenden Erlaubnistatbestands oder dem Vorliegen einer Einwilligung möglich. ${ }^{840}$ Das ergibt sich daraus, dass auch jedes scheinbar irrelevante Einzeldatum durch eine Zweckänderung eine Bedeutung erlangen kann. ${ }^{841}$ Entsprechende Erlaubnistatbestände, die eine nachträgliche Zweckänderung billigen, finden sich in $\ 14$ Abs. 2 TMG, der eine grundsätzliche Ermächtigung zu einer Auskunftsanfrage für einen bestimmten Personenkreis enthält, und dem Erlaubnistatbestand der Übermittlung von Abrechnungsdaten an Dritte $(\mathbb{\Omega} 15$ Abs. 5 S. 1). Dem Betroffenen soll das Zweckbindungsgebot einen Überblick über den Verarbeitungsprozess geben, damit er sich ein verlässliches Bild über die potenziellen Folgen der Verarbeitung machen und die Verarbeitung seiner Daten selbst steuern und rekonstruieren kann. ${ }^{842}$

\section{cc) Transparenz}

Um sich darüber informieren zu können, ,wer was wann und bei welcher Gelegenheit über [...]“ den Betroffenen weiß, ${ }^{843}$ und dadurch dem Recht auf informationelle Selbstbestimmung zu entsprechen, ist es Voraussetzung der Datenverarbeitung, dass diese transparent für den Betroffenen gestaltet ist. Ohne Wissen um eine etwaige Datenverarbeitung ist der Betroffene weder in der Lage die Rechtmäßigkeit der Datenverarbeitung zu überprüfen noch seine Rechte diesbezüglich geltend zu machen. ${ }^{844}$ Dieses notwendige Wissen um-

838 Dieser Grundsatz wurde im Volkszählungsurteil durch das BVerfG formuliert, BVerfGE 65, 1 (46).

839 BVerfGE 65, 1 (46) - Volkszählung; Hornung, S. 157; Bizer, DuD 2007, 352.

840 Roßnagel/Bizerer, RMD, Teil 3, 33 TDDSG Rn. 138; Schmitz, in: Hoeren/Sieber, Hdb. MultimediaR, Teil 16.4 Rn. 79; Spindler/Schuster/Spindler/Nink, \12 TMG Rn. 7; Bizer, DuD 2007, 352; Roßnagel/Laue, DÖV 2007, 546; Roßnagel/Müller, CR 2004, 630.

841 Roßnagel/Laue, DÖV 2007, 545; Hornung, S. 157; allgemeiner auch BVerfGE, NJW 2008, 822 (826) - Online-Durchsuchung.

842 Spindler/Schuster/Spindler/Nink, \12 TMG Rn. 7; Spindler/Schmitz/Geis/Schmitz, 』3 TDDSG Rn. 31; Simitis/Simitis, Einleitung Rn. 35; Roßnagel, NVZ 2006, 283; Roßnagel/Laue, DÖV 2007, 545.

843 BVerfGE 65, 1 (43).

844 Roßnagel/Roßnagel, Kap. 7.9 Rn. 88; Roßnnagel/Müller, CR 2004, 628; Scholv, S. 371; Hornung, S. 162. 
fasst ausreichende Informationen hinsichtlich der Erhebung personenbezogener Daten über Umstände, Verfahren und Struktur ihrer Verarbeitung sowie die Zwecke ihrer Verwendung. ${ }^{845}$ Transparenz gewinnt insbesondere dann an Bedeutung, wenn das Risiko besteht, dass das Kommunikationsverhalten umfassend und unbemerkt dokumentiert werden kann. ${ }^{846}$ Durch die leichte Möglichkeit der Koppelung von auf Web Services basierenden Diensten, besteht die Möglichkeit, dass auf einem Web Service-Portal sehr viele Web ServiceAnbieter integriert sind. Dadurch kann für den Betroffenen die Zahl der potenziellen Datensammler unüberschaubar werden. Es wird dem Portalnutzer daher realistisch betrachtet, kaum möglich sein, bemerken zu können, wer welche Informationen über sie sammelt und dokumentiert. Daher erlangt der Transparenzgrundsatz für die vorliegende Arbeit eine besondere Bedeutung. Ausprägungen des Transparenzprinzips finden sich beispielsweise in der Unterrichtungspflicht des $\ 13$ Abs. 1 TMG, bei der die datenerhebende Stelle selbst aktiv werden muss, der Auskunftspflicht des $₫ 13$ Abs. 7 TMG - die Auskunft muss der Betroffene selber geltend machen - und dem Grundsatz der Direkterhebung in $\ 4$ Abs. 2 BDSG.

dd) Grundsatz der Direkterhebung

$\int 4$ Abs. 2 S. 1 BDSG bestimmt, dass personenbezogene Daten beim Betroffenen zu erheben sind. Es wird daher vom Grundsatz der Direkterhebung gesprochen. Dieser setzt freilich im Sinne des $\int 4$ Abs. 1 BDSG voraus, dass die Erlaubnis zur Erhebung personenbezogener Daten in Form eines gesetzlichen Tatbestands oder einer Einwilligung des Betroffenen vorliegt. ${ }^{847}$ Das Erheben von Daten wird in $₫ 3$ Abs. 3 BDSG legaldefiniert als das Beschaffen von Daten über den Betroffenen. Der Grundsatz der Direkterhebung ist eine einfachgesetzliche Ausprägung des Transparenzgrundsatzes und damit letztlich ein Ausfluss des Rechts auf informationelle Selbstbestimmung, der dem Betroffenen das Wissen verschaffen soll, wer sich für seine Daten interessiert. ${ }^{848}$ Bei der Direkterhebung der personenbezogenen Daten ist die erhebende Stelle auf die aktive oder passive Mitwirkung, in Form der ausdrücklichen bewussten Duldung, des Betroffenen angewiesen. ${ }^{849}$ Ausnahmen vom

845

846

847

848

849

Scholz, S. 371

Hornung, S. 163.

Spindler/Schuster/Spindler/Nink, \& 4 BDSG Rn. 6.

Simitis/Sokol, \ 4 Rn. 20; Gola/Schomerus, \ 4 Rn. 21; Gola/Klug, S. 49; Hornung, S. 164, 198; Iraschko-Luscher, DuD 2006, 706.

Simitis/Sokol, \ 4 Rn. 23; Iraschko-Luscher, DuD 2006, 706. 
Grundsatz der Direkterhebung sind nur in den speziellen Fällen des $₫ 4$ Abs. 2 S. 2 BDSG zulässig. Eine solche liegt nach der für den Untersuchungsgegenstand relevanten Nr. 1 vor, wenn eine Rechtsvorschrift dies vorsieht oder zwingend voraussetzt. Darunter fallen insbesondere auch die gesetzlichen Erlaubnistatbestände zum Umgang mit personenbezogenen Daten im Bundesdatenschutzgesetz und im Telemediengesetz. ${ }^{850}$

Werden daher Daten im Rahmen des Untersuchungsgegenstands nicht direkt vom Betroffen beschafft, so ist dies nur dann zulässig, wenn eine Datenerhebung ohne Mitwirkung des Betroffenen durch einen gesetzlichen Tatbestand gestattet oder zwingend vorausgesetzt wird. Gerade wenn es, wie im Rahmen der Web Service-Portale, verschiedene potenzielle datenerhebende Stellen gibt, ist der Grundsatz der Direkterhebung stets bei der Zulässigkeit der Datenerhebung zu prüfen.

\section{ee) System- und Selbstdatenschutz}

In Anbetracht dessen, dass die nationalen Gesetze nur begrenzt in der Lage sind, im weltweit abrufbaren Internet Datenschutz zu gewährleisten und auch die Kontrollressourcen der Datenschutzbehörden bescheiden sind, stellt der Selbstdatenschutz eine wichtige Ergänzung zur eigenen Sicherung und Wahrung des Rechts auf informationelle Selbstbestimmung dar. ${ }^{851}$ Nur durch zusätzliche eigene Maßnahmen des Betroffenen können die Schutzziele auch tatsächlich verwirklicht werden. ${ }^{852}$ Durch diese eigenen Maßnahmen, ${ }^{853}$ flankiert durch technische Maßnahmen, wie Anonymisierungs- oder Pseudonymisierungstechniken, ${ }^{854}$ soll der Betroffene in die Lage versetzt werden, die ihm erwünschte Verarbeitung seiner Daten zu ermöglichen und unzulässigen Datenumgang zu verhindern. 855

\footnotetext{
850 Gola/Klug, S. 51.

851 Schaar, Rn. 922; Roßnagel/Pfitzmann/Garstka, DuD 2001, 260; Roßnagel, NVZ 2006, 286; Roßnagel/Roßnagel; Hdb. DatenschutzR, Kap. 3.4 Rn. 1 ff.

852 Roßnagel/Roßnagel; Hdb. DatenschutzR, Kap. 3.4 Rn. 3; Roßnagel, NVZ 2006, 286.

853 S. ausführlich zu den technischen Möglichkeiten des Selbstdatenschutzes, wie Verschlüsselung, Zugangskontrollen, Verteidigungen der eigenen Daten, das Vermeiden von Datenspuren etc. Hansen/Krause, in: Roßnagel, Technik für Nutzer, S. 94 ff.
}

854 Büllesbach/Garstka, CR 2005, 724; Roßnagel, NVZ 2006, 286.

855 Roßnagel/Roßnagel; Hdb. DatenschutzR, Kap. 3.4 Rn. 35; Roßnagel, NVZ 2006, 286. 
Der Systemdatenschutz ergänzt das Mittel des Selbstdatenschutzes, um einen effektiven Datenschutz zu erreichen. ${ }^{856}$ Das Konzept des Systemdatenschutzes zielt darauf, die technischen Systeme so zu gestalten, dass das System nur zu der Datenverarbeitung in der Lage ist, zu der es rechtlich auch ermächtigt ist. ${ }^{857}$ Durch den Einsatz einer entsprechenden Technik und Organisation bereits in der Entwicklungsphase wird so Datenschutz gewährleistet beziehungsweise ermöglicht und Datenmissbrauch verhindert. 858 Die Konzepte des System- und Selbstdatenschutzes finden sich in der Regelung des $₫ 13$ Abs. 6 TMG, der besagt, dass dem Nutzer im Rahmen der technischen Zumutbarkeit eine anonyme oder pseudonyme Nutzung ermöglicht werden muss, sowie in \15 Abs. 3 und 5 S. 3 TMG. Bereits bei der Entwicklung von Web ServicePortalen ist das Konzept des Systemdatenschutzes zu berücksichtigen.

\section{ff) Datensicherheit}

Die Erhebung und Verwendung personenbezogener Daten birgt immer das Risiko unberechtigter Zugriffe Dritter auf die entsprechenden Daten. Darüber hinaus besteht das Risiko einer zweckwidrigen Verwendung der Daten sowie einer damit verbundenen Verletzung des Rechts auf informationelle Selbstbestimmung. So können Cookies unter Umständen von den Web ServiceAnbietern unbefugt ausgelesen werden, obwohl sie nur der Portalnutzung dienen. ${ }^{859}$ Auch für den Fall, dass die erhobenen Daten, sei es durch Portalbetreiber oder Web Service-Anbieter auf einem Server vorgehalten werden, besteht die Gefahr von Angriffen unbefugter Dritter, durch das Abfangen oder Ausspionieren ungesicherter Kennungen und Passwörter, 860 oder einer missbräuchlichen Verwendung der Daten durch den Portalbetreiber oder Web Service-Anbieter selbst. Ebenfalls könnte in einen eventuell vorhandenen Account, der gespeicherte Daten des Betroffenen enthält, durch einen Dritten unbefugt eingedrungen werden. Um personenbezogene Daten vor Missbrauch zu schützen und dadurch Datenschutz zu gewährleisten, ist es stets erforderlich den Umgang mit Daten sicher zu gestalten. ${ }^{861}$ Dementsprechend wurde

\footnotetext{
856 Hansen/Krause, in: Roßnagel, Technik für Nutzer, S. 92; Büllesbach/Garstka, CR 2005, 724.

857 Roßnagel, NVZ 2006, 286; Büllesbach/Garstka, CR 2005, 724; Hornung, S. 88 f.

858 Roßnagel/Pfitzmann/Garstka, DuD 2001, 255; Rasmussen, CR 2002, 38.

859 Dazu mehr unter B.IV.2.b)bb)i) Cookies.

860 S. Schaar, Rn. 66.

861 Roßnagel/Federrath/Pfitzmann, Hdb. DatenschutzR, Kap. 2.2 Rn. 5; Roßnagel/Ernestus, Hdb. DatenschutzR, Kap. 3.2 Rn. 2; Bizer, DuD 2007, 355.
} 
der Aspekt der Datensicherheit schon im Volkszählungsurteil des Bundesverfassungsgerichts mit dem Datenschutz in Verbindung gebracht. ${ }^{862}$ Die Datensicherheit umfasst dabei die Gesamtheit aller organisatorischen und technischen Regelungen sowie Maßnahmen, um die Vertraulichkeit, Integrität und Verfügbarkeit der Daten zu wahren. ${ }^{863}$

Das Schutzziel der Vertraulichkeit soll sicherstellen, dass nur berechtigte Personen mit einer bestimmten Aufgabe auf Daten Zugriff haben, die sie zur Erledigung dieser Aufgabe benötigen, und unbefugte Dritte indes weder von den Daten noch von den Kommunikationsprozessen Kenntnis erlangen. ${ }^{864}$ Integrität hat die Richtigkeit, Vollständigkeit und Widerspruchsfreiheit personenbezogener Daten innerhalb eines Sachzusammenhangs zum Ziel. ${ }^{865}$ Die Verfügbarkeit personenbezogener Daten wird gewährleistet, wenn dem Betroffenen alle Leistungen und Funktionalitäten zum richtigen Zeitpunkt zur Verfügung stehen; 866 dazu gehört auch die Datensicherung in Form eines Back-up. ${ }^{867}$ Die Regelung des $\int 9$ BDSG samt Anlage bezieht die Datensicherheit ausdrücklich in den Regelungsbereich des Datenschutzes mit ein.

IV. Datenschutzrechtliche Anforderungen des TMG und BDSG

Einfachgesetzlich regeln neben den Landesdatenschutzgesetzen, dem Telekommunikationsgesetz sowie zahlreichen Einzelbestimmungen in unterschiedlichen Gesetzen, 868 insbesondere das Telemediengesetz und das Bundesdatenschutzgesetz Befugnisse und Grenzen des Umgangs mit personenbezogenen Daten. Im Folgenden werden die Voraussetzungen und Grenzen des Umgangs mit personenbezogenen Daten bei Web Service-Portalen anhand des für den Untersuchungsgegenstand relevanten Telemedien- sowie des Bundesdatenschutzgesetzes untersucht.

\footnotetext{
862 BVerfGE 65, $1(44,57,59)$.

863 Simitis/Ernestus, If Rn. 2; Bizer, DuD 2007, 355; Roßnagel/Federrath/Pfitzmann, Hdb. DatenschutzR, Kap. 2.2 Rn. 6; Hornung, S. 300; Scholz; S. 381; Schultze-Melling, CR 2005, 73 f.; ferner Schmitz/Eckhardt, CR 2007, 176.

864 Schmitz, S. 121; Scholz, S. 381; Schultze-Melling, CR 2005, 73.

865 Scholz, S. 381; Schultre-Melling, CR 2005, 74.

866 Schultre-Melling, CR 2005, 74; Scholy, S. 382.

867 Roßnagel/Heibey, Hdb. DatenschutzR, Kap. 4.5 Rn. 20.

868 S. nur $\int 24 c$ KWG; $\int 73$ SGB V; $\int 140 \mathrm{a}$ Abs. 2 SGB V.
} 


\section{Personenberogene Daten}

Voraussetzung des Greifens datenschutzrechtlicher Schutzkonzepte ist zunächst einmal das Vorliegen von personenbezogenen Daten ( $\mathbb{S} 11$ Abs. 1 TMG, $\int 1$ BDSG). Handelt es sich nicht um personenbezogene Daten, so stellt der Datenumgang keinen Eingriff in das Recht auf informationelle Selbstbestimmung dar und die Daten können grundsätzlich ohne Berücksichtigung der datenschutzrechtlichen Vorschriften ausgetauscht und verwertet werden.

\section{a) Definition und Inhalt}

Personenbezogene Daten werden in $₫ 3$ Abs. 1 BDSG legaldefiniert. Danach sind personenbezogene Daten Einzelangaben über persönliche oder sachliche Verhältnisse einer bestimmten oder bestimmbaren Person. Einzelangaben sind Informationen, die sich auf eine bestimmte natürliche Person beziehen oder geeignet sind, einen Bezug zu ihr herzustellen. ${ }^{869}$ Eine Differenzierung zwischen sachlichen und persönlichen Verhältnissen ist letztlich nicht erforderlich, da die Grenzen fließend sind. ${ }^{870}$ Als Beispiele seien nur Name, Anschrift, Telefonnummer, E-Mail-Adresse, Familienstand, Geburtsdatum, Staatsangehörigkeit, Konfession, Beruf, Kundennummern, Passwörter und Nutzerkennungen genannt. ${ }^{871}$ Das bedeutet, dass auch alle Daten, die der Portalnutzer bei der Anmeldung - etwa Name, Kontaktdaten, Geburtstag und unter Umständen auch persönliche Präferenzen sowie Interessen - auf dem Portal angibt, grundsätzlich personenbezogene Daten sein können. Im Rahmen der Telemediendienstenutzung sind personenbezogene Daten regelmäßig auch alle Daten, die während der Nutzung entstehen und die den Bezug auf eine bestimmbare natürliche Person ermöglichen. ${ }^{872}$ Bestimmt ist eine dem Datum zugehörige Person dann, wenn sich unmittelbar aus den Angaben ein Rück-

869 Roßnagel, in: Roßnagel/Banzhaf/Grimm, Teil B S. 149; Scholz, S. 183; Schmitz, S. 91; Gola/Schomerus, \3 Rn. 3; Spindler/Schuster/Spindler/Nink, \11 TMG Rn. 5.

870 Roßnagel/Tinnefeld, Hdb. DatenschutzR, Kap. 4.1 Rn. 18; Simitis/Dammann, \ 3 Rn. 7; i.d.S. auch Roßnagel, in: Roßnagel/Banzhaf/Grimm, Teil B S. 149.

871 Simitis/Dammann, \& 3 Rn. 61 ff.; Roßnagel, in: Roßnagel/Banzhaf/Grimm, Teil B S. 149; weitere Beispiele für personenbezogene Daten speziell bei Telemedien s. Spindler/Schuster/Spindler/Nink, \$11 TMG Rn. 6 ff.

872 Spindler/Schmitz/Geis/Schmitz, $\$ 1$ TDDSG Rn. 24; Scholv, S. 184 f.; Spindler/Schuster/Spindler/Nink, \$11 TMG Rn. 6. 
schluss auf die Person ergibt. ${ }^{873}$ Wann eine Person bestimmbar ist, ist seit der Entscheidung des AG Berlin Mitte, ${ }^{874}$ das im Herbst 2007 alle IP-Adressen als personenbezogene Daten einordnete, umstritten. Während die bis dahin noch vorherrschende Auffassung den Personenbezug relativ bestimmt, ${ }^{875}$ will die vordringende neuere Auffassung den Personenbezug objektiv bestimmen. ${ }^{876}$ Bislang stehen sich in der Rechtsprechung lediglich die Auffassung des $A G$ Berlin Mitte sowie die des $A G$ München, das in einem orbiter dictum explizit in gegensätzlicher Auffassung zu der Rspr. des $A G$ Berlin Mitte, den Personenbezug relativ bestimmte, 877 gegenüber. Instanzgerichtliche Entscheidungen existieren noch nicht. ${ }^{878}$ Bei der relativen Beurteilung des Personenbezugs kommt es für die Bestimmbarkeit auf die Kenntnisse, Mittel und Möglichkeiten (dementsprechend ein etwaiges Zusatzwissen) der speichernden Stelle an. Nur wenn diese in der Lage ist, die Zuordnung zu einer Person vorzunehmen, liegt auch Personenbezug vor. Die Theorie der Relativität des Personenbezugs hat zur Konsequenz, dass dieselben Daten für den einen anonym (d.h. nicht personenbezogen) und für den anderen der betroffenen Person zuordenbar sind und die Person daher bestimmbar ist (personenbezogen). ${ }^{879}$ Dagegen wollen die Vertreter der objektiven Bestimmung des Personenbezugs bereits die theoretische Möglichkeit der Bestimmbarkeit einer Person genügen lassen, um von einem personenbezogenen Datum auszugehen, auch wenn die Person nur durch einen Dritten bestimmt werden kann und dieser das notwendige Zusatzwissen nicht an die verarbeitende Stelle weitergibt. Der Personenbezug soll

873 Simitis/Dammann, $\int 3$ Rn. 21; Roßnagel/Tinnefeld, Hdb. DatenschutzR, Kap. 4.1 Rn. 20; Roßnagel, in: Roßnagel/Banzhaf/Grimm, Teil B S. 149.

874 AG Berlin Mitte, K\&R 2007, 600 f.

875 Simitis/Dammann, \ 3 Rn. 22 ff.; Gola/Schomerus, \ 3 Rn. 10; Roßnagel/Tinnefeld, Hdb. DatenschutzR, Kap. 4.1 Rn. 21; Roßnagel/Bär, Hdb. DatenschutzR, Kap. 5.7 Rn. 18; Scholv, S. 185; Meyerdierks, MMR 2009, 10; Härting, ITRB 2009, 37; Voigt, MMR 2009, 379; Köcher, Anm. zu LG Berlin, abgedruckt in: MMR 2007, 800 (799), MMR 2007, 801; Eckhardt, Anm. zu LG Berlin abgedruckt in: K\&R 2007, 601, K\&R 2007, 602 ff.; Roßßnagel/Schols, MMR 2000, 723; AG München, K\&R 2008, 767 f.; LG Frankenthal, Beschl. v. 21.05.2008 - 6 O 156/08, Rn. 15.

876 AG Berlin Mitte, K\&R 2007, 600 (601); Pablen-Brandt, K\&R 2008, 289; i.d.S., wenn auch nicht explizit Art. 29-Datenschutzgruppe, Stellungnahme 4/2007, S. 14.

877 AG München, K\&R 2008, 767 f.

878 Die Berufung beim LG Berlin, MMR 2007, 799 f. bestätigte die Auffassung des AG Berlin Mitte, K\&R 2007, 600 ff. entgegen der Auffassung der Vertreter der objektiven Auslegung nicht, da sich das LG lediglich mit einer prozessualen Frage beschäftigte und den Personenbezug von IP-Adressen gar nicht untersuchte.

879 Gola/Schomerus, $\ 3$ Rn. 10; Simitis/Dammann, $\ 3$ Rn. 35. 
daher ohne Berücksichtigung der Möglichkeiten der verarbeitenden Stelle bestimmt werden.

Aus dem Wortlaut des $₫ 3$ Abs. 1 BDSG ergeben sich weder für die einen noch die andere Auffassung Anhaltspunkte im Wortlaut. Auch Art. 2 a) der EG-Datenschutzrichtlinie, wonach „personenbezogene Daten“ alle Informationen über eine bestimmte oder bestimmbare natürliche Person (,,betroffene Person")" 880 sein sollen, schafft diesbezüglich keine Klarheit. Als bestimmbar sieht die EG-Datenschutzrichtlinie eine Person an, „die direkt oder indirekt identifiziert werden kann, insbesondere durch Zuordnung zu einer Kennnummer oder zu einem oder mehreren spezifischen Elementen, die Ausdruck ihrer physischen, physiologischen, psychischen, wirtschaftlichen, kulturellen oder sozialen Identität sind“. .881 Zur Bestimmung der Bestimmbarkeit „sollen alle Mittel berücksichtigt werden, die vernünftigerweise entweder von dem Verantwortlichen für die Verarbeitung oder von einem Dritten eingesetzt werden könnten, um die betreffende Person zu bestimmen." 882 Bestimmt man den Personenbezug rein objektiv, denn dies wäre die logische Konsequenz der Vertreter der objektiven Theorie, würde dies zu zahlreichen Nachteilen führen. Denn nicht geklärt ist, wo die Grenzen bei der Einbeziehung von Drittwissen zu ziehen sind. In der Konsequenz würde dies bedeuten, dass jedes Datum, bei dem auch nur irgendein beliebiger Dritter die dahinter stehende Person durch Zusatzwissen oder Schlüsseldaten bestimmen könnte, für jede speichernde Stelle ein personenbezogenes Datum darstellen würde, das regelmäßig - mangels Erlaubnistatbestand - rechtswidrig erhoben und gespeichert würde. ${ }^{883}$ Ohne Wissen der Daten verarbeitenden Stellen, hätte es derjenige, der die Schlüsseldaten besitzt, in der Hand, die Rechtswidrigkeit der Speicherung entfallen zu lassen, indem er die Schlüsseldaten löscht. Ob und wie viele Schlüsseldatenbesitzer existieren, ist für Daten verarbeitende Stelle meist gar nicht ersichtlich und somit auch nicht, ob sie die Daten erheben und speichern dürfen oder nicht. Letztlich führt die objektive Auslegung des Personenbezugs

880 Erwägungsgrund 26 der Richtlinie 95/46/EG des Europäischen Parlaments und des Rates vom 24. Oktober 1995 zum Schutz natürlicher Personen bei der Verarbeitung personenbezogener Daten und zum freien Datenverkehr, ABl. L 281 vom 23.11.1995, S. 31 ff.

881 Erwägungsgrund 26 der Richtlinie 95/46/EG des Europäischen Parlaments und des Rates vom 24. Oktober 1995 zum Schutz natürlicher Personen bei der Verarbeitung personenbezogener Daten und zum freien Datenverkehr, ABl. L 281 vom 23.11.1995, S. 31 ff.

882 Erwägungsgrund 26 der Richtlinie 95/46/EG des Europäischen Parlaments und des Rates vom 24. Oktober 1995 zum Schutz natürlicher Personen bei der Verarbeitung personenbezogener Daten und zum freien Datenverkehr, ABl. L 281 vom 23.11.1995, S. 31 ff. 
für die Daten verarbeitende Stelle zu großen Rechtsunsicherheiten, währenddessen bei Anwendung der Relativität des Personenbezugs die Daten verarbeitende Stelle für sich klar bestimmen kann, welche Daten für sie personenbezogen sind und welche nicht. Daher ist der Personenbezug richtigerweise relativ zu bestimmen und die Daten zur Bestimmung des Personenbezugs immer in Relation zu dem Datenverwender betrachtet werden.

Die Relativität des Personenbezugs zeigt sich auch bei Web Service-Portalen. Für den Portalbetreiber können daher unter einem Nickname angefallene Daten, sofern eine Registrierungspflicht auf dem Portal besteht, ohne besondere weitere Zusatzinformationen zuordenbar sein. Denn speichernde Stelle der Anmeldedaten ist der Portalbetreiber, so dass dieser die unter dem Nickname angefallenen Daten unproblematisch den Anmeldedaten des NicknameInhabers zuordnen kann. Dagegen werden diese Daten für im Portal integrierte Web Service-Anbieter erst dann zuordenbar, wenn ihnen - beispielsweise durch Übermittlung der Anmeldedaten - Zusatzinformationen über den Inhaber des Nicknames verschafft werden.

b) Anonyme und pseudonyme Daten

Keinen Personenbezug weisen anonyme Daten auf. Nach der Legaldefinition des $₫ 3$ Abs. 6 BDSG versteht man unter Anonymisieren das derartige Verändern personenbezogener Daten, „dass die Einzelangaben über persönliche oder sachliche Verhältnisse nicht mehr oder nur mit einem unverhältnismäßig großen Aufwand an Zeit, Kosten und Arbeitskraft einer bestimmten oder bestimmbaren natürlichen Person zugeordnet werden können“. Anonyme Daten zeichnen sich dadurch aus, dass die Wahrscheinlichkeit der Zuordenbarkeit zu einer Person nach der Lebenserfahrung oder dem Stand der Wissenschaft praktisch ausscheidet. ${ }^{884} \mathrm{Da}$ es sich bei Web ServicePortalen, im Falle einer Registrierungspflicht, um geschlossene Systeme handelt, bei denen alle beteiligten Akteure, je nach Ausgestaltung des Systems, Zugriff auf die Anmeldedaten, und somit die Identifizierungsdaten, haben können, liegen für die beteiligten Akteure regelmäßig keine anonymen Daten vor.

884 Roßnagel, in: Roßnagel/Banzhaf/Grimm, Teil B S. 150; Simitis/Dammann, 33 Rn. 196; Roßnagel/Scholi, MMR 2000, 723. 
Pseudonyme Daten 885 dagegen sind ein klassischer Fall der Relativität des Personenbezugs. Das heißt, pseudonyme Daten sind lediglich für denjenigen, der die Zuordnungsregel kennt beziehungsweise für den sich aus den gesammelten Daten oder aus anderen Informationen ein Schluss auf den Betroffenen ergibt, ${ }^{886}$ personenbezogene Daten, für alle anderen hingegen nicht, so dass sie in letzterem Fall grundsätzlich nicht den Datenschutzgesetzen unterfallen. ${ }^{887}$ Sollte das Web Service-Portal derart ausgestaltet sein, dass der Portalnutzer - nach erfolgter Registrierung - unter einem Nickname agiert, stellt dies für den Portalbetreiber selbst kein Hindernis zur Zuordnung der unter dem Nickname angefallenen Daten dar. Denn durch die Zugriffsmöglichkeit auf die hinterlegten Anmeldedaten, kann der Portalbetreiber die angefallenen Daten unmittelbar den Registrierungsdaten zuordnen und somit den Personenbezug herstellen. Die Web Service-Anbieter können dem Pseudonym spätestens dann den entsprechenden Portalnutzer zuordnen, wenn sie in irgendeiner Art und Weise, zum Beispiel durch die Übermittlung mit Hilfe ihrer Web Services, Zugriff auf die Registrierungsdaten des entsprechenden Portalnutzers erhalten.

\section{c) Zusammenfassung}

Da der Personenbezug im Falle einer Registrierungspflicht und Portalnutzung unter Nickname bei dem Portalbetreiber ohne Zusatzwissen und bei den Web Service-Anbietern durch die Übermittlung der Registrierungsdaten, zum Beispiel mit Hilfe der Web Services automatisiert, hergestellt werden kann, handelt es sich bei allen während der Portalnutzung anfallenden Daten, einschließlich den Registrierungsdaten für Portalbetreiber und Web Service-Anbieter regelmäßig um personenbezogene Daten, die den Anwendungsbereich der Datenschutzgesetze, speziell der $\int \mathbb{1 1} \mathrm{ff}$. TMG eröffnen, und somit auch beachtet werden müssen. Daneben müssen die allgemeinen Datenschutzgrundsätze, die zum Teil auch in den Datenschutzgesetzen konkretisiert wurden, beachtet werden.

885 Das Pseudonymisieren von Daten wird in $\ 3$ Abs. 6a BDSG legaldefiniert: „Pseudonymisieren ist das Ersetzen des Namens und anderer Identifikationsmerkmale durch ein Kennzeichen zu dem Zweck, die Bestimmung des Betroffenen auszuschließen oder wesentlich zu erschweren.“ 


\section{Datenverarbeitungsbefugnisse und Grenzen der Datenverarbeitung}

Eingriffe in das Recht auf informationelle Selbstbestimmung durch den Umgang mit personenbezogenen Daten bedürfen einer Rechtfertigung durch einen gesetzlichen Tatbestand oder eine Einwilligung des Betroffenen. ${ }^{888}$ Die gesetzlichen Ausprägungen greifen diesen Grundsatz des Eingriffs der einer Rechtfertigung bedarf auf, indem sie die entsprechenden Datenschutzvorschriften als „Verbot mit Erlaubnisvorbehalt" ausgestaltet haben, siehe nur $\$ 12$ Abs. 1, 1. Alt. TMG, $\ 4$ Abs. 1, 1. Alt. BDSG. ${ }^{889}$

Daher kann der Umgang mit personenbezogenen Daten in den gesetzlich bestimmten Fällen zulässig sein und durch Portalbetreiber und die Web Service-Anbieter im Rahmen der jeweiligen Vorschrift, auch automatisiert mittels Web Service, erfolgen. Das deutsche Datenschutzrecht ist nicht nur im Bundesdatenschutzgesetz normiert, sondern verteilt sich auf zahlreiche Spezialgesetze. ${ }^{890}$ Das Bundesdatenschutzgesetz bildet dabei das lex generalis, dass, wie $\int 1$ Abs. 3 S. 1 BDSG durch das Wort „soweit“ expressis verbis festlegt, immer nur dann herangezogen wird, wenn keine spezielleren Vorschriften greifen. Im Bereich des E-Commerce spielen die Vorschriften des Telemediengesetzes eine wichtige Rolle. Welches Gesetz Anwendung findet, bestimmt sich in erster Linie nach der Art der Daten, wobei aber auch die Einordnung der beteiligten Akteure von Relevanz ist. Da die Art der Daten immer anhand des Einzelfalls untersucht und kategorisiert werden muss, soll hier zunächst eine Einordnung der Akteure erfolgen, ${ }^{891}$ um den groben rechtlichen Rahmen abstecken zu können. Anschließend erfolgt die Untersuchung der einzelnen Erlaubnistatbestände anhand der konkreten Datenart. ${ }^{892}$

\section{a) Web Service-Portale als Telemediendiensteanbieter}

Der Web Service-Anbieter, dessen Web Service im Portal integriert ist, unterscheidet sich nicht vom Web Service-Anbieter in der Serviceorientierten Architektur. Es kommt auch hier entscheidend auf die erbrachte Leistung an und die liegt, wie auch im Rahmen der Serviceorientierten Architektur, in der Ser-

\footnotetext{
888 S. bereits B.III.3.b) Eingriff.

889 Schaar, Rn. 120; Spindler/Schuster/Spindler/Nink, \12 TMG Rn. 2; so auch - allerdings kritisch zur Terminologie - Simitis/Walz, 4 Rn. 3.

890 Dabei seien nur beispielhaft die $\iint 11 \mathrm{ff}$. TMG, $\int \mathbb{S} 91 \mathrm{ff}$. TKG, die Landesdatenschutzgesetze und $\int \$ 284 \mathrm{ff}$. SGB V genannt.

891 S. B.IV.2.a)Web Service-Portale als Telemediendiensteanbieter.

892 B.IV.2.b)Erlaubnistatbestände.
} 
viceleistung, der Übermittlung von Inhalten. ${ }^{893}$ Daher ist der Web ServiceAnbieter gegenüber dem Portalbetreiber sowie dem Portalnutzer als Telemediendiensteanbieter im Sinne des $₫ 2$ Nr. 1 TMG einzuordnen. Der Portalbetreiber wird häufig über die Web Services der Web Service-Anbieter hinaus auch eigene Inhalte und Services anbieten. Daher ist der Portalbetreiber im Sinne des $\$ 2$ Nr. 1 TMG sowohl aufgrund des Bereithaltens eigener Telemedien wie auch des Bereithaltens fremder Telemedien (der Web Services der Web Service-Anbieter) als Telemediendiensteanbieter zu qualifizieren. 894 Dementsprechend finden die $\iint 11 \mathrm{ff}$. TMG als datenschutzrechtliche Regelungen auf Web Service-Portale Anwendung.

\section{b) Erlaubnistatbestände}

Das Telemediengesetz differenziert bei der Zulässigkeit der Datenverarbeitungsvorgänge zwischen unterschiedlichen Erlaubnistatbeständen für die Verarbeitung personenbezogener Daten. Können sich Portalbetreiber und Web Service-Anbieter nicht auf einen gesetzlichen Erlaubnistatbestand berufen, so ist eine Datenerhebung und -verarbeitung nur dann zulässig, wenn der Betroffene in die entsprechende Maßnahme eingewilligt hat, $\$ 12$ Abs. 1, 2. Alt. TMG. Eine entsprechende Regelung existiert in $₫ 4$ Abs. 1 BDSG, der bei der Datenerhebung, -nutzung und -verarbeitung von Inhaltsdaten ${ }^{895}$ Anwendung findet.

Im Rahmen der Erlaubnistatbestände ist zwischen Bestands-, Nutzungs- und Abrechnungsdaten zu differenzieren. Konkret beziehen sich die Erlaubnistatbestände auf die Verarbeitung von Bestands-, Nutzungs- und Abrechnungsdaten unter den Voraussetzungen der $\$ ₫ 14,15$ TMG. Keine ausdrückliche gesetzliche Erwähnung finden die so genannten Inhaltsdaten. 896

\section{aa) Bestandsdaten}

\14 Abs. 1 TMG enthält eine Legaldefinition der Bestandsdaten. Danach handelt es sich bei Bestandsdaten um personenbezogene Daten, die „für die Begründung, inhaltliche Ausgestaltung oder Änderung eines Vertragsverhältnisses zwischen dem Diensteanbieter und dem Nutzer über die Nutzung von

\footnotetext{
893 S. ausführlicher A.II.1 Web Service-Anbieter.

894 Zur Einordnung als Telemediendiensteanbieter bereits ausführlicher A.II Einordnung der Dienste.

895 S. B.IV.2.b)cc)i) Einordnung.

896 S. zur Einordnung B.IV.2.b)cc) Inhaltsdaten.
} 
Telemedien erforderlich sind“. Dementsprechend dürfen Daten, die für die Abwicklung des Vertragsverhältnisses erforderlich sind, vom Web ServiceAnbieter ohne Einwilligung des Nutzers im Sinne des $₫ 14$ Abs. 1 TMG erhoben und verwendet werden. Für die Qualifikation als Bestandsdatum genügt bereits die abstrakte Geeignetheit zur Begründung, Ausgestaltung und Veränderung eines Vertrags. ${ }^{897}$ Dies allein genügt aber nicht für eine rechtmäßige Erhebung und Verarbeitung der Daten, denn der Begriff der „Erforderlichkeit" muss eng auslegt werden. Erforderlichkeit liegt daher nur vor, wenn die personenbezogenen Daten für die Gestaltung des Vertragsverhältnisses unerlässlich sind. ${ }^{898}$ Dies ergibt sich bereits aus dem allgemeinen Gebot der Datensparsamkeit in $\int 3$ a BDSG. ${ }^{899}$ Entscheidend ist dabei, dass der Telemediendienst Leistungsgegenstand des Vertrags und nicht nur Anbahnungs- oder Abwicklungsmedium ist. ${ }^{900}$ Sonstige personenbezogene Daten, die bei elektronischen Geschäften anfallen und dem Begriff der Bestandsdaten entsprechen, aber bei denen der Telemediendienst nicht den Leistungsgegenstand des Vertrags darstellt, können unter besonderen Voraussetzungen unter den Anwendungsbereich des $₫ 15$ TMG oder den des $₫ 28$ Abs. 1 Nr. 1 BDSG fallen, 901 der die Verarbeitung erlaubt, „wenn es für die Begründung, Durchführung oder Beendigung eines rechtsgeschäftlichen oder rechtsgeschäftsähnlichen Schuldverhältnisses mit dem Betroffenen erforderlich ist" und dadurch die Fälle auffängt, die nicht vom Regelungsbereich des Telemediengesetzes erfasst werden.

Der Begriff der Bestandsdaten in $\$ 14$ Abs. 1 TMG setzt demnach das Vorliegen eines Vertragsverhältnisses über die Nutzung von Telemedien voraus. ${ }^{902}$

897 Scholv, S. 232.

898 Roßnagel/Dix, RMD, Teil 3, \5 TDDSG Rn. 36; Roßnagel/Roßnagel, Hdb. DatenschutzR, Kap. 7.9 Rn. 69; Scbmitr, in: Hoeren/Sieber, Hdb. MultimediaR, Teil 16.4 Rn. 111; Schaar, Rn. 389; Scholv, S. 233; Stadler, S. 167; Zscherpe, K\&R 2005, 266; so auch Begr. RegE BT-Drs. 13/7385, S. 24. Anders Stellungnahme BR BT-Drs. 13/7385, S. 55, die nur von „benötigt werden" sprechen.

899 Anders kann es sich aber dann verhalten, wenn es sich um eine Parteivereinbarung handelt und der Nutzer in der Lage ist, zu überblicken, wie umfangreich die Datenverarbeitung erfolgen soll: in diesem Fall muss der Privatautonomie gegenüber dem Kriterium der Datensparsamkeit Vorrang gewährt werden, s. Spindler/Schmitz/Geis/Schmitz, \5 TDDSG Rn. 5 ff.; Spindler/Schuster/Spindler/Nink, \14 TMG Rn. 4.

900 Roßnagel/Roßnagel, Hdb. DatenschutzR, Kap. 7.9 Rn. 70; jurisPK-Internetrecht/Heckmann, Kap. 1.14 Rn. 5.

901 Gola/Schomerus, $\ 28$ Rn. 13 ff.; Simitis/Simitis, $\ 28$ Rn. 75 ff.; jurisPK-Internetrecht/Heckmann, Kap. 1.14 Rn. 6.

902 jurisPK-Internetrecht/Heckmann, Kap. 1.14 Rn. 5. 
Welche personenbezogenen Daten dabei konkret als Bestandsdaten in Betracht kommen, hängt von dem Zweck des jeweiligen Vertragsverhältnisses $\mathrm{ab}$, daher hat der Gesetzgeber auch von einer katalogartigen Aufzählung von Bestandsdaten abgesehen. ${ }^{903}$ Typische, praktisch relevante Arten von Bestandsdaten können Name, Anschrift, Rufnummer, E-Mail, IP-Adresse, Kenn- und Passwörter, Zahlungsart und -daten, Geburtsdatum, Zuordnung der vereinbarten Leistungsmerkmale oder -beschränkungen, User-ID etc. sein. ${ }^{904}$ Dabei muss es sich nicht zwangsläufig um ein entgeltliches Verhältnis handeln - obwohl ein solches ein starkes Indiz für das Vorliegen eines Vertrags darstellt -, auch die unentgeltliche Nutzung eines elektronischen Warenangebots kann ein Vertragsverhältnis begründen, wenn eine Anmeldung oder Registrierung verlangt wird. ${ }^{905}$ Anhaltspunkte für ein solches Vertragsverhältnis bestehen, wenn die Anmeldung mit besonderen Bestimmungen für die Nutzung des Dienstes verknüpft wird oder dem Kunden besondere Dienste, wie die Erzeugung persönlicher Nutzungsumgebungen, die Teilnahme an Bonussystemen oder gesonderte Hinweise auf Sonderangebote, offeriert werden. 906

In zeitlicher Hinsicht gilt die Erlaubnis der Verarbeitung der Bestandsdaten nur für den Abschnitt der Vertragsdauer. Ist das Vertragsverhältnis beendet und bestehen keine nachvertraglichen Ansprüche mehr, so müssen die Daten gelöscht werden. ${ }^{907}$ Das ergibt sich aus $\ 35$ Abs. 2 BDSG, der mangels Spezialregelungen zur Löschung im Telemediengesetz greift. 908

Begr. RegE BT-Drs. 13/7385, S. 24.

Scholz, S. 233; Spindler/Schuster/Spindler/Nink, \14 TMG Rn. 3; Roßnagel/Dix, RMD, Teil 3, $\int 5$ TDDSG Rn. 29; Schmitz, in: Hoeren/Sieber, Hdb. MultimediaR, Teil 16.4 Rn. 113; jurisPKInternetrecht/Heckmann, Kap. 1.14 Rn. 14; Biæ̌er, DuD 2002, 429; ferner Roßnagel, in: Roßnagel/Banzhaf/Grimm, Teil B S. 166; Zscherpe, K\&R 2005, 266; für IP-Adressen LG Berlin, CR 2006, 418 (420).

05 Roßnagel, in: Roßnagel/Banzhaf/Grimm, Teil B S. 167; jurisPK-Internetrecht/Heckmann, Kap. 1.14 Rn. 7.

S. dazu ausführlicher Scholv, S. 235.

Roßnagel, in: Roßnagel/Banzhaf/Grimm, Teil B S. 167 f.; Scholz, S. 238; OLG Bamberg, MMR 2006, 481 (482) .

OLG Bamberg, MMR 2006, 481 (482); Scholz, S. 238. Offen bzgl. der Frage, ob sich die Pflicht zur Löschung der Daten direkt aus TMG ergibt oder aus \35 BDSG: jurisPKInternetrecht/Heckmann, Kap. 1.14 Rn. 13. 


\section{i) Bestandsdaten bei der Portalnutzung}

Die Anmeldung - unabhängig von den weiteren Tätigkeiten - für die Nutzung des Web Service-Portals begründet einen entsprechenden Nutzungsvertrag zwischen Portalbetreiber und Portalnutzer. ${ }^{909}$ Darin wird in aller Regel - meist durch die Allgemeinen Geschäftsbedingungen des Portalbetreibers - geregelt, welche Rahmenbedingungen für die Nutzung des Portals gelten und unter Umständen auch, durch welche Handlungen Verträge zustanden kommen und wie diese abgewickelt werden, insbesondere bei der Nutzung von externen Dienstleistungen via Web Services. Darüber hinaus ist es Sinn und Zweck derartiger Portale, durch besondere Zusatzleistungen, wie das Nutzen externer Dienste auf dem Portal, oder das Offerieren gezielter, auf die Interessen abgestimmter, Werbung sowie der Gestaltung einer persönlichen Nutzerumgebung, eine besondere Bindung an das Portal zu schaffen. Selbst im Falle eines unentgeltlichen Nutzungsverhältnisses handelt es sich daher zumindest um einen Auftrag nach $\ 662$ BGB. Sollte bereits der Nutzungsvertrag entgeltlich ausgestaltet sein, so steht im Vordergrund des Vertrags, dass die Nutzungsvoraussetzungen durch den Portalbetreiber geschaffen werden. Somit steht in diesem Fall eine dienstvertragliche Komponente im Vordergrund. Ein entgeltlicher Portalnutzungsvertrag ist demnach regelmäßig als Dienstvertrag im Sinne des $₫ 611$ BGB zu qualifizieren. In beiden Fällen bildet die Nutzung des Portals den Leistungsgegenstand des Vertrags, so dass die Anmeldung als entscheidender Teil des Vertragsschlusses über die Portalnutzung Bestandsdaten enthalten kann. Entsprechend sind die für den Nutzungsvertrag unerlässlichen personenbezogenen Daten in erster Linie Name und Anschrift, um die Identität des Portalnutzers feststellen zu können sowie gegebenenfalls das Geburtsdatum, ${ }^{910}$ um zumindest einen irgendwie gearteten Anhaltspunkt für eine gegebenenfalls gewünschte Volljährigkeit zu haben. ${ }^{911}$ Darüber hinaus sind zu einer beschleunigten Kontaktaufnahme auch E-Mail-Adresse und Rufnummer erforderlich, sowie Teilnehmerkennung und Passwort als Zugangsberechtigung für die Portalnutzung, sofern das Portal derart ausgestaltet ist, dass es einer solchen Zugangsrestriktion unterliegt. ${ }^{912}$ Was die anderen personenbe-

909 S. dazu auch die Ausführungen bei Wiebe, in: Spindler/Wiebe, Kap. 4 Rn. 16 zum Teilnahmevertrag bei Internetauktionshäusern zwischen Plattformbetreiber und Bieter.

910 Scholz, S. 234. Die Möglichkeit einen falschen Namen und eine andere Anschrift anzugeben, spielt für die Beurteilung der Erforderlichkeit keine Rolle.

911 Ist eine Altersverifikation aus Jugendschutzgesichtspunkten von Gesetzes wegen erforderlich, wie bspw. in $\ 4$ Abs. 2 S. 2 JMStV, so genügt die Angabe eines Geburtsdatums freilich nicht.

912 Scholz, S. 235. 
zogenen Daten, die häufig in Anmeldeformularen als Pflichtangaben gemacht werden müssen, anbelangt, so richtet sich dies danach, wie die Portalnutzung konkret ausgestaltet ist. Ist die Nutzung des Portals daher entgeltlich, so dürfen auch Daten zur Abwicklung der Zahlung als erforderliche Bestandsdaten erhoben werden. Handelt es sich indes um eine unentgeltliche Portalnutzung, so sind die Angaben zur Zahlungsabwicklung und den Zahlungsmodalitäten keineswegs Daten, die für die Gestaltung des Telemediendienstevertrags unerlässlich sind. ${ }^{913}$ Sonstige Daten, wie Hobbys, Interessen, Familienstand, Beruf und ähnliches sind für die Begründung, Ausgestaltung oder Änderung des Vertragsverhältnisses über die Nutzung des Portals regelmäßig nicht erforderlich. Die Zulässigkeit der Datenverarbeitung kann daher nicht aus $\int 14$ Abs. 1 TMG erfolgen. Etwas anderes kann allerdings dann gelten, wenn das Portal beispielsweise nur bestimmten Berufsgruppen oder einer bestimmten Interessengruppe zugänglich sein soll. ${ }^{914}$ In diesem Fall können die jeweils relevanten sonstigen Daten Bestandsdaten im Sinne des $\$ 14$ Abs. 1 TMG sein.

\section{ii) Bestandsdaten bei der Web Service-Nutzung}

Eine andere Problematik ist die Feststellung der Bestandsdaten im Rahmen des Zugriffs auf die beim Portal hinterlegten Nutzerdaten durch Web Services für die Nutzung der im Portal integrierten Dienste der Web Service-Anbieter durch den Portalnutzer. Dafür ist entscheidend, wer bei der Web ServiceNutzung denn überhaupt Vertragspartner des Portalnutzers wird und ob das Portal eine Registrierungspflicht vorsieht. Stellt die Web Service-Nutzung nämlich lediglich eine Funktion des registrierungspflichtigen Portals dar, die nur zwischen Web Service-Anbieter und Portalbetreiber abgerechnet wird, nicht aber gesondert zwischen Web Service-Anbieter und Portalnutzer, so sind die mittels des Web Service übermittelten Anmeldedaten für die Begründung oder Durchführung des Nutzungsvertrags nicht unerlässlich. Denn bereits die tatsächliche Nutzung des Web Service zeigt, dass es sich um einen angemeldeten, gegebenenfalls sogar zahlenden, Portalnutzer handelt, dessen Identifizierungsdaten beim Portalbetreiber hinterlegt sind. Eine Abrechnung kann durch das Portal, als Vertragspartner der Web Service-Anbieter, gewährleistet werden. In diesem Fall hat daher eine Übermittlung oder Weitergabe der Regist-

913 So auch Scholz, S. 235.

914 Z.B. könnte es sich um ein reines Portal für Biologen handeln oder ein Portal für Freizeithandballer. Dann ist es bereits für die Zulassung zur Portalnutzung erforderlich zu wissen, welchen Beruf bzw. welches Hobby der potenzielle Nutzer ausübt. 
rierungsdaten des Portalnutzers zu unterbleiben, die Übermittlung ist nicht durch $\$ 14$ Abs. 1 TMG gedeckt.

Anders könnte es sich hingegen verhalten, wenn zwischen Web ServiceAnbietern und Portalnutzern jeweils eigene Verträge - je nach Art der Leistung beispielsweise Dienst-, Kauf-, oder Werkverträge - geschlossen werden. In diesem Fall können die mittels des Web Service übermittelten Daten für die Nutzung des Web Service, als Telemediendienst, hinsichtlich des Vertragsschlusses über die Dienstenutzung durchaus erforderlich sein, wobei sich die Erforderlichkeit auf personenbezogene Daten, die die Identifizierung des Portalnutzers und die Abrechnungsmodalitäten betreffen, beschränkt. Jedoch ist auch der Grundsatz der Direkterhebung nach $\ 4$ Abs. 2 BDSG zu beachten. ${ }^{115}$ Zwar ist $\int 14$ Abs. 1 TMG eine Rechtsvorschrift im Sinne des $₫ 4$ Abs. 2 S. 2 Nr. 1 BDSG, jedoch gestattet $\ 14$ Abs. 1 TMG lediglich die Erhebung, Verarbeitung und Nutzung von Daten. Allerdings definiert $\int 3$ Abs. 4 S. 1 BDSG Verarbeiten als Speichern, Verändern, Übermitteln, Sperren und Löschen personenbezogener Daten. Übermitteln wird wiederum nach $₫ 3$ Abs. 4 S. 2 Nr. 3 BDSG als die Weitergabe von Daten an Dritte (lit. a)) oder als das Einsehen oder Abrufen von zur Einsicht oder zum Abruf bereitgehaltener Daten durch Dritte (lit. b)) definiert. Unter den Begriff der Übermittlung und somit auch den Verarbeitungsbegriff des $\ 14$ Abs. 1 TMG fällt dementsprechend sowohl das aktive Senden der Daten durch den Portalbetreiber an einen Dritten als auch der Zugriff beispielsweise mit Hilfe von Web Services der Web Service-Anbieter auf die entsprechenden Daten des Portalnutzers, ${ }^{916}$ die dieser beim Portalbetreiber hinterlegt hat. Wer Dritter im Sinne des $\int 3$ Abs. 4 S. 2 Nr. 3 BDSG ist definiert $₫ 3$ Abs. 8 S. 2 und 3 BDSG. Danach ist Dritter jede Person oder Stelle außerhalb der verantwortlichen Stelle. Keine Dritten sind der Betroffene selbst sowie Personen und Stellen, die im Inland, in einem anderen Mitgliedstaat der Europäischen Union oder in einem anderen Vertragsstaat des Abkommens über den Europäischen Wirtschaftsraum personenbezogene Daten im Auftrag erheben, verarbeiten oder nutzen. Die im Portal integrierten Web Service-Anbieter lassen sich als solche Dritten qualifizieren, da sie weder verantwortliche Stelle ${ }^{917}$ oder Betroffener sind noch im

915 S. dazu bereits B.III.3.c)dd) Grundsatz der Direkterhebung.

916 So zu automatisierten Abrufverfahren generell Simitis/Dammann, \3 Rn. 148.

917 Verantwortliche Stelle ist nach $\ 3$ Abs. 7 BDSG jede Person oder Stelle, die personenbezogene Daten für sich selbst erhebt, verarbeitet oder nutzt oder dies durch andere im Auftrag vornehmen lässt. Hinsichtlich der Anmeldedaten ist verantwortliche Stelle daher nur der Portalbetreiber, nicht indes die Web Service-Anbieter. Etwas anderes gilt nur dann, wenn die 
Auftrag Daten verwenden. Daher sieht $₫ 14$ Abs. 1 TMG auch die Übermittlung der erforderlichen Bestandsdaten an die Web Service-Anbieter vor, so dass die Ausnahme des Grundsatzes der Direkterhebung nach $₫ 4$ Abs. 2 S. 2 Nr. 1 BDSG greift.

Die Erforderlichkeit beschränkt sich auf die Bestandsdaten, die für eine Identifizierung des Portalnutzers und etwaige Abrechnungsmodalitäten benötigt werden. Keine (erforderlichen) Bestandsdaten sind indes die Informationen, die der Portalnutzer bei der Web Service-Nutzung als Suchanfrage stellt oder mittels des Web Service übermittelt.

bb) Nutzungs- und Abrechnungsdaten

\15 Abs. 1 S. 1 TMG erlaubt dem Diensteanbieter, personenbezogene Daten eines Nutzers zu erheben und zu verwenden, soweit dies erforderlich ist, um die Inanspruchnahme von Telemedien zu ermöglichen und abzurechnen. Der Begriff des Verwendens wird zwar nicht explizit legaldefiniert, jedoch findet er sich in $₫ 3$ Abs. 5 BDSG, wonach das Nutzen von Daten jede Verwendung personenbezogener Daten ist, soweit es sich nicht um Verarbeitung handelt. Daraus lässt sich ableiten, dass der Begriff des Verwendens weit zu verstehen ist und sowohl das Nutzen als auch das Verarbeiten von personenbezogenen Daten umfasst. ${ }^{918}$

\section{i) Nutzungsdaten}

Nach der Legaldefinition des $₫ 15$ Abs. 1 S. 1 TMG sind Nutzungsdaten personenbezogene Daten, die erforderlich sind, um „die Inanspruchnahme von Telemedien zu ermöglichen und abzurechnen“. Wie die Definition zeigt, unterliegt die Erhebung von Nutzungsdaten, wie auch die Erhebung von Bestandsdaten, dem Grundsatz der Erforderlichkeit. Dieser Grundsatz bezieht sich allerdings auf die Ermöglichung und Abrechnung des Telemediendienstes

Diensteanbieter keine selbstständigen Web Service-Anbieter sind, die lediglich mit dem Portalbetreiber vertraglich kooperieren, sondern rechtlich unselbstständige Subunternehmen des Portalbetreibers sind, s. Roßnagel/Dix/Schaar, RMD, Teil 3, \$ 6 TDDSG Rn. 168; Gola/Schomerus, $\S 3$ Rn. 34. In diesem Fall kann die Vorschrift des $₫ 15$ Abs. 5 S. 1 TMG nicht greifen. Vielmehr kann sich der Diensteanbieter dann auf dieselben Vorschriften stützen wie der Portalbetreiber.

918 Spindler/Schuster/Spindler/Nink, \12 TMG Rn. 6; Roßnagel/Scbild, Hdb. DatenschutzR, Kap. 4.2 Rn. 11, 16; Gola/Schomerus, $\$ 3$ Rn. 25. 
und nicht auf den Vertragsschluss. ${ }^{919}$ Die Terminologie „zu ermöglichen und abzurechnen" erweckt den Eindruck, dass beides kumulativ vorliegen müsste. $\mathrm{Da}$ dies aber die gesonderte Legaldefinition der Abrechnungsdaten in $\ 15$ Abs. 4 S. 1 TMG überflüssig machen würde und darüber hinaus niemals eine zulässige Erhebung von Nutzungsdaten aus einem unentgeltlichen Vertrag möglich wäre, kann die Formulierung nur als Alternative verstanden werden. ${ }^{920}$ Daher lassen sich Abrechnungsdaten als Unterfall der Nutzungsdaten einordnen. ${ }^{921}$ Nutzungsdaten lassen sich zusammenfassen als sämtliche Informationen, die bei der Interaktion zwischen Nutzer und Anbieter während und durch die Dienstenutzung notwendigerweise entstehen. ${ }^{922}$ \ 15 Abs. 1 S. 2 Nr. 1-3 TMG enthält einen nicht abschließenden Beispielskatalog für Nutzungsdaten. ${ }^{923}$ Diese Beispiele zeigen, dass Bestands- und Nutzungsdaten sich zum Teil überschneiden. So können Merkmale zur Identifikation des Nutzers im Sinne des $₫ 15$ Abs. 1 S. 2 Nr. 1 TMG, wie zum Beispiel statische IPAdressen, Teilnehmerkennung und Passwort zugleich auch erforderliche Bestandsdaten sein. ${ }^{924}$ Welche personenbezogenen Daten im Rahmen einer Web Service-Portalnutzung erforderliche Nutzungsdaten darstellen, wird im Folgenden genauer untersucht.

\section{Besondere Nutzungsdaten bei der Web Service-Portalnutzung}

Für die vorliegende Untersuchung sind, neben den Daten die bei der Nutzung des Portals sowie der Web Services anfallen, die beim Portalbetreiber anfallenden Internet-Protokolldaten relevant. Dies ergibt sich bereits aus den üblichen technischen Voreinstellungen der Server: ${ }^{25}$ bei jedem Zugriff auf das

919 Roßnagel, in: Roßnagel/Banzhaf/Grimm, Teil B S. 168; Spindler/Schmitz/Geis/Schmitz, \6 TDDSG Rn. 8; jurisPK-Internetrecht/Heckmann, Kap. 1.15 Rn. 10 f.; Engel-

Flechsig/Maennel/Tettenborn, NJW 1997, 2987.

920 So i.E. auch jurisPK-Internetrecht/Heckmann; Kap. 1.15 Rn. 11; Engel-

Flechsig/Maennel/Tettenborn, NJW 1997, 2987.

921 Spindler/Schmitz/Geis/Spindler, \$6 TDDSG Rn. 1.

922 Begr. RegE BT-Drs. 13/7385, S. 24; Roßnagel/Roßnagel, Hdb. DatenschutzR, Kap. 7.9 Rn. 55; Roßnagel/Dix/Schaar, RMD, Teil 3, \6 TDDSG Rn. 82 ff.; Spindler/Schuster/Spindler/Nink, \15 TMG Rn. 2; Scholv, S. 239; Zscherpe, K\&R 2005, 266.

923 So explizit die Begr. RegE BT-Drs. 14/6098, S. 29, nach der die beispielhafte Aufzählung der Klarheit des Gesetzes dienen soll.

924 Spindler/Schmitz/Geis/Schmitz $\ 6$ TDDSG Rn. 9; Spindler/Schuster/Spindler/Nink, $₫ 15$ TMG Rn. 2.

925 S. dazu Gundermann, K\&R 2000, 226. 
Portal überträgt der Browser des Portalnutzers automatisch einen definierten Datensatz, den so genannten Log-File, an den Server des Portalbetreibers; dieser speichert den Log-File dann standardmäßig. ${ }^{926} \mathrm{Zu}$ den Daten im gespeicherten Log-File gehören die IP-Adresse des Portalnutzers, die URL des Portals als aufgerufene Seite, die URL, über deren Link der Zugriff erfolgte vereinfacht, eine Information darüber, wer mit wem kommuniziert -, der Zeitpunkt des Seitenaufrufs und Angaben über benutzte Hard- und Software (insbesondere Browser). ${ }^{927}$ Dabei dient die Übermittlung der Absenderadresse einer Antwort durch den Empfänger und die Empfängeradresse, um den Routern zu sagen, wohin das Datenpaket weiterzuleiten ist. ${ }^{928}$ Darüber hinaus kann anhand des Log-File aber auch ausgewertet werden, wie oft und wie lange eine Seite aufgerufen worden ist und welcher Weg durch die Seiten gewählt wurde. ${ }^{929}$ Log-Files können daher als Grundlage für Nutzerprofile, die nur unter den bestimmten engen Voraussetzungen des $\$ 15$ Abs. 3 und gegebenenfalls Abs. 1 TMG rechtmäßig erstellt werden dürfen, dienen. 930 Datenschutzrechtlich relevant sind dabei aber nur die Daten, die geeignet sind, ein personenbezogenes Datum zu bilden. Die übrigen Daten können ohne Einschränkung des Telemediengesetzes erhoben und verarbeitet werden.931 Die Log-File-Daten des Portalnutzers müssen vorliegend aber nur für den Portalbetreiber auf die Rechtmäßigkeit der Erhebung hin untersucht werden, da der Web Service-Anbieter bei der Web Service-Nutzung lediglich mit dem Portalbetreiber kommuniziert und daher auch nur dessen Daten loggt, nicht die des Portalnutzers.

\section{IP-Adressen}

Da IP-Adressen zur gleichen Zeit immer nur einmal vergeben sind, können sie eindeutig einem Nutzer-Rechner zugeordnet werden und sind daher grundsätzlich geeignet, ein personenbezogenes Datum zu bilden. ${ }^{932} \mathrm{Zu}$ unterschei-

926 Wiese, in: Bäumler, S. 11; Scholz, S. 239.

927 Schaar, DuD 2001, 384; Wiese, in: Bäumler, S. 11; Scholz, S. 241 f.; Moos, in: Kröger/Gimmy, Hdb. InternetR, S. 521; Grimm, in: Roßnagel/Banzhaf/Grimm, Teil A S. 57; HillenbrandBeck/Greß, DuD 2001, 390.

Grimm, in: Roßnagel/Banzhaf/Grimm, Teil A S. 57 f.; Schaar, DuD 2001, 383 f.

Gundermann, K\&R 2000, 226; Krieg, jurisPR-ITR 14/2007 Anm. 2; Scholz, S. 240.

930 Roßnagel/Schulz, RMD, Teil 3, \$ 1 TDDSG Rn. 33; Schaar, DuD 2001, 384; Scholz, S. 240.

931 S. auch Scholv, S. 240.

932 Roßnagel/Roßnagel, Hdb. DatenschutzR, Kap. 7.9 Rn. 56; Scholz, S. 240. 
den ist allerdings zwischen statischen IP-Adressen, ${ }^{933}$ die fest einer bestimmten Person oder einem bestimmten Rechner zugeteilt sind ${ }^{934}$ und dadurch zusätzlich zum Rückschluss auf die Person und mitunter die Organisation auch einen Rückschluss auf das Herkunftsland ermöglichen, ${ }^{935}$ und dynamischen IP-Adressen, die einer bestimmten Person nur für den jeweiligen Nutzungszeitraum zugeordnet sind. ${ }^{936}$ Access-Provider verfügen in der Regel über mehr Kunden als IP-Adressen und verteilen daher die vorhandenen, verfügbaren IP-Adressen der Reihe nach auf die Kunden, die gerade in das Internet wollen und daher eine IP-Adresse benötigen, so dass jeder Kunde regelmäßig bei jeder neuen Einwahl eine neue IP-Adresse erhält. ${ }^{937}$ Insofern bilden dynamische IP-Adressen den Großteil der verwendeten IP-Adressen. Für den Log-File spielt die Unterscheidung zwischen dynamischen und statischen IPAdressen aus technischer Sicht keine Rolle, denn der Log findet unabhängig von dem Charakter der IP-Adresse bei allen IP-Adressen statt, sofern der Portalbetreiber bei seinem Server diese (Vor-)Einstellung entsprechend beibehalten hat, was regelmäßig der Fall sein wird. Entscheidend ist die Differenzierung aber für die Einordnung als personen- oder nicht personenbezogenes Datum.

Während statische IP-Adressen unstreitig wegen ihrer Zuordenbarkeit zu einer Person beziehungsweise einem Rechner ${ }^{938}$ für denjenigen, der das erforderliche Zusatzwissen besitzt, ${ }^{939}$ als personenbezogene Daten gelten können, wobei nach der hier vertretenen Auffassung immer die Relativität des Personenbezugs zu beachten ist, herrscht Streit über die Einordnung von dynamischen IP-Adressen. Die Vertreter der Relativität des Personenbezugs differenzieren richtigerweise zwischen dem Zugangsprovider, der aufgrund von Referenzdateien, die zum entsprechenden Zeitpunkt vergebene IP-Adresse einer bestimmten Person zuordnen kann, und sonstigen Web Service-Anbietern, für

933 Statische IP-Adressen werden meist nur an größere Institutionen, wie Hochschulen oder Unternehmen, vergeben.

934 Rasmussen, CR 2002, 37; Spindler/Schmitz/Geis/Schmitz, 1 TDDSG Rn. 27; Schaar, Rn. 169.

935 Spiegel, DuD 2003, 266; Rasmussen, CR 2002, 37.

936 Roßnagel/Roßnagel, Hdb. DatenschutzR, Kap. 7.9 Rn. 56; Gundermann, K\&R 2000, 226.

937 S. Wiese, in: Bäumler, S. 11; Schaar, Rn. 169; Gundermann, K\&R 2000, 226.

938 Ist bekannt wo der Rechner seinen physikalischen Standort hat, so ist auch eine Zuordnung zu der den Rechner nutzenden Person möglich, s. Gundermann, K\&R 2000, 226.

939 S. nur Roßnagel/Schulz, RMD, Teil 3, S 1 TDDSG Rn. 35; Spindler/Schmitz/Geis/Schmitz, \ 1 TDDSG Rn. 27; Scholz, S. 240 f.; Schaar, Rn. 171 ff.; Gundermann, K\&R 2000, 227. 
welche die dahinter stehende Person ohne fremde Hilfe kaum rekonstruierbar ist. ${ }^{940}$ Die Gegenauffassung will dynamische IP-Adressen per se als personenbezogene Daten einordnen. ${ }^{941}$

Der Auffassung, dass dynamische IP-Adressen grundsätzlich personenbezogene Daten seien, kann bei Zugrundelegung der allgemein vertretenen Definition von personenbezogenen Daten nicht entsprochen werden. ${ }^{942}$ Andernfalls würden für die schwerer zuordenbaren dynamischen IP-Adressen strengere datenschutzrechtliche Auflagen gelten als für die leichter zuordenbaren statischen IP-Adressen. Da der Personenbezug relativ ist, muss er immer in Bezug auf die jeweilige Person gesondert festgestellt werden. Über das Zusatzwissen zur Zuordnung einer dynamischen IP-Adresse verfügt aber nur der Zugangsprovider. Dritte können höchstens durch ein Auskunftsersuchen beim Zugangsprovider erfahren, wer sich hinter der geloggten IP-Adresse verbirgt. Ohne die Hilfe des Zugangsproviders ist ihnen eine Zuordnung in der Regel nicht möglich. Insofern stellt die dynamische IP-Adresse nach richtiger Auffassung nur für den Zugangsprovider ein personenbezogenes Datum dar; für alle Übrigen, insbesondere Web Service-Anbieter und Portalbetreiber, dagegen regelmäßig kein solches. Eine Ausnahme bildet lediglich der Fall, dass der Nutzer sich bereits mit seinen persönlichen Daten, wie Name, Adresse und ähnlichem, beim Portal registriert hat und dadurch eine Zuordnung der IPAdresse zum Portalnutzer erfolgen kann. ${ }^{943}$ Insofern ist der Personenbezug sowohl bei statischen als auch dynamischen IP-Adressen gleich einzuordnen: er ist immer nur bei demjenigen zu bejahen, der über das nötige Zusatzwissen verfügt. Der einzige Unterschied zwischen dynamischen und statischen IPAdressen besteht de facto darin, dass sich der Personenbezug bei statischen IP-Adressen häufig leichter herstellen lässt, da neben dem Zugangsprovider oft auch andere Stellen, wie beispielsweise die Verwaltung der Universität, über das notwendige Zusatzwissen verfügen.

940 Roßnagel/Roßnagel, Hdb. DatenschutzR, Kap. 7.9 Rn. 56; Roßnagel/Scbulz, RMD, Teil 3, $\$ 1$ TDDSG Rn. 36; jurisPK-Internetrecht/Heckmann, Kap. 1.12 Rn. 25; Spindler/Schmitz/Geis/Schmitz, \$1 TDDSG Rn. 26; Spindler/Schuster/Spindler/Nink, \11 TMG Rn. 8; Moos, in: Kröger/Gimmy, Hdb. InternetR, S. 521; Scholz, S. 241 f.; Meyer, WRP 2002, 1030; AG München, K\&R 2007, 767; LG Frankenthal, Beschl. v. 21.05 .2008 - 6 O 156/08, Rn. 15; Meyerdierks, MMR 2009, 10; Köcher, Anm. zu LG Berlin, abgedruckt in: MMR 2007, 800 (801); Eckhardt, Anm. zu LG Berlin abgedruckt in: K\&R 2007, 601, K\&R 2007, 602 ff.

941 AG Berlin, DuD 2007, 856 f.; Schaar, Rn. 174 ff.; wohl auch in diese Richtung tendierend Krieg, jurisPR-ITR 14/2007 Anm. 2.

942 Zur Definition s. B.IV.1.a) Definition und Inhalt.

943 Moos, CR 2003, 388; Dix, DuD 2003, 235. 
Das bedeutet, dass die Erhebung und Verarbeitung sowohl von dynamischen als auch regelmäßig statischen IP-Adressen im Falle der registrierungsfreien Portalnutzung grundsätzlich ohne Einschränkungen sowohl für Portalbetreiber als auch die Web Service-Anbieter zulässig wäre, da es in diesem Fall regelmäßig an den erforderlichen Zusatzinformationen fehlt. Da allerdings das Risiko der Herstellung eines Personenbezugs bei statischen IP-Adressen ungleich höher ist als bei dynamischen IP-Adressen und die Portalbetreiber aufgrund der automatisierten Speicherung nicht unterscheiden können, ob sich für sie ein Personenbezug herstellen lässt oder nicht, müssen alle IP-Adressen aus Datenschutzgesichtspunkten einheitlich behandelt werden. Daher müssen auch bei fehlender Portalregistrierungspflicht alle IP-Adressen so behandelt werden, als handele es sich in Gänze um personenbezogene Daten. ${ }^{944}$ Gleiches muss erst Recht für registrierungspflichtige Portale gelten, da sich hier das Risiko der Herstellung eines Personenbezugs bei statischen IP-Adressen spätestens bei der nochmaligen Portalnutzung durch den Betroffenen verwirklicht, denn dann kann die statische IP-Adresse den Registrierungsdaten zugeordnet werden. Die Speicherung der während der Portal- oder Web ServiceNutzung anfallenden IP-Adresse ist folglich nur im Rahmen der Voraussetzungen des $₫ 15$ TMG oder einer Einwilligung ${ }^{945}$ des Portalnutzers zulässig.

Das Internet ist ein so genanntes verbindungsloses Protokoll, daher ist eine Speicherung der IP-Adresse des Portalnutzers sowohl im Rahmen des LogFile als auch während der Portalnutzung durch den Portalbetreiber für die Ermöglichung der Portalnutzung unerlässlich, um die zu übertragenden Datenpakete entsprechend zu adressieren und somit von $\ 15$ Abs. 1 TMG gedeckt. ${ }^{946}$ Die Zulässigkeit der IP-Adressen-Speicherung durch Portalbetreiber ist zeitlich auf die Dauer des Nutzungsvorgangs begrenzt. Anschließend müssen die IP-Adressen wieder gelöscht werden, da es nunmehr an der weiteren Erforderlichkeit fehlt. ${ }^{947}$

Etwas anderes muss hinsichtlich der Web Service-Anbieter gelten. Denn die durch den Web Service übermittelten Daten werden an den Portalbetreiber

944 Gundermann, K\&R 2000, 227; Ibde, CR 2000, 417; Scholv, S. 243; Roßnagel, in: Roßnagel/Banzhaf/Grimm, Teil B S. 169.

$945 \mathrm{Zu}$ den Voraussetzungen der Einwilligung B.III.2.b)aa) Exkurs: Anforderungen an die Einwilligung.

946 Roßnagel/Dix/Schaar, RMD, Teil 3, \6 TDDSG Rn. 102; Roßnagel, in: Roßnagel/Banzhaf/Grimm, Teil B S. 169; Scholz, S. 243.

947 Roßnagel, in: Roßnagel/Banzhaf/Grimm, Teil B S. 169; Scholy, S. 243; Roßnagel/Dix/Schaar, RMD, Teil 3, § 6 TDDSG Rn. 102. 
gesendet und dieser stellt sie dem Portalnutzer auf seinem Webspace zu Ansicht. Daher genügt es für Web Service-Anbieter, die IP-Adresse des Portalbetreibers zu speichern. Eine Speicherung der IP-Adresse des Portalnutzers ist für die Ermöglichung der Web Service-Nutzung nicht unerlässlich und daher auch nicht durch den Erlaubnistatbestand des $₫ 15$ Abs. 1 TMG gedeckt.

\section{Nutzerprofile}

Einzelne Daten wie etwa die IP-Adresse, der Browsertyp, die verwendete Software, Zeitpunkt und Dauer des Besuchs einer Website, welche Dienste wann und wie häufig genutzt wurden sowie Zahlungsverhalten sind jeweils für sich allein genommen regelmäßig keine per se besonders interessanten oder aussagekräftigen Daten für Portalbetreiber und Web Service-Anbieter. Aussagekräftig in der Art, dass sie Aufschlüsse über die Präferenzen einer Person, wie Hobbys, Interessen, Kaufverhalten, Bedürfnisse, Vorlieben, und daraus resultierend unter Umständen sogar Rückschlüsse auf den Charakter der jeweiligen Person zulassen, geben solche Einzeldaten meist erst dann, wenn sie miteinander verbunden werden. Eine solche zielgerichtete Verknüpfung von Daten, die einer Person zugeordnet sind, durch ein logisches zueinander in Beziehung Setzen, wodurch zumindest eine Wiedergabe des Teilabbilds der Persönlichkeit erfolgt, wird auch als Profil bezeichnet. ${ }^{948}$ Nutzungsdaten sowie ihre individuelle Auswertung eignen sich in besonderer Weise für die Bildung von Persönlichkeitsprofilen. Für die Sammlung der für die Erstellung solcher Profile notwendigen Daten, lassen sich neben Log-Files auch Techniken wie Cookiess49 und Web-Bugs950 nutzen. Die damit gesammelten Daten können mit eventuell vorhandenen Bestandsdaten kombiniert werden. Aus diesen Verknüpfungen lassen sich für den Portalbetreiber und die Web ServiceAnbieter wertvolle Informationen gewinnen, auf denen basierend ein gezieltes Direktmarketing möglich wird, das Streuverluste minimiert und so Kosten einspart. ${ }^{951}$ Die Verknüpfungen müssen aber nicht per se für den Betroffenen

948 Zwar findet sich keine einheitliche Profildefinition, die genannten Kriterien dienen aber als Anhaltspunkte zur Klassifizierung als Persönlichkeitsprofil, s. Jandt/Laue, K\&R 2006, 318; Roßnagel, in: Roßnagel/Banzhaf/Grimm, Teil B S. 209; Scholz, S. 95 f.; Spindler/Schuster/Spindler/Nink, $\$ 15$ TMG Rn. 8; ferner speziell für Nutzungsprofile Lange, BB 2002, 564 .

949 S. B.IV.2.b)bb)i) Cookies.

950 S. B.IV.2.b)bb)i) Web-Bugs.

951 Mit konkreten Zahlen zu den Einsparungen Weichert, in: Bäumler, S. 163 f.; Scholz, S. 98; s. dazu allgemein Buxel, DuD 2001, 579 ff. 
schlecht sein, denn anhand dieser Informationen wird auch eine gezielte Betreuung und serviceorientierte Bedienung des Kunden möglich. ${ }^{952}$

\section{Risiken der Profilbildung}

Durch die Errungenschaft der automatischen Datenverarbeitung ist es technisch gesehen praktisch unbegrenzt möglich, Einzelangaben über persönliche oder sachliche Verhältnisse einer bestimmten oder bestimmbaren Person zu speichern und jederzeit ohne Entfernungsbarrieren in Sekundenschnelle abzurufen. ${ }^{953}$ Diese Einzelangaben lassen sich mit anderen Datensammlungen zu einem teilweise oder weitgehend vollständigen Persönlichkeitsbild zusammenfügen, ohne dass der Betroffene dessen Richtigkeit und Verwendung hinreichend kontrollieren kann. ${ }^{954}$ Bei Persönlichkeitsprofilen ist nicht mehr das einzelne Datum die Gefahr für das Recht auf informationelle Selbstbestimmung sondern vielmehr die aus diversen Einzeldaten zusammengefügte Gesamtinformation. ${ }^{955}$ Gerade auf Web Service-Portalen, wo die Anzahl der unterschiedlichen Akteure, die Daten über den Portalnutzer sammeln, austauschen und schlussendlich zusammenführen, für den Portalnutzer nicht immer überschaubar ist, können aus einzelnen, scheinbar unbedeutenden Einzelinformationen personalisierte Nutzer- und Kundenprofile gebildet werden, die mit hoher Aussagekraft Präferenzen, Bedürfnisse, Kaufgewohnheiten und sonstige Verhaltensweisen beschreiben. ${ }^{956}$ So kann das gesamte Nutzungsverhalten des Portalnutzers auf dem Portal in einer Art Bewegungsprofil aufgezeichnet werden. Bestellvorgänge können systematisch miteinander verknüpft werden. Gleiches gilt für die bei der Web Service-Nutzung der Web ServiceAnbieter anfallenden Daten. Die Analyse solcher Daten lässt mit ziemlicher Sicherheit auf bestimmte demografische Merkmale des Nutzers schließen. ${ }^{957}$ Authentische und möglichst exakte Nutzerprofile ermöglichen es dem Ersteller, zu messen ob und wie die entsprechenden Zielgruppen erreicht werden

\footnotetext{
952 Kramer/Hermann, Rn. 165; Lange, BB 2002, 562; ferner Fröble, S. 245.

953 So bereits BVerfGE 65, 1 (42) - Volkszählung.

954 BVerfGE 65, 1 (42) - Volkszählung; Engel-Flecbsig/Maennel/Tettenborn, NJW 1997, 1887, Hornung, S. 159.

955 Roßßnagel/Pfitzmann/Garstka, S. 117; Roßnagel, in: Roßnagel/Banzhaf/Grimm, Teil B S. 209.

956 Breinlinger, RDV 1997, 252; Schaar, DuD 2001, 385; Roßnagel/Pfitzmann/Garstka, S. 117; Roßnagel, in: Roßnagel/Banzhaf/Grimm, Teil B S. 209; Jandt/Laue, K\&R 2006, 317; zu den Risiken der Verknüpfung von Daten speziell bei virtuellen Videotheken Ladeur, MMR 2000, 715, 718 ff.

957 Schaar, DuD 2001, 385.
} 
können und Werbeaktionen effizient zu platzieren. ${ }^{558}$ Dies kann dem Portalbetreiber beziehungsweise dem Web Service-Anbieter unter Umständen Wettbewerbsvorteile gegenüber der Konkurrenz und zum Teil sogar erhebliche Kostenersparnisse einbringen. Darüber hinaus sind auch Manipulationen hinsichtlich des Kaufverhaltens der Betroffenen möglich. Bucht eine Person beispielsweise regelmäßig nur teure fünf Sterne-Hotels oder hochpreisige Mietwagen, so können die Preise für ihn bewusst angehoben werden, da er die vorgeschlagenen Objekte, gleich wie viel sie kosten, regelmäßig buchen wird.

\section{Zulässigkeit von Persönlichkeitsprofilen}

Das Bundesverfassungsgericht hat im Rahmen des Volkszählungsurteils bereits vor einem viertel Jahrhundert die besonderen Risiken, die durch eine Erstellung von Persönlichkeitsprofilen im Wege der automatisierten Datenverarbeitung für den Betroffenen entstehen, aufgezeigt ${ }^{959}$ und die Erstellung solcher Persönlichkeitsprofile grundsätzlich als unzulässig qualifiziert. ${ }^{960}$

Dabei wird unterschieden zwischen Totalabbildern und Teilabbildern der Persönlichkeit. Totalabbilder, bei denen der Mensch zwangsweise in seiner ganzen Persönlichkeit registriert und katalogisiert wird, selbst wenn es sich um eine anonyme statistische Erhebung handelt, sind mit der Würde des Menschen unvereinbar und daher per se unzulässig. ${ }^{961}$ Aber auch die Erstellung von Teilabbildern gegen den Willen des Betroffenen wurde vom Bundesverfassungsgericht als unzulässig gesehen, wenn es sich um umfassende Persönlichkeitsprofile handelt. ${ }^{962}$ Das grundsätzliche Verbot solcher Profile entspricht dem Grundsatz der Zweckbindung und wurzelt daher letztlich auf dem Verhältnismäßigkeitsprinzip. ${ }^{963}$ Das bedeutet allerdings auch, dass nicht jedes Profil grundsätzlich eine Gefahr für das Recht auf informationelle Selbstbe-

Spiegel, DuD 2003, 265; Schmitr, S. 56.

BVerfGE 65, 1 (41 ff.) - Volkszählung.

BVerfGE 65, 1 (53) - Volkszählung.

BVerfGE 27, 1 (6) - Mikrozensus.

BVerfGE 65, 1 (53 f.) - Volkszählung. Zustimmend auch die Lit.: Roßnagel/von Zeasschwitz, Hdb. DatenschutzR, Kap. 3.1 Rn. 1; Schaar, DuD 2001, 383; Jandt/Laue, K\&R 2006, 317 ff.; kritisch indes Ladeur, MMR 2000, 718.

Hornung, S. 160; Roßnagel/von Zęschwitz, Hdb. DatenschutzR, Kap. 3.1 Rn. 1; Roßnagel/Roßnagel, Hdb. DatenschutzR, Kap. 3.4 Rn. 71; Roßnagel/Pfitzmann/Garstka, S. 117; Jacob/Jost, DuD 2003, 622; ferner Schaar, DuD 2001, 383. 
stimmung darstellt und es daher auch Profile geben kann, die zulässig sind. ${ }^{964}$ Denn eine Profilbildung kann unter bestimmten Umständen im Interesse des Betroffenen liegen. Beispielsweise fühlen sich viele Internetnutzer durch die undurchsichtige Flut an Werbung überfordert und empfinden es als praktisch, aufgrund von Profilen erstellte personalisierte Werbeangebote zu erhalten. Allerdings besteht immer die Gefahr, dass ein derart umfangreiches Wissen über einen sozialen Interaktionspartner eine selbstbestimmte Persönlichkeitsdefinition unmöglich macht und dadurch ein starker Eingriff in das Recht auf informationelle Selbstbestimmung erfolgt. ${ }^{965}$ Deshalb müssen selbst im Falle einer freiwilligen Profilbildung gewisse Grenzen bestehen, die aber weiter gefasst sein dürfen als bei einer zwangsweisen Zusammenführung von Daten. ${ }^{966}$ Die Bildung von Profilen bedarf daher einer Rechtfertigung, 967 die in Form eines Erlaubnistatbestands oder der Einwilligung (im Rahmen der zulässigen Grenzen) ${ }^{968}$ erfolgen kann. ${ }^{969}$ Erlaubnistatbestände zur Erstellung beziehungsweise Übermittlung von so genannten Nutzungsprofilen finden sich in \15 Abs. 1 und Abs. 3 TMG.

\section{Voraussetzungen der Nutzungsprofilbildung}

Um den Eingriff in das Recht auf informationelle Selbstbestimmung durch die Erstellung personenbezogener Profile zu rechtfertigen, muss eine Erlaubnis zur Erstellung und Verwendung entsprechender Profile vorliegen. Eine solche findet sich in den Erlaubnistatbeständen des $₫ 15$ Abs. 1 oder Abs. 3 TMG. Jenseits des erlaubten Bereichs des $₫ 15$ Abs. 1 oder Abs. 3 TMG bedarf es

964 Jandt/Laue, K\&R 2006, 319; i.d.S. Moos, MMR 2006, 721 f. So auch bereits das BVerfGE 27, 1 (6) - Mikrozensus und 65, 1 (52) - Volkszählung, indem es davon spricht, dass selbst die zwangsweise Zusammenführung von Daten nicht als Anfertigung unzulässiger Persönlichkeitsprofile einzuordnen sei, wobei bei einer zwangsweisen Zusammenführung jedoch eine verfassungsrechtliche Obergrenze für die Profilbildung existiere.

965 Hornung, S. 159.

966 Jandt/Laue, K\&R 2006, 319; OLG Frankfurt, DuD 2001, 294 (296).

967 Ladeur, MMR 2000, 718.

$968 \mathrm{Zu}$ den Anforderungen an eine wirksame Einwilligung s. bereits B.III.2.b)aa) Exkurs: Anforderungen an die Einwilligung, sowie OLG Frankfurt, DuD 2001, 294 (296); Jacob/Jost, DuD 2003, 622.

969 Jandt/Laue, K\&R 2006, 322; Schaar, DuD 2001, 385; Ladeur, MMR 2000, 718 ff. 
einer Einwilligung ${ }^{970}$ durch den Betroffenen. Eine Erstellung von anonymen Nutzungsprofilen ist indes auch ohne Einwilligung zulässig. ${ }^{971}$

Da das in $\ 15$ Abs. 1 TMG aufgestellte Kriterium der Erforderlichkeit jeweils anhand des konkreten Vertragsverhältnisses auszulegen ist und in diesem, neben dem Vertragsinhalt, der Verarbeitungsumfang festgelegt werden kann, soweit es freiwillig und einvernehmlich geschieht, kann eine Erstellung auch nach $\int 15$ Abs. 1 TMG zulässig sein. ${ }^{972}$ Das bedeutet aber, dass eine solche Profilerstellung für die Ausgestaltung des Vertragsverhältnisses erforderlich sein muss. Ob und wann dies bei Web Service-Portalen zutrifft, muss immer anhand der konkreten Ausgestaltung des Plattformvertrags beurteilt werden. Denkbare Fälle, in denen $\int 15$ Abs. 1 TMG auch bei Web Service-Portalen als Rechtfertigung für das Erstellen von Nutzerprofilen greifen könnte, sind bisher eher noch nicht ersichtlich. ${ }^{973}$

Von größerer Relevanz für den Untersuchungsgegenstand ist $₫ 15$ Abs. 3 TMG. Dieser erlaubt eine Erstellung von Nutzungsprofilen zum Zwecke der Werbung, der Marktforschung oder zur bedarfsgerechten Gestaltung der Telemedien unter der Voraussetzung der Verwendung eines Pseudonyms. Darüber hinaus darf der Nutzer der Erstellung nicht widersprochen haben. Eine Zusammenführung des Nutzungsprofils mit Daten über den Träger des Pseudonyms wird durch $\ 15$ Abs. 3 S. 3 und $\ 13$ Abs. 4 Nr. 6 TMG untersagt. \15 TMG stellt eine abschließende Regelung zur Erhebung und Verwendung von Nutzungsdaten dar. ${ }^{974}$ Das bedeutet, dass Nutzungsprofile zu einem anderen Zweck als dem der Werbung, der Marktforschung oder zur bedarfsgerechten Gestaltung der Telemedien nicht erstellt werden dürfen - es sei denn ein solches ist zur Ausgestaltung des beidseitig und freiwillig vereinbarten Vertragsverhältnisses im Sinne des $\int 15$ Abs. 1 TMG erforderlich - und somit

970 S. zu den Voraussetzungen der Einwilligung B.III.2.b)aa) Exkurs: Anforderungen an die Einwilligung.

971 Lange, BB 2002, 564; Fröble, S. 257; Spindler/Schmitz/Geis/Schmitz, 』6 TDDSG Rn. 31; Schmitz, in: Hoeren/Sieber, Hdb. MultimediaR, Teil 16.4 Rn. 173.

972 S. dazu ausführlich erstmals Jandt/Laue, K\&R 2006, 320. Zuvor wurde dieser Erlaubnistatbestand von der Literatur nicht behandelt, da scheinbar immer vorausgesetzt wurde, dass die Profile bereits zwangsweise erstellt wurden.

973 Jandt/Laue, K\&R 2006, 320 haben \15 Abs. 1 TMG für LBS als Erlaubnistatbestand angeführt, da dabei die Profilbildung einen integralen Bestandteil des Vertrags bildet und sie daher auch i.S.d. $\$ 15$ Abs. 1 TMG erforderlich ist.

974 Schmitr, in: Hoeren/Sieber, Hdb. MultimediaR, Teil 16.4 Rn. 123; Schaar, Rn. 711. 
einer strengen Zweckbindung unterliegen. ${ }^{975}$ Ein Rückgriff auf Erlaubnistatbestände des Bundesdatenschutzgesetzes, wie \28 BDSG, ist nicht möglich. ${ }^{976}$ Nutzungsprofile dienen dem Portalbetreiber und den Web ServiceAnbietern insbesondere dazu, ihre Angebote auf den Portalnutzer abzustimmen, indem sie ihm beispielsweise bei einem erneuten Portalbesuch passende Produkte oder Dienstleistungen vorschlagen. ${ }^{977}$

Wie sich bereits aus der systematischen Stellung des $₫ 15$ Abs. 3 TMG und der Überschrift „Nutzungsdaten“ des \15 TMG ergibt, dürfen Nutzungsprofile nur aus Nutzungsdaten erstellt werden, eine Verwendung von Bestands-, Inhaltsdaten ${ }^{978}$ oder Informationen aus externen Quellen ist daher nicht vom Erlaubnistatbestand des $₫ 15$ Abs. 3 TMG gedeckt. ${ }^{979}$ Hinsichtlich der Arten von Nutzungsdaten wird im Gesetz keine Aussage getroffen, daher können alle denkbaren Nutzungsdaten für ein Nutzungsprofil verwendet werden. Voraussetzung ist „lediglich“, dass sie zulässig erhoben wurden; die Erhebung kann auch aus verschiedenen Nutzungsvorgängen stammen, wie die im Gesetz verwendete Mehrzahl „Nutzungsprofile“ deutlich macht. ${ }^{980}$ Erheben Portalbetreiber oder Web Service-Anbieter daher bei unterschiedlichen Portalbesuchen des Portalnutzers in zulässiger Art und Weise Nutzungsdaten, so können diese auch unter einem Pseudonym miteinander zu einem Nutzungsprofil verknüpft werden. Die Erhebung selbst muss dabei nicht unter Pseudonym erfolgen. ${ }^{981}$ Der Begriff der „Erstellung“ ist eng zu verstehen. Das bedeutet, dass eine über die Erstellung, Speicherung und Auswertung hinausgehende Verwendung zu Werbezwecken bereits nicht mehr von der Erlaubnis des $\$ 15$ Abs. 3 TMG

Roßnagel/Dix/Schaar, RMD, Teil 3, 56 TDDSG Rn. 142; Spindler/Schuster/Spindler/Nink, \15 TMG Rn. 8; Spindler/Schmitz/Geis/Schmitz, \6 TDDSG Rn. 27; Schaar, DuD 2001, 386.

976 Schmitz, in: Hoeren/Sieber, Hdb. MultimediaR, Teil 16.4 Rn. 123; Fröble, S. 247.

977 Kramer/Herrmann, Rn. 164.

978 Eine Erstellung von Profilen mit Inhaltsdaten ist nach der hier vertretenen Auffassung zur Einordnung von Inhaltsdaten, s. B.IV.2.b)cc) Inhaltsdaten, nach dem weniger strengen $\$ 28$ BDSG zu beurteilen. Ordnet man Inhaltsdaten dagegen als Unterfall der Nutzungsdaten ein, so kommt man freilich zu dem Ergebnis, dass die Erstellung von Nutzungsprofilen mit Inhaltsdaten von dem Erlaubnistatbestand des $₫ 15$ Abs. 3 TMG gedeckt ist.

979 Jandt/Laue, K\&R 2006, 322; Hillenbrand-Beck/Greß, DuD 2001, 392; Schaar, Rn. 694; Scholv, S. 254; Roßnagel/Pfitrmann/Garstka, S. 110; Roßnagel/Roßnagel, Hdb. DatenschutzR, Kap. 7.9 Rn. 84; Roßnagel/Dix/Schaar, RMD, Teil 3, \6 TDDSG Rn. 133; Schaar, Rn. 107; Spindler/Schuster/Spindler/Nink, \15 TMG Rn. 8.

980 Schaar, DuD 2001, 385; ders., Rn. 109; Roßnagel/Dix/Schaar, RMD, Teil 3, \6 TDDSG Rn. 135; Scholz, S. 254; Spindler/Schmitz/Geis/Schmitz, \$ 6 TDDSG Rn. 26.

981 Spindler/Schmitz/Geis/Schmitz \6 TDDSG Rn. 25. 
erfasst ist. ${ }^{982}$ Eine Übermittlung dieser Daten ist nach $\ 15$ Abs. 5 S. 3 TMG nur zum Zweck der Marktforschung und in anonymisierter Form zulässig. ${ }^{983}$

\section{Anbieterïbergreifende Nutzungsprofile?}

Der Wortlaut des $₫ 15$ Abs. 3 TMG „der Diensteanbieter“ legt die Interpretation nahe, dass lediglich jeweils der Anbieter die pseudonymen Nutzungsprofile aus der Inanspruchnahme der eigenen Dienste erstellen darf. ${ }^{984}$ Dennoch will ein Teil der Literatur anbieterübergreifende Nutzungsprofile akzeptieren, da die Aufdeckung des Pseudonyms nicht auf einfache Weise möglich und daher die informationelle Selbstbestimmung nur in geringen Maßen gefährdet sei. ${ }^{985}$ Dem mag dahingehend Recht gegeben werden, dass bei der Pseudonymisierung nach $\ 13$ Abs. 4 Nr. 6 TMG technisch und organisatorisch sichergestellt werden muss, dass eine nachträgliche Verknüpfung der pseudonymen Nutzungsprofile mit der Identität des Nutzers praktisch ausgeschlossen wird, zumindest aber eine strikte Datentrennung erfolgt und der Zugriff auf die Zuordnungsregel verwehrt wird. ${ }^{986}$ Das bedeutet aber auch, dass im pseudonymisierten Nutzungsprofil de facto überhaupt keine personenbezogenen Daten mehr für den Profilersteller vorliegen.987 Nichtpersonenbezogene Daten unterfallen bereits grundsätzlich nicht den Regelungen und insbesondere den Einschränkungen des Datenschutzes. Normalerweise müsste die Verwendung pseudonymisierter Daten daher frei erfolgen können, ${ }^{988}$ ein Erlaubnistatbestand wäre gar nicht notwendig. Auch gibt es keine mit $\$ 15$ Abs. 3 TMG vergleichbare Regelung, die ebenfalls die Verwendung von pseudonymisierten Daten regelt. Diese Überlegungen zeigen, dass \15 Abs. 3 TMG eigentlich gar kein klassischer Erlaubnistatbestand ist, der

982 Fröble, S. 247 f.; Schaar, DuD 2001, 386.

983 S. Übermittlung anonymisierter Nutzungsdaten.

984 Roßnagel/Roßnagel, Hdb. DatenschutzR, Kap. 7.9 Rn. 84; Schaar, Rn. 710; Scholz, S. 254; Hillenbrand-Beck/Greß, DuD 2001, 391.

So Hillenbrand-Beck/ Greß, DuD 2001, 392.

Scholy, S. 255; Schaar, Rn. 708; Roßnagel/Roßnagel, Hdb. DatenschutzR, Kap. 7.9 Rn. 84; Spindler/Schmitz/Geis/Schmits, \6 TDDSG Rn. 25; Roßnagel/Dix/Schaar, RMD, Teil 3, \6 TDDSG Rn. 138; Roßnagel/Schol, MMR 2000, 730 f.; ferner Scholz, in: Roßnagel, OnlineEinkauf, S. 61.

987 Werden die strikten Regeln für die Pseudonymisierung eingehalten, ist dem Ersteller auch die Zuordnung der Daten nicht mehr möglich, so dass nach der hier vertretenen Auffassung keine personenbezogenen Daten vorliegen, s. B.IV.1.b) Anonyme und pseudonyme Daten. 
eine sonst verbotene Handlung ausnahmsweise gestattet, sondern vielmehr als Einscbränkung der ansonsten freien Verwendung von nicht-personenbezogenen Daten gesehen werden kann. ${ }^{989}$ Diese Einschränkung ergibt sich vor dem Hintergrund der besonderen Gefahren, die von Profilbildungen ausgehen, und dient daher der Risikovorsorge. ${ }^{990}$ Flankiert wird die Einschränkung durch das zusätzliche Widerspruchsrecht des Betroffenen gegen die Erstellung der Nutzungsprofile nach $\ 15$ Abs. 3 S. 1, 2. Hs. TMG. Dies zugrunde gelegt, kann die Argumentation hinsichtlich einer Akzeptanz anbieterübergreifender Nutzungsprofile nicht überzeugen. Denn das Risiko, das der Einschränkung des $\int 15$ Abs. 3 TMG zugrunde gelegt wurde, liegt nicht in der tatsächlichen Möglichkeit der Aufdeckung des Pseudonyms, sondern vielmehr in der Gefahr, die von der Profilbildung ausgeht. $\$ 15$ Abs. 3 TMG muss daher dahingehend ausgelegt werden, dass streng nach dem Wortlaut lediglich die bei der Nutzung der eigenen Telemedien entstandenen Daten zu einem Nutzerprofil verknüpft werden dürfen. Diese Auslegung entspricht auch dem Regelungszweck der Vorgängervorschrift des $\int 4$ Abs. 4 TDDSG 1997. Damit sollte ein Kompromiss zwischen den Interessen des Nutzers und dem wirtschaftlichen Interesse des Diensteanbieters, die Inanspruchnahme des Telemediendienstes auszuwerten, geschaffen werden. ${ }^{991}$ Diese Ratio zeigt deutlich, dass damit aus der Natur der Sache nur die Daten gemeint sein können, die bei der Nutzung der eigenen Telemedien angefallen sind. Daran hat sich auch durch die Neufassungen in $\ 6$ Abs. 3 TDDSG 2001 und nunmehr $\ 15$ Abs. 3 TMG, der den $\ 6$ Abs. 3 TDDSG 2001 wörtlich übernommen hat, nichts geändert. 992

Unabhängig davon, dass eine andere Betrachtungsweise bereits aus den oben genannten Gründen nicht zu überzeugen vermag, sprechen im Rahmen der Web Service-Portale auch andere Gründe gegen die Zulässigkeit anbieterübergreifender Nutzungsprofile nach $\int 15$ Abs. 3 TMG. Aufgrund der technisch vielfältigen Möglichkeiten von Web Service-Portalen, würde die Erlaubnis von anbieterübergreifenden Nutzungsprofilen eine ganz besondere Gefahr für die informationelle Selbstbestimmung des Betroffenen darstellen. Erhebt der Portalbetreiber Nutzungsdaten des Betroffenen in zulässiger Art und Weise zum Beispiel mit Hilfe der Cookie-Technik - 993 und werden den Web Service-

\footnotetext{
989 Roßnagel/Roßnagel, Hdb. DatenschutzR, Kap. 7.9 Rn. 83; Jandt/Laue, K\&R 2006, 321; Scholz, S. 253.

990 Roßnagel/Roßnagel, Hdb. DatenschutzR, Kap. 7.9 Rn. 83; Jandt/Laue, K\&R 2006, 321.

991 Begr. zum IuKDG v. 9.4.1997, BT-Drs. 13/7385, S. 24.

992 I.d.S. Begr. zum EGG v. 17.5.2001, BT-Drs. 14/6098, S. 29.

993 S. dazu B.IV.2.b)bb)i) Cookies.
} 
Anbietern nun diese Nutzungsdaten zur Erstellung von Nutzungsprofilen übermittelt, so erhält der Web Service-Anbieter Daten des Betroffenen, die er selber nicht hätte erheben dürfen. Selbst wenn diese Daten bereits vom Portalbetreiber unter Pseudonym erhoben wurden, weil die Portalnutzung beispielsweise unter einem Nickname erfolgt, so wird der Web Service-Anbieter je nach Funktionsweise des Portals durch den möglichen Zugriff auf die Registrierungsdaten des Betroffenen in der Lage sein, die Nutzungsdaten der betroffenen Person zu zuordnen und spätestens dadurch einen Personenbezug herzustellen. Während die nachträgliche Zusammenführung der Nutzungsprofile mit den Daten des Pseudonymträgers wegen der Missbrauchsgefahr nach \15 Abs. 3 S. 3 TMG verboten ist und ein Verstoß gegen dieses Verbot sogar eine bußgeldbedrohte Ordnungswidrigkeit nach $₫ 16$ Abs. 2 Nr. 6 TMG darstellt, wird eine solche vorangehende Zusammenführung nicht gesetzlich verboten. Die Web Service-Anbieter kämen so in den Genuss personenbezogener Nutzungsdaten, ohne die Voraussetzungen des $₫ 15$ Abs. 1 TMG für eine zulässige Erhebung und Verwendung erfüllen zu müssen. Dadurch würden die strengen Voraussetzungen für die Erhebung und Verwendung von Nutzungsdaten konterkariert und die informationelle Selbstbestimmung des Betroffenen stark gefährdet. Dass dies durch die Regelung des $\$ 15$ Abs. 3 TMG nicht bezweckt wurde, zeigt auch $₫ 15$ Abs. 5 S. 3 TMG, der eine Übermittlung von Nutzungsdaten einzig zum Zwecke der Marktforschung zulässt und dies auch nur in anonymisierter Form. ${ }^{994}$ Eine anbieterübergreifende Profilbildung gegen den Willen des Betroffenen kann daher auch aus diesen Gründen nicht akzeptiert werden.

Greifen die Web Service-Anbieter auf Daten des Portalbetreibers zu, die bei der Nutzung von dessen Dienst (dem Portal) angefallen sind oder vom Portalbetreiber zur Erbringung der Portalnutzung erhoben wurden, so dürfen diese Daten von den Web Service-Anbietern nicht genutzt werden. Auch im Rahmen des $\int 15$ Abs. 3 TMG dürfen diese durch den Portalbetreiber erhobenen Daten nicht von den Web Service-Anbietern für die Erstellung pseudonymer Nutzungsprofile genutzt oder gar mit Daten, die bei der Nutzung der Web Services angefallen sind, verknüpft werden. Eine portal- und Web Service-anbieterübergreifende Verknüpfung der anfallenden Nutzungsdaten ist daher ausschließlich mit einer Einwilligung des Betroffenen möglich. ${ }^{995}$ Sollen auch besonders sensible personenbezogene Daten im Sinne des $₫ 3$ Abs. 9

994 Ähnlich auch Schaar, Rn. 710.

995 Scholz, S. 254; Roßnagel/Roßnagel, Hdb. DatenschutzR, Kap. 7.9 Rn. 84; Schaar, Rn. 712; Fröble, S. 249. 
BDSG, wie Angaben über die rassische und ethnische Herkunft, politische Meinungen, religiöse oder philosophische Überzeugungen, Gewerkschaftszugehörigkeit, Gesundheit oder Sexualleben, in das Profil einfließen, so ist insbesondere $\int 4 \mathrm{a}$ Abs. 3 BDSG zu beachten. ${ }^{996}$ Danach muss sich die Einwilligung ausdrücklich auf die sensiblen Daten beziehen.

\section{Grenzen der Einwilligung}

Die Einwilligung stellt zum einen ein wirksames Mittel dar, einen Verstoß gegen ein Datenverarbeitungsverbot zu vermeiden, und zum anderen ermöglicht die Einwilligung eine sehr viel breitere Datenverarbeitung als sie die gesetzlichen Erlaubnistatbestände gestatten. Daher könnte man meinen, dass jede Art der Datenverarbeitung durch das Einholen einer wirksamen Einwilligung legitimiert wird. Aber auch der Einwilligung sind gewisse Grenzen gesetzt. Eine solche Begrenzung der Einwilligungsmöglichkeit erscheint zunächst zwar nicht einleuchtend, da das Recht auf informationelle Selbstbestimmung durch eine, allen Anforderungen entsprechende, Einwilligung ausgeübt wird. ${ }^{997}$ Allerdings wird das Recht auf informationelle Selbstbestimmung aus dem Allgemeinen Persönlichkeitsrecht abgeleitet und somit auf Art. 2 Abs. 1 GG in Verbindung mit Art. 1 Abs. 1 GG gestützt. Daraus ergibt sich zwangsläufig, dass eine Einwilligung in die Datenverarbeitung nicht unbegrenzt erfolgen darf. Es muss daher untersucht werden, ob es bestimmte Weisen der Datenverarbeitung hinsichtlich einer Profilbildung gibt, die der Einwilligung nicht zugänglich sind.

Dabei ist zunächst einmal der Grundsatz zu beachten, dass grundsätzlich jeder, gerade als Ausdruck des Rechts auf informationelle Selbstbestimmung, selber darüber bestimmen können muss, wem er welche Daten zur Verfügung stellt. Insofern darf eine Einwilligungsgrenze keinesfalls zu weit gezogen werden. Daher kann hier, wie teilweise im Schrifttum angeführt, 998 nicht die in der Verhältnismäßigkeit zu prüfende Sphärentheorie ${ }^{999}$ des Bundesverfassungsgerichts maßgeblich sein, sondern allein die Frage inwieweit die Menschenwürde

996 S. Roßnagel/Dix/Schaar, RMD, Teil 3, \6 TDDSG Rn. 136; ferner muss $₫ 4$ d Abs. 5 BDSG beachtet werden.

997 Die Einwilligung in die Beeinträchtigung des Rechts auf informationelle Selbstbestimmung ist dabei als Grundrechtsausübung geschützt, Klass, AfP 2005, 507; s. dazu allgemein Jarass/Pieroth/Jarass, Vorb. vor Art. 1 Rn. 36.

998 So scheinbar Iraschko-Luscher, DuD 2006, 709.

999 St. Rspr. BVerfGE 6, 32 (41); 6, 389 (433); 27, 1 (6 f.); 32, 373 (378 f.); 34, 238 (245); BVerfG, NJW 1990, 1980; NJW 2008, 39 (41 f.) - Esra. 
„disponibel“ ist. Hinsichtlich des Kernbereichs ist die Menschenwürde jedenfalls nicht disponibel. ${ }^{1000}$ Bei Eingriffen in den Kernbereich der Menschenwürde ändert auch eine Zustimmung des Betroffenen nichts an dem Eingriff. 1001 Wird in diesen geschützten Bereich eingegriffen wird, ist die Einwilligung rechtlich unbeachtlich. Wann diese Grenze überschritten wird, ist allerdings immer anhand des Einzelfalls zu beurteilen. 1002

Bei der Bildung von Persönlichkeitsprofilen, anhand der auf dem Web Service-Portal gesammelten Daten, erscheint zwar aufgrund der dargestellten Risiken grundsätzlich eine Verletzung der Menschenwürde denkbar. Weil die Daten, welche die Grundlage für die Profile bilden, aber aus einem begrenzten inhaltlichen Spektrum geschöpft werden, erscheint diese Grenze aktuell allerdings noch nicht überschritten, da es immer noch zahlreiche Facetten gibt, die von solchen Online-Profilen nicht erfasst werden können. Insofern ist die Erstellung von Profilen im Rahmen der Web Service-Portal-Nutzung gegenwärtig verfassungsrechtlich noch nicht einer Einwilligung unzulänglich und zu beachtende Grenzen der Einwilligung (noch) nicht ersichtlich.

\section{Widerspruchsrecht und Unterrichtungspflicht}

Zum Schutz des Betroffenen vor den besonderen Gefahren, die von einer Profilbildung ausgehen, enthält $₫ 15$ Abs. 3 S. 1, 2. Hs. TMG ein Widerspruchsrecht gegen die Verwendung pseudonymisierter Profildaten. Damit der Betroffene auch tatsächlich eine Möglichkeit hat, dieses Widerrufsrecht auszuüben, muss er darüber informiert werden. Diese Pflicht zur Information ist in \15 Abs. 3 S. 2 TMG als Unterrichtungspflicht nach den Vorgaben des $₫ 13$ Abs. 1 TMG ausgestaltet. Insofern wurde das Widerrufsrecht als Opt-OutLösung normiert. ${ }^{1003} \mathrm{Um}$ eine zulässige Erstellung von pseudonymen Profilen zu gewährleisten, muss der Profilersteller bereits zu Beginn des Nutzungsvor-

\footnotetext{
1000 Der Verzicht auf den Kernbereich der Menschenwürde ist nicht möglich BVerwG, NJW 1982, 664 - Peep-Shows; VG Neustadt, NVwZ 1993, 98 (99) - Zwergenweitwurf; Jarass/Pieroth/Jarass, Art. 1 Rn. 13; Sachs/Höfling, Art. 1 Rn. 17; s. auch zur Disponibilität des Rechts auf informationelle Selbstbestimmung Jandt/Laue, K\&R 2006, 319.

1001 BVerwG, NJW 1982, 664 - Peep-Shows; VG Neustadt, NVwZ 1993, 98 (99) Zwergenweitwurf; Jarass/Pieroth/Jarass, Art. 1 Rn. 13; Sachs/Höfling, Art. 1 Rn. 17; SchmidtBleibtreu/Hofmann/Hopfauf/Hofmann, Art. 1 Rn. 7; von Münch/Kunig/Kunig, Art. 1 Rn. 12.

1002 Jarass/Pieroth/Jarass, Art. 1 Rn. 13; i.d.S. auch SchmidtBleibtreu/Hofmann/Hopfauf/Hofmann, Art. 1 Rn. 7.

1003 Spindler/Schuster/Spindler/Nink, \15 TMG Rn. 8; Spindler/Schmitz/Geis/Schmitz, \6 TDDSG Rn. 28; Schaar, Rn. 718; ders., DuD 2001, 386.
} 
gangs allgemein über Art, Umfang und Zwecke der Erhebung und Verwendung personenbezogener Daten sowie über die Verarbeitung seiner Daten informieren und dabei auch darauf hinweisen, dass man sich die Erstellung pseudonymisierter Nutzungsprofile vorbehält, sofern der Nutzer dem nicht widerspricht. ${ }^{1004}$ Das bedeutet für den Portalbetreiber und die Web ServiceAnbieter, dass sie bereits vor den ersten Datenerhebungsmaßnahmen, wie zum Beispiel dem Setzen von Cookies oder Web-Bugs, neben ihrer allgemeinen Unterrichtungspflicht aus $\ 13$ Abs. 1 TMG auch über die Bildung möglicher Nutzungsprofile informieren müssen. Zugleich muss dabei auch aus Gründen der Wahrung der informationellen Selbstbestimmung der Betroffene auf sein Recht auf Widerspruch hingewiesen werden.

Der Widerruf kann jederzeit formlos erklärt werden. ${ }^{1005}$ Dem Portalnutzer muss allerdings eine angemessene Möglichkeit zum Widerruf eingeräumt werden. Eine solche Möglichkeit lässt sich beispielsweise in Form eines Widerspruchsbuttons 1006 oder eines „Opt-Out-Cookie“1007 realisieren. Macht der Portalnutzer von seinem Widerspruchsrecht Gebrauch, so muss die Profilbildung unterbleiben. ${ }^{1008}$

\section{Cookies}

Cookies ${ }^{1009}$ sind kurze Einträge, meist in Form von Textdateien, in einer kleinen Datenbank oder einem speziellem Datenverzeichnis auf dem Computer des Nutzers und dienen dem Austausch von Informationen zwischen Computerprogrammen oder der zeitlich beschränkten Archivierung von Informationen. ${ }^{1010}$ Cookies werden häufig von Website-Betreibern hinterlegt, um Nutzer beim erneuten Einloggen wieder zu erkennen und dann gezielt Werbung ein-

\footnotetext{
1004 Spindler/Schmitz/Geis/Schmitz \6 TDDSG Rn. 28.

1005 Roßnagel/Roßnagel, Hdb. DatenschutzR, Kap. 7.9 Rn. 85.

1006 Spindler/Schmitz/Geis/Schmitz, § 6 TDDSG Rn. 28; Roßnagel/Dix/Schaar, RMD, Teil 3, § 6 TDDSG Rn. 148.

1007 Roßnagel/Dix/Schaar, RMD, Teil 3, $\int 6$ TDDSG Rn. 148; Schaar, DuD 2001, 387; ders., Rn. 718; Scholy, S. 256.

1008 Kramer/Herrmann, Rn. 197.

1009 Wörtliche Übersetzung: Kekse. Die deutsche Entsprechung kann als „Profildatei“ bezeichnet werden.

1010 Wichert, DuD 1998, 273; Bizer, DuD 1998, 277; Hillenbrand-Beck/Greß, DuD 2001, 390; Lange, BB 2002, 564; Probst, DSB 6/2007, 11; Gundermann, K\&R 2000, 227 Fn. 23; Wiese, in: Bäumler, S. 13; jurisPK-Internetrecht/Heckmann, Kap. 1.12 Rn. 30.
} 
schalten oder konkrete Angebote machen zu können. ${ }^{1011}$ Dies erfolgt derart, dass die Cookies beim erneuten Besuch an den Portalbetreiber zurück versandt werden, so dass dieser die im Cookie enthaltenen Daten dem registrierten Profil des Portalnutzers zuordnen kann, ${ }^{1012}$ sofern es ein solches Profil gibt. Fehlt es an Zusatzinformationen, so ist eine Identifikation des Portalnutzers durch Cookies nicht möglich. In diesem Fall kann durch die Cookies lediglich festgestellt werden, dass es sich um denselben Nutzer handelt. ${ }^{1013}$ Zeitlich können Cookies derart eingerichtet werden, dass sie nach dem Besuch gelöscht werden oder aber ein darüber hinausgehendes Verfallsdatum haben. ${ }^{1014}$ Cookies lassen sich zudem dafür verwenden, den Nutzer, meist unbemerkt, zu überwachen und ein Profil von seinem Surfverhalten zu erstellen. ${ }^{1015}$ Ebenso können die in den Log-Files gespeicherten Daten in den Cookies abgelegt und damit umfangreiche Nutzungsprofile des Portalnutzers erstellt werden. ${ }^{1016}$ Dies ermöglicht dem Portalbetreiber ein effizientes Direktmarketing. Darüber hinaus können Cookies auch derart programmiert sein, dass sie sich „anbieterübergreifend“ nutzen lassen. Das bedeutet, dass nicht nur der Cookie-Setzer (zum Beispiel der Portalbetreiber) sondern auch andere Anbieter (wie zum Beispiel die Web Service-Anbieter) die Daten auf den Cookies - vom Portalnutzer unbemerkt - auslesen können. ${ }^{1017}$

\section{Unterrichtungspflicht des Cookie-Setzers}

$\int 13$ Abs. 1 S. 2 TMG verpflichtet den Telemediendiensteanbieter, für den Fall, dass er ein automatisiertes Verfahren verwendet, das eine spätere Identifizierung des Nutzers ermöglicht und eine Erhebung oder Verwendung personenbezogener Daten vorbereitet, den Nutzer zu Beginn dieses Verfahrens

1011 Pierrot, in: Ernst, Rn. 200; Scholy, S. 57; Probst, DSB 6/2007, 11; Spiegel, DuD 2003, 266; Meyer, WRP 2002, 1029.

1012 Lapp, ITRB 2001, 113; Pierrot, in: Ernst, Rn. 200; Selk, S. 5; Spindler/Schuster/Spindler/Nink, \13 TMG Rn. 4.

1013 Lapp, ITRB 2001, 113.

1014 Wiese, in: Bäumler, S. 13; Gundermann, K\&R 2000, 228; Meyer, WRP 2002, 1029; Spindler/Schuster/Spindler/Nink, \13 TMG Rn. 4.

1015 Probst, DSB 6/2007, 11; Bizer, DuD 1998, 277; Meyer, WRP 2002, 1029; Woitke, MMR 2003, 310; Pierrot, in: Ernst, Rn. 200; Wiese, in: Bäumler, S. 13; Scholv, S. 58; jurisPKInternetrecht/Heckmann, Kap. 1.12 Rn. 30; Moos, in: Kröger/Gimmy, Hdb. InternetR, S. 522.

1016 Bizer, DuD 1998, 277.

1017 Spiegel, DuD 2003, 266; Scholr, S 246; Hillenbrand-Beck/Greß, DuD 2001, 390; s. dazu mit technischen Erläuterungen Wichert, DuD 1998, 274 f. 
zu unterrichten. Diese Pflicht bezieht sich auch auf die automatisierten Verfahren, bei denen ein Personenbezug erst zu einem späteren Zeitpunkt festgestellt werden kann. ${ }^{1018}$ Damit ist insbesondere das Setzen von Cookies gemeint. ${ }^{1019}$ Zwar erfolgt durch das Setzen des Cookie nicht notwendigerweise eine Datenerhebung, eine solche sowie eine Datenverwendung werden jedoch dadurch zumindest ermöglicht, sei es auch zeitlich nachfolgend. Für den Betroffenen ist indes keineswegs ersichtlich, wann welche Daten aus dem Cookie zurückgesendet werden, daher ist es notwendig, diesen Vorgang für den Nutzer transparent zu machen, indem der Cookie-Setzer verpflichtet wird, den Betroffenen über ein gewünschtes Setzen zu unterrichten. ${ }^{1020} \mathrm{Um}$ dem Grundsatz der Transparenz vollständig zu entsprechen, müssen Portalbetreiber und Web Service-Anbieter, sofern auch diese Cookies setzen, den Portalnutzer bereits vor dem Setzen über Art, Umfang und Zweck ${ }^{1021}$ dieses Vorhabens informieren und nicht erst zum Zeitpunkt der Rückmeldung an den Server. ${ }^{1022}$

\section{Cookies des Portalbetreibers}

Ob ein Cookie aber Daten mit Personenbezug enthält, hängt von der Art der gespeicherten Daten und der Ausgestaltung des Portals ab. Erfordert das Portal eine Registrierung und zeichnet der Cookie entsprechend das Surfverhalten des Portalnutzers auf, so handelt es sich für den Portalbetreiber um personenbezogene Daten, da er den Bezug durch die Registrierungsdaten herstellen kann. ${ }^{1023}$ Ohne eine solche Registrierung erlangt ein Cookie in der Regel keine datenschutzrechtliche Relevanz, da eine Zuordnung und Identifizierung der Person meist nicht möglich ist. Insofern handelt es sich um datenschutzrechtlich zulässige anonyme Nutzerprofile. ${ }^{1024}$ Datenschutzrechtlich relevant sind die in den Cookies gespeicherten Daten daher nur, wenn das Portal eine Registrierung erfordert. In diesem Fall richtet sich die Zulässigkeit der Speiche-

1018 Begr. RegE BT-Drs. 13/7385, S. 22.

1019 Fröble, S. 235; Spindler/Schmitz/Geis/Schmitz, \4 TDDSG Rn. 5; Roßnagel/Roßnagel, Hdb. DatenschutzR, Kap. 7.9 Rn. 91; Zscherpe, K\&R 2005, 266.

$1020 \rrbracket 13$ Abs. 1 S. 2 TMG stellt insofern eine besondere Umsetzung des Transparenzgrundsatzes dar, s. B.III.3.c)cc) Transparenz.

1021 Der Inhalt der Unterrichtungspflicht ist in $₫ 13$ Abs. 1 S. 1 TMG festgelegt.

1022 Roßnagel/Roßnagel, Hdb. DatenschutzR, Kap. 7.9 Rn. 91.

1023 So auch Lapp, ITRB 2001, 113; Schaar, Rn. 186.

1024 Woitke, MMR 2003, 311; ferner Meyer, WRP 2002, 1029 f. 
rung der Daten auf dem Cookie nach $\ 15$ TMG. Das Setzen eines Cookie erfüllt den Tatbestand des Speicherns von Daten nach $₫ 3$ Abs. 4 S. 2 Nr. 1 BDSG, der einen Unterfall der Datenverarbeitung bildet. $\int 15$ Abs. 1 S. 1 TMG spricht allerdings nicht von ,verarbeiten“ sondern von verwenden. Der Begriff des Verwendens findet sich in $\int 3$ Abs. 5 BDSG, der besagt, dass Nutzen jede Verwendung personenbezogener Daten darstellt, soweit es sich nicht um Verarbeitung handelt. Dieser Nebensatz zeigt, dass der Begriff des Verwendens weit zu verstehen ist und sowohl Verarbeitung als auch Nutzung umfasst. ${ }^{1025}$ Aufgrund der gleichen Zweckrichtung lässt sich diese Auslegung auch auf das Telemediengesetz übertragen. ${ }^{1026}$ Insofern stellt das Setzen eines Cookie ein Verwenden im Sinne des $\ 15$ Abs. 1 S. 1 TMG dar. Das spätere Zurücksenden der im Cookie hinterlegten Daten beim erneuten Aufruf des Portals, kann als Erhebung personenbezogener Daten im Sinne des $₫ 3$ Abs. 3 BDSG und somit auch des $\int 15$ Abs. 1 S. 1 TMG, der den identischen Begriff verwendet, qualifiziert werden. ${ }^{1027}$ Daher eröffnet das Setzen des Cookie und die spätere Übermittlung der Daten, im Falle einer Registrierungspflicht auf dem Portal, den Anwendungsbereich des $\int 15$ TMG.

Der Einsatz der Cookies muss entsprechend $\ 15$ Abs. 1 S. 1 TMG erforderlich sein, um die Inanspruchnahme des Portals zu ermöglichen. Bezogen auf die reine Aufzeichnung des Nutzerverhaltens mit dem Zweck, Interessenprofile zu bilden und gezielte Werbung zu platzieren, kann nicht von einer Erforderlichkeit der Cookies, die streng auszulegen ist, gesprochen werden. ${ }^{1028}$ Gleiches gilt für die bloße Erleichterung der Portalnutzung durch Cookies, sofern eine Nutzung auch ohne Cookies möglich wäre. Derartige Einsätze von Cookies können somit nur durch eine Einwilligung des Portalnutzers gedeckt werden, \12 Abs. 1 TMG. ${ }^{1029}$

Hingegen können Cookies für die Nutzung des Portals erforderlich sein, wenn sie der Verbindungssteuerung eines einheitlichen Nutzungsvorgangs dienen, worunter man beispielsweise auch die Ablage des aktuellen Inhalts des Waren-

1025 Spindler/Schuster/Spindler/Nink, \12 TMG Rn. 6; Gola/Schomerus, \3 Rn. 25; Roßnagel/Scbild, Hdb. DatenschutzR, Kap. 4.2 Rn. 11, 16.

1026 Spindler/Schuster/Spindler/Nink, \12 TMG Rn. 6.

1027 Roßnagel, in: Roßnagel/Banzhaf/Grimm, Teil B S. 171; Scholz, S. 248; Bestmann, K\&R 2003, 500; Gundermann, K\&R 2000, 228. S. ausführlich noch zum TDDSG 1997 Bizer, DuD 1998, 279

1028 Bizer, DuD 1998, 280; Scholr, S. 248; Roßßnagel, in: Roßnagel/Banzhaf/Grimm, Teil B S. 171.

1029 S. zu den Voraussetzungen einer wirksamen Einwilligung B.III.2.b)aa) Exkurs: Anforderungen an die Einwilligung. 
korbs fasst, damit dieser während des gesamten Einkaufs verfügbar gehalten wird. ${ }^{1030}$ Auch können Cookies erforderlich sein, festzuhalten, dass bestimmte Elemente bereits einmal an den Portalnutzer übertragen wurden, um eine erneute automatisierte Übermittlung zu vermeiden. ${ }^{1031}$ Zeitlich genügt dafür in aller Regel allerdings auch das Setzen eines temporären Cookie, der nach Beendigung der Portalnutzung automatisch wieder gelöscht wird. ${ }^{1032}$

\section{Cookies und die Web Service-Anbieter}

$\mathrm{Da}$ Web Services in der Lage sind, Cookies zu lesen, können Cookies theoretisch auch vom Web Service-Anbieter auf dem Portal gesetzt werden, um Informationen über das Verhalten der Portalnutzer zu sammeln. Dies wird jedoch praktisch eher den Ausnahmefall bilden, da der Portalbetreiber den Web Service-Anbietern wohl nur in Ausnahmefällen derart viel Spielraum auf der eigenen Plattform geben wird und dies für den Web Service-Anbieter auch einen technisch höheren (eventuell sogar sehr kostspieligen) Aufwand bedeuten würde. Daher soll hier nur der Fall behandelt werden, dass der Portalbetreiber seine Cookies derart programmiert, ${ }^{1033}$ dass sie von den Web Services der Web Service-Anbieter ausgelesen werden können.

Die Erforderlichkeit des Auslesens der Daten auf den Cookies, zur Ermöglichung der Inanspruchnahme der Web Services, hängt von den Nutzungsvoraussetzungen der Web Services ab. Erfolgt die Abrechnung der Web ServiceAnbieter allein mit dem Portalbetreiber und können die Portalnutzer die Dienste der Web Service-Anbieter frei nutzen, so wird das Auslesen weder zum Zwecke der Abrechnung noch zur Inanspruchnahme der Web Services erforderlich sein. Das Auslesen der in den Cookies gesammelten Daten würde den Web Service-Anbietern ein fast vollständiges Abbild des Nutzungsverhaltens der Portalnutzer schaffen. Die einzelnen Web Service-Anbieter wären dadurch nicht nur in der Lage, alle Bewegungen und damit das gesamte Verhalten des Nutzers auf dem Portal auszuwerten, sondern auch zu wissen, welche Web Services der externen Anbieter er wann, wie oft und wie lange genutzt hat. Dadurch wäre es ihm möglich, auch ohne ein besonderes vertragliches Rechtsverhältnis, den Portalnutzer gezielt mit personalisierter

\footnotetext{
1030 Enzmann/Roßnagel, CR 2002, 148; Roßnagel, in: Roßnagel/Banzhaf/Grimm, Teil B S. 172; Schol\%, S. 249; Schmitz, S. 153 Fn. 728; a.A. noch Bizer, DuD 1998, 280 und Meyer, WRP 2002, 1029 f.

1031 Enzmann/Roßnagel, CR 2002, 148 f.; Roßnagel, in: Roßnagel/Banzhaf/Grimm, Teil B S. 172.

1032 Roßßnagel, in: Roßnagel/Banzhaf/Grimm, Teil B S. 172.

1033 Lapp, ITRB 2001, 114.
} 
und auf sein Surfverhalten abgestimmter Werbung zu konfrontieren und gegebenenfalls durch die Analyse seines Nutzungsverhaltens zu manipulieren. Der Ermöglichung der Nutzung der einzelnen Web Services dagegen dient das Auslesen der Cookie-Daten indes nicht. Eine Zulässigkeit des Auslesens der Cookies kann daher in diesem Fall dem Erlaubnistatbestand des $\int 15$ Abs. $1 \mathrm{~S}$. 1 TMG nicht entnommen werden. Ein solches Auslesen kann nur dann rechtmäßig sein, wenn eine entsprechende Einwilligung eingeholt wurde. ${ }^{1034}$ Darüber hinaus ist eine Erstellung von Nutzungsprofilen auch nicht von $\ 15$ Abs. 3 TMG gedeckt. Denn für die Rechtfertigung nach $\ 15$ Abs. 3 TMG dürfen lediglich Nutzungsdaten verwendet werden, die bei der eigenen Telemediennutzung angefallen sind, eine anbieterübergreifende Verwendung ist unzulässig. Das Auslesen der Cookies des Portalbetreibers würde aber eine solche unzulässige anbieterübergreifende Verwendung der Daten darstellen.

Denkbar erscheint lediglich, dass der Portalbetreiber - oder der jeweilige Web Service-Anbieter mit Erlaubnis des Portalbetreibers selbst - jeweils für jeden Web Service-Anbieter temporäre Cookies setzt, in denen die Informationen darüber gespeichert werden, welche Informationen durch den Web Service bereits an den jeweiligen Portalnutzer übermittelt worden sind, um dadurch eine erneute Übermittlung an den Portalnutzer zu vermeiden. ${ }^{1035} \mathrm{Da}$ dies aber einen erheblichen Aufwand für den Portalbetreiber bedeuten würde, wird das praktisch, wenn überhaupt, mit einer Erlaubnis für das Setzen eines Cookie durch die Web Service-Anbieter selbst gelöst werden. Darüber hinaus würde es, im Fall des Setzens eines Cookie für diese Zwecke durch den Portalbetreiber, im Sinne des $₫ 4$ Abs. 2 S. 2 Nr. BDSG an einer Rechtsvorschrift, die vorsieht oder zwingend voraussetzt, dass die Datenerhebung auch ohne Mitwirkung des Betroffenen erfolgen kann, fehlen. Demnach würde gegen den Grundsatz der Direkterhebung verstoßen, da die Erhebung nicht durch den Web Service-Anbieter sondern den Portalbetreiber als Cookie-Setzer erfolgt. Ein solches Vorgehen wäre dementsprechend unzulässig. Um doppelte Übermittlungen durch den Web Service-Anbieter zu vermeiden ist es daher nur möglich, dass dieser mit Erlaubnis des Portalbetreibers seine Cookies selber setzt.

Zusammenfassend lässt sich festhalten, dass das Auslesen von Cookies durch den Web Service-Anbieter in den meisten Fällen für die Ermöglichung der Web Service-Nutzung nicht erforderlich und somit auch nicht von $₫ 15$ Abs. 1

1034 S. B.III.2.b)aa) Exkurs: Anforderungen an die Einwilligung.

1035 So Enzmann/Roßnagel, CR 2002, 148 f.; Roßnagel, in: Roßnagel/Banzhaf/Grimm, Teil B S. 172. 
TMG gedeckt ist. Eine Ausnahme könnten lediglich spezielle temporäre Cookies des Web Service-Anbieters bilden, die nur das Verhalten des Portalnutzers bei der Web Service-Nutzung aufzeichnen und nach dem Nutzungsvorgang direkt wieder gelöscht werden, um doppelte Übermittlungen von Informationen durch den Web Service-Anbieter an den Portalnutzer zu vermeiden.

\section{Web-Bugs}

Ähnlich eingesetzt wie Cookies, aber bei der breiten Masse der Internetnutzer weniger bekannt, werden so genannte Web-Bugs ${ }^{1036}$. Dabei handelt es sich in der Regel um 1x1 Pixel kleine GIF-Dateien. Diese sind für den Nutzer unsichtbar, da sie transparent oder dem Hintergrund angepasst sind. ${ }^{1037}$ WebBugs werden nicht vom angewählten Server abgerufen, sondern vom Portalnutzer und sind lediglich in den HTML-Quelltext des angeforderten Dokuments eingebunden. ${ }^{1038}$ Web-Bugs senden die IP-Adresse, die InternetAdresse der besuchten Website, die Internet-Adresse des Web-Bug, den Zeitpunkt, an dem das Dokument mit dem Web-Bug angesehen wurde, den Browsertyp aber vor allem die in einem vorher gesetzten Cookie gespeicherten Informationen. ${ }^{1039}$ Insofern können durch Web-Bugs noch über die im Cookie gespeicherten Daten hinaus Informationen gesammelt und dem Web-BugSetzer geliefert werden. Ferner können auch durch Web-Bugs Cookies beim Portalnutzer gesetzt werden, ${ }^{1040}$ die dann Informationen sammeln und wiederum durch den Web-Bug auslesbar sind. Daraus resultiert die Gefahr, dass unterschiedliche Anbieter, Website-Betreiber etc. zusammenarbeiten und mit Hilfe der Web-Bug - Cookie-Kombination exakte statische Informationen

1036 Zu deutsch: Web-Wanze (s. Kramer/Herrmann, Rn. 161; Kramer, DSB 11/2001, 8); teilweise auch als Webkäfer bezeichnet.

1037 Wiese, in: Bäumler, S. 15; Schulqki-Haddouti, in: Bäumler, S. 22; Woitke, MMR 2003, 310; Spiegel, DuD 2003, 266; Hillenbrand-Beck/Greß, DuD 2001, 390; Köhntopp/Köhntopp, CR 2000, 253; Kremp, abrufbar unter: http://www.spiegel.de/netzwelt/web/0,1518,490827-2,00.html $<25.11 .2009>$; Fröble, S. 254.

1038 Wiese, in: Bäumler, S. 15; Fröhle, S. 254; Köhntopp/Köhntopp, CR 2000, 253; Hillenbrand-Beck/ Greß, DuD 2001, 390; Woitke, MMR 2003, 310; Kremp, abrufbar unter: http://www.spiegel.de/netzwelt/web/0,1518,490827-2,00.html <25.11.2009>.

1039 Kramer, DSB 11/2001, 8; Hillenbrand-Beck/Greß, DuD 2001, 390; Schul₹ki-Haddouti, in: Bäumler, S. 22; Köhntopp/Köhntopp, CR 2000, 253; Woitke, MMR 2003, 310 f.

1040 Köhntopp/Köbntopp, CR 2000, 253; Woitke, MMR 2003, 311. 
über den Nutzer erfassen und genaue Nutzungs- oder unter Umständen sogar Persönlichkeitsprofile über ihn erstellen. ${ }^{1041}$

Web-Bugs können sowohl von Browsern als auch HTML-fähigen E-MailProgrammen gelesen werden. Entscheidend ist dabei nur, dass es sich um eine HTML-fähige Technik handelt. Da die Informationen, die mit Hilfe eines Web Service übermittelt werden, durchaus auch im HTML-Format übermittelt werden können, ist es möglich, Web-Bugs direkt mit diesen Informationen durch die Web Service-Anbieter mit zusenden. Dies bringt für die Web Service-Anbieter den Vorteil, dass die Web-Bugs dann sogleich die gegebenenfalls durch das Portal gesetzten Cookies auslesen und die darin enthaltenen Informationen mit übermitteln können. Die Web Service-Anbieter können so mit geringem Aufwand zahlreiche Informationen über den Nutzer sammeln und dadurch exakte Profile über diesen erstellen, um ihn beispielsweise gezielt zu bewerben. Web Service-Anbietern, deren Dienste über Web Services mit einem Portal verknüpft sind, eröffnen sich dadurch, je nach Größe des Portals, beispiellose Datenpoole aus denen sie schöpfen können.

Neben dem Versenden eigener Web-Bugs durch die Web Service-Anbieter, ist es auch durchaus möglich, dass Web Service-Anbieter Web-Bugs Dritter, beispielsweise gegen Entgelt, in ihre Web Services integrieren, so dass diese, ohne mit dem Portalbetreiber oder den Portalnutzern in vertraglichen Kontakt zu treten und dadurch ihre Identität preisgeben zu müssen, Zugriff auf einen großen Datenpool erhalten. Dadurch kann dem Datenmissbrauch Tür und Tor geöffnet werden. Gleiches wäre im Rahmen von größeren Konzernen mit Tochtergesellschaften möglich. Datenschutzrechtlich werden Konzernverbunde nach $\$ 15 \mathrm{AktG}$ nicht als einheitliche datenverarbeitende Stellen qualifiziert. ${ }^{1042}$ Jede Weitergabe personenbezogener Daten muss daher als Übermittlung an Dritte behandelt werden. ${ }^{1043}$ Schleust beispielsweise die Tochtergesellschaft für die Muttergesellschaft einen Web-Bug mit Hilfe des im Portal implementierten Web Service ein, so kann dies nicht anhand der Zulässigkeit der Datenerhebung und -verwendung für die Tochtergesellschaft als Web Service-Anbieter beurteilt werden, sondern die Zulässigkeit muss selbstständig für die Muttergesellschaft beurteilt werden. Weil es aber regelmäßig an den Voraussetzungen eines Erlaubnistatbestands mangeln wird, ist das

\footnotetext{
1041 Schul₹ki-Haddouti, in: Bäumler, S. 22; Kramer, DSB 11/2001, 8; Fröble, S. 254.

1042 Roßnagel/Wedde, Hdb. DatenschutzR, Kap. 4.3 Rn. 50; Simitis/Simitis, \2 Rn. 142.

1043 Simitis/Simitis, $\ 2$ Rn. 142; Roßnagel/Büllesbach, Hdb. DatenschutzR, Kap. 6.1 Rn. 65 ff.
} 
Mitsenden eines Web-Bug des Mutterunternehmens grundsätzlich nur mit Einwilligung der Portalnutzer zulässig.

Gegebenenfalls wäre es auch möglich, dass sich die unterschiedlichen Anbieter, der auf dem Portal implementierten Web Services, zu einer Gemeinschaft zusammenschließen, um ihre mit Hilfe von Web-Bugs übermittelten Informationen auszutauschen. Denkt man diese Möglichkeiten der Verknüpfung von Web-Bugs und Cookies im Rahmen der Web Service-Portale weiter, wäre es sogar möglich, dass Web Service-Anbieter ihren Web Service dem Portal und dem Portalnutzer kostenlos zur Verfügung stellen, nur um so an kostenlose umfangreiche Daten zu gelangen, die wiederum ein Vielfaches der durch die kostenlose Zurverfügungstellung des Web Service entstandenen Kosten einbringen.

Sobald der Web Service-Anbieter in der Lage ist, hinsichtlich der durch den Web-Bug übermittelten Daten einen Personenbezug herzustellen, müssen die Vorschriften des Telemediengesetzes sowie subsidiär die des Bundesdatenschutzgesetzes beachtet werden. Da es sich um ähnliche Daten wie bereits bei der Erhebung durch Cookies handelt, kann bezüglich der Daten, bei denen sich ein Personenbezug herstellen lässt, auf die dortigen Ausführungen verwiesen werden. ${ }^{1044}$ Eine Erhebung oder Verwendung dieser Daten ist daher nur dann zulässig, wenn sie nach $\ 15$ Abs. 1 TMG wirklich erforderlich ist. Die Voraussetzungen sind aber eng auszulegen. Die Erhebung und Verwendung dieser Daten dürfte in der Regel nicht für die Ermöglichung der Web Service-Nutzung erforderlich sein. Eine Datenerhebung und -speicherung mit Hilfe von Web-Bugs ist somit regelmäßig nicht durch den Erlaubnistatbestand des $₫ 15$ Abs. 1 TMG gedeckt.

Eine Erstellung von Nutzungsprofilen durch Auslesen der Cookies des Portalbetreibers stellt eine unzulässige anbieterübergreifende Profilbildung dar. ${ }^{1045}$ Eine Rechtfertigung der Datenerhebung und -speicherung kann dementsprechend nur durch eine Einwilligung erfolgen. Werden mit Hilfe von Web-Bugs dagegen anonyme Nutzerprofile erstellt, so bestehen keinerlei datenschutzrechtliche Bedenken, eine solche Erstellung ist immer zulässig. ${ }^{1046} \mathrm{Da}$ dem Web Service-Anbieter durch eine Kombination mit den Cookie-Daten oder gegebenenfalls durch den Zugriff auf die Registrierungsdaten des Portalnutzers beim Portalbetreiber in der Regel eine Herstellung des Personenbezugs

\footnotetext{
1044 S. B.IV.2.b)bb)i) Cookies.

1045 S. dazu ausführlich B.IV.2.b)bb)i) Cookies.

1046 Woitke, MMR 2003, 311; Fröble, S. 257.
} 
möglich sein wird, bildet die Erstellung von anonymen Profilen die Ausnahme. Eine Erstellung von Nutzerprofilen ist demnach regelmäßig nur unter den strengen Voraussetzungen des $₫ 15$ Abs. 3 (Abs.1) TMG unter einem Pseudonym zulässig.

\section{Übermittlung anonymisierter Nutzungsdaten}

Haben Web Service-Anbieter oder Portalbetreiber dennoch Interesse an den Nutzungsdaten der Portalnutzer, die durch den jeweils anderen mittels Cookies, Web-Bugs oder ähnlichen Techniken erhoben wurden, so bietet $\ 15$ Abs. 5 S. 4 TMG eine weitere Möglichkeit, ohne Einwilligung des Betroffenen an dessen Nutzungsdaten zu gelangen. Danach dürfen rechtmäßig erhobene Nutzungsdaten zum Zwecke der Marktforschung anderen Web ServiceAnbietern übermittelt werden. Neben der Einhaltung des engen Zwecks der Marktforschung ist Voraussetzung, dass die Nutzungsdaten vor der Übermittlung anonymisiert werden. Da es sich bei anonymen Daten nicht um personenbezogene Daten handelt ${ }^{1047}$ und eine Verwendung frei erfolgen könnte, mag diese Vorschrift zunächst überraschen. Pseudonymisierten Daten ist aber die Gefahr der Aufdeckung immanent, wodurch eine unzulässige Profilbildung ermöglicht werden könnte. Vor diesem Hintergrund muss diese Vorschrift als Begrenzung der grundsätzlich möglichen freien Verwendung nichtpersonenbezogener Daten verstanden werden. ${ }^{1048}$ Im Umkehrschluss bedeutet dies, dass eine Übermittlung pseudonymisierter Nutzungsdaten, die gleichfalls mangels Personenbezug für den jeweiligen Empfänger nach der hier vertretenen Auffassung zulässig wäre, ${ }^{1049}$ durch $\ 15$ Abs. 5 S. 4 TMG ausgeschlossen wird. ${ }^{1050}$ Weiter begrenzt die Vorschrift die Zwecke der zulässigen Übermittlung auf solche die allein die Marktforschung zum Gegenstand haben. ${ }^{1051}$

Hinsichtlich des Anonymisierungserfordernisses gilt der Maßstab des $₫ 3$ Abs. 6 BDSG, wonach eine faktische Anonymisierung genügt. Die Anonymisierung muss derart gestaltet sein, dass es praktisch ausgeschlossen ist, die den Nut-

1047 B.IV.1.b) Anonyme und pseudonyme Daten.

1048 So auch Scholz, S. 257; Roßnagel/Roßnagel, Hdb. DatenschutzR, Kap. 7.9 Rn. 87.

1049 S. B.IV.1.b) Anonyme und pseudonyme Daten.

1050 Roßnagel/Roßnagel, Hdb. DatenschutzR, Kap. 7.9 Rn. 87; Roßnagel/Dix/Schaar, RMD, Teil 3, \} 6 \text { TDDSG Rn. 194; Scholv, S. 257; Schmitz, S. } 1 4 1 \text { f. }

1051 Schmitr, in: Hoeren/Sieber, Hdb. MultimediaR, Teil 16.4 Rn. 119; Scholr, S. 257; Schmitz, S. 142; Roßnagel/Dix/Schaar, RMD, Teil 3, s 6 TDDSG Rn. 192. 
zungsdaten zugehörige Person zu re-identifizieren. ${ }^{1052}$ Eine Übermittlung von Einzelangaben, die eine Re-Identifizierung der Person ermöglichen würden, sei es durch eventuell vorhandenes Zusatzwissen des Empfängers, wie Name, E-Mail-Adressen, und Nutzerkennungen, ist dementsprechend nicht zulässig; solche Daten müssen aus den Datensätzen entfernt werden. ${ }^{1053}$ Darüber hinaus ist auch eine Übermittlung von nach einzelnen pseudonymisierten Nutzern aufgeschlüsselten Nutzungsdaten nicht zulässig, da andernfalls durch die Verknüpfung dieser Informationen eine Pseudonymisierung ermöglicht würde, die aber gerade durch $\int 15$ Abs. 5 S. 4 TMG ausgeschlossen werden soll. ${ }^{1054}$ Das bedeutet allerdings auch, dass durch $₫ 15$ Abs. 5 S. 4 TMG den Web Service-Anbietern und Portalbetreibern die Möglichkeit der Auswertung, welcher Nutzer wie oft und wie lange einen Dienst genutzt hat, verwehrt werden soll. Einzig eine abstrakte statistische Auswertung der Portal- und Web Service-Nutzung wird durch die Vorschrift gestattet. Dadurch können Datenempfänger den Markterfolg des jeweiligen Dienstes abschätzen und Aussagen über einzelne Käufergruppen (keinesfalls den Einzelnen) treffen. 1055 Auswertungen, die andere Daten benötigen und für andere Zwecke erfolgen sollen, bedürfen indes einer Einwilligung des Portalnutzers. ${ }^{1056}$

\section{ii) Abrechnungsdaten}

Abrechnungsdaten werden in $\$ 15$ Abs. 4 S. 1 TG legaldefiniert als Nutzungsdaten, die „für Zwecke der Abrechnung mit dem Nutzer erforderlich sind“. Abrechnungsdaten sind insofern ein Unterfall der Nutzungsdaten, ${ }^{1057}$ die dem besonderen Zweck der Abrechnung dienen und bei denen für diesen Zweck eine weitere Verarbeitungs- und Nutzungserlaubnis gewährt wird. Die Zulässigkeit der Erhebung richtet sich allein nach $\ 15$ Abs. 1 TMG. ${ }^{1058}$ Eine Spei-

1052 Scholy, S. 258; Simitis/Dammann, \& 3 Rn. 196; Roßnagel/Roßnagel, Hdb. DatenschutzR, Kap. 7.9 Rn. 51, 87; Spindler/Schuster/Spindler/Nink, $\int 13$ TMG Rn. 11; Roßßnagel/Scholv, MMR 2000, 723; ferner Bižer, DuD 1998, 278; Schmitz, S. 113 f.

1053 Spindler/Schmitz/Geis/Schmitz, \6 TDDSG Rn. 47; Scholz, S. 258; Roßnagel/Scholz, MMR 2000, 723.

1054 Scholv, S. 258.

1055 Schols, S. 258.

$1056 \mathrm{Zu}$ den Voraussetzungen einer wirksamen Einwilligung s. B.III.2.b)aa) Exkurs: Anforderungen an die Einwilligung.

1057 Zscherpe, K\&R 2005, 266; Kramer/Herrmann, Rn. 148; Spindler/Schmitz/Geis/Schmitr, \6 TDDSG Rn. 1; Schmitr, in: Hoeren/Sieber, Hdb. MultimediaR, Teil 16.4 Rn. 124.

1058 Roßnagel/Dix/Schaar, RMD, Teil 3, § 6 TDDSG Rn. 151. 
cherung dieser Daten über die Erforderlichkeit für die Abrechnung hinaus darf nicht erfolgen. ${ }^{1059}$ Die Erforderlichkeit muss dabei, ebenso wie bei den Bestands- und Nutzungsdaten, eng ausgelegt werden und richtet sich nach dem jeweiligen Vertragsverhältnis hinsichtlich der Telemediendienstenutzung. ${ }^{1060}$ Geht es bei der Abrechnung um die Bezahlung von Waren, so findet $\ 15$ Abs. 4 TMG keine Anwendung und die Vorschriften des Bundesdatenschutzgesetzes müssen beachtet werden. ${ }^{1061}$

\section{Zulässigkeit der Erhebung und Verwendung}

Bei kostenlosen Diensten ist eine Erhebung und Verwendung von Abrechnungsdaten schon aufgrund der Natur der Sache nicht erforderlich. ${ }^{1062}$ Daraus folgt allerdings nicht der Umkehrschluss, dass bei entgeltlichen Diensten immer eine Erforderlichkeit zur Erhebung von Abrechnungsdaten gegeben ist. Bei entgeltlichen Diensten muss vielmehr anhand des Einzelfalls entschieden werden. Insbesondere konkretisiert $\ 15$ Abs. 6 TMG dies dahingehend, dass die Abrechnung über die Inanspruchnahme von Telemedien Anbieter, Zeitpunkt, Dauer, Art, Inhalt und Häufigkeit bestimmter von einem Nutzer in Anspruch genommener Telemedien nicht erkennen lassen darf, es sei denn, der Nutzer verlangt einen Einzelnachweis. Dies entspricht und konkretisiert den Grundsatz der Datenvermeidung des $₫ 3$ a BDSG. ${ }^{1063}$ Grundsätzlich können Verbindungszeiten und Angaben über die Zahlungsmodalitäten des Nutzers erforderliche Abrechnungsdaten sein. ${ }^{1064}$ Je nach den Besonderheiten des Einzelfalls können davon auch nur einzelne Daten erforderlich sein. Uneinigkeiten bestehen beispielsweise hinsichtlich der Notwendigkeit der Speicherung der IP-Adresse im Rahmen einer nutzungsabhängigen Flatrate über den Vorgang der Verbindung hinaus. ${ }^{1065}$ Dieser Streit bedarf für den Untersuchungs-

\footnotetext{
1059 Engel-Flechsig, DuD 1997, 14.

1060 Dix, DuD 2003, 235; Schmitr, S. 125; Scholz, S. 250; Spindler/Schmitz/Geis/Schmitz, $\int 6$ TDDSG Rn. 33; Spindler/Schuster/Spindler/Nink, \15 Rn. 9.

1061 Scholy, S. $250 \mathrm{f}$.

1062 Roßnagel/Roßnagel, Hdb. DatenschutzR, Kap. 7.9 Rn. 79; Spindler/Schuster/Spindler/Nink, \15 Rn. 9; Scholr, S. 251.

1063 Roßnagel/Dix/Schaar, RMD, Teil 3, \6 TDDSG Rn. 218; Schols, S. 251.

1064 Scholv, S. 250.

1065 Die h.M. sieht die Speicherung der IP-Adresse als nicht erforderlich an, s. Dix, DuD 2003, 235 f.; Schmitz, Anm. zu RP Darmstadt, MMR 2005, 213 f., MMR 2005, 214 (215); Scholv, S. 251, 258; Schaar, Rn. 444; Roßnagel/Roßnagel, Hdb. DatenschutzR, Kap. 7.9 Rn. 79; Roßnagel/Dix/Schaar, RMD, Teil 3, \6 TDDSG Rn. 156; Spindler/Schuster/Spindler/Nink,
} 
gegenstand aber keiner weiteren Untersuchung, da die Speicherung von IPAdressen im Rahmen der Web Service-Portal-Nutzung, wie nachfolgend aufgezeigt wird, für die Abrechnung nicht erforderlich ist.

Sind die Web Service-Portale derart ausgestaltet, dass der Portalnutzer auf der Plattform mit einer Benutzerkennung agiert, genügt es für die Abrechnung, diese Kennung zu speichern. Damit kann eine Zuordnung der Nutzungsdaten erfolgen und eine Abrechnung erstellt werden. ${ }^{1066}$ Etwas anderes könnte lediglich dann gelten, wenn die Portal- und/oder Web Service-Nutzung ohne gesonderte Registrierung erfolgen, die Dienste aber dennoch kostenpflichtig sind. In diesem Fall stellt die dynamische IP-Adresse allerdings kein für den Portalbetreiber oder den Web Service-Anbieter zuordenbares Datum dar, so dass die IP-Adresse keine unmittelbare Hilfe zur Erstellung der Abrechnung bietet. Vielmehr muss der Portalnutzer hier seine Zahlungsdaten übermitteln, um eine Abrechnung zu ermöglichen. Greift der Web Service-Anbieter auf die beim Portalbetreiber hinterlegten Daten zu, so könnte man meinen, dass hier nun eine Speicherung der IP-Adresse erforderlich ist, um den Portalnutzer den entsprechenden Daten zuordnen zu können. Aber auch dieser Gedanke geht fehl, da dynamische IP-Adressen immer wieder anderen Nutzern zugeteilt werden, eine erfolgreiche Zuordnung damit lediglich bei Portalnutzern möglich wäre, die statische IP-Adressen verwenden. Indes ist die Speicherung selbst bei statischen IP-Adressen keineswegs erforderlich. Greift der Web Service-Anbieter nämlich auf die beim Portalbetreiber hinterlegten Daten zu, so bedeutet dies, dass der Nutzer sich beim Portalbetreiber registriert hat, er also in der Regel unter einem Nickname, einer Kundennummer oder ähnlichem auf der Plattform agiert. Das bedeutet für die Web Service-Anbieter, dass es zum Zwecke der Abrechnung der Web Service-Nutzung genügt, die entsprechende Kennung des Portalnutzers zu registrieren und damit eine Verknüpfung zu den Daten, die zur Abrechnung erforderlich sind, zu ermögli-

\15 Rn. 9; Spindler/Schmitz/Geis/Schmitz, \6 TDDSG Rn. 35; hinsichtlich der Speicherung von dynamischen IP-Adressen zustimmend LG Darmstadt, MMR 2006, 330 (331); AG Darmstadt, MMR 2005, 634 (636), allerdings mit der Einschränkung, dass eine Speicherung wegen der Möglichkeit eines anderweitigen Zugangs, der dann nutzungsabhängig abgerechnet wird, zumindest bis zur Erstellung der Abrechnung erforderlich sei, nicht aber zur Durchsetzbarkeit oder Beweisbarkeit der Richtigkeit der Abrechnung. Dem für Content-Anbieter zustimmend Moos, CR 2003, 387. Dagegen tendiert das RP Darmstadt in die Richtung, dass eine Speicherung erforderlich sei, da dadurch die Fehlersicherheit der Datenverarbeitung sowie die Nachweisbarkeit und Durchsetzbarkeit von Forderungen gewährleistet würden, RP Darmstadt, MMR 2003, 213 m. Anm. Schmit\%.

1066 Roßnagel/Dix/Schaar, RMD, Teil 3, § 6 TDDSG Rn. 158. 
chen. Eine Speicherung der IP-Adresse ist für die Abrechnung der WebService-Portalnutzung daher nicht erforderlich.

Nach Erfüllung der entsprechenden Verbindlichkeit besteht eine Pflicht zur Löschung. Das ergibt sich aus dem Grundsatz der Erforderlichkeit sowie dem Gebot der Zweckbindung und darüber hinaus aus dem in $\ 12$ Abs. 1 TMG verankerten Grundsatz des Verbots mit Erlaubnisvorbehalt. 1067 Denn ist die Speicherung nicht mehr zur Erfüllung der Verbindlichkeit erforderlich, so greift auch der Erlaubnistatbestand des $\$ 15$ Abs. 4 TMG nicht mehr und eine fortwährende Speicherung ist mangels Erlaubnis unzulässig. Eine Ausnahme gilt für Abrechnungsdaten, die für die Erstellung von Einzelnachweisen über die Inanspruchnahme bestimmter Angebote auf Verlangen des Nutzers verarbeitet werden. Hier legt $₫ 15$ Abs. 7 S. 1 TMG eine Höchstfrist zur Speicherung fest, nach der diese Abrechnungsdaten höchstens bis zum Ablauf des sechsten Monats nach Versendung der Rechnung gespeichert werden dürfen. Ausnahmsweise dürfen diese Abrechnungsdaten nach $\$ 15$ Abs. 7 S. 2 TMG dann weiter gespeichert werden, wenn gegen die Entgeltforderung innerhalb der Frist Einwendungen erhoben oder diese trotz Zahlungsaufforderung nicht beglichen wurden. Die Abrechnungsdaten dürfen dann solange weiter gespeichert werden, bis die Einwendungen abschließend geklärt sind oder die Entgeltforderung beglichen ist. Verlangt der Nutzer keinen Einzelverbindungsnachweis, so sind die Daten mit Begleichung der Rechnung zu löschen.

\section{Abrechnungsdaten und Cookies}

Das Setzen von Cookies durch Web Service-Anbieter beziehungsweise deren Auslesen ist nicht für die Abrechnung erforderlich, wenn die Portale derart ausgestaltet sind, dass die Abrechnung immer zwischen Portalbetreiber und Portalnutzer erfolgt. Anders könnte es sich verhalten, wenn die Abrechnung der Web Service-Nutzung nicht mit dem Portalbetreiber, sondern direkt mit dem Portalnutzer erfolgt. Denn in diesem Fall kann sich der Web ServiceAnbieter nicht darauf verlassen, dass der Portalbetreiber für die Abrechnung der durch die Web Service-Nutzung entstandenen Kosten aufkommt. Insofern könnte man annehmen, dass er die Daten der Cookies für die Abrechnung benötigt. Cookies speichern allerdings nur bei der Nutzung anfallende Daten, nicht aber Name, Adresse, Bankverbindung etc. Diese Daten über das Nutzungsverhalten sind dem Zwecke der Abrechnung aber regelmäßig, freilich abhängig vom Geschäftsmodell des Portals, weder dienlich noch sind sie

1067 Spindler/Schuster/Spindler/Nink, \15 Rn. 10; Spindler/Schmitz/Geis/Schmitz, 』6 TDDSG Rn. 34. 
dafür erforderlich. Darüber hinaus können die hier untersuchten Portale derart eingerichtet sein, dass die Web Service-Anbieter mittels Web Services auf die Registrierungsdaten der Nutzer zugreifen können und daher selbst wenn in den Cookies zur Abrechnung grundsätzlich erforderliche Daten gespeichert würden, ein Zugriff auf diese nicht erforderlich wäre, da die Abrechnung bereits durch den Zugriff auf die Registrierungsdaten sichergestellt wird. Dies verstößt auch nicht gegen den Grundsatz der Direkterhebung, da das Übermitteln vom Begriff des Verwendens ( $\$ 3$ Abs. 5 in Verbindung mit Abs. 4 BDSG) in $\int 15$ Abs. 1 erfasst wird und somit eine Ausnahmevorschrift im Sinne des $₫ 4$ Abs. 2 S. 2 Nr. 1 BDSG vorliegt.

\section{Übermittlung von Abrechnungsdaten}

\ 15 Abs. 5 S. 1 TMG erlaubt dem Telemediendiensteanbieter die Übermittlung von Abrechnungsdaten, soweit dies zur Ermittlung des Entgelts und zur Abrechnung mit dem Nutzer - im Verhältnis zu diesem - ${ }^{1068}$ erforderlich ist. Dabei ist $\ 15$ Abs. 5 S. 1 TMG eine zusätzliche Befugnisnorm hinsichtlich der Abrechnungsdaten, die sich jedoch auf das Übermitteln beschränkt. Nicht erfasst wird das Erheben und Verwenden von Abrechnungsdaten. Übermitteln ist nach $\int 3$ Abs. 4 Nr. 3 BDSG legaldefiniert als das „Bekannt geben gespeicherter oder durch Datenverarbeitung gewonnener personenbezogener Daten an einen Dritten in der Weise, dass die Daten an den Dritten weitergegeben werden oder der Dritte zur Einsicht oder zum Abruf bereitgehaltene Daten einsieht oder abruft". Das bedeutet, dass nicht lediglich ein aktives Senden der Daten durch den Telemediendiensteanbieter unter die Übermittlungsbefugnis des $₫ 15$ Abs. 5 S. 1 TMG fällt, sondern auch der Zugriff mit Hilfe von Web Services der Web Service-Anbieter auf die notwendigen Daten, ${ }^{1069}$ die der Portalnutzer beim Portalbetreiber hinterlegt hat, unter den Begriff des Übermittelns subsumiert werden kann. Wer Dritter ist bestimmt $\int 3$ Abs. 8 S. 2 und 3 BDSG. Danach ist Dritter jede Person oder Stelle außerhalb der verantwortlichen Stelle. Keine Dritten sind der Betroffene sowie Personen und Stellen, die im Inland, in einem anderen Mitgliedstaat der Europäischen Union oder in einem anderen Vertragsstaat des Abkommens über den Europäischen Wirtschaftsraum personenbezogene Daten im Auftrag erheben, verarbeiten oder nutzen. Die externen Web Service-Anbieter im Rahmen von Web Service-Portalen lassen sich als solche Dritten qualifizieren, da sie weder

\footnotetext{
1068 Spindler/Schmitz/Geis/Schmitz, \6 TDDSG Rn. 46.

1069 So zu automatisierten Abrufverfahren generell Simitis/Dammann, S 3 Rn. 148.
} 
verantwortliche Stelle ${ }^{1070}$ oder Betroffener sind noch im Auftrag Daten verwenden.

Die Erforderlichkeit der Übermittlung, und somit eine Ausnahme vom Grundsatz der Direkterhebung nach \4 Abs. 2 S. 2 Nr. BDSG, ergibt sich immer dann, wenn die Abrechnung der Web Service-Nutzung nicht pauschal zwischen Portalbetreiber und Portalnutzern erfolgt, sondern zwischen Web Service-Anbietern und Portalnutzer, sofern der Portalnutzer ihm die erforderlichen Daten nicht selbst übermittelt. Eine solche separate Übermittlung ist zwar möglich, würde aber die barrierefreie und kostengünstige Lösung der Portale mit integrierten externen Diensten stark einschränken und somit einen Rückschritt zu der vollautomatisierten Übermittlung bedeuten. Eine separate Übermittlung ist daher im Rahmen der Web Service-Portale aus ökonomischen Gesichtspunkten weitaus ungünstiger als die Ermöglichung einer Web Service-Nutzung ohne weitere Datenübermittlungen durch den Portalnutzer. Die durch den Web Service-Zugriff übermittelten Daten unterliegen freilich einer strengen Zweckbindung. ${ }^{1071}$ Eine Verwendung zum Zwecke einer Nutzungsprofilbildung oder ähnlichem ist nicht zulässig. Die „übermittelten“ Daten dürfen lediglich zur Abrechnung der Web Service-Nutzung verwendet werden.

Zusammenfassend lässt sich festhalten, dass $₫ 15$ Abs. 4 S. 1 TMG dem Portalbetreiber die Befugnis gibt, die zur Abrechnung der Web Service-Nutzung erforderlichen Daten an den Web Service-Anbieter zu übermitteln, sofern dieser sie nicht selbst vom Portalnutzer erhält. In dem Fall würde die Erforderlichkeit entfallen. Unter die Befugnis fällt auch die Variante, dass der Portalbetreiber dem Web Service-Anbieter den Zugriff auf die entsprechenden Daten des Portalnutzers gestattet und nicht selbst aktiv übermittelt. Denn der Abruf von zum Abruf bereitgehaltener Daten wird ebenfalls vom Begriff des Übermittelns in $\ 15$ Abs. 4 S. 1 TMG erfasst und stellt insofern eine Ausnahme vom Grundsatz der Direkterhebung im Sinne des $₫ 4$ Abs. 2 S. 2 Nr. 1

1070 Verantwortliche Stelle ist nach $₫ 3$ Abs. 7 BDSG jede Person oder Stelle, die personenbezogene Daten für sich selbst erhebt, verarbeitet oder nutzt oder dies durch andere im Auftrag vornehmen lässt. Hinsichtlich der Anmeldedaten ist die verantwortliche Stelle daher nur der Portalbetreiber, nicht die Web Service-Anbieter. Etwas anderes gilt lediglich dann, wenn die Web Service-Anbieter keine selbstständigen Anbieter, die lediglich mit dem Portalbetreiber vertraglich kooperieren, sondern rechtlich unselbstständige Subunternehmen des Portalbetreibers sind, s. Roßnagel/Dix/Schaar, RMD, Teil 3, \$ 6 TDDSG Rn. 168; Gola/Schomerus, \3 Rn. 34. In diesem Fall kann die Vorschrift des $₫ 15$ Abs. 5 S. 1 TMG nicht greifen. Vielmehr kann sich der Web Service-Anbieter dann auf dieselben Vorschriften stützen, wie der Portalbetreiber.

1071 Roßnagel/Dix/Schaar, RMD, Teil 3, § 6 TDDSG Rn. 184. 
BDSG dar. Voraussetzung ist jedoch immer, dass die Daten für den Web Service-Anbieter zu Abrechnungszwecken erforderlich sind.

iii) Zusammenfassendes Resümee

Die Zulässigkeit der Erhebung von Nutzungs- und Abrechnungsdaten richtet sich gesetzlich allein nach $\ 15$ TMG. Geprägt wird die Norm, die weitestgehend als Erlaubnistatbestand ausgestaltet ist, ${ }^{1072}$ durch den Grundsatz der Erforderlichkeit. Die Erhebung und Verwendung der spezifischen Daten ist grundsätzlich nur dann zulässig, wenn sie zur Ermöglichung der Telemediennutzung oder deren Abrechnung unerlässlich sind. Die Voraussetzungen des $\$ 15$ TMG müssen in der Regel auch für die Erhebung und Verwendung von IP-Adressen beachtet werden. Eine Speicherung ist danach nur durch den Portalbetreiber und auch lediglich für die Dauer des Nutzungsvorgangs erforderlich. Die Web Service-Anbieter indes benötigen regelmäßig nicht die IPAdresse des Portalnutzers zur Ermöglichung des Web Service, so dass sie sich grundsätzlich nicht auf $₫ 15$ Abs. 1 TMG berufen können.

Der Einsatz von Cookies und Web-Bugs ist grundsätzlich zumindest temporär für die Dauer des Nutzungsvorgangs zulässig, sofern der Portalnutzer nicht auf dem Portal registriert ist und kein Agieren unter einem zuordenbaren Pseudonym erfolgt. Anders verhält es sich dagegen, wenn die Portalnutzung eine Registrierung voraussetzt oder zulässt. In diesem Fall ist ein Einsatz von Cookies und Web-Bugs durch den Portalbetreiber nur dann zulässig, wenn es der Verbindungssteuerung eines einheitlichen Nutzungsvorgangs dient, beispielsweise um den aktuellen Inhalt des Warenkorbs während des gesamten Einkaufsvorgangs verfügbar zu halten. Ein Auslesen der vom Portalbetreiber gesetzten Cookies durch die Web Service-Anbieter ist hingegen regelmäßig weder zur Ermöglichung der Web Service-Nutzung noch zur Abrechnung dieser erforderlich und daher auch nicht durch $₫ 15$ Abs. 1 TMG als zulässige Handlung gedeckt. Setzen Web Service-Anbieter Web-Bugs ein, so sind dadurch nur anonyme Nutzungsdatenerhebungen zulässig. Ein Auslesen der Cookies, die der Portalbetreiber beim Portalnutzer gesetzt hat, ist indes auch nicht mit Hilfe von Web-Bugs zulässig.

Auch die Bildung von Nutzungsprofilen ist von Gesetzes wegen lediglich unter den strengen Voraussetzungen des $₫ 15$ Abs. 3 TMG sowie gegebenenfalls

1072 Wobei allerdings $\int 15$ Abs. 3 TMG, s. B.IV.2.b)bb)i) Nutzerprofile, u. $\int 15$ Abs. 5 S. 4, s. B.IV.2.b)bb)i Übermittlung anonymisierter Nutzungsdaten, Begrenzungen der ansonsten freien Verwendung nicht personenbezogener Daten darstellen. 
denen des $₫ 15$ Abs. 1 TMG zulässig, wenn der Vertragsgegenstand entsprechend freiwillig und beidseitig vereinbart wurde und die Nutzungsprofilbildung zur Erfüllung des Vertragsverhältnisses unerlässlich ist. Dies schließt insbesondere anbieterübergreifende Nutzungsprofile aus. Web ServiceAnbieter dürfen daher folgerichtig auch nicht zum Zwecke der Nutzungsprofilbildung auf Nutzungsdaten, die der Portalbetreiber zur Ermöglichung der Portalnutzung erhoben hat, zugreifen. Eine Übermittlung von Nutzungsdaten durch den Portalbetreiber an die Web Service-Anbieter ist nach $\int 15$ Abs. 5 S. 4 TMG lediglich in anonymisierter Form und einzig zum Zwecke der Marktforschung zulässig. Datenumgang außerhalb dieser Vorschriften ist nur mit einer wirksamen Einwilligung ${ }^{1073}$ des Betroffenen zulässig.

\section{cc) Inhaltsdaten}

Während Bestands-, Nutzungs- und Abrechnungsdaten ausdrücklich im Telemediengesetz erwähnt werden, fehlt eine Erwähnung der Inhaltsdaten. Ob diese dennoch vom Anwendungsbereich des Telemediengesetzes erfasst werden, ist umstritten. Einigkeit besteht indes darüber, was unter Inhaltsdaten zu verstehen ist. Inhaltsdaten sind alle Daten, die mit Hilfe eines Telemediendienstes übermittelt werden, um die durch den Telemediendienst begründeten Leistungs- und Rechtsverhältnisse zu erfüllen. ${ }^{1074}$

\section{i) Einordnung}

Teile der Literatur ordnen die Inhaltsdaten dem Telemediengesetz zu, mit der Begründung, dass sie ein Unterfall der Nutzungsdaten seien und sich dies aus \12 Abs. 1 TMG ( 3 Abs. 1 TDDSG a.F.) ergebe. ${ }^{1075}$ Das übrige Schrifttum sieht Inhaltsdaten gerade nicht als Unterfall der Nutzungsdaten und subsumiert sie daher, mangels fehlender Spezialregelung, unter den Anwendungsbereich des Bundesdatenschutzgesetzes. ${ }^{1076}$

1073 S. zu den Anforderungen an eine wirksame Einwilligung B.III.2.b)aa) Exkurs: Anforderungen an die Einwilligung.

1074 Spindler/Schmitz/Geis/Schmitz \3 TDDSG Rn. 8; Roßnagel/Roßßnagel, Hdb. DatenschutzR, Kap. 7.9 Rn. 59; Scholr, S. 258; Stadler, S. 169; Spindler/Schuster/Spindler/Nink, \15 TMG Rn. 3.

1075 So z.B. Spindler/Schmitz/Geis/Schmitz, \6 TDDSG Rn. 18, 20 f.; Schmitz, in: Hoeren/Sieber, Hdb. MultimediaR, Teil 16.4 Rn. 140 ff.; Imhof, CR 2000, 113 ff.

1076 Roßnagel/Roßnagel, Hdb. DatenschutzR, Kap. 7.9 Rn. 37; Roßnagel/Bizer, RMD, Teil 3, \3 TDDSG Rn. 61; jurisPK-Internetrecht/Heckmann, Kap. $1.14 \quad$ Rn. 9; Spindler/Schuster/Spindler/Nink, \15 TMG Rn. 3; Niedermeier, in: Gounalakis, Rhb. Electronic Busi- 
Legt man die unumstrittene Definition von Inhaltsdaten zugrunde, so wird deutlich, dass Inhaltsdaten gerade nicht erforderlich sind, um die Inanspruchnahme von Telemediendiensten zu ermöglichen. Dies ist aber Voraussetzung für eine Einordnung als Nutzungsdaten nach der Legaldefinition des $₫ 15$ Abs. 1 S. 1 TMG. Darüber hinaus fallen Inhaltsdaten weder notwendig während noch durch die Nutzung der Telemedien an. Insofern entsprechen sie nicht der Legaldefinition der Nutzungsdaten. Legt man beispielsweise die Daten, die der Portalnutzer zur Bestellung einer Ware eingibt, zugrunde, so werden diese Daten zwar technisch übermittelt, sie dienen aber nicht der Übermittlung selbst, sondern vielmehr dem Zustandekommen eines Kaufvertrags, welchen der Telemediendienst ermöglicht. ${ }^{1077}$ Das bedeutet, dass lediglich die Daten, die beim Versenden des Bestellformulars anfallen, Nutzungsdaten im Sinne des Telemediengesetzes sind. Daten, die lediglich die Bestellung selbst und somit den Vertragsgegenstand betreffen, sind hingegen Inhaltsdaten, die unter den Anwendungsbereich des Bundesdatenschutzgesetzes subsumiert werden. Dem lässt sich zwar entgegen halten, dass dadurch ein einheitlicher Lebensvorgang auseinander gerissen wird, ${ }^{1078}$ dies kann aber nicht dazu führen, dass man künstlich versucht, unterschiedliche Sachverhalte in eine Definition zu pressen, obwohl der Wortlaut dem eindeutig entgegensteht. Darüber hinaus werden auch in anderen Bereichen einheitliche Lebensvorgänge auseinander gerissen, weil es der Wortlaut erfordert: ${ }^{1079}$ so fallen beispielsweise bei der Nutzung eines Mobilfunktelefons zugleich Nutzungs- und Bestandsdaten an. ${ }^{1080}$ Ferner entspricht die Einordnung von Inhaltsdaten in das Bundesdatenschutzgesetz auch dem Telos des Telemediengesetzes. Dieses soll den besonderen Risiken, die durch die Erbringung von Leistungen durch Telemedien entstehen, Rechnung tragen, nicht aber datenschutzrechtliche Schranken für Kundenbeziehungen errichten. ${ }^{1081}$ Andernfalls würden hinsichtlich der Be-

ness, $\int 19$ Rn. 33; Roßnagel, in: Roßnagel/Banzhaf/Grimm, Teil B S. 138; Scholz, S. 156, 258; Selk, S. 65; Kramer/Herrmann, Rn. 194; Jandt/Laue, K\&R 2006, 320; Scböttle, BRAK-Mitt 2004, 255; Zscherpe, K\&R 2005, 266; Gola/Mütblein, RDV 1997, 196; Bäumler, DuD 1999, 259; 18. Tätigkeitsbericht des Bundesbeauftragten für Datenschutz BT-Drs. 14/5555.

1077 S. Schöttle, BRAK-Mitt 2004, 255; Roßnagel, in: Roßnagel/Banzhaf/Grimm, Teil B S. 159 f.; Stadler, S. 169; Spindler/Schuster/Spindler/Nink, \15 TMG Rn. 3; i.d.S. auch Stellungnahme BR, BT-Drs. 13/7385, S. 53.

1078 I.d.S. Schmitr, in: Hoeren/Sieber, Hdb. MultimediaR, Teil 16.4 Rn. 151.

1079 I.d.S. allgemein Scholz, S. 157.

1080 Stadler, S. 166 ff.; Spindler/Schuster/Spindler/Nink, \15 TMG Rn. 3.

1081 Kramer/Herrmann, Rn. 194. 
stelldaten für telefonisch oder postalisch aufgegebene Bestellungen andere Datenschutzbedingungen gelten, als für elektronisch aufgegebene Bestellungen. Eine solche Ungleichbehandlung kann nur hinsichtlich der bei der Bestellung anfallenden Daten gerechtfertigt sein, da bei diesen im elektronischen Bereich höhere Risiken für das Recht auf informationelle Selbstbestimmung bestehen als in der analogen Welt, nicht aber bei den Daten der Bestellung selbst.

\section{ii) Inhaltsdaten und die Web Service-Nutzung}

Im Rahmen der Web Service-Nutzung gestaltet sich die Abgrenzung zwischen Inhalts- und Nutzungsdaten problematischer. Relevante abzugrenzende Daten sind die Daten einer Suchanfrage über einen Web Service, wie beispielsweise die Eckdaten einer Reise (Ort, Zeitraum, Präferenzen rund um den Komfort etc.), der Ort oder die Postleitzahl für eine Wetterauskunft und Standortdaten für eine Navigation. $\mathrm{Ob}$ es sich bei diesen Daten um Inhaltsdaten, die lediglich mit Hilfe des Telemediums Web Service übermittelt werden, oder gar um Nutzungsdaten handelt, weil sie bei die Ermöglichung der Web ServiceNutzung unerlässlich sind, bedarf einer weiteren Erörterung.

Für eine Einordnung als Inhaltsdaten spricht der Charakter der übermittelten Daten. Diese ähneln in gewisser Form denen einer Warenbestellung und werden, wie auch eine elektronische Warenbestellung, mit Hilfe einer Software übermittelt. Hingegen deutet die Funktionsweise von Web Services eher auf eine Einordnung als Nutzungsdaten hin. Denn die Art und Weise der Suchanfrage, die vollautomatisiert erfolgt, ist allein mittels der Nutzung des konkreten Telemediums „Web Service“ möglich. Die Suchanfrage mit den Web Services lässt sich teilweise mit den so genannten „Location Based Services“ vergleichen. ${ }^{1082}$ Bei diesen standortbasierten Diensten stellt die gesamte personalisierte und ortsbezogene Dienstleistung das eigentliche Ziel des Telemediums dar, so dass Location Based Services unter den Anwendungsbereich des Telemediengesetzes subsumiert werden. ${ }^{1083}$ Auch bei dem mittels Web Service erbrachten Dienst stellt die Suchanfrage selbst das Ziel des Web Service dar. Die „Suchdaten“ sind insofern notwendig für die Ermöglichung der

1082 LBS sind Mobilfunk-Online-Dienstleistungen, die dem Nutzer entweder in Abhängigkeit von seinem Standort zur Verfügung gestellt werden, die gleichen Anfragen in Abhängigkeit des Standorts des Nutzers unterschiedlich beantworten oder deren Inhalt sich auf den Standort anderer Nutzer beziehen, s. dazu ausführlich mit weiteren Beispielen Jandt/Laue, K\&R 2006, 316; Jandt, MMR 2007, 74; Gola, NZA 2007, 1142; Hellmich, MMR 2002, 152.

1083 Jandt/Laue, K\&R 2006, 320. 
Diensteerbringung mit Hilfe des Web Service. In gewisser Weise bedingt dabei die Suchanfrage die vollautomatisierte Diensteerbringung und umgekehrt, denn bei der Diensteerbringung mit Hilfe von Web Services wird im Gegensatz zu herkömmlichen elektronischen Bestellungen auch gerade der Inhalt technisch verarbeitet, da die Verarbeitung rein automatisiert zwischen Maschinen abläuft. Man kann sogar davon sprechen, dass die Suchanfrage unerlässlich für die Erbringung des Dienstes mittels Web Service ist. Eine Trennung von Inhalt und Übermittlung ist folglich nicht möglich. Die spezifischen Risiken der Nutzung des Telemediums Web Service beziehen sich aufgrund der technischen Funktionsweise sogar in besonderem Maße auf den Inhalt der Suchanfrage. Die Datenschutzvorschriften des Telemediengesetzes dienen gerade der Kontrolle der durch die Nutzung von Telemedien entstehenden Risiken. Daher ist es unerlässlich, das Telemediengesetz auch auf die Daten der Suchanfrage anzuwenden. Eine Anwendung des Bundesdatenschutzgesetzes kommt hinsichtlich der „Suchdaten“ nicht in Frage.

Der gegenüber $₫ 15$ TMG weniger strenge $\ 28$ BDSG findet daher keine Anwendung. Dies bedeutet starke Einschränkungen für den Umgang mit den Daten der Suchanfrage. Während $\ 28$ Abs. 1 Nr. 1 BDSG das Erheben, Speichern, Verändern oder Übermitteln personenbezogener Daten oder ihre Nutzung als Mittel für die Erfüllung eigener Geschäftszwecke bereits zulässt, wenn es der „Zweckbestimmung eines Vertragsverhältnisses oder vertragsähnlichen Vertrauensverhältnisses mit dem Betroffenen dient" oder nach Nr. 2 „zur Wahrung berechtigter Interessen der verantwortlichen Stelle erforderlich ist und kein Grund zu der Annahme besteht, dass das schutzwürdige Interesse des Betroffenen an dem Ausschluss der Verarbeitung oder Nutzung überwiegt", ist das Erheben und Verwenden von Nutzungsdaten nach $\ 15$ Abs. 1 S. 1 TMG nur zulässig, wenn es für die Inanspruchnahme des Telemediums Web Service erforderlich und damit unerlässlich ist. Der Datenumgang nach \15 Abs. 1 S. 1 TMG ist daher in deutlich engerem Rahmen zulässig.

\section{c) Exkurs: Die Einwilligung von Minderjährigen}

Die Einwilligung wird im Rahmen von Web Service-Portalen praktisch den Hauptrechtfertigungsgrund für den Eingriff in das Recht auf informationelle Selbstbestimmung darstellen, da die gesetzlichen Datenverarbeitungserlaubnisse sehr eng gefasst sind. Da auch Minderjährige rein faktisch in der Lage sind, Web Service-Portale zu nutzen und dabei eine Einwilligung in die Datenverarbeitung abzugeben, muss untersucht werden, inwiefern dies überhaupt rechtlich zulässig ist. 
Die Problematik der Einwilligung durch Minderjährige beginnt bereits bei der umstrittenen Rechtsnatur der datenschutzrechtlichen Einwilligung. Während eine Auffassung im Schrifttum die Einwilligung als rechtsgeschäftliche Erklärung ${ }^{1084}$ qualifiziert, ordnet der andere Teil der Literatur sie als Realakt ${ }^{1085}$ ein. Geht man davon aus, dass die Einwilligung eine rechtsgeschäftliche Erklärung darstellt, so finden die $\iint 105$ ff. BGB Anwendung und es muss grundsätzlich auf den Willen der Eltern abgestellt werden. ${ }^{1086}$ Qualifiziert man die Einwilligung hingegen als Realakt, finden die $\int S 104$ ff. BGB mangels Analogiefähigkeit grundsätzlich keine Anwendung. ${ }^{1087}$ Dies ergibt sich daraus, dass Realakte einen natürlichen Willen begründen und einen solchen auch Geschäftsunfähige haben können. Minderjährige können nach dieser Auffassung, wenn und soweit sie über die notwendige Einsichtsfähigkeit verfügen, wirksam in die Datenverwendung einwilligen.

Bei der Einwilligung willigt der Betroffene in einen Eingriff in das Allgemeine Persönlichkeitsrecht ein. Eine Einwilligung stellt daher eine Rechtfertigung des an sich unzulässigen Eingriffs in das Recht auf informationelle Selbstbestimmung dar. Dabei wird nicht, wie bei der rechtsgeschäftlichen Erklärung, auf einen rechtsgeschäftlichen Erfolg abgezielt, sondern vielmehr eine Art „Bestimmung“"1088 über das Allgemeine Persönlichkeitsrecht getroffen. ${ }^{1089}$ Konse-

1084 LG Bremen, DuD 2001, 620; Helfrich, in: Hoeren/Sieber, Teil 16.1 Rn. 52; Simitis/Simitis, $\ 4$ a Rn. 10; Spindler/Schmitz/Geis/Schmitr, \4 TDDSG Rn. 15; Fröble, S. 234; Klass, AfP 2005, 511.

1085 Zscherpe, MMR 2004, 724; Gola/Schomerus, \4a Rn. 10; Spindler/Schuster/Spindler/Nink, \4a BDSG Rn. 2; Scholz, S. 281, der von „tatsächlicher Handlung“ spricht; dies voraussetzend Arlt, MMR 2007, 684 und Schaar, Rn. 579. Mit etwas anderer Terminologie: Roßnagel/Holznagel/Sonntag, Hdb. DatenschutzR, Kap. 4.8 Rn. 21 ff., die die Einwilligung als „geschäftsähnliche Handlung“ einordnen, aber auch festhalten, dass sich die Erklärung auf eine tatsächliche Handlung bezieht. Durch die Einordnung als geschäftsähnliche Handlung sind allerdings, im Gegensatz zur Einordnung als Realakt, auch die $\$ \int 105$ ff. BGB entsprechend anwendbar, s. Ulrici, NJW 2003, 2054; Bamberger/Roth/Wendtland, \105 Rn. 3;

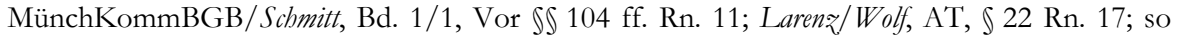
dass die Einordnung als geschäftsähnliche Handlung praktisch die gleiche Konsequenz hat, wie eine Einordnung als rechtsgeschäftliche Erklärung.

1086 Ausnahmen von diesem Grundsatz bestehen nur dann, wenn der Minderjährige durch die Willenserklärung lediglich einen rechtlichen Vorteil erlangt (\$107 BGB) oder der so genannte Taschengeldparagraph (\$110 BGB) greift, s. Laren₹/Canaris, AT, \25 Rn. 16 ff.

1087 Larenz/Wolf, AT, $\ 22$ Rn. 21 f.; Bamberger/Roth/Wendtland, \105 Rn. 3; Erman/Palm, Einl. \S 104 ff. Rn. 7; Spindler/Schuster/Spindler/Nink, \4a BDSG Rn. 2.

1088 Ohne dabei freilich eine absolute Herrschaft über die Daten begründen zu können.

1089 Spindler/Schuster/Spindler/Nink, \ 4a BDSG Rn. 2. 
quenterweise muss die Einwilligung als Realakt eingeordnet werden, mit der Konsequenz, dass auch Minderjährige, mit der notwendigen Einsichtsfähigkeit, ${ }^{1090}$ wirksam in die Datenverarbeitung einwilligen können. Sofern Minderjährige Web Service-Portale nutzen, können sie dementsprechend auch grundsätzlich in eine umfassende Datenverarbeitung einwilligen.

\section{aa) Wirksamkeit des Portalnutzungsvertrags}

Nutzen Minderjährige registrierungsfreie Web Service-Portale, so können Portalbetreiber und Web Service-Anbieter nach eingeholter Einwilligung in die Datenverarbeitung auch Profile über das Nutzungsverhalten des Minderjährigen erstellen und diesen dann gezielt Werbung und Angebote zukommen lassen, die sich an Altersgruppenspezifika in Kombination mit seinen individuellen Präferenzen anhand des Nutzungsverhaltens orientieren.

Ist indes eine Registrierung erforderlich, so stellt bereits die Registrierungspflicht - noch vor dem Einholen einer Einwilligung in die Datenverarbeitung - die erste Hürde für die Datenverarbeitung dar. Denn Minderjährige können sich nur dann wirksam registrieren, wenn ihre Eltern eingewilligt haben oder die Registrierung lediglich einen rechtlichen Vorteil für sie darstellt $(\mathbb{} 107$ BGB). Da ein lediglich rechtlicher Vorteil bereits dann nicht gegeben ist, wenn durch die Willensklärung, hier in Form der Registrierung, zugleich Pflichten ${ }^{1091}$ geschaffen werden, ${ }^{1092}$ erscheint die Zulässigkeit der Registrierung, sofern die Eltern nicht zugestimmt haben, fraglich. Wird bereits die Nutzung des Portals an ein Entgelt geknüpft, so kann keineswegs von einem lediglich rechtlichen Vorteil gesprochen werden, da der vorteilhaften Portalnutzung eine Entgeltpflicht gegenübersteht. ${ }^{1093}$ Sind dem Minderjährigen Mittel, in Form des Taschengelds, zur freien Verfügung überlassen worden, so kann der Nutzungsvertrag jedoch nach $\ 110$ BGB wirksam sein, wenn der Minderjährige das Nutzungsentgelt mit den überlassenen Mitteln bewirkt. Geschäfte nach $\ 110$ BGB werden aber erst dann wirksam, wenn der Minderjährige die Leistung tatsächlich erfüllt hat, da der Minderjährige durch das Geschäft nicht Schuldner werden soll. ${ }^{1094}$ Die Wirksamkeit des Vertrags hängt daher zum

\footnotetext{
1090 Hinsichtlich der Einsichtsfähigkeit gelten individuelle Maßstäbe, s. Arlt, MMR 2007, 684 f.

1091 Dabei müssen sowohl Haupt- als auch grds. Nebenpflichten berücksichtigt werden.

1092 MünchKommBGB/Schmitt, Bd. 1/1, \107 Rn. 29; Erman/Palm, \107 Rn. 5; Laren₹/Wolf, AT, \ 25 Rn. 19.

1093 BGH, NJW 2003, 514; Laren₹/Wolf, AT, \25 Rn. 19; Bamberger/Roth/Wendlandt, \107 Rn. 4.

1094 MünchKommBGB/Schmitt, Bd. 1/1, \110 Rn. 1; Staudinger/Knothe, $₫ 110$ Rn. 9; Derleder/Thielbar, NJW 2006, 3237; Klees/Lange, CR 2005, 630; Stacke, NJW 1991, 876.
} 
einen von der Bewirkung durch den Minderjährigen und zum anderen von den Zahlungsmodalitäten des Nutzungsvertrags ab. Handelt es sich um einen Nutzungsvertrag, bei dem jeweils kontinuierlich in bestimmter Taktung Nutzungsgebühren (monatlich, vierteljährlich, halbjährlich etc.), auf einen bestimmten Zeitraum (Ein- bis Zwei-Jahres Vertrag oder ähnliches), fällig werden, so kann maximal Teilwirksamkeit des Vertrags vorliegen, immer für die bereits erfolgte Zahlung, hinsichtlich der übrigen Gebühren bleibt der Vertrag nach $\int 108$ Abs. 1 BGB schwebend unwirksam. ${ }^{1095}$ Werden die Nutzungsgebühren hingegen einmalig beglichen, so wird der Vertrag durch die Zahlung des Minderjährigen nach $\ 110$ BGB wirksam.

Fehlt es indes an einem zur freien Verfügung überlassenen Taschengeld, so ist der Nutzungsvertrag unwirksam. Die Unwirksamkeit des Nutzungsvertrags hat keine Auswirkung auf die Wirksamkeit einer etwaigen Einwilligung in die Datenverarbeitung. Da diese als Realakt unabhängig von der Wirksamkeit der Willenserklärung hinsichtlich des Nutzungsvertrags ist, können sowohl Portalbetreiber als auch gegebenenfalls Web Service-Anbieter trotz der Unwirksamkeit des Nutzungsvertrags im Rahmen der Einwilligung das Nutzungsverhalten des Minderjährigen aufzeichnen und gegebenenfalls Profile erstellen. Insoweit fallen die zivilrechtliche Wirksamkeit des Vertrags und die datenschutzrechtliche Wirksamkeit der Einwilligung auseinander. ${ }^{1096}$

Handelt es sich um einen unentgeltlichen, registrierungspflichtigen Vertrag, so hat der Minderjährige zwar keine Nachteile durch eine etwaige Entgeltzahlung, jedoch treffen ihn zumindest hinsichtlich der Portalnutzung Rücksichtnahmepflichten sowie die „Pflicht“ zur Beachtung der Portalregeln. Bei Missachtung dieser „Spielregeln“ ist neben dem Ausschluss vom Portal freilich eine Haftung nicht ausgeschlossen. Würde allerdings jeder irgendwie geartete rechtliche Nachteil darunter fallen, so gäbe es de facto gar keine einwilligungsfreien Geschäfte und somit auch keinen lediglich rechtlichen Vorteil, da letztlich irgendwie geartete rechtliche Nachteile bei keinem Rechtsgeschäft auszuschließen sind. ${ }^{1097}$ Nach der überwiegenden Ansicht wird als lediglich rechtlicher Nachteil daher nur der Nachteil eingestuft, der eine unmittelbare

\footnotetext{
1095 Staudinger/Knothe, \110 Rn. 10; Klees/Lange, CR 2005, 630 f.; Derleder/Thielbar, NJW 2006, 3237.

1096 I.d.S. auch Arlt, MMR 2007, 684 f.; Zscherpe, MMR 2004, 724; Spindler/Schuster/Spindler/Nink, $\int 4$ a BDSG Rn. 2.

1097 Stürner, AcP 173 (1973), 416 f.; Staudinger/Knothe, \107 Rn. 5.
} 
Folge des Rechtgeschäfts darstellt. ${ }^{1098}$ Etwaige Haftungen aufgrund von Verstößen gegen die Portalordnung sind jedenfalls keine unmittelbaren Folgen des Portalnutzungsvertrags, daher bedarf es im Fall eines registrierungspflichtigen aber unentgeltlichen Portalnutzungsvertrags keiner Einwilligung durch den gesetzlichen Vertreter.

\section{bb) Anforderungen}

Ein spezieller Minderjährigenschutz hinsichtlich der Risiken durch die Datenverarbeitung für die informationelle Selbstbestimmung über die normalen Datenschutzvorschriften hinaus, existiert nicht. Verfügt der Minderjährige daher über die notwendige Einsichtsfähigkeit, so kann er - unabhängig von der Wirksamkeit etwaiger Nutzungsverträge - wirksam in die Datenverarbeitung einwilligen. Hinsichtlich der Einsichtsfähigkeit gibt es keine starren, mit dem Strafrecht oder der Geschäftsfähigkeit vergleichbaren, Grenzen. Abzustellen ist immer auf die individuelle Einsichtsfähigkeit. Da aber insbesondere die elektronische Datenverarbeitung ein äußerst unüberschaubares intransparentes Feld darstellt und für Kinder häufig auch dann nicht verständlich ist, wenn es kindgerecht aufbereitet ist, ${ }^{1099}$ empfiehlt es sich in der Praxis dennoch, gewisse Richtwerte als objektive Standards festzulegen und so einem Missbrauch mit dem Alter entgegenzuwirken. Dabei können bestehende Normen, die an das Alter der Minderjährigen knüpfen, als Richtwerte herangezogen werden. So kann man Kinder bis zum siebten Lebensjahr in Anlehnung an $\ 828$ Abs. 1 BGB und $\ 10$ StGB in Verbindung mit $\iint 1$ Abs. 2, 3 JGG keine Einsichtsfähigkeit in dieser Hinsicht zusprechen, ${ }^{1100}$ gleiches sollte in Anlehnung an $\ 19$ StGB auch bis zum 14. Lebensjahr gelten. ${ }^{1101}$ Schwieriger ist die Differenzierung zwischen dem 14. Lebensjahr und der Volljährigkeit. Per se ab dem 14. Lebensjahr von der Einsichtsfähigkeit auszugehen, erscheint hingegen unangemessen. So nimmt beispielsweise das Strafgesetzbuch in $\ 182$ StGB hinsichtlich des sexuellen Missbrauchs eine Unterscheidung zwischen der Altersstufe 16-18 Jahre und unter 16 Jahre vor. Darüber hinaus haben Mecklenburg-Vorpommern und Nordrhein-Westfalen das aktive

\footnotetext{
1098 MünchKommBGB/Schmitt, Bd. 1/1, 『107 Rn. 32; Staudinger/Knothe, \107 Rn. 8; Palandt/Heinrichs/Ellenberger, \107 Rn. 2 f; Erman/Palm, \107 Rn. 3 ff.; a.A. bspw. Stürner, AcP 173 (1973), 409, wobei die Gegenvorschläge mangels Rechtsklarheit nur schwer überzeugen können.

1099 S. Schaar, Rn. 580.

1100 So Zscherpe, MMR 2007, 724; Fröble, S. 234 f.; i.d.S. auch Scholz, S. 282.

1101 Arlt, MMR 2007, 684 f.; i.d.S. auch Scholz, S. 282.
} 
Wahlrecht bei den Kommunalwahlen auf 16 Jahre gesenkt. Dies entspricht auch dem grundsätzlichem technischen Verständnis von Jugendlichen, die durch das Aufwachsen mit den Internet und seinen Möglichkeiten häufig ein besseres (technisches) Verständnis haben als viele Erwachsene. Dies zugrunde gelegt, ist davon auszugehen, dass ab dem 16. Lebensjahr die Einsichtsfähigkeit in aller Regel vorliegen wird. ${ }^{1102}$ Das wird auch durch die Regelung des \828 Abs. 3 BGB unterstützt, die in der Regel von der Einsichtsfähigkeit des Minderjährigen ausgeht und das Fehlen als Ausnahme sieht („wenn“). Dagegen kann im Alter von 14-16 Jahren nicht regelmäßig von der erforderlichen Einsichtsfähigkeit, gerade vor dem Hintergrund der Unüberschaubarkeit der Datenverarbeitung und der fehlenden Lebenserfahrungen, die sie die inhaltlichen Konsequenzen einer solchen Einwilligung oft nur schwer überschauen lassen, ${ }^{1103}$ ausgegangen werden. Die notwendige Einsichtsfähigkeit ist lediglich dann anzunehmen, wenn entsprechende Anhaltspunkte dafür bestehen. ${ }^{1104}$

Unter Beachtung dieser Richtwerte ist trotz der mangelnden Anwendbarkeit der $\int \mathbb{S} 104$ ff. BGB ein interessengerechter Minderjährigenschutz hinsichtlich der Datenverarbeitung und dem daraus resultierenden Eingriff in die informationelle Selbstbestimmung möglich.

V. Folgen der Missachtung der Datenschutzvorgaben

Werden die datenschutzrechtlichen Regeln des Telemedien- und subsidiär des Bundesdatenschutzgesetzes missachtet, so kann dies, neben der Unwirksamkeit der Einwilligung und etwaiger Datenverarbeitungen sowie Löschungspflichten hinsichtlich bereits erhobener Daten, eine Sanktionierung mit Bußgeld zur Folge haben, das aufgrund der Summen bis zu 50.000 Euro, siehe nur \16 Abs. 3 TMG, und im Einzelfall bis zu 250.000 Euro nach $\ 43$ Abs. 3 BDSG ein durchaus scharfes Schwert darstellt und den „Datenschutzsünder“ empfindlich treffen kann. $\int 44$ BDSG enthält sogar eine Strafvorschrift, die allerdings nur dann greift, wenn eine in $₫ 43$ Abs. 2 BDSG bezeichnete vorsätzliche Handlung gegen Entgelt oder in der Absicht, sich oder einen anderen zu bereichern oder einen anderen zu schädigen, begangen wird. Da die Voraussetzungen sehr hoch sind und bereits Verstöße aus Unkenntnis oder Interpretationsunsicherheiten der datenschutzrechtlichen Bestimmungen aus dem Anwendungsbereich herausfallen, spielt diese Vorschrift, die sich auf

\footnotetext{
1102 Arlt, MMR 2007, 684 f.

1103 S. Schaar, Rn. 581.

1104 So auch Arlt, MMR 2007, $684 \mathrm{f}$.
} 
besonders hartnäckige und zielgerichtete Verstöße beschränkt, ${ }^{1105}$ in der Praxis keine bedeutende Rolle. Praktisch relevant sind daher vor allem die Ordnungswidrigkeitentatbestände, die bei vorsätzlichen oder fahrlässigen Verstößen greifen und mit Bußgeld sanktioniert werden können.

Der Tatbestand des $₫ 43$ BDSG, der sich in zwei Tatbestandskreise untergliedert, wird im Rahmen der Web Service-Portale lediglich hinsichtlich des Missbrauchs bei der Datenverarbeitung von Inhaltsdaten Anwendung finden, da die übrige Datenverarbeitung dem Telemediengesetz und somit der Vorschrift des $\$ 16$ TMG unterfällt. Dabei wird regelmäßig eher der in Abs. 3 mit „,nur“ bis zu 25.000 Euro sanktionierte $₫ 43$ Abs. 1 BDSG Anwendung finden. Von größerer Relevanz ist indes $\ 16$ Abs. 2 TMG. Danach stellen Verstöße gegen das Koppelungsverbot, die Unterrichtungspflichten des $\$ 13$ Abs. 1 S. 1 und 2 TMG, die Pflichten zu technischen und organisatorischen Vorkehrungen nach \13 Abs. 4 S. 1 Nr. 1-5 TMG, die Erlaubnistatbestände zur Erhebung von Bestands- und Nutzungsdaten sowie des Verbots der Zusammenführung von Nutzungsprofilen mit dem Träger des Pseudonyms Ordnungswidrigkeiten dar, die nach $\ 16$ Abs. 3 TMG mit bis zu 50.000 Euro sanktioniert werden können.

Exkurs: Verstoßgegen $\int 13$ Abs. 2 Nr. 1-4 TMG

Im Vergleich mit der Vorgängervorschrift des $\int 9$ TDDSG fällt auf, dass in dieser Aufzählung eine Sanktionierung des Verstoßes gegen Pflichten hinsichtlich der elektronischen Einwilligung ( $\$ 13$ Abs. 2 Nr. 1-4 TMG) fehlt. Noch nach $\int 9$ Abs. 1 Nr. 3 TDDSG führte das Nichteinhalten dieser Pflichten zu einer Ordnungswidrigkeit, die nach \9 Abs. 2 TDDSG mit bis zu 50.000 Euro Bußgeld geahndet werden konnte. Das Fehlen der Sanktionierung dieses Verstoßes mutet daher seltsam an. Die Heranziehung der Gesetzesbegründung zeigt, dass dies keine bewusste Entscheidung des Gesetzgebers gegen eine Bußgeldbewährung bei entsprechendem Pflichtenverstoß darstellen kann. Ausweislich der Gesetzesbegründung wollte der Gesetzgeber \13 Abs. 2 TMG selbst lediglich hinsichtlich des Wortlauts an die Parallelvorschrift in \94 TKG angleichen. ${ }^{1106}$ Darüber hinaus führt die Gesetzesbegründung hinsichtlich \16 TMG folgendes aus: ${ }^{1107}$

1105 Bestmann, K\&R 2003, 497.

1106 So ausdrücklich die Gesetzesbegründung zu \13 Abs. 2 TMG, BT-Drs. 16/3078, S. 16.

1107 BT-Drs. 16/3078, S. 16. 
„\$16 TMG enthält die bisherigen Bußgeldtatbestände, die bis auf redaktionelle Anpassungen unverändert übernommen wurden. In $\int 16$ Abs. 1 wird eine neue Bußgeldvorschrift im Hinblick auf das in $\ 6$ Abs. 3 enthaltene Verbot eingeführt.“

Zusammen mit der Begründung zu $₫ 13$ Abs. 2 TMG zeigt diese Ausführung, dass der Gesetzgeber am bisherigen Ordnungswidrigkeitenkatalog inhaltlich nichts ändern wollte. Auch hat sich der Gesetzeskontext der elektronischen Einwilligung im Gegensatz zum alten $\int 4$ Abs. 2 TDDSG nicht verändert. Dass der Verweis auf $\int 13$ Abs. 2 TMG dennoch fehlt, kann daher nur als Redaktionsversehen ${ }^{1108}$ gewertet werden. Im Rahmen der nächsten Novellierung des Telemediengesetzes sollte der Verweis auf $₫ 13$ Abs. 2 TMG daher aufgenommen werden. Normalerweise können Gerichte Redaktionsversehen korrigierend auslegen, ${ }^{1109}$ da hier allerdings eine Einordnung als Ordnungswidrigkeit Gegenstand ist, gilt der Grundsatz des Art. 103 Abs. 2 GG „nulla poena sine lege“. ${ }^{1110}$ Abstellend auf den Zeitpunkt der Handlung, kann ein Pflichtenverstoß der ab dem 1.3.20071111 erfolgte oder erfolgt nicht als Ordnungswidrigkeit mit dem Hinweis auf ein Redaktionsversehen und die alte Regelung in $\int 9$ Abs. 1 Nr. 3 TDDSG geahndet werden. Ohne Gesetzesänderung kann daher auch keine korrigierende Auslegung der Gerichte erfolgen. Vor diesem Hintergrund sollte eine Ergänzung des $\$ 13$ Abs. 2 TMG im Rahmen des $₫ 16$ TMG schnellstmöglich erfolgen.

VI. Rechte der betroffenen Portalnutzer

Voraussetzung für die Ausübung des Rechts auf informationelle Selbstbestimmung ist das tatsächliche Wissen um die Erhebung und Verarbeitung von personenbezogenen Daten. ${ }^{1112}$ Dem Betroffenen werden zur Stärkung des Grundrechts durch das Datenschutzrecht umfassende individuelle Kontrollund Mitwirkungspflichten an die Hand gegeben, ${ }^{1113}$ die nicht durch Rechtsge-

\footnotetext{
1108 S. zum Begriff des Redaktionsversehens Kramer, Methodenlehre, S. 126; Laren₹/Canaris, Methodenlehre, S. 219; Holzer, NZI 1999, 44.

1109 Roth-Stelow, S. 46; Laren₹/Canaris, Methodenlehre, S. 219 f.

1110 Der Wortlaut, der explizit nur von „Strafbarkeit“ spricht, erfasst auch das Ordnungswidrigkeitenrecht, st. Rspr. BVerfGE 48, 348 (371); 55, 144 (152); 81, 132 (135); 87, 399 (411); Jarass/Pieroth/Pieroth, Art. 103 Rn. 41.

1111 An diesem Tag trat das TMG mit dem neuen $₫ 16$ in Kraft, s. BGBl. I Nr. 6 v. 28.2.2007, S. 179 ff.

1112 Roßnagel/Wedde, Hdb. DatenschutzR, Kap. 4.4 Rn. 12.

1113 Scholz, S. 313; Tinnefeld/Ehmann/Gerling, S. 410 f.
} 
schäft ausgeschlossen oder eingeschränkt werden können. ${ }^{1114}$ Zentrales Sicherungsrecht ist das mit den Rechten auf Datenkorrektur und Unterrichtungspflichten der verantwortlichen Stellen verbundene Auskunftsrecht des Betroffenen. ${ }^{1115}$

\section{Unterrichtungspflicht}

$\int 13$ Abs. 1 TMG setzt das Leitbild des informierten und aufgeklärten Nutzers voraus, ${ }^{1116}$ denn nur ein solcher kann wirksam in die Datenverarbeitung einwilligen. Darüber hinaus ist eine umfassende Information über die Datenverarbeitung auch erforderlich, um dann gegebenenfalls Auskunftsrechte ausüben zu können. Um dieses Ziel zu erreichen, wird der Telemediendiensteanbieter verpflichtet, den Nutzer zu Beginn des Nutzungsvorgangs über Art, Umfang und Zwecke der Erhebung und Verwendung personenbezogener Daten in allgemein verständlicher Form zu unterrichten. Erfolgt die Datenverarbeitung im EU-Ausland muss zudem auch über den Ort der Datenverarbeitung informiert werden, innerhalb der Europäischen Union ist dies nicht notwendig. Diese Unterrichtungspflicht dient letztlich der Ermöglichung der aktiven Mitwirkung des Betroffenen an der Preisgabe seiner Daten. Dabei unterscheidet sich die Unterrichtungspflicht von der in $\int 4 a$ Abs. 1 S. 2 BDSG verankerten Hinweispflicht insofern, dass der Hinweis immer vor der Einwilligung erfolgen muss, währenddessen die Unterrichtungspflicht von der Einwilligung völlig unabhängig ist, wobei sie natürlich dennoch zeitlich zusammenfallen können. ${ }^{1117}$

Auch in anderen Stellen des Telemediengesetzes sind Informationspflichten vorgesehen. So muss der Nutzer nach $₫ 13$ Abs. 3 TMG auf sein Widerrufsrecht hingewiesen, nach $₫ 13$ Abs. 6 S. 2 TMG über mögliche anonyme oder pseudonyme Nutzungsmöglichkeiten informiert sowie auf die Widerspruchsmöglichkeit gegen das Erstellen von Nutzungsprofilen nach $\int 15$ Abs. 3 S. 2 TMG hingewiesen werden. Indes hat die Unterrichtung über die Datenlöschung nach $\ 15$ Abs. 8 S. 3 TMG nichts mit der Unterrichtungs-

\footnotetext{
1114 Schaar, Rn. 486; Tinnefeld/Ehmann/Gerling, S. 411; speziell für das Auskunftsrecht ausdrücklich \6 Abs. 1 BDSG; Simitis/Dix, \34 Rn. 3.

1115 BVerfGE 65, 1 (46) - Volkszählung; Scholz, S. 313; Roßnagel/Wedde, Hdb. DatenschutzR, Kap. 4.4 Rn. 14 ff.

1116 Gola/Mütblein, RDV 1997, 196.

1117 Schols, S. 313; Roßnagel/Bizer, RMD, Teil 3, \4 TDDSG Rn. 92 ff.
} 
pflicht des $\int 13$ Abs. 1 TMG zu tun, denn dabei handelt es sich um eine nachträgliche Pflicht, die lediglich die Überprüfung ermöglichen soll. 1118

Die Unterrichtung muss in verständlicher Form erfolgen, wobei es erforderlich ist, zu erläutern, welche Techniken wofür eingesetzt werden, wie sie funktionieren und was mit den gesammelten Daten geschieht. Eine wiederholte Unterrichtung vor jeder erneuten Nutzung ist indes nicht erforderlich, da der Unterrichtungstext ohnehin zum jederzeitigen Abruf bereitgehalten werden muss. ${ }^{1119}$ Relevanter Zeitpunkt der Unterrichtung ist „zu Beginn des Nutzungsvorgangs“. In der erstmaligen Fassung in \3 Abs. 5 TDDSG 1997 hieß es, dass die Unterrichtung ,,vor der Erhebung“ erfolgen muss. Durch die Neufassung in $\ 4$ Abs. 1 TDDSG 2001, die $\ 13$ Abs. 1 TMG entspricht, sollte die Handhabbarkeit optimiert werden, da bei Abruf des Angebots durch den Nutzer bereits eine automatische Erhebung von Nutzungsdaten erfolgen kann und eine Unterrichtung vor der Erhebung dann nicht möglich ist. ${ }^{1120}$ Diese Beweggründe entsprechen auch den vorangegangen Erläuterung, denn bereits der Aufruf einer Website führt regelmäßig zur Speicherung des Log-File, ${ }^{1121}$ daher ist es sachgerecht auf den Beginn der Nutzung abzustellen, es sei denn es ist bereits eine Unterrichtung vor der Datenerhebung für den Diensteanbieter möglich. ${ }^{1122}$

Die Vorschrift des $\int 13$ Abs. 1 TMG bezieht sich ausschließlich auf die Datenverarbeitung zur Durchführung von Telemedien. Sollen auch Inhaltsdaten erhoben werden, muss die Vorschrift des $₫ 4$ Abs. 3 S. 1 BDSG beachtet werden. Bei automatisierten Verfahren, die lediglich die Erhebung, Verarbeitung und Nutzung von Daten vorbereiten, wie Cookies, muss dennoch nach $\ 13$ Abs. 1 S. 2 TMG bereits zu Beginn des Nutzungsvorgangs unterrichtet werden. ${ }^{1123}$

Für Web Service-Portale mit und ohne Registrierungspflicht ist es aus Effektivitätsgründen sinnvoll, die Unterrichtung über sämtliche eventuellen Datenverarbeitungsvorgänge, insbesondere auch das Setzen von Cookies und dem Einsatz von Web-Bugs, in eine Datenschutzerklärung, die für den Nutzer

\footnotetext{
1118 Roßnagel/Bizer, RMD, Teil 3, \4 TDDSG Rn. 90; von Lewinski, DuD 2002, 396.

1119 BT-Drs. 14/6098, S. 28; Roßnagel/Bizer, RMD, Teil 3, \4 TDDSG Rn. 122.

1120 BT-Drs. 14/6098, S. 28.

1121 B.IV.2.b)bb) Nutzungs- und Abrechnungsdaten.

1122 S. dazu ausführlich Scholz, S. 314.

1123 S. ausführlicher B.IV.2.b)bb)i) Cookies.
} 
entsprechend dem Erfordernis der jederzeitigen Abrufbarkeit in $₫ 13$ Abs. $1 \mathrm{~S}$. 3 TMG auf dem Portal gut sichtbar, ständig abrufbar zur Verfügung steht, zu integrieren und mit einer etwaigen Einwilligung zu verbinden. Damit würde auch dem Erfordernis der Unterrichtung „zu Beginn des Nutzungsvorgangs“ Rechnung getragen.

\section{Auskunftsrecht}

Das Auskunftsrecht ist für die Wahrnehmung des Rechts auf informationelle Selbstbestimmung von fundamentaler Bedeutung, da nur das Wissen des Betroffenen, welche Daten über ihn gespeichert worden sind und wohin sie übermittelt werden, ${ }^{1124}$ die Möglichkeit eröffnet, gegen die Datenverarbeitung vorzugehen. ${ }^{1125}$ Auch die Europäische Datenschutzrichtlinie sieht vor, dass jede Person ein Auskunftsrecht hinsichtlich der sie betreffenden Daten, die Gegenstand einer Verarbeitung sind, haben muss, damit sie sich insbesondere von der Richtigkeit dieser Daten und der Zulässigkeit ihrer Verarbeitung überzeugen kann. ${ }^{1126}$ Das Auskunftsrecht steht demjenigen zu, dessen personenbezogene Daten gespeichert sind und gilt für alle gespeicherten Daten.

\13 Abs. 7 TMG enthält eine dem allgemeinen Auskunftsanspruch in $\iint 19$, 34 BDSG sowie den Landesdatenschutzgesetzen vorgehende Spezialregelung zur Auskunftserteilung bei Telemedien. Vorrang hat die Regelung nur hinsichtlich der Auskunftserteilung über Bestands-, Nutzungs- und Abrechnungsdaten. ${ }^{1127}$ Inhaltsdaten indes fallen unter den Anwendungsbereich der Vorschriften des Bundesdatenschutzgesetzes beziehungsweise der Landesdatenschutzgesetze. ${ }^{1128}$ Das Telemediengesetz regelt den Inhalt (Auskunft über die zu seiner Person oder zu seinem Pseudonym gespeicherten Daten) und die Art und Weise (auf Verlangen des Nutzers auch elektronisch) der Auskunftserteilung, im Übrigen wird nach $\ 13$ Abs. 7 S. 1 TMG auf $\ 34$ BDSG („,nach Maßgabe") verwiesen. Das bedeutet, dass sich der Auskunftsanspruch insbesondere auch an $\$ 34$ Abs. 5 BDSG zu orientieren hat. Danach muss zwar die

1124 BVerfGE 65, 1 (43) - Volkszählung.

1125 Simitis/Dix, \34 Rn. 1; Schaar, Rn. 487; Roßnagel, in: Roßnagel/Banzhaf/Grimm, Teil B S. 226; i.d.S. auch Roßnagel/Wedde, Hdb. DatenschutzR, Kap. 4.4 Rn. 38.

1126 So Erwägungsgrund 41 der Richtlinie 95/46/EG, ABl. EG L 281 v. 23.11.1995, S. 31-50.

1127 Roßnagel/Schaar, RMD, Teil 3, \4 TDDSG Rn. 450; jurisPK-Internetrecht/Heckmann, Kap. 1.13 Rn. 92; Schaar, Rn. 501.

1128 Im Rahmen der Web Service-Nutzung fallen allerdings regelmäßig keine Inhaltsdaten an, s. B.IV.2.b)cc)ii) Inhaltsdaten und die Web Service-Nutzung. 
Auskunftserteilung grundsätzlich kostenlos erfolgen, ausnahmsweise lässt Satz 2 unter den einschränkenden Voraussetzungen der Sätze 3 und 4 aber dann ein Entgelt zu, wenn der Betroffene die Auskunft gegenüber Dritten zu wirtschaftlichen Zwecken nutzen kann. Darüber hinaus greift aber auch $₫ 34$ Abs. 4 BDSG, der eine Pflicht zur Auskunftserteilung dann verneint, wenn der Betroffene nach $\int 33$ Abs. 2 S. 1 Nr. 2, 3 und 5 bis 7 BDSG nicht zu benachrichtigen ist. Die in $₫ 33$ Abs. 2 S. 1 Nr. 2, 3 und 5 bis 7 BDSG aufgelisteten Ausnahmen werden zwar den privaten Portalnutzer eher am Rande tangieren. Davon unabhängig wird der Auskunftsanspruch durch die dort aufgelisteten Ausnahmen aber sehr weitgehend ausgeschlossen, dies erscheint ohne Begründung des Gesetzgebers so nicht nachvollziehbar. ${ }^{1129}$

Problematisch ist der Zeitrahmen der Auskunftserteilung, da weder $\int 13$ Abs. 7 TMG noch $₫ 34$ BDSG dazu Angaben machen. Noch in der Vorgängervorschrift des $\int 4$ Abs. 7 TDDSG war die Rede von ,unverzüglich“, was kommentarlos in der Neufassung gestrichen wurde. Für eine zeitliche Begrenzung muss daher aufgrund des Verweises auf die in der Literatur ausgearbeiteten Grenzen des $\int 34$ BDSG zurückgegriffen werden. ${ }^{1130}$ Dort wird vertreten, dass der Betroffene aufgrund des Schutzzwecks der Norm eine - wie auch in der Formulierung des $\int 4$ Abs. 7 TDDSG a.F. - unverzügliche und schnelle Auskunftserteilung verlangen kann, da das Risiko mit der Länge der Wartezeit steigt, dass aufgrund von unrichtigen Daten beispielsweise nicht mehr rückgängig zu machende Entscheidungen zu seinem Nachteil getroffen werden. ${ }^{1131}$ So könnten Web Service-Anbieter aufgrund falscher Bonitätsdaten wichtige zeitabhängige Dienstleistungen verweigern. Unter unverzüglich wird auch noch eine aus Kostengründen gesammelte Bearbeitungszeit von ein bis zwei Wochen verstanden, unter besonderen Umständen kann auch ein längerer Zeitraum gerechtfertigt sein. ${ }^{1132}$

Der Auskunftsanspruch gilt verantwortungsunabhängig sowohl gegenüber Portalbetreiber als auch den integrierten Web Service-Anbietern. ${ }^{1133}$ Grund-

1129 Vor dem pauschalen Verweis auf $\int 34$ BDSG bereits im Gesetzgebungsverfahren warnend, Bizer, A-Drs. 16(9)530, S. 2 u. Breyer, A-Drs. 16(9)517, S. 34 f.

1130 Ebenso jurisPK-Internetrecht/Heckmann, Kap. 1.13 Rn. 98.

1131 Simitis/Dix, \ 34 Rn. 36; Gola/Schomerus, \34 Rn. 16; Roßnagel, in: Roßnagel/Banzhaf/Grimm, Teil B S. 229.

1132 Gola/Schomerus, $₫ 34$ Rn. 16; Simitis/Dix, $₫ 34$ Rn. 36; so auch für $\ 13$ Abs. 7 TMG jurisPKInternetrecht/Heckmann, Kap. 1.13 Rn. 98.

1133 So allgemein Schaar, Rn. 503. 
sätzlich erfolgt die Auskunft nach $₫ 34$ Abs. 3 BDSG schriftlich, sie kann auf Verlangen des Nutzers aber nach $₫ 13$ Abs. 7 S. 2 TMG auch auf elektronischem Wege erfolgen, sie muss dann allerdings nach $₫ 13$ Abs. 4 TMG technisch und organisatorisch abgesichert werden. ${ }^{1134}$

Viel kritisiert ist die Möglichkeit nach $\int 13$ Abs. 7 S. 1, 2. Alt. TMG Auskunft zu den unter seinem Pseudonym gespeicherten Daten zu erteilen. ${ }^{1135}$ Anknüpfend an die Regelung des $₫ 13$ Abs. 6 TMG, wonach der Diensteanbieter dem Nutzer die Nutzung der Telemedien und deren Bezahlung, soweit möglich, anonym oder unter Pseudonym ermöglich soll, steht dies im Konflikt mit $\ 15$ Abs. 3 S. 3 TMG, der eine Zusammenführung der Nutzungsprofile mit den Daten über den Träger des Pseudonyms verbietet. Um dieses Problem zu lösen, werden verschiedene Wege vorgeschlagen. Während die einen eine Auskunftserteilung gleichfalls unter Pseudonym als ausreichend ansehen, ${ }^{1136}$ wollen andere die Vorschrift derart auslegen, dass eine Information über die Aufdeckungsrisiken erfolgen muss, aufgrund derer dann im Falle eines fortwährenden Auskunftsbegehrens eine Einwilligung in die Zusammenführung gesehen wird. ${ }^{1137}$ Zwar erscheint die erste Lösung praktisch leichter handhabbar, jedoch wird dabei der Fall übersehen, dass im Falle einer Pseudonymisierung durch den Diensteanbieter, der Nutzer keine Kenntnis von dem ihm zugeordneten Pseudonym hat, so dass die Zusammenführung letztlich doch vom Diensteanbieter zu bewerkstelligen wäre, ${ }^{1138}$ was wiederum dem Verbot des $₫ 15$ Abs. 3 S. 3 TMG widerspricht. Daher ist es notwendig, die Vorschrift entsprechend verfassungskonform auszulegen und eine umfassende Informationspflicht hineinzulesen.

\section{Datenkorrektur}

Um wirksam gegen den Datenmissbrauch vorgehen zu können, sind vor allem so genannte Folgenbeseitigungsansprüche erforderlich. Solche enthält das Telemediengesetz selber nicht, jedoch gewährt \6 Abs. 1 BDSG in Verbin-

1134 Schaar, Rn. 510.

1135 S. dazu nur Scholz, S. 334; Schaar, Rn. 507 ff.; jurisPK-Internetrecht/Heckmann, Kap. 1.13 Rn. 99; Spindler/Schmitz/Geis/Schmitz, \4 TDDSG Rn. 51; Schmitz, S. 135; Roßnagel/Schaar, RMD, Teil 3, § 4 TDDSG Rn. 473 f.

1136 jurisPK-Internetrecht/Heckmann, Kap. 1.13 Rn. 100; Roßnagel/Schaar, RMD, Teil 3, 』 4 TDDSG Rn. 474; Schaar, Rn. 508; Scholz, S. 334 f.

1137 Spindler/Schmitz/Geis/Schmitz, \& 4 TDDSG Rn. 52; Schmitz, S. 135 f.

1138 Spindler/Schmitz/Geis/Schmitz, 』4 TDDSG Rn. 51. 
dung mit $₫ 35$ BDSG, ${ }^{1139}$ die über $\ 12$ Abs. 4 TMG Anwendung finden, dem Betroffenen unabdingbare Ansprüche auf Berichtigung, Löschung und Sperrung der Daten.

Sind personenbezogene Daten unrichtig, ${ }^{1140}$ so müssen sie nach $₫ 35$ Abs. 1 BDSG berichtigt werden. Durch die Formulierung wird deutlich, dass die Stelle auch von sich aus - unabhängig von einem Begehren des Betroffenen tätig werden muss, wenn sie von der Unrichtigkeit erfährt. ${ }^{1141}$ Bestehen Uneinigkeiten hinsichtlich der Unrichtigkeit, so sieht das Bundesdatenschutzgesetz in $₫ 35$ Abs. 4 eine Sperrung der Daten vor. Berichtigungsfälle treten insbesondere auf, wenn sich der Nutzer bei einer Dateneingabe, zum Beispiel im Anmeldeformular, vertippt hat ${ }^{1142}$ oder sich Bestandsdaten durch Umzug ändern. Web Service-Anbieter und Portalbetreiber müssen die unrichtigen Daten dann durch korrekte Daten ersetzen.

\35 Abs. 2 BDSG regelt, wann Daten zu löschen sind. Eine Löschung von Daten, die in $\int 3$ Abs. 4 Nr. 5 BDSG legaldefiniert wird als das Unkenntlichmachen gespeicherter personenbezogener Daten, muss grundsätzlich immer dann erfolgen, wenn ihre Speicherung unzulässig war oder wenn sie für den Zweck der Speicherung nicht mehr erforderlich sind, darunter fällt auch die Beendigung des Vertragsverhältnisses. ${ }^{1143} \mathrm{Zu}$ der Löschungspflicht gehören auch Daten, die durch den Telemediendiensteanbieter auf dem Rechner des Nutzers abgelegt wurden. ${ }^{1144}$ Die Daten in Cookies müssen vom Portalbetreiber und gegebenenfalls den Web Service-Anbietern daher im Falle einer fehlenden Einwilligung oder nach Zweckerfüllung umgehend gelöscht werden. Was die Daten der Web Service-Nutzung betrifft, so dürfen die Da-

1139 Handelt es sich um öffentliche Stellen, so findet \20 BDSG Anwendung.

1140 Die Berichtigung ist eine besondere Form der in $\int 3$ Abs. 4 Nr. 2 BDSG legaldefinierten Veränderung von Daten. Von unrichtigen Daten spricht man, wenn sie Informationen enthalten, die mit der Wirklichkeit nicht übereinstimmen oder nur ein unvollständiges Abbild der Wirklichkeit abgeben und deswegen falsch sind, darüber hinaus auch, wenn zwar Einzeldaten richtig sind, sie jedoch in einem anderen Zusammenhang verwendet werden und dadurch ein falsches Gesamtbild entsteht, s. Schol\%, S. 335 Fn. 744; Gola/Schomerus, J 20 Rn. 3.

1141 Simitis/Dix, \35 Rn. 9; Gola/Schomerus, \ 35 Rn. 3; Scholz, S. 335.

1142 Scholy, S. 336. Allerdings ist der Portalbetreiber auch aufgrund von $\int 312$ e Abs. 1 S. 1 Nr. 1 BGB gehalten, dem Kunden wirksame und zugängliche technische Mittel zur Verfügung zu stellen, mit deren Hilfe der Kunde Eingabefehler vor Abgabe seiner Bestellung erkennen und berichtigen kann.

1143 Schaar, Rn. 529; Scholy, S. 336.

1144 Schaar, Rn. 534. 
ten nur so lange vorgehalten werden wie etwaige Sekundärrechte bestehen. ${ }^{1145}$ Besteht eine Registrierungspflicht, so müssen Portalbetreiber und Web Service-Anbieter alle Daten des Portalnutzers nach Beendigung des Nutzungsvertrages umgehend löschen. ${ }^{1146}$ Eine Ausnahme ist lediglich möglich, wenn noch Abrechnungen offen sind und die Daten dafür benötigt werden. Dann dürfen allerdings auch nur die dafür erforderlichen Daten bis zur Erfüllung aufbewahrt werden, \15 Abs. 4 S. 1 TMG.

Ausnahmen hinsichtlich der Löschungspflicht bestehen in den Fällen des $₫ 35$ Abs. 3 Nr. 1-3 BDSG. In den dort genannten Fällen tritt anstelle der Löschung eine Sperrung der Daten. Sperren wird in $\int 3$ Abs. 4 Nr. 4 BDSG legaldefiniert als das Kennzeichnen gespeicherter personenbezogener Daten, um ihre weitere Verarbeitung oder Nutzung einzuschränken.

Probleme bereitet die Datenkorrektur bei Web Service-Portalen durch die Vielzahl der Beteiligten, denn berichtigen muss sie jeder Telemediendiensteanbieter, der die Daten gespeichert hat. Da im Rahmen des Web Service-Portals unter den Beteiligten eine gewisse Verbindung besteht, kann erwartet werden, dass derjenige, der von der Unrichtigkeit erfährt, auch die anderen darüber informiert oder zumindest die Information zentral an den Portalbetreiber weitergibt, der sie dann wiederum den anderen zukommen lässt; andernfalls sind die Rechte zur Datenkorrektur für den Betroffenen ein stumpfes Schwert.

\section{Zivilrechtliche Ansprüche}

Da $\int 35$ BDSG hinsichtlich auf Sperrung und Löschung gerichteter Korrekturansprüche, die sich aus der Verletzung des Allgemeinen Persönlichkeitsrechts oder einem Eingriff in das Recht am eingerichteten und ausgeübten Gewerbebetrieb begründen, abschließend ist, ${ }^{1147}$ kommen Beseitigungsan-

\footnotetext{
1145 So für den elektronischen Kauf Schol₹, S. 337.

1146 Anders wollte indes unzulässigerweise zunächst das Portal StudiVZ vorgehen. In einer ersten Version einer neuen Datenschutz-Erklärung versicherte das Portal nur, das die Daten im Falle der Löschung des Profils lediglich nicht mehr über die Plattform einsehbar seien, das Recht zur Löschung wurde dort nicht mehr erwähnt, s. dazu Heise-Newsmeldung v. 14.12.2007, abrufbar unter: http://www.heise.de/newsticker/Werbung-und-persoenliche-Daten-neue-AGB-fuerStudiVZ--/meldung/100579 <25.11.2009>. Nach zahlreichen Protesten ist nunmehr wieder ein Recht auf Löschung vorgesehen, s. http://www.studivz.net/1/policy/info/ <25.11.2009> unter Punkt 11. Mitgliedschaft beenden.

1147 BGH, NJW 1984, 1886 (1887); NJW 1986, 2505 (2507); Gola/Schomerus, \ 35 Rn. 25; Simitis/Dix, $\ 35$ Rn. 69.
} 
sprüche aus $₫ 1004$ BGB analog in Verbindung mit $₫ 823$ BGB oder $\int 823$ Abs. 1 oder 2 BGB nicht in Betracht. Indes können neben $₫ 35$ BDSG Ansprüche aus Kreditgefährdung nach \824 BGB und Auskunft wegen Kreditgefährdung greifen. ${ }^{1148}$ Darüber hinaus kann gegen künftige rechtswidrige Datenspeicherung vorbeugend Unterlassung nach \$1004 BGB analog geltend gemacht werden, ${ }^{1149}$ ferner auch Beseitigung und Unterlassung nach $\$ 8$ UWG. ${ }^{1150}$

VII. Schutzmöglichkeiten des Betroffenen

Der Vollständigkeit halber sei hier darauf hingewiesen, dass dem Nutzer neben dem Datenschutz auch eigene technische Möglichkeiten zur Verfügung stehen, um bereits eine Datenerhebung zu verhindern.

So kann der Nutzer die Einstellungen seines Browsers derart einstellen, dass ein Setzen von Cookies nicht zugelassen wird. Ist das Zulassen von Cookies Voraussetzung für die Portalnutzung, so bleibt ihm weiterhin die Möglichkeit, die Cookies bei Schließen des Browsers automatisch löschen zu lassen oder manuell zu löschen. Dieses Vorgehen verhindert zumindest, dass auch fremde Cookies, die einen solchen Zugriff zulassen, ausgelesen werden können. Darüber hinaus können im Rahmen der Einstellungen auch die Löschung des Browserverlaufs, des Cache sowie die Liste der gespeicherten URLs geregelt werden. Dadurch verhindert der Nutzer, dass die Web Service-Portale noch über die Portalnutzung hinausgehende Nutzungspräferenzen vom Nutzer erhalten. ${ }^{1151}$ Auch gegen Web-Bugs gibt es einfache Abhilfe, so sollten nur Bilder zugelassen werden, die von demselben Server wie die aufgerufene Seite stammen. ${ }^{1152}$

Das Nutzen von Anonymisierungsdiensten, wie beispielsweise JonDonym, ${ }^{1153}$ die die Herkunft des Nutzers durch das Verwenden fremder IP-Adressen verbergen, wobei der Webserver nicht direkt sondern verschlüsselt über eine oder

\footnotetext{
1148 BGH, NJW 1986, 2505 (2507); OLG Frankfurt, NJW-RR 1988, 562 ff.

1149 Gola/ Schomerus, \35 BDSG Rn. 26.

1150 In diesem Fall muss die Erhebung und Verwendung der Daten eine unlautere Wettbewerbshandlung darstellen.

$1151 \mathrm{Zu}$ weiteren Möglichkeiten s. ausführlich Spiegel, DuD 2003, 268 f.

1152 Spiegel, DuD 2003, 269.

1153 Zur Funktionsweise dieses im Rahmen des Projekts AN.ON entwickelten Anonymisierungsdienstes s. https://www.jondos.de/de/ <25.11.2009> und http://anon.inf.tu-dresden.de/ $<25.11 .2009>$.
} 
mehrere Zwischenstationen, so genannte Mixe, sendet oder die Identität des Nutzers auf sonstige Weise verschleiert wird, ${ }^{1154}$ ermöglicht dem Portalnutzer hingegen nur bedingten Schutz, da die Web Service-Portale regelmäßig eine Registrierung voraussetzen werden und auf diese Weise bereits an interessantere Daten als die IP-Adresse gelangen.

VIII. Sonstige datenschutzrechtliche Anforderungen

Der Plattformbetreiber ist letztlich die unmittelbare Kontaktperson zum Nutzer. Als Verantwortlicher, der von ihm betriebenen Plattform, obliegt im die technische Sicherheit, worunter Aufgaben wie Authentifizierung und Authentisierung fallen. ${ }^{1155}$ Neben den technischen Sicherheitspflichten obliegt ihm aber auch die Umsetzung datenschutzrechtlicher Vorkehrungen.

Darunter fällt insbesondere die Pflicht des $₫ 13$ Abs. 6 TMG, die Nutzung von Telemedien und ihre Bezahlung anonym oder unter Pseudonym zu ermöglichen, soweit dies technisch möglich und zumutbar ist. Über diese Möglichkeit muss er den Nutzer dann auch informieren. Die Erfüllung dieser Pflicht ist den integrierten Web Service-Anbietern indes nicht möglich, da sie keinerlei Verfügungsgewalt über die Plattform haben, sondern lediglich an diese lose gekoppelt sind.

Sowohl Portalbetreiber als auch den Web Service-Anbietern obliegen die technisch-organisatorischen Pflichten des $₫ 13$ Abs. 4 Nr. 1-6 TMG. Danach müssen sie durch technische und organisatorische Vorkehrungen sicherstellen, dass der Nutzer die Nutzung des Dienstes jederzeit beenden kann (Nr. 1), die anfallenden personenbezogenen Daten über den Ablauf des Zugriffs oder der sonstigen Nutzung unmittelbar nach deren Beendigung gelöscht oder in den Fällen des Satzes 2 gesperrt werden (Nr. 2), der Nutzer Telemedien gegen Kenntnisnahme Dritter geschützt in Anspruch nehmen kann (Nr. 3), die personenbezogenen Daten über die Nutzung verschiedener Telemedien durch denselben Nutzer getrennt verwendet (Nr. 4), Daten nach $\ 15$ Abs. 2 nur für Abrechnungszwecke zusammengeführt (Nr. 5) und Nutzungsprofile nach $\ 15$ Abs. 3 nicht mit Angaben zur Identifikation des Trägers des Pseudonyms zusammengeführt werden können (Nr. 6).

\footnotetext{
1154 Spindler/Schmitz/Geis/Schmitz, \4 TDDSG Rn. 46; Roßnagel/Hansen, Hdb. Datenschutz, Kap. 3.3 Rn. 94; Spindler/Schuster/Spindler/Nink, $\$ 13$ TMG Rn. 12; Federratb/Golembiewski, DuD 2004, 486; Raabe, CR 2003, 269.
}

1155 Melzer, S. 14. 


\section{Resümee: Teil 3}

Das Gesetz stellt hohe Hürden für den Umgang mit Daten auf. Dem technisch möglichen unbegrenzten Datensammeln setzt das Gesetz Grenzen. Zweckänderungen hinsichtlich der Datenverwendung sind nur mit Zustimmung des Betroffenen möglich. Auch der unbegrenzte Zugriff auf die beim Portalbetreiber gespeicherten Daten durch die Web Service-Anbieter ist keineswegs möglich. Ein Zugriff ist nur unter bestimmten Voraussetzungen zum Zwecke der Web Service-Nutzung oder gegebenenfalls der Abrechnung möglich.

Wollen Portalbetreiber und Web Service-Anbieter daher Web Service-Portale zum effektiven Direktmarketing nutzen und den Portalnutzern eine personalisierte Umgebung mit exklusiven Diensten schaffen, so sind sie regelmäßig auf das Instrument der Einwilligung angewiesen. Aber auch dabei sind strenge Anforderungen zu beachten. So darf dem Portalnutzer die Einwilligung vor allem nicht versteckt in einem Paket anderer Erklärungen und Bestimmungen „untergeschoben“ werden, um ein mögliches Veto im Falle des aufmerksamen Lesens der Datenschutzerklärung zu vermeiden. 


\section{Teil Schlussbetrachtungen}

Das Auslagern von Aufgaben und Funktionen ist in den globalisierten Strukturen der heutigen Wirtschaft unlängst gängige Praxis, da es Zeit und Kosten spart, die Konzentration auf Kernkompetenzen ermöglicht, Prozesse flexibilisiert und neue Organisationsstrukturen an die neuen Realitäten der Märkte anpasst. Durch schnelle Kommunikation und wettbewerbsintensive Märkte beschleunigt sich der Bedarf nach Anpassungen und Veränderungen, Innovationszyklen, insbesondere für Geschäftsprozesse und daraus resultierend die Informationstechnologie, werden kürzer. Aufgrund der vielfältigen Vergleichsmöglichkeiten wollen Kunden Leistungen zum besten Preis in höchster Qualität und möglichst sofort. Können Unternehmen in diesem stetigen Prozess der Verbesserung und Erneuerung von Produkten und Dienstleistungen nicht Schritt halten, kann dies schnell zum Abstieg führen. Insofern zahlt sich Innovation und Schnelligkeit im World Wide Web häufig aus. Dabei dürfen aber auch die Kosten nicht außer Acht gelassen werden. Explodieren die Ausgaben, so nützen auch Schnelligkeit und Innovation dem Unternehmen wenig.

Eine optimale Unterstützung zur Umsetzung schneller und innovativer Geschäftsprozesse bieten mit Web Services umgesetzte Serviceorientierte Architekturen, die es ermöglichen, auf bereits vorhandene Leistungen externer Anbieter zurückzugreifen und diese leicht in den eigenen Prozess zu 
implementieren. Durch die Gewährleistung von Schnelligkeit (hinsichtlich der Einbindung der Dienste) und Kostenersparnissen (keine eigene Entwicklung und Implementierung der notwendigen Anwendungen) steht der Umsetzung innovativer Geschäftsideen wenig im Wege.

Unternehmen nutzen Serviceorientierte Architekturen indes hauptsächlich innerhalb ihrer eigenen Vertrauenszirkel, also in geschlossenen Systemen, und schöpfen dadurch nicht die komplette Breite der Möglichkeiten aus, die Serviceorientierte Architekturen bieten. Unter Zugrundelegung der Ausführungen der vorliegenden Arbeit verwundert dies. Setzen sich Serviceorientierte Architekturen mit Web Services in offenen Systemen breitflächig durch und entwickelt sich daraus ein echter Wettbewerb unter den Web Service-Anbietern hinsichtlich Vielfalt und Qualität der Dienste, so würde dies für Unternehmen wirtschaftlich rentable Vorteile bedeuten.

Das Recht stellt für den Einsatz Serviceorientierter Architekturen keine unüberwindbaren Hürden. Serviceorientierte Architekturen mit Web Services lassen sich - unter Beachtung bestimmter Anforderungen - in die bestehende Rechtsordnung eingliedern. Es bestehen umfassende Regelungen auf nationaler Ebene, die den Einsatz von Serviceorientierten Architekturen ermöglichen. Die (freilich) mit einer solchen automatisierten Struktur im Mehrpersonenverhältnis entstehenden Probleme und Missbrauchsmöglichkeiten sind keine neuen Probleme. Es kann vielmehr auf Altbekanntes zurückgegriffen werden.

So ist unlängst anerkannt, dass auch vollautomatisierte Erklärungen, rechtsverbindliche Willenserklärungen sein können und ein Vertragsschluss somit auch vollautomatisiert unter den Beteiligten der Serviceorientierten Architektur zustande kommen kann. Hinsichtlich des Web Service-Nutzungsvertrags zwischen Web Service-Anbieter und Web Service-Nutzer kann zwar nicht auf einen klassischen Vertragstyp des Bürgerlichen Gesetzbuchs zurückgegriffen werden, dafür hilft aber das Konstrukt des gemischttypischen-Vertrags mit dienst- und werkvertraglichen Elementen. Besonderheiten bestehen hinsichtlich der Einbeziehung von Allgemeinen Geschäftsbedingungen, da zwischen Web Service-Anbieter und Web Service-Nutzer kein unmittelbarer Kontakt erfolgt. Der Web Service-Anbieter stellt lediglich eine Schnittstelle zur Verfügung, die der Web Service-Nutzer in seine eigene Plattform integriert oder nicht. Das Fehlen der Möglichkeit, den Web Service-Nutzer über die Vertragsmodalitäten vor Vertragsschluss zu informieren, lässt sich dabei nur über das UDDI lösen. Wünscht der Web Service-Anbieter daher eine Einbeziehung seiner Geschäftsbedingungen, so muss er diese bereits in seine Dienstebeschreibung, die im UDDI abgelegt ist, beispielsweise durch einen Link integrieren. 
Auch im Bereich der Haftung für Fehler im Web Service, Fehlfunktion der UDDI-Suche oder einem Missbrauch des UDDI kann unter anderem auf Parallelprobematiken, wie den Missbrauch von Kennzeichen und Unternehmensnamen durch Metatags oder die so genannte Weiß-auf-Weiß-Schrift, zurückgegriffen und Lösungsansätze übertragen werden. Neben den einschlägigen Spezialvorschriften verstößt derartiges Verhalten zudem gegen das Wettbewerbsrecht. Mitbewerber und Geschädigte haben daher umfangreiche rechtliche Möglichkeiten gegen missbräuchliches Verhalten im Zusammenhang mit dem UDDI vorzugehen.

Problematisch gestaltet sich indes der Missbrauch von Kennzeichen, Firmennamen oder der Übermittlung von rechtswidrigen Inhalten durch den Web Service-Anbieter für den Web Service-Nutzer. Dieser haftet gemäß \10 TMG analog nach den Grundsätzen der Störerhaftung im Falle des Vorliegens zumutbarer Prüfungs- und Kontrollpflichten hauptsächlich auf Unterlassung der Übermittlung kerngleicher rechtswidriger Inhalte durch die Web Services, obwohl er realiter keinen Einfluss auf die Inhalte des Web Service-Anbieters hat. Da ihm de facto eine solche Unterlassung aber nicht möglich ist, muss er, um einen Verstoß gegen den Unterlassungstitel zu vermeiden, den Web Service des Web Service-Anbieters aus seiner Web-Präsenz entfernen. ${ }^{1156}$

Diese Risiken sollten aber nicht dazu führen, in Gänze auf einen Einsatz Serviceorientierter Architekturen mit Web Services zu verzichten. Vielmehr könnte beispielsweise der Einsatz von Selbstregulierungsmechanismen helfen, Missbräuche in Grenzen zu halten. So könnte beispielsweise der Web ServiceVermittler den Web Service-Nutzer eine Bewertungsmöglichkeit des genutzten Dienstes im Sinne des eBay-Bewertungssystems anbieten, ${ }^{1157}$ wo der Web Service-Nutzer angeben kann, ob der jeweilige Dienst tatsächlich das gehalten hat, was er nach seiner Dienstebeschreibung versprochen hat.

Web Services bieten auch für den Bereich des Direktmarketing zahlreiche Möglichkeiten. Im Rahmen so genannter Web Service-Portale können riesige Datenpoole entstehen, die eine Vielfalt an Datensammlungs- und vor allem Verwendungsmöglichkeiten bieten. Das gilt vor allem für die Web ServiceAnbieter, die gegebenenfalls selber nur einen simplen Service anbieten, der an ein Web Service-Portal gekoppelt wird. Durch diese Verbindung ist ihnen mit Hilfe der Web Services unter Umständen technisch sowohl der Zugriff auf die

1156 B.V Haftung des Web Service-Nutzers.

1157 S. zur Funktionsweise bei eBay http://pages.ebay.de/services/forum/feedback.html $<25.11 .2009>$. 
Portalprofildaten als auch auf mögliche Nutzungsdaten der Portalnutzer möglich. Web Services können sowohl Cookies des Portalanbieters auslesen als auch Web-Bugs mitsenden, die wiederum eigene Informationen über den Portalnutzer in Erfahrung bringen können. Hier ist Vorsicht geboten. Die technischen Möglichkeiten, die Web Services aufgrund ihrer einfachen Implementierungsmöglichkeit bieten, dürfen nicht das geltende Recht ignorieren. Die dadatenschutzrechtlichen Grenzen zum Umgang mit personenbezogenen Daten sind stets zu beachten. Hinsichtlich der Suchdaten bei einer Web ServiceNutzung gilt die Besonderheit, dass diese aufgrund der Funktionsweise von Web Services ausnahmsweise nicht als Inhaltsdaten, die den weniger strengen Anforderungen des $₫ 28$ BDSG unterfallen, sondern vielmehr als Nutzungsdaten, die nach $\int 15$ TMG zu beurteilen sind, einzuordnen sind.

Es empfiehlt sich insgesamt notwendiges Vertrauen in den Einsatz Serviceorientierter Architekturen zu schaffen, um den Weg für eine breitflächige Nutzung innerhalb offener Systeme wie dem Internet zu ebnen. Ein solcher Vertrauensfaktor lässt sich neben Selbstregulierungsmechanismen bereits auf technischer Ebene durch den Einsatz von (qualifiziert) elektronischen Signaturen verwirklichen. Die speziell auf die Kommunikation mit Web Services zugeschnittene Spezifikation XML Signature stellt dabei eine entsprechende Umsetzungsmöglichkeit dar.

Die rechtlichen und technischen Rahmenbedingungen für die breite Nutzung Serviceorientierter Architekturen innerhalb offener Systeme sind abgesteckt. Dagegen ist die Fortentwicklung in allen diesen Bereichen noch offen. An Wissenschaft und Technik stellt sich deshalb die Herausforderung der Begleitung, Analyse und Systematisierung. Die vorliegende Arbeit hofft, dazu einen Beitrag geleistet zu haben. Werden die im Rahmen der Untersuchung aufgezeigten Grenzen beachtet, so steht dem Einsatz von Serviceorientierten Architekturen mit Web Services auch innerhalb offener Systeme nichts im Weg. 


\section{Teil Anhang}

\section{Muster-Datenschutzerklärung}

Unter Berücksichtigung der Ausführungen des 0 könnte sich die folgende Datenschutzerklärung im Rahmen der Web Service-Portale anbieten. Je nach Portalausgestaltung und Umfang der Datenverarbeitung sind allerdings weitere Informationen notwendig. Der folgende Vorschlag dient lediglich als Orientierung.

\section{Verantwortliche Stelle}

Verantwortliche Stelle im Sinne der Datenschutzgesetze ist die Portalbetreiberin [genaue Bezeichnung]. 
Hinweis: Handelt es sich um ein Unternehmen im Nichteuropäischen Ausland, so sollte hier erläutert werden, wie das erforderliche Datenschutzniveau sichergestellt wird (zum Beispiel Safe Harbor-Principles bei einem Unternehmen mit Sitz in den Vereinigten Staaten).

\section{Datenverarbeitung auf dem Web Service-Portal}

Persönliche Informationen verwenden wir für [genaue Auflistung der Verwendung].

Hinweis: Zum Beispiel, um individuelle Produkt- und Dienstleistungsempfehlungen aussprechen zu können etc.

\section{Folgende Informationen sammeln wir:}

\section{Automatische Informationen:}

Log-Files

Die Portalbetreiberin erhebt und speichert automatisch in ihren Server LogFile- Informationen, die Ihr Browser an die Portalbetreiberin übermittelt. Zu den Log-File-Informationen gehören Browsertyp und Browserversion, das verwendete Betriebssystem, die zuletzt besuchte Seite (Referrer URL), die IPAdresse (Hostname des zugreifenden Rechners) und die Uhrzeit der Serveranfrage.

\section{Cookies und Web-Bugs}

Die Internetseiten verwenden an mehreren Stellen so genannte Cookies. Sie dienen dazu, unser Angebot nutzerfreundlicher, effektiver und sicherer zu gestalten. Cookies sind kleine Textdateien, die auf Ihrem Rechner abgelegt werden und die Ihr Browser speichert. Die meisten der von uns verwendeten Cookies sind so genannte „Session-Cookies“. Sie werden nach Ende Ihres Besuchs automatisch gelöscht. Langfristige Cookies dienen dazu, [genauen Zweck der Speicherung angeben]. Diese Cookies werden [Zeitpunkt angeben] ge- 
löscht. Cookies richten auf Ihrem Rechner keinen Schaden an und enthalten keine Viren.

Bei Web-Bugs handelt es sich um kleine unsichtbare Bilddateien, die innerhalb von unseren Portalseiten oder in den durch unsere Partner-Web ServiceAnbieter [genaue Aufzäblung, Firmenbezeichnung etc.] übermittelten Dokumenten enthalten sind. Web-Bugs übermitteln die IP-Adresse, die Internet-Adresse der besuchten Website, die Internet-Adresse des Web-Bug, den Zeitpunkt, an dem das Dokument mit dem Web-Bug angesehen wurde und den verwendeten Browsertyp. Darüber hinaus übermitteln sie die in den Cookies gespeicherten Informationen. Web-Bugs werden verwendet für [genauen Zweck der Speicherung angeben]. Eine Löschung erfolgt [Zeitpunkt angeben].

\section{Sonstige Informationen}

Wir und unsere im Portal implementierten Web Service-Anbieter [genaue Aufzählung, Firmenbezeichnung etc.] erfassen und speichern alle Informationen, die Sie auf unserer Website, bei der Nutzung der Dienste unserer auf dem Portal integrierten Partnerunternehmen eingeben oder uns in anderer Weise übermitteln. Es steht Ihnen aber frei, uns bestimmte Informationen nicht zu geben. Dies kann allerdings dazu führen, dass Sie einige unserer Angebote (wie zusätzliche Dienste oder Features) nicht nutzen können. Diese gespeicherten Informationen verwenden wir für [genauen Zweck, der Speicherung angeben].

\section{Informationen aus Dritt-Quellen}

Gelegentlich nutzen wir auch Informationen über Sie, die wir aus anderen Quellen erhalten haben, und fügen diese unseren internen Informationen über Ihr Kundenprofil hinzu. Bei den Informationen, die wir von Dritten erhalten, handelt es sich um [genaue Aufzä̈blung].

\section{Datenübermittlung}

Wir arbeiten auf unserem Portal mit folgenden Web Service-Anbietern [genaue Aufzäblung, Firmenbezeichnung etc.] zusammen. Diese Web Service-Anbieter sind technisch in unser Portal implementiert. Um Ihnen die Nutzung dieser ange- 
botenen Dienste einfacherer zu gestalten, werden die zur Abrechnung der Dienstenutzung notwendigen persönlichen Informationen von uns an die entsprechenden Web Service-Anbieter übermittelt.

Hinweis: Sollen den Web Service-Anbietern noch weitere Datenverwendungsmöglichkeiten eingeräumt werden, müssen diese ebenfalls aufgeführt werden.

\section{Auskunftsrecht}

Sie haben jederzeit das Recht auf Auskunft über die zu Ihrer Person gespeicherten Daten, deren Herkunft und Empfänger sowie den Zweck der Speicherung. Auskunft über die gespeicherten Daten gibt Ihnen unser Datenschutzbeauftragter.

Hinweis: Kontakt zum Datenschutzbeauftragten entweder als E-Mail-Link ausführen oder E-Mail-Adresse direkt danach platzieren.

\section{Dauer der Datenvorhaltung}

Hinweis: Hier muss eine Erläuterung erfolgen, wie lange die Daten vorgehalten werden beziehungsweise wann ihre Löschung erfolgt.

\section{Weitere Informationen}

Da uns Ihr Vertrauen wichtig ist, möchten wir Ihnen jederzeit alle offenen Fragen bezüglich der Verarbeitung Ihrer personenbezogenen Daten erläutern. Bei Fragen oder weitergehenden Informationswünschen wenden Sie sich bitte an unseren Datenschutzbeauftragten. 
Hinweis: Kontakt zum Datenschutzbeauftragten entweder als E-Mail-Link ausführen oder E-Mail-Adresse direkt danach platzieren.

\section{Widerrufrecht}

Eine erteilte Einwilligung in die Datenverarbeitung können Sie jederzeit widerrufen.

Hinweis: E-Mail-Adresse, Kontakt für Widerruf angeben

\section{Einwilligungserklärung}

Ich willige in die Verarbeitung und Nutzung meiner personenbezogenen Daten gemäß der vorstehenden Datenschutzerklärung ein.

Hinweis: Kasten zum Setzen eines Häkchens platzieren und danach noch zusätzlicher Button zu Bestätigung der erklärten Einwilligung. 


\begin{abstract}
er Einsatz von Serviceorientierten Architekturen (SOA) mit Web Services ermöglicht Unternehmen, zahlreiche (externe) Dienste flexibel und kostengünstig ohne Systemanpassungen an ihr eigenes Angebot zu koppeln. Die noch junge Technik erlangt daher sowohl in der unternehmensinternen Organisation als auch im E-Commerce zunehmend an Bedeutung.

udith Nink befasst sich mit den rechtlichen Anforderungen, die an den Einsatz von SOAs mit den Softwarekomponenten Web Services zu stellen sind. Die Untersuchung ist in drei größere Komplexe gegliedert, die sich mit Problemen beim Einsatz von SOAs mit Web Services im vertraglichen Bereich, Fragen der außervertraglichen Haftung der Beteiligten sowie datenschutzrechtlichen Anforderungen befassen.
\end{abstract}

